\title{
Atlas propedéutico
}

El Curso Básico de la Hochschule für Gestaltung UIm

Autor

Angel Luis Fernández Campos

Arquitecto

Directora

Atxu Amann Alcocer

Doctora Arquitecta 

Tribunal nombrado por el Magnífico y Excelentísimo Sr. Rector de la Universidad Politécnica de Madrid, el dia de

de

\section{Presidente}

Vocal

Vocal

Vocal

Secretario

Realizado el acto de defensa y lectura de la tesis doctoral "Atlas Propedéutico. El Curso Básico de la Hochschule für Gestaltung Ulm el día de en

\section{Calificación}

Presidente

Secretario Vocales 



\section{Índice}

Ointroducción 12

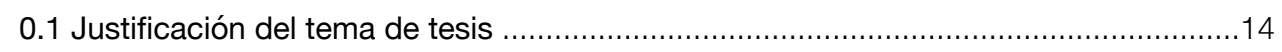

0.2 Objetivos. Hipótesis de trabajo.................................................................... 17

0.3 Estado de la cuestión. Antecedentes....................................................................19

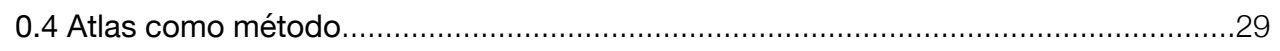

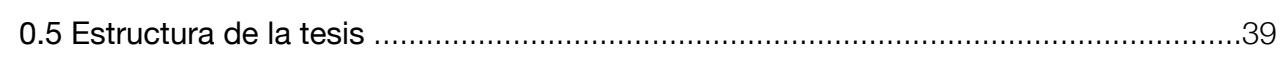

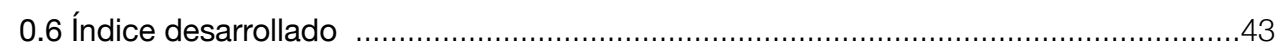

marco didáctico 49

1.1 Enseñanzas visuales en los cursos básicos precursores .......................................53

1.2 Estructura y docencia en la Hochschule für Gestaltung........................................67

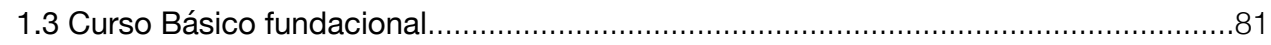

1.4 Relevo Bill-Maldonado en el Curso Básico .........................................................103

1.5 Reafirmación de la propuesta de Maldonado y la consolidación del

Visuelle Einführung en el Curso Básico.................................................................115

1.6 Reforma del curriculum del Curso Básico: hacia un nuevo Formgeber......................123

1.7 Metodología en el Curso Básico........................................................................129

1.8 La disolución del Curso Básico en el curriculum de los departamentos ....................141

Atlas Curso Básico .....................................................................................149

\section{2 atlas color $\quad 151$}

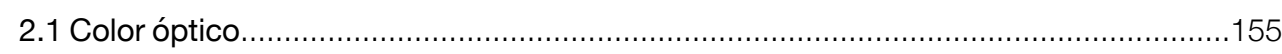

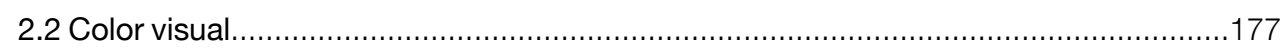

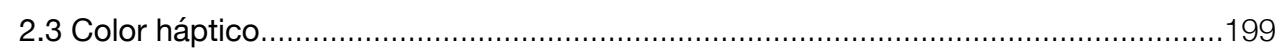

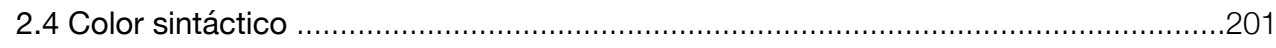

2.5 Contribución a la didáctica del color conclusiones parciales atlas color.....................219

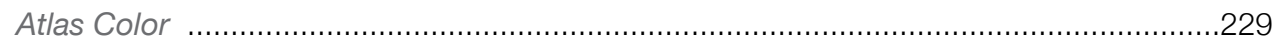

\section{3 atlas forma 231}

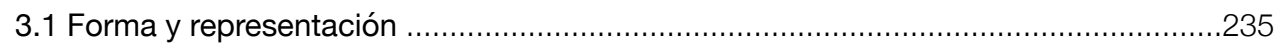

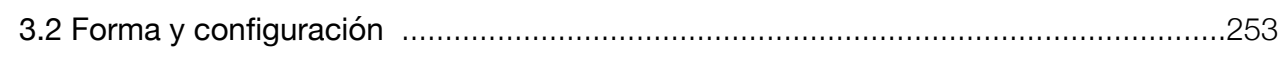

3.3 Contribución a la didáctica de la forma conclusiones parciales atlas forma.................345

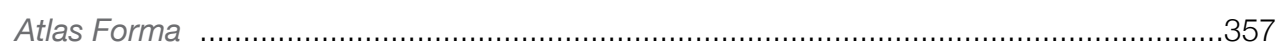


Nota sobre la numeración de imágenes, citas a pie de página y referencias bibliográficas:

Las imágenes aparecen numeradas con una referencia al capítulo al que pertenecen antes de los dígitos.

Las numeración de las citas a pie de página y referencias bibliográficas son correlativas e independientes en cada capítulo, y figuran en la página en la que se consignan. 


\section{4 atlas materia}

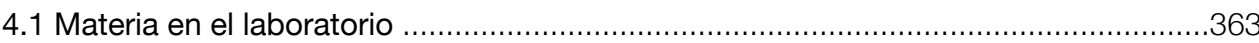

4.2 Materia en el taller $\quad$.

4.3 Contribución a la didáctica de la materia conclusiones parciales atlas materia..........445

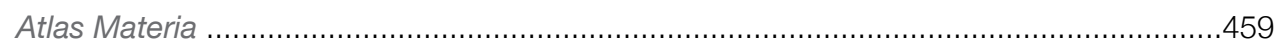

\section{5 atlas proyecto $\quad 461$}

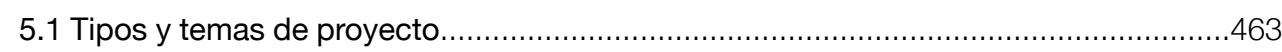

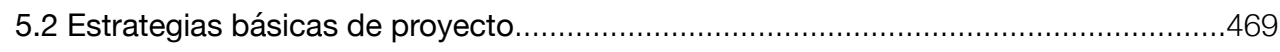

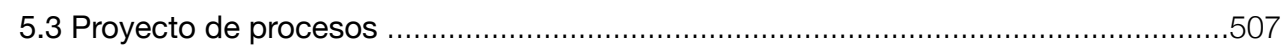

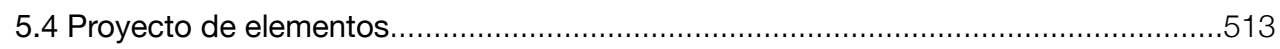

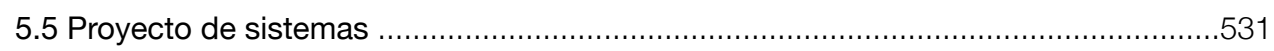

5.6 Contribución a la didáctica del proyecto conclusiones parciales atlas proyecto.........587

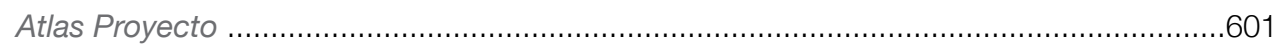

\section{6 conclusiones generales $\quad 603$}

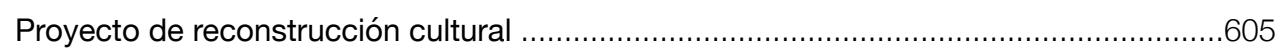

La superación del mito Bauhaus desde la didáctica .....................................606

Tomás Maldonado y Walter Zeischegg como catalizadores del Grundlehre..................607

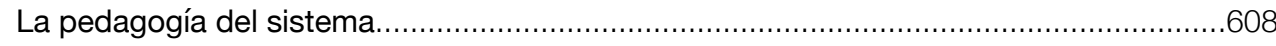

El Grundlehre como experimento de transformación didáctica................................609

\section{7 bibliografía 611}

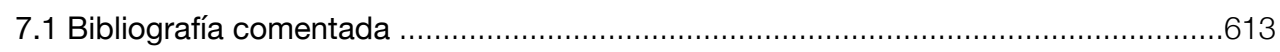

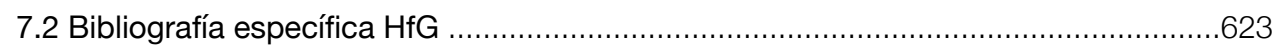

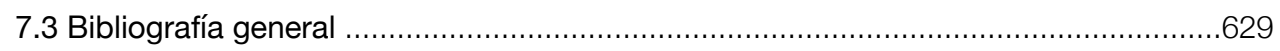





\section{Resumen}

El 3 de agosto de 1953 acuden los primeros estudiantes de la Hochschule für Gestaltung Ulm a participar de un seminario de Walter Peterhans organizado en la sede provisional de la Ulmer Volkshochschule. Comienza así el primer año de Curso Básico, organizado por Max Bill y bajo su rectorado. La fundación de la escuela significa el relevo y la recogida del testigo de la mítica Bauhaus, no para revivirla y continuarla, sino para hacer evolucionar su legado. La HfG Ulm siempre se caracterizó por crecer en un estado de ósmosis constante entre un cuerpo docente formado por artistas, científicos, técnicos, arquitectos y teóricos, y un conjunto de estudiantes que acudieron desde todas partes del mundo. Los ámbitos de desarrollo de la escuela -Arquitectura, Diseño Industrial, Comunicación Visual e Información-, buscaron situarse en la vanguardia cultural y tecnológica de su tiempo.

Desde su gestación hasta su cierre, el devenir de la escuela estuvo sujeto a una sucesión de relevos generacionales e intelectuales que tuvieron como consecuencia la redefinición continua de los objetivos y con ellos, cambios en los métodos de enseñanza. Sin embargo, y a pesar de que existieron diferencias y discrepancias entre los responsables de la escuela, todas las posturas que se asumieron con respecto a los objetivos de la institución estuvieron basados en el carácter y la vocación social con la que se había gestado el proyecto: la contribución a la reconstrucción moral y cultural de Alemania tras la Segunda Guerra Mundial.

El Curso Básico o Grundlehre fue uno de los ejes metodológicos de la escuela, el que albergó las propuestas docentes más experimentales y en el que más transformaciones se llevaron a cabo. A diferencia del Vorkurs de la Bauhaus, en la estructura del Curso Básico de la HfG se incorporan formalmente disciplinas de carácter científico y sociológico, además de las de tipo proyectual. Su superación era obligatoria para todos los estudiantes que aspiraban a ingresar en los departamentos de especialización en la HfG. En el curso 19611962 el Curso Básico desaparece como departamento independiente tras un interesante debate y sus enseñanzas se integran como primer año de estudios preparatorios en cada una de las especializaciones.

La tesis se propone como objetivo contribuir al conocimiento y a la difusión del conjunto de disciplinas visuales que se ejercitaron en el Curso Básico, durante la etapa en la que funcionó como un curso común a todos los estudiantes y un departamento independiente, entre los años 1953 y 1961. La investigación busca determinar y contextualizar aquellos principios didácticos de las enseñanzas visuales que contribuyeron a definir una propedéutica del aprender a proyectar para unos fines concretos. Atendiendo al carácter iniciador del primer curso, y teniendo en cuenta que, en su devenir, el Curso Básico experimentó varios cambios, la tesis pretende vislumbrar qué invariantes didácticos definen el carácter preparatorio en cada disciplina a lo largo de este periodo.

El trabajo parte del estudio de las fuentes originales del Curso Básico, que se encuentran en el archivo de la Hochschule für Gestaltung, ubicado en la sede de la escuela, proyectada por Max Bill. El archivo contiene los trabajos gráficos y maquetas, enunciados, documentos, cuadernos de apuntes, biografía de profesores, fotografías y documentación administrativa. La tesis se sostiene sobre la base documental formada en una fase de investigación en la sede del archivo entre los años 2014 y 2018. 
La tesis se concreta en la forma de un atlas como método visual de conocimiento y como montaje dinámico de heterogeneidades, de carácter compilatorio y narrativo que estructura, muestra y difunde un vasto material hasta ahora inédito.

La estructura se organiza en dos bloques, el marco didáctico, que explica de forma cronológica el origen, evolución y final del Curso Básico, y el Atlas Propedéutico, ordenado en base a las analogías reconocidas en la primera fase de exploración del Curso Básico, que ha dado como resultado cuatro ejes de trabajo: el Atlas Color, Atlas Forma, Atlas Materia y Atlas Proyecto.

Cada uno de los capítulos del Atlas profundiza en la didáctica de los temas que vertebran el Curso Básico. Los ejercicios se ordenan en virtud de los objetivos que persiguen o las herramientas que utilizan. Se evalúa la contribución a la didáctica de la escuela, por su repercusión una vez finalizado el Grundlehre, y su incorporación a la enseñanza de cursos superiores en los Departamentos. Forman parte de esta revisión crítica las experiencias previas vinculadas con el Grundlehre, tanto de la Bauhaus, New Bauhaus y Vkhutemas. La experiencia de estas escuelas señalan el punto de partida y un instrumento más de medida para determinar la evolución de la propuesta didáctica de la HfG.

Se puede considerar el Grundlehere de la HfG como un referente pedagógico para la enseñanza del proyectar por la finalidad exploratoria de los trabajos, la transversalidad de las enseñanzas mediante la integración de disciplinas científicas, y el rol de profesor como guía del aprendizaje autónomo del alumno. Esto hace del Grundlehre la experiencia más singular y completa de las escuelas de diseño y arquitectura de su entorno y contexto temporal, que no ha sido estudiada en profundidad y en todas sus fases hasta esta investigación. 


\section{Abstract}

On August 3, 1953, the first students of the Hochschule für Gestaltung Ulm attended a seminar by Walter Peterhans at the temporary headquarters of the Ulmer Volkshochschule. Thus begins the first year of the Basic Course, organised by Max Bill and under his rectorship. The foundation of the school means the relay and the hand over the baton of the mythical Bauhaus, not to revive it and continue it but to make its legacy evolve. The HfG Ulm has always been characterized by a constant state of osmosis between a faculty of artists, scientists, technicians, architects, and theorists and a group of students from all over the world. The school's areas of development - Architecture, Industrial Design, Visual Communication and Information - sought to position itself at the cultural and technological forefront of its time.

From its gestation until its closure, the faculty's development experienced a succession of generational and intellectual relays that resulted in the continuous redefinition of the objectives and with them, changes in teaching methods. However, despite the differences and discrepancies between the faculty leaders, all the positions taken with regard to the objectives of the institution were based on the character and social vocation with which the project was conceived: the contribution to the moral and cultural reconstruction of Germany after the Second World War.

The Basic or Grundlehre Course was one of the faculty's methodological core lines, the one that hosted the most experimental teaching proposals and in which the most transformations were carried out. Unlike the Bauhaus' Vorkurs, the structure of the HfG Basic Course formally incorporates disciplines of a scientific and sociological nature, in addition to those of a design nature. It was compulsory to pass for all students wishing to access the specialization departments of the HfG. In the 1961-1962 academic year, the Basic Course disappeared as an independent department after an interesting debate and its teachings were integrated as the first year of preparatory studies in each of the specializations.

The thesis aims to contribute to the knowledge and dissemination of visual disciplines that were exercised in the Basic Course, during the stage in which it functioned as a common course to all students and as an independent department, between 1953 and 1961. The research seeks to determine and contextualise those didactic principles of the visual teachings that contributed to define a propaedeutic of learning to project for specific purposes. Considering the initiating character of the first course, and taking into account that, in its evolution, the Basic Course underwent several changes, the thesis aims to glimpse which didactic invariants define the preparatory character in each discipline throughout this period.

This work is based on the study of the original sources of the Basic Course, which can be found in the archive of the Hochschule für Gestaltung, located in the faculty's headquarters, designed by Max Bill. The archive contains the graphic works and models, statements, documents, notebooks, biographies of teachers, photographs and administrative documentation.

The thesis is supported on the basis of documentary evidence from a research phase at the archive's headquarters between 2014 and 2018. 
The thesis takes the form of an atlas as a visual method of knowledge and as a dynamic montage of heterogeneities, of a compilation and narrative nature that structures, shows and disseminates a vast and hitherto unpublished material.

The structure is organised in two blocks, the didactic framework, which explains in chronological order the origin, evolution and the end of the Basic Course, and the Propaedeutic Atlas, ordered on the basis of the analogies recognised in the first phase of exploration of the Basic Course, which has resulted in four strands of work: The Atlas Color, Atlas Form, Atlas Matter and Atlas Project.

Each one of the chapters of the Atlas delves into the didactics of the topics that form the backbone of the Basic Course. The exercises are arranged according to the objectives they pursue or the tools they use. The contribution to the school's didactics is evaluated, due to its repercussion once the Grundlehre is finished, and its incorporation into the teaching of higher courses in the Departments. Included in this critical review are the previous experiences linked to the Grundlehre, of both Bauhaus, New Bauhaus and Vkhutemas. The experience of these schools provides a starting point and another measuring method to determine the evolution of the HfG's teaching approach.

The Grundlehere of the HfG can be considered as a pedagogical reference for the teaching of project planning due to the exploratory purpose of their works, the transversality of the teachings through the integration of scientific disciplines, and the role of the teacher as a guide/advisor for the autonomous learning of the student. This makes Grundlehre the most unique and complete experience of the schools of design and architecture of their environment and temporal context, which has not been studied in depth and in all its phases until this research. 


\section{Ontroducción}

0.1 Justificación del tema de tesis

0.2 Objetivos. Hipótesis de trabajo

0.3 Estado de la cuestión. Antecedentes

0. 3. 1 Documentos del periodo HfG 1948-1968

0. 3. 2 Documentos tras el cierre de la HfG

\subsection{Atlas como método}

0.4.1 Identificación de lagunas

0.4.2 Recopilación del Atlas

0.4.3 Atlas Panóptico

0.4.4 Atlas Sinóptico

\subsection{Estructura de la tesis}

0.5.1 Marco didáctico

0.5.2 Atlas propedéutico

0.6 Índice ampliado 



\subsection{Justificación del tema de tesis}

\section{La enseñanza sin investigación está condenada a la repetición ${ }^{1}$}

Ser docente implica convivir de forma habitual con la pregunta de cómo se debe enseñar. Es imprescindible la revisión continua de los objetivos y los métodos de enseñanzaaprendizaje mediante la investigación. Esta tesis pretende profundizar en referentes metodológicos fundamentales en el ámbito de la enseñanza del proyecto y con ello contribuir a la actualización de la docencia.

El aprendizaje consiste un proceso de profundización sucesiva en un ámbito de conocimiento. Este camino parte en su primera etapa de un conjunto de nociones básicas de introducción a cualquier disciplina. Esta primera base de conocimiento recibe el nombre de propedéutica ${ }^{2}$, o enseñanza preparatoria. En el caso de las disciplinas del proyecto, la propedéutica se enmarcaba en los cursos básicos o cursos preparatorios, que tenían el objetivo de propiciar el desarrollo de las habilidades suficientes mediante la realización de ejercicios de naturaleza gráfica y visual y así preparar a los alumnos para un posterior internamiento en la acción de proyectar.

En el terreno del proyecto, se puede considerar que el referente que más influencia ha ejercido ha sido la Bauhaus, si bien su influjo ha sido convenientemente sometido a crítica y revisión. Esta influencia puede deberse a que la Bauhaus es la primera escuela que institucionaliza y define la enseñanza propedéutica del proyectar y difunde este legado en nuestra cultura occidental. Desde esta primera formalización son muchas las escuelas que, basándose o no en esta referencia originaria, han organizado sus cursos básicos.

En la Unión Soviética post-revolucionaria los Vkhutemas adoptaron una fórmula análoga a la del Curso Básico de la Bauhaus. Otras experiencias posteriores retoman, reinterpretan, adaptan la propedéutica definida en la Bauhaus, en parte por ser iniciadas por profesores antiguos miembros del Bauhaus: Moholy-Nagy en la New Bauhaus de Chicago, Peterhans en el ITT y Josef Albers en el Black Mountain College.

Max Bill ${ }^{3}$, primer director de la Hochschule für Gestaltung de Ulm -en adelante, HfG Ulm-, definió un Curso Básico en parte heredado de su antigua escuela, e impartidos por varios de los profesores del Bauhaus Josef Albers, Walter Peterhans y también antigua alumna Heléne Nonné-Schmidt. La marcada misión social de esta escuela desencadena sucesivas y profundas transformaciones que definen y adaptan a estos fines las metodologías y los currículos de los distintos departamentos. Resulta especialmente significativa la evolución de la propedéutica desde una estructura heredada de la Bauhaus a un modelo singular y único de la HfG.

El Curso Básico o Grundlehre fue uno de los ejes metodológicos de la escuela. A diferencia del Vorkurs de la Bauhaus, en la estructura y el currículum del Curso Básico de la HfG se incorporaron formalmente disciplinas de carácter científico y sociológico, además de las de tipo proyectual. Implantado en el primer curso de funcionamiento de la escuela (1953-1954), era de estudio obligatorio para todos los alumnos, tras el que un estudiante podía acceder
1 Schnaidt, Claude. "La escuela conectada a la vida no es una utopía". Temes de Disseny $\mathrm{n}^{\circ}$ 6, 1981, pp. $261-276$.

Claude Schnaidt fue alumno y posteriormente profesor de la HfG UIm.

2 Real Academia de la Lengua Española (2014).

3 Max Bill fue alumno de la Bauhaus mientras esta escuela estuvo bajo la dirección de Hannes Meyer en Dessau. 
a otros tres de especialización en la HfG. Constituía uno más de los departamentos con los que contaba la Escuela a nivel organizativo. En el curso 1961-1962 desaparece como departamento y sus enseñanzas se integran como primer año de estudios preparatorios en cada una de las especializaciones, para llevar a cabo una enseñanza más específica desde inicio de los estudios.

Se puede considerar el Curso Básico un referente pedagógico para la enseñanza del proyectar por la finalidad exploratoria de los trabajos, la transversalidad de las enseñanzas mediante la integración de disciplinas científicas, y el rol de profesor como guía del aprendizaje autónomo del alumno. Esto hace del Grundlehre la experiencia más singular y completa de las escuelas de diseño y arquitectura de su entorno y contexto temporal.

La tesis establece por tanto, como tema central de investigación, las disciplinas visuales y su evolución en el Curso Básico de la HfG y las enseñanzas preparatorias de la Bauhaus, Vkhutemas y New Bauhaus como precedentes y contexto periférico. 


\subsection{Objetivos. Hipótesis de trabajo}

Tomás Maldonado, uno de los profesores que asumió el rectorado de la Hochschule für Gestalgung de Ulm, considera que con frecuencia, las teorías educativas han sido instrumentalizadas por las clases dominantes en aras de un control social y manifiesta que resulta esencial aclarar una filosofía de la educación que preceda a la educación misma. ${ }^{4}$ La educación tiene que tener claros sus fines.

De acuerdo con la idea de Maldonado, ¿Qué fines se ha marcado como objetivo la HfG?. La escuela nace en la ciudad alemana de Ulm en un contexto de profundo cambio social y como evolución de la Ulm Volkshochschule -universidad popular- creada por Inge Scholl y Otl Aicher ${ }^{5}$ tras el fin de la Segunda Guerra Mundial. En este momento, resulta urgente volver a definir la identidad cultural y la moral del pueblo alemán, también devastada como consecuencia del régimen fascista y posterior guerra. Otl Aicher se aparta de sus estudios superiores de arte en Munich consciente de que no se ajustan al contexto social del momento:

"Habíamos regresado de la guerra, y en la academia nos encontrábamos con que debíamos trabajar en la estética por la estética. Aquello no podía seguir. Quienquiera que tenía ojos para ver y oídos para oír hubo de reconocer que el arte era una huida de las múltiples tareas que aguardaban también a la cultura tras el derrumbe del régimen nazi."6

En este contexto de profundo debate y reflexión acerca del futuro de la nación, surge la HfG y con ella, su primer curso, denominado Grundlehre o Grundkurs.

El primer objetivo que se propone esta tesis, consiste en contribuir a un conocimiento amplio y riguroso de las enseñanzas desarrolladas en las disciplinas visuales del Curso Básico, durante la etapa en la que funcionó como un curso común a todos los estudiantes como un departamento independiente. Esta aportación se concreta en la forma de un Atlas como método visual de conocimiento y como montaje dinámico de heterogeneidades, de carácter compilatorio y narrativo que estructura, muestra y difunde un vasto material hasta ahora inédito.

El segundo objetivo consiste en investigar, determinar y contextualizar aquellos principios didácticos de las enseñanzas visuales que contribuyeron a definir una propedéutica del aprender a proyectar para unos fines concretos. Atendiendo al carácter iniciador del primer curso, y teniendo en cuenta que, en su devenir, el Curso Básico experimentó varios cambios, la tesis busca vislumbrar qué invariantes didácticos definen el carácter preparatorio en cada disciplina a lo largo de este periodo.

Se trabaja con la hipótesis de que subyacen una serie de enseñanzas constantes en el conglomerado de ejercicios que integraron la instrucción de los alumnos en las disciplinas visuales. Estas enseñanzas fueron constantes a lo largo de todo el desarrollo del Grundlehre y es su metodología la que cambia y se adapta a la idea del tipo de profesional que la escuela define en cada etapa. Se parte de la idea de la existencia de una primera fase del Curso Básico integrado por un aprendizaje intuitivo y heredado de metodologías importadas de otras escuelas. Esta base importada experimenta en Ulm una gran transformación didáctica basada en el hallazgo de nuevos métodos y la incorporación de nuevas disciplinas, que pretenden servir de forma más consecuente a los fines de la escuela y a la formación de los responsables de construir el entorno material de la nueva Alemania.
4 Maldonado, T. "Der Krise der Pädagogik un die Philosophieder Erziehung". Melkur, n 13, septiembre 1959). Citado en: Maldonado, Tomás. Vanguardia y Racionalidad. Artículos, ensayos y otros escritos: 1946-1974. Barcelona: Gustavo Gill. pag. 89.

5 La escuela de UIm nace vinculada al movimiento de la resistencia anti nazi. Sophie y Hans Scholl, dos estudiantes de la universidad de Munich, fueron dirigentes y activistas del grupo de resistencia no violenta la Rosa Blanca. Este grupo de convicciones cristinas, se oponía al régimen de Hitler no sólo por motivos políticos sino fundamentalmente por cuestiones éticas y morales. Acusados de traición, los hermanos Scholl fueron ejecutados en 1943 y se convirtieron posteriormente en símbolos de la lucha antifascista. Su hermana, Inge Scholl junto con su marido Otl Aicher fueron dos de los fundadores de la escuela de Ulm y principales ideólogos de la escuela en sus inicios.

6 Aicher, Otl. "La Bauhaus y Ulm", en : Lindinger, Herbert. Die moral der Gegenstände, Berlin: Ernst \& Sohn, 1987. 

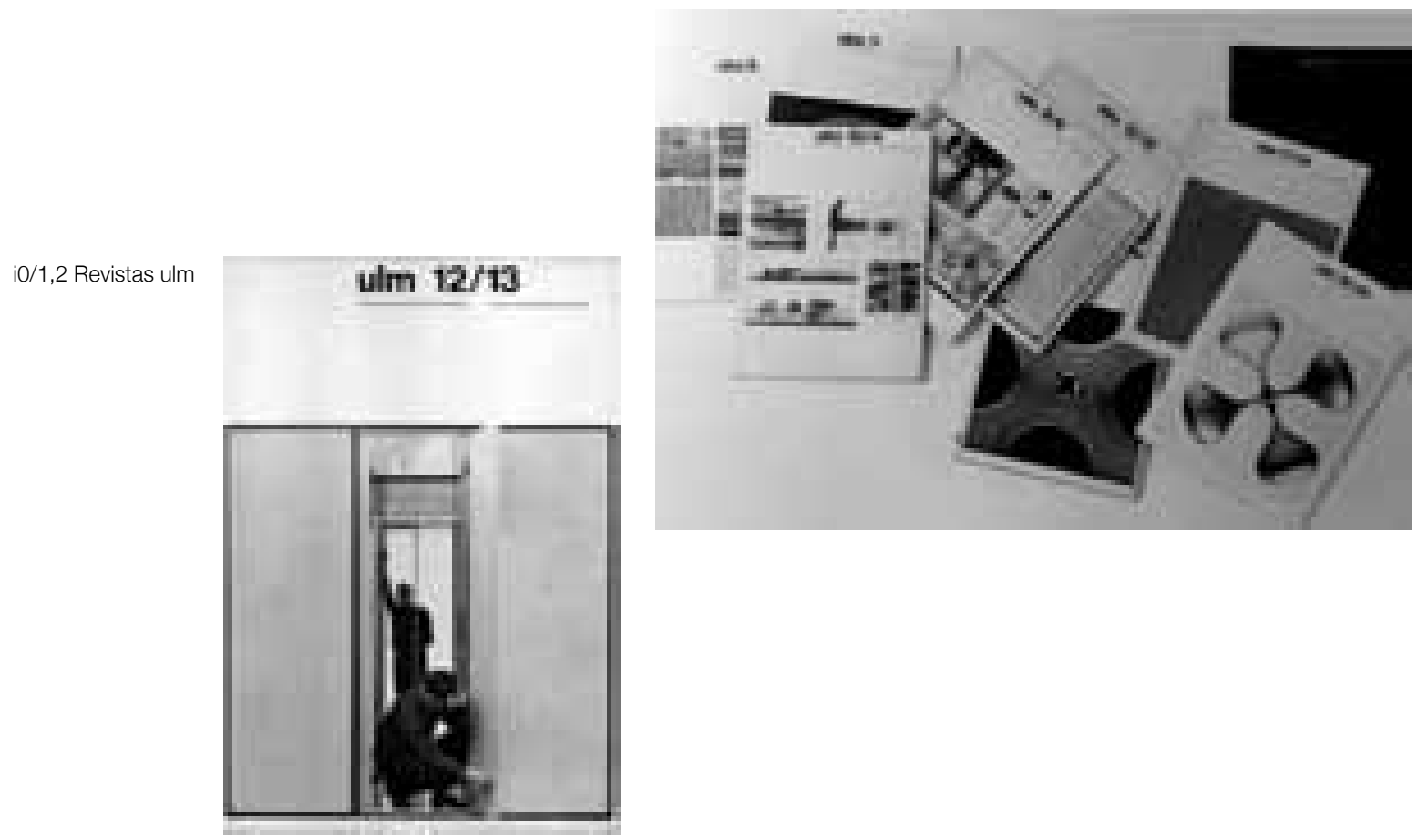

i0/3,4 Revistas output
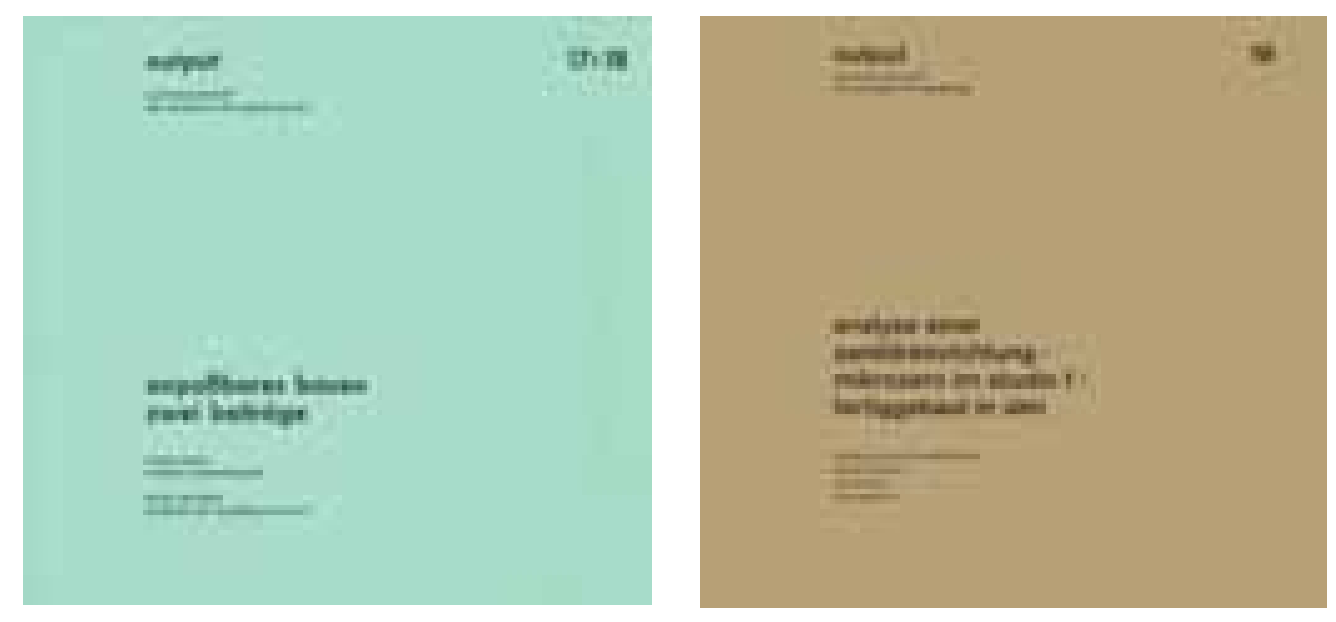

i0/5 Exposición Curso Básico

con trabajos de los docentes: Aicher, Maldonado, Itten, Gugelot, Bill, Albers, Nonné-Schmidt. [HfG archiv]

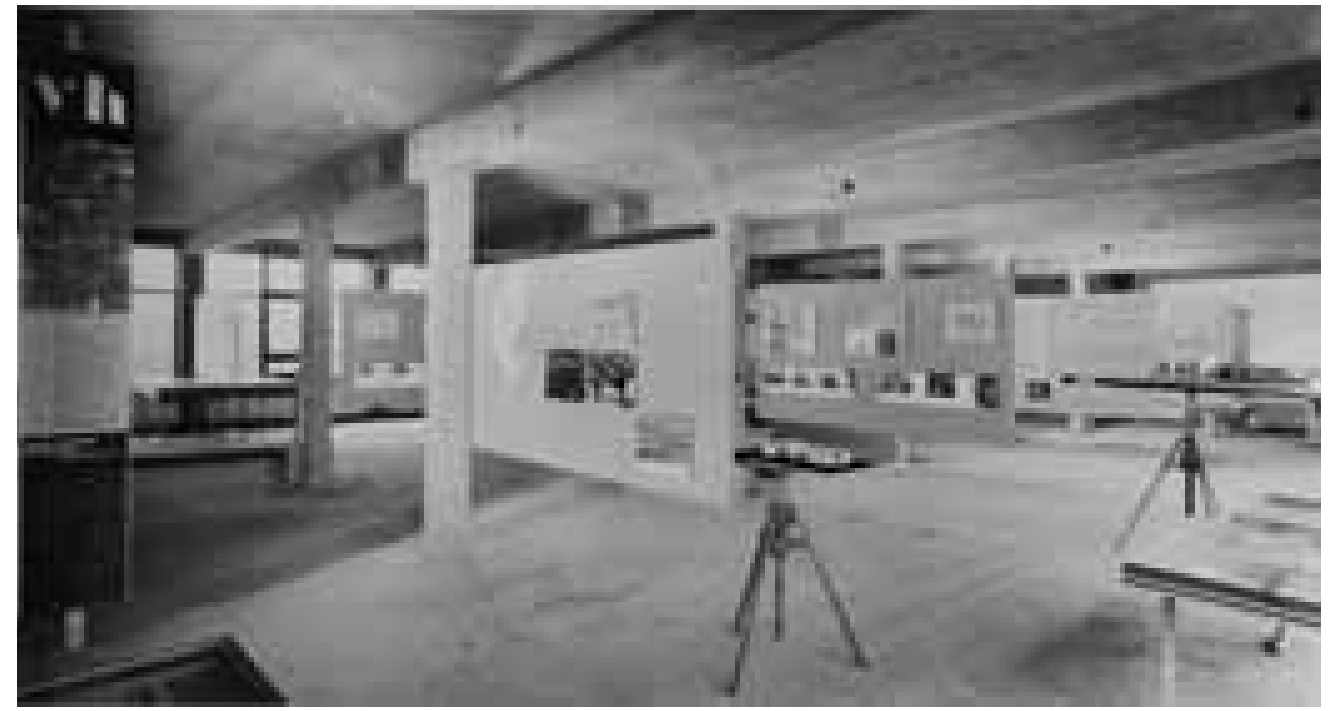




\subsection{Estado de la cuestión. Antecedentes}

El Curso Básico de la escuela de Ulm no ha sido objeto de un estudio de carácter monográfico, profundo y sistemático. Existen diversas publicaciones en las que se aborda de manera general o en las que aparece de forma tangencial en el contexto más amplio del conjunto de la escuela. Se aporta una bibliografía comentada con las publicaciones y trabajos más relevantes y completos sobre esta cuestión. Las fuentes originales que representan el contenido didáctico del Curso Básico y su evolución son, en gran parte, inéditas.

Las fuentes originales del Curso Básico se encuentran en el archivo de la Hochschule für Gestaltung, ubicado en la sede de la escuela, proyectada por Max Bill. El archivo contiene los trabajos gráficos y maquetas, enunciados, documentos, cuadernos de apuntes, biografía de profesores, fotografías y documentación administrativa. La colección se compone del material que permaneció en la sede tras el cierre de la escuela y las progresivas incorporaciones a la colección promovidas por el club off-ulm. ${ }^{7}$ Se distingue el origen del documento dependiendo si proviene de donaciones de antiguos alumnos -Schenkung-, depósitos en préstamo -Depositum- o los legados, como los de los profesores Aicher y Zeischegg y el antiguo alumno y profesor Hans Nick Roericht.

La difusión de la producción de la escuela ha experimentado dos fases, una durante su existencia, y la segunda tras su cierre. Mientras que la HfG existió como escuela, la difusión fue un aparato propagandístico para su presentación como una institución educativa y productiva de vanguardia y como un instrumento de comunicación de la ideología que guiaba sus pasos. Tras su cierre, las publicaciones y exposiciones más significativas se han centrado bien en su historia política o bien en su legado como conjunto, haciendo especial énfasis en los departamentos de especialización, con un carácter marcadamente compilatorio. El Curso Básico no se ha tratado como tema protagonista ni monográfico anteriormente.

A continuación se desgranan las publicaciones, las exposiciones y la documentación difundida sobre el Curso Básico en los dos periodos mencionados.

\subsubsection{Documentos del periodo HfG 1948-1968}

La HfG comienza su andadura en el curso 1953-1954, sin embargo, la escuela nace como idea varios años antes. Desde el final de la Segunda Guerra Mundial y hasta que se materializa el proyecto, el matrimonio Scholl-Aicher elabora y difunde varios folletos y documentos destinados a la búsqueda de financiación, en los que se que registran los fines didácticos de la futura escuela y su estructura incipiente.

Durante el funcionamiento de la HfG se editan folletos promocionales en los que aparecen descripciones de los contenidos del Curso Básico junto con el resto de los departamentos. Algunos de los folletos aparecen ilustrados con trabajos de los alumnos del Curso Básico.

Dos son las publicaciones de referencia de esta época: la revista ulm editada por la escuela como institución, y output, elaborada por los alumnos.
7 El club off Ulm es una asociación fundada en 1985 por antiguos alumnos y profesores que pronto alcanza los 150 miembros, repartidos por todo el mundo. Uno de sus objetivos es la obtención de los fondos documentales y su recopilación a través de legados y préstamo en depósito. 

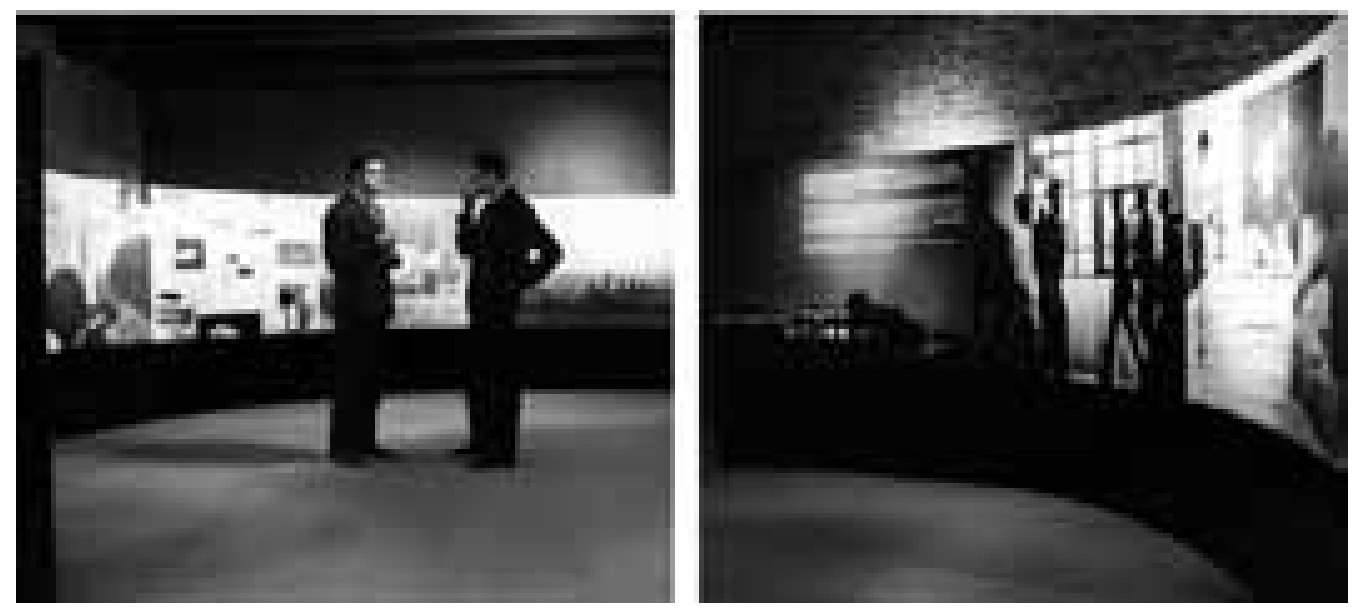

i0/6 La HfG en la Trienal de Milán, 1960

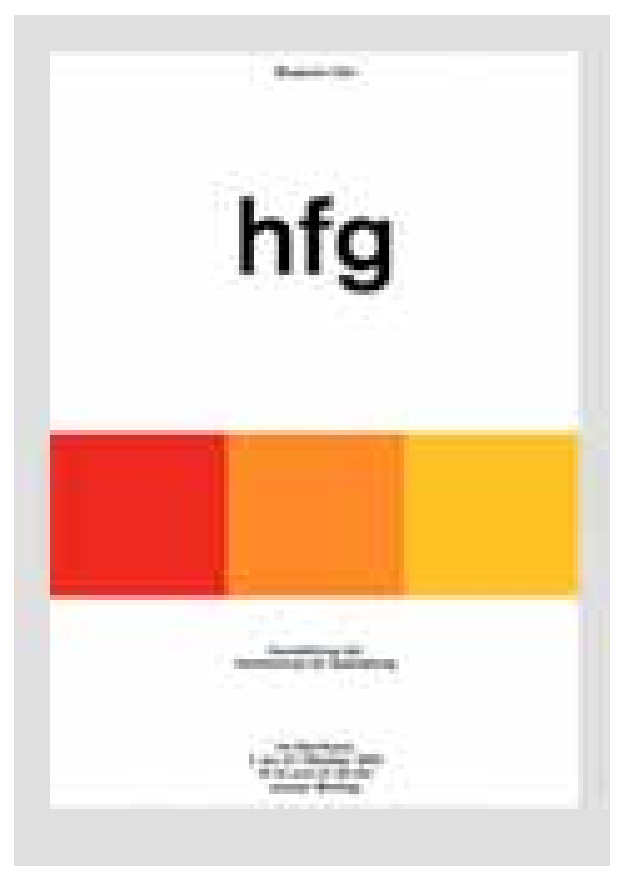

i0/7 Exposición 1958

i0/8 Cartel de la Exposición itinerante de 1963 diseñado por el alumno Herbert Lindinger
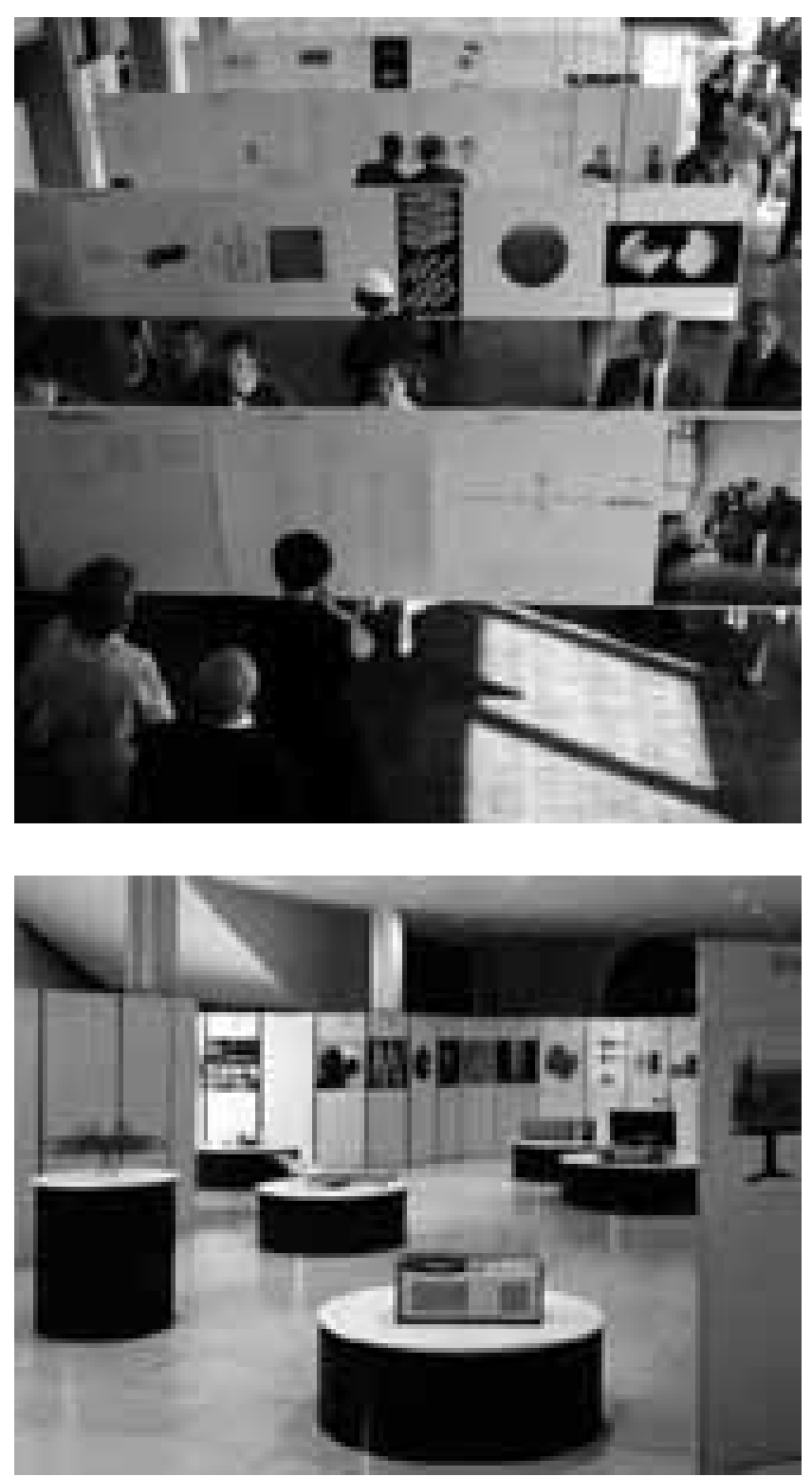
La revista ulm se publicó en dos épocas, entre 1958 y 1968, como medio de difusión de las actividades de escuela y sus colaboradores. En la revista se publican distintas informaciones relativas a las enseñanzas, entre las que apenas aparecen cuestiones relativas al Curso Básico. El $n^{\circ} 1$ contiene los objetivos y la organización de la docencia. El $n^{\circ} 4$, publicado en abril de 1959, muestra algunos trabajos de alumnos del Grundlhere. El número doble 12-13 contiene un extenso artículo de William S. Huff ${ }^{8}$ sobre el Curso Básico. En algunos de los números sucesivos se publican trabajos de los alumnos del primer curso de cada departamento, una vez que el Grundlehre se integró en éstos.

Los alumnos de la escuela desarrollaron la publicación output en veintiséis números, desde 1961 hasta 1964. En los números $6+7$ de noviembre y diciembre de 1961 se dedica a la enseñanza básica en el momento en el que se está planteando la desaparición del Curso Básico común a los departamentos. ${ }^{9}$

Las exposiciones más relevantes que se organizan durante el funcionamiento de la escuela tienen como objetivo presentar en público la producción de la institución. La primera de ellas tiene lugar en 1955, en el edificio aún en construcción de Max Bill, y está dedicada exclusivamente al trabajo del Grundlehre. La segunda exposición es la más difundida en fotografías y libros sobre la HfG, tuvo lugar en el junio de 1958 en el comedor de la sede. Tiene como objetivo mostrar el método de enseñanza y la producción de la escuela. Se inauguró con una conferencia de Karl Korn, director del Frankfurter Allgemeine Zeitung, titulada "Reflejos de la situación cultural". ${ }^{10} \mathrm{Ni}$ la exposición de 1955 ni la de 1958 dan lugar a publicaciones. En 1960 la escuela es invitada a formar parte de la trienal de Milán y allí se exhiben unos paneles en forma de panorama. Con motivo de la conmemoración del décimo aniversario de la HfG se organiza una nueva y última gran exposición itinerante que recopila los logros de los 10 primeros años y se edita un catálogo. ${ }^{11}$

\subsubsection{Documentos tras el cierre de la HfG}

Tras el cierre de la escuela a finales del 1968, los archivos documentales y el material de las clases permanecieron depositados en el edificio al cargo de la Fundación Hermanos Scholl, institución de la que dependía la HfG. De forma análoga a la Bauhaus, la HfG comenzaba su diáspora por todo el mundo.

El primer artículo que mira a una HfG ya en el pasado lo publica Kenneth Frampton en mayo de 1974 en la revista OPPOSITIONS 3, y se titula "A propos Ulm"12. El artículo contiene una relectura crítica de la revista $u l m$.

En 1975, el número 15 de la revista Architese $^{13}$ dedica una restrospectiva a la HfG. En ella Otl Aicher describe una cronología en nueve fases de la historia de la escuela, en la que aborda las transformaciones ideológicas que influyeron en el desarrollo de las enseñanzas. Además es la primera vez que se publican documentos sobre el plan de estudios de la escuela: el currículo del Grundlehre para el primer curso de la Escuela, 1953-1954 y una descripción de las materias y profesores para los cursos 1958-1959 y 1966-1967.
$8 \mathrm{~S}$. Huff, William. "Argumente für eine Grundkurs". Ulm: Journal of the Ulm School of Design, marzo(12/13), 1965, pp. 25-39.

9 Czemper, K. A. "Die pädagogischen Ziele der Grundlehre"

Output, (6+7), 1-10 (1961, p. 1-10)

10 "Chronik" en: Ulm n², Vierteljahresbericht der Hochschule für Gestaltung UIm. Hg. v. Hochschule für Gestaltung. (Revista editada por el rectorado de la $\mathrm{HfG}$ ), pp. 41

11 Ulm. Hochschule für Gestaltung 1963. Katalog einer Wanderausstellung. UIm, 1963

12 Frampton, Kenneth. "A propos UIm”. OPPOSITIONS, (3). Cambridge: MIT press, 1974.

13 Schnaidt, Claude; Aicher, Otl; Ohl, Herbert, \& Frampton, Kenneth. hfg ulm ein rückblick (número monográfico architese 15), Niederteufen: Verlag Arthur Niggli, 1975. 

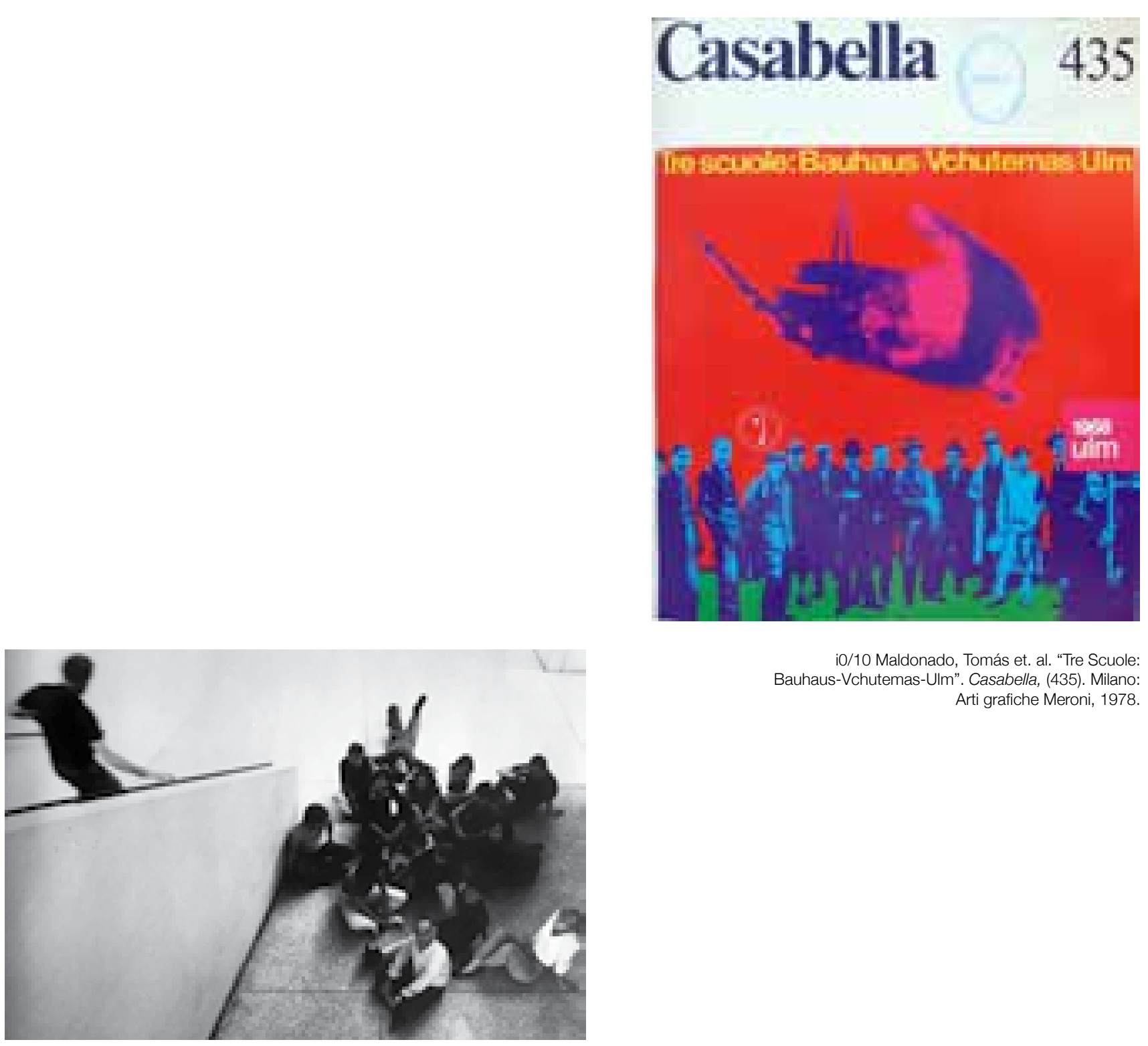

i0/10 Maldonado, Tomás et. al. "Tre Scuole: Bauhaus-Vchutemas-UIm". Casabella, (435). Milano: Arti grafiche Meroni, 1978.

i0/11 Fragmento de uno de los paneles del HfG Synopse

i0/12 Fragmento de uno de los paneles del HfG Synopse
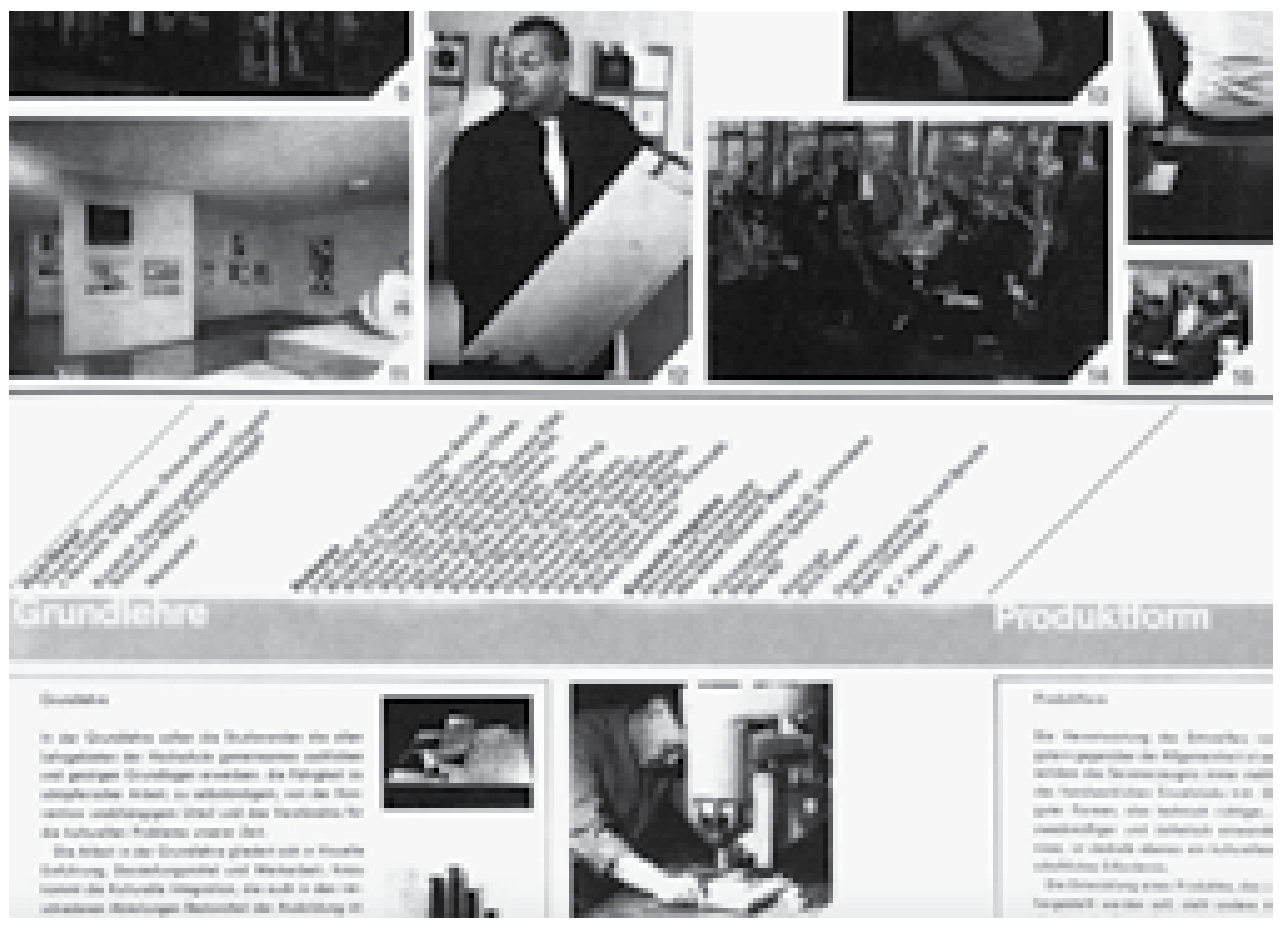
El número 435 de la revista Casabella ${ }^{14}$, publicado en 1978, dedica un monográfico a las tres escuelas: Bauhaus-Vchutemas-Ulm y repasa los aspectos sociopolíticos y pedagógicos comunes que marcaron su historia. Resulta de interés la información relativa al Curso Básico de los Vkhutemas. El artículo "Il contributo di la escuela de Ulm", publicado en el número de 19 de la revista Raseggna ${ }^{14}$ (septiembre de 1984), se puede considerar como un artículo complementario al difundido por Casabella. Su contenido es monográfico sobre la HfG y aporta un repaso sobre el alcance y la influencia de la Escuela a través de la trayectoria de algunos de sus protagonistas.

En la década de los 80 empieza a gestarse la recuperación de la memoria de la Escuela de Ulm. En 1982 el antiguo alumno y profesor Hans Nick Roericht obtiene fondos públicos para formar un grupo de trabajo y elaborar la primera gran recopilación de la historia y desarrollo de la HfG: la HfG Synopse. La exposición se prepara en nueve meses y se convierte en la crónica más completa que desgrana las cuestiones docentes, políticas y la producción del instituto de diseño a lo largo de la vida de la escuela. Los paneles se editan todavía hoy en dos formatos y constituyen el documento recopilatorio imprescindible para cualquier investigador sobre la HfG. Respecto al Curso Básico, los paneles de la exposición contienen materiales e información acerca de su organización a lo largo de los años. ${ }^{16}$

En la mitad de la década de los 80 ven la luz las primeras producciones científicas sobre la historia de la HfG en forma de tesis escritas por dos historiadores: La primera ${ }^{17}$, titulada "Geschichte der Hochschule für Gestaltung Ulm 1953-1968. Ein Beitrag zur Entwicklung ihres Programms und der Arbeiten im Bereich der Visuellen Kommunikation", es defendida en Colonia por Hartmut Seeling. La segunda"18, "die Hochschule für Gestaltung in Ulm. Gründung (1949 - 1953) und Ära Max Bill (1953 - 1957)"; es elaborada por Eva von Seckendorff en la Universidad de Hamburgo y posteriormente publicada en 1989. Seeling aporta una primera y sucinta revisión del desarrollo del Curso Básico como departamento independiente y su incorporación como primer año de estudios en los departamentos y muestra material gráfico de interés. Aunque de mayor difusión, la tesis de Eva von Seckendorff no añade más información sobre el Grundlehre.

Al final de la década, en 1987, el club off ulm promueve la fundación del archivo y reclama al Ulmer Museum su custodia y la rehabilitación del edificio de Bill. El archivo pasa a ser propiedad de esta institución en 1993 y permanece en sus dependencias hasta que en el año 2011 vuelve al Kühberg, donde se encuentra actualmente.

Este mismo año, y coincidiendo con la formación del archivo, se organiza una gran exposición retrospectiva de la Escuela en cuyo catálogo, "HfG Ulm. Die moral der Gegestande", ${ }^{19}$ se publican algunos de los trabajos del Grundlehre más representativos que se conservaban en los archivos de la HfG. El autor es el antiguo profesor Herbert Lindinger. Desde la pertenencia del archivo al Ulmer Museum se realizarán una serie de exposiciones y se editarán seis catálogos con material inédito de los alumnos de la escuela. De especial interés será la exposición de 1993 "Bauhäusler in Ulm. Grundlehere an der HfG 1953-1955", exposición dedicada a las enseñanzas desarrolladas por los profesores de la HfG vinculados a la Bauhaus. En el catálogo ${ }^{20}$ se publican trabajos de los alumnos durante los cursos de Walter Peterhans, Josef Albers, Helene Nonnè-Schmidt, Johannes Itten y Max Bill.
14 Maldonado, Tomás et. al. "Tre Scuole: Bauhaus-Vchutemas-Ulm". Casabella, (435). Milano: Arti grafiche Meroni, 1978.

15 Maldonado, Tomás, et. al.: "II contributo della scuola di Ulm =: The legacy of the school of Ulm". Rassegna 19, 1984. Número monográfico con textos de de Tomás Maldonado, Marina Bistolfi, Martin Krampen, Giovanni Anceschi, Piero G. Tanca.

16 Roericht, Hans Nick. \& Arbeitsgruppe HfG-Synopse. HfG Synopse. Ulm, 1982 Catálogo de la exposición del mismo nombre celebrada en Ulm en 1982. Los paneles se organizan en forma de un panorama cronológico, un curso académico por panel. El equipo estuvo formado por personas que después continuaron la labor de formación el archivo, como Marcela Quijano.

17 Seeling, Hertmurt. Geschichte der Hochschule für Gestaltung in Ulm, 1953 - 1968. Ein Beitrag zur Entwicklung ihres Programmes und der Arbeiten im Bereich der Visuellen Kommunikation. (Tesis doctoral). Köln, 1985

18 Seckendorff, Eva. von. Die Hochschule für Gestaltung in UIm. Gründung (1949 - 1953) und Ära Max Bill (1953 - 1957) (Tesis Doctoral). Universität Hamburg, 1986.

19 Lindinger, Herbert. Ulm Design: The Morality of Objects (catálogo de exposición). Massachusetts: MIT Press. 1991.

20 Albers, Ingela; Quijano, Marcela; \& Wachsmann, Chirstianne. Bauhaüsler in Ulm. Die Grundlehre an der Ulmer HfG zwischen 1953 und 1955 (catálogo de exposición). Ulm: HfGarchiv UIm Dokumentationen 4, 1993. 
i0/13 Albers, Ingela; Quijano, Marcela; \& Wachsmann, Chirstianne. Bauhaüsler in UIm. Die Grundlehre an der Ulmer HfG zwischen

1953 und 1955 (catálogo de exposición). Ulm: HfG-archiv Ulm Dokumentationen 4, 1993. i0/14 Müller-Krauspe, G., \& et. al. Die Grundlehre von 1953 bis 1960. 16 Rückblicke und 6 Kurzporträts. Ulm: Club Off Ulm, 2011

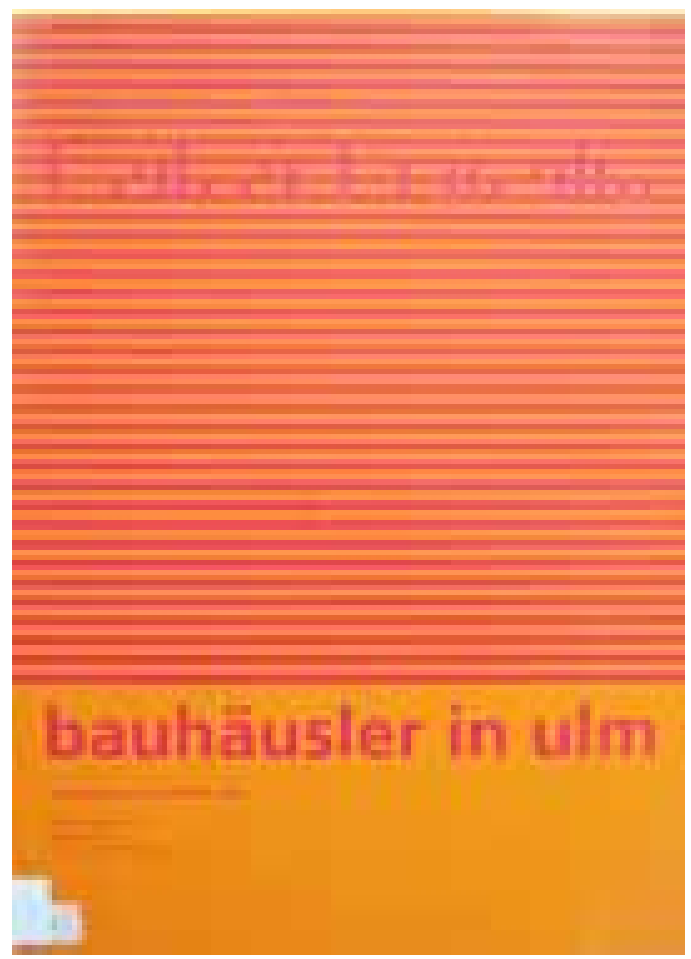

i0/15 W.AA. Ulmer modelle - Modelle nach ulm, hochschule für gestaltung ulm 1953-1968. Ostfildern-Ruit: Hatje Cantz Verlag, 2003. (Catálogo de exposición).

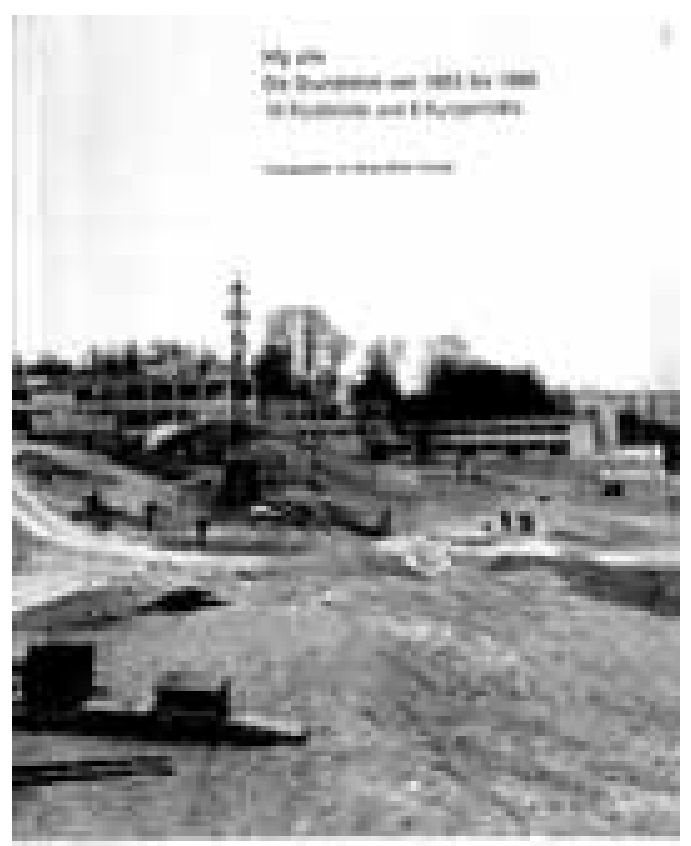

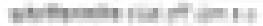

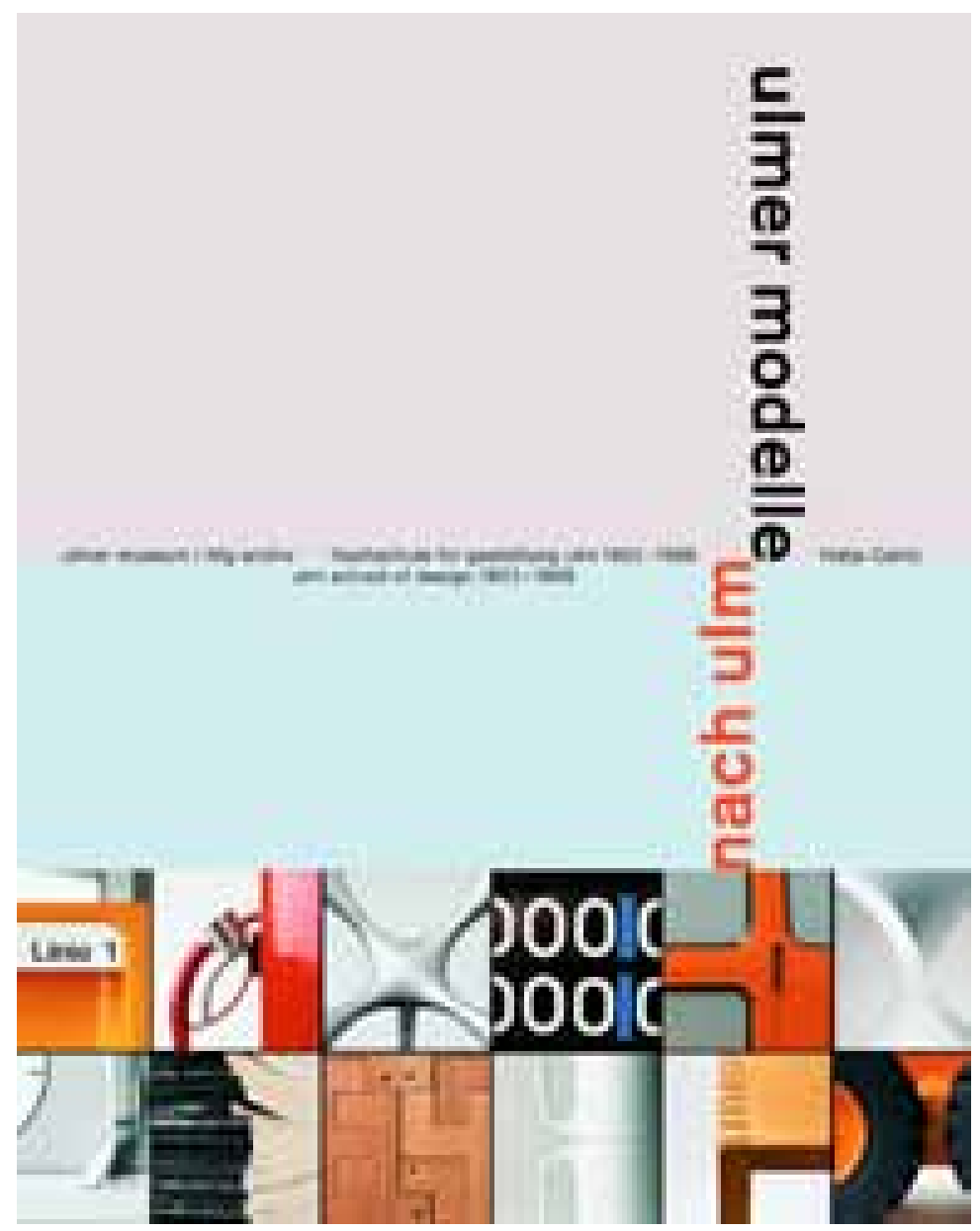


René Spitz defiende su tesis ${ }^{21}$ en Colonia en 1997 bajo el título "Die politische Geschichte der Hochschule für Gestaltung UIm (1953-1968) Ein Beispiel für Bildungs- und Kulturpolitik in der Bundesrepublik Deutschland”. Posteriormente se publicará en 2002 para convertirse en el texto de referencia ${ }^{22}$ más reciente sobre la historia política de la Escuela. El valor de este documento para la investigación reside en que se desgranan de forma exhaustiva acontecimientos históricos relativos a la configuración y trayectoria del Curso Básico y sus personajes, pero exclusivamente desde un punto de vista político y circunstancial, no didáctico.

De mayor interés para esta tesis es la información contenida en la monografía del antiguo alumno Martin Krampen, The Ulm School of Design-Beginings of a Project of Unyielding Modernity, ${ }^{23}$ en la que se profundiza en los aspectos organizativos y pedagógicos de los departamentos, y se hace referencia al Curso Básico como departamento independiente y su incorporación como primer año de estudios.

Con el cambio de milenio, la editorial Hypen Press edita una recopilación de trabajos ${ }^{24} y$ textos del antiguo profesor de la HfG Anthony Froshaug. La monografía recoge el texto Foundation course, Ulm 1953-61, dedicado íntegramente al desarrollo del Grundlehre como curso independiente. Froshaug aporta una valiosa visión crítica sobre la didáctica del Curso Básico y los cambios de rumbo que marcaron su trayectoria.

Tras la HfG Synopse, el segundo gran impulso al legado de la escuela se produce en el $50^{\circ}$ aniversario de su fundación. En el año 2003 se organiza la exposición Modelos de Ulm, que fue concebida inicialmente como una exposición en el museo municipal de la ciudad. "Modelos de Ulm" cosechó un éxito sin precedentes y pasó a convertirse en una exposición itinerante que recorrería el mundo a lo largo de 11 años y lograría un formidable impulso a la difusión de la escuela. La selección, a cargo de Marcela Quijano y Dagmar Rinker, configura una muestra panorámica de la didáctica y producción de la escuela. En su catálogo, diversos autores se refieren a las enseñanzas. En lo que concierne al Curso Básico, resulta de especial interés un artículo del catálogo escrito por William S. Huff, ${ }^{25}$ quien fuera estudiante y profesor de la escuela, que desgrana el Curso Básico de Tomás Maldonado.

Coincidiendo con este impulso se publicarán gracias a la iniciativa del club off ulm una serie de seis monografías con caracter restrospectivo sobre la historia de los departamentos de la HfG. En la dedicada al Curso Básico ${ }^{26}$ entre 1953 y 1960, "Die Grundlehre an der hfg ulm 1953-1960, 16 Rückblicke und 6 Kurzporträts”, publicada en 2011, se encuentran testimonios de antiguos alumnos que describen su paso a lo largo del Grundlehre.

Form $+Z w_{e c k^{27}}$ se une a la conmemoración del 50 aniversario del nacimiento de la escuela y dedica un número monográfico en el que se recoge un documento inédito de Tomás Maldonado en el que establece una nueva configuración del Curso Básico en 1956.

Con el traslado definitivo del material del archivo a su sede en el edificio de Max Bill, se inaugura en el 2013 la exposición permanente que todavía puede visitarse. En ella se encuentra una selección icónica de trabajos del Grundlehre.
21 Spitz, René. Die politische Geschichte der Hochschule für Gestaltung UIm (1953-1968) (tesis doctoral). Köln, 1997.

22 Spitz, René. HfG UIm. The view venid the foreground. The political history of the UIm school of design 1953-1968. Stuttgart-London: Alex Menges, 2002

23 Krampen, Martin., \& Hörmann Günter. Die Hochschule für Gestaltung Ulm - Anfänge eines Projektes der unnachgiebigen Moderne. Berlin: Ernst \& Sohn. 2003.

24 Froshaug, Anthorny "Foundation course, Ulm 1953-61" (1961) en Kinross, Robin Ed. Anthony Froshaug. Typography \& texts. Londres: Hyphen Press, 2000. p. 133-146.

25 Huff, William S. "Grundlehre at the HfG - with a focus on the "Visuelle Grammatik" publicado en W.AA. Ulmer modelle - Modelle nach ulm, hochschule für gestaltung ulm 1953-1968. Ostfildern-Ruit: Hatje Cantz Verlag, 2003. (Catálogo de exposición).

26 Müller-Krauspe, G., \& et. al. Die Grundlehre von 1953 bis 1960. 16 Rückblicke und 6 Kurzporträts. Ulm: Club Off UIm, 2011

27 Maldonado, Tomás (1956) Grundlehre. En: W.AA. (2003). HfG Ulm: form+zweck 20 (número monográfico). form + zweck. 


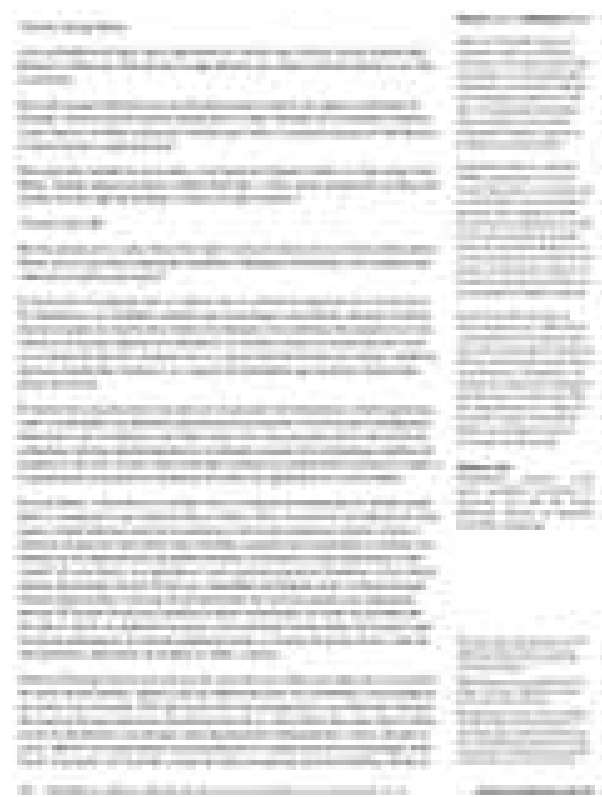

i0/16 Fernández Campos, Ángel Luis; Benito Roldán, Emilia; Sánchez Moya, María Dolores, (2015). "From intuition to methodology. Design propaedeutic in HfG Ulm basic course". Rita Revista Indexada de Textos Académicos, 4, 2015

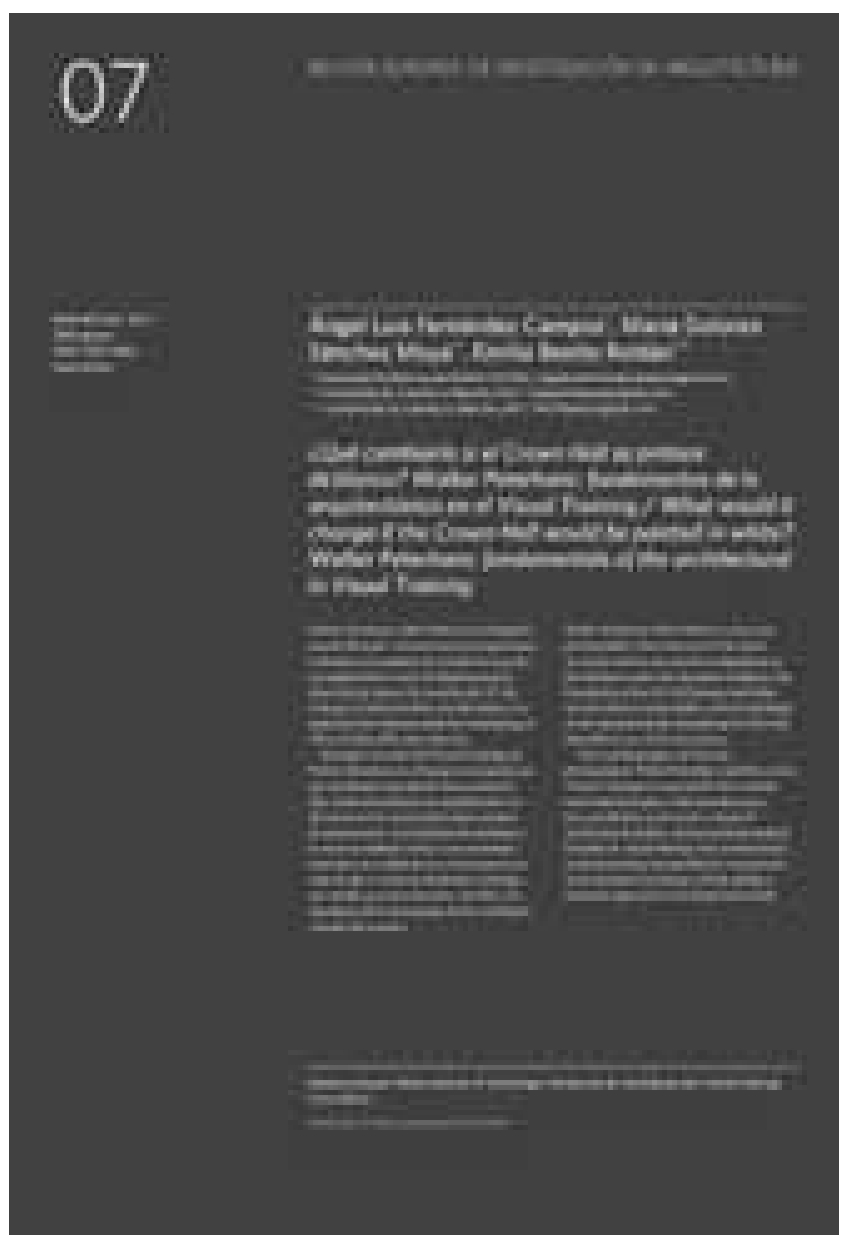

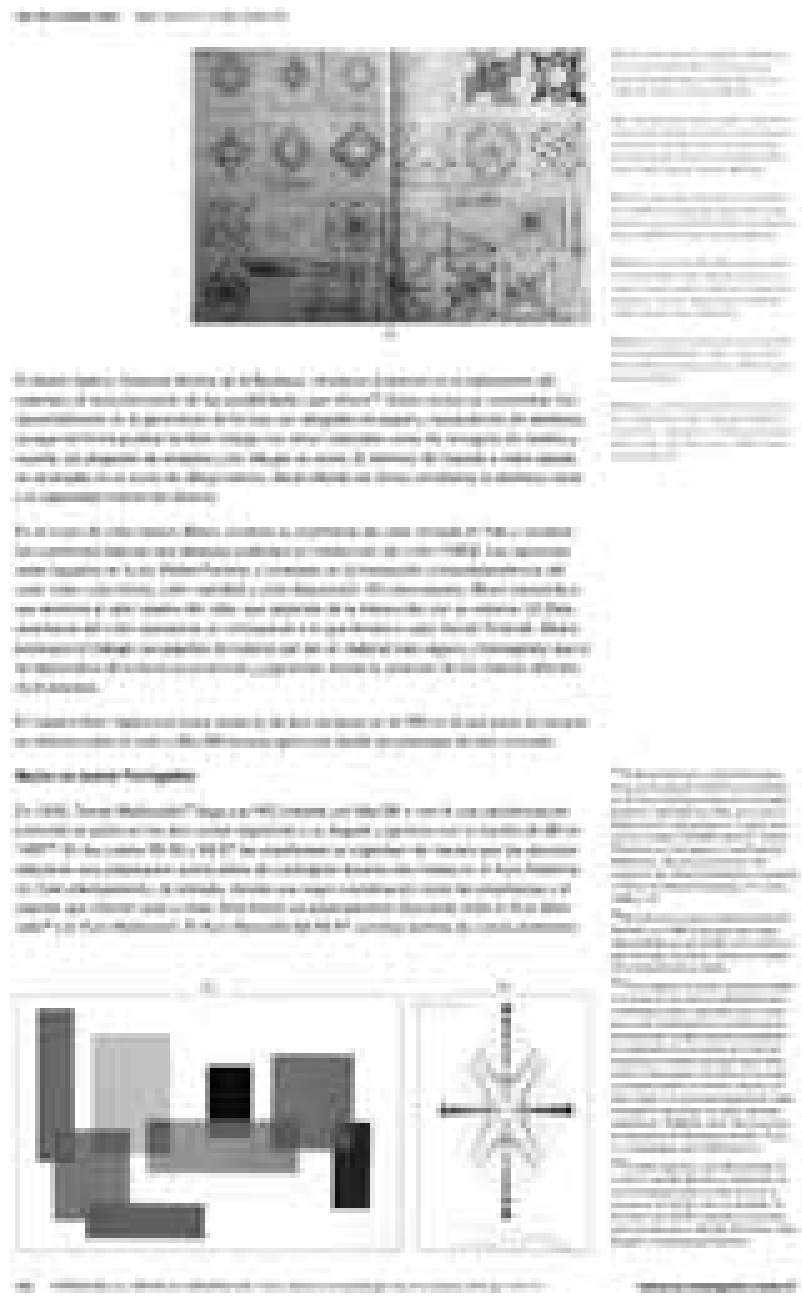

iO/17 Fernández Campos, Ángel Luis; Benito Roldán, Emilia; Sánchez Moya, María Dolores, "¿Qué cambiaría si el Crown Hall se pintara de blanco?

Walter Peterhans : fundamentos de lo arquitectónico en el Visual Training What would it change if the Crown Hall would be painted in white? Walter Peterhans: fundamentals of the architectural in Visual." REIA. Revista Europea de Investigación En Arquitectura, (7-8), 2016, 115-129. Retrieved from http:// hdl.handle.net/11268/6273.

Artículo sobre la didáctica de W. Peterhans en la HfG de Ulm importada del IIT. 
De las tesis que se han publicado recientemente sobre la didáctica de la escuela, la que guarda una estrecha relación con la presente investigación es la defendida en el año 2016 por Emilia Benito Roldán, ${ }^{28}$ titulada "La geometría como lenguaje de las formas. Hermann von Baravalle en la HfG de Ulm". Esta tesis expone la revolucionaria enseñanza de la geometría que llevó a cabo Hermann von Baravalle en el Curso Básico de la HfG en diferentes periodos y de forma panorámica, recoge otras enseñanzas de geometría a cargo de los docentes implicados en el Grundlehre como Emde, Ohl, Schatz, Bill, Leowald y Gugelot. Otra tesis defendida en el mismo año, "Industria y Diseño. Ideología de la Hochschule für Gestaltung Ulm 1953-1968”29 propone una visión cronológica de la didáctica de la HfG de los departamentos y en las etapas de los rectorados, en la que recoge episodios del Curso Básico, pero por su carácter panorámico y recopilatorio, no profundiza en los invariantes didácticos ni aporta material gráfico inédito del Grundlehre.

En definitiva, la relevancia del Curso Básico es tal que ha encontrado eco en cada exposición y cada publicación monográfica sobre la HfG. Sin embargo, debido a su variación y complejidad a lo largo de su existencia, todas estas publicaciones abordan inevitablemente de forma secundaria o tangencial el tema. Como ya se ha visto, únicamente existen dos publicaciones monográficas parciales sobre el Grundlehre: "Bauhäusler in Ulm (1993)" y "Die Grundlehre an der HfG Ulm 1953-1960, 16 Rückblicke und 6 Kurzporträts (2011)". No existe por tanto ninguna publicación ni investigación que ofrezca un estudio sistemático de las enseñanzas desarrolladas en el Curso Básico de la HfG en su conjunto. Esta tesis pretende aportar una organización metódica de todo el material inédito y la visión del Curso Básico desde su aspecto más esencial: el de la enseñanza y los métodos docentes experimentados en el Grundlehre. La propedéutica del proyectar.

El desarrollo de esta investigación ha dado lugar a tres artículos. El primero, "Proyectar es decidir. Ética y pedagogía del proyectar" 30 ahonda en la finalidad social de la escuela de Ulm y las herramientas pedagógicas que se ponen al servicio de sus objetivos. El segundo, "De la intuición a la metodología", ${ }^{11}$ identifica las etapas en las que se organizó el Grundlehre y los principales rasgos de su didáctica. El último, “¿Qué cambiaría si el Crown Hall se pintara de blanco? Walter Peterhans: fundamentos de lo arquitectónico en el Visual Training", ${ }^{32}$ desarrolla el contenido de la didáctica que Walter Peterhans lleva a cabo durante su estancia en la HfG importado de sus cursos en IIT de Chicago.
28 Benito-Roldán, Emilia. La geometría como lenguaje de las formas. Hermann von Baravalle en la hfg de Ulm. (Tesis doctoral inédita) Universidad Politécnica de Madrid, 2016 https://doi.org/https://doi. org/10.20868/UPM.thesis.43027.

29 Moyano Miranda, Neus. Industria y diseño. Ideología de la Hochschule Für Gestaltung UIm 1953-1968. Universitat Rovira i Virgili 2016.

30 Fernández-Campos, Ange Luis; Sánchez Moya, María Dolores "Proyectar es decidir. Ética y pedagogía del proyectar". Sociedad Y Utopía. Revista de Ciencias Sociales, (43), 2014, 392-411.

31 Fernández Campos, Ángel Luis; Benito Roldán, Emilia; Sánchez Moya, María Dolores, "From intuition to methodology. Design propaedeutic in HfG Ulm basic course". Rita Revista Indexada de Textos Académicos,4, 2015, pp. 110-117.

32 Fernández Campos, Ángel Luis; Benito Roldán, Emilia; Sánchez Moya, María Dolores, “¿Qué cambiaría si el Crown Hall se pintara de blanco? Walter Peterhans : fundamentos de lo arquitectónico en el Visual Training [What would it change if the Crown Hall would be painted in white? Walter Peterhans: fundamentals of the architectural in Visual." REIA. Revista Europea de Investigación En Arquitectura, (7-8), 2016, 115-129. Retrieved from http://hdl.handle. net/11268/6273. 

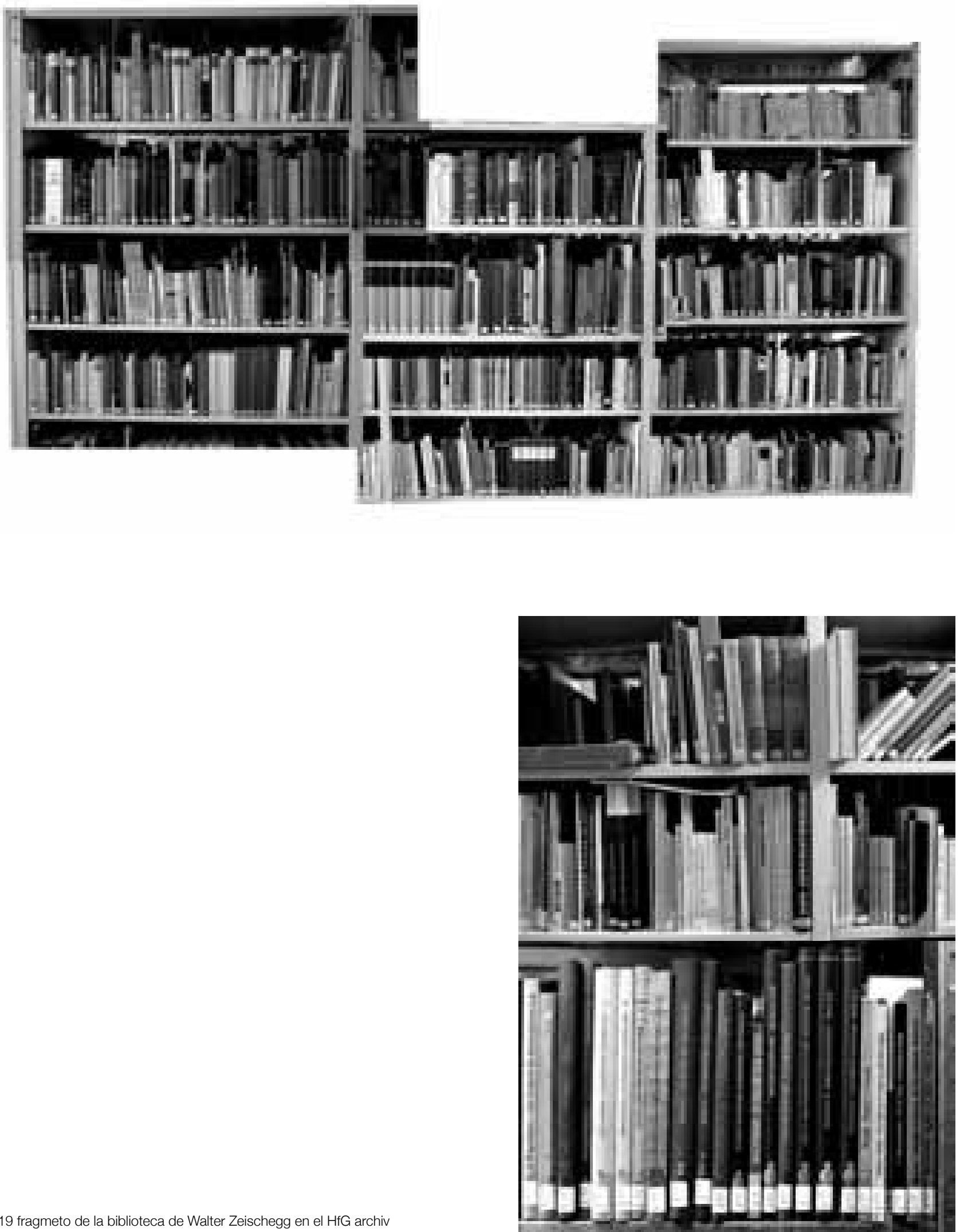


\subsection{Atlas como método}

El tema de tesis se intuye durante el periodo de preparación del trabajo para el Diploma de Estudios Avanzados en 2011, que trataba sobre la relación entre las pedagogías de los cursos básicos de Bauhaus - Vjutemas - Hfg Ulm. En esos meses llega a Madrid la exposición itinerante "Modelos de Ulm", donde tiene lugar un primer contacto con el material del Curso Básico. Tres años más tarde, en enero de 2015, se realiza la primera visita al archivo de la HfG, situado en la antigua sede de la escuela, en el Kuhberg, una colina de la ciudad de Ulm. Esta visita señala el comienzo de la inmersión durante varios años en las fuentes originales de la escuela. La formidable cantidad de material inédito, el descubrimiento de asignaturas de gran valor didáctico, unido al vacío de conocimiento alrededor del Grundlehre, confirmado por el entorno de la HfG, acaban confirmando y cercando el tema sobre la didáctica del Curso Básico de la escuela de UIm.

\subsubsection{Identificación de lagunas}

Es necesario señalar la significativa falta de documentación sobre la HfG y el Curso Básico en cualquiera de las bibliotecas en la península dedicadas al diseño o a la arquitectura. La biblioteca del Museo de Artes decorativas en Madrid es en el que se han podido consultar más libros relativos a la HfG. Se realiza una búsqueda sistemática de artículos, monografías, catálogos de exposiciones, tesis y libros sobre la HfG y sus etapas para la formación de una base bibliográfica en continuo crecimiento y actualización. Es necesario recurrir a la adquisición de muchas referencias en librerías de anticuario, dado que los libros sobre este tema son escasos y se encuentran agotados.

El estudio de la documentación manifiesta la existencia de lagunas y discontinuidades en lo difundido del Curso Básico. Los escritos de Maldonado y algunas recopilaciones más extensas como las de Martin Krampen, William S. Huff y Anthony Froshaug - alumnos y profesores de la escuela-, dejan ver que existen unas enseñanzas más complejas con diferentes objetivos en cada época. Monografías de antiguos profesores como Josef Albers, ponen de manifiesto la continuidad de sus enseñanzas en Estados Unidos y la HfG.

Ha sido fundamental el acceso a la biblioteca propia del HfG Archiv donde se han podido consultar muchas de las referencias que no estaban disponibles por otras vías. Además, esta biblioteca cuenta con otra importante colección de monografías y tesis doctorales que ha permitido completar la bibliografía de esta investigación. La importancia de la biblioteca propia de la HfG también reside en su sección histórica, donde se encuentran los manuales de referencia de alumnos y profesores que forman la base teórica de las enseñanzas allí desarrolladas. Este hecho es clave en el contexto de una investigación pedagógica puesto que se pueden consultar los manuales y referentes contemporáneos a la escuela y que se manejaban como material docente.

Esta biblioteca también acoge colecciones de libros donadas que han sido de interés para su consulta, como la biblioteca personal de Otl Aicher y de Walter Zeischegg. 
i0/20 Calendario del Grundlehre como base para la reconstrucción del Curso Básico. Christianne Wachsmann. Hfg Archiv.

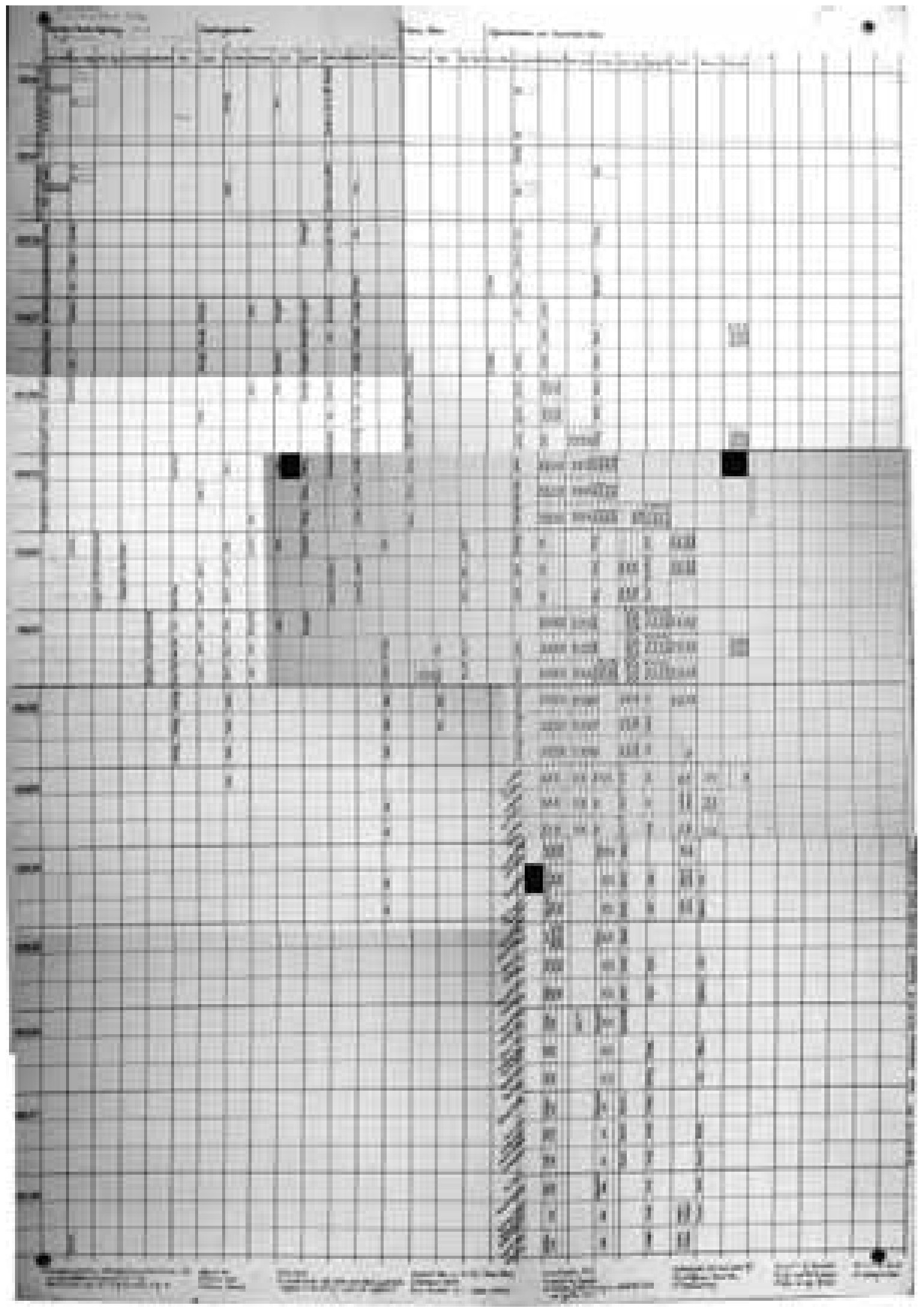




\subsubsection{Recopilación del atlas}

El atlas, en su acepción más general del término, es una "colección de láminas descriptivas pertenecientes a ciertas disciplinas, y que suele aparecer encuadernada como libro". ${ }^{33} \mathrm{El}$ discurso de la tesis se asienta sobre la base del material gráfico del Grundlehre, por lo que el atlas resulta imprescindible como herramienta que ordena y muestra dicha información.

La recopilación comienza en la sede de el Archivo de la Hochschule für Gestaltung, que actualmente es una institución vinculada al museo municipal de la ciudad. ${ }^{34}$

Durante las seis estancias realizadas en los años 2014, 2015, 2017 y 2018, en periodos que oscilan entre una semana y un mes, se recaba toda la información necesaria y más completa posible sobre las disciplinas visuales del Curso Básico y temas relacionados. El volumen de información recopilada en este periodo de tiempo es considerable y difícil de manejar. Esta información consiste en unos documentos generales relativos a la organización del Curso Básico -horarios, actas de reuniones docentes- y material didáctico -cuadernos de apuntes, enunciados, fotografías y trabajos de alumnos de cada año-; sin embargo, no siempre tienen una relación evidente con el Curso Básico ni con la asignatura a la que pertenecen. Esto, unido al hecho de que la base de datos no está completamente formada, hace que la búsqueda sea enormemente complicada. Por ejemplo, en numerosas ocasiones los ejercicios aparecen como parte del "Kurs Maldonado" o del "Kurs Aicher" pero no se hace referencia a la asignatura o al desarrollo concreto de la materia. Fue clave contar con otros documentos para escudriñar entre la ingente cantidad de material que custodia el archivo e interpretar, identificar y ubicar cada ejercicio.

El HfG Synopse sirve de guía y referencia fundamental para la organización del propio archivo y para esta investigación, porque incluye unos listados completos del nombre de los alumnos que cursaron el Grundlehre cada año. La búsqueda en el archivo se realiza por años y apellidos de los alumnos de nuevo ingreso.

Los listados obtenidos de esta manera constituyen una guía básica para la obtención de los documentos, que están organizados en forma de depósitos temporales o legados de cada titular. Por tanto, un depósito o legado se compone de un conjunto de documentos de diversa naturaleza: producción de diferentes asignaturas, fotografías personales o vinculadas a los trabajos, memorias, cuadernos de apuntes, etcétera.

La segunda herramienta fundamental para la reconstrucción del Curso Básico y sus asignaturas consiste en un horario elaborado por Christiane Wachsmann ${ }^{35}$ que relaciona las materias, la concatenación de éstas durante el periodo estudiado y el profesor que las imparte, tomando como base la fuente de documentos y trabajos existentes en el archivo.

El contacto directo con el trabajo gráfico y de modelado ha sido fundamental para apreciar la pedagogía de los distintos profesores basada en el planteamiento sistemático de las tareas, el rigor en la ejecución de las mismas y la coherencia entre los fines propuestos por el docente y los resultados obtenidos por los alumnos.
33 Segunda acepción de la palabra "Atlas". Real Academia Española Diccionario de la lengua española (23.a ed.). Madrid, 2014. http://dle. rae.es/?id=4GOMWuY (consultado en abril 2018).

34 Es importante señalar que, a diferencia del Bauhaus Archiv que depende del Estado Alemán, el HfG

- Archiv es una institución municipa y en proporción, los recursos personales y económicos son más limitados.

35 Christiane Wachmann forma parte del personal del HfG Archiv que ha dirgido entre los años 1989-1997. 

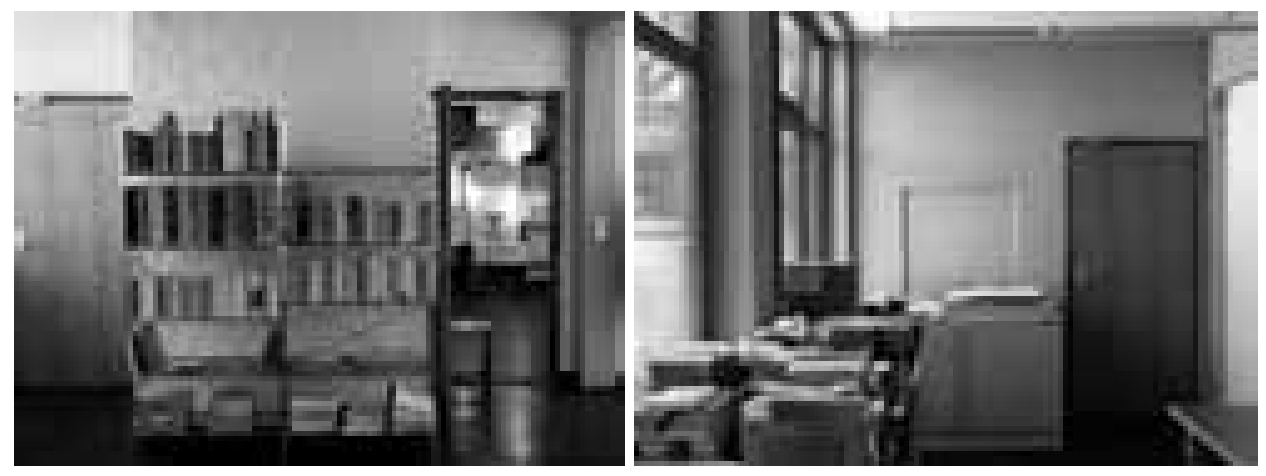

i0/21, 22, 23 Archivo HfG
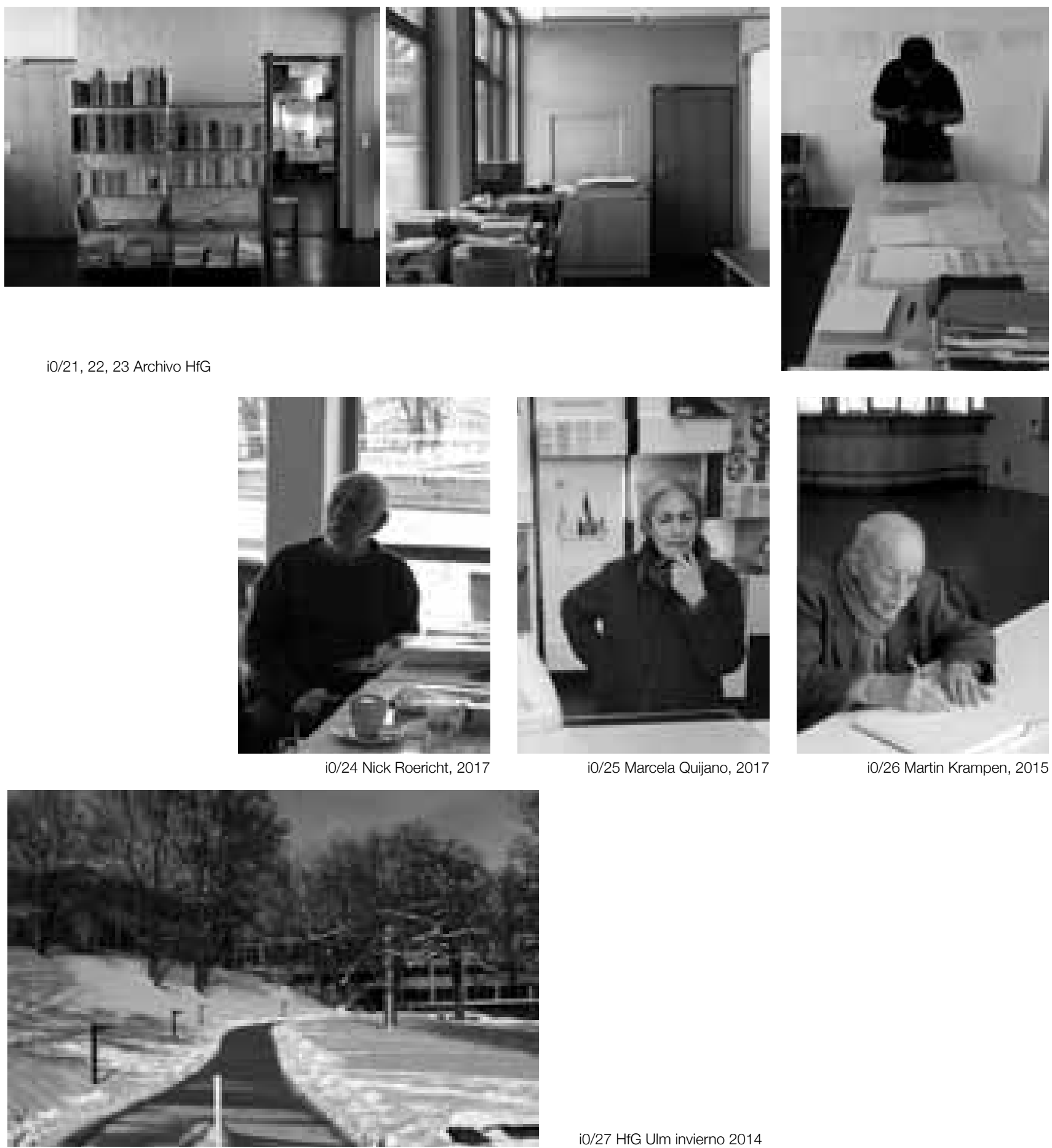

iO/27 HfG Ulm invierno 2014

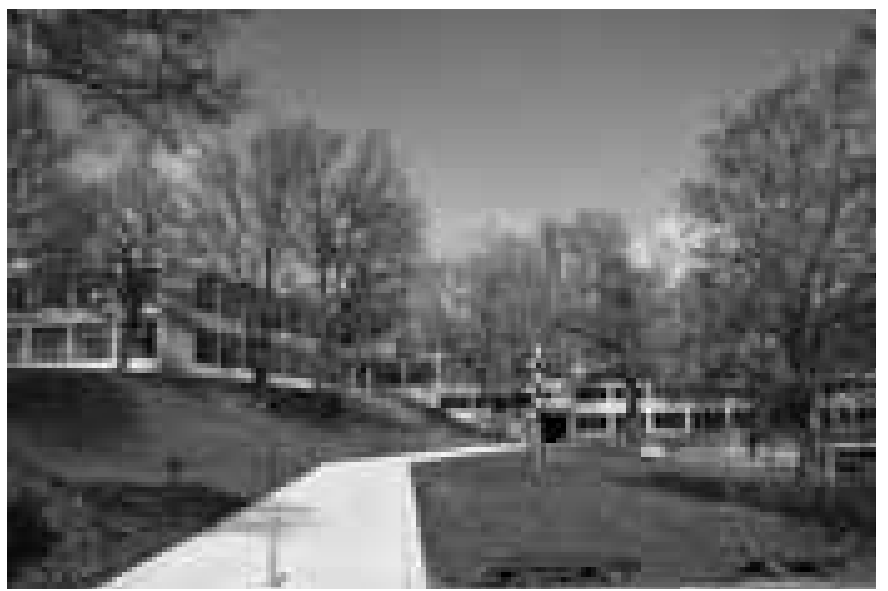

iO/28 HfG Ulm primavera 2016 
Toda esta documentación se fotografía, ${ }^{36}$ ya que el archivo no cuenta con un servicio de reproducción y digitalización de planos y documentos.

El trato directo y la convivencia con las personas relacionadas con la $\mathrm{HfG}$ ha resultado ser fundamental en el trabajo de investigación. En primer lugar es necesario destacar la importante labor de Marcela Quijano. Estudia Diseño industrial en la Hochschüle der Künste Berlin donde Hans Nick Roericht es docente. Colabora con él en la redacción del HfG Synopse y desde ese momento se vincula a la memoria de la escuela y al archivo. Contribuye a recopilar los fondos de antiguos alumnos dispersos en varios países. Es comisaria de exposiciones tan significativas como "Modelos de Ulm" y viaja alrededor del mundo difundiendo el legado de la escuela en conferencias. Su dilatada experiencia ha sido un soporte imprescindible para esta investigación, facilitando el acceso a los trabajos, siendo un referente primordial de consulta en cualquier duda y ha encauzando las intuiciones que guían la investigación.

La asistencia diaria al archivo ha permitido un trato diario y charlas con su director, Martin Mäntele y antiguos alumnos y profesores como Nick Roericht o Martin Krampen, testigos directos de la HfG, su apertura, su cierre y la gestión de su legado.

\subsubsection{Atlas panóptico}

A medida que se recoge e identifica la información, se lleva a cabo una primera organización cronológica de toda la documentación, como sistema más eficiente para una revisión y análisis inicial del considerable volumen de material obtenido. Esta clasificación inicial da lugar a un atlas panóptico, que ofrece una secuencia panorámica y cronológica del Grundlehre.

Este atlas, como herramienta de trabajo personal, supone una primera reconstrucción completa del Curso Básico. Su carácter eminentemente compilatorio, permite confirmar y poner en cuestión la información de referencia obtenida en la bibliografía y mencionada en el estado de la cuestión y también sirve para identificar con claridad los periodos del Curso Básico, así como sus lagunas y discontinuidades. ${ }^{35}$ En definitiva, este análisis sistemático y ordenado ha constituido un panorama de referencia, una base contrastada y depurada sobre la que trazar la estructura de la tesis, la base para la elaboración del discurso. De la reconstrucción del Curso Básico se obtiene la certeza que el núcleo didáctico principal de las enseñanzas iniciales se sustenta en las disciplinas visuales. Las materias que formaron el denominado Visuelle Einführung -introducción visual- fueron las que tuvieron más importancia y trascendencia en todas las fases del Grundlehre y también las que experimentaron una mayor evolución. Por este motivo, se acota la tesis en el ámbito de las disciplinas visuales.

Para la confección de este atlas se cuenta como hoja de ruta fundamental los horarios realizados por Christiane Wachsmann.

Este formato panóptico permite trabajar en uno de los objetivos principales de la tesis: identificar los principios didácticos.
36 La investigación se desarrolla en paralelo a la de Emilia Benito Roldán y junto con Maria Dolores Sánchez Moya. Los créditos de las imágenes del archivo se nombarán con el acrónimo F.S.B; haciendo referencia al trabajo compartido.

37 De esta primera fase de trabajo surge el artículo mencionado en el estado de la cuestión que identifica y establece las fases del Curso Básico que se desarrollan en el capítulo segundo de esta tesis.

Fernández Campos, Ángel Luis Benito Roldán, Emilia; Sánchez Moya, María Dolores, op. cit. pp. 110-117. 


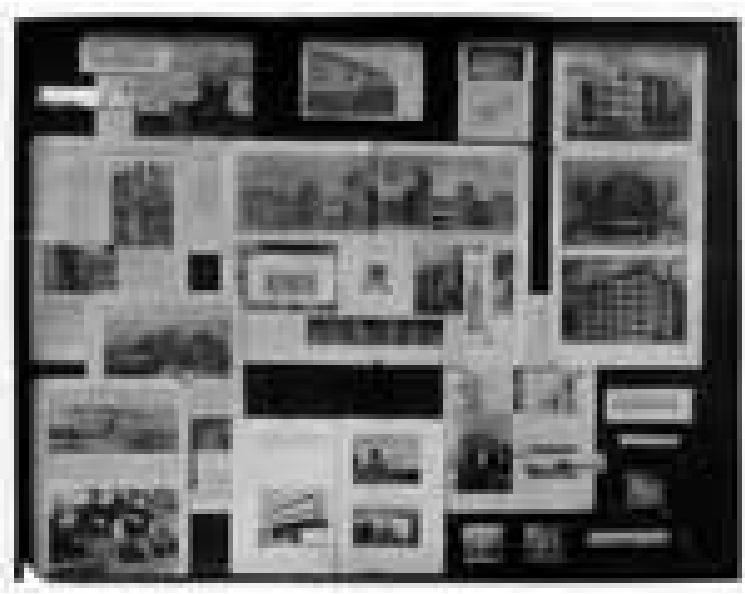

i0/29 Paneles didácticos utilizados en los Vkhutemas.

Khan-Magomedov, Selim O. Vhutemas. Moscou 1920-1930. Paris: Editions du

Regard, 1990.
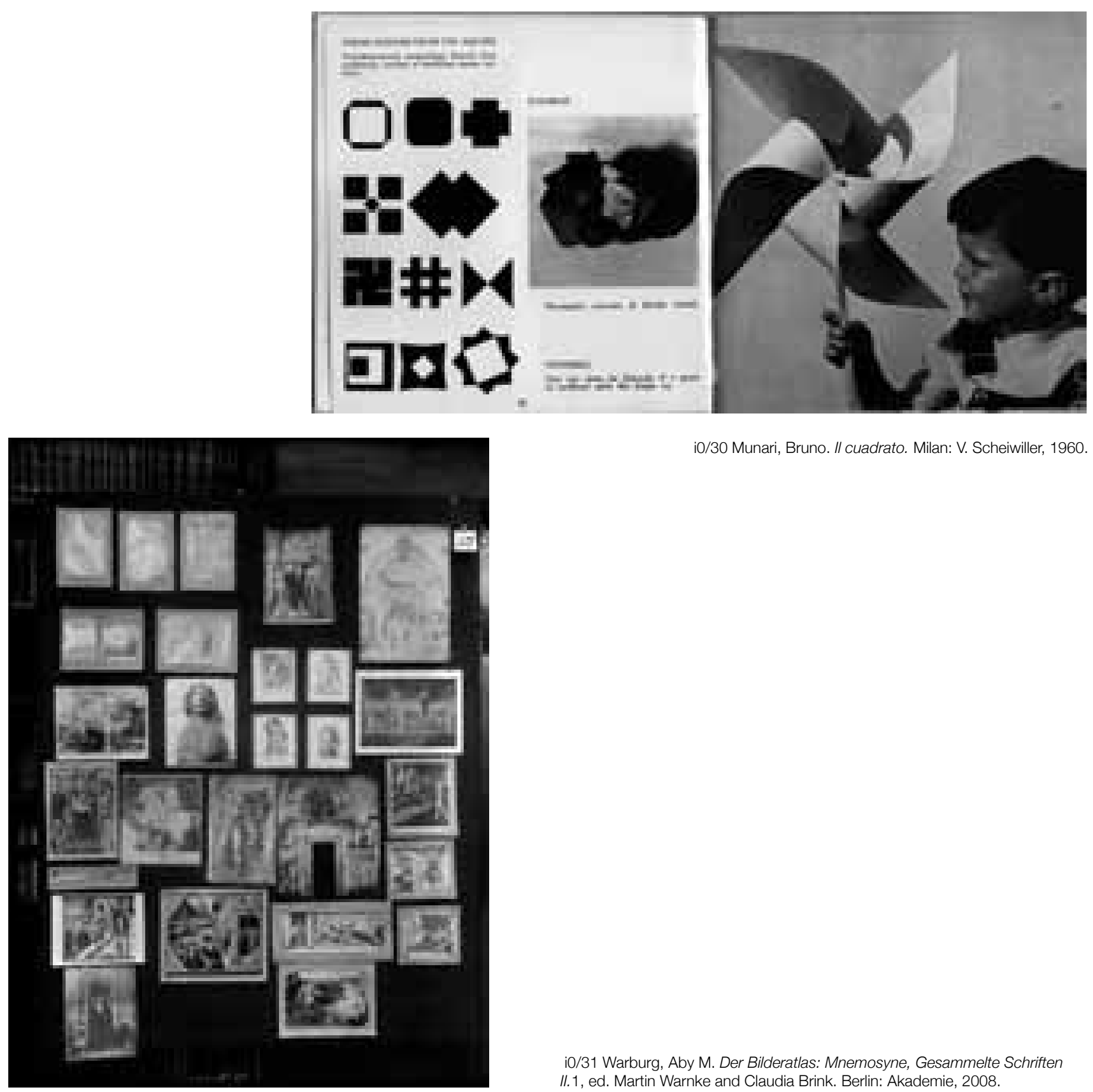

i0/30 Munari, Bruno. Il cuadrato. Milan: V. Scheiwiller, 1960. 
El atlas para Walter Benjamin es un montaje dinámico de heterogeneidades, una "forma que hace operar extremos alejados, excesos aparentes de la evolución, configuración (...) donde tales oposiciones pueden coexistir de una manera que cree sentido". ${ }^{38}$

El proceso de investigación toma el atlas como formato metodológico porque la capacidad enunciada por Walter Benjamin constituye en sí un proceso de trabajo; la recopilación y su yuxtaposición como método de análisis da lugar a un orden no evidente en primer lugar que surge de las analogías visuales y conceptuales que contienen. El atlas, por su carácter, se considera un formato-contenedor, una herramienta eficaz para establecer una recopilación histórica y dialéctica, el soporte para la construcción de un panorama de los momentos y conceptos que se desarrollan a lo largo de la tesis.

En los Vkhutemas, los profesores elaboraban paneles didácticos sobre los que se fijaban imágenes que guiaban el discurso teórico de las clases. La situación de una imagen al lado de otra es un recurso muchas veces empleado, que favorece que broten las concomitancias, las simultaneidades y analogías entre la didáctica de cada enunciado y sus resultados. En paralelo, se realiza un estudio de la trayectoria docente de los profesores implicados en el Grundlehre y de la enseñanza en otras instituciones de referencia de los temas abordados en la HfG. De esta forma, se identifican los invariantes didácticos y su continuidad o ruptura con otras escuelas, así como las experiencias que constituyen una novedad en la enseñanza y pueden considerarse una aportación histórica de las disciplinas visuales en el Grundlehre.

Del atlas panóptico y cronológico se salta a la dimensión sinóptica del Atlas, sobre el que se configura la estructura final de esta tesis.

\subsubsection{Atlas sinóptico}

La identificación y profundización en los invariantes didácticos del Grundlehre es uno de los principales objetivos de la tesis. Una narración cronológica y compilatoria dificultaría el discurso ordenado y la revisión crítica bajo esta óptica. La tesis alcanza el formato de atlas sinóptico en su última fase y con ello pretende trascender la mera recopilación.

El atlas sinóptico se organiza en base a las analogías reconocidas en la primera fase de exploración del Curso Básico, que ha dado como resultado cuatro ejes de trabajo: el color, la forma, la materia y los proyectos. Si sinopsis significa "Disposición gráfica que muestra o representa cosas relacionadas entre sí, facilitando su visión conjunta" ${ }^{39}$, la organización del atlas en estas categorías es una herramienta para profundizar en la didáctica de los temas que vertebran el Curso Básico.

Aby Warburg construye su Atlas Mnemosine ${ }^{40}$ como una colección abierta, en la que la afinidad pone en relación sus elementos por encima de cualquier otra categoría jerárquica. Con notables diferencias pero con aspectos en común, Bruno Munari publica sus manuales "el cuadrado" 41 y "el círculo" 42 en formato atlas, en los que reúne elementos de cualquier ámbito visual bajo estas formas básicas. Lo que comparten los atlas de Warburg y Munari es la construcción de una urdimbre cuya jerarquía emana de los elementos en si, de la imagen o de lo que representa. En otro polo se situarían, por ejemplo, atlas como el de Owen Jones y su "The grammar of ornament" ${ }^{43}$, en los que los elementos se subordinan a
38 Didi-Humbermann, Georges. Atlas, ¿cómo llevar el mundo a cuestas? (Catálogo exposición MNCARS 2010). Madrid: TF Editores/ Museo Reina Sofía, 2010, p. 109.

39 Primera acepción de la palabra "Sinopsis". Real Academia Española. Diccionario de la lengua española (23.a ed.). Madrid, 2014. http://dle rae.es/?id=4GOMWuY (consultado en abril 2018).

40 Warburg, Aby M. Der Bilderatlas: Mnemosyne, Gesammelte Schriften II.1, ed. Martin Warnke and Claudia Brink. Berlin: Akademie, 2008.

41 Munari, Bruno. I/ cerchio.Milan: V Scheiwiller, 1964.

42 Munari, Bruno. I/ cuadrato. Milan: V. Scheiwiller, 1960.

43 Jones, Owen et. al. The grammar of ornament. London: Day and Son, 1856. 

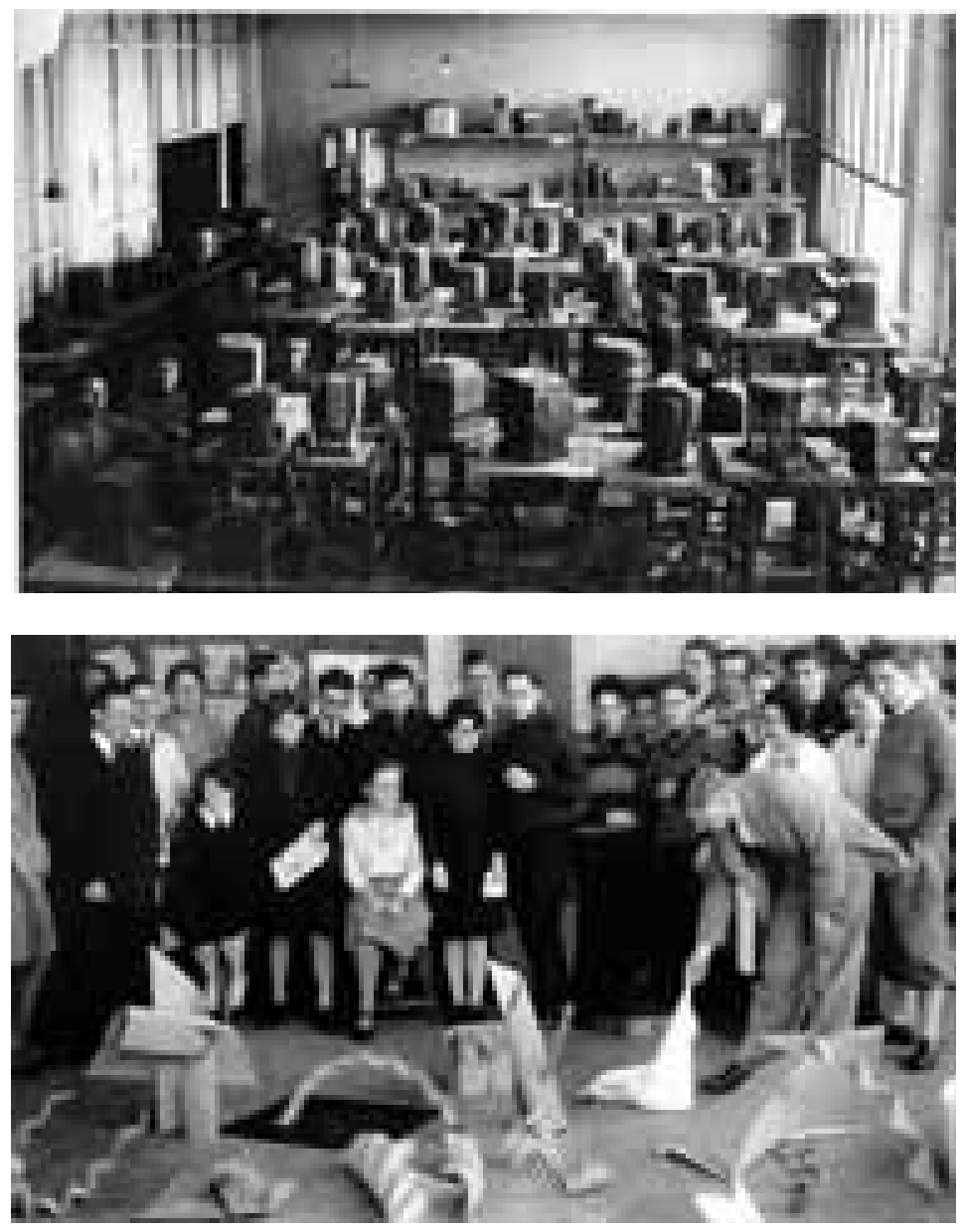

i0/32 Curso Básico Vkhutemas.

Ivanova-Veen, L.I; Ovsiannikova, E.B. From Vkhutemas to Markhi 1920-1936. Moscow: Museum of the History of the Moscow School of Architecture, 2005

i0/34 Curso Básico Hochschule für Gestaltung Ulm. Taller de yeso de Otto Schild. Archivo H. G. Conrad.

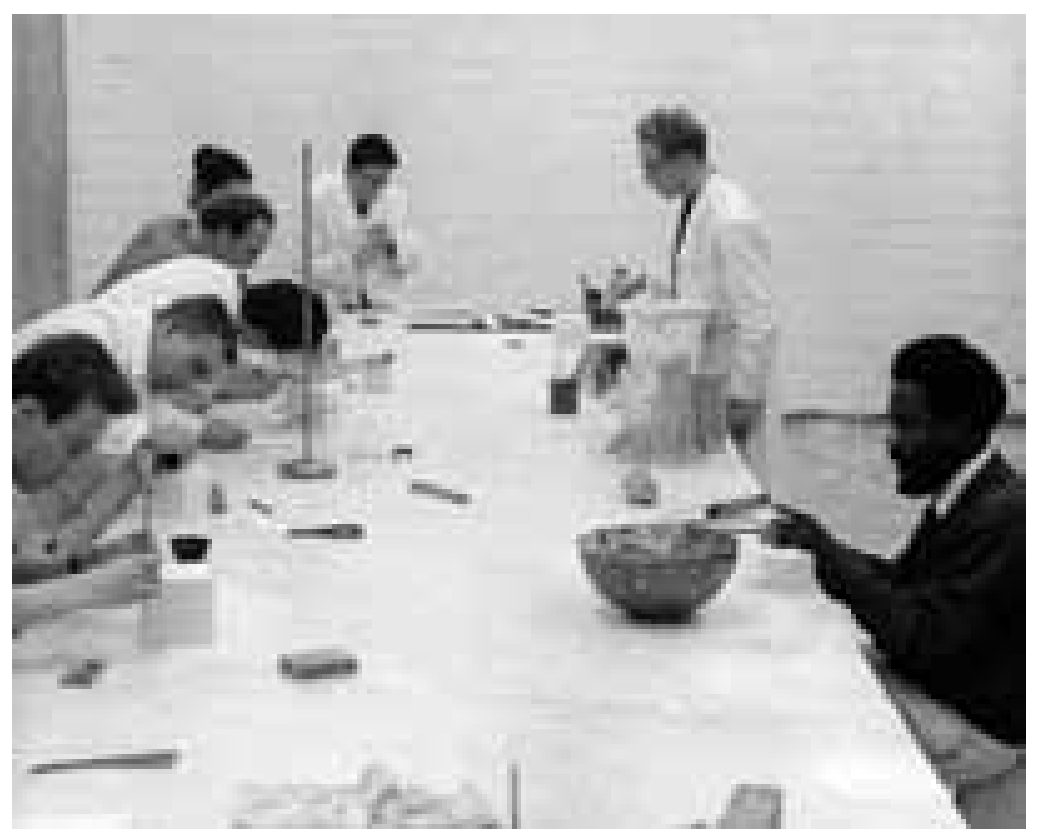


estilos - el ornamento egipcio, el ornamento asirio y persa, etc.-. La jerarquía se traza desde un tiempo y un lugar geográfico concreto, y no surge del elemento propio.

Los cuatro campos en los que se organiza el Atlas Propedéutico se determinan en base a la identificación de analogías en la exploración previa de la primera reconstrucción del Grundlehre y además, tomando en consideración varios aspectos. Por un lado, se tiene en cuenta el ejemplo de la organización del Curso Básico del Vkhutemas, que en el curso 19221923 se constituye como una facultad independiente con las disciplinas de color, dibujo, volumen y espacio. ${ }^{44}$ Otras escuelas, como la de diseño de Basilea, también organizan su curso preparatorio en disciplinas visuales - dibujo -objetos, museo, naturaleza y memoria-, dibujo técnico y perspectiva, rotulación, estudios del material, diseño textil y color-. ${ }^{45}$ Josef Albers describe sus enseñanzas concentradas en tres cursos que denomina "diseño básico, dibujo básico, color básico" en el informe que elabora tras su primera estancia en Ulm. ${ }^{46}$

La HfG no representa una experiencia histórica aislada, tiene herencias y se proyecta hacia el futuro. Formarán parte, por tanto, de esta revisión crítica, las experiencias previas vinculadas con el Grundlehre, tanto de la Bauhaus, New Bauhaus y Vkhutemas. La experiencia de estas escuelas señalarán el punto de partida y un instrumento más de medida para determinar la evolución de la propuesta didáctica de la HfG.

La tesis, por tanto, se estructura atendiendo a estas capacidades, panóptica y sinóptica del Atlas, dando lugar a dos bloques principales: el Marco Didáctico y el Atlas Propedéutico.
44 Colón Llamas, Luis Carlos. Las vanguardias artísticas y la enseñanza de la Rusia de los años 20. Valladolid: Secretariado de publicaciones e intercambio editorial. Universidad de Valladolid, 2002, p. 105

45 Maier, Manfred. Basic Principles of Design. The Foundation Program at the School of Design in Basel, Switzerland. New York: Van Nostrand Reinhold Company, 1980.

46 El curso así estructurado es el que Albers imparte en todas facultadas a la que es invitado en los años 50 y 60. Horowitz, F., \& Danilowitz, B. Josef Albers: to open eyes. New York: Phaidon, 2006. pp. 276, 277. 

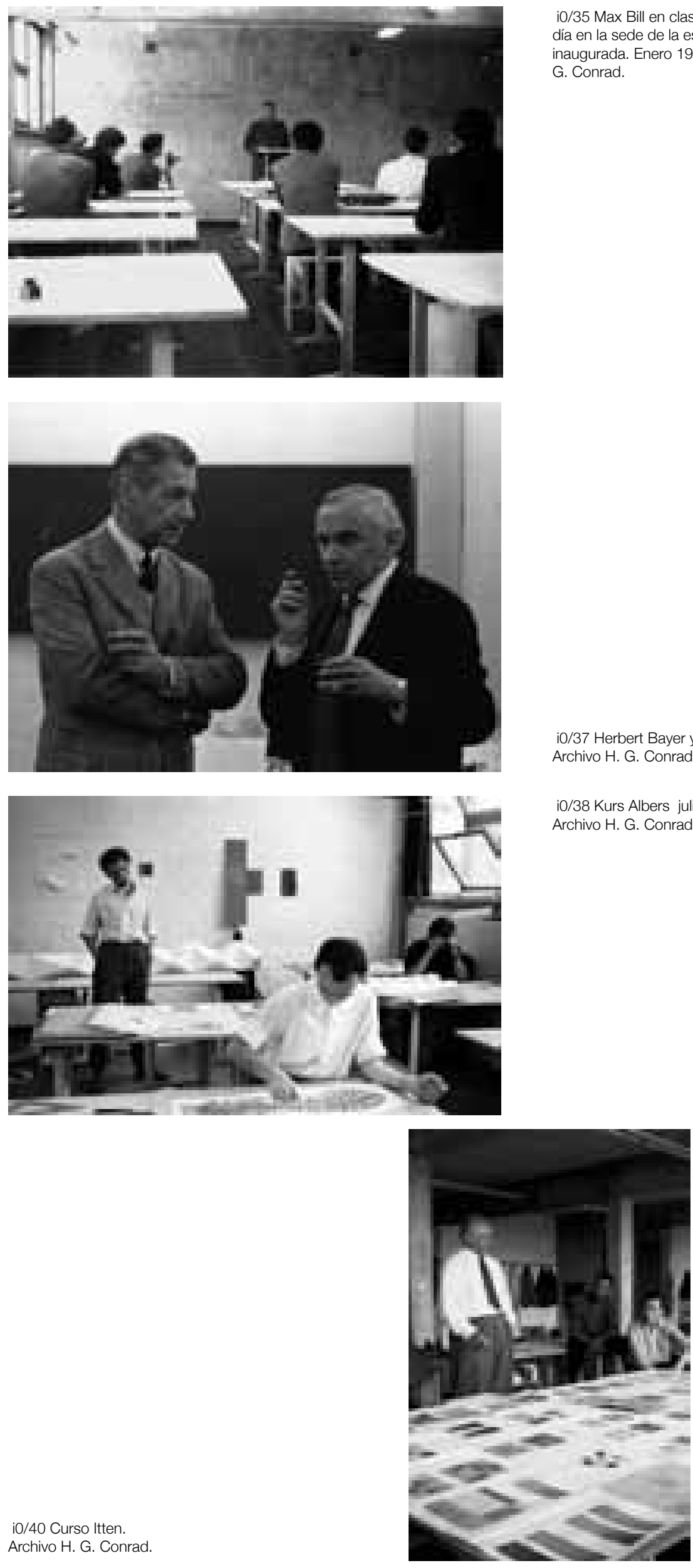

i0/39 Curso Albers.

Archivo H. G. Conrad.

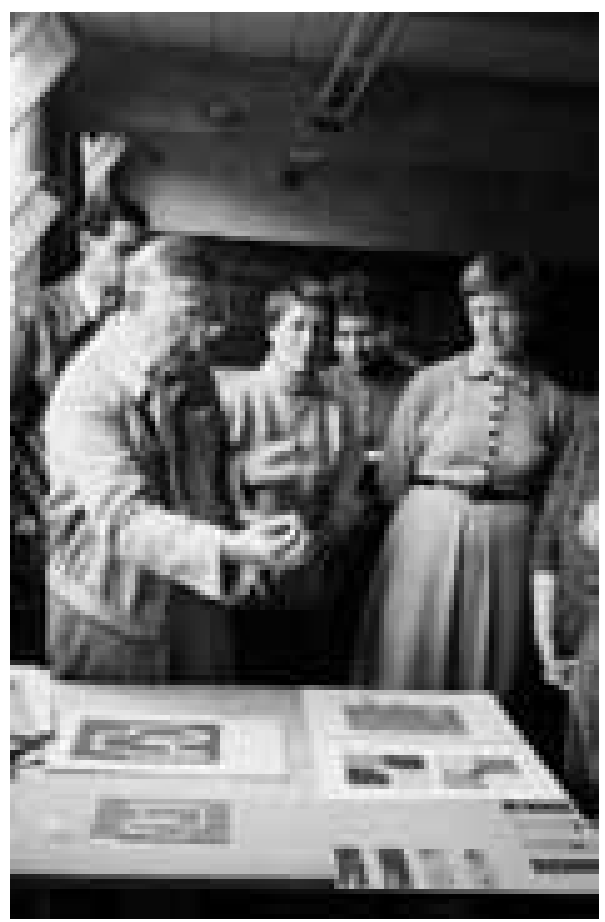

dia en la sede de la escuela recién

inaugurada. Enero 1955. Archivo H.

G. Conrad.

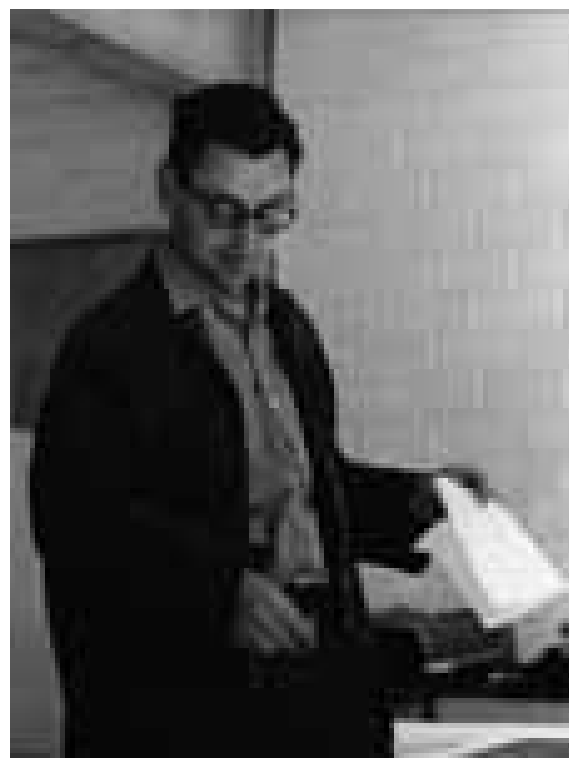

i0/36 Hans Gugelot. Archivo H. G. Conrad.

i0/37 Herbert Bayer y Vomdemberge-Gildewart. Archivo H. G. Conrad.

i0/38 Kurs Albers julio 1955.

Archivo H. G. Conrad. 


\subsection{Estructura de la tesis}

La estructura de la tesis se desarrolla en cinco capítulos que abordan el Marco Didáctico de partida de la investigación y el Atlas Propedéutico que desgrana los invariantes didácticos de las disciplinas visuales del Grundlehre.

\subsubsection{Marco didáctico}

El marco didáctico tiene un carácter compilatorio y consiste en la reconstrucción cronológica del Grundlehre en la HfG a partir del material recopilado en el archivo. El análisis y recorrido por el desarrollo de las enseñanzas visuales esboza un panorama de la didáctica y sus personajes en las fases que marcan los hitos de la evolución del Curso Básico, desde su fundación hasta su disolución en los departamentos.

El capítulo comienza con un compendio histórico, ideológico y pedagógico de las disciplinas visuales precursoras del Curso Básico de la HfG como base fundamental de las enseñanzas preparatorias para el estudio del arte, el diseño y la arquitectura. El epígrafe expone la raíz y la procedencia de las materias de lo visual en las escuelas pioneras directamente vinculadas con la HfG.

El capítulo va introduciendo paulatinamente a los profesores a medida que participan en el Curso Básico. El texto recoge el momento didáctico de partida en cada caso, ya que algunos habían esbozado en otras escuelas la didáctica que desarrollan en el Grundlehre. Otros, recogen temas que habían surgido anteriormente en otras enseñanzas que hacen evolucionar en Ulm. El marco didáctico permite localizar los cursos básicos precedentes y sus temas, para después valorar la repercusión de la enseñanza en Ulm.

En la primera fase participan los antiguos miembros de la Bauhaus y está liderada por Max Bill. Los repertorios visuales consisten en conjuntos de ejercicios de carácter gráficoplástico planteados a lo largo de un curso que responden a determinados enunciados -Peterhans-, o se ejecutan en función de determinados análisis formales -Albers, Nonné-, así como ejercicios de carácter exploratorio. Se caracterizan por proporcionar al alumno el desarrollo de habilidades para la ideación gráfica y la sensibilización frente a aspectos expresivos y visuales del color, la forma y la materia.

La fase intermedia es la protagonizada por Tomás Maldonado y su transformación del Curso Básico. Mediante sus ejercicios planteados como tareas orientadas introduce aspectos del arte concreto y las formula mediante enunciados en los que se incorporan cuestiones matemáticas o perceptivas como desencadenantes para la configuración de la solución.

En la fase final del Curso Básico, las Methodische Übungen y los Abteilungsarbeiten se plantean como tareas metodológicas en las que los alumnos analizan una serie de factores como modo de aproximación previo al desarrollo del proyecto. Los ejercicios son pequeños proyectos, desarrollados bajo la supervisión y coordinación profesores del departamento asociado a cada enunciado, como un ejercicio preparatorio de cada especialidad: diseño de producto, comunicación visual, arquitectura e información.

La justificación de este capítulo en la tesis es la de proporcionar una base panorámica, ordenada y coherente en la que se puedan contextualizar los invariantes pedagógicos que se desgranan a lo largo de los cuatro capítulos del Atlas Propedéutico. 
i0/41 Color. Ejercicio del curso de Nonné Schmidt. HfG. Archiv. FBS
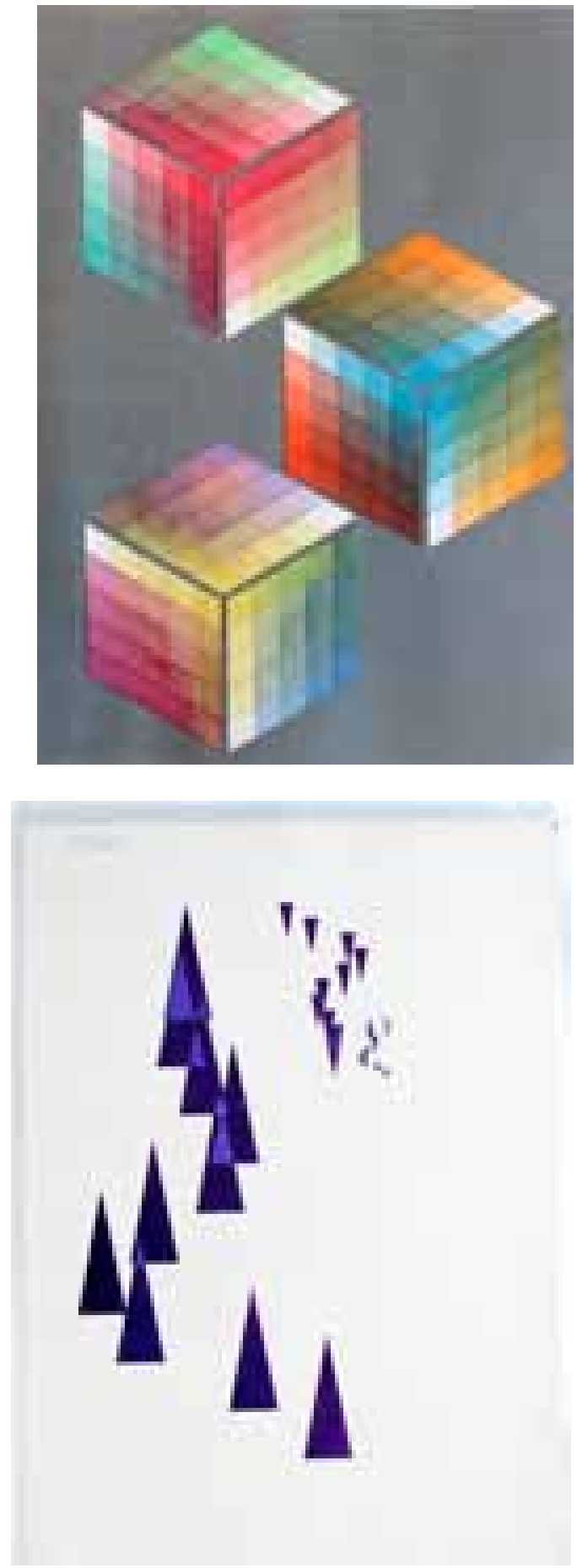

i0/43 Forma. Ejercicio de simetría del Kurs Maldonado. HfG. Archiv. FBS i0/42 Materia. Ejercicio del curso de Walter Zeischegg. HfG. Archiv. FBS
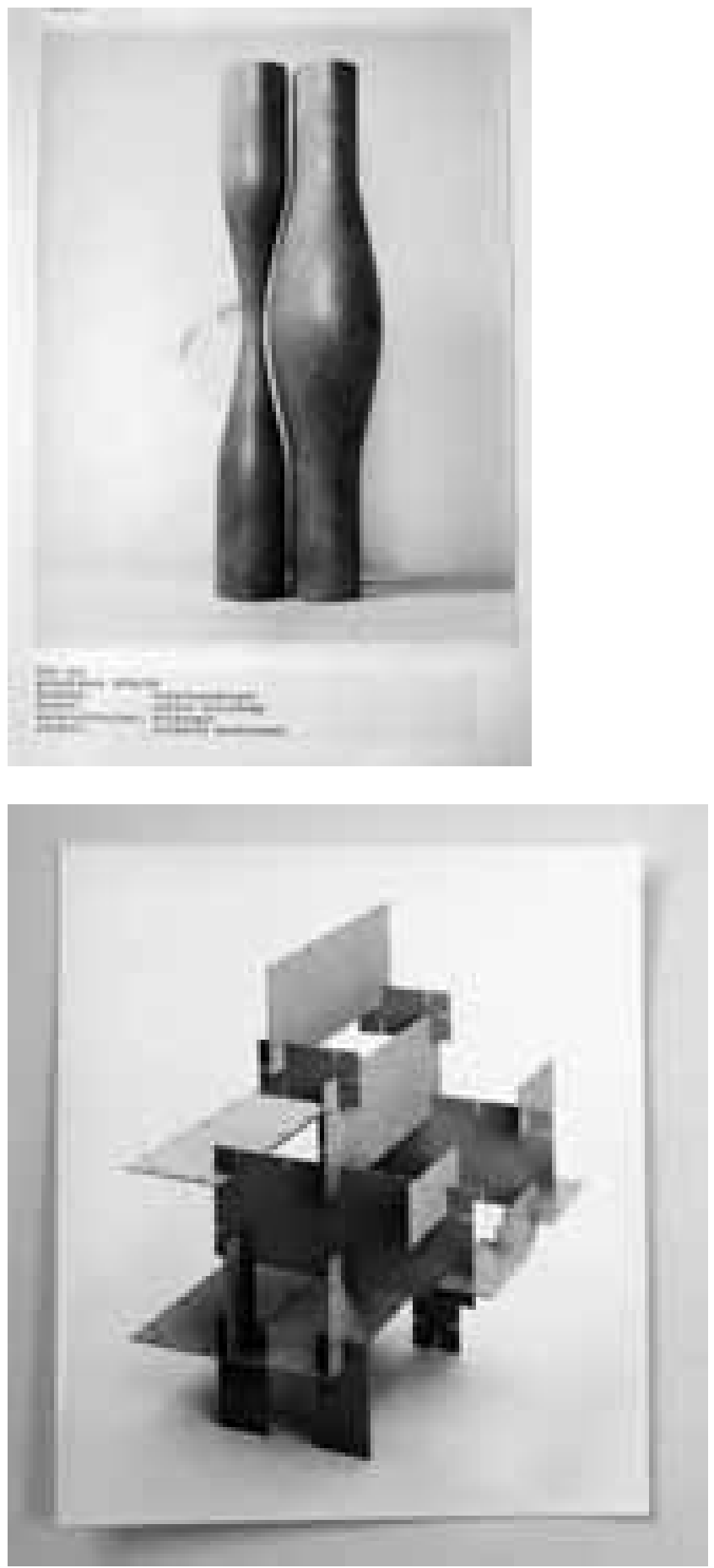

i0/44 Proyecto. Ejercicio del curso de Froshaug. HfG. Archiv. FBS 


\subsubsection{Atlas propedéutico}

Esta parte construye un marco postreferencial organizado como una taxonomía de cuatro grandes ámbitos en los que se sintetizarán los invariantes didácticos que vertebraron la existencia del Curso Básico a lo largo de la etapa estudiada.

Los ámbitos sobre los que se configura el discurso son cuatro grandes temas comunes presentes en la didáctica de las disciplinas visuales de cada fase: color, forma, materia y proyecto.

En cada categoría, los ejercicios se sitúan en un orden secundario que atiende a cualidades específicas de cada grupo y que han surgido del formato atlas. Del archivo se extrae un abundante material que tiene implícito distintos órdenes: cronológico, temático, de profesor, del alumno que ejecuta la tarea... cada ejemplo es singular. Si se observan los ejercicios yuxtapuestos, se desvelan de forma automática y espontánea, afinidades y divergencias que sugieren un camino para recomponer de nuevo el orden de estudio. Cada ámbito se concreta como un capítulo que sintetiza la contribución específica de cada docente a partir de las herramientas, procesos y métodos del aprender a proyectar propios de su didáctica, para configurar de modo panorámico una propedéutica del Curso Básico de la HfG. El análisis comparativo y su contextualización en el marco teórico conveniente en cada caso, fija el nuevo orden.

La revisión crítica de cada campo está basada en el análisis comparativo de los ejercicios que se proponen en torno al tema, de esta forma, se evidencia la evolución en la didáctica de una disciplina. Cada campo acoge una diversidad de ejercicios que buscan profundizar en las dimensiones del desarrollo de las enseñanzas. Por ejemplo, el color comienza impartiéndose con didácticas heredadas de la Bauhaus y otras escuelas, profundiza en experiencias basadas en la Gestalt en otra época, para acabar incorporándose a las metodologías matemáticas de base combinatoria en la última etapa. La estructura dentro de cada tema sigue un orden de subtemas -color óptico, color visual, color háptico, color sintáctico- que responden a la evolución en la metodología de enseñanza y a la incorporación de nuevos enfoques. La estructura evita categorizar los cursos en referencia a sus docentes. ${ }^{47}$ Se pretende evitar de esta forma personalizar en la figura del docente y su particular didáctica, a favor de proporcionar una lectura global del Curso Básico en su conjunto.

Los cuatro capítulos que integran el atlas tienen el objetivo de trascender el carácter compilatorio y aportar una revisión crítica que sirva para calibrar la validez didáctica de los ejercicios propuestos en cada ámbito mediante su análisis panorámico y contextualizado. Maldonado ${ }^{48}$ afirma que la $\mathrm{HfG}$ ha heredado de la Bauhaus la idea de trabajo con una gramática visual que constituye una herramienta para aprender principios abstractos -es decir, sin aplicación práctica- que puedan aplicarse a problemas concretos de diseño. Las tres primeras categorías -color, forma y materia- proporcionan los elementos que integran la dimensión sintáctica de la escuela de Ulm y la última categoría -proyectos- introduce cuestiones como la componente semántica y pragmática de las enseñanzas. El recorrido se realiza desde la evolución de las componentes de ejercitación abstracta hasta la componente proyectual.
47 Existen manuales como el de Rainer Wick que realizan un recorrido por las enseñanzas de la Bauhaus en base a una estructura de la docencia de cada profesor. Esta forma de organizar un libro sobre pedagogía establece una predominancia de la figura -el profesor- sobre la idea global de la existencia de una serie de enseñanzas concatenadas y en evolución.

Wick, Rainer. Pedagogía de la Bauhaus. Madrid: Alianza Editorial, 1982.

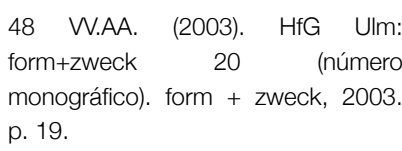


A lo largo del Atlas Propedéutico se acude a ejemplos de lo que se ha definido como periferia de la investigación, referentes del marco histórico que se utilizan como mecanismos de confrontación paralela para evaluar la validez de los ejercicios propuestos en la HfG. Si bien estos cuatro capítulos contienen conclusiones parciales, las concusiones generales se recogen en el séptimo y último capítulo previo a la bibliografía.

La bibliografía se ordena en tres partes; bibliografía comentada, que destaca las publicaciones de referencia para la elaboración de esta tesis, bibliografía específica de la $\mathrm{HfG}$ y bibliografía general. 


\section{6 Índice desarrollado}

introducción

0.1 Justificación del tema de tesis

0.2 Objetivos. Hipótesis de trabajo

0.3 Estado de la cuestión. Antecedentes

0.3.1 Periodo HfG 1948-1968

0.3.2 Tras el cierre de la HfG

0.4 Atlas como método

0.4.1 Identificación de lagunas

0.4.2 Recopilación del Atlas

0.4.3 Atlas Panóptico

0.4.4 Atlas Sinóptico

0.5 Estructura de la tesis

39

0.5.1 Marco didáctico

0.5.2 Atlas propedéutico

0.6 Índice ampliado

43 
1.1.1 Precedentes de las disciplinas visuales

1.1.2 Bauhaus

1.1.3 Vkhutemas-Vkhutein

1.1.4 New Bauhaus Chicago - Institute of Design

1.1.5 Illinois Institute of Technology

1.2 Estructura y docencia en la Hochschule für Gestaltung

1.2.1 Génesis del proyecto docente. Hitos fundacionales.

1.2.2 Objetivos de la escuela. Los fines.

1.2.3 Organización pedagógica por departamentos. Los medios.

1.2.4 Gestación, evolución y estructura del Grundlehre

\subsection{Curso Básico fundacional}

1.3.1 Contexto espacio-temporal del Curso Básico fundacional

1.3.2 Docentes y alumnos del periodo

1.3.2 Max Bill desde el arte concreto

1.3.3. Los cursos de Josef Albers en la HfG

1.3.4. Las enseñanzas de Nonné Schmidt

1.3.5. El seminario de Itten

1.4 Relevo Bill-Maldonado en el Curso Básico

1.4.1 Contexto espacio-temporal del relevo Bill-Maldonado

1.4.2 Docentes y alumnos del periodo

1.4.3 Docencia compartida entre Max Bill y Tomás Maldonado

1.4.4 Colaboración entre Tomás Maldonado y Herbert Ohl

1.4.5 Tomás Maldonado como docente autónomo

1.5 Reafirmación de la propuesta de Maldonado y la consolidación del Visuelle Einführung en el Curso Básico.

1.5.1 Contexto espacio-temporal del Visuelle Einführung

1.5.2 Docentes y alumnos del periodo

1.5.3 Programa de seminarios

1.5.4 Fusión didáctica de los primeros años con los temas del arte concreto

1.6 Reforma del curriculum del Curso Básico: hacia un nuevo Formgeber

1.6.1 Contexto espacio-temporal de la reforma

1.6.2 Docentes, materias y alumnos del periodo

1.6.3 Incorporación de Anthony Froshaug y el trabajo con grafos en Visuelle Methodik

\subsection{Metodología en el Curso Básico}

1.7.1 Contexto espacio-temporal de la etapa de la metodología

1.7.2 Docentes, materias y alumnos del periodo

1.7.3 La llegada de Rittel y la introducción de la metodología

1.7.4 Proyectos concretos: el abandono de la abstracción en las Metodische Übungen.

1.7.5 Pensamiento metodológico en las Grafische Darstellung

1.8 La disolución del Curso Básico en el curriculum de los departamentos

1.8.1 Contexto espacio-temporal de la etapa final del Curso Básico

1.8.2 Docentes y alumnos del periodo

1.8.3 Los departamentos en el Curso Básico y el papel de Bruce Archer

1.8.4 La madurez del Curso Básico en los departamentos 
Luz, oscuridad. Color. Círculo cromático

Colores primarios, secundarios, terciarios, cuaternarios Sólido de color de Oswald

\subsubsection{Escalas}

Escalas acromáticas y monocromáticas

Escalas polícromas

Escalas de colores con blanco y negro

\subsubsection{Teoría de contrastes}

\subsection{Color visual}

2.2.1 Color visual: enfoque experimental

$$
\text { Un color parece dos }
$$

Dos colores parecen uno

Mezcla ilusoria y transparencia

Colores progenitores

Intersección de colores

Transformaciones

Cantidad de color

Borde vibrante

Intensidad luminosa

Estudios libres

2.2.2 Color visual: enfoque sistemático

Proximidad-ambigüedad-semejanza

Fondo-ambigüedad-primer plano

Estructuras y superficies de color en equilibrio visual

Cantidad de color

\subsection{Color Háptico}

\subsection{Color sintáctico}

2.4.1 Topología y color

Superficie de Sierpinski

La Curva de Peano como activadora del color

Curva de Peano: sistema de brillo de color equivalente, sistema

de figura-fondo positivo-negativo

Escala de color lineal sobre la Curva de Peano

Relatividad del color en la Supercie de Peano

Superficie de Peano: negro como color

\subsubsection{Distribución sistemática o aleatoria}

Distribución de color mediante un patrón

Comparativa entre campos coloreados al azar

Similitud de los campos por brillo y/o color

Escala de grises aleatoria / sistemática y con dado al azar

2.5 Contribución a la didáctica del color conclusiones parciales atlas color

El color pragmático

El color y otras variables

Entre procesos exploratorios y sistemáticos

Color, función y significado

La disección del color

Arquetipos didácticos en la enseñanza del color 
3.1.1 El contorno como representación de la forma

3.1.2 El dibujo del natural como representación de la forma

3.1.3 La luz en la fotografía para la representación de la forma

3.2 Forma y configuración

3.2.1 Estructura

Distribución visual: proporción y dinámica

Subdivisión de la forma: arquetipos estructurales

Simetría como ley generativa

3.2.2 Movimiento

Trayectorias

Estelas

3.2.3 Iteración

Iteración visual aleatoria

Iteración visual programada

3.2.4 Espacio

Solapamiento

Gradientes

Perspectiva, encuadre y ángulo

3.3 Contribución a la didáctica de la forma conclusiones parciales atlas forma

Atlas Forma

\subsection{Materia en el laboratorio}

4.1.1 Analizar la materia

Evidenciar las cualidades mediante el medio gráfico

\section{El dibujo}

La iluminación en la fotografía

Organizar las cualidades de la materia mediante el medio gráfico

Collage de texturas gráficas

Collage de papeles de colores

Fotografía en serie

4.1.2 Experimentar con la materia

Alambre
Arena
Pigmento

\subsection{Materia en el taller}

4.2.1 Construcción de superficies

Superficies plegadas

Estudios de transición

Superficies no orientables

4.2.2 Construcción de sólidos

Sólidos por simetría

Sólidos por rotación

Sólidos por intersección

4.2.3 Contrucción de redes espaciales

Redes espaciales construidas con dos materiales

Redes espaciales construidas con un material 
5.1 Tipos y temas de proyecto

5.1.1 Áreas temáticas

proyecto

5.1.2 Invariantes del proyecto

5.2 Estrategias básicas de proyecto

469

5.2.1 Orden de la información

5.2.2 Comparación de magnitudes

5.2.3 Abstracción de la información

5.2.4 Síntesis de datos

5.3 Proyecto de procesos

5.3.1 Representación de un fenómeno con intervención del azar

5.3.2 Representación de estrategias de trabajo y protocolos de actuación

5.4 Proyecto de elementos

5.4.1 Expositor para un ejercicio de taller

5.4.2 Bastidor de soporte para una esfera

\subsection{Proyecto de sistemas}

5.5.1 Sistemas de patrones

Rueda de trinquete

Contenedor de transporte en aluminio

Lingotes apilables de aluminio

Juguete constructivo

5.5.2 Sistemas modulares

Sistema legible por hombres y máquinas

Formato de composición para revista

Sistema de pesas

Sistema de configuración del espacio mediante unidades funcionales

5.6 Contribución a la didáctica del proyecto

conclusiones parciales atlas proyecto

Atlas Proyecto

Proyecto de reconstrucción cultural

La superación del mito Bauhaus desde la didáctica

Tomás Maldonado y Walter Zeischegg como catalizadores del Grundlehre

La pedagogía del sistema

El Grundlehre como experimento de transformación didáctica

7.1 Bibliografía comentada

7.2.4 Documentos audiovisuales 



\section{1 marco didáctico}

\subsection{Enseñanzas visuales en los cursos básicos precursores}

1.1.1 Precedentes de las disciplinas visuales

1.1.2 Bauhaus

1.1.3 Vkhutemas-Vkhutein

1.1.4 New Bauhaus Chicago - Institute of Design

1.1.5 Illinois Institute of Technology

\subsection{Estructura y docencia en la Hochschule für Gestaltung}

1.2.1 Génesis del proyecto docente. Hitos fundacionales.

1.2.2 Objetivos de la escuela. Los fines.

1.2.3 Organización pedagógica por departamentos. Los medios.

1.2.4 Gestación, evolución y estructura del Grundlehre

\subsection{Curso Básico fundacional}

1.3.1 Contexto espacio-temporal del Curso Básico fundacional

1.3.2 Docentes, materias y alumnos del periodo

1.3.3 Peterhans y el Visual Training

1.3.4 Max Bill desde el arte concreto

1.3.5 Las enseñanzas de Nonné-Schmidt

1.3.6 Enseñar a mirar. Josef Albers en la HfG

1.3.7 El seminario de Itten

\subsection{Relevo Bill-Maldonado en el Curso Básico}

1.4.1 Contexto espacio-temporal del relevo Bill-Maldonado

1.4.2 Docentes, materias y alumnos del periodo

1.4.3 Docencia compartida entre Max Bill y Tomás Maldonado

1.4.4 Colaboración entre Tomás Maldonado y Hans Gugelot

1.4.5 Tomás Maldonado como docente autónomo 

1.5 Reafirmación de la propuesta de Maldonado y la consolidación del Visuelle Einführung en el Curso Básico.

1.5.1 Contexto espacio-temporal del Visuelle Einführung

1.5.2 Docentes, materias y alumnos del periodo

1.5.3 Programa de seminarios

1.6 Reforma del curriculum del Curso Básico: hacia un nuevo Formgeber

1.6.1 Contexto espacio-temporal de la reforma

1.6.2 Docentes, materias y alumnos del periodo

1.6.3 Incorporación de Anthony Froshaug y el trabajo con grafos en Visuelle

Methodik

\subsection{Metodología en el Curso Básico}

1.7.1 Contexto espacio-temporal de la etapa de la metodología

1.7.2 Docentes, materias y alumnos del periodo

1.7.3 La llegada de Rittel y la introducción de la metodología

1.7.4 Proyectos concretos: el abandono de la abstracción en las Methodische Übungen.

1.7.5 Pensamiento metodológico en las Grafische Darstellung.

1.8 La disolución del Curso Básico en el curriculum de los departamentos

1.8.1 Contexto espacio-temporal de la etapa final del Curso Básico

1.8.2 Docentes, materias y alumnos del periodo

1.8.3 Los departamentos en el Curso Básico y el papel de Bruce Archer

1.8.4 La madurez del Curso Básico en los departamentos 


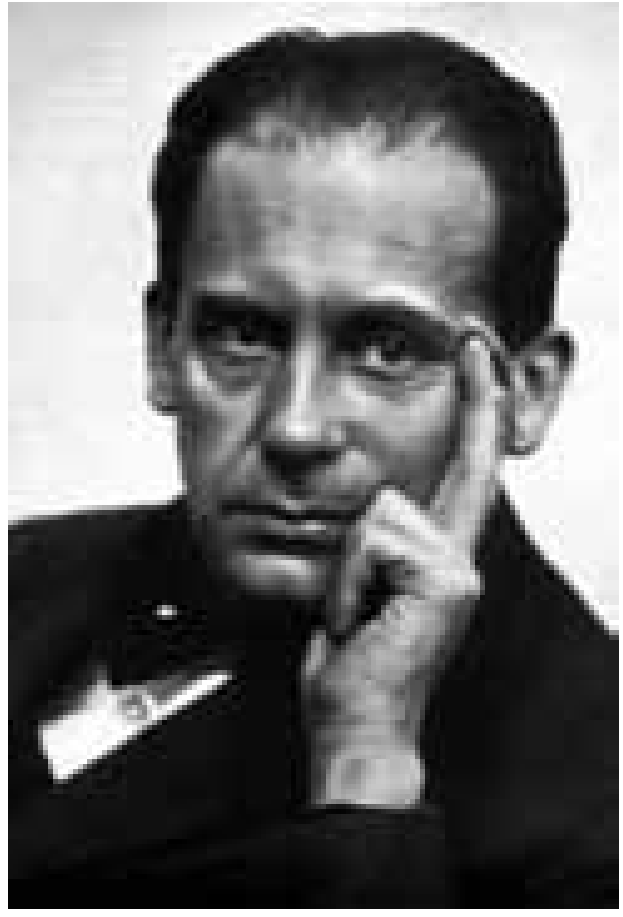

i1/1 Walter Gropius, 1920 


\subsection{Enseñanzas visuales en los cursos básicos precursores}

Porque actualmente la adaptación a una época que ha cambiado completamente exige una gran elasticidad por parte de todos; puesto que han desaparecido totalmente las antiguas premisas... y los hombres han cambiado, sobre todo por causa de la guerra, la reconstrucción es siempre experimento en el sentido literal de la palabra. ${ }^{1}$

Tras el primer año de existencia de la Bauhaus, Gropius defiende su experimento ante la Dieta de Turingia, justificando la condición innovadora de la Bauhaus en su tiempo. Pone de manifiesto que su proyecto no parte de cero; la Bauhaus pertenece al conjunto de iniciativas surgidas durante el primer tercio del siglo XX bajo la reforma de las escuelas de arte, como respuesta a la inquietud de la época que requería de un cambio profundo en las enseñanzas artísticas.

Rainer Wick identifica cinco cvaracterísticas comunes a las distintas experiencias llevadas a cabo en la reforma. ${ }^{2}$ La primera es la "superación de la academia" incapaz de ajustarse con realismo a la situación de la época, por la obsolescencia de su planteamiento didáctico. La "fusión de formación artística con la artesanía" es la segunda idea que actúa como estrategia para aunar el potencial pedagógico y económico de la técnica y el oficio artesano. La tercera es el "principio de formación en talleres" como espacios tanto para el aprendizaje como para la producción. La integración de la arquitectura con las demás artes libres y aplicadas como idea de la escuela unificada de arte, es el cuarto eje de la reforma que gira entorno a la fusión entre arte y técnica para la formación de artistas libres y aplicados. La última idea presente en el movimiento reformista es la idea de un nivel o escuela preliminar, un espacio iniciático en el que ponerse a prueba en el inicio de unos estudios para conocer las capacidades propias y para facilitar la continuidad posterior.

Wick afirma que la "idea de un nivel o escuela preliminar", un primer curso común de estudios o Curso Básico, tiene como objetivo el de "servir para la propia práctica y autoexploración de los que empiezan sus estudios, para la comprobación de sus aptitudes creadoras y como ayuda con vistas a la decisión en un momento especialmente crítico de los estudios"3

Estos programas tenían la intención de ayudar a los nuevos estudiantes en la autoexperiencia y el auto-descubrimiento, para poner a prueba sus posibilidades creativas, para introducirlos en cuestiones básicas de diseño, y para ayudarlos a elegir un área específica de estudio o un énfasis específico de estudio. ${ }^{4}$

A lo largo de este capítulo se identifican los principales referentes históricos del Curso Básico en la HfG. El objetivo es crear un panorama de acercamientos didácticos a las disciplinas visuales como enseñanzas preparatorias para el proyecto que permitan vislumbrar los vínculos y las diferencias existentes entre estas escuelas con la didáctica del Grundlehre.
1 Walter Gropius. Discurso pronunciado ante la Dieta de Turingia, en Weimar, el 9 de julio de 1920. Acta de la LXXXIII sesión de la Dieta de Turingia, 9 de julio de 1920. Punto 1: Primera lectura del presuepuesto de la Bauhaus Estatal de Weimar.

En: Wingler, Hans M. La Bauhaus. Weimar, Dessau, Berlín. Barcelona: Gustavo Gili, 1975. p. 57. Edición original: Bramsche, Gebr. Rasch. \& Co., 1962.

2 Wick, Rainer. Pedagogía de la Bauhaus. Madrid: Alianza Editorial, 1982.p. 58-61.

3 Ibidem p. 61.

4 Wick, Rainer K. Marginalia on the History of the Vorkurs, en Krampen, M. Beobachten und formulieren. Grundkurs mit Übungen, nach einem Filmskript von Josef Albers. Zentrum für Kunst und Medientechnologie Karlsruhe ZKM, 2009, p.3 

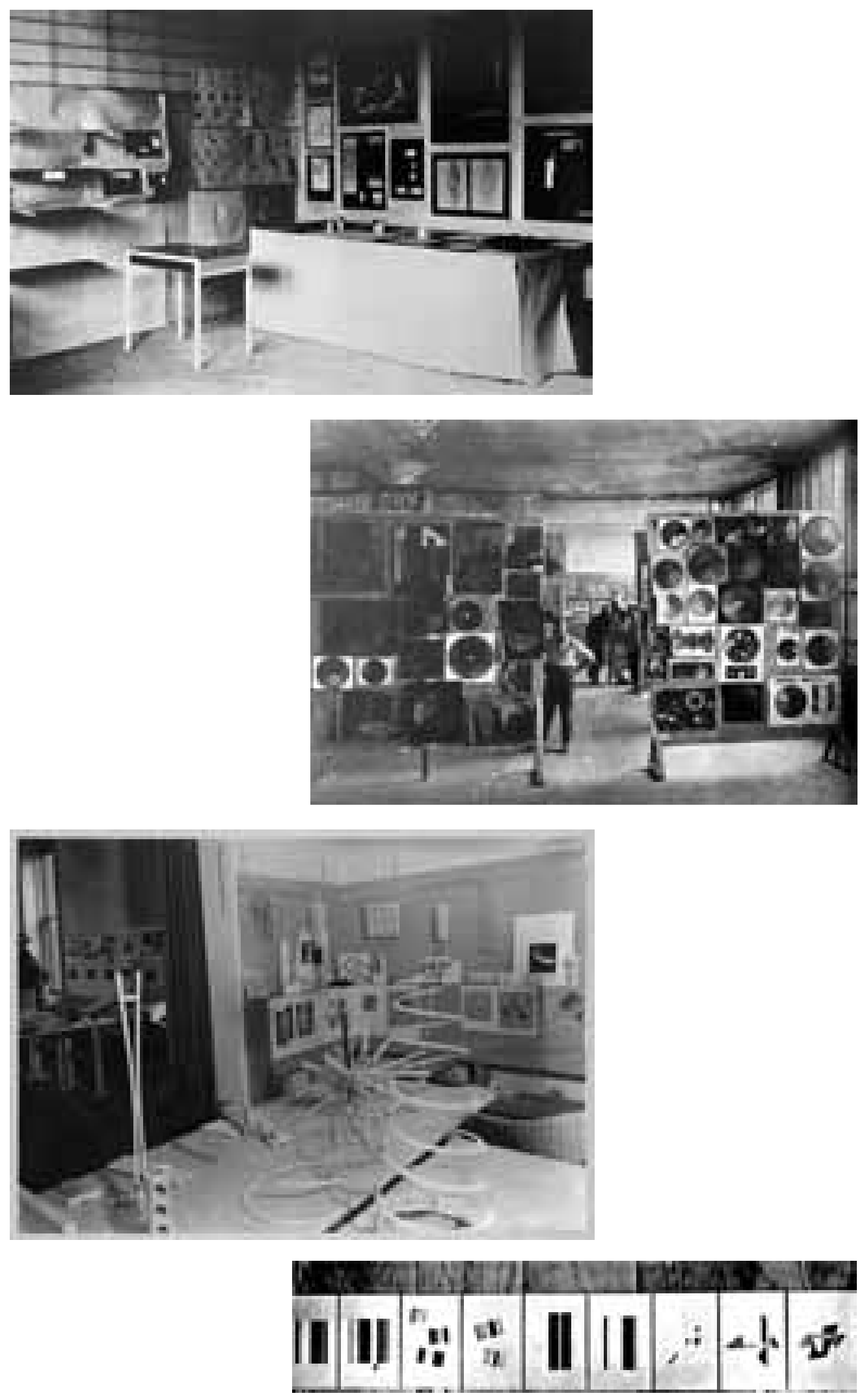

Exposiciones de trabajos de las disciplinas visuales.

11/2 Vorkurs, Bauhaus, Dessau 1927.

11/3 Disciplina Color, Vkhutemas, Moscú, 1926.

i1/4 Curso Básico, New Bauhaus, Chicago, 1930.

i1/5 Visual Training, Illinois Institute of Technology, 1944 


\subsubsection{Precedentes de las disciplinas visuales}

La bibliografía señala al Vorkurs de la Bauhaus como el referente más directo de las enseñanzas visuales que se desarrollan en el Curso Básico de la Hochschule für Gestaltung Ulm.

El propio Tomás Maldonado vincula el origen de su disciplina Visuelle Einführung ${ }^{5}$ con una tradición pedagógica que asocia a tres momentos. El primero es el "tiempo del expresionismo", que enmarca en las dos primeras décadas del siglo XX, vinculado al movimiento de las escuelas de trabajo y al movimiento de la educación artística. El segundo momento lo representan en la Bauhaus las figuras de Itten, Albers, Moholy-Nagy, Klee y Kandisnky. Y, finalmente, resalta los referentes posteriores a la Bauhaus en las figuras de Moholy-Nagy con su extensión de la educación a la periferia de la ciencia a través de la semántica y de Albers como una continuación de la idea de la Bauhaus bajo una mayor consideración de los problemas que surgen de las nuevas ciencias.

El Vorkurs de la Bauhaus es un proceso de síntesis sucesivas a partir de las experiencias didácticas de los responsables a cargo del Vorkurs o Curso Preliminar.

Los Vkhutemas son la institución dentro de la Rusia post-revolucionaria con una historia paralela a la Bauhaus en la que se logra sistematizar una enseñanza preparatoria organizada por áreas.

La New Bauhaus Chicago-Insitute of Design representa la primera sucesora de la Bauhaus tras su desaparición y fue coetánea a la HfG. La organización del curso básico se proyecta a partir de las experiencias de Moholy-Nagy en Alemania y se amplía y sistematiza por parte de sus sucesores.

En la enseñanza de la Arquitectura a cargo de Mies van der Rohe en el Illinois Institute of Technology no existe un curso básico como tal, sin embargo, Walter Peterhans imparte un seminario, Visual Training, que está incorporado como enseñanza preparatoria para la formación de los arquitectos en el IIT.

La reconstrucción de la didáctica de las enseñanzas visuales del Curso Básico de la HfG parte necesariamente de revisión contextualizada de estos y otros referentes que pertenecen al marco de influencia del Bauhaus y entroncan directamente con la historia del Grundlehre.
5 Ulrich Burandt. Apuntes del curso Visuelle Einführung de Tomás Maldonado, 1956-1957. Dp_033.012, p. 1. 
Ejercicio del Vorkurs de Itten

i1/6 Estudio del ritmo. Werner Graeff, 1920

11/7 Estudio de luz y oscuridad con el caracter del cuadrado y el círculo. Friedl Dicker, 1919

i1/8 Estudio de materiales n. 4. Vincent Weber, 1920.

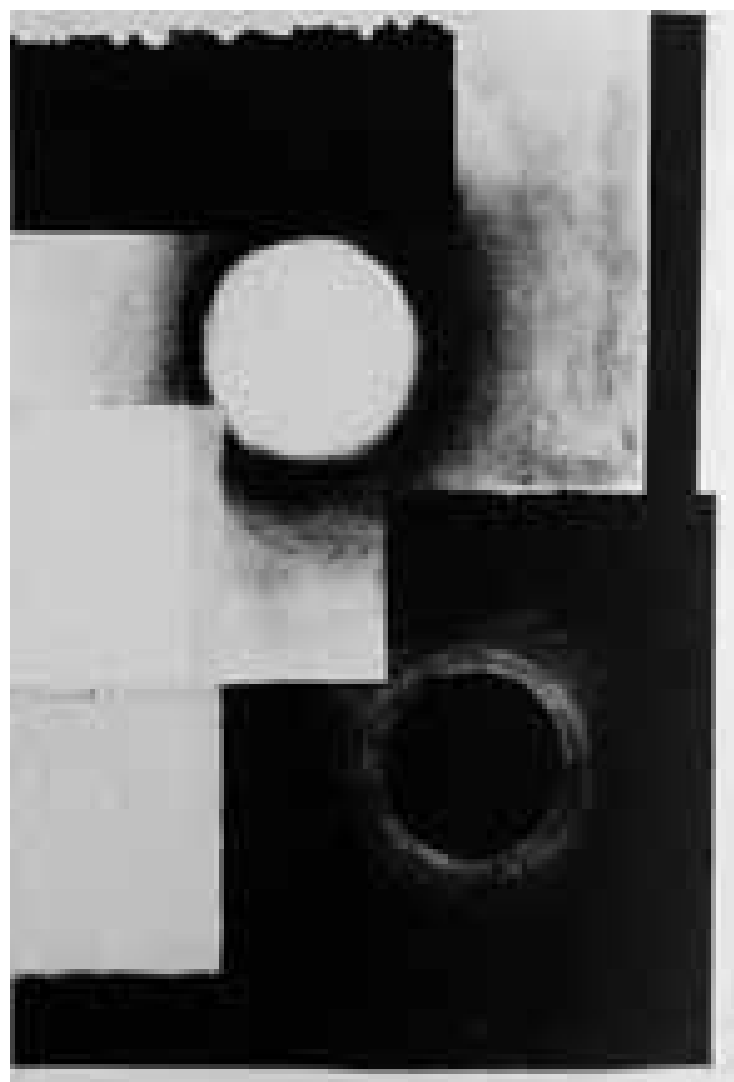

$i 1 / 7$

$i 1 / 9$

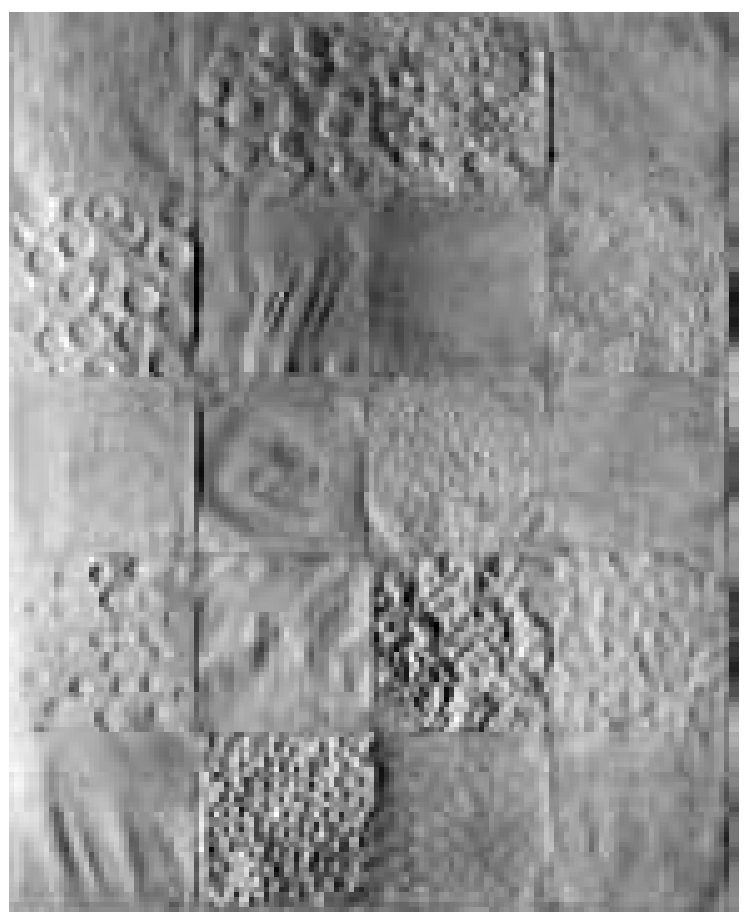

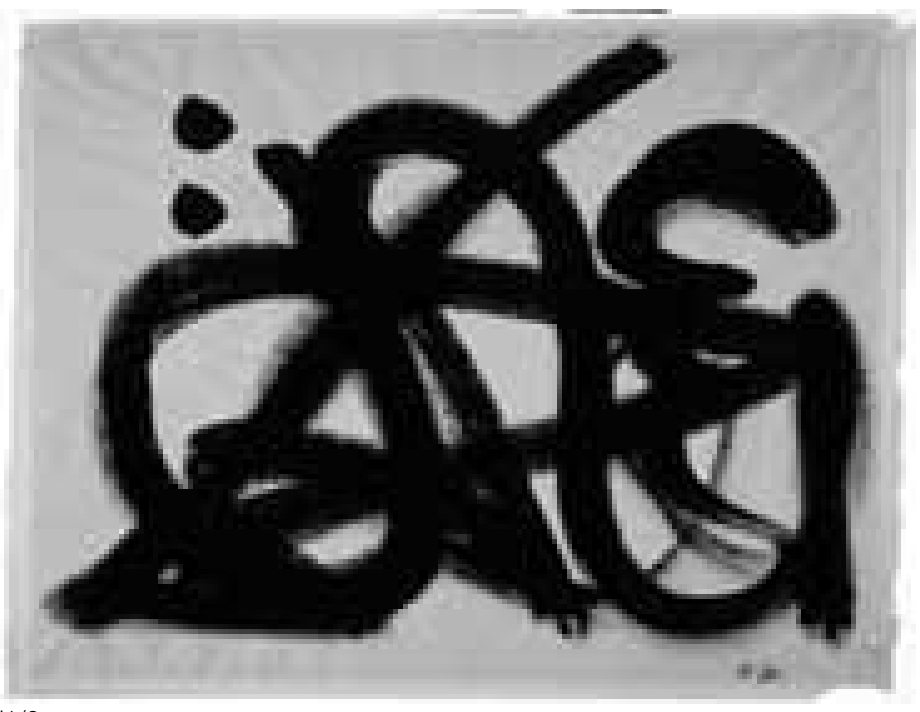

$i 1 / 6$

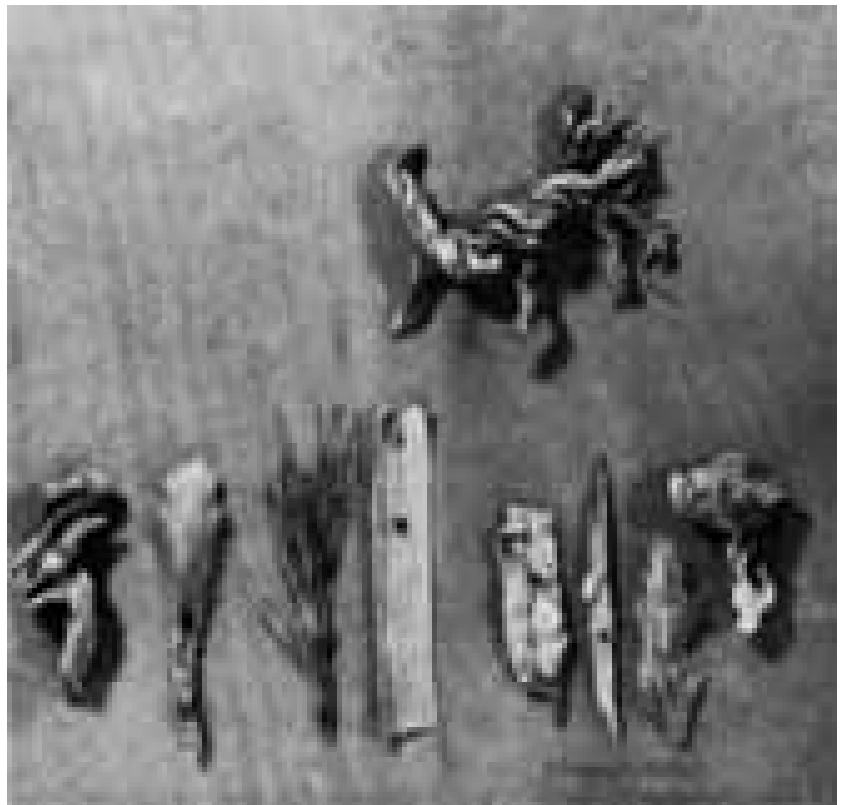

$i 1 / 8$

Ejercicios del Vorkurs de Moholy-Nagy

i1/9 Ejercicios de factura en papel con distintos utensilios. Gerda Marx, 1927 i1/10 Tabla táctil de dos celdas con diagramas para el intento de su traducción gráfica. Willy Zierath, 1927

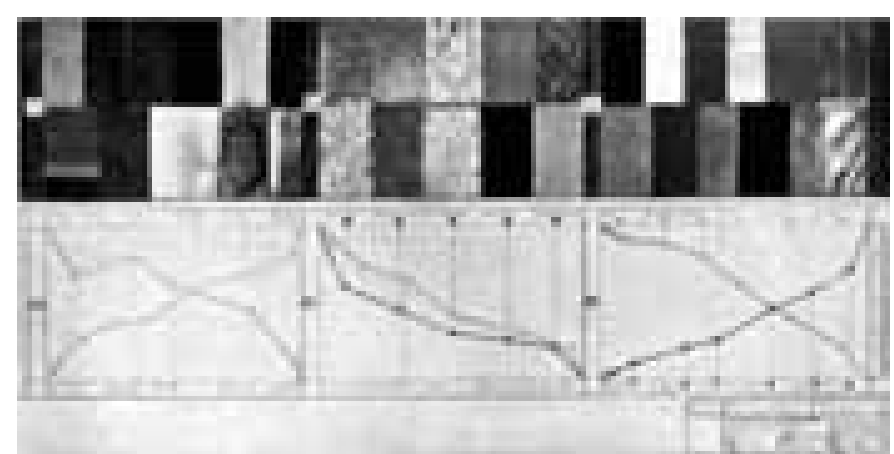




\subsubsection{Bauhaus}

El Vorkurs es tradicionalmente el referente inicial de las enseñanzas visuales en el Grundlehre de la HfG. A pesar de su transcendencia posterior y su mitificación ${ }^{6}$ como seña de identidad del Bauhaus, no se puede afirmar que existiera un único curso básico. Muy al contrario, el Vorkurs es un espacio didáctico en permanente redefinición por los tres profesores que lo dirigieron ${ }^{7}$. La sucesión y transformación de la didáctica es el fruto indisociable ${ }^{8}$ entre el bagaje artístico y docente de Itten, Moholy-Nagy y Albers. Cada uno construyó su espacio didáctico como enseñanza preparatoria contribuyendo a los objetivos de la escuela. La identificación de los elementos singulares de cada docente proporciona los hitos más significativos de la evolución de la didáctica del Vorkurs en la Bauhaus a modo de referentes para el Grundlehre de la HfG

\section{Johannes Itten: pedagogía de la divergencia}

Itten es el pionero capaz de crear un Vorkurs con un lenguaje y metodología propias sobre los fundamentos de la pedagogía reformista y a partir del influjo de los movimientos de vanguardia. Su enseñanza en la Bauhaus es una reelaboración de las experiencias desarrolladas en su etapa formativa como maestro de primera enseñanza y como artista. ${ }^{9}$ Centra el protagonismo de su labor en el desarrollo de las capacidades del alumno. Sus tareas contribuyen al triple objetivo del Vorkurs de "liberar sus fuerzas creadoras", facilitar la elección de un taller para su formación posterior, así como el conocimiento de las leyes fundamentales de la creación plástica. ${ }^{10}$

La secuencia didáctica de Itten comienza por los ejercicios gimnásticos para "despertar en el cuerpo la expresividad"11 y "ejercicios rítmicos de las formas" (i1/6) para "experimentar físicamente el movimiento y el ritmo como el principio existencial básico y como principio organizativo plástico". ${ }^{12}$ El estudio general de contrastes es el fundamento teórico del Vorkurs, ya que "todo lo perceptible es perceptible por su divergencia". ${ }^{13}$ Itten somete el aprendizaje de los alumnos a una dialéctica en la que los elementos visuales se organizan a partir de conceptos extremos. (i1/7) Esta lógica de opuestos se aplica a las condiciones de la forma bidimensional y tridimensional -recto-curvado, puntiagudo-romo, superficie-línea, superficie-cuerpo-; a la dimensión y la cantidad -mucho-poco, grande-pequeño, largocorto, ancho-estrecho- y a las cualidades físicas -áspero-liso, ligero-pesado-. En el caso del color, Itten plantea el uso de siete tipo de contrastes ${ }^{14}$ que permiten que los alumnos afinen y armonicen su agudeza visual con su destreza técnica.

Los estudios de material y la textura son la herramienta para adiestrar el sentido visual y táctil que facilita la decisión por uno de los talleres. (i1/8) El conjunto de tareas se completa con los estudios de la naturaleza como dibujos fotográficamente exactos contribuyen a "ampliar el pensamiento concreto". ${ }^{15}$

\section{Laszlo Moholy-Nagy: dialéctica de la materia}

El horizonte educativo de Moholy-Nagy es el "hombre total" y el camino para su educación es la "educación de los sentidos."16 Para ello construye un repertorio de tareas orientadas a la sensibilización del sentido táctil y visual de los alumnos que recoge en Von Material zu Architektur, una recopilación de su labor y pensamiento pedagógico en el Vorkurs.
6 Wick, Rainer. Pedagogía de la Bauhaus, op. cit, p. 79.

7 Itten dirige el Vorkurs entre 1919 y 1923. Laszlo Moholy Nagy lo asume entre 1923 y 1928 . Entre 1925 y 1928 Albers impartía el primer semestre y Moholy-Nagy el segundo. A partir de 1928 y hasta el cierre de la Bauhaus en 1933, Albers se hizo cargo del Vorkurs.

8 Wick, Rainer. Pedagogía de la Bauhaus, op. cit, p. 118

9 Entre 1909 y 1912 se forma con Gilliard en Ginebra de quien adquiere los fundamentos para la interacción entre las Formas. Entre 1914 y 1916 en Stuttgart, aprende con Holzel la teoría de contraste de los colores -que se remonta a Goethe- el análisis de las obras de los antiguos maestros, el claroscuro, los collages a partir de fragmentos, los ejercicios gimnásticos y el estímulo hacia el dibujo automático. De regreso a su academia privada en Viena pone en práctica estas enseñanzas previamente a su llegada a la Bauhaus en 1919. Wick, R op. cit. p. 80-81.

10 Ibidem, p. 88.

11 (Itten, , "Tagebuch", 2 de marzo de 1918, en Rotzler, Willy (ed.) Johannes Itten. Werke und Schriften, Zúrich, 1978 citado en Wick, p89)

12 lbidem, p.89

13 Ibidem, p. 89

14 Contraste del color en sí mismo, contraste de claroscuro, contraste fríocaliente, contraste complementario, contraste simultáneo, contraste de calidad, contraste de cantidad.

15 (Itten, , "Tagebuch", 2 de marzo de 1918, en Rotzler, Willy (ed.) Johannes Itten: Werke und Schriften, Zúrich, 1978 citado en Wick, Rainer. Pedagogía de la Bauhaus, op. cit, $\mathrm{p}$. 93.

16 Ibidem, p. 129 


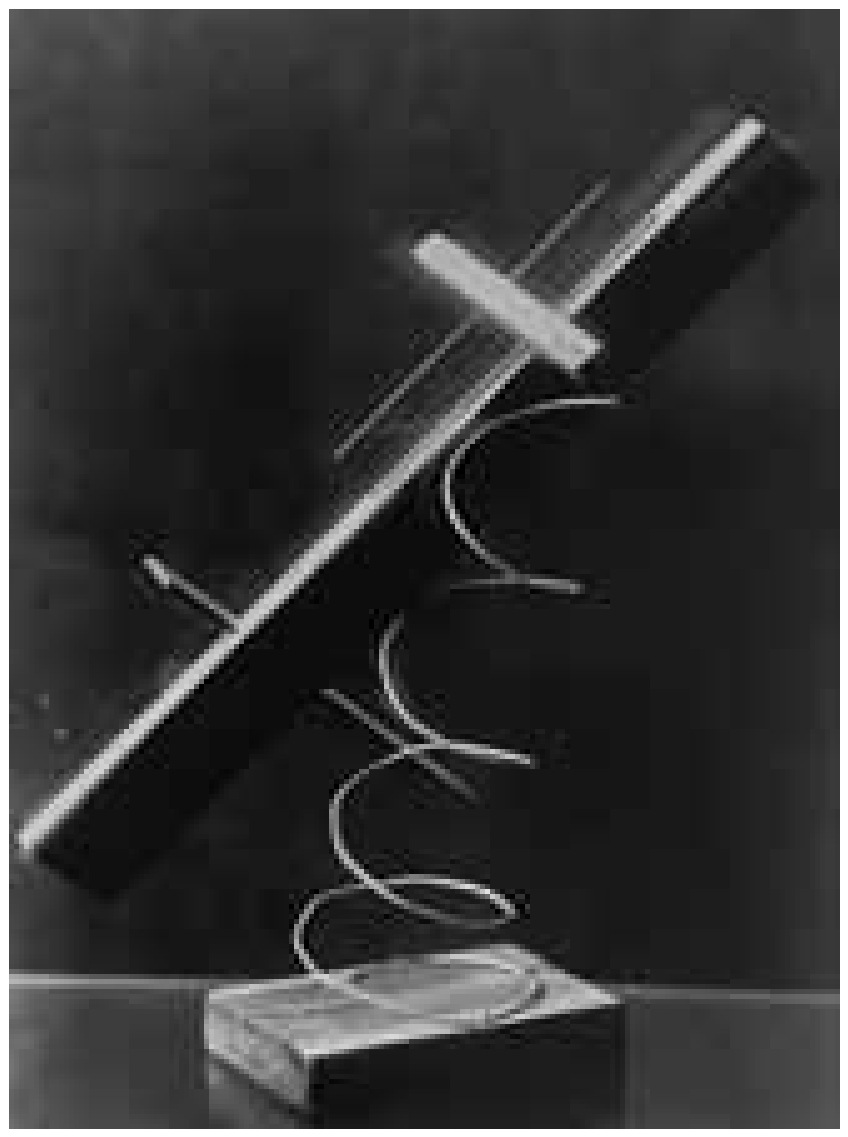

Ejercicio del Vorkurs de Moholy-Nagy

i1/11 Escultura suspendida. Estudio ilusionista y de equilibrio. Corona Krause,
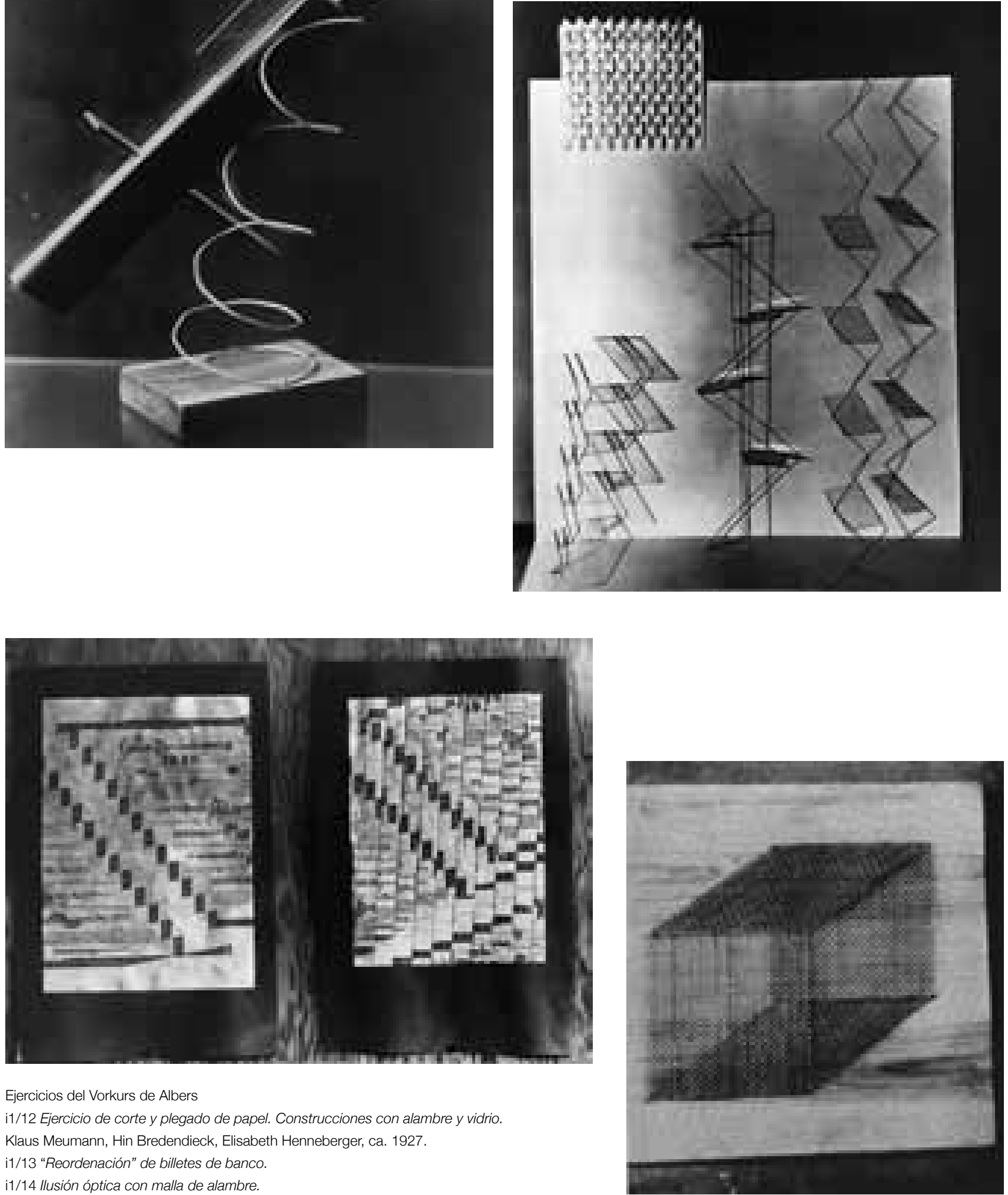

Ejercicios del Vorkurs de Albers

i1/12 Ejercicio de corte y plegado de papel. Construcciones con alambre y vidrio.

Klaus Meumann, Hin Bredendieck, Elisabeth Henneberger, ca. 1927.

i1/13 "Reordenación" de billetes de banco.

i1/14 llusión óptica con malla de alambre. 
Las tablas sensoriales son ejercicios para la sensibilización del sentido del tacto que se organizaban de forma sistemática y graduada conforme a un criterio de escalas. Los materiales "se transformaban inmediatamente en cualidades abstractas y en relaciones de estas"17 mediante los diagramas táctiles que acompañan el montaje de materiales. (i1/10)

En las transformaciones graficas o pictóricas de los materiales, lo estudiantes "copian" el montaje de materiales para agudización sensorial óptica y práctica de la técnica gráfica.

El siguiente paso en la secuencia didáctica de Moholy-Nagy son los ejercicios de faktura, como ejercicios con el material "transformado desde fuera". ${ }^{18}$ (i1/9) Tras ellos, los ejercicios de construcción tridimensional abordan el tratamiento del material a partir de cinco fases que sistematizan la evolución histórica de la plástica desde la idea "de la masa al movimiento". ${ }^{19}$ Este proceso consiste en la progresiva reducción del volumen y una tendencia hacia la ligereza y el movimiento a modo de secuencia morfológica. Los trabajos del curso preliminar se centran principalmente en las tres fases más avanzadas en las que los alumnos utilizan madera, metal, cristal y plástico para la creación de esqueletos estructurales bajo la directriz de la economía de medios. Los estudios de equilibrio se enmarcan en la "fase flotante". Introducen al alumno mediante la experimentación al conocimiento más profundo del comportamiento de los materiales y le proporcionan elementos para composición a partir de categorías estéticas: dimensión y proporción, estática y dinámica y tensión y contraste. (i1/11) La fase cinética la representan esculturas móviles que incorporan mediante la idea "relación situacional de cuerpos"20 la ocupación del espacio en sentido arquitectónico.

\section{Josef Albers: fusión didáctica}

El Vorkurs alcanza su madurez gracias Albers, que consigue sistematizar la didáctica de sus precursores. ${ }^{21}$ El análisis de los materiales de trabajo orientado como una educación visual ${ }^{22}$ es el tema central de su enseñanza en la Bauhaus. Albers crea una metodología propia basada en los principios racionales de economía de medios -minimizando los procesos, utilizando de forma limitada los instrumentos- y economía del material -utilizando de forma óptima los materiales, sin recortes ni sobrantes. Bajo estas condiciones como límites de trabajo los estudiantes exploran las posibilidades constructivas y visuales de los materiales.

Albers redefine la labor de Itten y Moholy-Nagy con dos objetivos. Los Materialstudie o ejercicios con el material, son tareas para la construcción intuitiva a partir de las "energías internas" del material. (i1/12). A través de una selección de materiales comunes y accesibles los estudiantes experimentan de forma no convencional sus posibilidades constructivas. ${ }^{23}$ Al mismo tiempo se propicia el Materialgerecht o trabajo adecuado a sus propiedades que revela nuevas posibilidades desconocidas en su uso. ${ }^{24}$

La segunda intención fundamental de Albers es la sensibilización visual a través de los Materiestudie. Estos ejercicios con la materia se centran en el estudio sensorial de la superficie del material. Los estudiantes ordenan los materiales en "cadenas de progresión entre polaridades" ${ }^{25}$ Con ello Albers persigue agudizar la capacidad del estudiante para establecer relaciones entre materiales a partir de sus analogías o contrastes.

Pero la gran innovación de Albers en el Vorkurs son las tareas que exploran los umbrales de la percepción visual a modo de experimentos gestálticos. (i1/13, i1/14) Los alumnos manipulan los materiales para crear la ilusión de movimiento, transparencia y volumen, o para experimentar la ambigüedad entre fondo y figura. Con ello se completa el germen de su curso de Diseño Básico que madurará en el Black Mountain College y con el que llegará al Grundlehre en Ulm.
17 Ibidem, p. 133

18 Moholy-Nagy Von Material zu Architektur p. 43 La faktura es junto a la estructura, la textura y la acumulación, uno de las cuatro categorías que vinculan la acción plástica con la configuración visual de los materiales.

Estructura: "la forma inalterable de construcción de la estructura del material", esto es, la relación estructural del material.

Textura: "superficie final orgánica de cada estructura hacia afuera. epidermis, orgánico"

Faktura: "la forma y la apariencia, el resultado sensorialmente perceptible -la influencia- que se muestra en toda transformación de material. Esto es, la superficie del material transformado desde fuera -epidermis, artístico-".

Acumulación regular, irregular rítmicamente organizada: estado del material en el que el rasgo típico es la adición y que -sin una fuerte coherencia interna- es "generalmente fácil de modificar."

Moholy-Nagy, L. Von Material zur Architektur citado en Wick, Rainer Pedagogía de la Bauhaus, op. cit, p. 134-135

19 Moholy-Nagy presenta estas fases acompañadas de obras propias, de ejemplos históricos y de artistas coétaneos, así como de alumnos de la Bauhaus Las cinco fases son: fase del bloque, fase del modelado, fase de perforado (agujereado), fase flotante y fase cinética (móvil). Moholy-Nagy, L. Von Material zur Architektur citado en Wick p. 137.

20 Wick, R. op. cit 140.

21 Maldonado, Tomás: "Opinions: Is the Bauhaus relevant today?" Ulm 8/9, p 12. Citado en: Huff, W. S. "Grundlehere at the HfG-with a focus in "Visuelle Grammatik", en ulmer modelle-modelle nach ulm, op. cit, p 175. Además, bajo el mandato de Hannes Meyer, junto a las clases de Albers se incluyen disciplinas como geometría descriptiva, física, química, escritura o gimnasia.

22 Wick, Rainer K. Marginalia on the History of the Vorkurs, en: Krampen, Martin, Beobachten und formulieren. Grundkurs mit Übungen, nach einem Filmskript von Josef Albers. Zentrum für Kunst und Medientechnologie Karlsruhe ZKM, 2009, p.2

23 Horowitz, F. "Albers The Teacher". en: Horowitz, F y Danilowitz B. "Josef Albers: To open eyes. The Bauhaus, Black Mountain College, and Yale" NewYork: Phaidon, 2006. p.103.

24 ibidem, p 106

25 Albers, J. "La enseñanza de la forma en el taller" en catálogo Josef Albers medios mínimos, efecto máximo. publicado originalmente bajo el título Werklicher Formunterricht en la revista Bauhaus 2/3. 

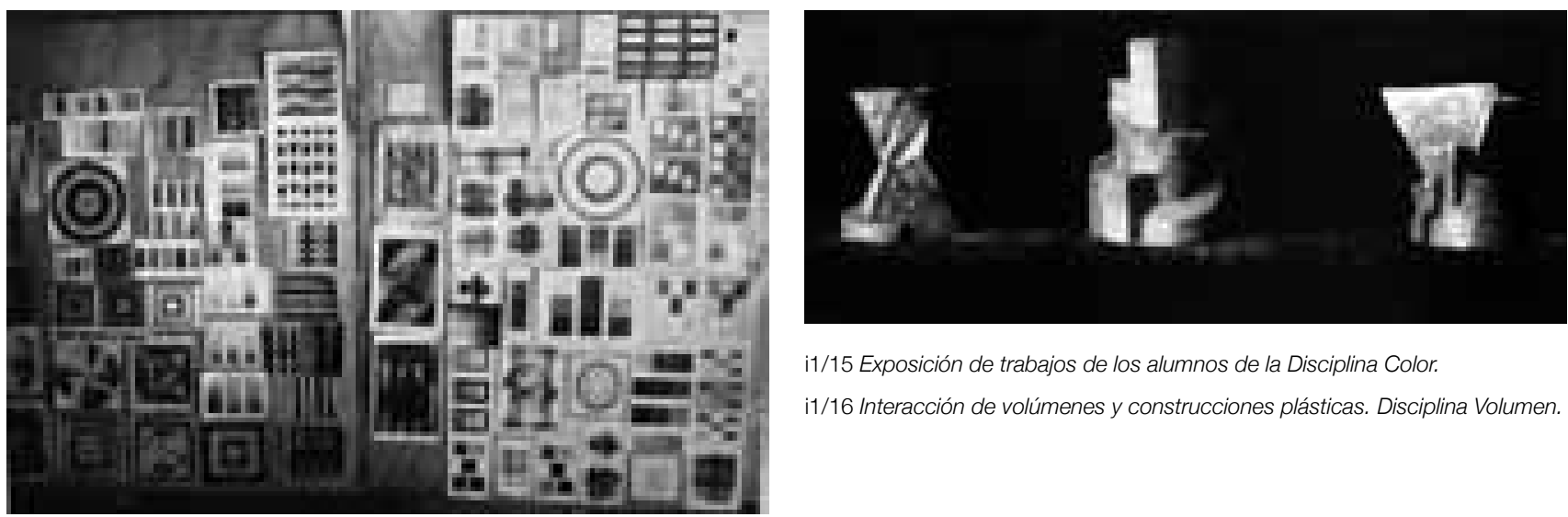

i1/15 Exposición de trabajos de los alumnos de la Disciplina Color.

i1/16 Interacción de volúmenes y construcciones plásticas. Disciplina Volumen.
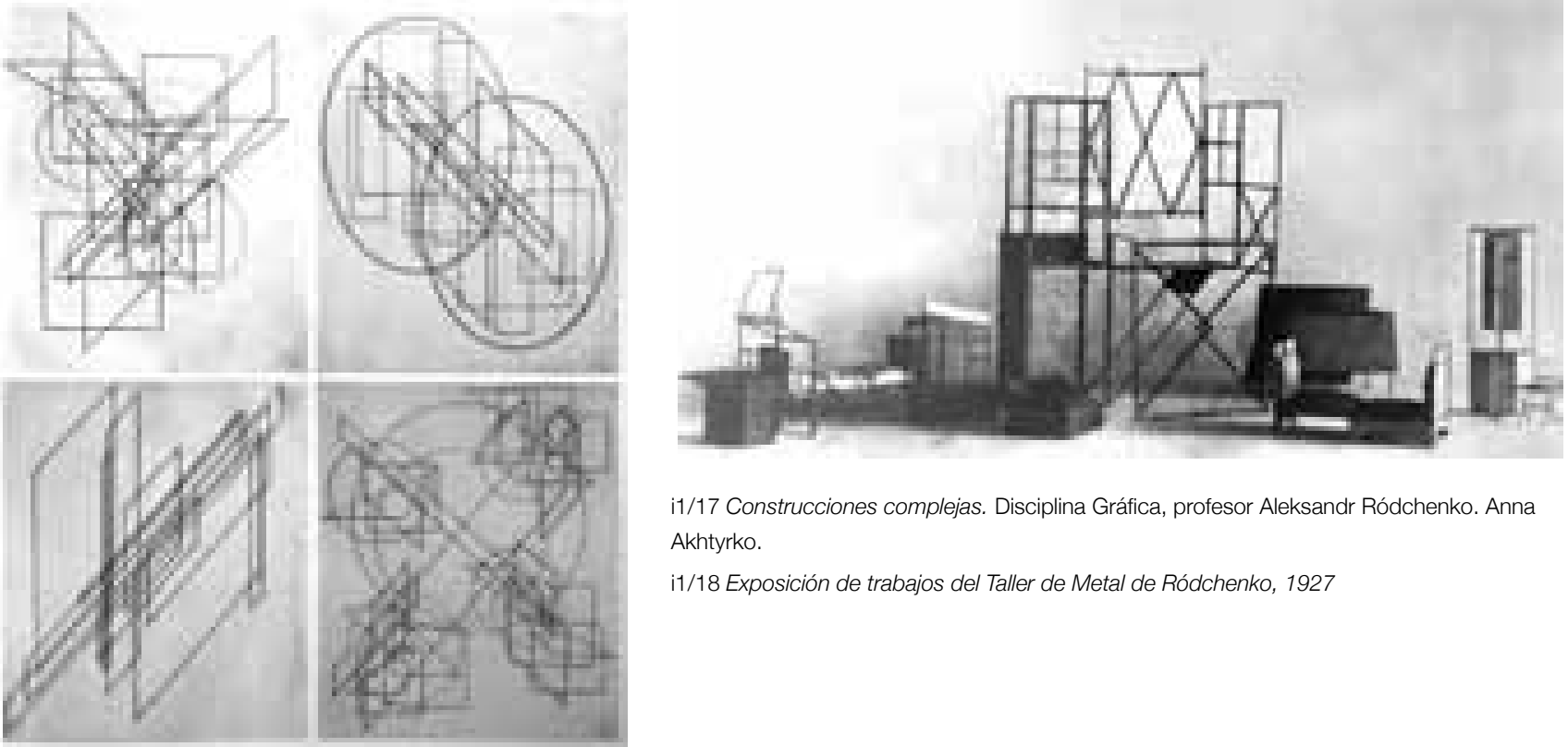

i1/17 Construcciones complejas. Disciplina Gráfica, profesor Aleksandr Ródchenko. Anna Akhtyrko.

i1/18 Exposición de trabajos del Taller de Metal de Ródchenko, 1927
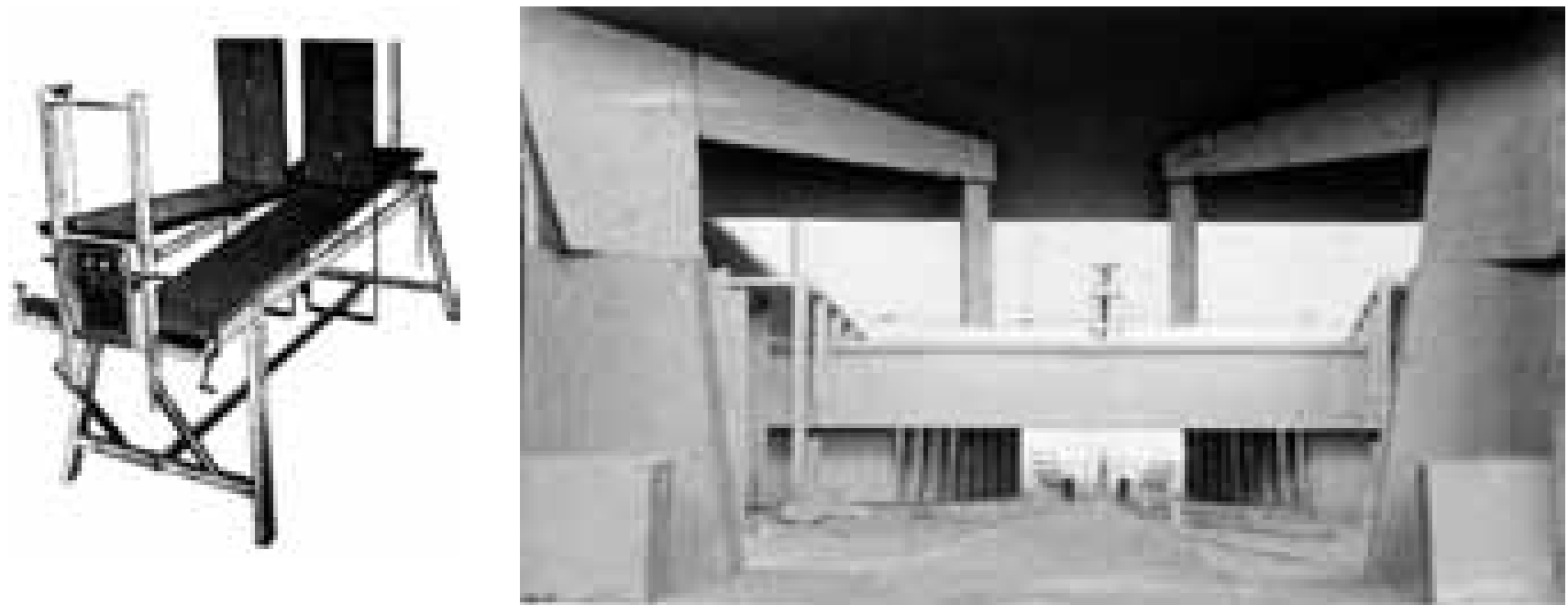

11/19 Prostómetro. Aparato para medir la capacidad de la percepción de la profundidad del espacio, 1927. i1/20 Maqueta de un ejercicio sobre el tema espacio en profundidad con énfasis en el primer plano. Trabajo de la disciplina Espacio. Anónimo, ca. 1920-1926. 


\subsubsection{Vkhutemas / Vkhutein}

Frente a la labor individual de Itten, Moholy-Nagy y Albers en la Bauhaus, los Vkhutemas llevan a cabo la búsqueda de unos principios objetivos de enseñanza de forma colectiva. ${ }^{26}$ Los estudiantes debían realizar un curso básico obligatorio antes del ingreso en una facultad de especialización. El trabajo de investigación realizado por los artistas de vanguardia en el Inkhuk ${ }^{27}$ fructificó en el establecimiento de cuatro disciplinas visuales básicas y comunes para todos los estudiantes concebidas como las enseñanzas preparatorias para el proyecto en el curso básico: Color, Gráfica, Volumen y Espacio. ${ }^{28}$

El Color se estudia como una disciplina física y óptica. (i1/15) En los ejercicios de aplicación práctica los alumnos de Gustav Klutsis, tras estudiar el comportamiento físico del color, lo incorporan como un elemento preciso para la configuración del resultado y no como un añadido decorativo.

En la disciplina Gráfica, el aprendizaje del dibujo se desarrolla en dos vertientes. La primera parte del dibujo del natural como análisis de las formas. La otra vertiente está liderada por Aleksandr Ródchenko quien extiende su propuesta en la facultad de Madera y Metal. Considera el dibujo como una herramienta de proyecto que parte del estudio de su estructura interna y desvela la realidad funcional y constructiva de los objetos. (i1/17) En sus cursos de dibujo técnico en el taller de Metal y Madera (i1/18 trabajos del taller) elabora un programa de dibujo de objetos con diversos niveles de complejidad orientado a la construcción de objetos. ${ }^{29}$

La creación e interacción entre las formas tridimensionales se estudia en la disciplina Volumen. Se plantea un programa de trabajo progresivo que parte de las formas geométricas elementales. Una vez asumidas, se fusionan, creando sistemas más complejos que se sintetizan en composiciones basadas en la figura humana.

La disciplina Espacio está dominada por los discípulos de Ladovsky, líder del racionalismo. Se puede considerar como una enseñanza que participa del análisis de formas, la composición y la introducción al proyecto de forma simultánea. La materia Espacio representa el desencadenante del nuevo enfoque de la enseñanza del proyecto arquitectónico.

La necesidad de orientación del hombre en el espacio es una cuestión esencial para Nikolay Ladovsky, y aborda este aspecto en la enseñanza preliminar en la disciplina Espacio. Con un enfoque marcadamente científico desarrolla un método psicoanalítico que tiene como objetivo el despertar de los estudiantes a la agudización de su sentido de la percepción de las proporciones y las dimensiones.

Ladovsky se basa en una serie de experimentos que lleva a cabo en su laboratorio psicotécnico con un conjunto de aparatos que le permiten medir y realizar un seguimiento de las capacidad perceptivas de los alumnos. ${ }^{30}$ (i1/19) Estas investigaciones tienen su continuidad en la disciplina Espacio. Los estudiantes exploran las relaciones entre las variables de carácter físico y visuales de la forma y la materia: superficie, estructura, peso, masa, volumen y equilibrio. ${ }^{31}$ Para ello construyen maquetas de arcilla, papel, metal, madera y otros materiales durante el primer año como ejercicio abstracto. (i1/10)

Superada esta fase y ya en el segundo curso, el dominio de las cualidades de estructura, peso, masa y equilibrio se aplica a proyectos sencillos de arquitectura caracterizados por alguna de estas cuatro variables, incorporando además aspectos funcionales y constructivos.
26 Los Vkhutemas - Talleres Artísticos y Técnicos Superiores del Estadose fundaron en 1920 sobre la base de los Primeros y Segundos Talleres Libres Artísticos del Estado, que a su vez se constituyeron por la fusión de la Escuela de Pintura, Escultura y Arquitectura de Moscú y la Escuela de Artes Industriales Stroganov. Los Vkhutemas se organizan en 8 facultades: Pintura, Escultura, Arquitectura, Artes gráficas, Cerámica, Textil, Madera, Metal. Estas dos últimas se fusionarán en la etapa final y junto con la de Arquitectura son las que contribuyeron en mayor medida a la redefinición de la disciplina del proyecto. En 1926 los Vkhutemas se transformarán en el Vkhutein,

Instituto Superior del Arte y la Técnica, hasta su desaparición en 1930. La estructura y duración de las enseñanzas del curso básico se vio sometida a los cambios en la organización y orientación ideológica de los Vkhutemas.

27 El Inkhuk, Insituto de Cultura Artistica de Moscú, fue un colectivo de artistas cuya labor fue la de crear un espacio de debate para la reflexión entorno a la creación de una "ciencia del arte". Colón Llamas, L. C. Las Vanguardias Artísticas y la enseñanza en la Rusia de los años 20. Valladolid: Universidad de Valladolid. Secretariado de Publicaciones e Intercambio Editorial, 2002. p. 37

28 Además de estas enseñanzas de tipo artístico los alumnos cursan otras de tipo teórico y científicoeconomía, historia del arte, geometría, matemáticas, física, química. KhanMagomedov, Selim O. Vhutemas. Moscou 1920-1930. Paris: Editions du Regard, 1990, p. 475.

29 Colón Llamas, L.C. op. cit. p. 195

30 Ibidem p. 235. Entre otras la apreciación visual de líneas, superficies y volúmenes; el sentido de las proporciones, el grado de percepción de las dimensiones espaciales.

31 Ibidem, p. 195. Se realizan cuatro tipos de ejercicios: expresividad de la superficie, expresividad del volumen, expresividad de la masa y el peso de un volumen y expresividad de la forma en un espacio circunscrito. 

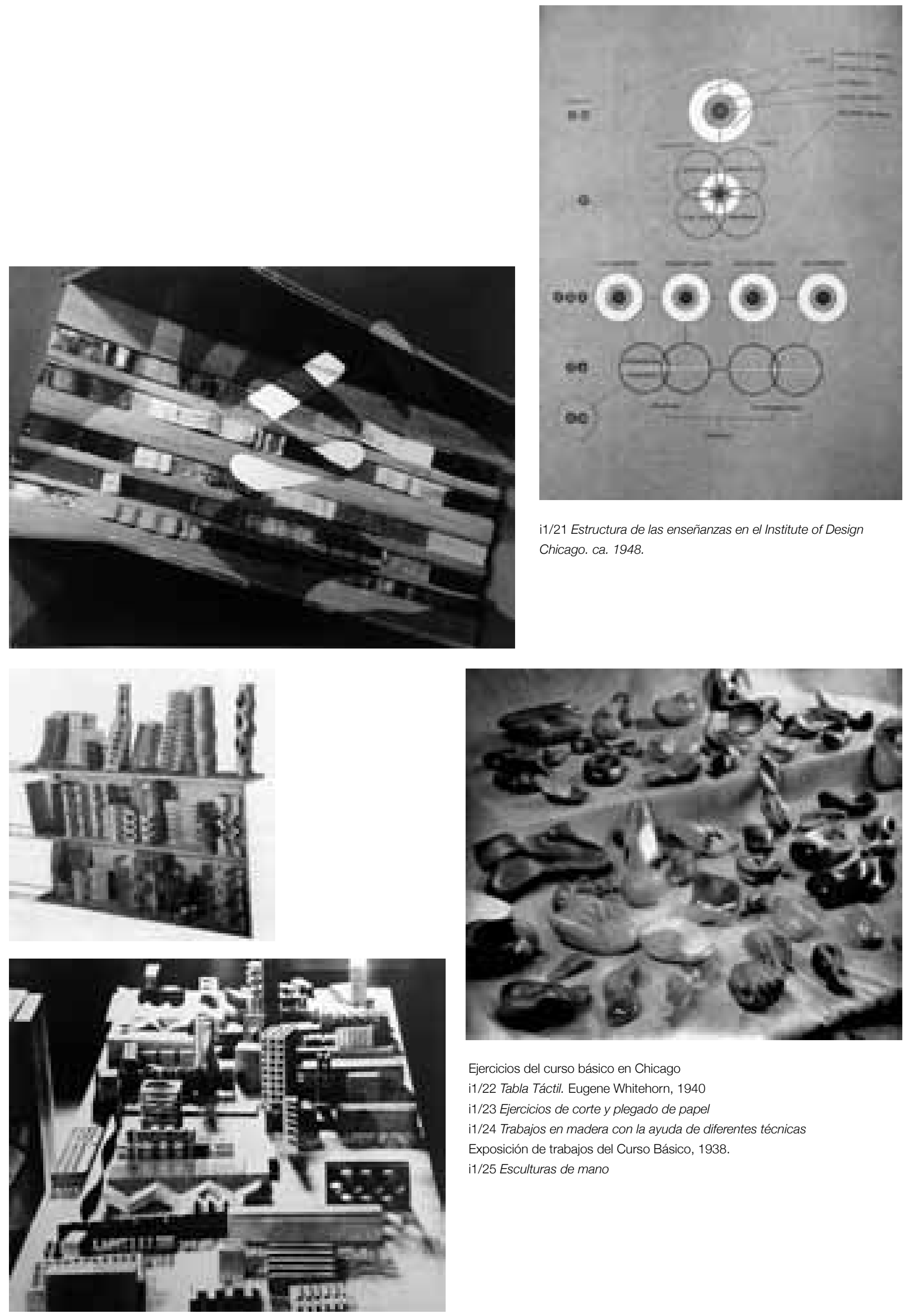

11/21 Estructura de las enseñanzas en el Institute of Design Chicago. ca. 1948

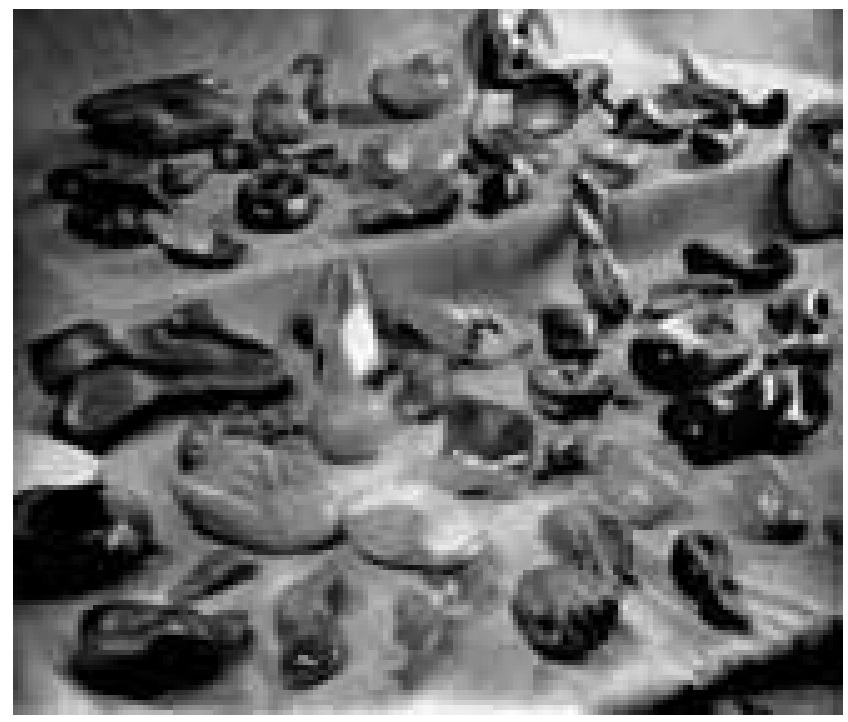

Ejercicios del curso básico en Chicago

i1/22 Tabla Táctil. Eugene Whitehorn, 1940

i1/23 Ejercicios de corte y plegado de papel

i1/24 Trabajos en madera con la ayuda de diferentes técnicas

Exposición de trabajos del Curso Básico, 1938.

i1/25 Esculturas de mano 


\subsubsection{New Bauhaus Chicago - Institute of Design}

La New Bauhaus Chicago-Insitute of Design ${ }^{32}$ es la primera escuela sucesora de la Bauhaus que se enfrenta al conflicto de intereses en torno al diseño en los años ' 30 y ‘ 40 . En Estados Unidos el debate se polariza entre el sector artístico, que lo contemplan como un fenómeno cultural, y el sector económico, que lo aprovechan como una herramienta para satisfacer las demandas del mercado.

Ante esta situación, Moholy-Nagy defiende la formación del diseñador desde un entendimiento de la profesión como un desempeño creativo en el que "ver, sentir y pensar en relaciones y no en fenómenos aislados." ${ }_{33}$

La renovación cultural mediante la formación de diseñadores anima a Moholy-Nagy a incluir en el currículo de la New Bauhaus disciplinas como Historia, Sociología, Literatura, Matemáticas y Física. Además invita a participar a profesores de la Universidad de Chicago que pertenecían al movimiento Unity of Science ${ }^{34}$ como ponentes en las asignaturas científicas entre los que se encuentran el semiótico Charles W. Morris. Las otras innovaciones principales consisten en la incorporación de cursos científicos en el programa del curso preliminar y la apertura de las disciplinas artísticas a otras como la música y la poesía.

Al igual que en la Bauhaus, los estudiantes ingresan en un Curso Básico de dos semestres. (i1/21) Tras este periodo, los alumnos continúan su formación como diseñadores durante tres años en algunos de los departamentos. Las enseñanzas preliminares tienen un triple objetivo: poner a prueba las aptitudes técnicas, artísticas e intelectuales de los estudiantes; desarrollar un método de trabajo creativo, y que se familiaricen con las herramientas del diseño -los materiales, las técnicas artesanales e industriales, la técnicas de representación gráfica y las estructuras del pensamiento científico, artístico y técnico. ${ }^{35}$

La estructura inicial del Curso Básico consta de tres partes: el taller básico, los cursos de comunicación visual y los cursos de ciencias. ${ }^{36}$ Tras la muerte de Moholy-Nagy, se amplia la formación durante un semestre más y las enseñanzas se optimizan y se orientan en con un propósito concreto por cada semestre: experimento, control y aplicación. ${ }^{37}$ Permanecen las materias científicas y como materias artísticas Fundamentos Visuales, Taller Básico y Escultura. La novedad significativa es la sustitución de estas últimas en el tercer trimestre por enseñanzas básicas en Arquitectura, Diseño de Producto, Diseño Visual y Fotografía como paso previo a la incorporación a estos departamentos.

Hin Bredendieck, antiguo alumno de Albers y Moholy-Nagy en Dessau, es invitado por este último para hacerse cargo del curso básico en Chicago. Mantiene algunos ejercicios del Vorkurs como las tablas táctiles e introduce algunas novedades en el repertorio importado de la Bauhaus. (i1/22) Su objetivo es sistematizar el trabajo de los alumnos a partir de tres fases: experimentación, análisis y exploración. Así, al plantear los ejercicios de pliegues de papel de Albers, el trabajo inicial se realiza sin restricciones como en el Vorkurs. (i1/23) A continuación, se analizan los principios que conducen a las soluciones obtenidas y, finalmente, los hallazgos se aplican en la "construcción de una estructura bien organizada." 38

Como otra novedad, Bredendieck propone nuevos ejercicios como los "cortes en madera" o la "esculturas de mano" planteados como investigaciones sobre la relación entre la forma y los procesos de mecanización. ${ }^{39}$ (i1/24) Las esculturas de mano tienen la finalidad de compensar el énfasis en lo visual en las tareas de diseño y llamar la atención sobre la importancia de la relación entre el hombre y su entorno físico..$^{40}(\mathrm{i} 1 / 25)$.
32 En 1939 se funda la New Bauhaus Chicago a instancias Chicago Association of Arts and Industries, y nombra a Moholy-Nagy su director. El proyecto atraviesa numerosas vicisitudes en su primeros años. En 1939 se renombra como Insitute of Design en 1944. En 1946, Serge Chermayeff sucede a Moholy tras la muerte de éste. Chermayeff mantuvo el currículo propuesto por Moholy y durante su mandato se establecieron departamentos especiales: Diseño Visual, Diseño de Producto, Arquitectura y Fotografía Esta estructura fue adoptada posteriormente por un gran número de escuelas de diseño, entre ellas la HfG. En 1949 pasa a formar parte del Illinois Institute of Technology y obtiene el estatus universitario.

33 Grawe, Gabriele Diana, "Continuity and Transformation: Bauhaus Pedagogy in North America". En: Wick, Rainer, Teaching at the Bauhaus. Ostfildern-Ruit: Hatje Cantz, 2000, p. 360

34 Betts, Paul "La New Bauhaus y la School of Design de Chicago" en: Fiedler, Jeannine y Feierabend, Peter. op. cit, p. 72

35 Findelli, A. Le Bauhaus de Chicago: l'oeuvre pédagogique de Làszlò Moholy-Nagy. Quebec, Editions du Septentrion, 1995, p. 55.

36 Ibidem, p. 56

37 Ze_AZ 300-1. The Institute of Design. Chicago. Folleto informativo, ca. 1948 , p. 10

38 Bredendieck, $\mathrm{H}$. Beyond Bauhaus. The evolving man-made environment. Atlanta: Georgia Tech College of Architecture, 2009. p 14

39 Bredendieck, H. "The Legacy of the Bauhaus", The Art Journal volumen XXII $n^{\circ} 1,1962$, p. 16

40 Bredendieck, $\mathrm{H}$. The evolving manmade environment. op. cit. p. 14 


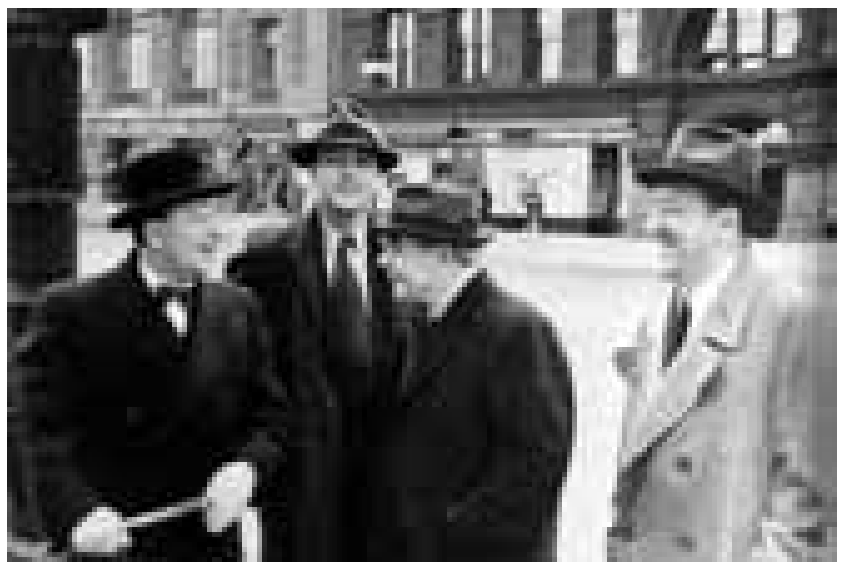

¡1/26 Eric Mendelsohn, Walter Peterhans, Ludwig Hilbersheimer y Mies van der Rohe en Chicago, ca. 1938
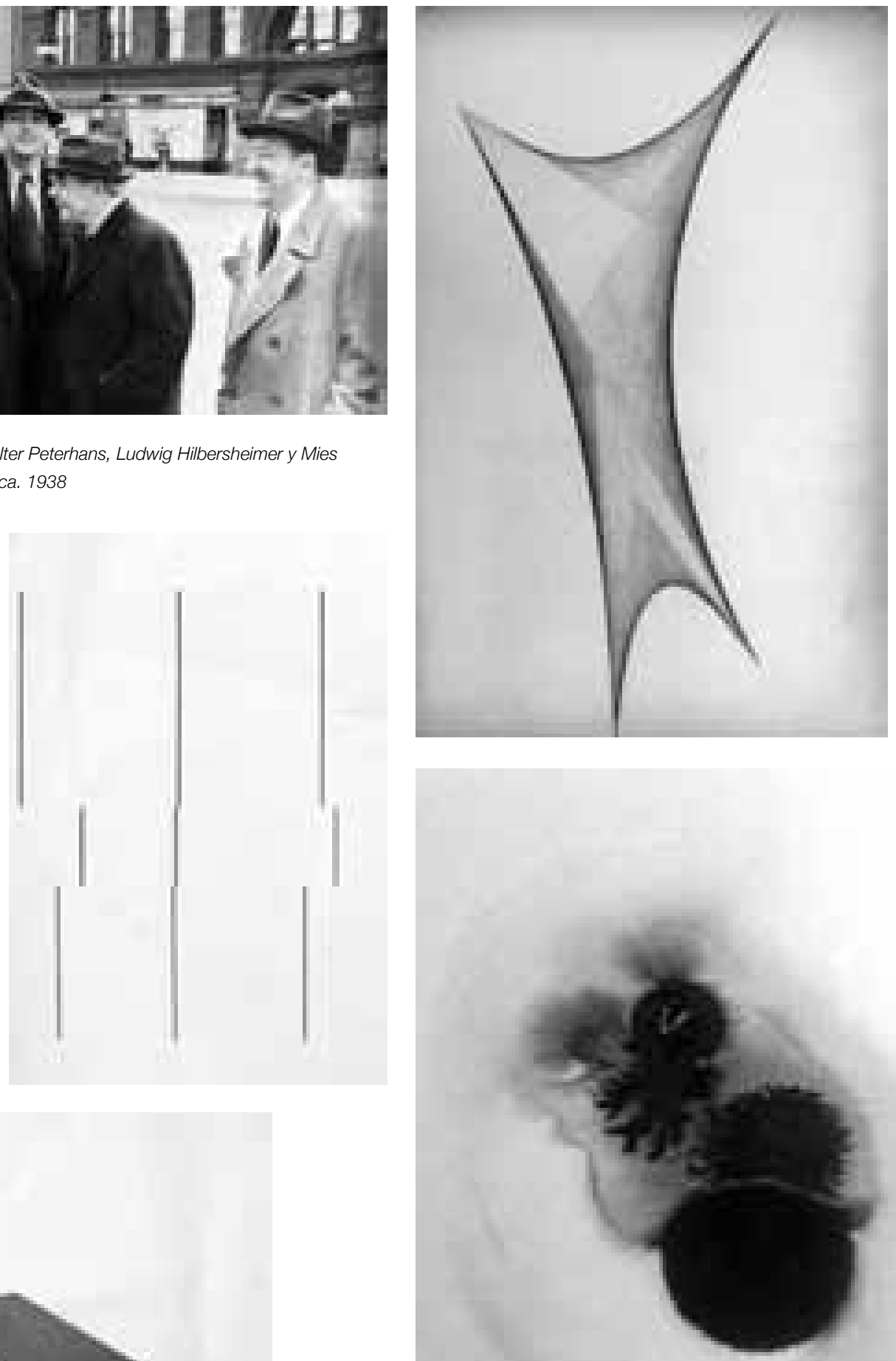

Ejercicios del seminario Visual Training en Chicago

11/27 Tarea n. 2. Tres franjas de espacios superpuestos, equilibrados e interconectados.

11/28 Tarea $n$. 5 Planos en caída libre que atrapan entre sí el espacio.

i1/29 Tarea n. 6 Superficies alabeadas a partir de haces de reactas de distinta densidad. i1/30 Tarea n. 10 Nubes de color al dispersar tinta china y acuarela sobre el cartón húmedo. 


\subsubsection{Illinois Institute of Technology}

Mies Van der Rohe, que había coincidido con Peterhans en la última etapa de la Bauhaus ${ }^{41}$, se hace cargo de las enseñanzas de Arquitectura en el Armour Institute de Chicago ${ }^{42}$, que posteriormente se transformará en el IIT. Una de las mayores dificultades que encuentra es la coexistencia de estudiantes de diferentes niveles de formación y su escasa madurez en cuestiones básicas sobre la percepción y el tratamiento de las formas. ${ }^{43}$ Emerge entonces la necesidad proporcionar una base para que los alumnos asimilen el Estilo Internacional.

Peterhans, a petición de Mies, creó un nuevo curso llamado Visual Training con el objetivo de enseñar a mirar, desarrollar el sentido crítico en la toma de decisiones y aprehender las cuestiones básicas de la forma en relación a la arquitectura: la proporción, el ritmo y el equilibrio. El curso fue introducido en 1938 e inicialmente se desarrollaba mediante ejercicios de dibujo y fotografía, y posteriormente estos medios desaparecieron para dar lugar a otras técnicas. ${ }^{44}$

El Visual Training se inserta en la secuencia de aprendizaje del currículo del IIT en cuatro seminarios distribuidos entre el segundo y tercer año de los cinco en los que se organizó finalmente la carrera. Está precedido por la enseñanza del dibujo durante el primer y el segundo año que se concibe como la adquisición de un medio, un lenguaje y un instrumento para la expresión y comunicación de ideas. Junto con las habilidades desarrolladas en el Visual Training, constituyen los fundamentos necesarios para abordar el estudio del espacio arquitectónico.

Peterhans trabaja con un repertorio de diez ejercicios que tratan sobre invariantes de carácter visual vinculados a lo arquitectónico. Plantea cada tarea con un enunciado en el que se describen las variables cuya manipulación dará lugar a las posibles respuestas.

\section{Perseguimos aislar cualidades estéticas entre sí para mostrarlas de una forma intensificada. Después, se combinan en un todo bastante diferente en el que trascienden, un espacio formado a partir de ellas. ${ }^{45}$}

El repertorio se diseña y planifica a partir del estudio de cinco elementos del lenguaje visual, cinco cualidades estéticas - la proporción, la forma, el espacio, el color y la textura-, que se emplean como herramientas de trabajo. La manipulación de estas categorías y sus variables se lleva a cabo sobre un cartón para ilustración, de color blanco de $20 \mathrm{x}$ 30 pulgadas. Sobre este espacio virtual los estudiantes manipulan diferentes materiales mediante operaciones gráfico-plásticas -pegar, dibujar, recortar, estampar, componer- que les permite agudizar su visión para educar la mirada hacia el desarrollo de un sentido crítico de la proporción y el espacio en la búsqueda de la solución óptima a cada enunciado.

Esta metodología de trabajo es el primero de los dos pilares fundamentales sobre los que se asienta la didáctica de Peterhans. El segundo es la creación de un entorno de aprendizaje en el que el maestro ejerce de guía dentro de un clima de "interacción sensibilizante"46 que propicia la estimulación de la percepción, la concentración en el trabajo y la reflexión sobre los resultados obtenidos. Una vez alcanzados estos, se ponían en común, y desde el grupo se valoraban las soluciones hasta lograr un consenso sobre el acierto en la ejecución de las mismas. Esta última práctica fue especialmente valorada por los alumnos de Peterhans en Ulm por el carácter "democrático"47 de este proceso.
41 "En 1930 cuando me hice cargo de la Bauhaus en Dessau, Walter Peterhans estaba al frente del Departamento de Fotografía. Allí me di cuenta de su esmerado trabajo con los estudiantes, y la gran disciplina que les demandaba. No solo era un fotógrafo insuperable, también tenía una fuerte personalidad con una amplia educación en muchos campos, especialmente en matemáticas, historia y filosofía".

Mies van der Rohe en: Wingler H. M. et al. Walter Peterhans. Elementarunterricht und photographische Arbeiten (Exposición celebrada en Darmstadt, Ernst-Ludwig-Haus del 10-II-1967 al 5-III-1967). Darmstadt: BauhausArchiv, 1967.

42 Dos años después del cierre de la Bauhaus, David Adler y John A. Hollabird, dos reconocidos arquitectos de Chicago, forman parte del comité para la búsqueda del nuevo director para el Armour Institute. Conocedores de la labor profesional y docente de Mies le ofrecen el cargo. Finalmmente acepta en 1937, con la condición de reformar el curriculum docente del Armour Institute. El Illinois Institute of Technology es el resultado de la fusion en 1940 de dos instituciones educativas de finales de la segunda mitad del siglo XIX: El Armour Institute of Technology y el Lewis Institute, una escuela de adultos con un marcado carácter social.

43 "Los estudiantes parecían entender lo que decía acerca de la importancia de la proporción, pero no demostraban el más mínimo sentido de ella en sus ejercicios. Descubrí que, sencillamente, sus ojos no percibían la proporción". Van Der Rohe, L.M. (1937) "The architecture curriculum at IIT", en: Swenson, A; Pao-Chi (1980) C. Architectural education at IIT, 1938-1978, Chicago: Illinois Institute of Technology, 1980, p. 47.

44 Jones, Kristin. 2016. "Research in Architectural Education: Theory and Practice of Visual Training." Enquiry 13 (1): 8. Architectural Research Centers Consortium. http://dx.doi. org/10.17831/enq:arcc.v13i2.404

45 Wingler, H. M. et al. 1967 op. cit.

46 Krumrey, I. ( 1993 ). Grundlehre and der HfG Ulm. Conferencia en la inauguración de la exposición Bauhausler in Ulm, en el HfG-Archiv Ulm.

47 Krumrey, I. Op. cit. 


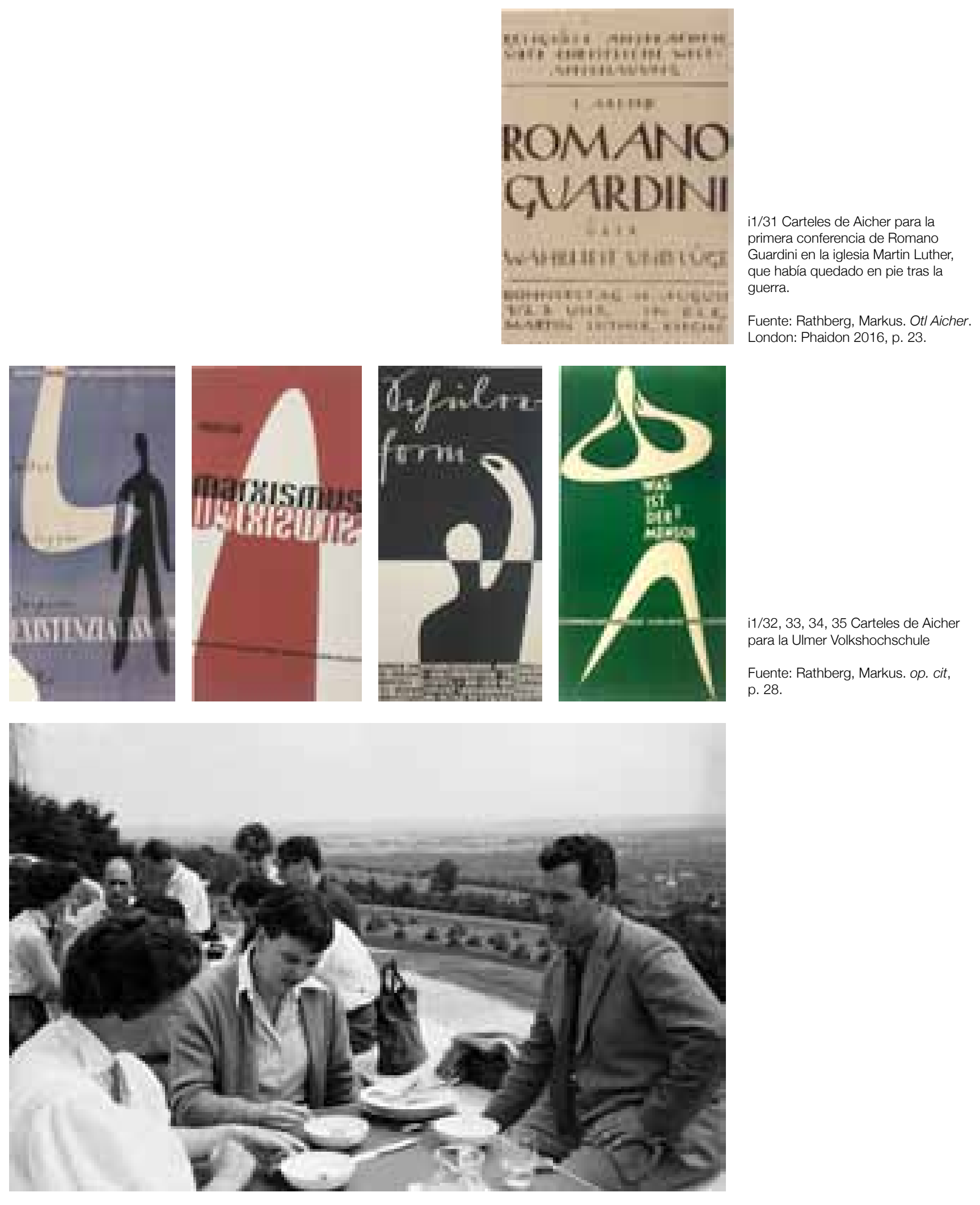

11/36 Inge Scholl y Otl Aicher en la terraza del comedor de la sede de la HfG en el Kuhberg, 1955. Archivo Hans Gugelot Konrad. 


\subsection{Estructura y docencia en la Hochschule für Gestaltung}

La Hochschule für Gestaltung Ulm nace en 1953, en un contexto de profundo cambio social. En este momento de extraordinaria crisis para Alemania, no solo es necesario recuperar la economía de una nación y reconstruir las ciudades devastadas -dos tercios de la ciudad de Ulm fueron destruidos por los bombardeos-, resulta todavía más urgente volver a definir la identidad cultural y la moral del pueblo alemán, también devastada como consecuencia del gobierno Nacionalsocialista y posterior guerra.

Tras la finalización de la Segunda Guerra Mundial, Inge Scholl y Otl Aicher ${ }^{48}$ dedicaron todos sus esfuerzos a perpetuar el espíritu pacifista y de resistencia anti-nazi de la Rosa Blanca. Otl Aicher se aparta de sus estudios superiores de arte en Múnich consciente de que no se ajustan al contexto social del momento. "Habíamos regresado de la guerra, y en la academia nos encontrábamos con que debíamos trabajar en la estética por la estética. Aquello no podía seguir. Quienquiera que tenía ojos para ver y oídos para oír hubo de reconocer que el arte era una huída de las múltiples tareas que aguardaban también a la cultura tras el derrumbe del régimen nazi" ${ }^{49}$. Otl Aicher fue la primera persona autorizada en Ulm para organizar una conferencia tras la guerra. Esta conferencia, "verdades y mentiras", fue ofrecida por el teólogo y profesor en Múnich Romano Guaridini, y le sucedieron otras muchas con el objetivo de trasladar a la población la necesidad del resurgimiento de una nueva sociedad..$^{50}$ Con el convencimiento de que el futuro común dependía de la educación, Scholl y Aicher fundaron junto a otros intelectuales la Ulm Volkshochschule. Esta universidad popular ofrecía no solo formación profesional, también se concentraba en la enseñanza de la economía, la política, las ciencias y el arte y promovía los grupos de debate y pensamiento crítico. El centro se organizaba en talleres y éstos colaboraron con el municipio en proyectos de planeamiento y reconstrucción de la ciudad. La Volkshochschule llegó a ser considerada en 1949 uno de los mejores centros de educación para adultos en Alemania. Es en este lugar donde crece el germen de lo que después sería la Hochschule für Gestaltung UIm.

\subsubsection{Génesis del proyecto docente. Hitos fundacionales}

Las tropas americanas liberan la ciudad de Ulm el 24 de abril de 1945. Otl Aicher e Inge Scholl forman parte del Ilamado "Ulmer Freudenkreis" -círculo de amigos de Ulm- que se encuentran para proponer una serie de acciones encaminadas a la reconstrucción de la ciudad. Este grupo participaba de un sentimiento colectivo basado en la creencia de estar viviendo una Stunde Null, la auténtica hora cero de un mundo mejor que deje atrás definitivamente la oscuridad y la barbarie de la guerra. Un nuevo comienzo basado en los valores humanitarios, morales y justos que defendía la Rosa Blanca.

En Enero de 1948, en paralelo a la actividad de la Volkshochschule, Otl e Inge Scholl fundan Studio Null, un grupo de personas con un fuerte carácter crítico y propositivo, que servía para revisar y poner en cuestión los objetivos colectivos en la definición de este nuevo mundo. ${ }^{51}$ Los Aicher pronto se dieron cuenta del corto alcance que tenía la Universidad Popular, a pesar de su éxito en la ciudad, y fue en este grupo donde surge la idea de dar un paso más y crear una institución de enseñanza superior centrada en las ciencias políticas. Los fundadores de la escuela de Ulm tenían la firme convicción de que la tarea de educar a los jóvenes debía depender de los propios alemanes y no de los aliados. "La juventud
48 La Hochschule für Gestaltung de Ulm nace vinculada al movimiento de la resistencia anti nazi. Sophie y Hans Scholl, dos estudiantes de la universidad de Múnich fueron dirigentes y activistas del grupo de resistencia no violenta la Rosa Blanca. Este grupo, de convicciones cristinas, se oponía al régimen de Hitler no sólo por motivos políticos sino fundamentalmente por cuestiones éticas y morales. Acusados de traición, los hermanos Scholl fueron ejecutados en 1943 y se convirtieron posteriormente en símbolos de la lucha antifascista. Su hermana, Inge Scholl junto con su marido Ot Aicher fueron dos de los fundadores de la escuela de Ulm y principales ideólogos de la escuela en sus inicios.

49 Aicher, Otl, "La Bauhaus y Ulm", El Mundo como Proyecto. Barcelona: Gustavo Gili, 1991, pag. 81

50 Spitz, René. HfG Ulm The View behind the Foreground The Political History of the UIm School of Design 1953 -1968. Stuttgart/Londres: Axel Menges, 2002, pág. 48

51 Ver Rathgeb, Markus. Otl Aicher Design as a method of action. Tesis doctoral, Universidad de Reading, Septiembre 2002. Versión publicada: Otl Aicher. Londres: Phaidon, 2006. 


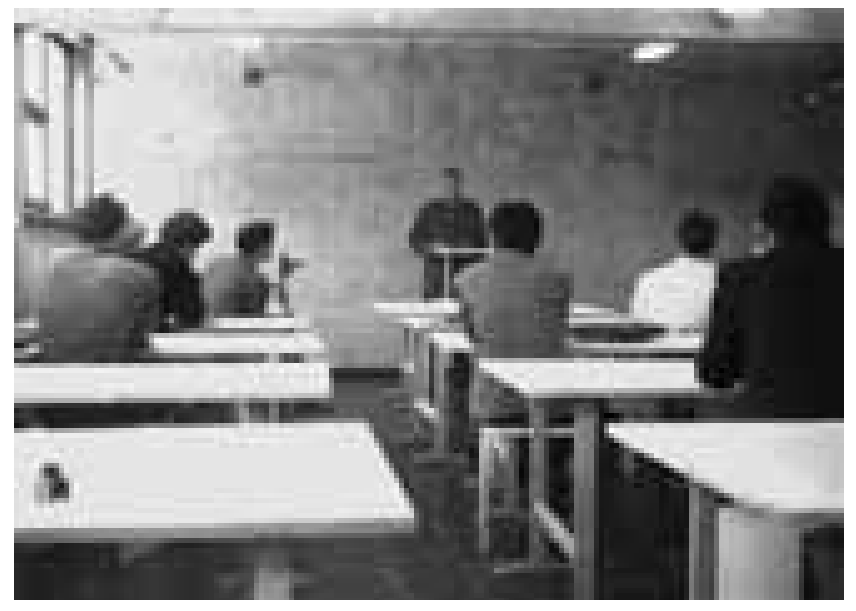

i1/37 Primer dia de clase. Max Bill en el aula. 10 de enero de 1955. Colección Hans G. Conrad.

i1/38 Ceremonia inauguración de la sede en el Kuhberg. 2 octubre 1955 Colección Hans G. Conrad.

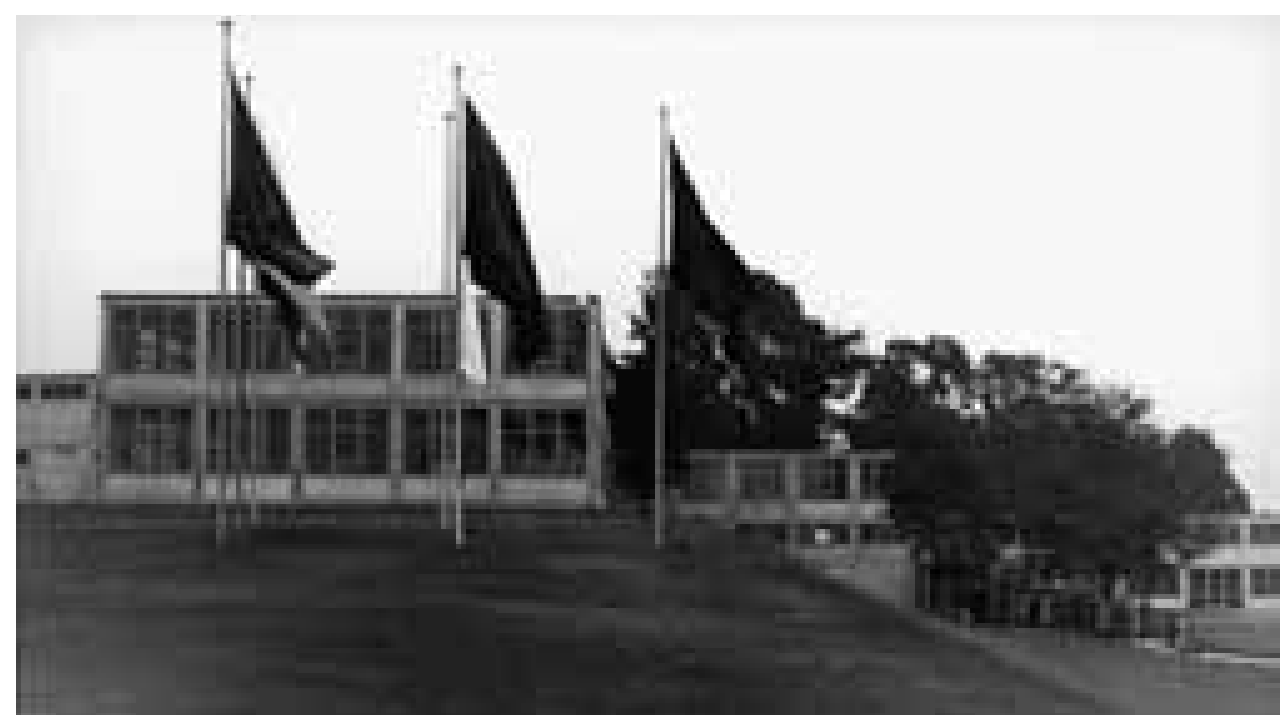

¡1/39 Ceremonia inauguración de la sede en el Kuhberg. 2 octubre 1955 Dp. 117.4 Eva Koch Weser

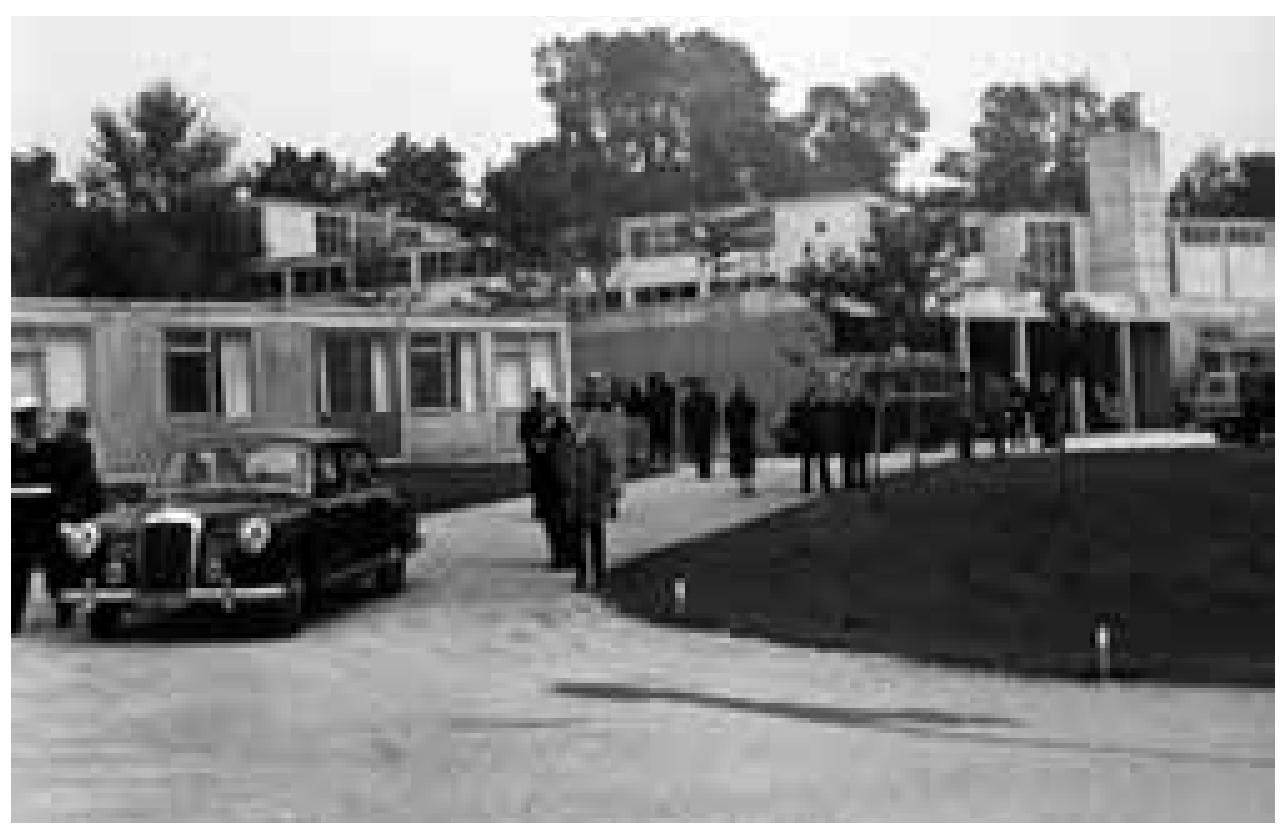


está desolada pero no es nihilista. Todavía deben encontrarse las respuestas propias a la dictadura, la guerra y la muerte de millones de personas". ${ }^{52}$ La reforma educativa que propone la HfG tenía como objetivo principal la formación de individuos comprometidos con la sociedad. "Debemos educar mujeres y hombres cuyas actitudes éticas y políticas estén de nuevo en armonía". ${ }^{33}$ La escuela se proyecta como una institución pequeña de 150 estudiantes y 16 profesores permanentes, seleccionados por sus valores y capacidades independientemente de la educación previa recibida. El objetivo inicial de la HfG se planteó sobre la base de "experiencias comunes, debate libre, exploración e investigación en grupo (...) en vez de llenar la cabeza de conocimiento, esperamos afilar la percepción del ojo y la mente". ${ }^{54}$

Los programas iniciales de la HfG previos a su fundación contemplaban siete enseñanzas principales: política, periodismo, radiodifusión, fotografía, publicidad, diseño industrial y planificación de la ciudad. En la descripción de cada una de ellas se alude a los objetivos que la escuela persigue: el interés público, la responsabilidad social, el impulso para la paz, la conquista de una vida más feliz.

Este proyecto de escuela impulsado por el matrimonio Aicher y Hans Werner Richter experimenta un golpe de timón cuando el arquitecto suizo Max Bill entra en escena. Los Aicher invitaron a Bill a llevar la exposición Die Gute Form a la sede de la Volkshochschule en 1949. Existía una evidente concomitancia entre el trabajo y las ideas de Bill y el proyecto social de los Aicher, y le piden formar parte del consejo de formación de la futura escuela. Bill propuso un cambio de orientación definitivo, la escuela debía ser de arquitectura y diseño y su producción tendría un impacto diario en la vida cotidiana en Alemania. Tras una reunión en Meersburg, cerca del lago Constanza, Max Bill pronto pasa a formar parte del núcleo del proyecto, e Inge Scholl le pide detalles de la organización de la Bauhaus de Dessau y también el desarrollo del programa docente..$^{55}$

Desde el año 1949 a 1951 tiene lugar un intenso periodo de concreción del proyecto y de búsqueda y obtención de la financiación. El desarrollo de los acontecimientos está recogido de forma exhaustiva en la tesis doctoral sobre la historia política de René Spitz. ${ }^{56}$

Max Bill tuvo un papel primordial junto a los Aicher en la fundación de la escuela. No solo por el cambio del objetivo y porque se hiciera cargo de la construcción de la sede en el Kuhberg, también por su influencia para que las diferentes instituciones que financiaron el proyecto creyeran en él. Junto con la solvencia y el trabajo incansable de Inge Scholl, el hecho de que el arquitecto suizo fuera delegado para el programa de reeducación americano y que contara con el apoyo de Walter Gropius ${ }^{57}$, ayudaron a que la escuela se hiciera una realidad en 1953 con Max Bill nombrado primer rector el 1 de abril de 1953.

Cuatro meses más tarde, las clases dan comienzo el lunes 3 de agosto de 1953 en la sede de la Ulmer Volkshochschule ${ }^{58}$ con el seminario de Walter Peterhans en el Curso Básico. El 10 de enero de 1955 las aulas se trasladan a la sede definitiva en el Kuhberg, una colina situada al suroeste de la ciudad, con el edificio aún en construcción. La ceremonia de inauguración del edificio se celebra finalmente el 2 de octubre de 1955.
52 Inge Scholl, "Geschwister Schoil Hochschule. An active school for science, art and politics in Germany. A Project". Panfleto del proyecto de fundación de la HfG, 1950, Archivo de la HfG Ulm.

53 Ibidem, pag. 8.

54 Ibidem, pag. 7.

55 Spitz, René; op. cit. pag 79

56 Spitz, René. Die politische Geschichte der Hochschule für Gestaltung UIm (1953-1968). Tesis doctoral. Universidad de Colonia, 1997.

57 Ver Rathgeb, Markus (2006). pag. 42.

58 La sede estaba situada en el centro de la ciudad, en Marktplatz 1. 

Desde su gestación hasta su cierre, el devenir de la escuela estuvo sujeto a una sucesión de relevos generacionales e intelectuales que tuvieron como consecuencia la redefinición continua de los objetivos y con ellos, cambios en los métodos de enseñanza. Sin embargo, y a pesar de que existieron diferencias y discrepancias entre los diferentes responsables de la escuela, todas las posturas que se asumieron con respecto a los objetivos de la institución estuvieron basados en el carácter moral y la vocación social con la que se había gestado el proyecto.

Como se ha mencionado, se parte de la idea de la educación como herramienta eficaz para la recuperación moral después de la guerra. La escuela está llamada a educar a los diseñadores y arquitectos que darán forma al nuevo entorno material del país. Para Otl Aicher, el oficio del diseñador consiste en valorar. "El diseñador es una especie de moralista. El diseñador valora. Su actividad consiste en hacer valoraciones". ${ }^{59}$

En el acto de entrega del cheque de millón de marcos que formará parte de la financiación de la escuela, el alto comisionado norteamericano John McCoy, enuncia el carácter experimental, propositivo y novedoso de la escuela que está a punto de nacer y su contribución a la generación de una nueva democracia. ${ }^{60}$ Max Bill explica que el diseñador que se eduque en la HfG Ulm tendrá una dimensión social como ciudadano responsable y contribuirá elevar el nivel de vida de gran parte de la población, diseñando productos -casas, utensilios, coches, trenes- mejores y más baratos que el resto. ${ }^{61}$

El carácter eminentemente político del comienzo, y como eco de la memoria de la lucha antifascista de los hermanos Scholl, se va reorientando en los años posteriores hacia la preocupación por el papel que debe ocupar el diseñador en una sociedad inmersa en la industrialización. La escuela se presenta en el primer número de su revista como una escuela superior de diseño con un nivel científico y tecnológico adecuado para la colaboración con la industria, y con un sentido de la responsabilidad sobre las consecuencias culturales y sociológicas del trabajo. ${ }^{62}$

Maldonado también señala esta misión ética del diseñador: "La escuela de Ulm quiere señalar el camino a seguir para lograr el más alto nivel de creatividad, y para señalar cuál ha de ser la finalidad social de esta creatividad, es decir, cuáles son las formas que merecen ser creadas y cuáles no". ${ }^{63}$ En su reformulación de las enseñanzas tras la marcha de Max Bill expone su creencia en un nuevo tipo de formgeber ${ }^{64}$ que necesitará el planteamiento de los problemas y su análisis científico con herramientas metodológicas.

Desde este momento y hasta su cierre, la actividad productiva y docente de la escuela se encamina hacia una mayor competitividad en términos de especialización, con un espíritu eminentemente positivista, apoyado en la ciencia y en otras disciplinas transversales. La transformación de la didáctica, en constante evolución, persigue situarse del lado de la vanguardia y se adentra en territorios nunca antes explorados.
59 Aicher, Otl. «Hans Gugelot». Catálogo de la exposición Systemdesing, Múnich, 1983 Artículo recogido en: Aicher, Otl. El mundo como proyecto. (3a edición). Barcelona: Gustavo Gili, 1994. p. 63.

60 Hausman, Brigitte. "Experiment 53/68" en: W.AA. Ulmer modelle Modelle nach ulm, hochschule für gestaltung ulm 1953-1968. OstfildernRuit: Hatje Cantz Verlag. (Catálogo de exposición), 2003, p. 16.

61 Ibidem.

62 ulm. 1, Oktober 1958 - 5, Oktober 1959. Vierteljahresbericht der Hochschule für Gestaltung UIm. Hg. v. Hochschule für Gestaltung. (Revista editada por el rectorado de la HfG)

63 Maldonado, Tomás. «Ulm 1955». Nueva visión, n 7, 1955

64 Maldonado, Tomás. Bericht von Herr Tomás Maldonado in der Verwaltungsratsssitzung am 21 April 1958. (Informe de D. Tomás Maldonado en la reunión del consejo del 21 de abril de 1958). Discurso inédito. HfG Ulm, 1958 p.4. 


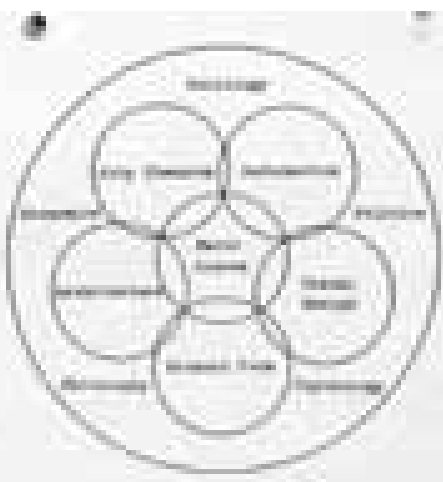

11/40 Diagrama de áreas

dependientes de la fundación

Geschwister-Scholl. 1951.

Geswister-Scholl Stiftung. School of design. Research Institute of Product Form. Ulm (Donau), Germany, 1951. Documento inédito. Fuente: HfG Archiv. Az 619. Document 14

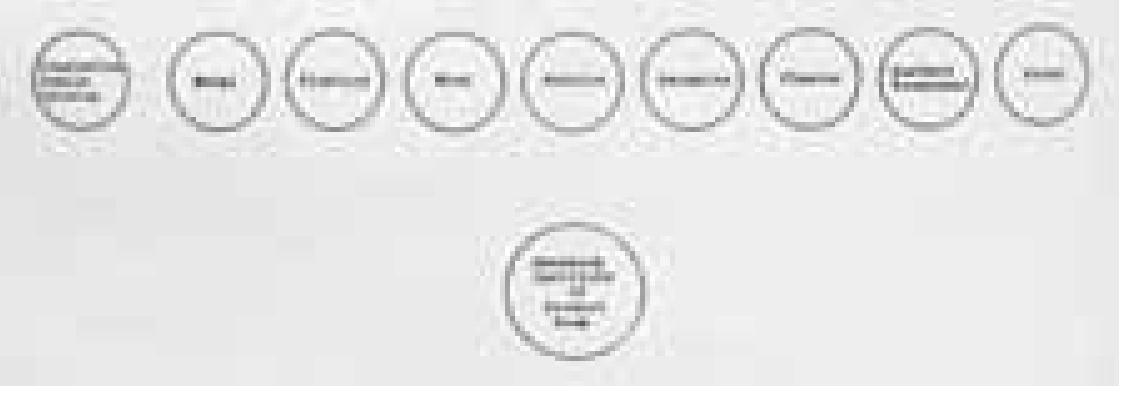

i1/41 Diagrama de departamentos y materias.

Geswister-Scholl Stiftung. School of design. Research Institute of Product Form. Ulm (Donau), Germany, 1951. Documento inédito. Fuente: HfG Archiv. Az 619. Document 14.
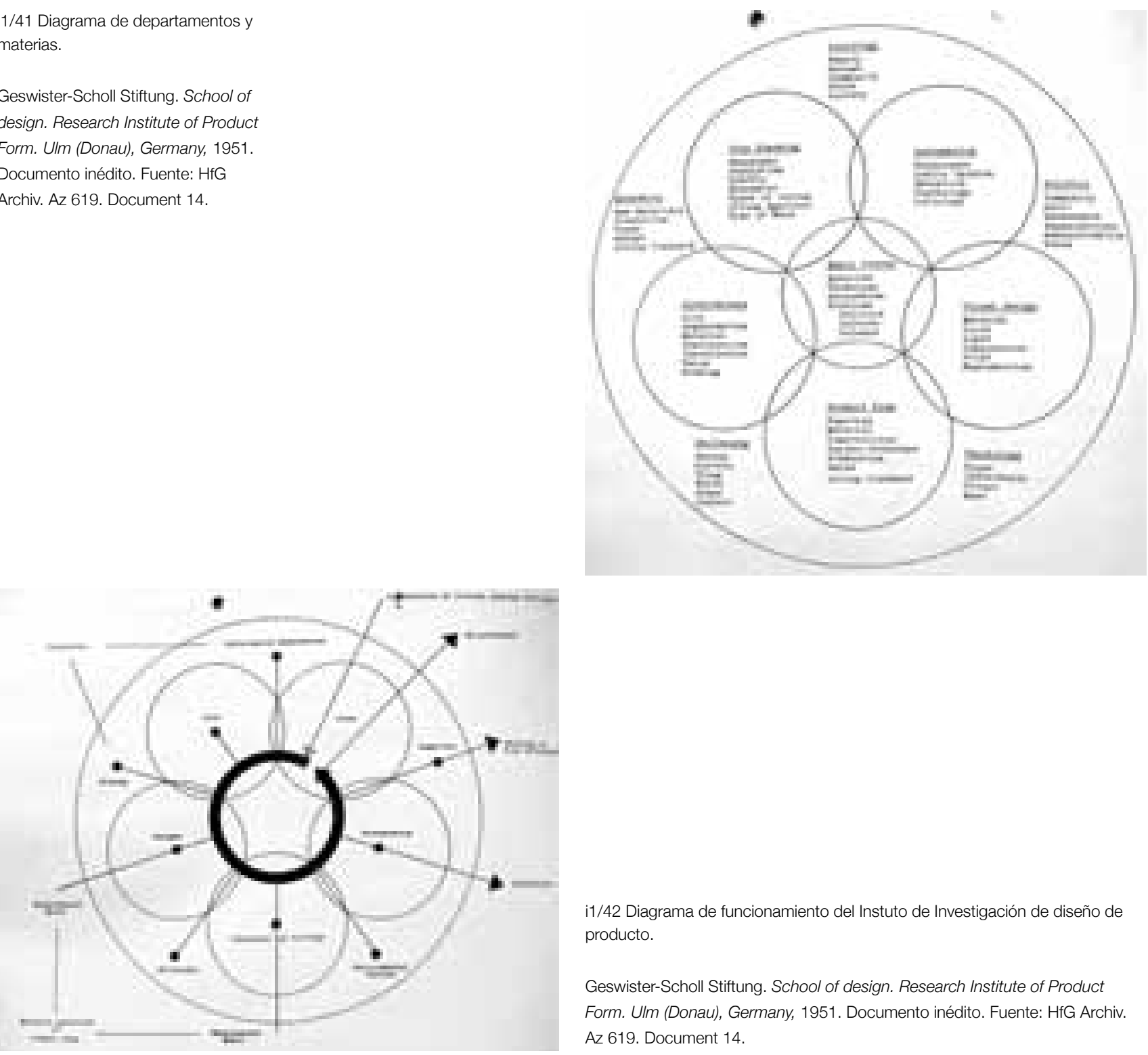

¡1/42 Diagrama de funcionamiento del Instuto de Investigación de diseño de producto.

Geswister-Scholl Stiftung. School of design. Research Institute of Product Form. Ulm (Donau), Germany, 1951. Documento inédito. Fuente: HfG Archiv. Az 619. Document 14. 


\subsubsection{Organización pedagógica por departamentos. Los medios.}

El plan de estudios presentado por Max Bill y los Aicher en 1951 para la obtención de la financiación, ${ }^{65}$ establecía que la fundación Geschwister-Scholl, sin ánimo de lucro, tendría el objetivo fundamental de fundar y mantener una escuela de diseño en conexión con un instituto de investigación de diseño industrial. Este primer documento incluía un diagrama circular muy difundido que sintetizaba en una sola imagen la estructura de la escuela. El diagrama esbozaba un círculo central ocupado por el Curso Básico, y a su alrededor cinco departamentos: planificación urbana, información, diseño visual, diseño de producto y arquitectura. En el documento se nombra a Inge Scholl como directora de la fundación, a su marido Otl Aicher como asistente a dirección, a Max Bill como director de la escuela y a Walter Zeischegg como director del instituto de investigación del diseño industrial.

\section{Departamentos}

El Curso Básico o Grundkurs se concibe como un conjunto de enseñanzas de carácter propedéutico o preparatorio que proporcionaba una base común a los alumnos procedentes de varios países y desde distintas ramas de la enseñanza o del mundo profesional, artístico, técnico o artesanal. El paso por este primer curso orientaba a aquellos que lo superaban a continuar sus estudios en los departamentos de especialización. Los departamentos estaban dirigidos por un profesor responsable del planteamiento docente y de la coordinación con el resto de profesores. Era frecuente el cambio de responsable así como del cuerpo docente; el grupo de profesores contratados representaba un porcentaje muy bajo frente a los invitados, que impartían cursos esporádicamente. La consecuencia fue una continua renovación que proporcionó una importante riqueza y la posibilidad de contar con los mejores profesionales y docentes del momento.

Cuando la escuela abre oficialmente en 1953, existen cuatro departamentos: Grundlehre, Produktform, Architektur, y Visuelle Kommunikation. Max Bill figura a cargo del departamento de arquitectura, que se dedica a llevar a cabo la obra de la sede de la HfG los dos primeros cursos. En el segundo curso ya existe un responsable de cada departamento: Tomás Maldonado en Grundlehre, Friedirch Vordemberge-Gildewart en Visuelle Kommunikation y Max Bill en Produkt Form y Architektur que amplía su campo de acción a Architektur-Stadtbau. En el curso 1956-1957 surge un nuevo departamento denominado Information a cargo de Max Bense. Una vez que Max Bill abandona la escuela, el departamento de Architektur / Stadtbau cambia su nombre por Bauen y se queda temporalmente sin responsable hasta que en el 1958-1959 se hace cargo Herbert Ohl. En el curso 1960-1961 acontecen importantes cambios en la estructura de los departamentos: se crea el efímero departamento de especialización Film que es absorbido por Visuelle Kommunication dos cursos después, es el último años de Grundlehre como departamento independiente y el departamento de arquitectura cambia su nombre de nuevo a Industrialisiertes Bauen, expresando de forma explícita su objetivo.

El departamento Produktform, - denominado Produktgestaltung a partir del curso 1962/63fue el más numeroso de la escuela, con 249 estudiantes. Los principales docentes de este departamento fueron Max Bill, Hans Gugelot, Georg Leowald, Herbert Lindinger, Tomás Maldonado y Walter Zeischegg. El Diseño industrial que se imparte en la escuela tiene el objetivo de formar a diseñadores capaces de resolver cualquier proyecto por complejo que sea, desde objetos cotidianos y bienes de consumo hasta máquinas complejas y medios
65 Geswister-Scholl Stiftung. School of design. Research Institute of Product Form. Ulm (Donau), Germany, 1951. Documento inédito. Fuente: HfG Archiv. Az 619. Document 14. 


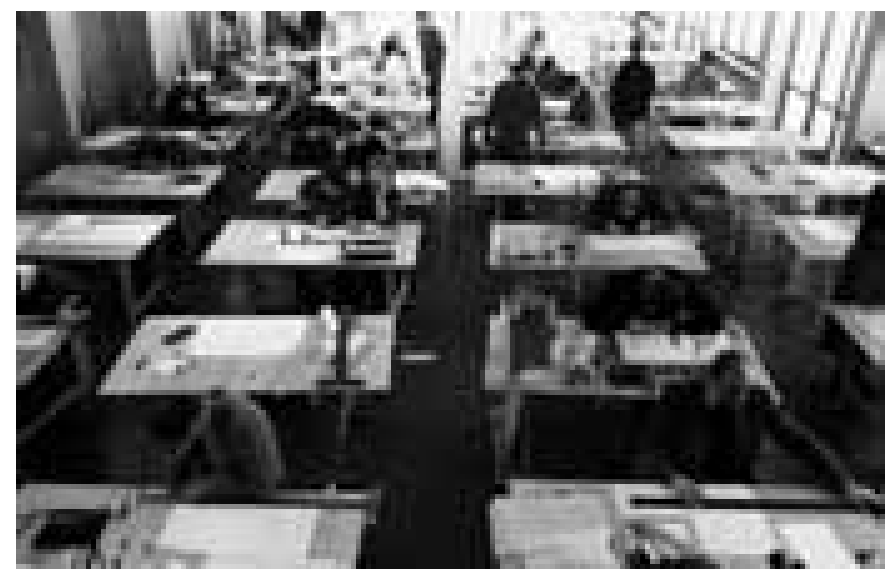

i1/43 Departamento Grundlehre

Archivo Hans G. Conrad

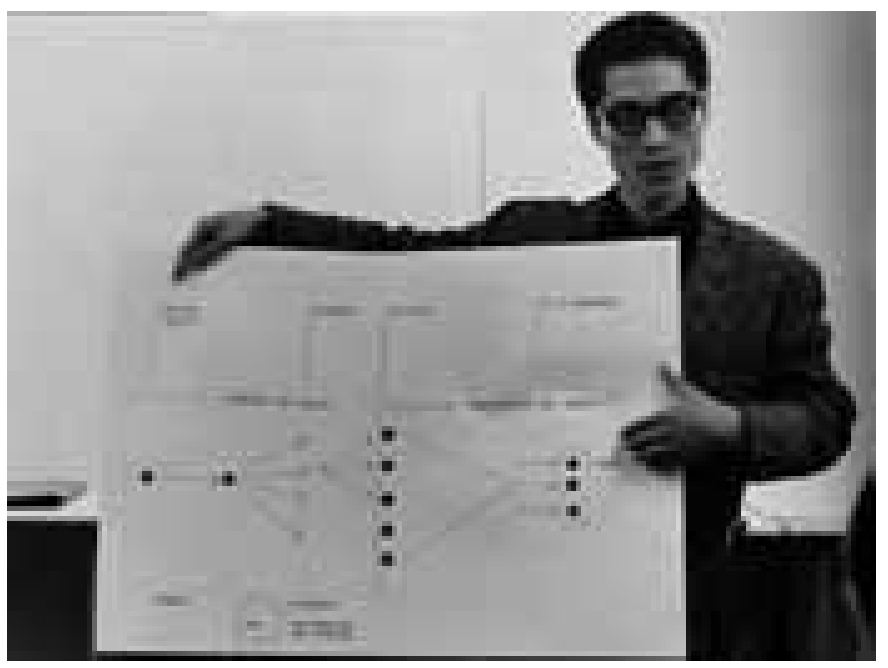

i1/44 Departamento de Diseño de Producto

F.58.0475
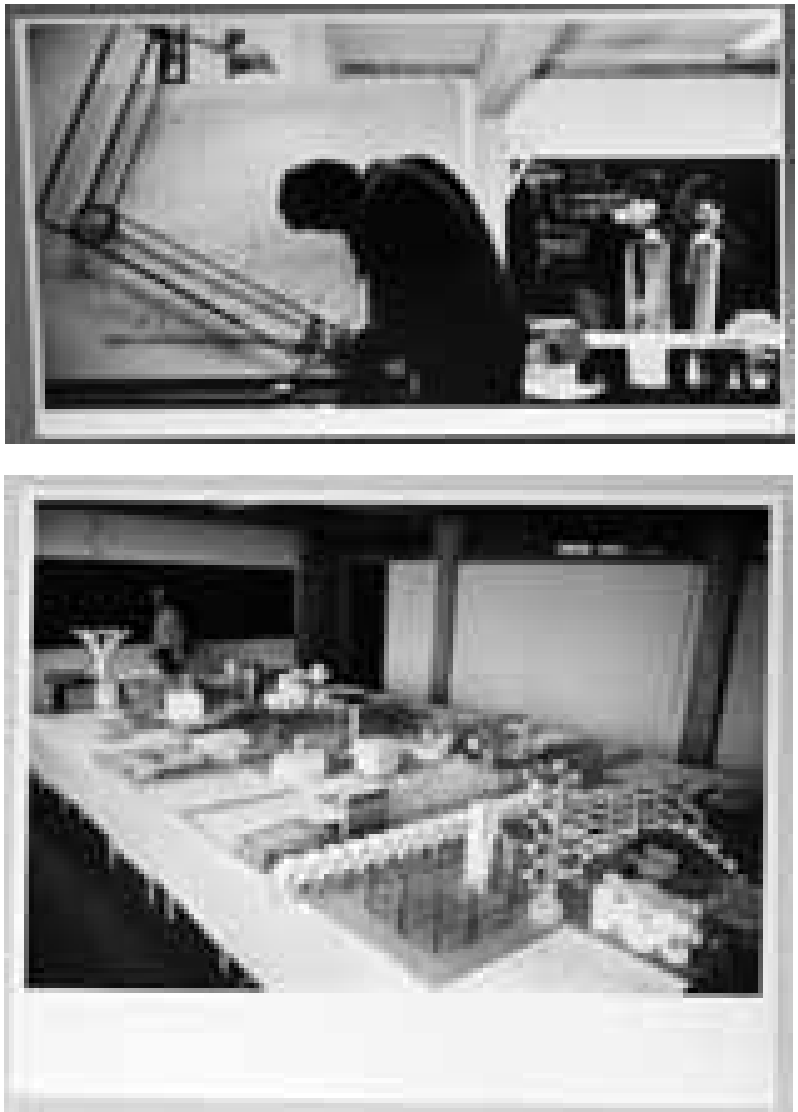

i1/45 Departamento de Construcción industrializada

Exposición de trabajos de alumnos del primer curso 1962-1963

Dp 120.002 fotografía del alumno Futuwakari FBS

i1/46 Departamento de Comunicación Visual. Profesor Kohei Sugiura.

Fuente: Quijano, Marcela "Ist der Weg das Ziel? en: W.AA. Modelos de

UIm, op. cit. p. 68

i1/47 Departamento de Información

F 58_0460_3 
de transporte. La superación del entorno doméstico significa dar un paso más allá de la Bauhaus, ${ }^{66}$ que solo se implica en el diseño muebles, enseres o lámparas. La progresiva complejidad de los enunciados que tienen que resolver los estudiantes determina que la enseñanza sea específica y que responda adecuadamente a los retos que se plantean. Los alumnos estudian teoría de la producción, materiales, construcción técnica, MOA, filosofía de la cienca, ergonomía, sociología y mecánica.

El departamento Visuellekomunikation, con 158 estudiantes, fue el segundo departamento más solicitado de la HfG. Los principales profesores fueron Otl Aicher, Anthony Froshaug, Gui Bonsieppe, Herbert W. Kapitzki y Tomas Gonda, Tomás Maldonado y Friedrich Vordemberge-Wildewart. El diseño gráfico se impartía en dos vertientes, una dirigida a las artes gráficas, fotografía y tipografía, diseño de exposiciones, paquetería, tipografía y gráfica para dispositivos tecnológicos. ${ }^{67}$ La segunda linea se dedicó a cine y televisión, que en 1962-1963 se convirtió en un departamento independiente para más tarde integrarse en Information.

El departamento Bauen experimentó varios cambios de nombre que fueron concretando su carácter. Dirigido por Max Bill, comenzó llamándose Arquitectura y desarrollo urbanístico. La ciudad para Bill constituía sin duda un asunto de primer orden. En sus escritos, manifestó la necesidad de proyectar un espacio urbano para el bienestar de las personas, y denunció que la práctica habitual del urbanismo no tomaba en consideración este objetivo. ${ }^{68}$ Tras la marcha de Max Bill, pasó a llamarse Departamento de Construcción. Bajo la influencia de Konrad Wachmann, la industrialización de la arquitectura había tomado mucha relevancia. Cuando Herbert Ohl toma el mando el departamento fue rebautizado Construcción Industrializada, que hace explícita la especialización que había tomado el departamento. Herbert Ohl reflexiona años después que el nombre adecuado habría sido Arquitectura Industrializada. ${ }^{69}$ El departamento acotó el objetivo inicialmente planteado y se propuso formar arquitectos capaces de aplicar al proyecto de arquitectura modernos métodos de producción industrial, como respuesta válida a cuestiones necesarias para la sociedad como la construcción de vivienda con unos estándares tecnológicos y de confort del máximo nivel. Pasaron por sus clases 170 estudiantes, la enseñanza se dividía en materias teóricas y prácticas. La base teórica se componía de cuestiones relacionadas con las instalaciones y el confort: fisiología aplicada -calidad del aire, óptica, acústica- técnica física y servicios domésticos -calefacción, iluminación-; también sobre estructuras, matemáticas, y sociología. Los profesores más relevantes del departamento fueron Abraham A. Moles, Max Bill, Herbert Ohl, Frei Otto, Claude Schnaidt, Konrad Wachsmann y Werner Wirsing.

El departamento Information, con 27 estudiantes, fue el menos numeroso de la escuela. El objetivo de este departamento era formar personas del ámbito de los medios de comunicación y el periodismo -radio, televisión, prensa escrita-, publicidad y cine. Con un firme creencia en la importancia y el poder de la opinión pública, se pretende que los alumnos del departamento sean profesionales con una responsabilidad social en su desempeño, del lado de la objetividad y en clara oposición a la manipulación. Este departamento se consideraba un complemento de Comunicación Visual. El docente con más influencia fue Max Bense que determina el plan de estudios del departamento en 1956 con el título "Un curriculum experimental para la Escuela de Diseño de Ulm". ${ }^{70} \mathrm{La}$ parte experimental de la docencia se desarrollaba en los textos, los juegos de palabras, la semiótica, la sintáctica con influencia de la poesía concreta, las teorías de Wittgenstein y Bertolt Brecht, Goethe, Arno Schmidt y Edmund Husserl. Gui Bonsiepe se refiere a este departamento como "cocina de teoría". ${ }^{71}$
66 Entrevista con Herbert Lindinger en Krampen, Martin, \& Hörmann, Günther. Die Hochschule für Gestaltung UIm - Anfänge eines Projektes der unnachgiebigen Moderne. Berlin: Ernst \& Sohn, 2003, p. 113.

67 Paneles-resumen de los departamentos en la exposición permantente del archivo de la HfG Ulm. Hochschule für Gestaltung UImVon der Stunde Null bis 1968.

68 Bill, Max. "El barrio vivienda como elemento urbano diferenciado". En Maldonado, Tomás. Max Bill. Buenos Aires: Nueva Visión, 1955, p. 101.

69 Ohl, Herbert. "Industrilized Building at Ulm”. En: Lindinger, Herbert. UIm Design: The Morality of Objects. Massachusetts: MIT Press. (Catálogo de exposición). 1991 Versión inglesa del original: LINDINGER, Herbert Die Moral der Gegenstände, Berlin: Ernst \& Sohn Verlag, 1987, p. 197.

70 El plan de estudios ideado por Max Bense fue publicado en la revista Texte und Zeischen, citado en: Krampen, Martin, \& Hörmann, Günther, op. cit. pag. 153.

71 Ver entrevista con Gui Bonsiepe en: Krampen, Martin, \& Hörmann, Günther, op. cit. pag. 159. 


\section{(ब) L Lufth hansa}

i1/48 Proyecto identidad corporativa Lufthansa. Grupo E5.

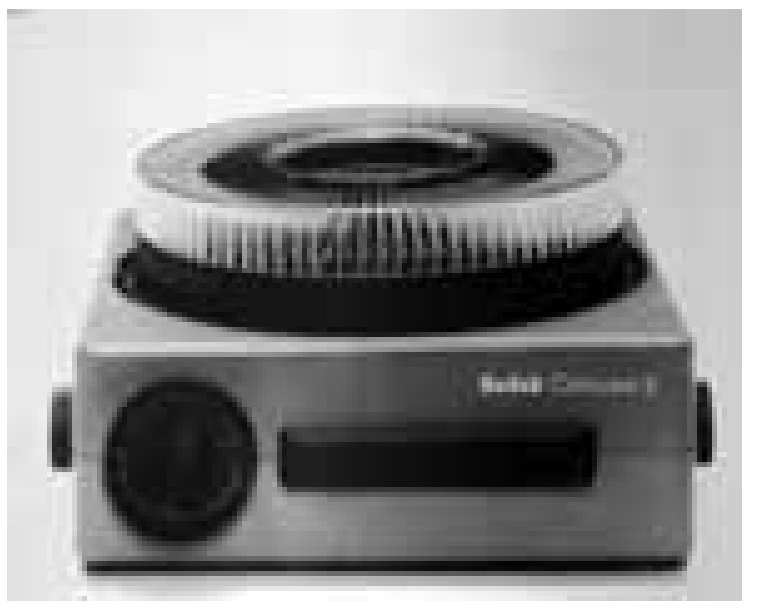

11/50 Proyector Carousel S para Kodak. Lufthansa. Grupo E2

i1/51 Módulo de construcción Grupo IIB O
11/49 Sistema M125.

Grupo E2
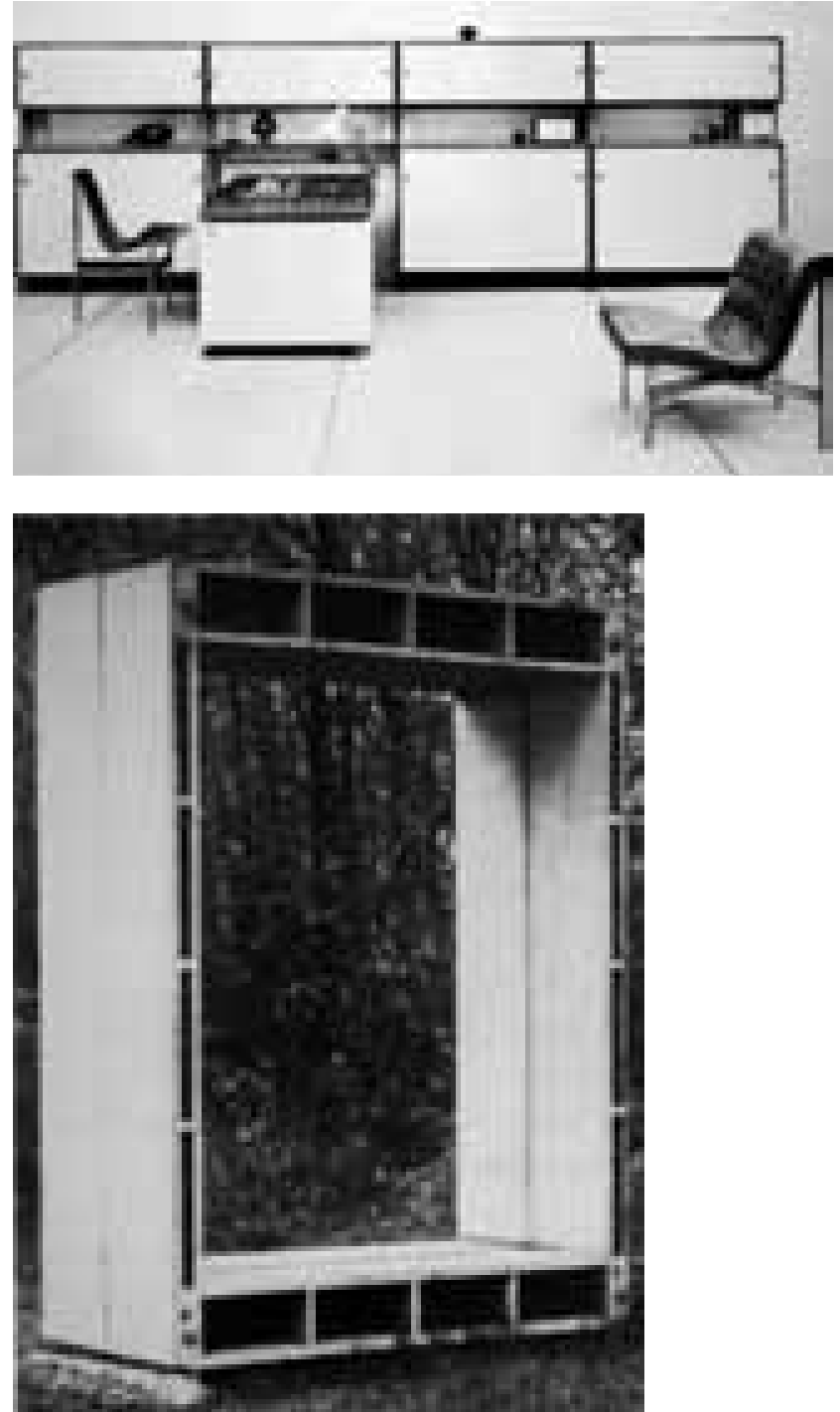
El departamento de cine, integrado en Información hasta 1962, fue una de las primeras escuelas de cine de la República Federal Alemana, influido por la Nouvelle Vague. Al igual que el departamento de información, tuvo un bajo número de estudiantes (18), pero debido a que solo se admitían 5 por curso, de 1962 a 1968.

\section{Instituto de investigación y grupos de desarrollo}

El Instituto de Investigación de Diseño de Producto se proyecta vinculado al proyecto de escuela desde el primer momento. Los fundadores prevén que se desarrolle en paralelo al proyecto docente pero de forma independiente.

Walter Zeischegg, escultor de origen austriaco, desarrolla un planteamiento para el Instituto a petición de Inge Scholl. El proyecto de Zeischegg prevé la necesidad de talleres para el desarrollo de prototipos que se compartirían con la escuela: madera, metal, plástico, textil, cerámica, yeso, color, y un departamento de investigaciones sociológicas. Los estudiantes en los departamentos desarrollan encargos reales que reciben del Instituto. A partir del año 1958 se establecen varios grupos de desarrollo bajo el paraguas del Instituto de Investigación, que desarrollan proyectos propios y también se unen en colaboración. En 1968, cuando cierra la escuela, los grupos de desarrollo que han existido en el Instituto son los siguientes: ${ }^{72}$

Instituto de Construcción Industrializada (IIB O) - Director Herbert Ohl. Proyectos para Aluminum Walzwerk, Goldschmidt GmbH, Metzeler AG, Rhenus AG y Wellit GmbH. Pabellón alemán para la Exposición Universal de Montreal.

E2: Hasta 1966, director Hans Gugelot. ${ }^{73}$ Desde la aparición de Gugelot en la HfG se había iniciado una fructífera colaboración con Braun. El grupo E2 desarrolla la afeitadora Braun Sixtant, un sistema de alta fidelidad -proyecto fin de carrera de Herbert Lindinger-; una máquina de coser para Pfaff, proyector de disapositivas Carrousel F para Kodak, sistema de amueblamiento M125, trenes del metro de Hamburgo.

E3: director Walter Zeischegg. Proyectos para Philips, patentes de adhesivos magnéticos para German Refined Steel Works, iluminación para Max Braun, desarrollo de celosías tridimensionales, utensilios de oficina y de hogar en plástico para Helit.

EL: director Herbert Lindinger. Bancos y sillas de plástico para BASF, platos de plástico, autobús para Büssing Automobile Works, motoniveladora para Machine Construction UIm $\mathrm{GmbH}$, escala de precisión para laboratorio para Sauter, helicóptero para MBB GmbH, máquinas plegadoras de papel para Stahl, logotipo de Mont Blanc.

E5: director Otl Aicher, se convierte en el E5A. Identidad corporativa para Lufthansa, sistemas de color de BASF, y publicidad para Herman Miller.

E6: director Tomás Maldonado, se convierte en el E6B con Gui Bonsiepe. Diseñan sistemas electrónicos de procesos de datos para Olivetti, dispositivos electromecánicos para Erbe Electromedical, mobiliario de oficina para UniData, máquina de escribir eléctrica Tekne 3 para Olivetti en colaboración con Ettore Sottsass. 
E7K: director Herbert Kapitzki. Imagen corporativa de Westdeutscher Verlag, posters y publicidad para el diario Stuttgarter Nachrichten, imagen corporativa del Canal de Teledifusión del Suroeste Baden-Baden, proyecto de señalización de los aereopuertos de Alemania, que se extendió a los aereopuertos de Austria y a otros en Europa.

Instituto del Proyecto de Cine: director Alexander Kluge. Se realizaron varios proyectos sin finaciación. El único financiado fue un documental sobre la situación universitaria en Alemania.

La producción previa del Instituto de diseño de producto tuvo mucha repercusión y ha sido muy difundida. Los proyectos más destacados fueron la cama HfG para Dunlop -1954, Bill-Aicher-; Reloj de cocina para Junghans -1956, Bill-Moeckl-, G11 radio y giradiscos para Max Braun AG, -1955 Gugelot-, SK4 radio y giradiscos para Braun -1956 GugelotAicher-, lámpara y lavabo para el edificio de la HfG -1954 Walter Zeischegg-, manilla para las puertas del edificio HfG -1954 Ernst Moeckl-.

\section{Talleres}

En el primer curso se abrieron los talleres de madera, yeso, metal y plástico. Los maestros de taller no cambian a lo largo de toda la trayectoria de la escuela: Paul Hindinger se dirigió el Taller de madera, Otto Schild el de yeso, Josef Schlecker el de metal y Cornelius Uittenhout el de plástico. En el curso 1955-1956 se incorporan dos talleres de fotografía a cargo de Fritz Hahn y Wolfgang Siol; este último continúa hasta el final.
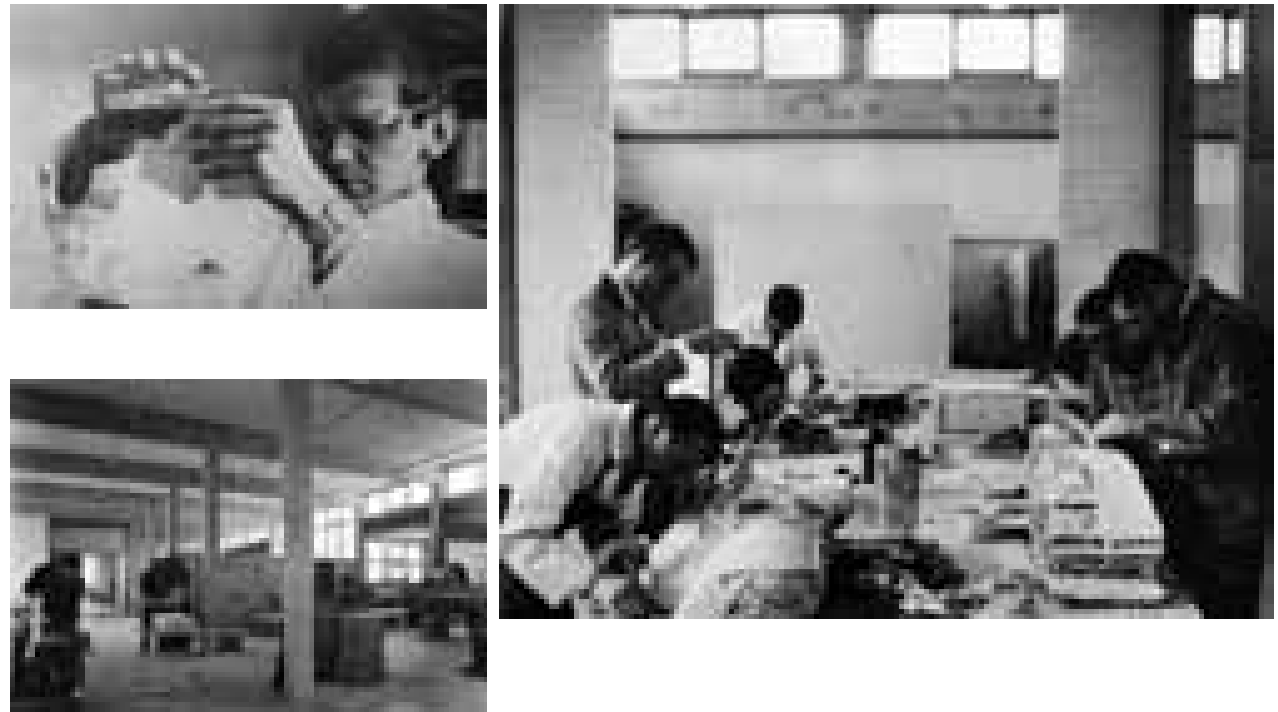


\subsubsection{Gestación, evolución y estructura del Grundlehre}

La idea de un Curso Básico estuvo presente en la propuesta docente de Max Bill desde su primer esbozo. El primer documento que establece el Curso Básico y sus objetivos, enuncia su carácter obligatorio, y a la vez panorámico. El Curso Básico debía estar compuesto por una variedad de asignaturas tal que los estudiantes pudieran probar ese primer año para qué departamento tenían más aptitudes. ${ }^{74}$

Cuando se edita el primer número de la revista Ulm, ha comenzado el curso 1958-1959, y ya han transcurrido cinco años de Grundlehre. Los objetivos han sido redefinidos y decantados por el tiempo.

La fundación del Curso Básico tiene cuatro objetivos:

1. Inicia a los estudiantes en el trabajo de los departamentos, sobre todo en los métodos en los que se basa el trabajo.

2. Pone en contacto a los estudiantes con las cuestiones más importantes de nuestra civilización técnica, y de esta forma transmite los horizontes de los actuales problemas de diseño.

3. Educa a los estudiantes en el trabajo en varias disciplinas y los prepara para el trabajo en equipo, por ejemplo, para trabajar en comités de especialistas, en los que cada uno de ellos entiende el problema y la visión de sus colaboradores.

4. Ajusta los niveles de la educación previa, debido a que los estudiantes no solo provienen de diferentes profesiones sino también de muchos países con diferentes sistemas de educativos. ${ }^{75}$

Los alumnos, al finalizar el Curso Básico, eran examinados por un claustro de profesores que decidía si habían alcanzado el nivel suficiente para ingresar en los departamentos en el curso siguiente.

A pesar de que el Grundlehre registra variaciones, se mantiene en el tiempo una estructura en cuatro grupos de disciplinas: Visuelle Einführung -aprendizaje del lenguaje visual-, el bloque llamado Darstellungstechniken o técnicas y herramientas de representación, las ciencias exactas y las asignaturas teóricas. El Visuelle Einführung constituía el núcleo fundamental y el centro de gravedad del Grundehre, donde se establecían las bases y las enseñanzas fundamentales del proyecto para los futuros diseñadores. El resto de asignaturas tenían un carácter de apoyo técnico -bien de representación, elaboración de modelos en taller- y teórico.

Es imposible describir el curso fundacional de la escuela de Ulm. Todo lo que puede describirse son una sucesión de varios cursos entre los años 1953-4, cuando la escuela comenzó, y 1960-61 después del cual el Curso Básico fue abolido. ${ }^{76}$
74 Geswister-Scholl Stiftung. School of design. Research Institute of Product Form. Ulm (Donau), Germany, 1951. Documento inédito. Fuente: HfG Archiv. Az 619. Document 14.

75 ulm. 1, Oktober 1958 - 5, Oktober 1959. Vierteljahresbericht der Hochschule für Gestaltung Ulm. Hg. v. Hochschule für Gestaltung. (Revista editada por el rectorado de la $\mathrm{HfG}$ ).

76 Froshaug, Anthony. "Foundation Course, Ulm 1953-61”. En: Kinross, Robin (ed.). Anthony Froshaug. Typography \& texts. Londres: Hyphen press, 2000. p. 133. Anthony. 
77 Froshaug, Anthony. "Foundation course, Ulm 1953-61. En: Kinross, op. cit. pag. 133

78 Bonsiepe, Gui. "Sobre la relevancia de la HfG UIm”, en: W.AA. Ulmer modelle - Modelle nach ulm, hochschule für gestaltung ulm 19531968. Ostfildern-Ruit: Hatje Cantz Verlag, 2003.
Anthony Froshaug explica ${ }^{77}$ que más que de una evolución, habría que hablar de una sucesión de varios cursos básicos, debido a que las diferencias entre sus contenidos y sus protagonistas fueron muy marcadas. ¿Por qué varios? Son los protagonistas y sus diferentes enfoques del proyecto los que operan las transformaciones de un Curso Básico que nace bajo la influencia de los bauhäusler y se extingue como departamento independiente y común, durante el curso 1960-1961 tras experimentar una radicalización en la aplicación de la metodología científica en el proyecto.

Gui Bonsiepe ${ }^{78}$ recuerda la indefinición que en aquellos días existía acerca de la profesión de diseñador industrial y de su falta de concepto. Formgeber-dador de forma- era la denominación común de la persona que proyectaba los elementos producidos en la industria en los países de habla alemana.

La evolución del Curso Básico por tanto, consiste en la búsqueda individual de los sucesivos responsables por alcanzar la preparación de forma óptima de los futuros formgeber de la HfG para su actividad proyectual en los departamentos. El primer año de una profesión todavía por inventar. i1/55, 56 Folletos del Institute of Design Chicago en el archivo de Zeischegg. HfG Archiv Ze AZ 300.1-2 FBS
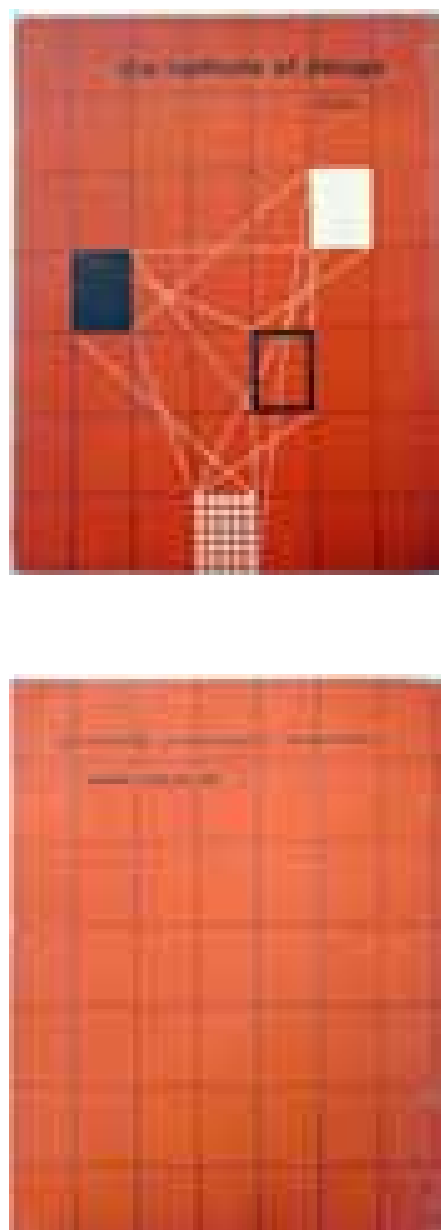

i1/57, 58 Documento manuscrito que examina el número de horas dedicado a cada materia del Institut of Design de Chicago. No se ha podido establecer la autoría.

Documento del HfG Archiv Ze AZ 31.1-2
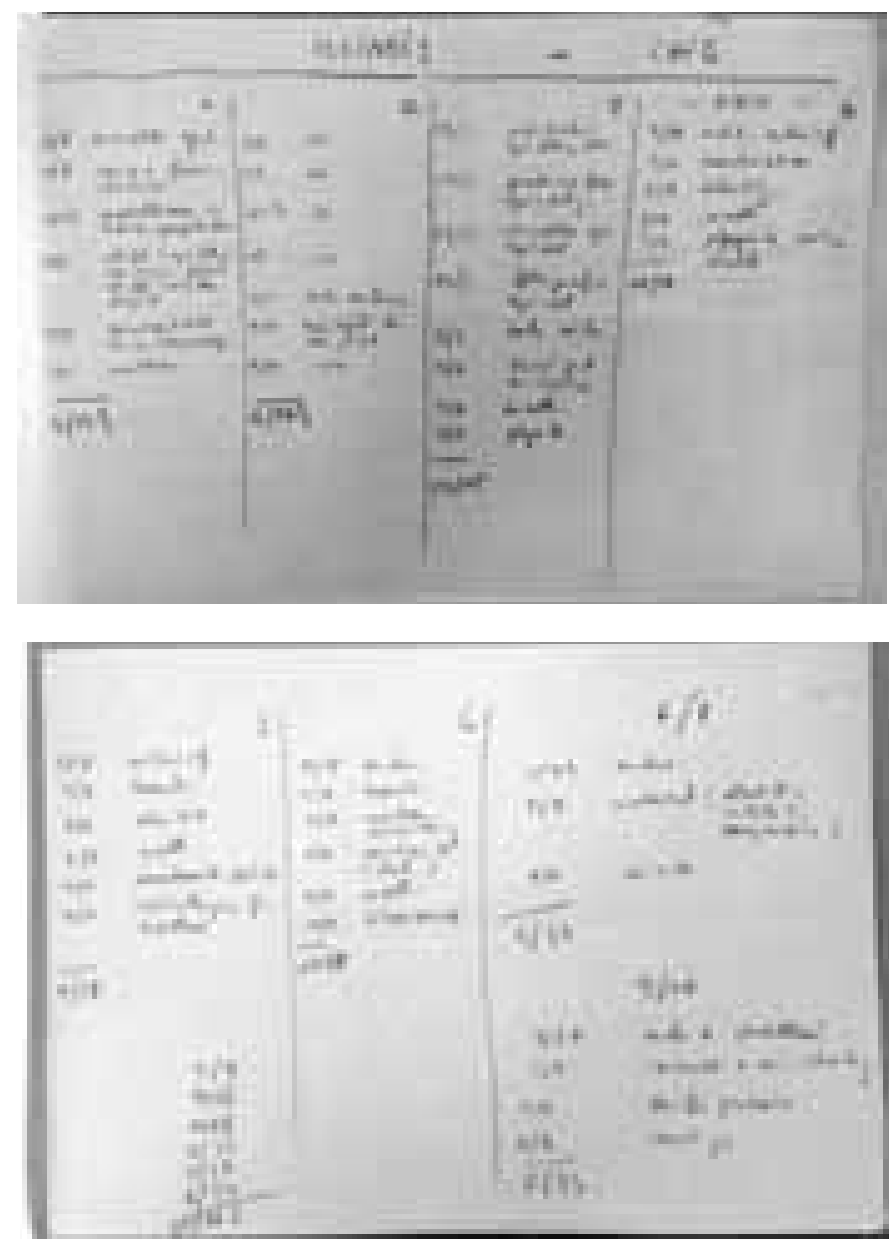


\subsection{Curso Básico fundacional}

\subsubsection{Contexto espacio-temporal del Curso Básico fundacional}

"querido gyorgy kepes,

como probablemente sepa, estoy organizando un instituto que continúa nuestra querida vieja Bauhaus en Alemania. naturalmente es algo diferente que usted encontrará adjunto en un folleto preliminar.

esta carta es para informarle que necesitaríamos para un primer año algunos profesores de prestigio. tenemos buenos jóvenes artistas pero no están formados como docentes. nuestros cursos básicos necesitan profesores invitados para instruir a pequeños grupos de estudiantes y al mismo tiempo a nuestros jóvenes.

para esta labor también he convocado a venir desde los estados unidos a mi viejo amigo josef albers. también planeamos llamar a walter peterhans. y ahora quiero preguntarle si podría serle posible venir por algunas semanas o meses y en qué momento". ${ }^{79}$

"Querido señor Bill,

Muchas gracias por su carta. Estuvo bien saber acerca de usted y de sus emocionantes planes. Pienso que es una tarea fundamental restablecer la Bauhaus en Alemania, y me complace que usted sea el espíritu que lo guía". ${ }^{80}$

El intercambio de palabras entre un director que no culmina la trayectoria de la Hochschule für Gestaltung y un candidato a profesor que nunca llega a concretarse ${ }^{81}$, expresan la intensidad de la puesta en marcha del primer Curso Básico que inauguraría la HfG Ulm. La escuela siempre se caracterizó por crecer en un estado de ósmosis constante entre un cuerpo docente formado por artistas, científicos, técnicos, arquitectos y teóricos, y un conjunto de estudiantes que acudieron desde todas partes del mundo. Max Bill cree en la importancia de la incorporación de profesores experimentados que sean una garantía en la puesta en marcha del Grundlehere y que contribuyan a la formación docente de los artistas que ya integran la escuela, como Aicher y Zeischegg. Bill acude a los profesores de la Bauhaus para formar este primer núcleo que confiera el impulso y la proyección necesarias a la nueva escuela.

El Curso Básico fundacional comienza el 3 de agosto de 1953 en la sede provisional de la Ulmer Volkshochschule, organizado por Max Bill y bajo su rectorado. Como se puede apreciar en la carta a Gyorgy Kepes, la fundación de la escuela significa el relevo, la recogida
79 Bill, Max. Carta a Gyorgy Kepes con copia a Inge Aicher-Scholl, Zürich, 11 de mayo 1953. Carta inédita, Archivo HfG Ulm. En minúsculas del original.

80 Kepes, Gyorgy. Carta a Max Bill, MIT Cambridge, 13 de mayo 1953 Estados Unidos. Carta inédita, Archivo HfG Ulm.

81 Gyorgy Kepes en aquel momento enseñaba en el MIT invitado por Moholy Nagy. En la carta explica que, a pesar de que desea colaborar con Max Bill en la escuela, sus compromisos laborales en Estados Unidos le impiden comprometerse a ser docente de la HfG. 


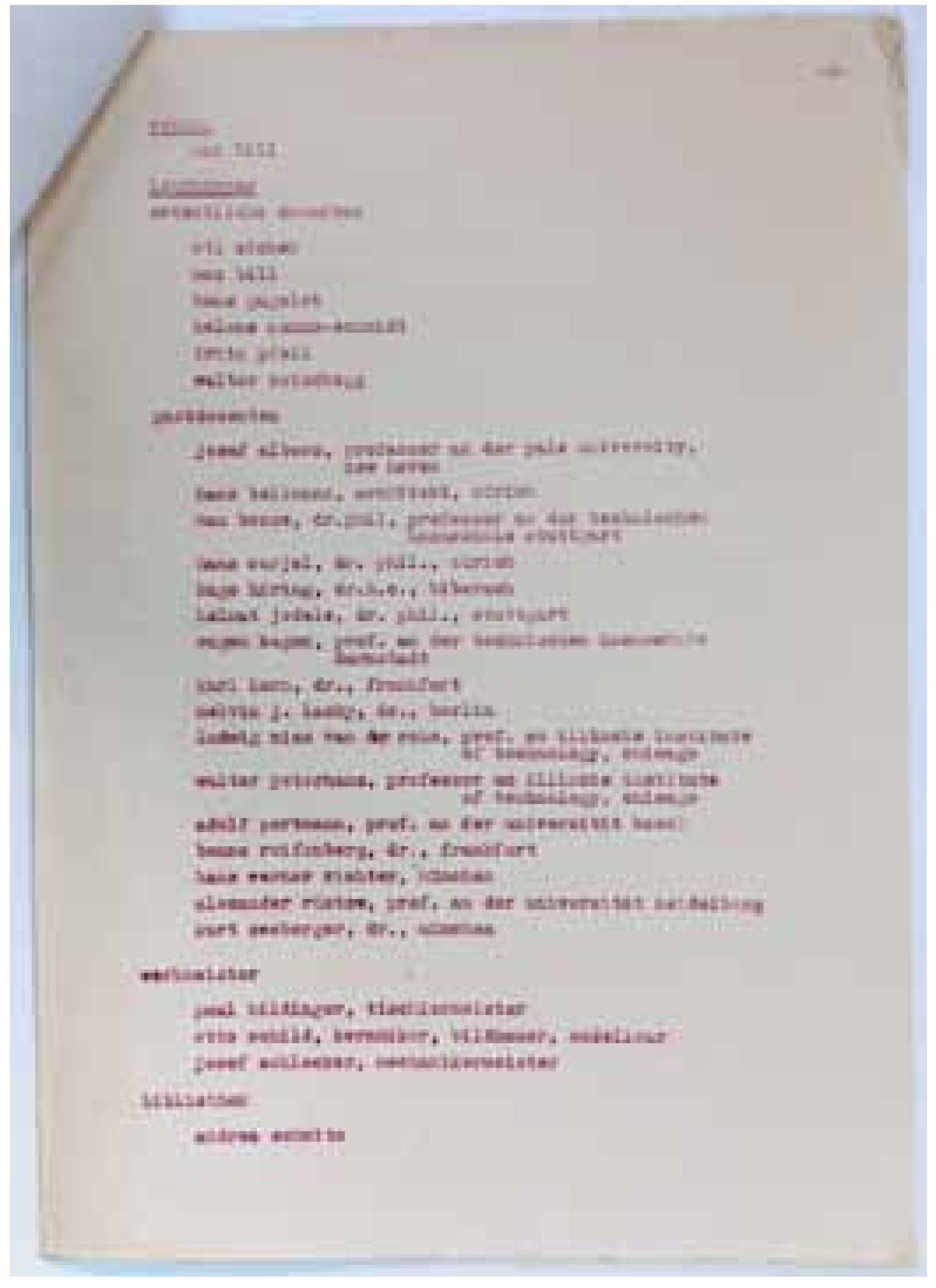

i1/59 Cuerpo docente del

primer curso.

Documento HfG Archiv Az 31.1 
del testigo de la mítica Bauhaus, no para revivirla y continuarla sino para evolucionar su legado.

La referencia a la Bauhaus se convierte en una manera sencilla de ubicar la escuela en un contexto y en un titular orientados a la difusión general. Sin embargo, esta herencia no es reflejo del planteamiento general de la educación y los objetivos que se propone la HfG. La prensa acentúa este vínculo con la vieja escuela anunciando en la inauguración del edificio en 1955 "La nueva Bauhaus", "Vuelve la Bauhaus" "la HfG asume la sucesión de la Bauhaus". ${ }^{34}$ Esta asociación es razonable si se tiene en cuenta que Max Bill fue exalumno de la Bauhaus de Dessau, donde estudió dos años, que Walter Gropius apoya explícitamente el proyecto y que el primer grupo de profesores tiene representación de un nutrido grupo de ex-Bauhaüsler. Sin embargo, la raíz didáctica y los objetivos tienen más que ver con el Institut of Design de Chicago que funda Moholy Nagy que con la propia Bauhaus.

Se considera en esta investigación que Curso Básico fundacional abarca el curso 19531954 y el 1954-1955. Durante el segundo curso, los ecos de los planteamientos del inicio perduran, pero ya se comienza a gestar el cambio de rumbo del Grundlehre bajo la dirección de Tomás Maldonado.

\subsubsection{Docentes, materias y alumnos del periodo}

En este apartado se incluye una relación de materias impartidas en Grundlehre y los docentes responsables, repartidas en las cuatro áreas temáticas mencionadas: Visuelle Einführung, Darstellungstechniken, o técnicas y herramientas de representación, las ciencias exactas y las asignaturas teóricas pueden organizarse en cuatro áreas: Visuelle Einführung -aprendizaje del lenguaje visual-; Darstellungstechniken -técnicas de representación-, Naturiuische Facher -ciencias exactas-, Allgemeinbildende und Theoretische Fascher -materias generales y teóricas-.

Asimismo, se recoge la relación de estudiantes que cursan en cada periodo Grundlehre y su campo de titulación, en caso que completaran los estudios. ${ }^{83}$

\section{Docentes contratados e invitados 1953-1954 ${ }^{84}$}

Otl Aicher

Josef Albers

Max Bill

Max Bense

Hans Bellmann

Hans Curje

Helene Nonné-Schmidt

Walter Peterhans

Adolf Portmann

Walter Zeischegg
Maestros de taller 1953-1954

Paul Hindinger - Madera

Otto Schild - Yeso

Josef Schlecker - Metal

Cornelius Uittenhout - Plástico
82 Albers, Ingela; Quijano, Marcela \& Wachsmann, Christianne. Baushaüsler in UIm. Die Grundlehre an der Ulmer HfG zwischen 1953 und 1955. Ulm: HfG-Archiv UIm, 1993, p. 4.

83 La relación de asignaturas impartidas y el docente responsalbe se extrae del horario realizado por la comisaria del Ulmer Museum Christianne Wachsmann.

El Atlas Synopse realizado por Hans Nick Roericht y su equipo es el documento de referencia de donde se extraen los datos de profesores y alumnos por curso.

84 La relación de profesores que se recoge en el documento HfG Archiv Az 31.1 -página izquierda-, incluye entre los profesores contratados a Fritz Pfeil, un arquitecto colaborador de Max Bill en el proyecto y dirección de obra de la sede de la $\mathrm{HfG}$ que no impartió docencia. También se incluyen en los profesores invitados a un número de docentes que solo impartieron una conferencia, como Hugo Häring con "Fábrica de formación orgánica” y Mies Van der Rohe con "La juventud como una fuerza para la nueva construcción”. 


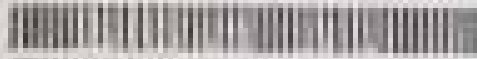
|||||||| | |||||||||||||||||||||||| |- | 1. 4.

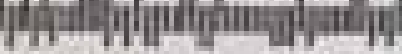

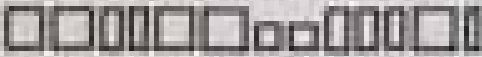
$\square 00000000$ OOOoooooogda hhddggelehrter ge ometerdada amen 口DL Laggerist ttt MModernnnnnnno
100 Oberschwei?

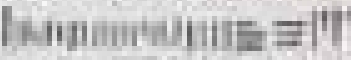
agoDLLeebben Rauh quu schaubidaubiauu schaubidaubiauu schhhhih Magenbauchaao agerecee

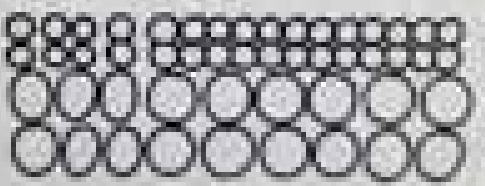

i1/60 Materia Schrift impartida por Otl Aicher del bloque Darstellungthechniken o técnicas de representación. Alumno Erler 1953-1954. Dp 009.16

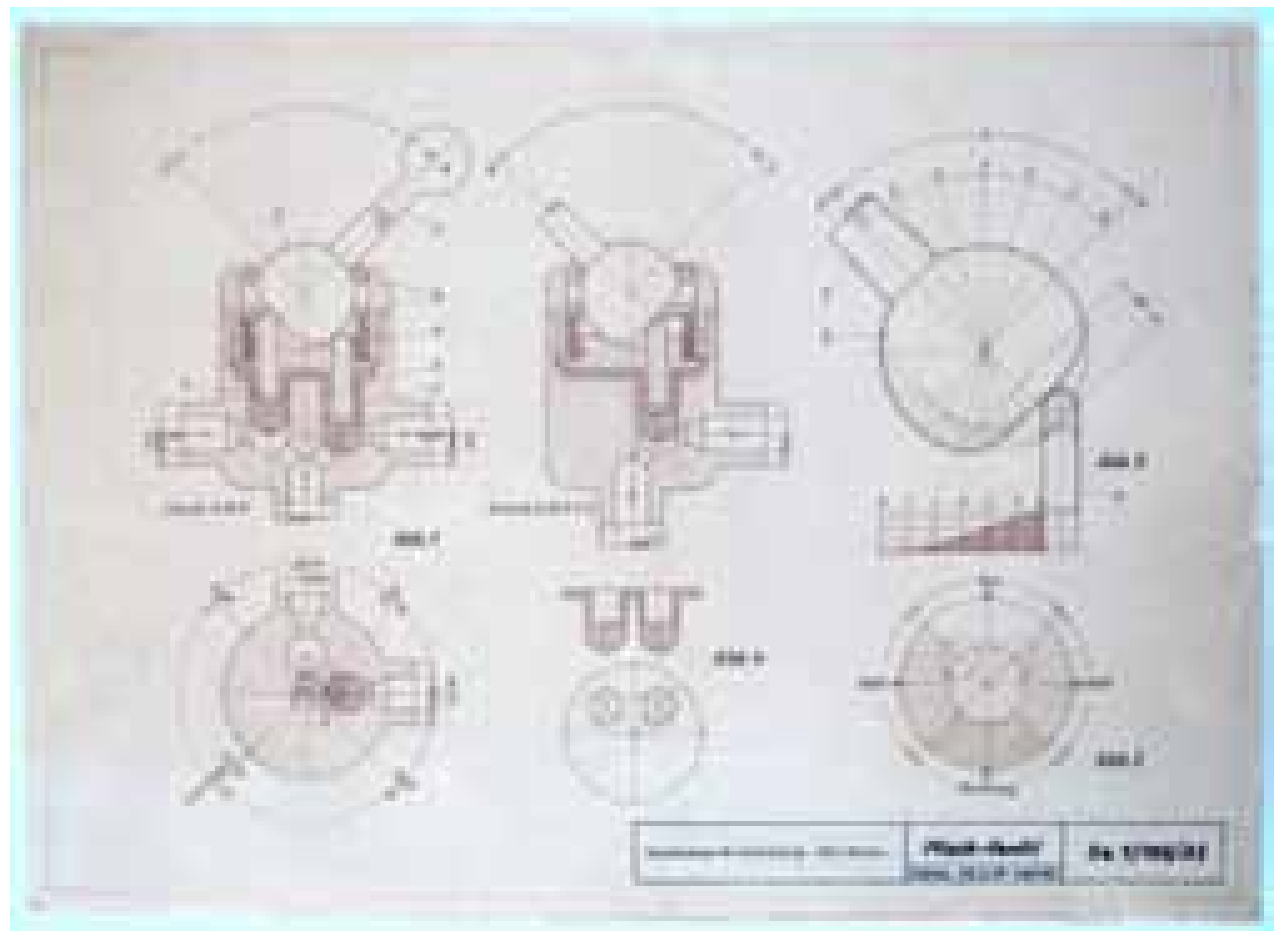

11/61 Materia Technische Zeichnen impartida por Walter Zeischegg del bloque Darstellungthechniken o técnicas de representación. Representación de válvula mezcladora Alumno Ernst Moeckl 1954-1955. Ze G1 


\section{Materias 1953-1954}

Visuelle Einführung - El curso 1953-1954 comienza con un seminario de tres meses dirigido por Walter Peterhans. Le sigue el curso de Albers de dos meses y tras él, Nonné-Schmidt imparte docencia los cinco meses de curso restante. Max Bill no aparece con un curso propio continuado sino en colaboración con Nonné-Schmidt.

Darstellungstechniken - Se imparten durante todo el periodo sin discontinuidades las asignaturas Technische Zeichnen -dibujo técnico- por Walter Zeischegg y Schrift -escritura- por Otl Aicher. En este bloque se incluyen las colaboraciones de alumnos en el proyecto y dirección de la sede de la HfG. El dibujo técnico aborda el dibujo de objetos desde la geometría descriptiva. Walter Zeischegg desarrolla varios de los elementos del edificio como el sistema de iluminación y el lavabo y se dibujan en Technische Zeischen. Los ejercicios de escritura de Aicher se centran en la ejercitación de la destreza del dibujo a mano alzada de tipos a diferentes escalas.

Allgemeinbildende und Theoretische Fächer - Se imparte Kulturgeschichte, o historia cultural, por el profesor Curjel, durante dos meses, en Noviembre entre Peterhans y Albers y junio, el último mes de curso. En los dos meses se realiza un breve recorrido por lo más destacado de la historia del arte y de la arquitectura del siglo XIX la primera mitad de siglo XX.

\section{Alumnos 1953-1954}

Rudolf Bleisch

Armin Bohnet *producto

Hans Conrad

Ermanno Delugan

Klaus Erler

Peter Gautel

Gerhard Gehle *arquitectura

Maurice Goldring

Alfred Griesser

Guido Herzog

Ello Hirschfeld

Fred Hochstrasser

Eva Maria Koch

Martin Krampen *comunicación visual

Imno Krumrey

Almir Mavignier *comunicación visual

Helmut Müller-Kühn *producto

Christoph Naske

Richard Rau

Christel Sztankovitz

Mary Viera 


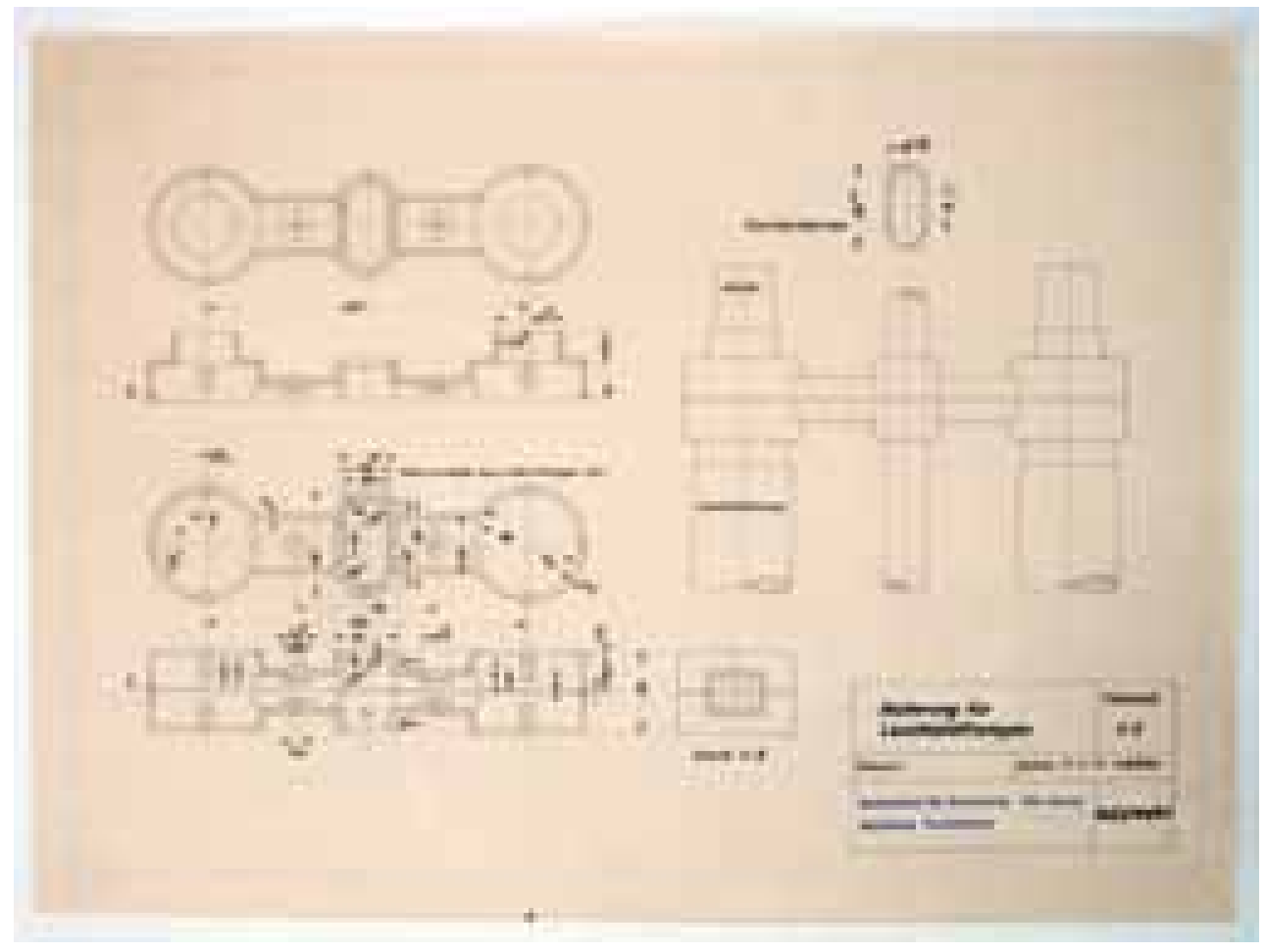

i1/62 Materia Technische Zeichnen impartida por Walter Zeischegg del bloque Darstellungthechniken o técnicas de representación.

Representación de lámpara y sistema de iluminación de la sede HfG Alumno Ernst Moeckl 1954-1955. Ze G2

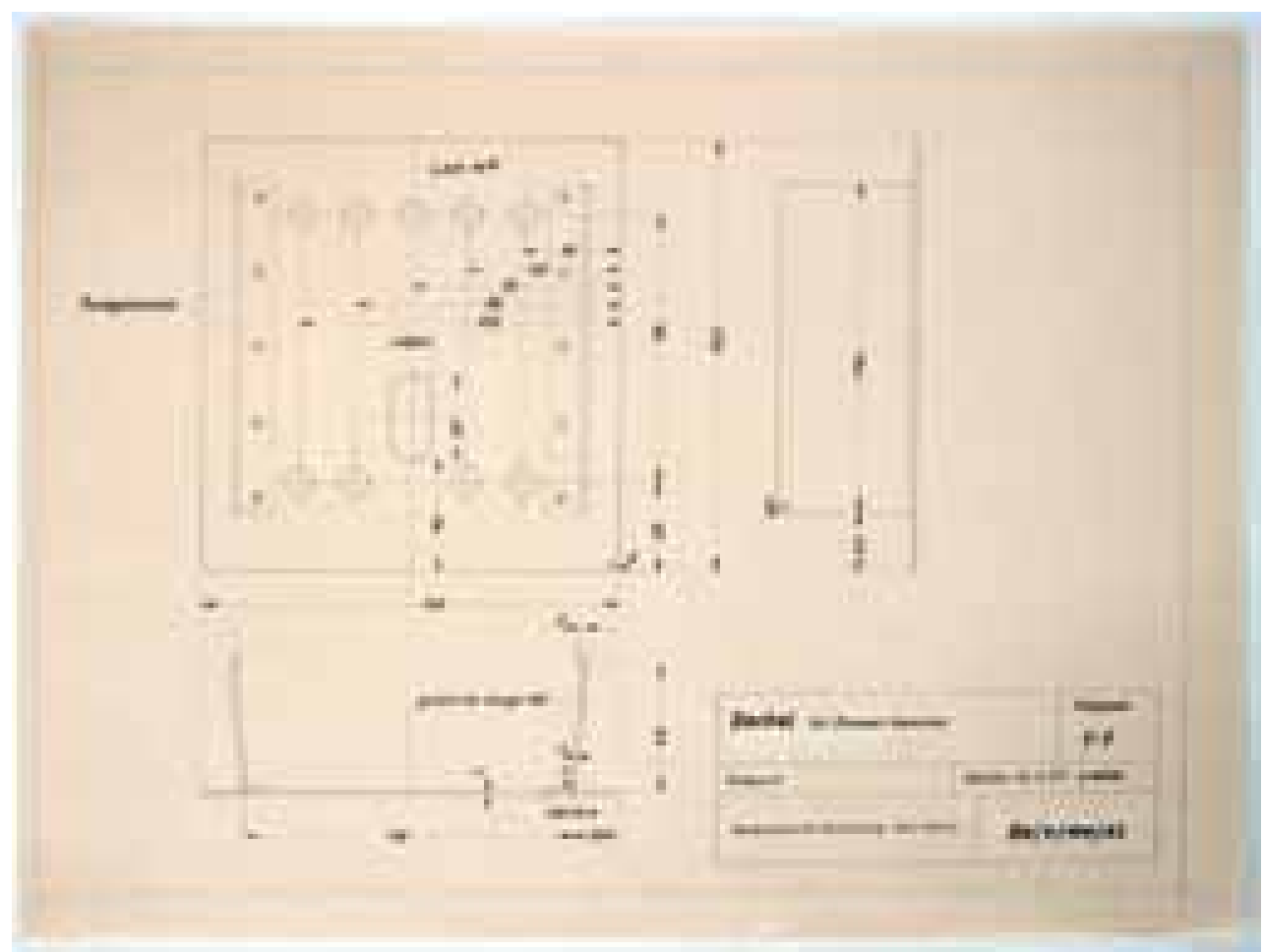

i1/63 Materia Technische Zeichnen impartida por Walter Zeischegg del bloque Darstellungthechniken o técnicas de representación. Representación de lámpara y sistema de iluminación de la sede HfG Alumno Ernst Moeckl 1954-1955. Ze G2 


\section{Docentes contratados 1954-1955}

\section{Otl Aicher}

Max Bill

Max Bense

Hans Gugelot

Tomás Maldonado

Helene Nonné-Schmidt

Fritz Pfeil

Walter Zeischegg

\section{Docentes invitados 1954-1955}

Josef Albers- Visuelle Einführung

Herman Von Baravalle - Visuelle Einführung

Hans Curjel, Kulturelle Integration

Johannes Itten, Visuelle Einführung

Aemilius Müller

Paul Schatz, Visuelle Einführung

Friedrich Vordemberge-Gildewart - Comunicación visual

Conrad Wachmann-Arquitectura

Podach

Heckelsberger

\section{Maestros de taller 1954-1955}

Paul Hindinger - Madera

Otto Schild - Yeso

Josef Schlecker - Metal

Cornelius Uittenhout - Plástico

\section{Materias 1954-1955}

Visuelle Einführung - Por los documentos que figuran en el archivo de la HfG, parece que el curso existe de forma irregular hasta que en Enero de 1955 se comienzan a impartir en la sede del Kuhberg. Tomás Maldonado aparece como responsable del seminario que imparte con Max Bill desde Enero hasta mediados de Abril. Albers continua desde mediados de Mayo hasta Julio. La enseñanza del color de Nonné-Schmidt aparecede forma continua en todo el periodo. Las enseñanzas de Konstruktive Darstellung -representación constructivaestán a cargo de Paul Schatz y Herman von Baravalle.

Darstellungstechniken - Se imparten durante todo el periodo sin discontinuidades las asignaturas Technische Zeichnen -dibujo técnico- por Hans Gugelot, que llega en este curso a la HfG y permanece hasta su fallecimiento en 1964. Comienzan los trabajos en los talleres de madera, metal y yeso. El taller de plástico estaba dedicado al Instituto de diseño de producto en este momento y a las investigaciones de Walter Zeischegg.

Allgemeinbildende und Theoretische Fächer - Se imparte Kulturgeschichte, o historia cultural, por el profesor Podach y Heckelsberger. Este último imparte su seminario en el otoño, antes del traslado al edificio. 


\section{Alumnos 1954-1955}

Mary Bauermeister

Carl Heinz Bergmüller * producto

Fritz Bühler

Walter Figle

Olivio Ferrari

Brigitte Gehle

Max Graf * arquitectura

Willi Herold * producto

Shoichi Kawai

Frauke Koch-Weser * producto

Heinz Kuhlmann

Herbert Lindinger * producto + comunicación visual

Margaret Marsenger

Ernst Möckl * producto

Eva Pfleil

Claude Schnaidt * arquitectura

Hans Schimmel

Walter Schneider

Rolf Schroeter

Fritz Sulzer

Peter Sulzer

Margit Staber * información

Colette Thienhaus-Dey

Alexandre Wollner

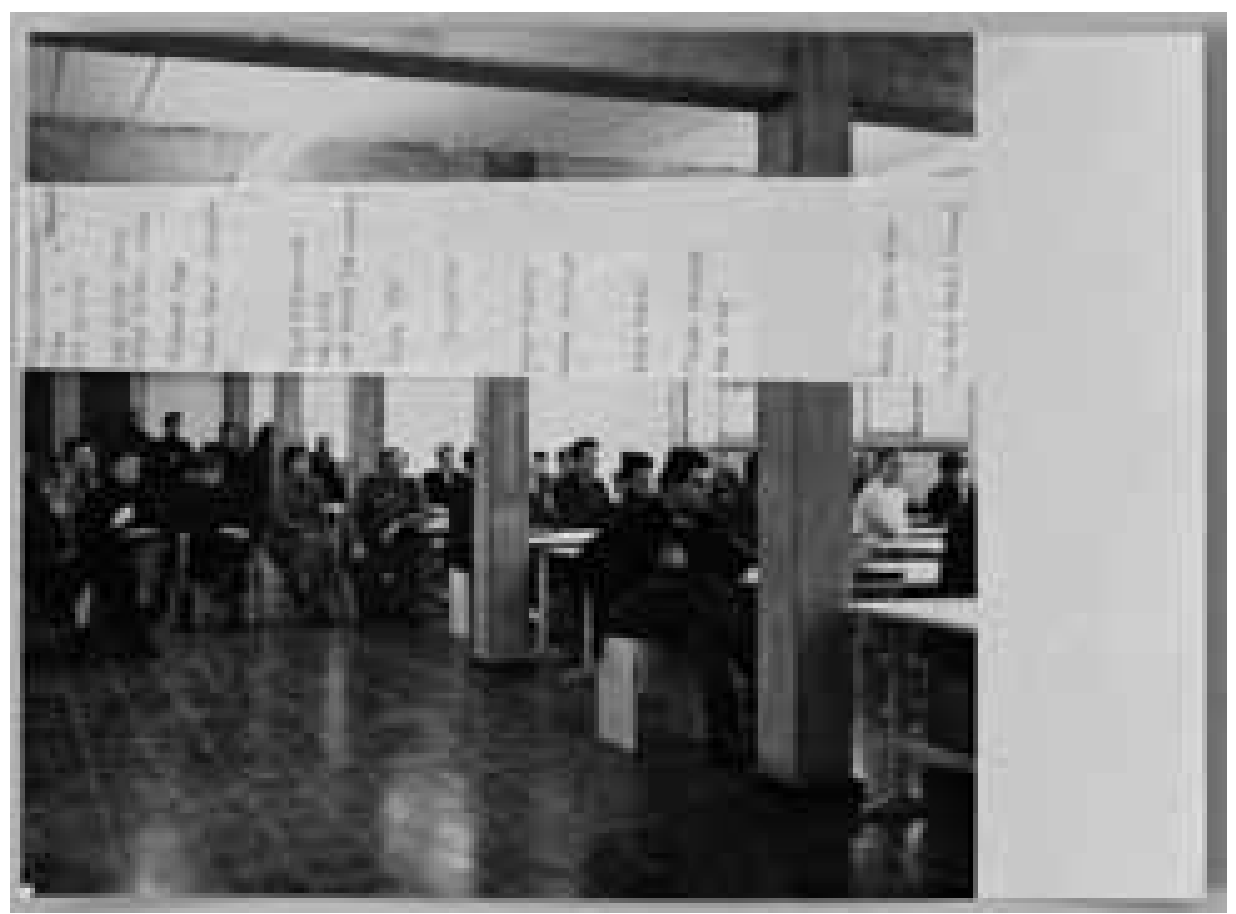




\subsubsection{Peterhans y el Visual Training ${ }^{85}$}

Walter Peterhans estudia ingeniería mecánica en la Universidad de Dresde y, posteriormente, obtiene la licenciatura en matemáticas y filosofía en Múnich y Göttingen. Su afición por la fotografía se convierte en profesión tras estudiar los aspectos de la técnica fotográfica, reproducción y procesos de impresión en Leipzig. En Berlín establece su comienzo profesional como fotógrafo y profesor, cuando está a punto de cumplir 30 años.

La llegada de Walter Peterhans a la Bauhaus se vincula a la profunda transformación de las enseñanzas que lleva a cabo Hannes Meyer. ${ }^{86}$ Allí imparte un curso de fotografía orientado a la publicidad y a la prensa. Es donde entra en contacto con Mies Van der Rohe quien le pide colaboración para esbozar el primer plan de estudios de las enseñanzas de Arquitectura en el Armour Institute of Chicago, que posteriormente se transforma en el IIT.87

En la HfG, Peterhans importa los ejercicios del Visual Training del IIT manteniendo la metodología y condensado su desarrollo en tres meses. Además, imparte un seminario filosófico sobre David Hume. Como se ha explicado en el epígrafe dedicado al IIT, el repertorio del Visual Training consta de diez ejercicios que tratan sobre invariantes de carácter visual vinculadas a lo arquitectónico. ${ }^{88}$

Las soluciones a los ejercicios propuestos no son únicas, depende de la investigación personal de cada alumno. Por ejemplo, el enunciado del ejercicio $n^{\circ} 4$ describe el trazado de una curva sobre el plano que divida el espacio, permitiendo que éste fluya o una curvamembrana que trabaje en el espacio atándolo y condensándolo. En los ejercicios de los alumnos de la Escuela de Ulm se pone de manifiesto que las respuestas al enunciado poseen analogías estructurales pero el tratamiento de la forma es el que diferencia el carácter final de la solución: la curva, que se cierra sobre sí misma, se hace permeable al espacio en que se inserta bien fragmentándose o bien variando su grosor.

El sentido de la proporción y el espacio bidimensional se trabaja en los primeros ejercicios mediante la manipulación total o parcial del plano de trabajo. Inicialmente, el plano se subdivide en cuatro áreas buscando el equilibrio del todo y las partes a través de una situación de máxima tensión y asimetría de los elementos. La proporción también subyace en el resto de los ejercicios tanto en el tratamiento de la propia forma, su grosor, movimiento y disposición en el plano, como en el tratamiento de la alternancia entre blanco y negro y las superficies flotantes de los planos de color.

La forma se trabaja con líneas curvas en el mencionado ejercicio 4. La forma se considera el medio para definir el espacio del plano de trabajo. Las variables que se manipulan son el cambio continuo en el grosor y dirección de la curva, su orientación, su apertura o conexión, la continuidad o fragmentación del trazo y el tratamiento como membrana.

En el ejercicio 5 y sus variantes, el desplazamiento de la línea da lugar a haces de rectas cuya densidad genera superficies alabeadas. En el ejercicio 6 se incorpora el sentido de la percepción tridimensional manipulando el contorno de los cuadriláteros que flotan configurando perspectivas y recreando la profundidad del espacio mediante relaciones de superposición, transparencia y opacidad.

El color es un elemento compositivo en el Visual Training. Peterhans elude el estudio de las cualidades cromáticas. En el último ejercicio de la serie, se trabaja de forma aleatoria
85 Se ha publicado un artículo que realiza un análisis comparativo de la docencia de Walter Peterhans en la Bauhaus, el IIT y en la HfG. Fernández Campos, Angel Luis, Sánchez Moya María Dolores; Benito Roldán, Emilia. "¿Qué cambiaría si el Crown Hall se pintara de blanco? Walter Peterhans : fundamentos de lo arquitectónico en el Visual Training" En: REIA, $n^{\circ} 7-8$ 2017.

86 El nuevo director busca potenciar uno de los mayores aciertos de Gropius: aumentar el vínculo de la escuela con la producción industrial y transformarla en una entidad productiva. Kieren, M. "La Bauhaus se convierte en una entidad productiva: el director Hannes Meyer" en: Fiedler J; Feierabend, P. Bauhaus. Colonia: Könemann, 2000 pág. 204.

87 "Los estudiantes parecían entender lo que decía acerca de la importancia de la proporción, pero no demostraban el más mínimo sentido de ella en sus ejercicios. Descubrí que, sencillamente, sus ojos no percibían la proporción". Van der Rohe, L. M. "The architecture curriculum at IT", 1937 en: Swenson, A; Pao-Chi; C Architectural education at IIT, 19381978, Chicago: Illinois Institute of Technology, 1980.

88 Ver página 65 de la tesis 


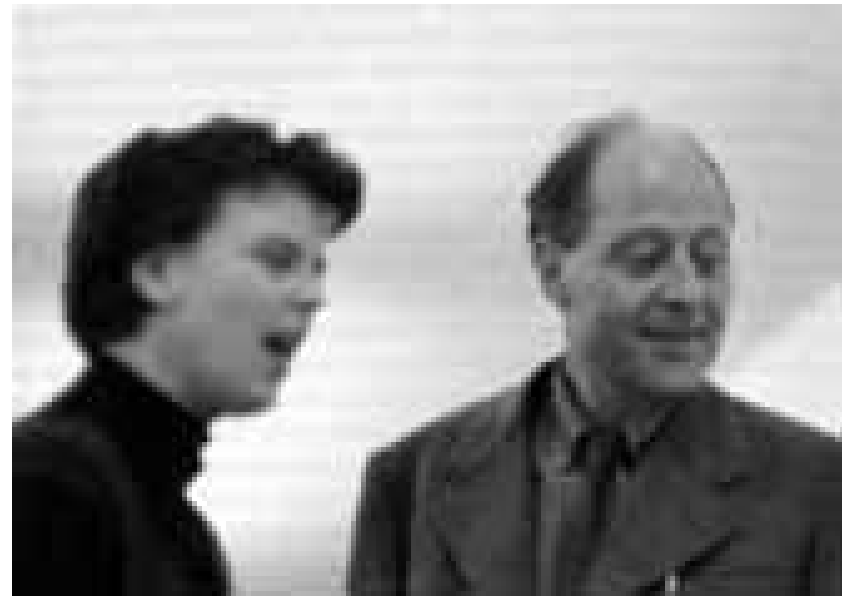

i1/65 Walter Peterhans en Ulm con

Inge Scholl

Hans G. Conrad

Fuente: Albers, Ingela; Quijano,

Marcela, \& Wachsmann, Christianne.

op. cit, p. 7.

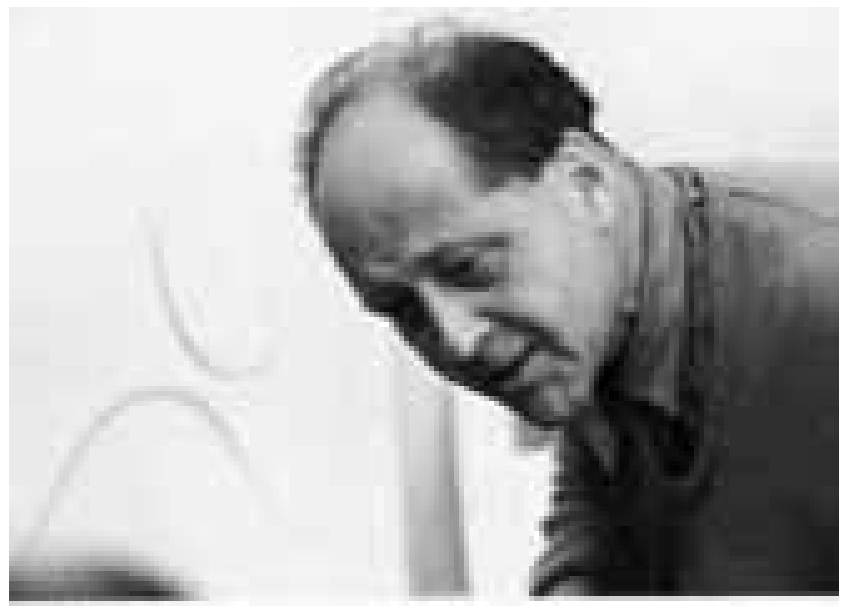

11/66 Walter Peterhans en UIm.

Donación Naske

Fuente: Albers, Ingela; Quijano,

Marcela, \& Wachsmann, Christianne.

op. cit, p. 6

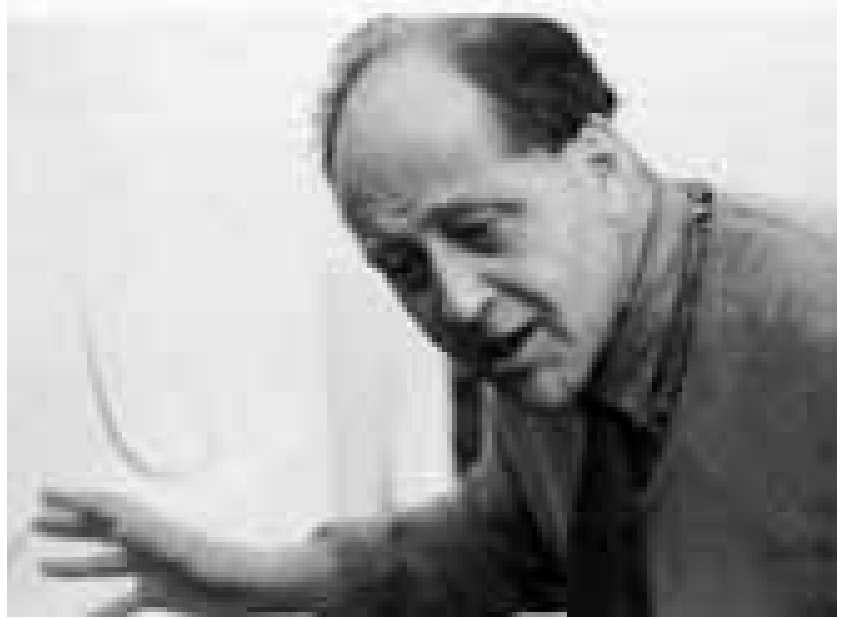


explotando las posibilidades visuales que ofrece la manipulación directa de los pigmentos haciendo que fluyan sobre el plano de soporte húmedo, generando formas y efectos visuales de transparencia, movimiento y densidad. En el ejercicio 6 el color es el elemento que potencia la profundidad del espacio. Su uso ponderado genera efectos de transparencia y solapamiento que articulan los planos entre sí. En el ejercicio 9 el color es una de las variables que otorgará más o menos peso visual y profundidad a los cuadriláteros determinando de este modo su distribución en el plano.

Uno de los vínculos entre el Visual Training y las enseñanzas inmediatamente posteriores del Curso Básico se fundamenta en los postulados del arte concreto, según los cuales la obra se construye con elementos puramente plásticos: planos y colores sin otro significado más que la forma que representan ni referencias a otras realidades.

El curso de Peterhans representa un primer repertorio sistemático e introductorio que no volverá a repetirse en los cursos siguientes. Quedarán, sin embargo, algunos ecos de sus enunciados en otros posteriores, como "Una linea que cambia continuamente de dirección y distribución" similar al que propone Max Bill "diferenciación de tres lineas en una superficie". El seminario introduce a los alumnos en la pulcritud y el esmero en el desarrollo del trabajo que representa una de las señas de identidad de la escuela y un denominador común de todos los trabajos del Visuelle Einführung. Se demanda un alto grado de exactitud y destreza gráfica en el dibujo, de forma que los resultados alcancen un valor estético. ${ }^{89}$
89 Max Bill marca como objetivo el valor estético que alcanzan los trabajos que dirige Peterhans. En el enunciado del examen del primer año de Grundlehre, las directrices que ejecución de los trabajos establecen que:

"estas tareas deben resolverse de ta manera que

su fundamento teórico sea evidente

su base teórica sea discernible

su representación técnica perfecta, ejecutada en tinta, pluma o pintura, el resultado tiene que alcanzar un valor estético como el tipo de láminas del profesor Peterhans"

Enunciado del examen de Grundlehre 1953-1954 en el Deposita de Klaus Erler, Archivo HfG. 
i1/67 Max Bill en el taller de yeso Dp 089.001 carpeta de originales y negativos de Ernst Scheidegger i1/68 Max Bill en clase

Fuente: Lindinger, Herbert, op. cit. pag 38.
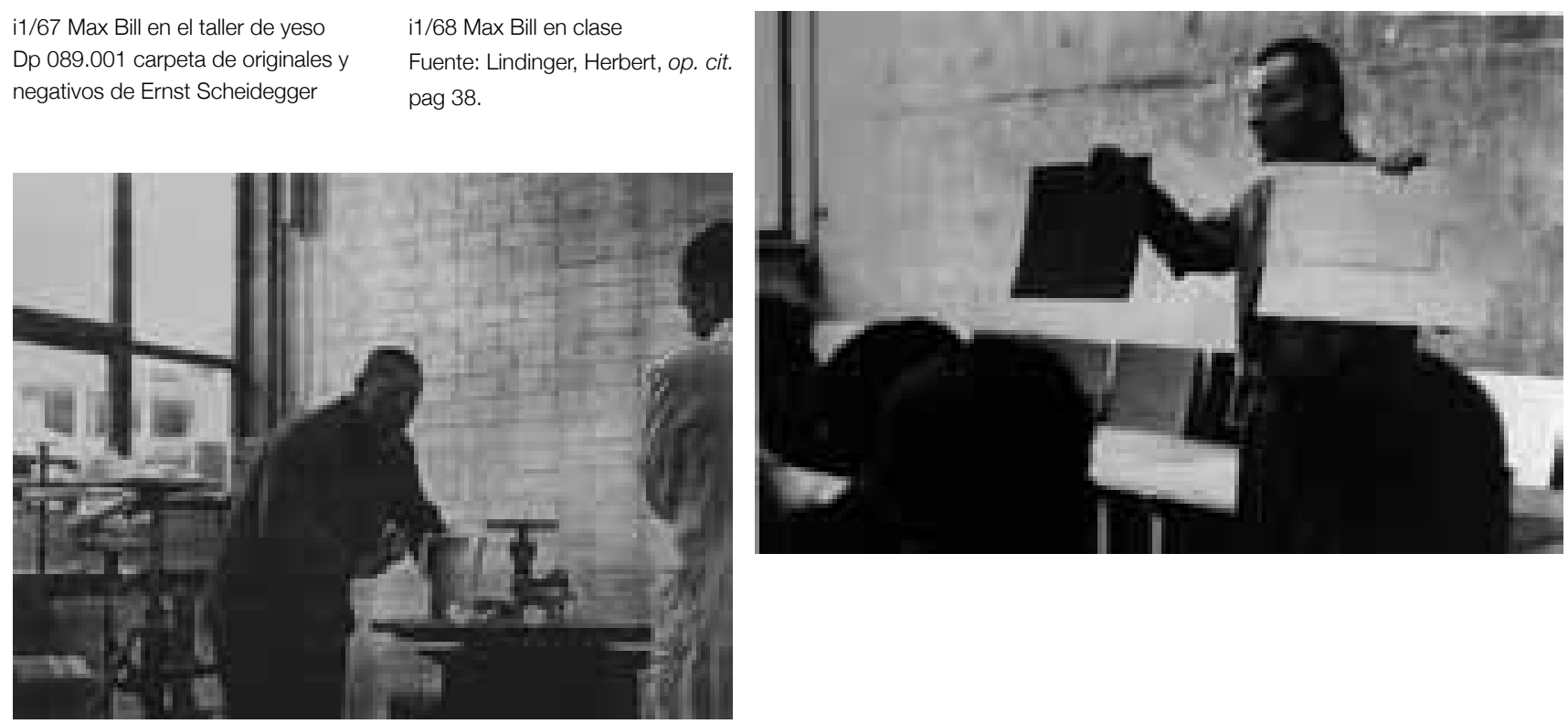

Instrucciones cuadrado $20 \times 20$ 1 fila de puntos horizontal y 1 vertical 1 distancias progresivas $1 \times$ distancias regulares Max Bill, 1942

Fuente: Maldonado, Tomás, op. cit. pag 11.

¡1/70 Solución de Klaus Erler a cuadrado 20×20 Dp 090.020

i1/71 Apuntes de Klaus Erler Dp 090.020

$x$ fila progresiva (expansión)

$1 \times$ tamaños regulares

$1 \times$ graduaciones regulares entre blanco y negro

$1 \times$ valor de brillo equivalente
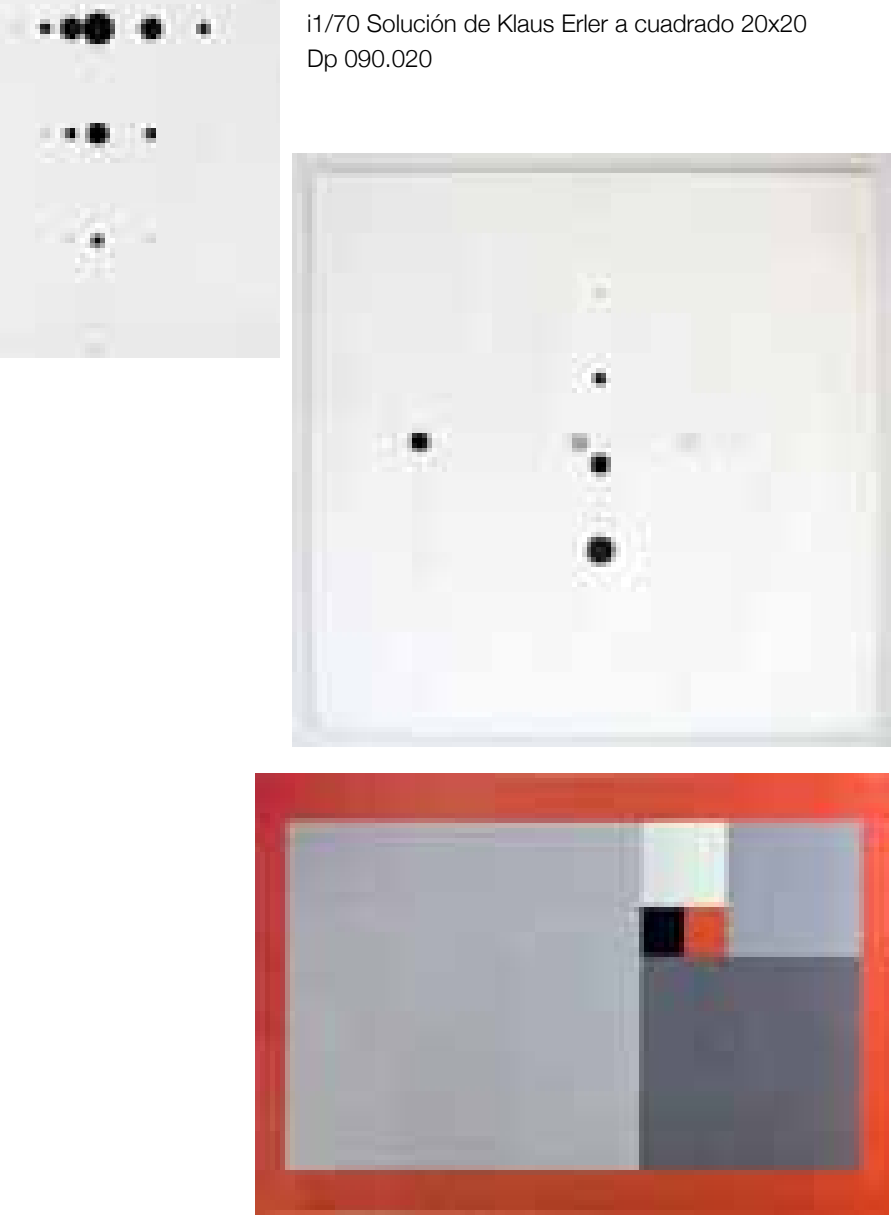

i1/72 Curso Bill.

Escala de grises en 5

cuadrados y un color

Alumno: Herbert

Lindinger
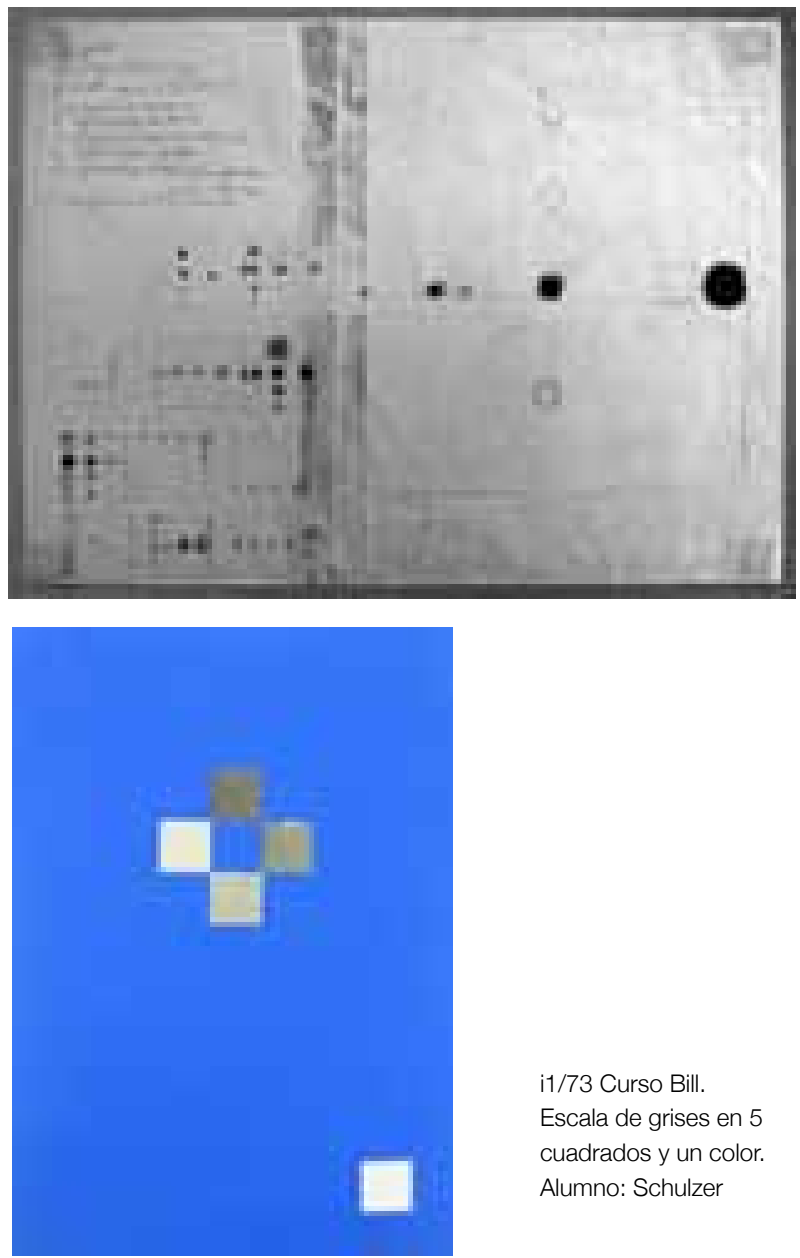

i1/73 Curso Bill.

Escala de grises en 5

cuadrados y un color.

Alumno: Schulzer 


\subsubsection{Max Bill desde el arte concreto}

Empieza el curso 1953-1954 y Max Bill está inmerso en una actividad frenética. No solo se dedica a poner en marcha toda una escuela, también se está ocupando de cumplir con el compromiso de la construcción de la sede en los plazos que han establecido las subvenciones y sigue atendiendo en su estudio de Zúrich los encargos que están en marcha; en ese periodo de tiempo también atiende exposiciones y conferencias en Sudamérica. ${ }^{90}$

Su papel en este Curso Básico fundacional está más dedicado a la gestión y a la búsqueda de profesores que a tener un protagonismo en la docencia. En el archivo, los ejemplos de ejercicios propuestos por Bill no aparecen formando un seminario completo como en el caso de Peterhans o Albers, sino como enunciados sueltos o bien intercalados entre ejercicios de otros docentes como Nonné-Schmidt en 1953-1954 o Baravalle 1954-1955.

Se observa tanto en los ejercicios que se custodian en el archivo de la $\mathrm{HfG}$, como los que han sido publicados, que el breve repertorio del primer año se amplía y completa en el segundo, probablemente porque ya cuenta con el apoyo de Tomás Maldonado en clase. Todos los ejercicios tienen un planteamiento fuertemente enraizado en el arte concreto. Tanto la forma como su composición entronca con los principios más radicales de este arte y con realizaciones plásticas propias de Max Bill. En los ejercicios se valora la elocuencia del proceso en el desarrollo del alumno y cómo éste responde al enunciado. Los estudiantes tienen que escribir una breve memoria justificando la solución propuesta, que es una elección personal en cada caso. La justificación con base teórica resulta ser una constante en el método de trabajo de los alumnos de la HfG, y llega a ser una parte obligatoria con el tiempo. La discusión y el razonamiento de las decisiones están en la base del trabajo.

Para Max Bill, el misterio de los problemas matemáticos, como el concepto de una infinitud finita, lo inefable del espacio, la sorpresa de un espacio que comienza por un lado y termina por otro, un campo de fuerza, las líneas paralelas que se encuentran en el infinito, son las más trascendentales para la humanidad. El arte concreto asume estos principios universales que subyacen en todas las cosas y los hace visibles. ${ }^{91}$

Los ejercicios parten de un formato básico -cuadrado o rectangular- sobre el que se organizan otras formas según principios de configuración que relacionan todos los elementos entre si por la modificación de su color, posición y ubicación. Las tareas llevan implícitas una serie de objetivos a conseguir que están formulados en el enunciado y están relacionados con principios matemáticos como las escalas de grises basadas en la progresión aritmética, las formas básicas y su transformación.

Los ejercicios de distribución de escalas de puntos sobre el plano, organización de seis elementos cuadrados en escalas de grises y la distribución de líneas ocupando el plano se repiten los dos años del Curso Básico fundacional. Tienen en común unos principios de trabajos comunes que integran una sintaxis propia, la ocupación de un plano de soporte mediante la disposición organizada de los elementos visuales. Max Bill extrae de su propia obra plástica los principios compositivos que utiliza como principios didácticos. En el ejercicio de distribución de la escala de puntos existe una relación directa con su obra $\mathrm{x}=7$ a través de la organización de los puntos en base a una progresión numérica. ${ }^{92}$

En este primer periodo se realiza un ejercicio en los talleres que consiste en la elaboración de un cubo con madera, metal o yeso. El objetivo consiste en que los estudiantes se habitúen a que la forma y la escala dependen en gran medida de las limitaciones y posibilidades que establezca el material o el Materialgerecht que también trabaja Albers. Si bien no hay documentos que vinculen este ejercicio a Bill, como resposable del Curso Básico, es bastante probable que fuera puesto en marcha por él. ${ }^{93}$
90 Albers, Ingela; Quijano, Marcela, \& Wachsmann, Christianne. op. cit, p. 23.

91 Bill, Max. "La concepción matemática en el arte de nuestro tiempo" en: Maldonado, Tomás. Max Bill. Buenos Aires: Nueva visión, 1955. p. 33-35.

92 "En $\mathrm{x}=7$, los puntos se ordenan según la progresión 1-3-5-7-5-3-1, numérica y geométricamente en forma de cruz, estableciendo un eje de coordenadas. Visualmente percibimos una sensación de orden, una simetría deformada horizontal y verticalmente donde los puntos perimetrales esbozan la figura de un romboide".

Martinez Castillo, Alberto. Max Bill: Variaciones sobre la búsqueda de la belleza. Tesis Doctoral. Universidad Politécnica de Madrid 2013, p. 63.

93 El ejercicio se realiza en los talleres, guiado por sus responsables. Este ejercicio bien podría haber partido de Walter Zeischegg pero el cubo no es un volumen de su repertorio. 
i1/74 Max Bill fotografiado por Josef Albers. Fuente: Horowitz, f., \& Danilowitz, B. Josef Albers: to open eyes. New York: Phaidon, 2006, p. 57

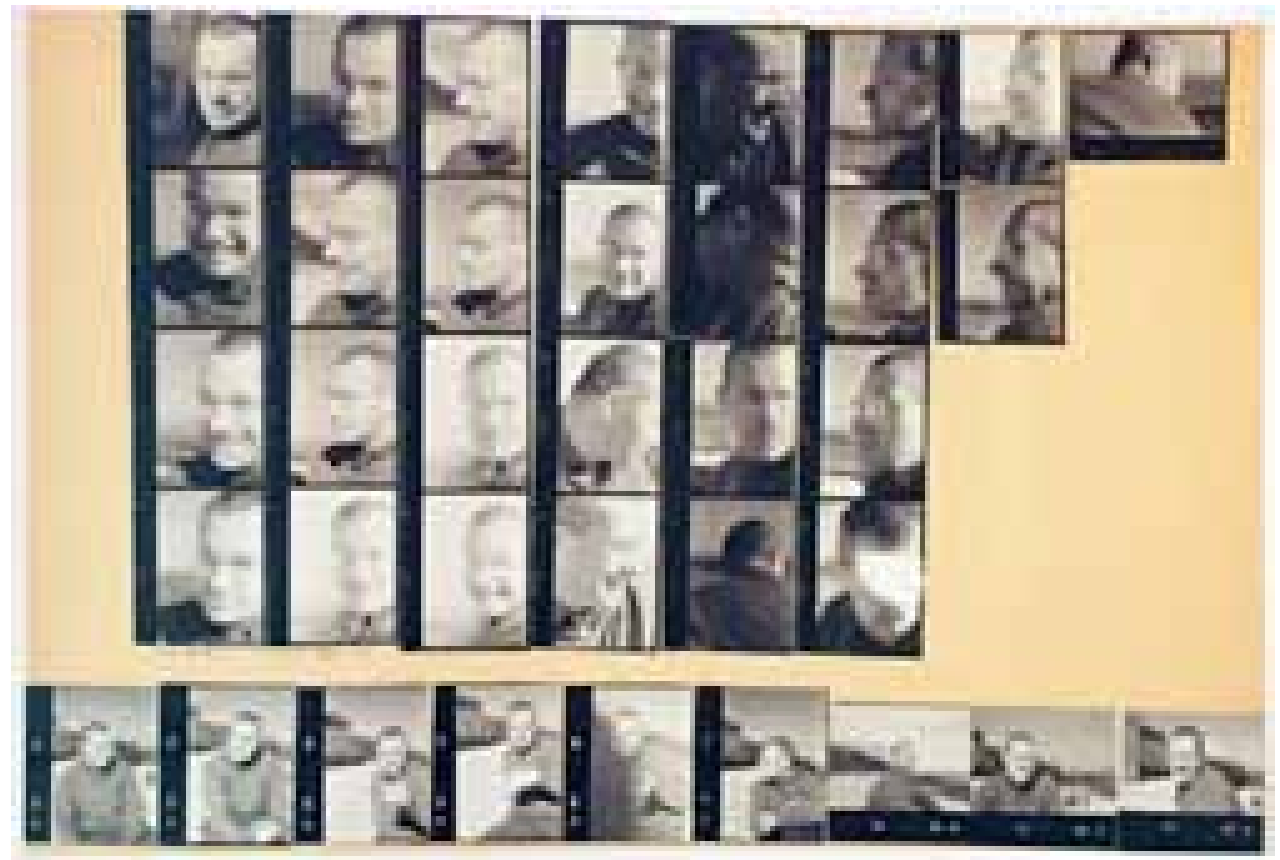

i1/75, 76 Aufgabe 5 Alumna: Eva Maria Koch

curso Bill 1954-1955 Archivo HfG, G 12 FBS
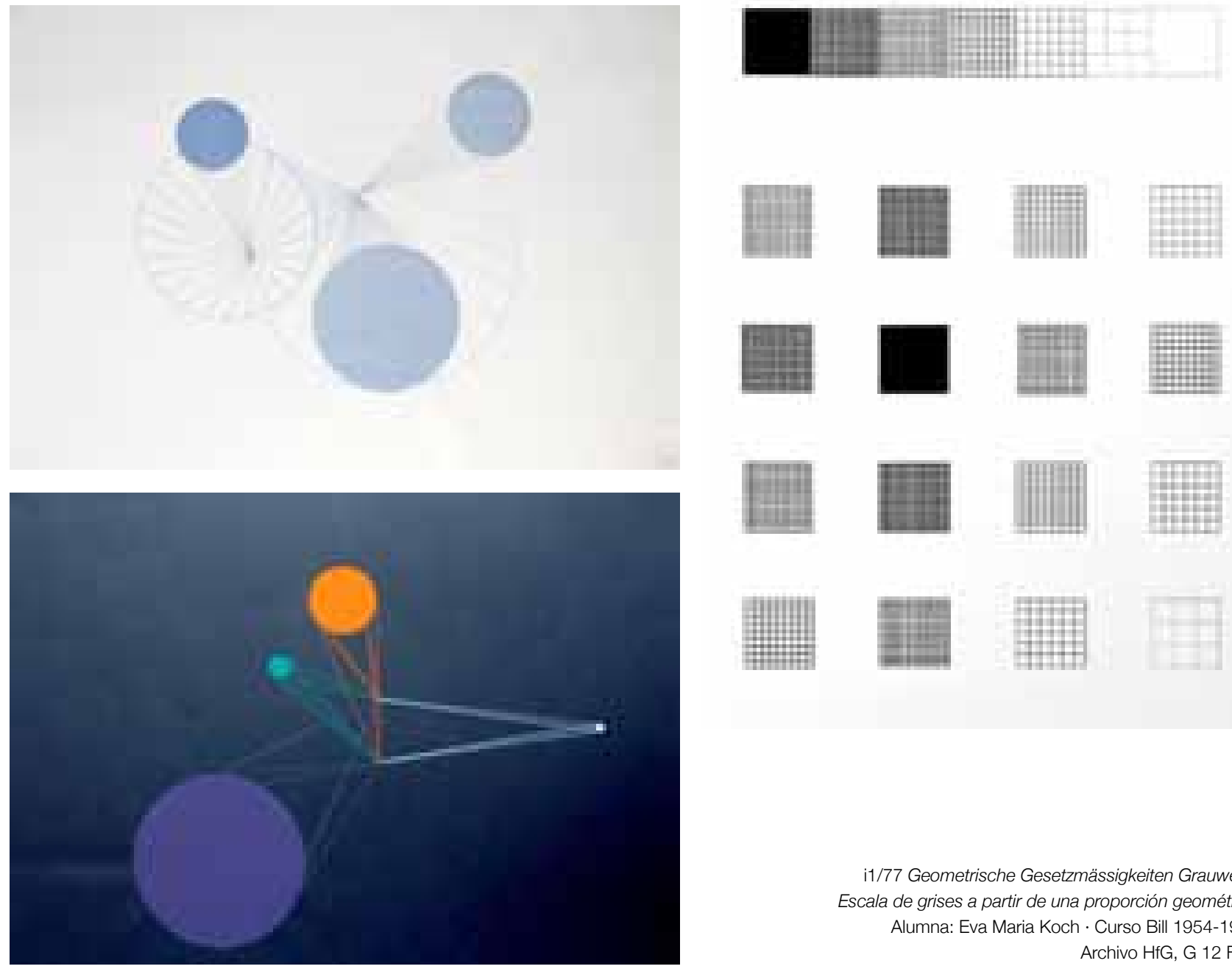

11/77 Geometrische Gesetzmässigkeiten Grauwerte Escala de grises a partir de una proporción geométrica Alumna: Eva Maria Koch · Curso Bill 1954-1955 Archivo HfG, G 12 FBS 


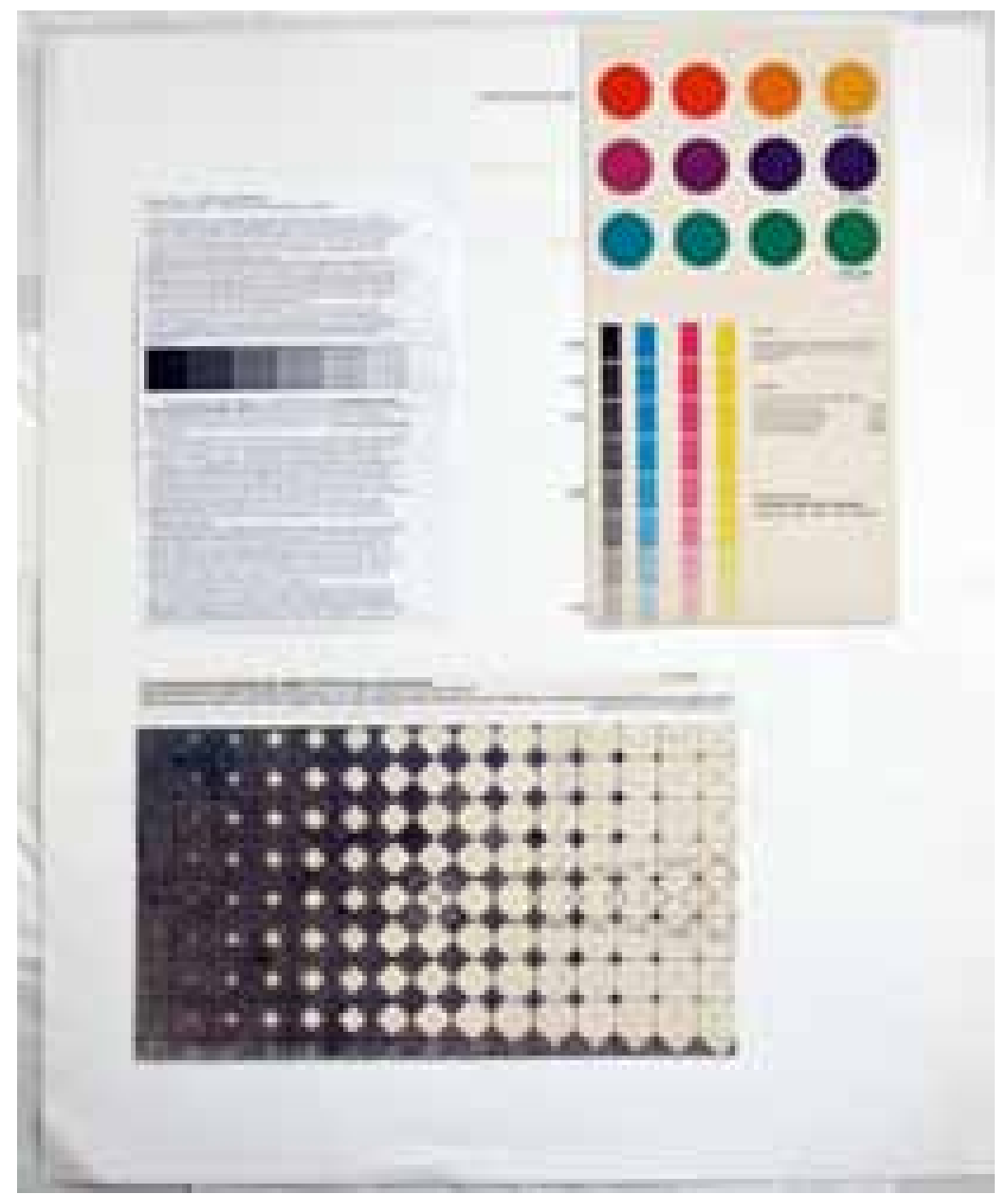

i1/82 Irmgard Philippi, taller de yeso

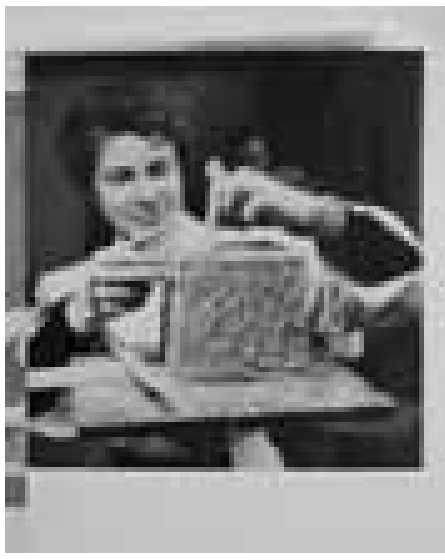

i1/84 Fritz Sulzer, taller de metal

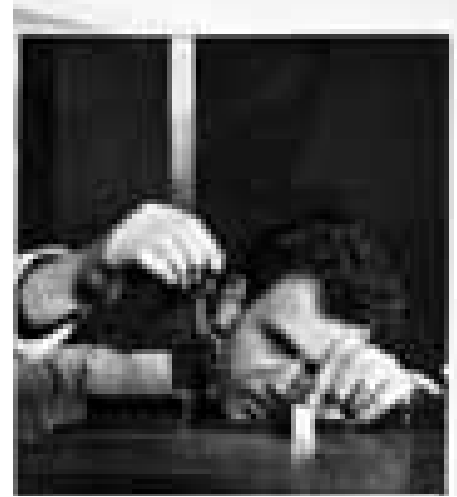

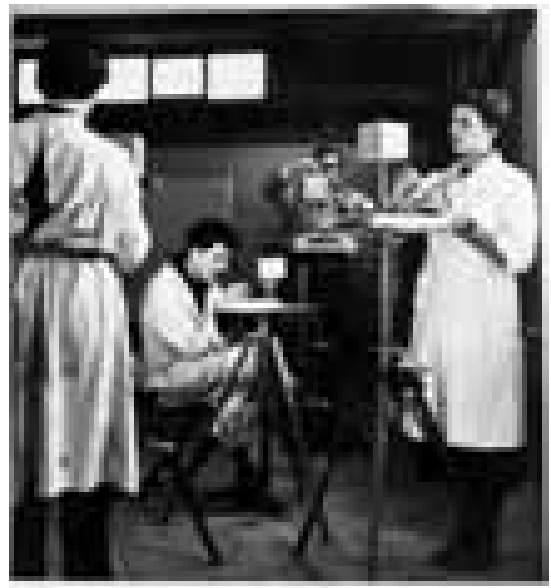

i1/83 Angela Hackelsberger, Burmeister, Thienhaus en el taller de yeso

Trabajo en los talleres 1945/55 Ernst Hahn. Dp 090.009 Archivo HfG
11/78 Trabajos de recuperación de Grundlehre 1953-1954, verano 1954 julio-septiembre Alumno: Erle

Docentes: Bill - Nonné-Schmidt

Dp 009.009

i1/79 Rolf Schröter, taller de metal
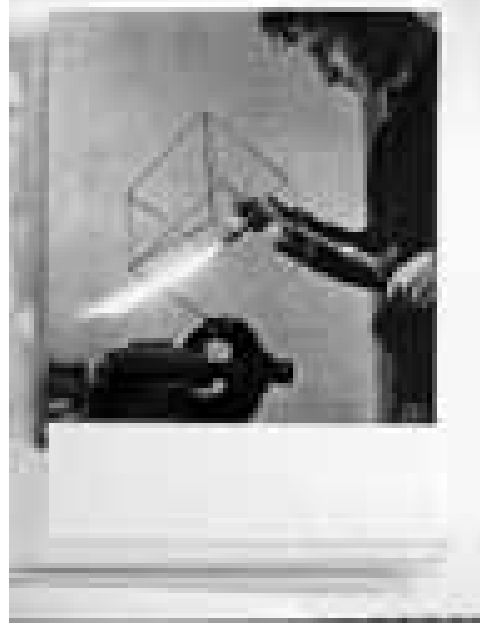

i1/80 Ermano Delugan, taller de madera

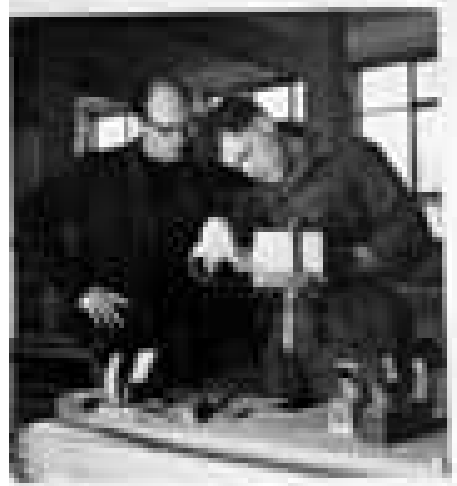

i1/81 Max Graf, taller de yeso

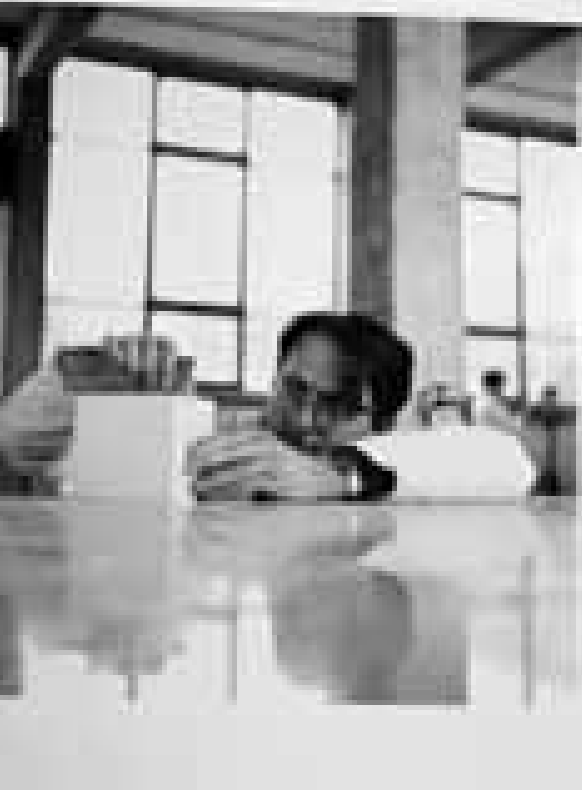




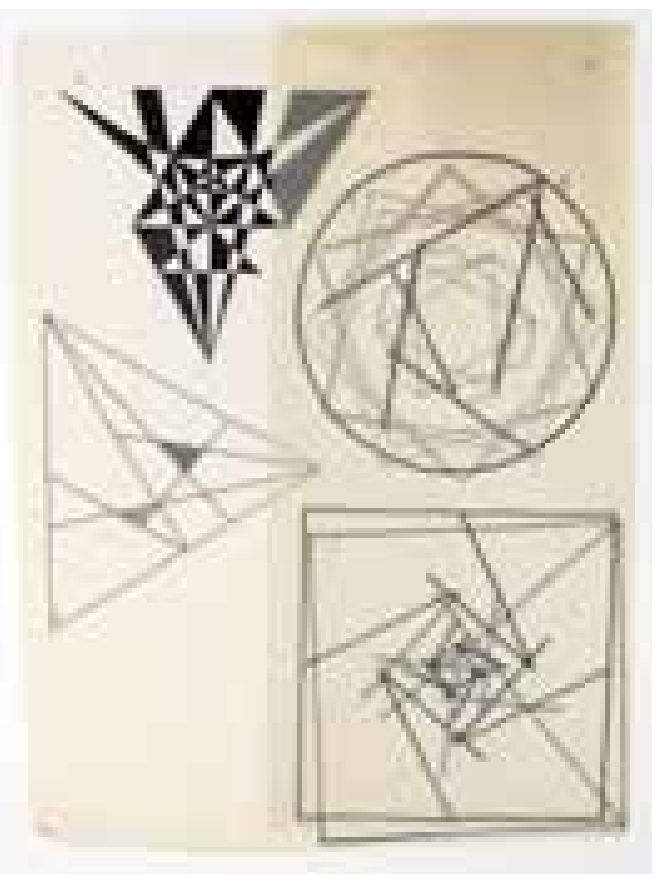

i1/85 Estudios de la forma a partir de

la subdivisión de las formas básicas

(Klee)

Alumno: Naske

curso Nonné-Schmidt 1953-1954

Dp 024.008 FBS

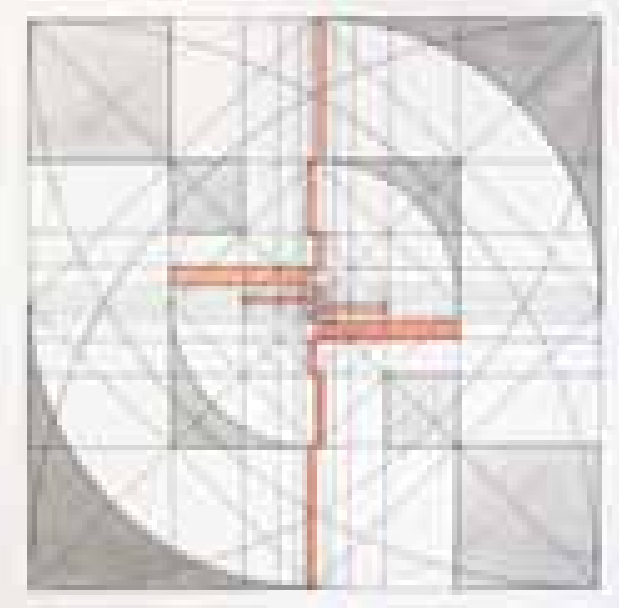

i1/86 Estudios de la forma, forma dentro del formato (Klee)

Alumno: Schroeter

curso Nonné-Schmidt 1954-1955 Dp 083.017 FBS

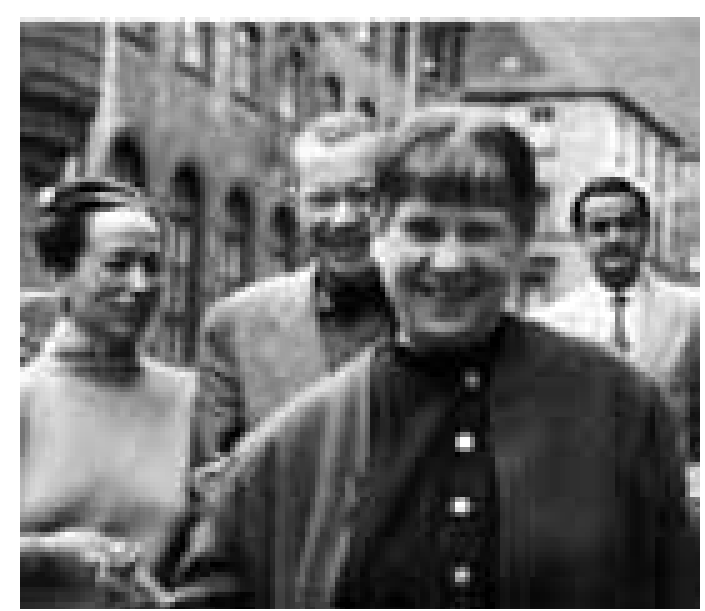

i1/87 Nonné-Schmidt en primer plano, Bill, Maldonado Alumna: Eva Maria Koch curso Bill 1954-1955 Archivo HfG, G 12 FBS
11/88 Análisis de las formas esquemáticas en el cuadrado, triángulo y círculo (Klee) Alumno: Naske curso Nonné-Schmidt 1953-1954 Dp 024.008 FBS

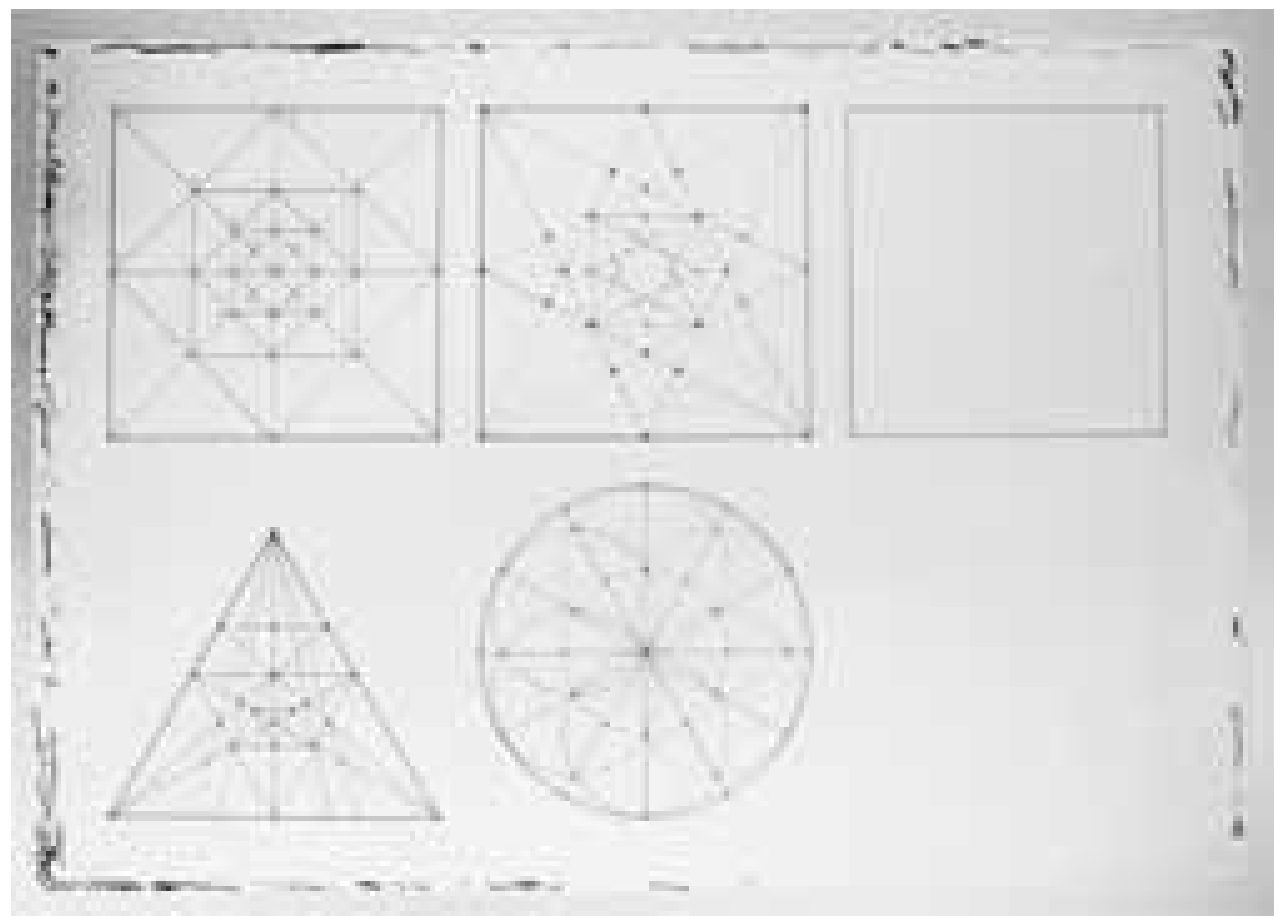




\subsubsection{Las enseñanzas de Nonné-Schmidt}

Heléne Nonné-Schmidt vivía en Wangen, una pequeña ciudad de la región de Allgau, en el lado alemán del Lago Constanza, cuando Max Bill le ofrece incorporarse al primer curso de la HfG. En aquel momento se dedicaba a la pintura y a la gestión del legado de su marido Joost Schmidt, maestro de la Bauhaus, fallecido en 1948.

La pintora acepta el ofrecimiento de Bill y se incorpora a la escuela en una de las colaboraciones didácticas más constantes y de mayor continuidad hasta el curso 1956-1957 que abandona la escuela, al igual que Max Bill. Sus seminarios ocupan en proporción a los demás, un gran número de semanas, en las que se trasladaba a vivir a Ulm.

Nonné-Schmidt había sido alumna de la Bauhaus en Dessau. Comenzó sus estudios en 1925 y llegó a estar muy próxima a Paul Klee. Se dedica a recoger sus lecciones transcribiendo las enseñanzas del maestro a lo largo de cuatro semestres, con la ilustración de cada uno de los ejercicios y su explicación oral ${ }^{94} \mathrm{Klee}$, a diferencia de otros maestros de la Bauhaus, no se preocupa por ordenar, sistematizar ni publicar su didáctica, por eso la figura de Nonné-Schmidt es relevante y lleva consigo este valor a la HfG.

La enseñanza de los cuatro cursos en Grundlehre de Nonné-Schmidt puede dividirse en dos ciclos. En el primero, su curso se organiza en tres bloques: las lecciones de Paul Klee, el dibujo del natural de la Bauhaus y la teoría del color de Joost Schmidt. De Paul Klee explica la teoría de la configuración pictórica, y especialmente la teoría de la forma pictórica. Los contenidos se centran en la subdivisión de las superficies del cuadrado, triángulo y círculo como formas básicas. Hace referencia a la organización del color de Klee como un conjunto de relaciones dinámicas pero desarrolla mucho más la enseñanza de color de Joost Schmidt, centrándose en el orden cromático y su aplicación sistemática. El dibujo del natural de texturas y elementos como la sección de una col eran ejercicios habituales en la Bauhaus. Tras un tiempo de observación, los estudiantes debían reproducir de memoria las texturas que habían observado, por lo que es un ejercicio muy vinculado a la morfología y al descubrimiento de patrones.

A partir del curso 1955-1956 deja de explicar las teorías de Klee y se centra de lleno en el color. Al igual que Joost Schmidt, su enseñanza está marcada por la ordenación del color de Ostwald, lo que significa un acercamiento científico y no artístico. Se trabaja con tipo de papel determinado y con unos colores normalizados, con técnica de acuarela y gouache. La acuarela se emplea en los ejercicios de superposición y el gouache en las escalas por etapas. Los alumnos reproducen escalas sistemáticas por colores complementarios y tríadas y también los ordenan. ${ }^{95}$

Los libros y documentos sobre la HfG presentan a Nonné-Schmidt en una posición secundaria, eclipsada por la prominencia de las figuras de la didáctica que ella personifica, Klee y su marido Joost Schmidt. Sin embargo, en los cuatro años que está en Ulm, llega a desarrollar y sistematizar una didáctica personal que nace de la síntesis de su propio aprendizaje. Sus enseñanzas proporcionan a los alumnos cuestiones básicas más allá del color y la forma, como el orden, la precisión, la auto exigencia y la programación de las tareas. Además, tiene una posición de importancia en la organización del Curso Básico los primeros años, ya que, hasta la llegada de Tomás Maldonado, Max Bill delega en ella las correcciones de sus trabajos y el seguimiento de las tareas del Curso Básico. ${ }^{96}$
94 Schilly, Katja. Die Bauhäuslerin Helene Schmidt-Nonné. Lehre, leben und werk. Tesis doctoral, Facultad de artes Nijmegen, 1999, p. 50.

95 Ejemplo de secuencia de tareas en el curso 1956-1957 extraído de los apuntes de Klaus Krippendorf:

Visuelle Einführung 1

1. curso de acuarela Nonné-Schmidt

1.1 escala de grises, escala de color, círculo cromático

1.2 colores primarios, secundarios, terciarios y cuaternarios

1.3 superposición de colores primarios y secundarios de primer y segundo orden

1.4 superposición de colores primarios y sus complementarios y adyacentes

1.5 leyenda de la lámina VE4

1.6 superposiciones

Visuelle Einführung 5

5.1 Sólido de color de Ostwald

5.2 Oscurecimientos con gris y blanco

5.3 Escalas de transición entre dos complementarios

5.4 Escalas de transición entre dos complementarios

5.5 Escalas de transición entre dos complementarios

5.6 Escalas de transición entre blanco, un color y negro

5.7 Colores mixtos

96 Max Bill designa a Nonné-Schmidt como la docente responsable del seguimiento y correcciones del examen.

Enunciado del examen para la recuperación del Curso Básico, Dp 009.009, Alumno Erler 1953-1954. 


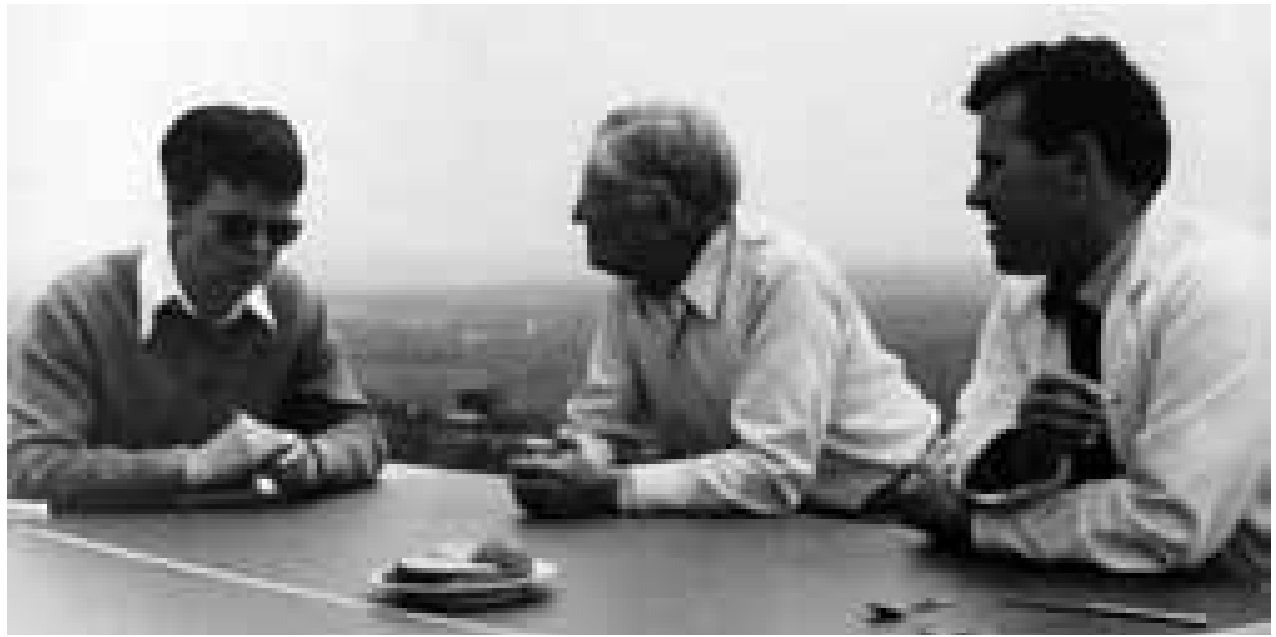

i1/89 Josef Albers con Inge Scholl y Otl Aicher en el verano de 1955.

Fuente: Horowitz, f., \& Danilowitz, B.

Josef Albers: to open eyes. New York: Phaidon, 2006, p. 53

¿1/90 Josef Albers con Otl Aicher e

Ingela Albers

Foto: Kock Weser

Dp 117.4

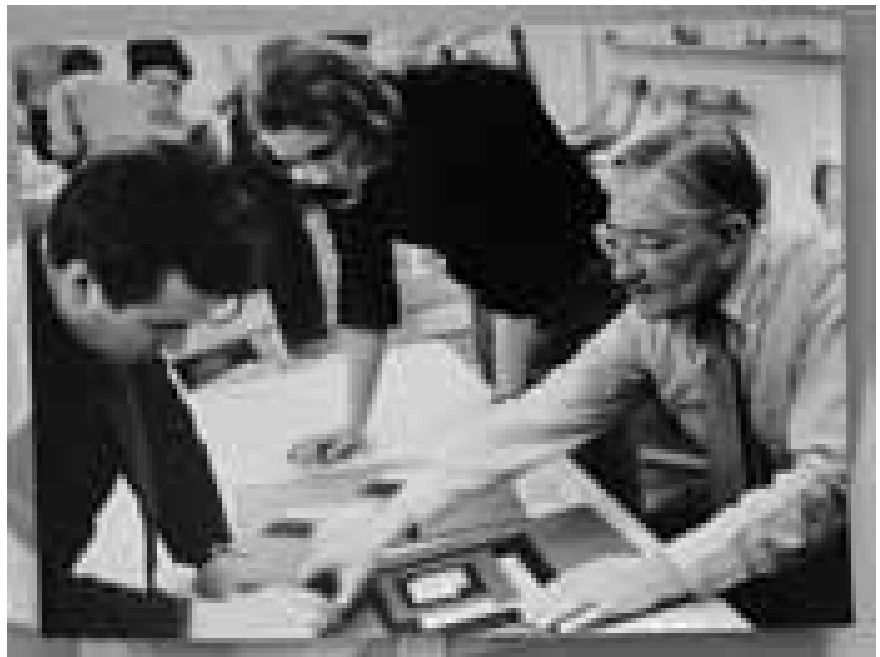

i1/91 Alumnas resolviendo ejercicios de color del curso de Albers Foto: Kock Weser Dp 117.4

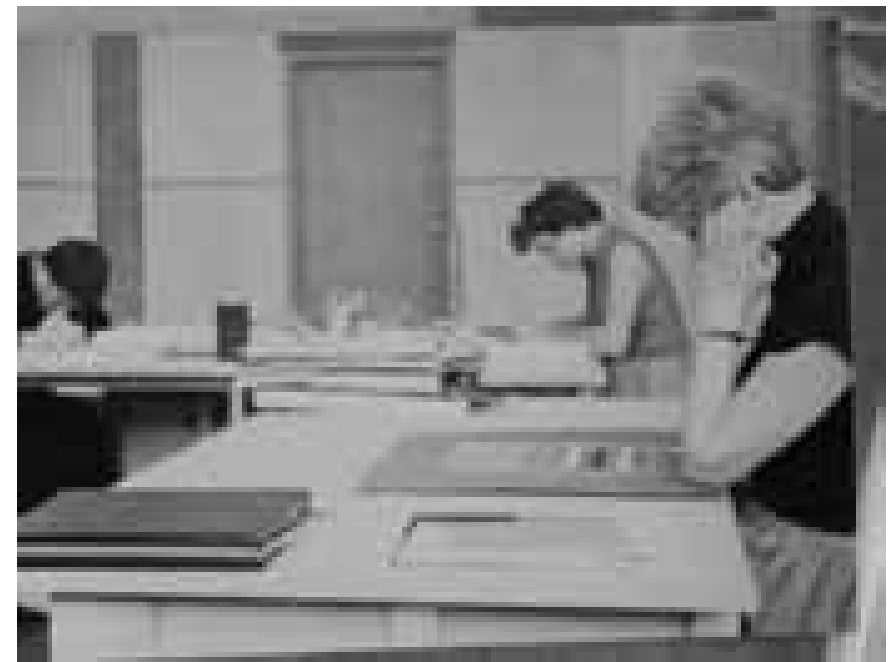




\subsubsection{Enseñar a mirar. Josef Albers en la HfG}

En el año 1933, el matrimonio Albers llega a Estados Unidos para participar en la fundación del Black Mountain College en Carolina del Norte. Tienen que pasar todavía 20 años hasta que vuelva de nuevo a Alemania para participar en la HfG. Albers, maestro de formación, había ingresado como alumno en la Bauhaus y rápidamente se hace responsable del taller de vidrio. Es profesor del Curso Básico de la Bauhaus desde 1923 hasta su exilio. En el periodo norteamericano, Josef Albers viaja de forma incansable e imparte conferencias por todo el país y también recorre Sudamérica. Enseña en Harvard y en el Armour Institute de Chicago, en la UNAM de la ciudad de Méjico, dirige la cátedra del departamento de diseño de la Universidad de Yale. ${ }^{97}$ Es un periodo de una intensa actividad docente, y cuando vuelve de nuevo a Alemania ha destilado un seminario para el Curso Básico basado en su experiencia.

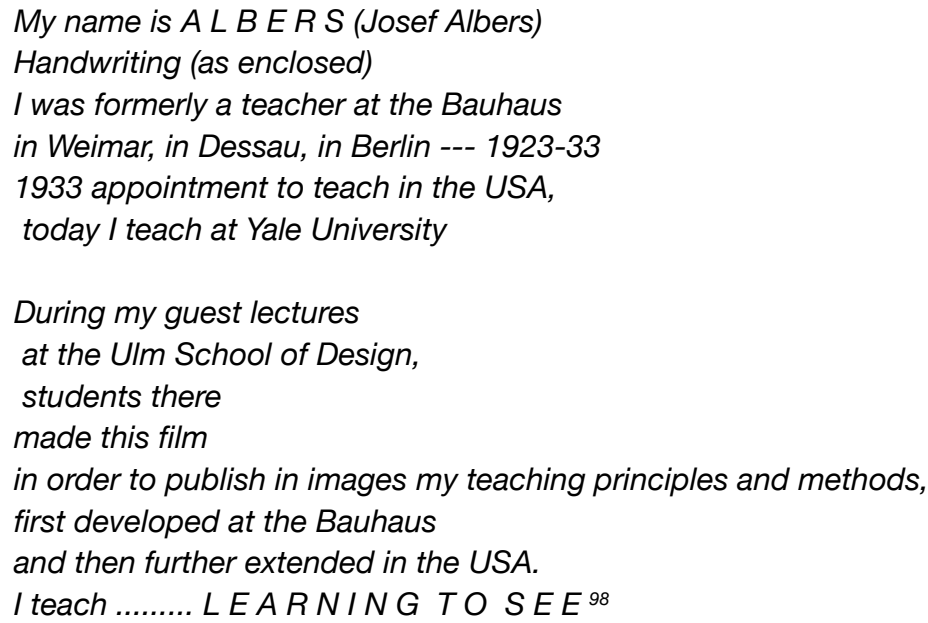

El seminario de Albers se imparte durante los dos primeros cursos del Grundelhre, en 19531954 -del 23 de Noviembre al 22 de Enero- y 1954-1955 -del 19 de mayo al 4 de agosto-. En este mismo periodo enseña en Nueva York, Santiago de Chile, Lima, Minneapolis, Hawaii, Honolulu, MIT Cambridge y Harvard. Es decir, su paso por la HfG representan dos paradas en el contexto de una gira docente mundial. Su estancia se financia con el programa amerciano "U.S. Specialist".

Albers idea un repertorio genérico, que imparte con la misma estructura en varias escuelas, que no diferencia entre los ejercicios bidimensionales y los tridimensionales. Son una combinación de trabajos de construcción -tres dimensiones-, combinación -dos dimensiones, collage- y dibujo. En el repertorio incluye ejercicios de interacción del color y se basa en la observación y no en una base teórica explícita. El objetivo último, como el propio Albers explica, es que el alumno aprenda a mirar.

Los cursos se estructuran en tres partes, denominadas Diseño Básico, Dibujo Básico y Color Básico. Diseño Básico -llamado construcción en otros cursos- se centra en la exploración intuitiva del material de trabajo, y de forma experimental, explorar qué puede hacerse con él y de esta forma profundizar en sus características. A este grupo pertenecen los ejercicios con arena, alambre, plegados de papel y estructuras de madera en tensegridad. Albers aplica aquí el método inductivo, reflexionar una vez que se ha llegado de forma intuitiva a los resultados.
97 Resumen del curso de diseño básico que Albers envía a la Universidad Pontificia de Santiago de Chile. Horowitz, f., \& Danilowitz, B. Josef Albers: to open eyes. New York: Phaidon, 2006, p. 51

98 Krampen, M. Beobachten und formulieren. Grundkurs mit Übungen, nach einem Filmskript von Josef Albers. Zentrum für Kunst und Medientechnologie Karlsruhe ZKM, 2009. 


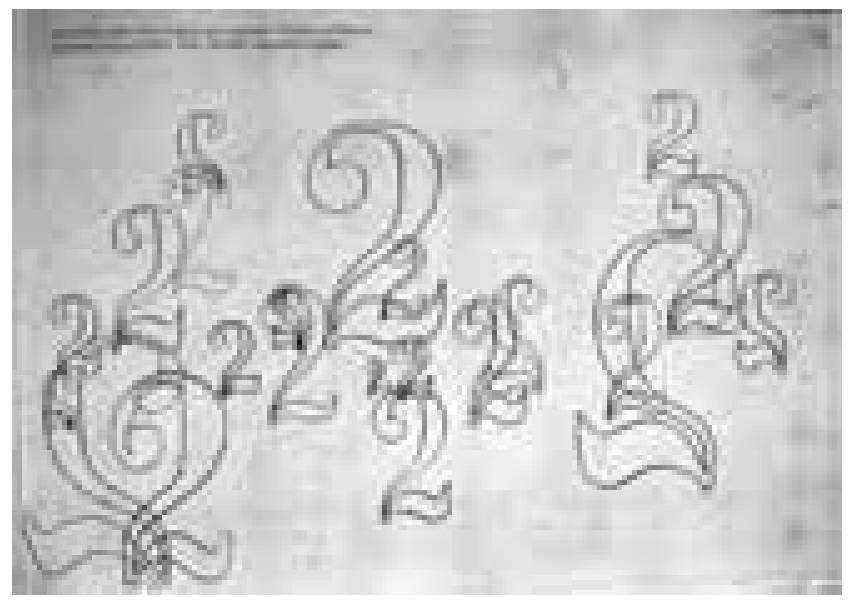

¡1/92 Ejercicios del curso color básico 1953-1954 de Josef Albers

Alumno: Erler Dp 009.012
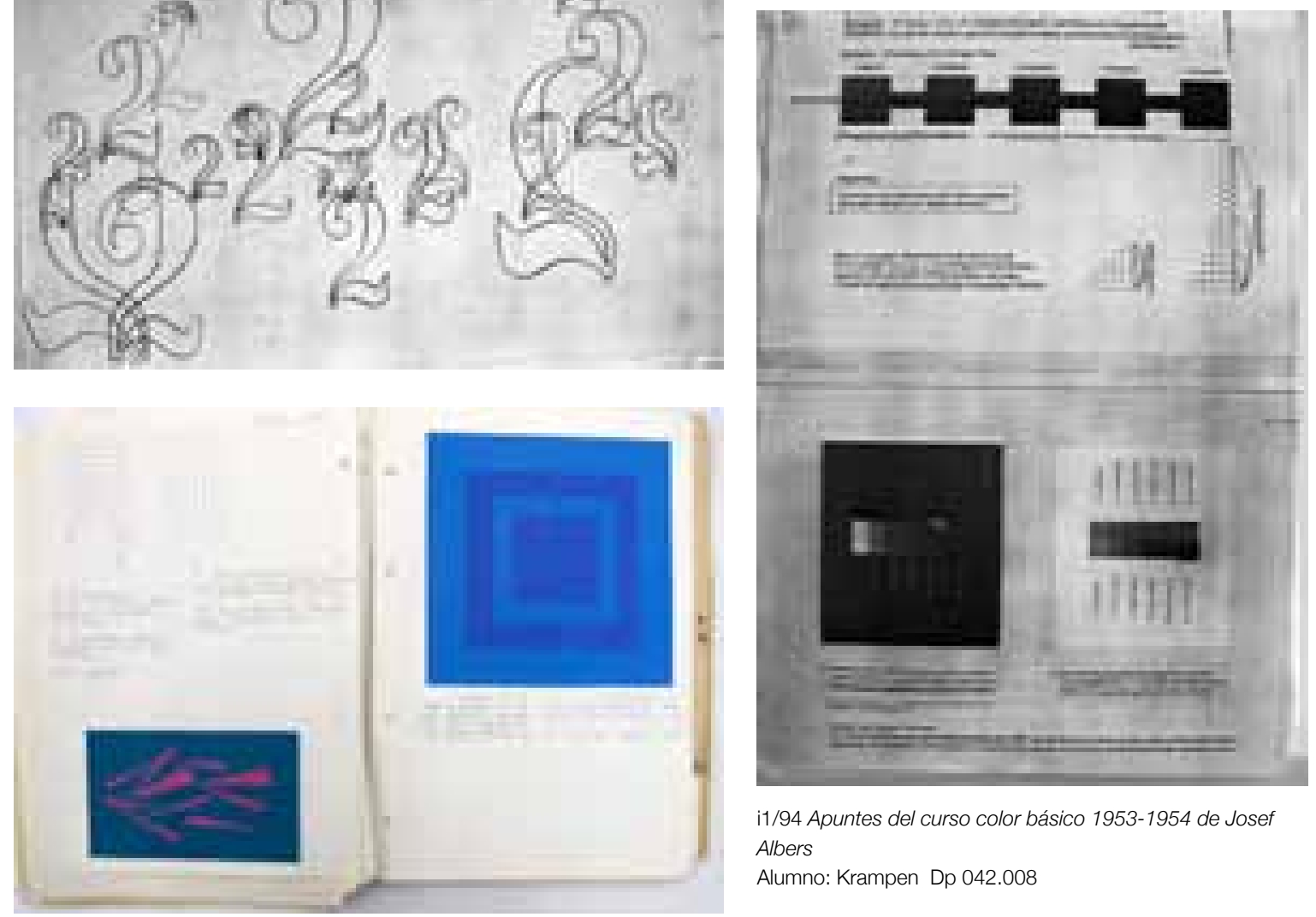

11/94 Apuntes del curso color básico 1953-1954 de Josef Albers

Alumno: Krampen Dp 042.008

i1/93 Ejercicios del curso dibujo básico1953-1954 de Josef Albers Alumno: Erler Dp 009.012
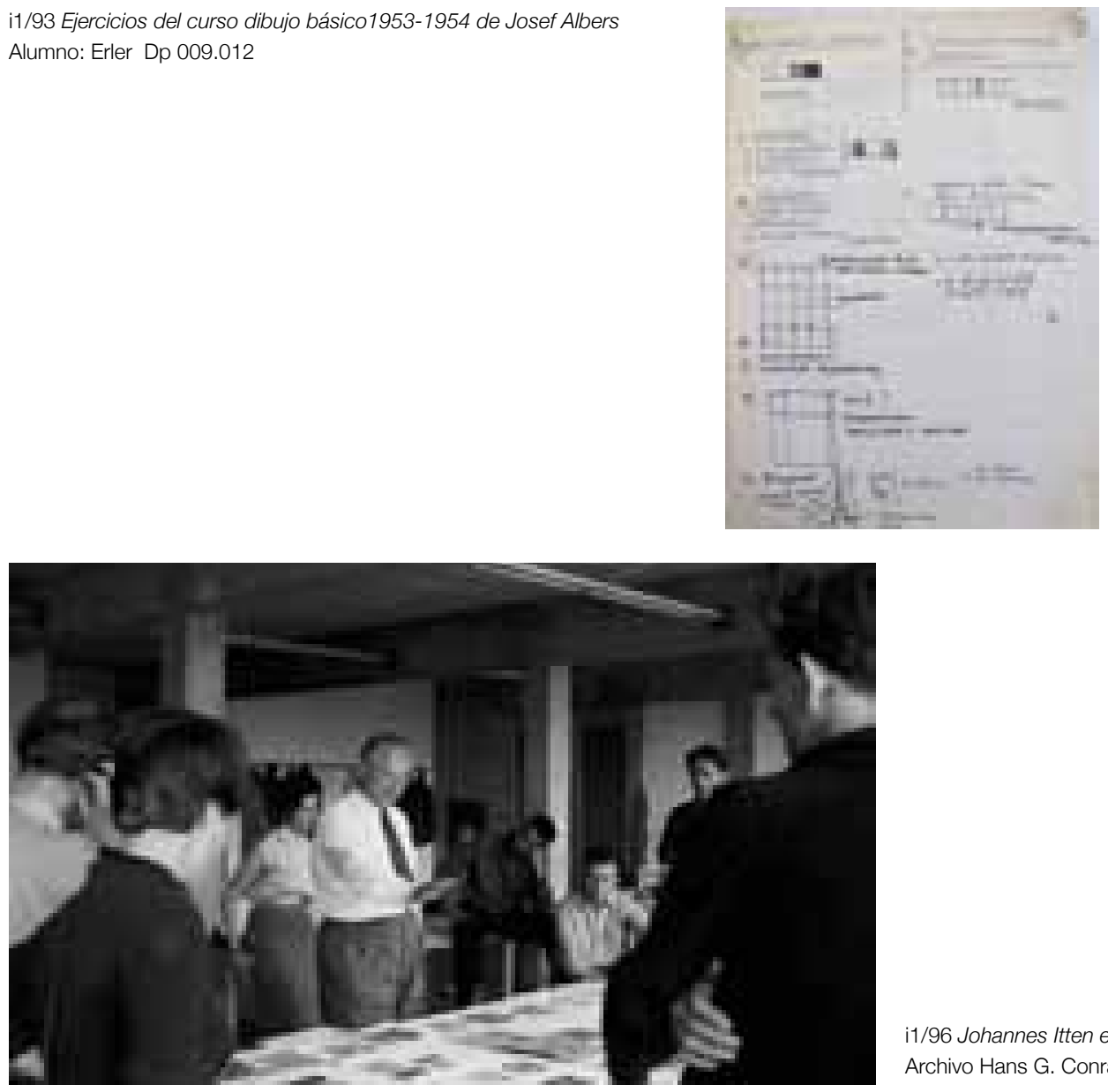

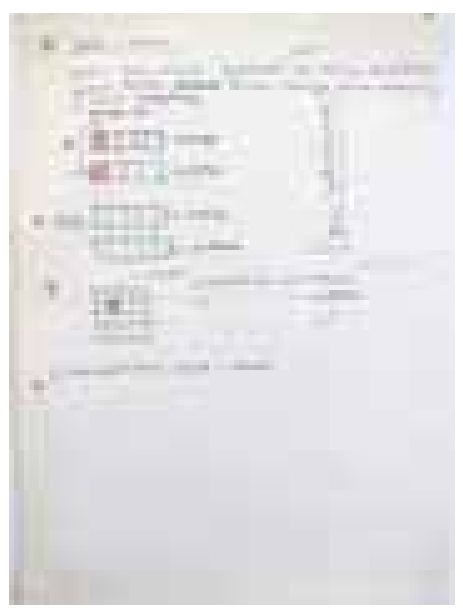

11/95 Apuntes curso Itten Max Graf. Archivo HfG 
En el curso de Dibujo Básico se ejercita el dibujo a mano alzada, para lograr el control del ojo y la mano. Albers realiza una serie de ejercicios para potenciar los aspectos motóricos del dibujo y entrenar el movimiento del brazo. ${ }^{99}$ Se dedican numerosos ejercicios a desarrollar este aspecto, como el dibujo en el aire, el dibujo con ojos cerrados, dibujos de izquierda a derecha y de derecha a izquierda, líneas moduladas enfatizando el trazo y el grosor, para practicar la fuerza.

El curso de dibujo se desarrolla a lápiz, puesto que Albers está más interesado en el ensayo y desarrollo del trazo y la observación más que la representación de los volúmenes. Se dibujan tipografías, líneas curvas y pliegues de ropa.

En el curso Color Básico se elude el aprendizaje del color a través de la teoría. Albers se basa en la idea de que el color puro es imposible de conocer físicamente, porque en la realidad, no se puede separar de su contexto. De esta forma, el color siempre dependerá de su percepción y de los factores que le acompañan y su interdependencia. Los ejercicios de color de Albers se basan en las mezclas ilusorias y las mezclas ópticas, partiendo de la ley de Weber-Fechner o la percepción de estímulos visuales.

Se trabaja con cartulinas de color para evitar errores en las mezclas y porque la técnica es más rápida.

Los estudiantes del primer curso, liderados por Martin Krampen, ponen en marcha la realización de una película que muestre el método de enseñanza del curso de Albers. La película no llega a realizarse pero Albers escribe un guión con sus objetivos y los ejercicios que emplea para alcanzarlos. ${ }^{100}$

\subsubsection{El seminario de Itten}

Es sorprendente la repercusión que ha tenido en las publicaciones sobre la HfG la existencia de un curso de Johannes Itten que solo dura una semana. Aemilius Müller, un especialista en color, da un seminario de la misma duración en el mismo curso y no tiene la misma repercusión en la historia contada del Curso Básico. ${ }^{101}$ Es probable que esto sea debido a que Itten, como personaje, viene a completar la representación Bauhausler con la que arranca la escuela.

El seminario de Itten tiene lugar a finales de abril de 1955. En aquel periodo era director de la Kunstgewerbeschule y de la Textilfachschule de Zúrich, y había comenzado sus estudios sobre el color, que habían tomado un camino más sistemático que el que empleaba en la Bauhaus. Al igual que Josef Albers, a lo largo de su vida fragua una personal teoría del color que acaba publicando en la década de los sesenta, al final de su vida.

Itten centra su enseñanza en la HfG en la teoría de contrastes, especialmente en el binomio claro-oscuro y el contraste de color en sí mismo, y cómo estas configuraciones transmiten ciertos estados de ánimo. Este matiz expresionista no convence a los estudiantes que están inmersos en la decisión de criterios objetivos.
99 Albers, Josef. Josef Albers: medios mínimos, efecto máximo. Madrid: Fundación Juan March, 2014. Catálogo de la exposición de la Fundación Juan March, p. 276.

100 Krampen, M. Beobachten und formulieren. Grundkurs mit Übungen, nach einem Filmskript von Josef Albers. Zentrum für Kunst und Medientechnologie Karlsruhe ZKM, 2009

101 No tiene la misma repercusión que Itten en las publicaciones sobre la HfG, pero en aquel momento su enseñanza es relevante, y recogida y desarrollada por docentes como Vordemberge-Gildewart. 


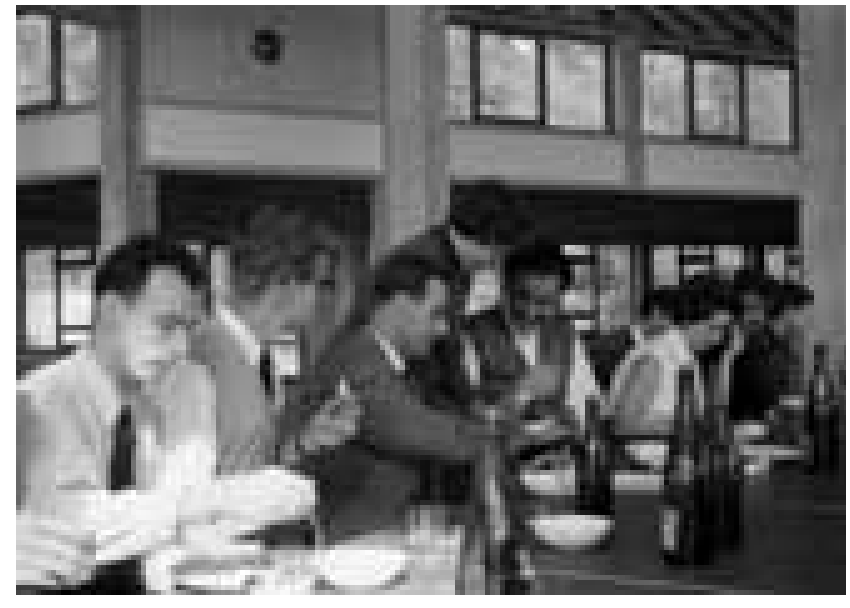

i1/97 Tomás Maldonado, alumnos

y profesores en el mensa de la HfG,

septiembre de 1955

Archivo Hans G. Conrad FBS

i1/98 Tomás Maldonado

Se 170.4 FBS
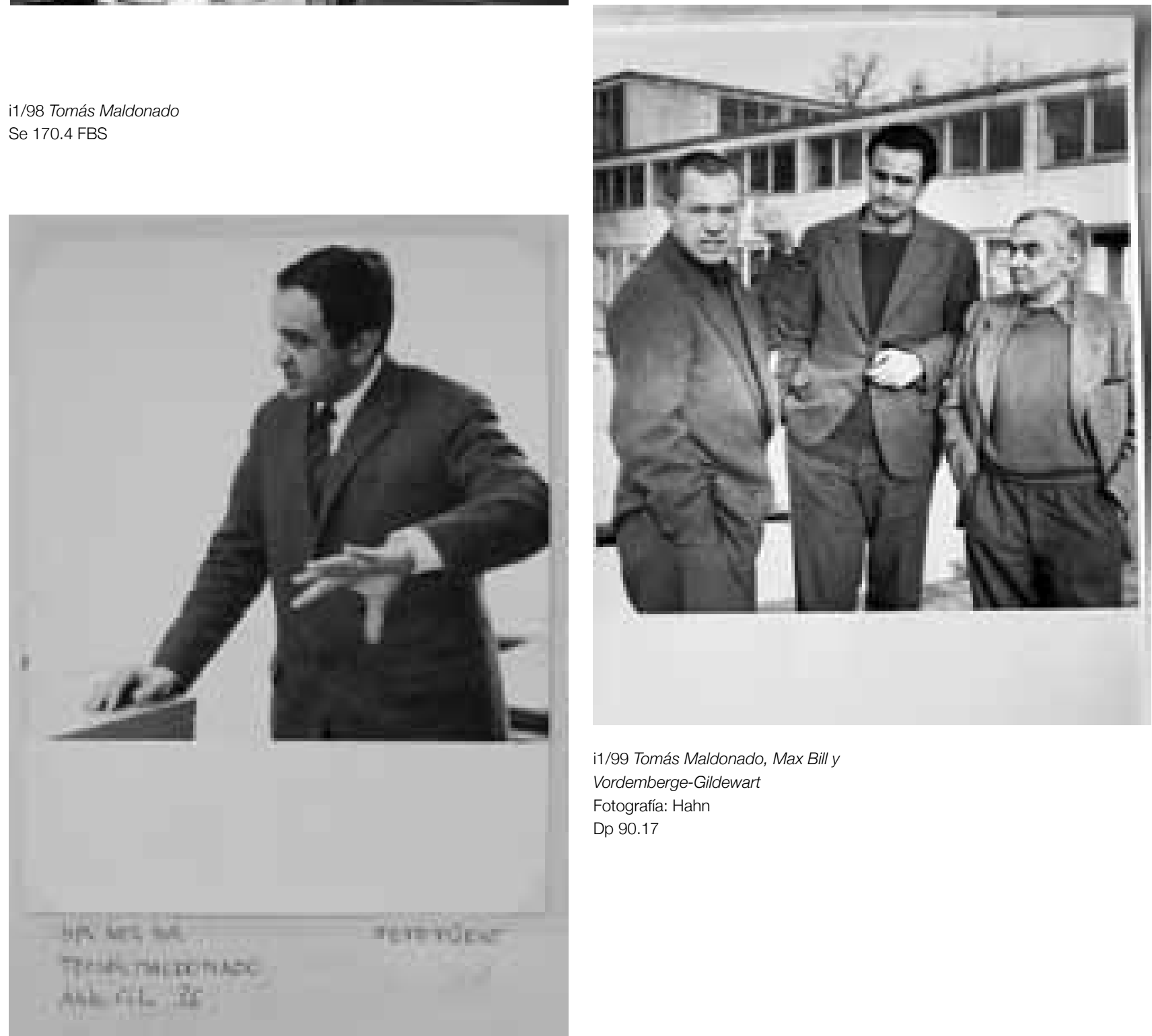

11/99 Tomás Maldonado, Max Bill y

Vordemberge-Gildewart

Fotografía: Hahn

Dp 90.17 


\subsection{Relevo Bill-Maldonado en el Curso Básico}

\subsubsection{Contexto espacio-temporal del relevo Bill-Maldonado}

\begin{abstract}
Max Bill ha sabido hacer efectiva una de las aspiraciones más ambiciosas del dilatado programa del espíritu moderno: la práctica de la totalidad de las artes visuales con un mismo y único sentido.
\end{abstract}

Pintor, escultor, arquitecto, gráfico, diseñador, es el tipo moderno del "artista total", anticipo el "hombre total" que todavía debe conquistarse. ${ }^{102}$
Tomás Maldonado viaja por primera vez a Europa en Abril de 1948. Viene de una Argentina aislada culturalmente, en la que el arte concreto se ha introducido tímidamente a través de escritos de exiliados que provenían principalmente de Berlín. Maldonado pasa un tiempo entre Milán y París, visitando a arquitectos y pintores abstractos, con el objetivo de ampliar sus horizontes. Uno de los propósitos de su viaje consiste en conocer personalmente a Max Bill, con quien se había estado escribiendo algún tiempo. Le visita en su casa estudio de Limmatstrasse en Zúrich y el argentino recuerda que, en aquel primer encuentro, también estaban presentes Inge Scholl y Otl Aicher. Cuando regresa a Buenos Aires, comienza la edición de la revista Nueva Visión. Continúa la correspondencia con Bill, que le va relatando la puesta en marcha de la HfG y le invita formalmente en 1954 a formar parte del desarrollo didáctico del curriculum básico. Lo que comienza como un proyecto de estancia de dos años, se convierte en trece años de vida y trabajo en Ulm. ${ }^{103}$

El periodo del relevo entre Bill y Maldonado en el Grundlehere comienza con la llegada de éste durante el curso 1954-1955. Sin embargo, no es hasta el curso siguiente cuando el cambio se evidencia; Maldonado comienza a ser autónomo para proponer sus propias tareas y los vestigios Bauhausler en el Curso Básico se han diluido. ${ }^{104}$ Las experiencias del relevo a las que se refiere este epígrafe, por tanto, trascurren entre el curso 1954-1955 y el 1955-1956.

En el curso 1955-1956 Max Bill continúa siendo rector pero los Aicher están fraguando la sustitución del modelo de dirección de la escuela por otro más democrático y colectivo, un rectorado de varios profesores elegido por los docentes de reelección anual, aconsejado por círculo de personas denominado kleiner Konvent, ${ }^{105}$ e integrado por representantes de alumnos, profesores y maestros de taller.

El proyecto de Curso Básico que Max Bill concibe desde el comienzo, se basa en la sucesión de seminarios de profesores experimentados acompañados por un profesor vinculado de forma permanente a la escuela, que se convertiría con el tiempo en el único docente que impartiría el Curso Básico. Si bien en el curso inaugural Bill no encontró la figura adecuada, Tomás Maldonado es invitado a Ulm en el segundo curso con esta misión específica. ${ }^{106}$
102 Maldonado, Tomás, op. cit. 1955 p. 7.

103 Ver entrevista a Maldonado "De Buenos Aires a Ulm" en : W.AA HfG Ulm: form+zweck 20 (número monográfico). form + zweck, 2003, p. 17.

104 En el curso 1955-1956 Josef Albers no está, tampoco vuelve Peterhans que solo estuvo un curso y Nonné-Schmidt ha creado su propio repertorio sobre el color en el que las enseñanzas de Klee ya no se imparten.

105 Círculo interno. Ver: Spitz, René Op. cit. pag 183.

106 Ibidem, pag 55 
i1/100 Apuntes del curso Konstruktive Darstellungen Methoden impartido por Herbert Ohl

Alumno: Max Graf
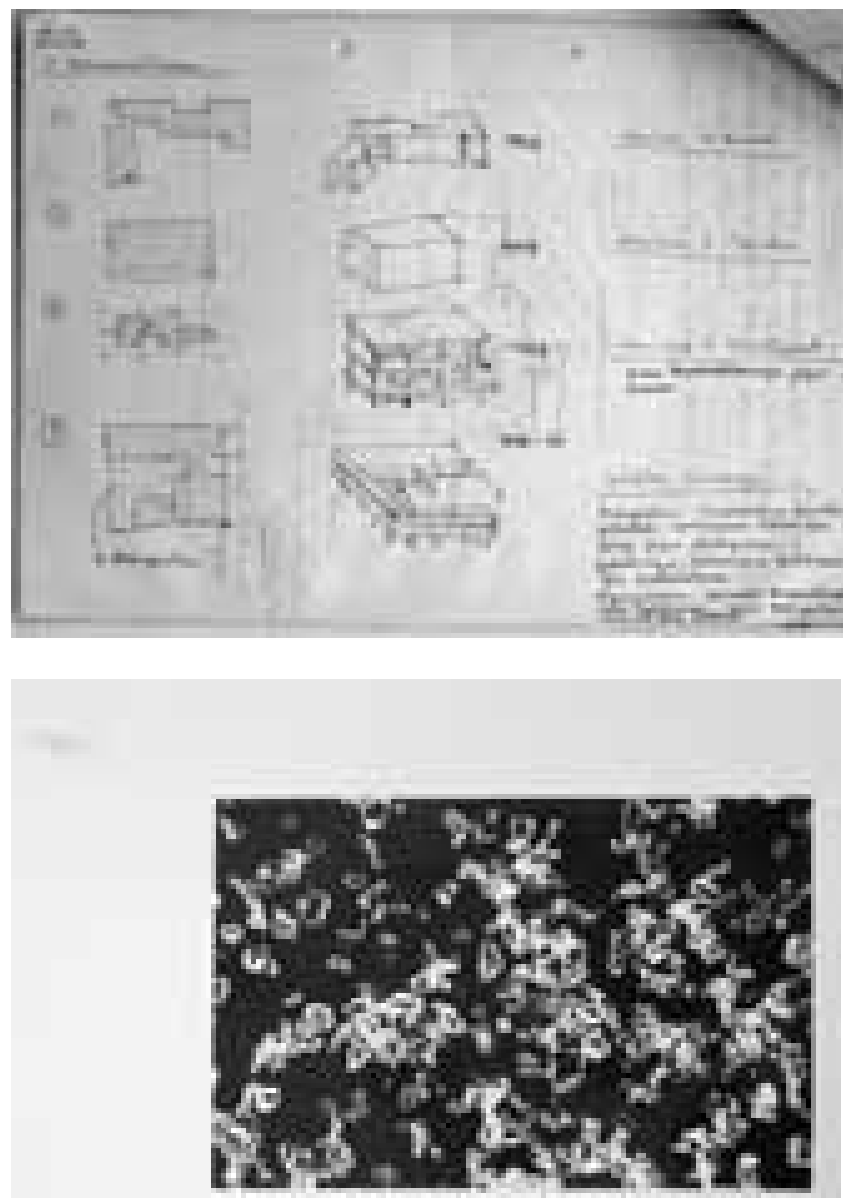

Cristales de Sal

Alumno: Peter Disch

Fotografía, Scheidegger 1955-1956

GS 037.001 FBS

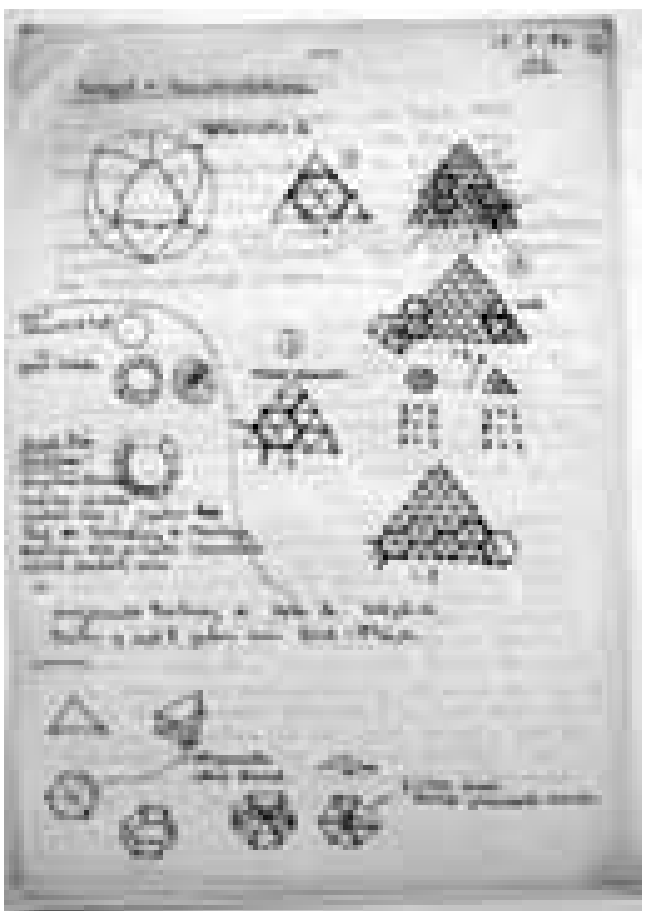

i1/101 Apuntes del curso Konstruktive Darstellungen Methoden impartido por

Herbert Ohl Alumno: Max Graf

¡1/103 Darstellende Geometrie

Alumna: Ilse Gubrich Kurs Gugelot 1955-1956 Dp 058.01 FBS

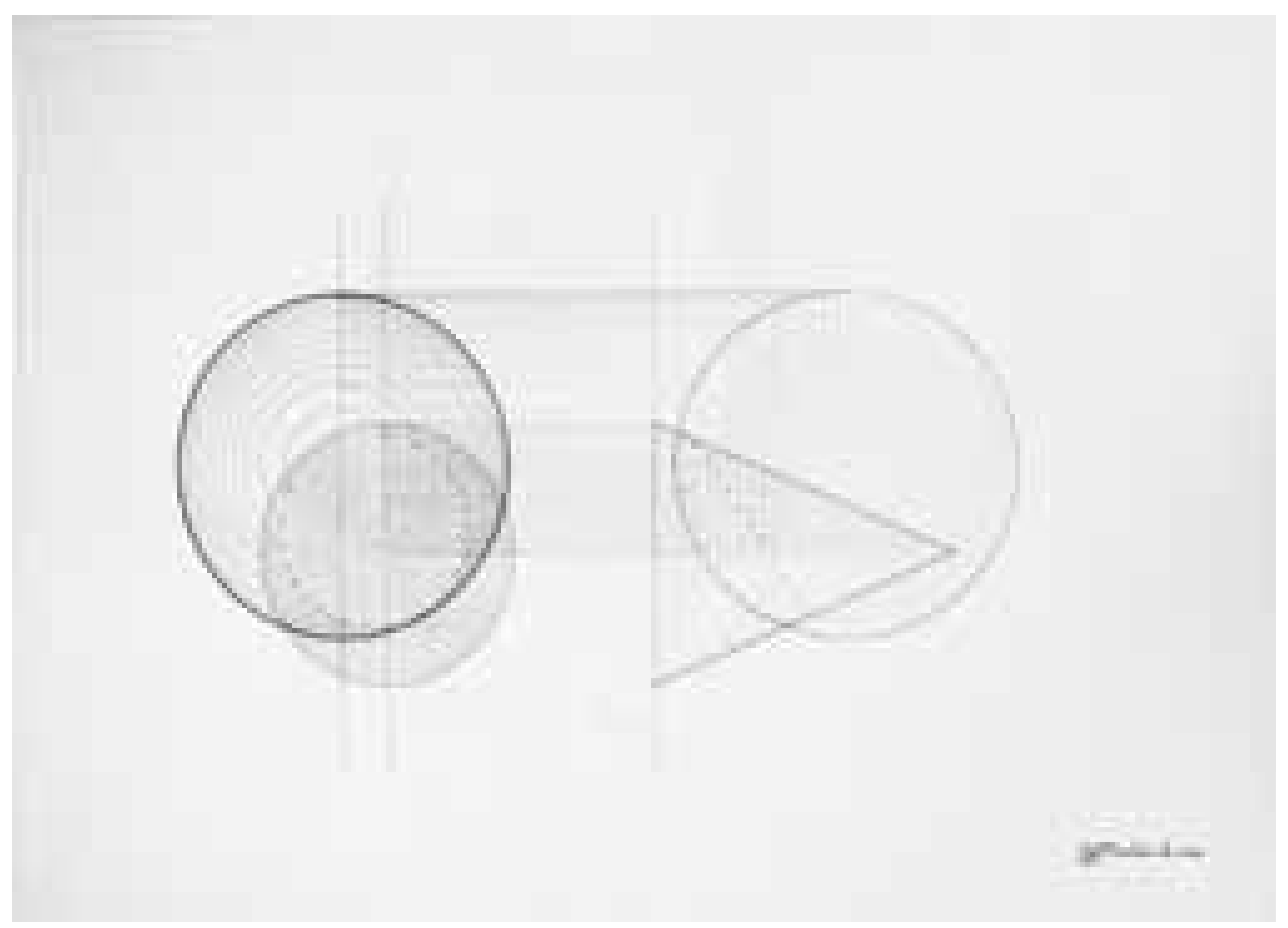




\subsubsection{Docentes, materias y alumnos del periodo ${ }^{107}$}

\section{Rectorado 1955-1956}

Rector: Max Bill, renuncia en marzo 1956

Rectorado posterior:

Otl Aicher, Max Bill,

Hans Gugelot, Tomás Maldonado,

Friedrich Vordemberge-Gildewart

Rectorado honorífico: Walter Gropius, Herny Van de Velde, Alvar Aalto, Le Corbusier, Mies van der Rohe, van Esteren,

Georg Schmidt.

\section{Departamentos 1955-1956}

Grundlehre - Tomás Maldonado

Producto - Hans Gugelot

Arquitectura y construcción de la ciudad- Max Bill

Comunicación Visual - Otl Aicher

\section{Docentes 1955-1956}

Otl Aicher

Max Bill

Hans Gugelot

Tomás Maldonado

Helene Nonnè Schmidt

Fritz Pfeil

Friedrich Vordemberge-Gildewart

Walter Zeischegg

\section{Materias 1955-1956}

Visuelle Einführung - El curso 1955-1956 es el primero que se organiza por quartal o trimestres. Nonné-Schmidt y Maldonado se hacen cargo de la enseñanza del color. Maldonado comienza a introducir ejercicios con cuestiones relativas a la simetría.

El segundo bloque de enseñanzas esencial del Visuelle Einführung está compuesto por las materias en torno a la geometría. El primer trimestre lo imparte Baravalle, en el que desarrolla la asignatura Konstruktive Darstellung Methoden KDM1. Baravalle trabaja con los alumnos en este periodo en su repertorio centrado en la serie geométrica del círculo, las concoides y cardioides, el caracol de Pascal, la construcción de elipses, espirales logarítmica y aritmética, y las inversiones de las curvas cónicas. Herbert Ohl, arquitecto colaborador de Egon Eiermann, imparte Konstruktive Darstellung Methoden KDM 2 y 3. Estas dos materias están orientadas a la representación de objetos y sistemas que se van a construir, bien en el taller, o que se proyectan como mallas tridimensionales de base a la construcción industrializada. También incorpora estudios de movimiento como la oscilación armónica del sonido. ${ }^{108}$

Hans Gugelot, arquitecto y diseñador industrial formado en Zúrich y Laussane, imparte la materia Darstellende Geometrie. En ese momento, su actividad principal consiste en desarrollar proyectos en el Instituto de investigación de diseño industrial.

Darstellungstechniken - Vordemberge-Gildewart enseña un curso de tipografía que abarca el primer trimestre. Se llevan a cabo dos talleres de fotografía, el primer trimestre a cargo de Hahn y de Scheidegger en el tercer trimestre. Durante todo el curso los alumnos trabajan en los talleres de madera, yeso y metal elaborando maquetas.
Maestros de taller 1955-1956

Fitz Hahn - Fotografía

Otto Schild - Yeso

Wolfgang Siol - Fotografía

Cornelius Uittenhout - Plástico
107 Como ya se ha explicado, el periodo del relevo abarca los cursos 1954-1955 y 1955-1956, la información relativa a profesores, alumnos y materias del 1954-1955 está recogida en el epígrafe anterior.

108 Benito, Emilia. Op. cit. pag 194.

\author{
Docentes invitados 1955-1956
Herman Von Baravalle - Visuelle Einführung \\ Max Bense: Información, Integración cultural \\ Werner Blaser - Departamento de Diseño de \\ Ernst Scheidegger - Comunicación Visual \\ Conrad Wachmann - Arquitectura
}



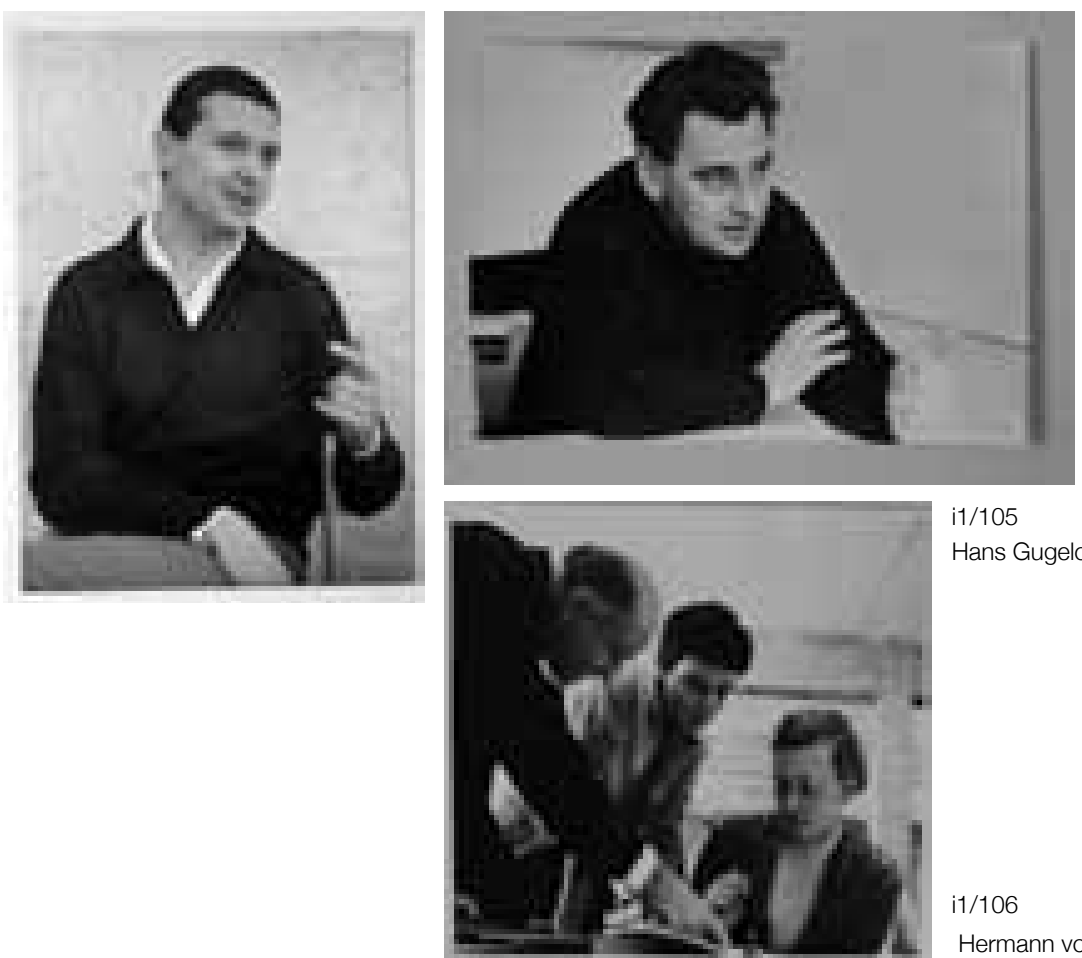

i1/105
Hans Gugelot

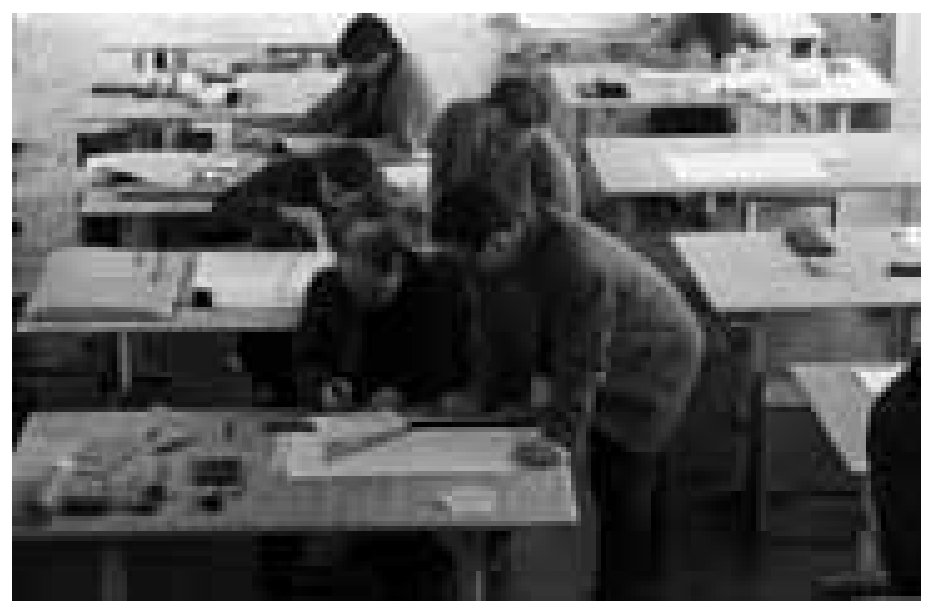

Hermann von Baravalle

i1/107

Aula Curso Básico febrero 1956 Archivo Hans G. Conrad

11/108 Aula Curso Básico 1956 Eva Maria Koch

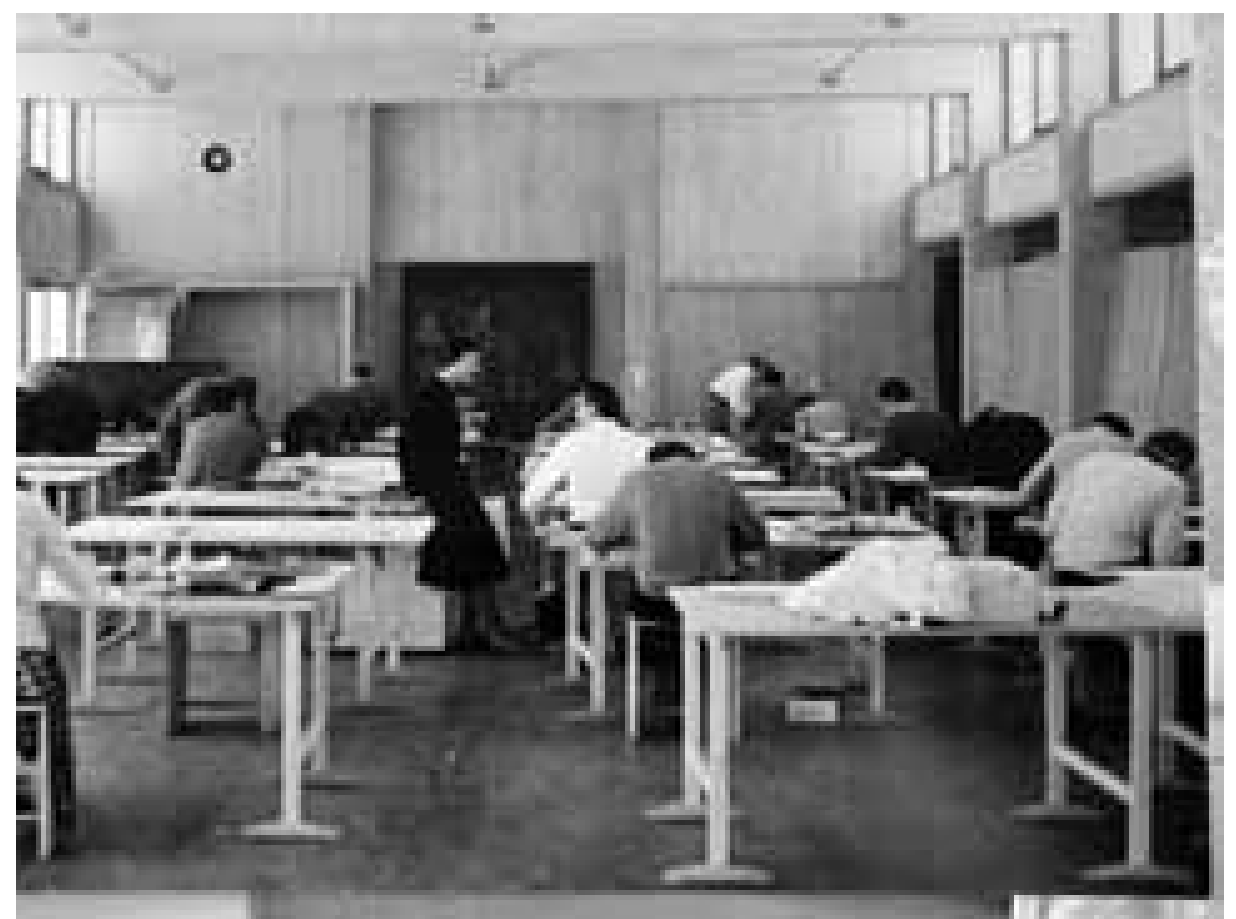


Allgemeinbildende und Theoretische Fächer - En este periodo comienzan a ocupar un mayor espacio lectivo las asignaturas teóricas. La materia Kulturgeschichte se afianza. Se suceden varios seminarios que ocupan todo el curso. Hans Curjel explica la Bauhaus y De Stijl, arquitectura y cultura general. Max Bill explica historia cultural del siglo XX. Curjel, Walter y Gomringer explican literatura. ${ }^{109}$

\section{Alumnos 1955-1956}

\section{Burkhardt Berger}

Gui Bonsiepe *información

Rido Busse * producto

Peter Disch

Hans Elmer

Richard Fischer

Claus Frank

Uwe Freidinger

Dominique Gilliard * arquitectura

Reinhart Graf

Ilse Gubrich * información

Peter Hofmann

Hans Kammermann

Hans von Klier

Cornelia Koch

Elke Koch-Weser *información

\author{
Meret Lindinger \\ Andres van Onck *producto \\ Wilhem Ritz *producto \\ Hans Roericht *producto \\ Hermann Roth *comunicación visual \\ Karen Saturski \\ Walter Schaer \\ Detten Schleimacher \\ Franziska Schörghuber \\ Peter Seitz *comunicación visual \\ Eva Seyfang *producto \\ Tilmann Strunz \\ Peter Uster \\ Reinhold Weiss *producto \\ Theodor Zeitler *producto \\ Adolf Zillmann *producto + comunicación visual
}

109 Según los programas recogidos en los apuntes del alumno Max Graf.

\subsubsection{Docencia compartida entre Max Bill y Tomás Maldonado}

En el archivo de la HfG solo se encuentra un ejercicio propuesto en común por Max Bill y Maldonado, del alumno Immo Krumrey con referencia Dp 019.009, en el que se nombra a los dos docentes. El ejercicio se titula "cinco escalas de grises continuas incluyendo blanco, negro y un color". El objetivo es ocupar un plano de soporte dado, que se considera un color de la escala, con una agrupación de cuadrados que forman una escala de grises. La ocupación no es aleatoria, sino que tiene que reunir una serie de requisitos que recoge el enunciado.

El cuadrado más grande posible dentro del formato dado tiene el lado estrecho igual a la longitud del lado.

Si se corta por la mitad dos o tres veces, se obtienen las longitudes laterales de las dos siguientes.

Al mismo tiempo, se consigue una relación más equilibrada entre la superficie de demostración y la superficie restante. 

incluyendo blanco, negro y un color

Alumna: Frauke Koch-Weser Curso Maldonado - Bill 1954-1955

Dp 042.039 i1/109 Cinco escalas de grises continuas
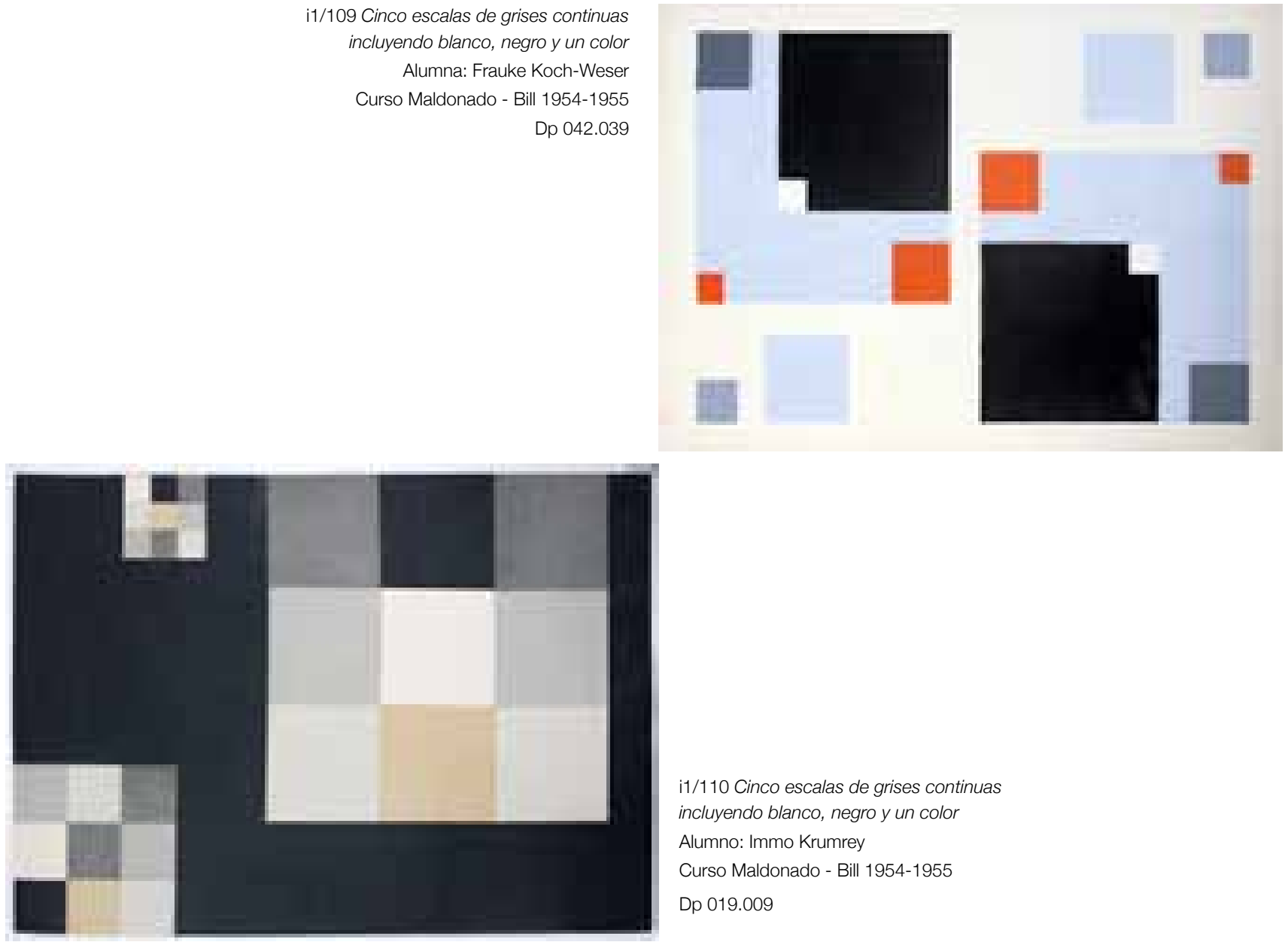

i1/110 Cinco escalas de grises continuas

incluyendo blanco, negro y un color

Alumno: Immo Krumrey

Curso Maldonado - Bill 1954-1955

Dp 019.009 i1/111 Reducción del efecto espacial de una estructura a un efecto bidimensional Curso Maldonado 1954-1955 Alumna: Frauke Koch Weser Dp 042.039G 
Max Bill ya había propuesto en el curso anterior un ejercicio con las mismas premisas y objetivos, titulado "seis elementos de acuerdo a una norma". La diferencia entre las soluciones del ejercicio en solitario de Bill y el compartido con Maldonado radica en que, en este último, existe una relación más intensa entre los elementos y el formato. La ocupación del espacio con los elementos adquiere una mayor importancia; la composición resultante es de una mayor unidad debido a que el sistema de relaciones de proporcionalidad y posición relativa entre elementos está mucho más estudiado y definido.

Tomás Maldonado vuelve a formular un ejercicio con la misma intención en el 1954-1955, a continuación de su docencia con Bill, en el que introduce nuevas variables que vienen a afianzar la predominancia de la ocupación del espacio independientemente de la escala cromática.

El ejercicio se titula "Reducción del efecto espacial de una estructura a un efecto bidimensional". Se plantea en dos pasos. El primero se puede considerar un estudio parcial del problema. Maldonado proporciona una estructura básica compuesta a partir de un grupo de cuadrados inscritos en uno de mayor tamaño. Esta estructura presenta una pregnancia visual dominante que los alumnos tendrán que desfigurar. El sombreado es la herramienta que permite romper la primacía formal de los cuadrados. El segundo paso consiste en la composición de seis figuras cuadradas sobre un rectángulo. Los cuadrados se agrupan en tres filas de tres, dos y un elemento. El conjunto presenta una fuerte identidad visual que se trata de diluir mediante los rallados y superficies sólidas. La proporción de color de los rallados y su dirección acaban formando la escala cromática de diferente intensidad que desfigura la predominancia de la figura cuadrada.

Los tres ejercicios mencionados tienen una relación evidente. Se puede apreciar una evolución en ellos, a partir de la intervención de Tomás Maldonado, se introducen recursos gráficos como los sombreados y la ruptura de la simetría que encaminan las soluciones hacia una cuestión de su interés: la disolución del fondo-figura.

\subsubsection{Colaboración entre Tomás Maldonado y Hans Gugelot}

El grueso de la formación en el Visuelle Einführung estuvo centrada desde el principio en el desarrollo de las habilidades de dibujo y representación y el dominio de mecanismos de composición y de generación de forma desde la práctica bidimensional. Sin embargo, algunos ejercicios anticipan la introducción al proyecto que se generalizará en la etapa final del Curso Básico. El enunciado que inicia la serie de pequeños proyectos, se propone en el año 1954-1955 por Bill y Maldonado.

Hans Gugelot, al igual que Tomás Maldonado, había sido invitado por Max Bill en las mismas fechas a formar parte de la escuela. Pero mientras Tomás Maldonado tenía la misión de organizar el Curso Básico, Hans Gugelot, que había sido colaborador de Max Bill en Zúrich, viene con el objetivo de colaborar en la ejecución de la obra de la sede y a poner en marcha desarrollos de productos y relaciones con la industria. Gugelot acaba convirtiéndose en un personaje de una aportación extraordinaria a la escuela. Con una declarada oposición al "estilo", le interesaba profundamente la conexión entre el diseño y la ingeniería, y muchos de los productos que forman la identidad de la producción de la HfG son producción suya. ${ }^{110} \mathrm{La}$ dedicación fundamental de Hans Gugelot está en la docencia del
110 Hans Gugelot fallece a los 45 años en 1964. Tomás Maldonado en su obituario, expresa que Gugelot, su producción, y sus ideas representan el núcleo de la identidad de la HfG.

Maldonado, Tomás. "In memoriam Hans Gugelot" en: Ulm no 14, 15 y 16, Zeitschrift der Hochschule für Gestaltung. Hg. v. Hochschule für Gestaltung, diciembre 1964 pag 93. 
i1/112, 113, 114, 115

Croquis desarrollo solución

Maldonado/Gugelot

Agosto 1955

Alumno: Max Graf

Curso 1954-1955

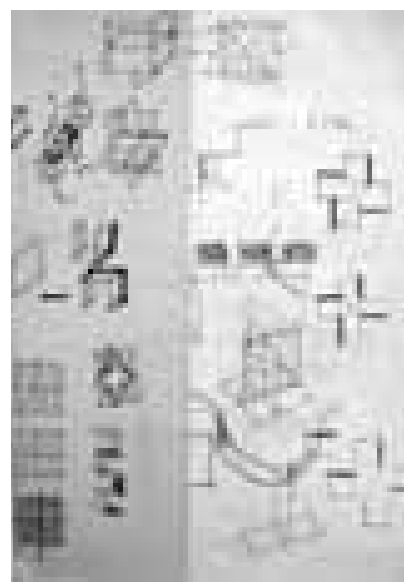

i1/116 Enunciado Maldonado/Gugelot Agosto 1955

Alumno: Max Graf

Curso 1954-1955

i1/117 Propuesta solución Maldonado/

Gugelot

Agosto 1955

Alumno: Frauke Koch-Weser

Curso 1954-1955

Dp 042 039G
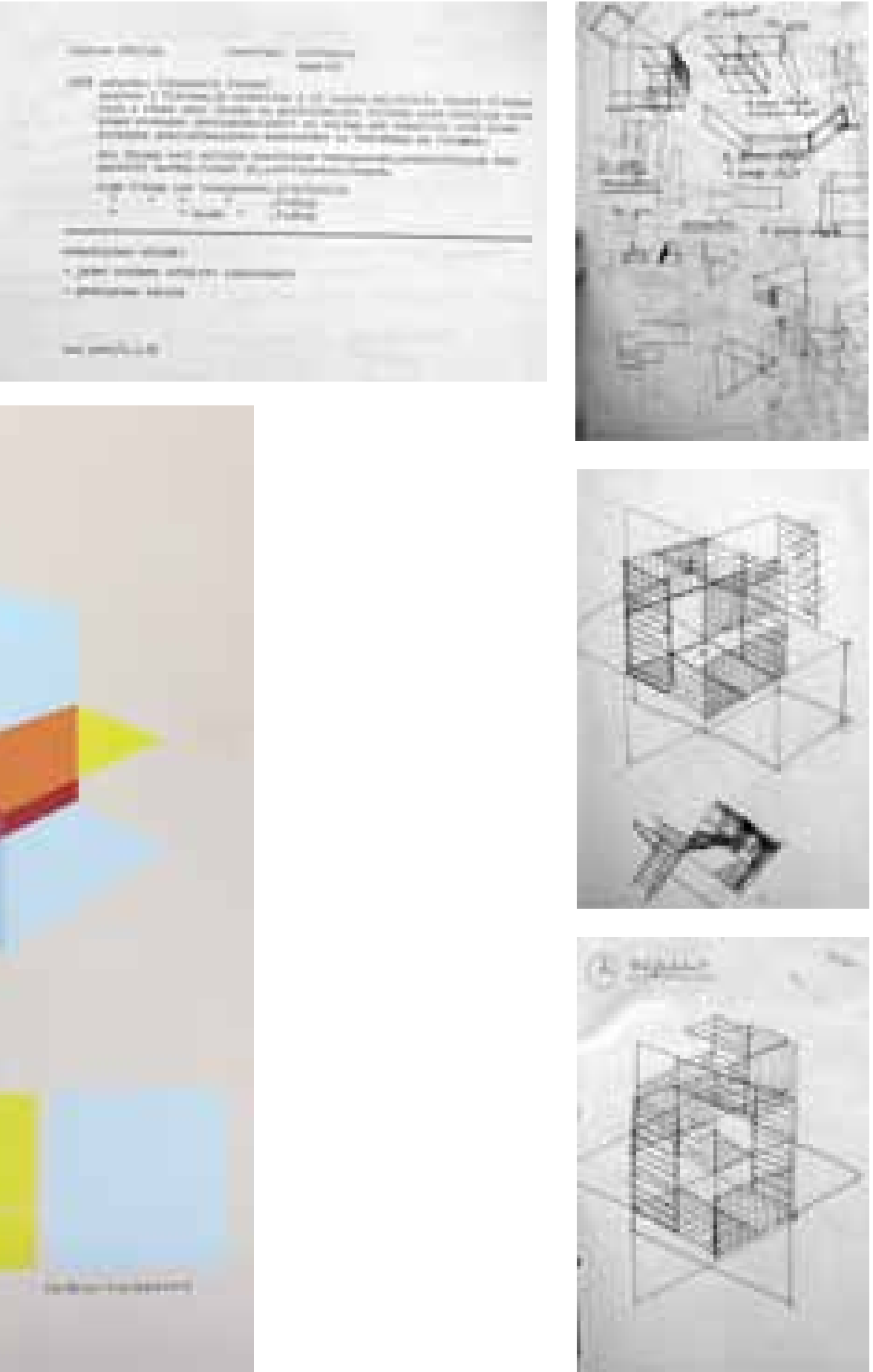
departamento Produktform y la dirección de uno de los grupos de desarrollo más prolíficos. Tiene una limitada participación en el Curso Básico, donde imparte Darstellende Geometrie, propone algunas tareas o pequeños proyectos desde los departamentos - Abteilungarbeit - y también dirige algunas Methodische Übungen.

La tarea tiene el objetivo de proyectar un objeto tridimensional a partir de la unión de tres superficies. Cada una de las superficies debe dividirse en 4 partes según una ley que decide y establece el alumno y que atiende a las cuestiones de proporcionalidad entre las partes y el todo y la capacidad de unión y de combinación de las superficies para la formación del objeto. Una de las superficies es transparente, otra también es transparente y está coloreada, y la tercera es opaca y de color. Los alumnos tienen que representar la solución en una axonometría sobre un cartón Schöll formato A2 con témpera.

En el ejercicio confluyen intereses de ambos docentes. La solución tiene un componente de modularidad y posibilidad de combinación y situación de las piezas que remite a Gugelot, que había desarrollado el sistema de mobiliario M125 con esas premisas. La concepción espacial y la definición de las relaciones entre elementos por leyes, así como el uso del color, son aspectos que Maldonado desarrolla en su didáctica.

Posteriormente, y antes de la llegada de las tareas metodológicas, Maldonado volverá a colaborar con otro docente, Herbert Ohl, para proponer un enunciado que se desvíe de las tareas comunes del Curso Básico y proponga el desarrollo de un nuevo proyecto.

\subsubsection{Tomás Maldonado como docente autónomo}

Muchos, por cierto, se sorprenden de estos tan frecuentes desplazamientos conceptuales del arte concreto. Se preferiría una doctrina más estable; un dogma si fuera posible. Pero el arte concreto no es ni más ni menos que un método. Un método, por otra parte, en continua renovación y verificación.

\section{(...)}

La nueva temática también se manifiesta en la producción pictórica de Bill. Ciertas nociones como continuo-discontinuo, preciso-impreciso, limitado-ilimita do, que constituyen su repertorio temático preferido son, sin duda, filtraciones provenientes de la expresión científica más actual. ${ }^{111}$

Maldonado había viajado a Europa por primera vez para entrevistarse con las personalidades más destacadas del arte concreto en Suiza e Italia a finales de la década de los 40. La concepción matemática del arte está en el origen de sus intereses plásticos y desde un primer momento, aborda su docencia desde esta posición.

Se puede considerar el ejercicio "Reducción del efecto espacial de una estructura a un efecto bidimensional", descrito en el epígrafe 1.4.3 , el primer enunciado que plantea Tomás Maldonado como docente autónomo, y ya aquí está tratando temas propios del arte concreto como la disolución fondo-figura.

En el curso 1955-1956 Maldonado y Nonné-Schmidt se hacen cargo de la docencia de Visuelle Einführung junto a los profesores de los seminarios de Konstruktive Darstellung
111 Maldonado, Tomás, op. cit, 1950 p. 12 


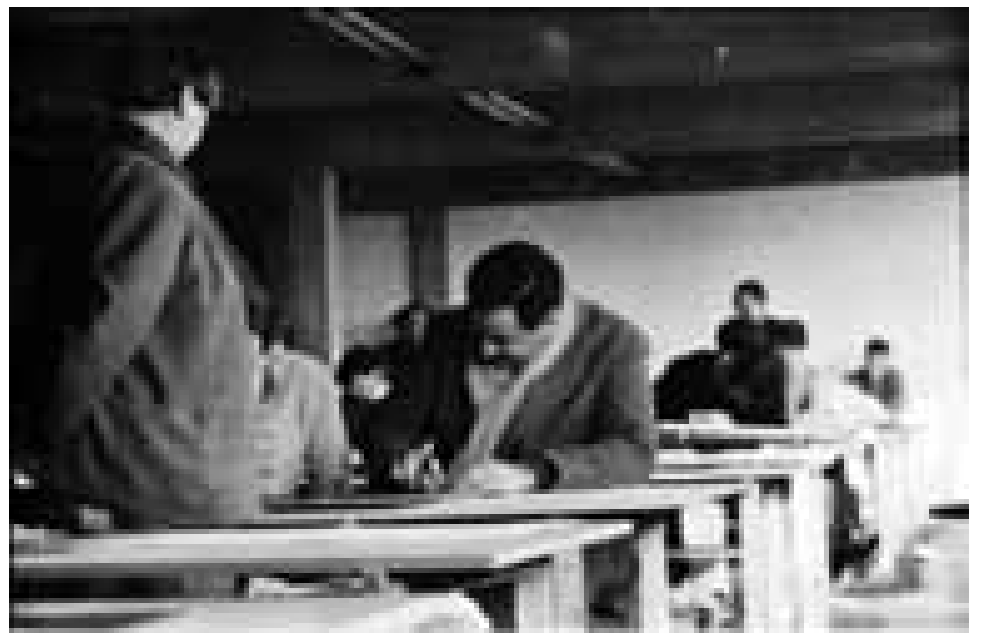

11/118 Helene Nonné-Schmidt y Tomás

Maldonado. Enero 1956

Fondo Hans G. Conrad

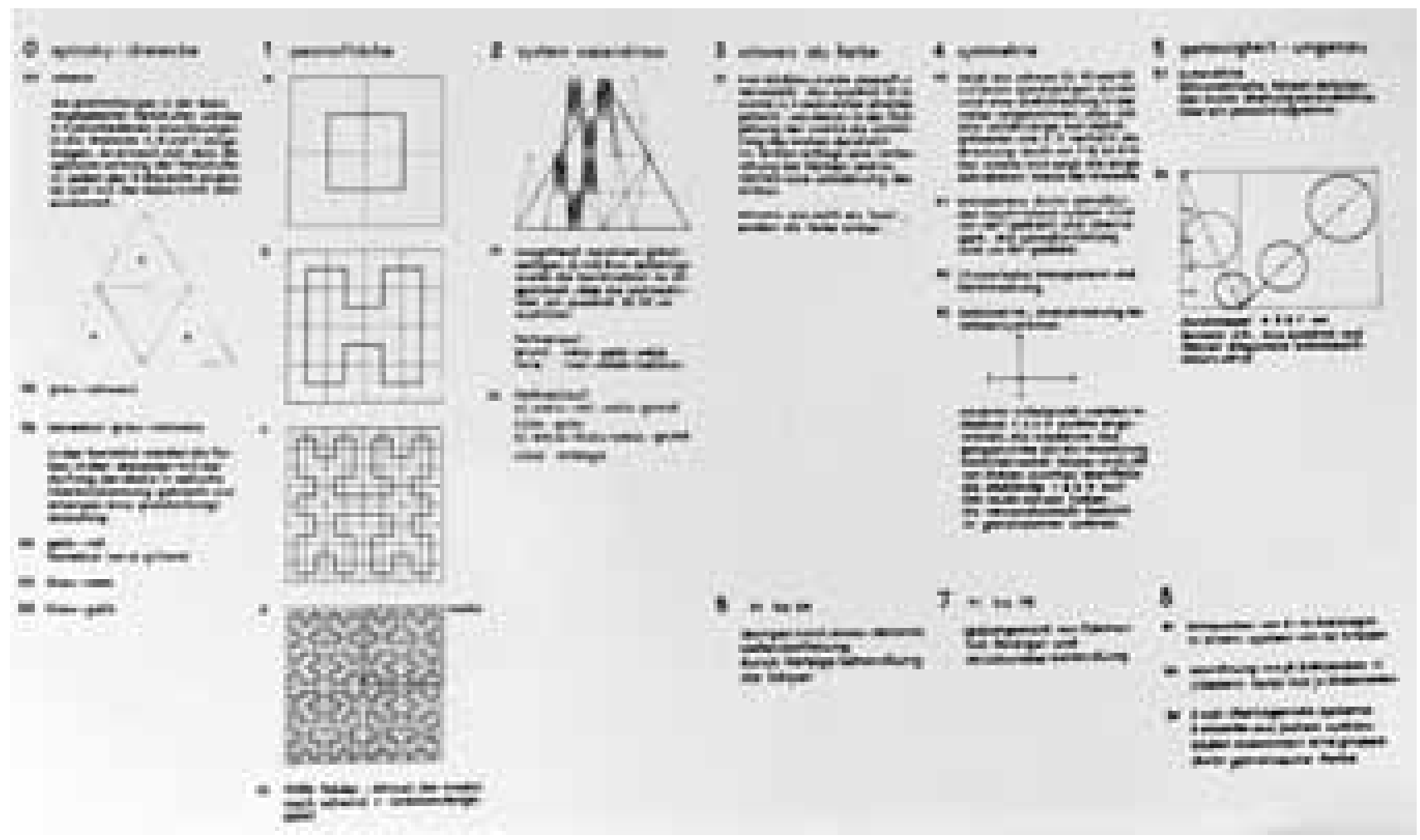

i1/119 Resumen del Kurs Maldonado elaborado por el alumno Klaus Frank.

Exposición permanente del archivo de la HfG 
Methoden y Darstellende Geometrie. La revisión de ejercicios de este curso evidencia que los papeles se reparten entre ambos, Nonné-Schmidt se centra en la enseñanza sistemática del color y abandona la enseñanza de la forma a partir de Klee, como ya se ha explicado. Maldonado toma los aspectos del color sintáctico y la generación de la forma e inicia un repertorio propio que desarrollará los siguientes años. Ambos profesores utilizan el mismo formato, y exigen un alto nivel de perfección técnica, lo que le confiere unidad al curso.

Maldonado en su primer repertorio independiente establece un sólido soporte científico para la enseñanza del color y la forma. Incorpora temas de la matemática en sus ejercicios, la topología -curva de Peano y Weierstrass-, el fractal -Sierpinski-; teoría de la percepción y teoría de la simetría. William S. Huff explica que es en este momento cuando el Curso Básico cambia de rumbo y comienza a hacerse interdisciplinar. ${ }^{112}$

Tareas del Kurs Maldonado en el Visuelle Einführung:

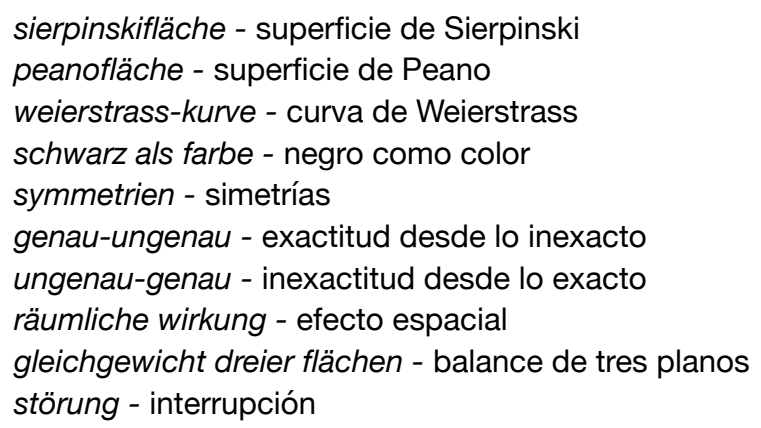

112 Huff, William S. "Grundlehre at the HfG" en: W.AA. Ulmer modelle - Modelle nach ulm, hochschule für gestaltung ulm 1953-1968. OstfildernRuit: Hatje Cantz Verlag, p. 117. 


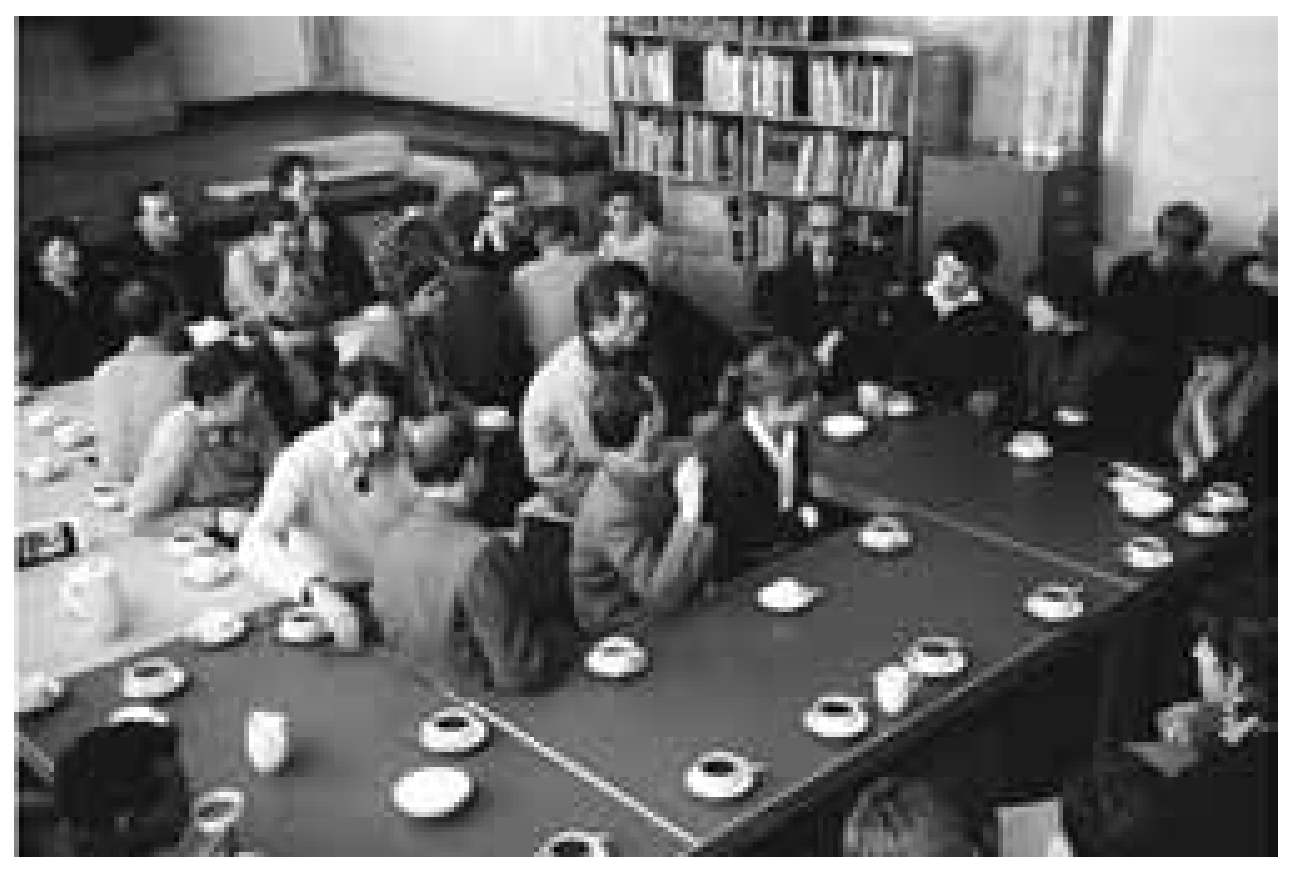

i1/120 Inge Aicher-Scholl en reunión

con los estudiantes para exponer

el distanciamiento de la HfG de Bill.

Febrero 1956

René Spitz, 2002 op. cit. p. 183.

i1/121 Max Bill y Tomás Maldonado Diciembre 1957

René Spitz, 2002 op. cit. p. 200.
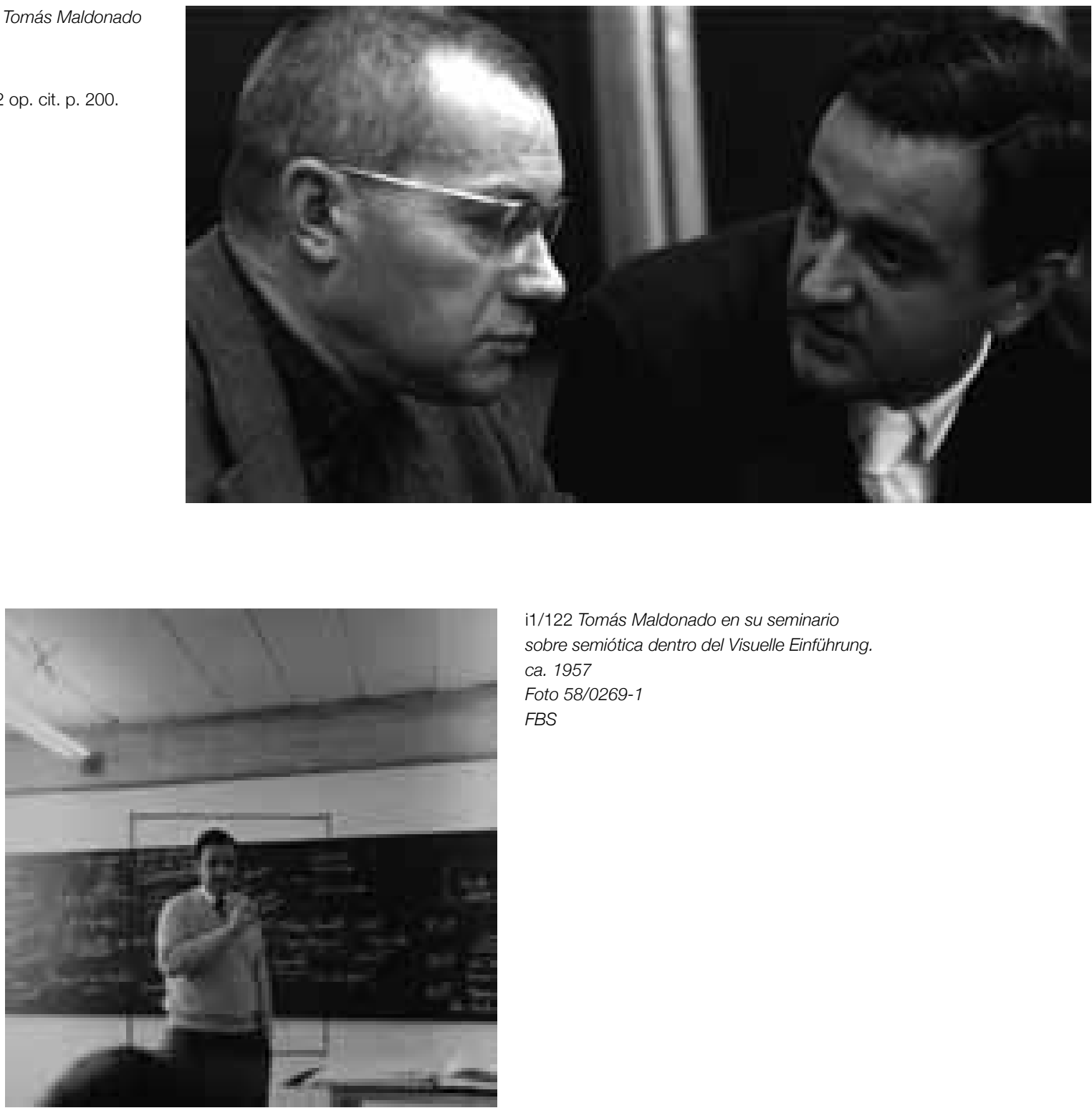

i1/122 Tomás Maldonado en su seminario

sobre semiótica dentro del Visuelle Einführung.

ca. 1957

Foto $58 / 0269-1$

FBS 


\subsection{Reafirmación de la propuesta de Maldonado y la consolidación del Visuelle Einführung en el Curso Básico}

\subsubsection{Contexto espacio-temporal del Visuelle Einführung}

Maldonado confirma su proyecto de Curso Básico en un momento de crisis institucional y de liderazgo. La escuela está dirigida por un rectorado colegiado de cinco profesores -Aicher, Bill, Gugelot, Maldonado y Vordemberge-Gildewart-, y Bill está contratado como profesor invitado. El contrato representa un distanciamiento formal entre Max Bill y la Fundación de los Hermanos Scholl, quien le otorgan un papel de consultor y colaborador autónomo. La versión oficial para justificar el cambio de contrato de profesor de la plantilla permanente a profesor visitante se apoya en el hecho de que Bill deseaba volver a dedicarse con intensidad a sus labores como artista y arquitecto tras los años intensos de puesta en marcha de la escuela. Max Bill se marcha definitivamente de la escuela el 30 de septiembre de 1957.

El curso 1956-1957 en el que se desarrolla la confirmación de la propuesta de Maldonado en el Grundlehre es el curso en el que se intensifica el conflicto entre Max Bill y la Fundación que acaba con su marcha a principio del curso siguiente. El conflicto que se evidencia y acrecienta en este periodo representa la escenificación final de un problema existente en la base y desde el principio de la fundación de la escuela.

Todas las fuentes documentales se refieren al hecho de que Max Bill nunca llega a residir en Ulm y mantiene su estudio y vivienda en Zúrich. Acude regularmente a la escuela en semanas alternas pero no siempre es accesible debido a que tiene que atender a labores no solo docentes, sino también de organización y gestión. Este hecho dificulta no solo la docencia sino la toma de decisiones. Claude Schnaidt se refiere a la ausencia de un programa concreto docente en el tiempo que Max Bill dirige el departamento de arquitectura. ${ }^{113}$

El nombramiento de un rectorado colegiado venía a solventar las dificultades de la gestión por itinerancia del rector Bill. El rectorado comienza a votar y tomar decisiones en su ausencia y precisamente este hecho es el detonante del conflicto final. Max Bill había comenzado a trabajar con un grupo de alumnos afines dentro de la escuela y lo había denominado "Atelier Bill". En un primer momento la Fundación le había permitido esta circunstancia y cierta autonomía, pero al poco tiempo este grupo comienza a ser excluyente, Bill no acepta intromisiones y veta la entrada al resto de profesores y alumnos. El rectorado -reunido sin él- le insta a cambiar el nombre del Atelier por considerarse una "escuela dentro de la escuela". Hasta la renuncia y marcha definitiva del profesor se desencadena un cruce de cartas y acusaciones cruzadas que salta de forma pública a la prensa. Max Bill considera que el asunto del Atelier es solo una excusa para librarse de su presencia. ${ }^{114}$

A esta serie de circunstancias se añade el debate sobre si el modelo Bauhuas es el modelo a seguir por la HfG tanto en la concepción pedagógica de la escuela, como en el modelo de Curso Básico que Max Bill había ideado. A pesar de los esfuerzos de Bill por inculcar el diseño "no-objetivo"115 como diseño no orientado hacia ningún objeto con el soporte de los antiguos bauhäusler, los nuevos docentes y los alumnos consideran que el modelo Bauhaus es un modelo obsoleto y extemporáneo. En realidad no existe tal modelo Bauhaus, no existe un "concepto coherente" ni existe tampoco coordinación entre los profesores ${ }^{116}$ quienes por otro lado contaban con una trayectoria propia posterior a la Bauhaus.

Para los profesores más jóvenes imbuidos de una "insaciable curiosidad" 117 hacia las nuevas disciplinas científicas, seguir la tradición Bauhaus implica distanciarse de ella de tal manera que se adopta un posicionamiento en el que aprovechar la libertad de acción que generan las circunstancias históricas del momento ${ }^{118}$ y lo que llevado al Curso Básico implica que su didáctica se haga "receptiva a la metodología científica" ${ }^{119}$ Este es el camino por el que se incorporan temas de la topología, la simetría y la teoría de la percepción ${ }^{120}$ que alcanzan su madurez en los ejercicios del Visuelle Einführung del curso 1956-1957.
113 "Cuando comenzó el nuevo semestre a mediadios de Octubre, Bill vino con la documentación de un concurso. Un poco como Hannes Meyer en la Bauhaus. Los participantes tenían que diseñar un edificio multiusos en Immendingen. Sin muchas explicaciones, Bill lanzó el enunciado del concurso sobre la mesa. Teníamos tres meses para trabajar en el proyecto. Durante este periodo, apenas le vimos". Claude Schnaidt se refiere a la división entre los estudiantes del Departamento de Arquitectura afines y críticos con Bill, división que se extendía al resto de la escuela.

Entrevista a Claude Schnaidt en: W.AA. HfG UIm: form+zweck 20 (número monográfico). form + zweck, 2003, p. 69

114 René Spitz recoge de forma cronológica las cartas y su contenido en el que se puede seguir el desarrollo del conflicto a lo largo de todo el curso. Spitz, René, op. cit. pag 200 204.

115 Ver entrevista con William S. Huff: Krampen, Martin, \& Hörmann, Günther, op. cit. pag. 97.

116 Ver entrevista con Herbert Lindingeren: Krampen, Martin, \& Hörmann, Günther, op. cit. pag. 95.

117 Tomás Maldonado: Lindinger op. cit. pag. 222.

118 Ver entrevista con Tomás Maldonado: Krampen, Martin, \& Hörmann, Günther, op. cit. pag. 95

119 Ibid. p. 241

120 Ver entrevista con Tomás Maldonado: form+zweck, op. cit. pag. 18. 

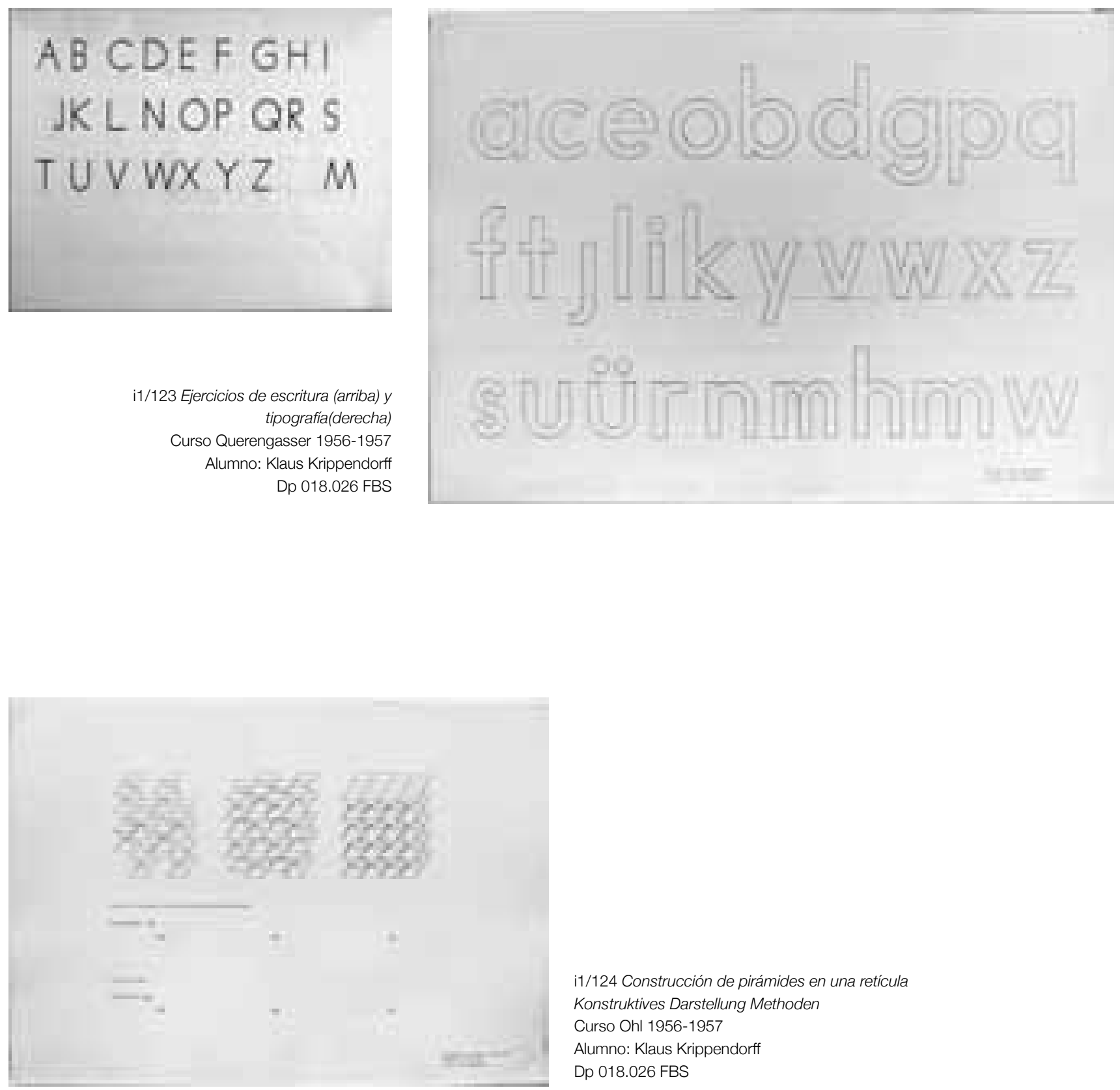

i1/124 Construcción de pirámides en una retícula Konstruktives Darstellung Methoden

Curso Ohl 1956-1957

Alumno: Klaus Krippendorff

Dp 018.026 FBS

i1/125 Estudios sobre la circunferencia en perspectiva Freihandzeichnung Curso Aicher 1956-1957 Alumno: Klaus Krippendorff Dp 018.026 FBS 


\subsubsection{Docentes, materias y alumnos del periodo}

\author{
Rectorado 1956-1957 \\ Otl Aicher \\ Max Bill \\ Hans Gugelot \\ Tomás Maldonado \\ Friedrich Vordemberge-Gildewart
}

\section{Docentes 1956-1957}

Otl Aicher

Max Bill

Hans Gugelot

Tomás Maldonado

Helene Nonnè Schmidt

Fritz Pfeil

Ernst Scheidegger

Friedrich Vordemberge-Gildewart

Konrad Wachsmann

Walter Zeischegg

\section{Maestros de taller 1956-1957}

Fritz Hahn - Fotografía

Paul Hindinger - Madera

Otto Schild - Yeso

Josef Schlecker - Metal

Wolfgang Siol - Fotografía

Cornelius Uittenhout - Plástico

\section{Departamentos 1956-1957}

Grundlehre - Tomás Maldonado

Producto - Hans Gugelot

Arquitectura y construcción de la ciudad-

Max Bill

Comunicación Visual - Otl Aicher

Información - Max Bense

\section{Docentes invitados 1956-1957}

Herman Von Baravalle - Visuelle Einführung

Max Bense: Información, Integración cultural

Max Bill

Werner Blaser - Departamento de Producto

Hans Curjel - Integración Cultural

Hans M. Enrensberger - Información

Albrech Fabri, Información

Enrich Franzen - Integración Cultural

Eugen Gomringer, Integración Cultural

Kate Hamburger, Información

Georg Leowald - Diseño de Producto

Helenne Nonné-Schmidt - Grundlehre

E.F. Podach - Integración Cultural

Elisabeth Walther - Información, I.Cultural

\section{Asistentes 1956-1957}

Herbert Ohl, Fritz Querengässer

\section{Materias 1956-1957}

Visuelle Einführung - Maldonado y Nonné-Schmidt continúan alternándose en el Visuelle Einfuhrung. Maldonado repite el repertorio iniciado el curso anterior y profundiza en los temas de simetría y teoría de la percepción. Nonné-Schmidt por su parte, aplica su repertorio de ejercicios sobre el color. En Konstruktive Darstellung Methoden, Baravalle repite su curso en el primer cuatrimestre. Herbert Ohl participa en la materia durante el tercero.

Darstellungstechniken - Eugene Gomringer, secretario personal de Max Bill y poeta concreto, imparte la materia Sprache en la que se trabaja el lenguaje como medio de representación y expresión como complemento a las disciplinas visuales. Otl Aicher inicia la materia de dibujo del natural. Fritz Querengasser, colaborador de Aicher, imparte sendos cursos de escritura y tipografía. Los trabajos en los talleres de Madera, Metal y Yeso se suceden a lo largo de los dos primeros cuatrimestres. El trabajo en el taller de fotografía lo dirige Ernst Scheidegger, colaborador de Max Bill.

Naturwissenschaften Fächer

En este curso se imparte por primera vez Matemáticas, que se incluye en el área de ciencias exactas y naturales. El profesor Eichhorn desarrolla su curso en el último cuatrimestre. 


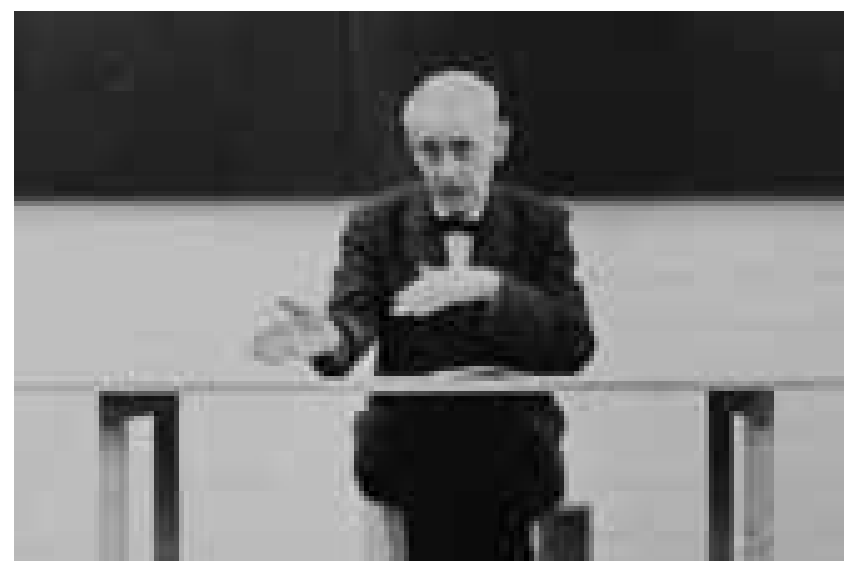

i1/126 Erich Franzen, profesor de Sociología

FBS

i1/127, 128 Elaboración de una memoria informativa para el Ulmer Hocker Sprache Curso Eugene Gomringer 1956-1957 Alumno: Ulrich Burandtf Dp 033.007 FBS
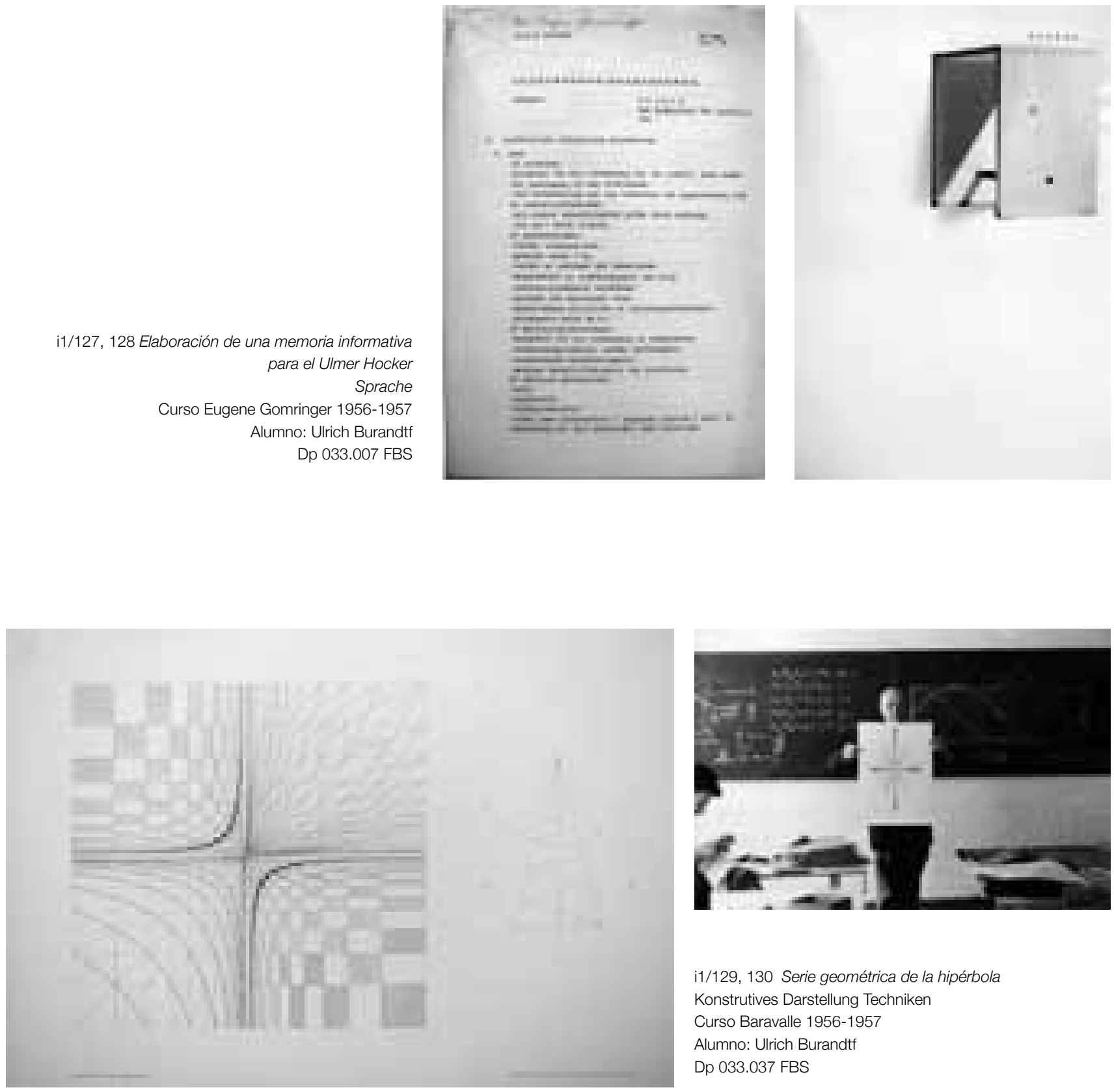

11/129, 130 Serie geométrica de la hipérbola

Konstrutives Darstellung Techniken

Curso Baravalle 1956-1957

Alumno: Ulrich Burandtf

Dp 033.037 FBS 
Allgemeinbildende und Theoretische Fächer - Durante este curso se amplían las materias del bloque. Gerhard Eichhorn imparte matemáticas en el tercer cuatrimestre. Elisabeth Walther, asistente de Max Bense, imparte Kulturelle Integration y Filosofía. Kulturgeschichte lo imparten Bill en el primer cuatrimestre y Hans Günther Sperlich en el tercero. En este curso aparece una materia de Methodologie a cargo de Elisabeth Walther. Erich Franzen imparte sociología en el segundo y tercer cuatrimestres. Derechos de autor a cargo de Hermsler en el segundo cuatrimestre.

La sencuencia en el tiempo entre las disciplinas visuales es el siguiente:

VE kdm 1, Kurs Baravalle

VE kdm 2, Kurs Ohl

VE kdm 3, kurs Ohl

DM schr, Kurs Querengässer

$D M$ spr, Kurs Gomringer (informes escritos)

WA (werkarbeit) Holz

WA metall

VE 1, kurs Nonné-schmidt

VE 2, kurs Maldonado

VE 3, kurs Aicher (tareas de dibujo)

VE 4, kurs Maldonado

VE 5, kurs Nonné-Schmidt

VE 6, kurs Maldonado-OhI

\section{Alumnos 1956-1957}

Josef Althoff

Urs Beutler *arquitectura

Günther Bischoff

Monika Buch

Ulrich Burandt

Nicholas Chaparos

Glean Chase

Thomas Dawo

Edgar Decurtins *arquitectura

Walter Dillis

James Dobson

Hermann Edel *arquitectura

Frank Geiser *arquitectura

Heinz Hess

Gilbert Hirt

William Huff

Werner Kilian

Erich Klein
Klaus Krippendorff *producto

Karl-Heinz Krug *producto

Bernd Meurer *arquitectura

Shutaro Mukai

Bertus Mulder

Eckhard Neumann

Yedda Pitanguy

Diego Peverelli *arquitectura

Ferdinand Porsche

Albert Renftle

Sennosuke Shimizu

Heinz Stempfle

Sven Weisshardt *comunicación visual

Klaus Wille *comunicación visual

Rudolf Winkler *arquitectura

Norishigue Yokomari

Hubert Zimmermann 

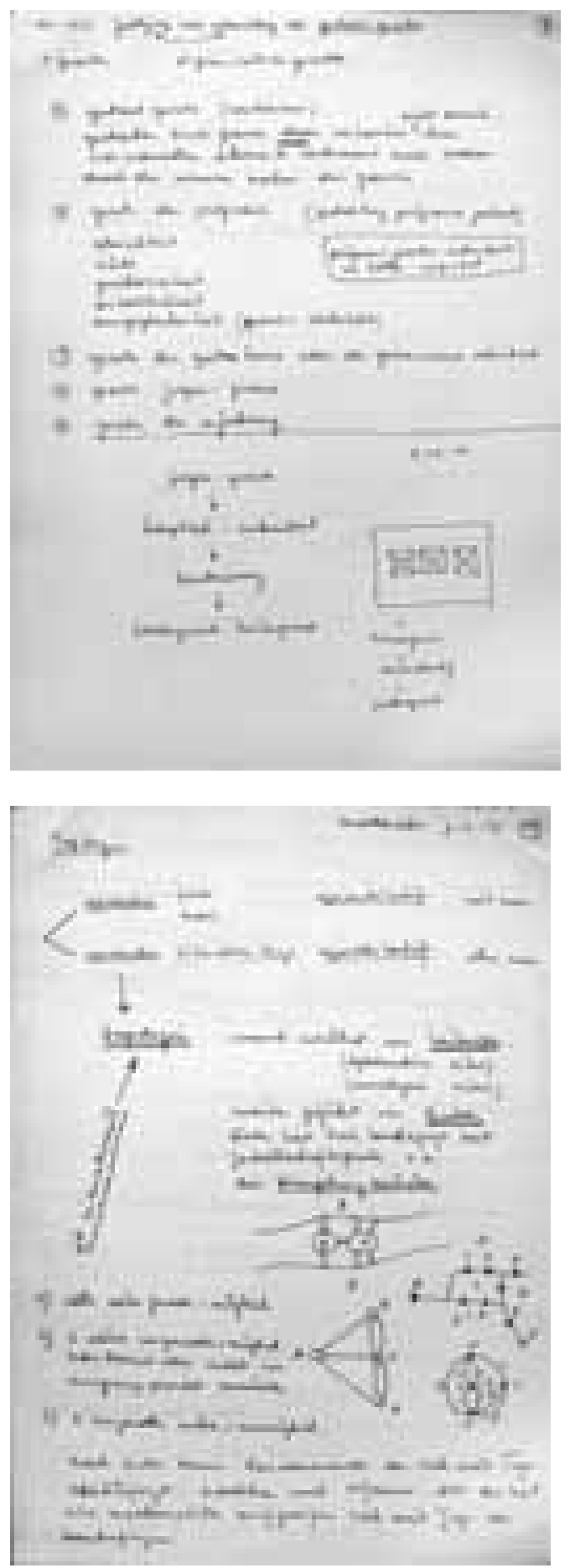
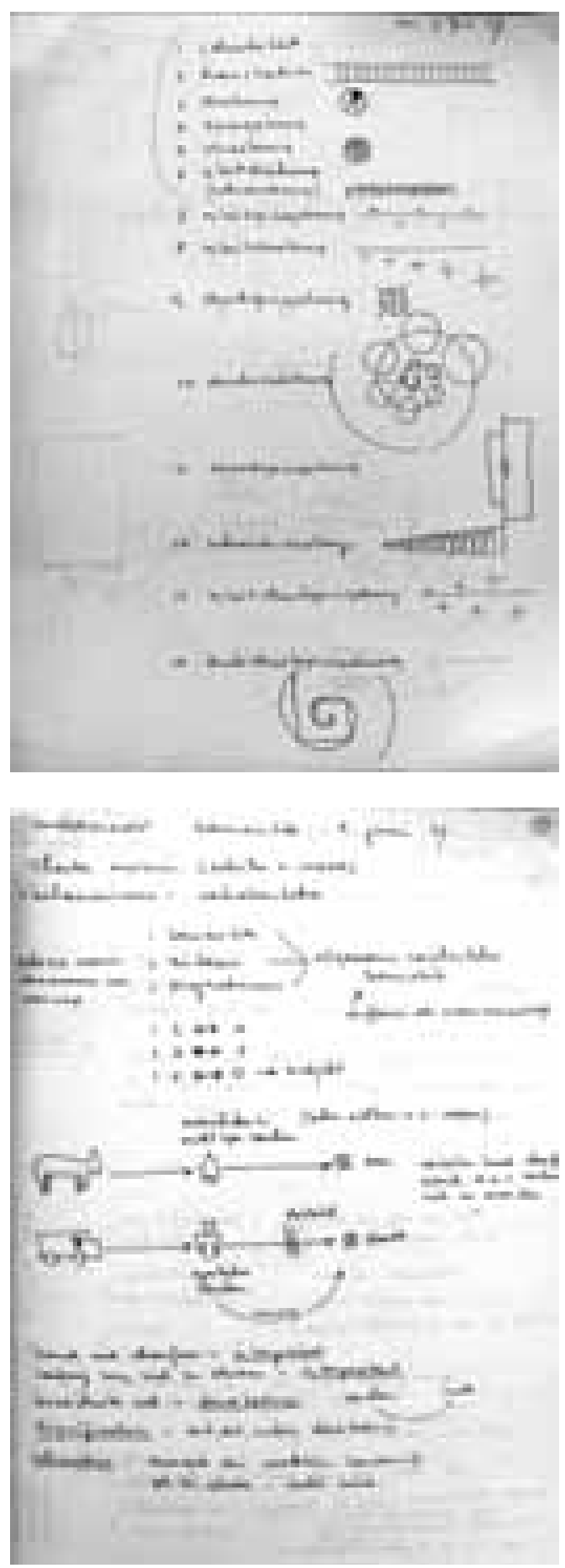

11/131, 132, 133, 134 Apuntes de los seminarios teóricos de Tomás

Maldonado en el Grundlehre del curso 1956-1957. Teoría de la Percepción,

Simetría, Topología y Semiótica. Alumno: Max Graff. 


\subsubsection{Programa de seminarios}

Dentro de su curso en el Visuelle Einführung, Maldonado incorpora unos seminarios con una secuencia destinada a estimular la actividad práctica. ${ }^{121}$

Cada seminario se programa para un mes y su desarrollo es paralelo a las tareas de los alumnos. El plan de trabajo que propone Maldonado para este curso es el siguiente:

1. Introducción elemental a la Teoría de la Percepción

2. Introducción elemental a la Teoría de Simetría

3. Introducción elemental a la Topología

4. Introducción elemental a la Teoría Visual del Significado Visual (semiótica)

Maldonado va más allá de sus predecesores en el Curso Básico -Bill, Albers, Peterhansporque incluye un componente teórico y lo vincula directamente con los ejercicios. La relación consiste en la puesta en práctica de los preceptos teóricos impartidos en los seminarios sobre topología, teoría de la percepción y simetría. Los ejercicios no tienen un enunciado estructurado como tal. Se formulan, como hiciera Peterhans, generando unas condiciones que han de cumplir los elementos visuales a partir de las directrices impartidas en los seminarios teóricos. De este modo las leyes de la Gestalt ${ }^{122}$ o las curvas topológicas forman parte del proceso de elaboración de los ejercicios.

La confirmación de un Curso Básico específico evolucionado por Maldonado en esta etapa se basa en que consigue por primera vez, en la HfG y sus escuelas precedentes, la transversalidad de la didáctica al incorporar estos núcleos de conocimiento científico en la práctica visual. ${ }^{123}$

Todos los seminarios desarrollados por Maldonado parten de una profusa revisión histórica de cada área para centrar los núcleos de interés coetáneos. En la introducción a la Teoría de la Percepción se remonta a Spinoza, Hobbes y Bacon. Al abordar la psicología experimental Menciona a Johannes Müller como el padre de la psicología experimental que establece un puente entre Fisiología y Psicología. Tras profundizar en la Psicología de la Gestalt ${ }^{123}$ y sus principales representantes -Wertheimer, Köhler y Koffka- finaliza introduciendo el desarrollo científico que se está llevando a cabo en la época a través de la Teoría de la información, la Neurología y la Cibernética.

En lo relativo a la Teoría de la Simetría, hace una revisión de su estudio desde la antigüedad clásica -Aristóteles, Policleto, Platón, Vitrubio- el uso como herramienta ornamental en las culturas antiguas -mayas, aztecas- y subraya la importancia que adquiere en el Renacimiento -Durero, Luca Paciolli. Sitúa el estudio científico de la simetría en el siglo XIX a partir de la Biología, la Cristalografía, y las Matemáticas, y se refiere a la obra de Wolf y sus 14 operaciones de superposición como el referente para el trabajo práctico.

En repaso histórico del seminario de Topología menciona a Moebius, Jordan, Dyck, Cantor y Poincaré. Presenta la situación del momento como una fusión entre las dos corrientes la topología algebraica y la topología general. Adopta como referente principal para el trabajo práctico la obra de Walther Lietzmann, Anschauliche Topologie, para estudiar las leyes generales, las superficies no orientables, las parqueterías y los grafos.

Max Bense había introducido la Semiótica en sus cursos de teoría de Grundlehre. Maldonado destaca las figuras importantes de la disciplina y se centra en Morris y Peirce.
121 Apuntes del curso de Maldonado en el Visuelle Einführung durante el curso 1956-1957. Ulrich Burandt. Dp_033.012.

122 Huff, W. Op cit. p. 180

123 En el seminario de Teoría de la Percepción recurre a las 5 leyes de la Gestalt como leyes organizativas que serán la herramienta para el trabajo en sus ejercicios del Visuelle Einführung:

"1.Ley de la forma. Las formas son un todo, cuyo comportamiento no está determinado por sus elementos individuales, sino por la naturaleza interna del todo. (Wertheimer)

2. Ley de Pregnancia: [principios de] Igualdad, Proximidad, Cercanía Unidad, Equilibrio (Estabilidad)

3. Ley de la buena curva: o el destino común

4. Ley de la Figura-Fondo

5. Ley de la experiencia."

Dp 033.012 


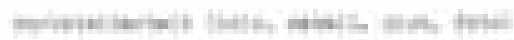
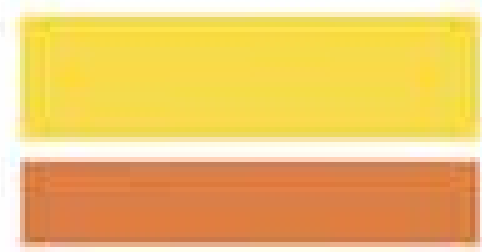

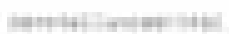

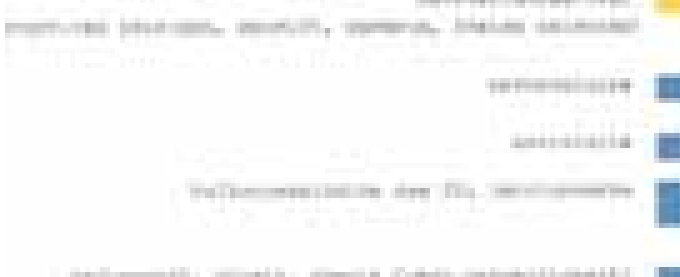

¡1/135 Gráfico de distribución de horas por materias del Grundlehre en uno de los paneles de la exposición de 1958.

T 097 FBS

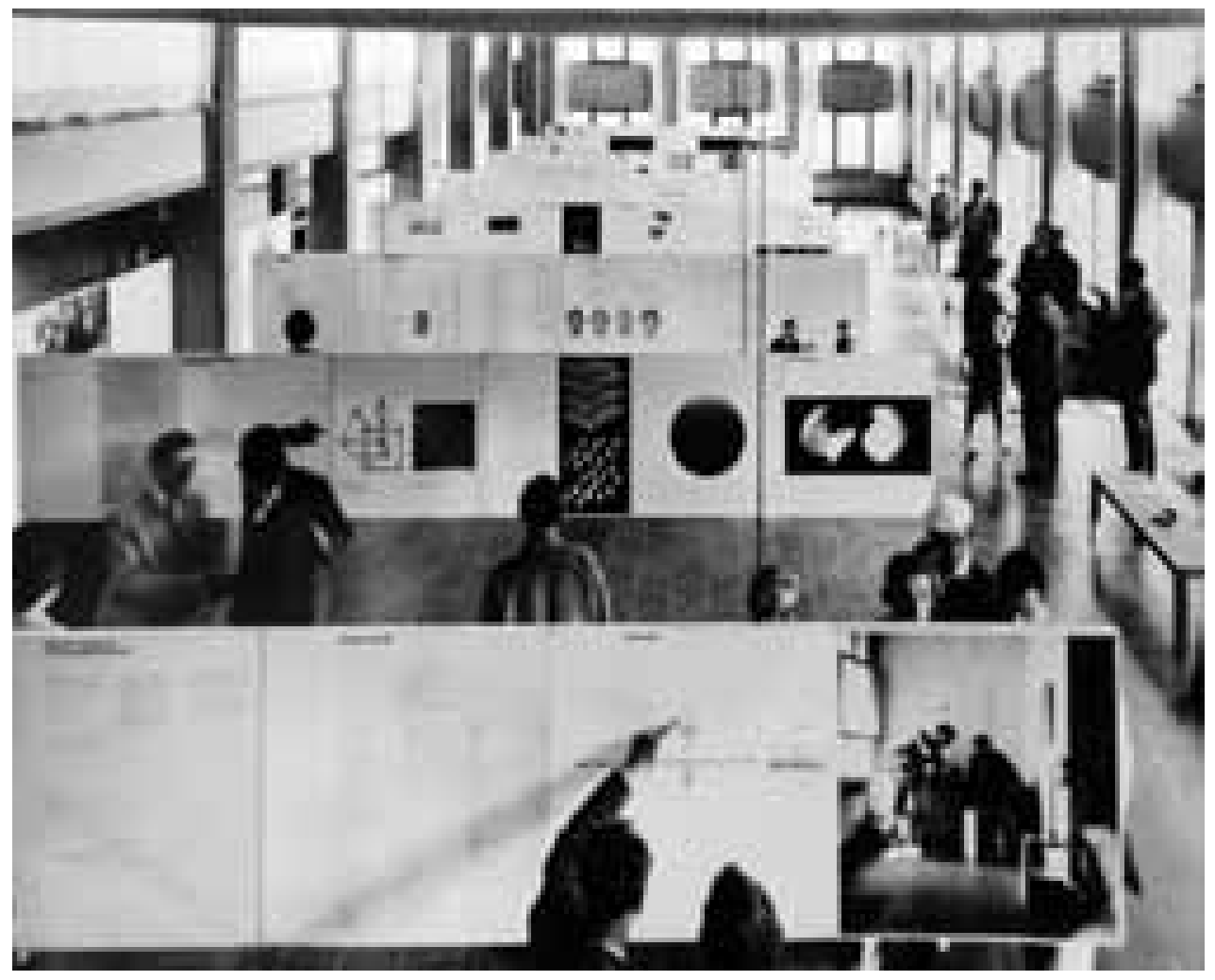

11/136 Exposición "Métodos y

resultados de trabajo", inaugurada en el mensa de la HfG el 6 de junio de 1958

Fotógrafo: Siol

F 58.0186 


\title{
1.6 Reforma del curriculum del Curso Básico: hacia un nuevo Formgeber
}

\author{
1.6.1 Contexto espacio-temporal de la reforma ${ }^{124}$
}

Maldonado enuncia la ruptura definitiva con la Bauhaus en el discurso de apertura del curso 1957-1958, y con ello, un cambio de rumbo: "Aceptamos solamente la actitud progresista y anti-convencional, el deseo de contribuir a la sociedad en su propia situación histórica. En este sentido, y sólo en este sentido, estamos continuando la labor de la Bauhaus". ${ }^{125}$ En este discurso clave para comprender el rumbo que tomaría la escuela, Maldonado se lamenta que hasta el momento, la "racionalidad" por la que se empieza a conocer a la HfG es tan sólo un disfraz científico. Anuncia que la escuela va a otorgar una mayor importancia a las asignaturas teóricas para la educación de un diseñador más modesto que conozca los materiales, las leyes de construcción y los procesos de fabricación con los que debe trabajar. De acuerdo a este objetivo, en el Grundkurs van a empezar a colaborar los profesores de los departamentos impartiendo docencia en el tercer cuatrimestre. Éste sin duda, es un adelanto de la aparición de las Methodische Übungen -tareas metodológicas-, el vuelco definitivo al enfoque propedéutico del Curso Básico. ${ }^{126}$

Max Bill renuncia en 1957, augurando un futuro oscuro para la HfG. Está decepcionado con una escuela que se ve seducida por un "misticismo tecnológico" 127 personificado en la figura de Maldonado. Éste concibe el Curso Básico con un nuevo sentido en el que prevé una introducción de los alumnos a la práctica y el análisis de los medios técnicos del diseño, de los medios visuales, y de los problemas culturales del momento. Por otro lado, le otorga mucha importancia a la promoción en el alumno de la conciencia de su futura responsabilidad social como formgestalter ${ }^{128}$ y además la liberación de prejuicios y deformaciones psicológicas de la formación anterior del alumno. El Curso Básico mantiene el carácter de filtro y de selección de los alumnos más capaces y motivados para su ingreso en los departamentos.

En el curso 1957-1958 la escuela celebra sus cinco años de existencia con una exposición en el comedor de la escuela sobre una completa representación de los métodos y resultados de la escuela llevados a cabo hasta entonces. ${ }^{129}$ La imagen de los paneles cuadrados en alineación, flotando en el espacio del comedor, se convierte en una de las señas de identidad de la escuela.
124 Parte del epígrafe es un extracto publicado anteriormente en: Fernández-Campos, Angel Luis; Sánchez Moya, María Dolores op. cit, 392-411.

125 Maldonado, Tomás "Rede des Vorsitzenden des Rektoratskollegiums der Hoschule für Gestaltung, Tomás Maldonado, zur Eröffnung des Studienjahres 1957-1958 am Donnerstag den 3. Oktober 1957". (Discurso del Presidente del Rector de la Escuela Superior de Diseño Hoschule, Tomás Maldonado, para la apertura del curso académico 1957 1958 en Jueves, 3 de octubre 1957). Discurso inédito. HfG Ulm, 1957 p. 1

126 En este curso, además, se prestará especial atención a la formación matemática con el Dr. Eichhorn, así como a la introducción a la metodología impartida por la profesora Dra. Walther. El Dr. Sperlich y el arquitecto Joseph Ryckwert dirigen el seminario histórico-cultural y el profesor Franzen la introducción a la sociología.

127 Carta de Max Bill a Walter Gropius, 10 de febrero de 1959. Spitz René. op. cit. p. 231

128 Diseñador, equivalente a Formgeber.

129 Quijano, Marcela “¿Será el camino la meta? en: W.AA. Ulmer modelle, op. cit, p. 56. 

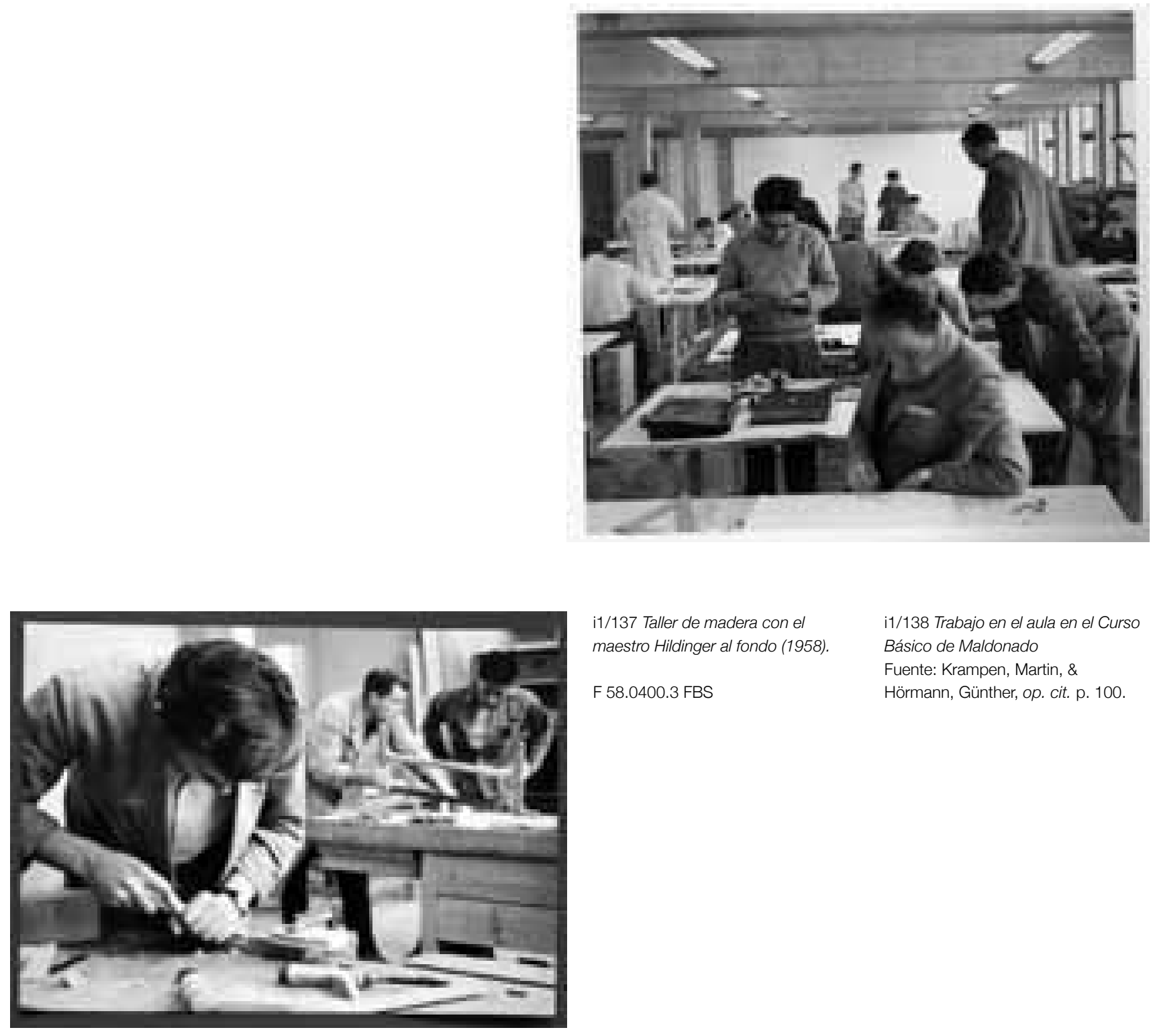

i1/137 Taller de madera con el

maestro Hildinger al fondo (1958).

F 58.0400.3 FBS
11/138 Trabajo en el aula en el Curso

Básico de Maldonado

Fuente: Krampen, Martin, \&

Hörmann, Günther, op. cit. p. 100

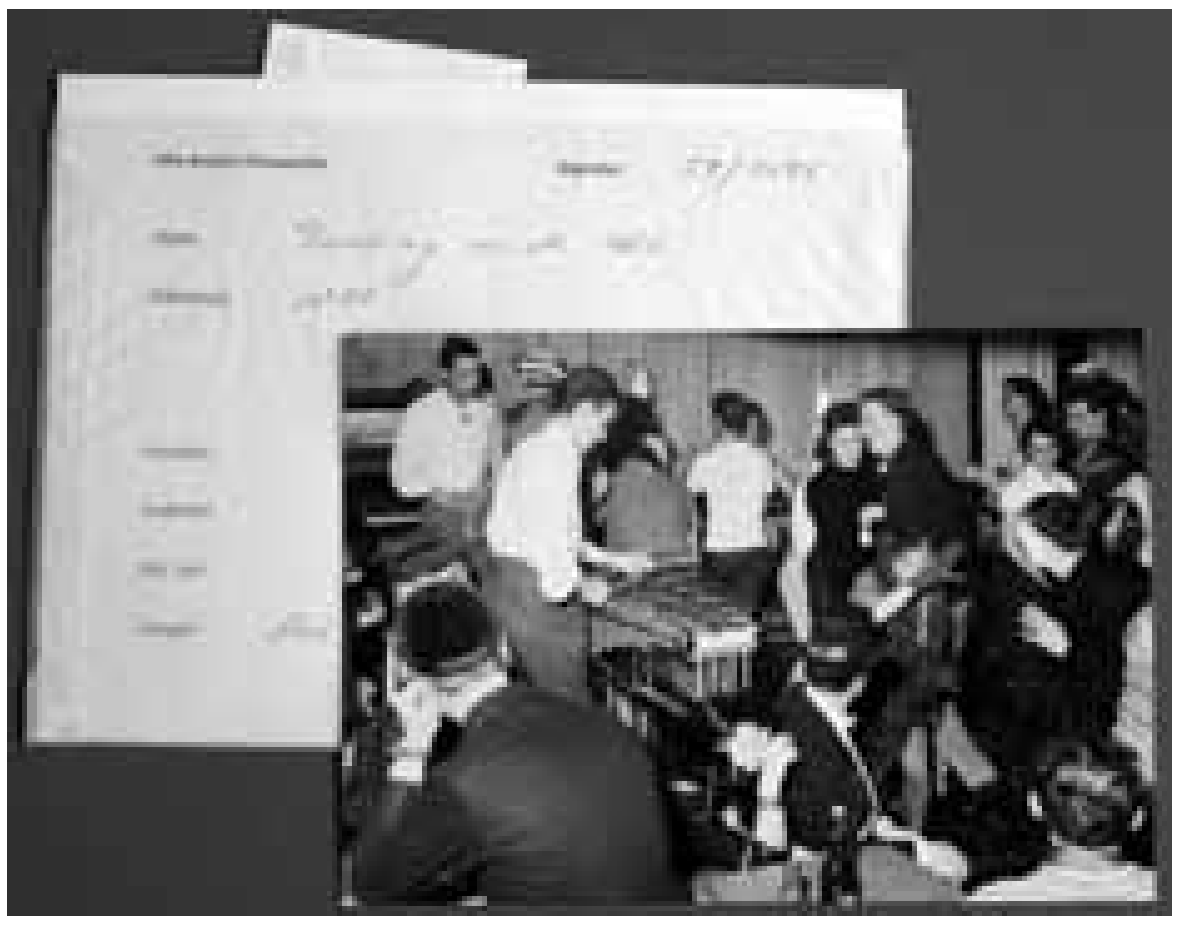

i1/139 Fiesta en la HfG. 1958

Fotógrafo: Siol

F 59.0198 FBS 


\subsubsection{Docentes, materias y alumnos del periodo}

\author{
Rectorado 1957-1958 \\ Otl Aicher \\ Hans Gugelot \\ Tomás Maldonado
}

\section{Departamentos 1957-1958}

Grundlehre - Tomás Maldonado

Construcción ${ }^{130}$

Producto - Hans Gugelot

Comunicación visual - Otl Aicher

Información - Max Bense

\section{Docentes 1957-1958}

Otl Aicher

Anthony Froshaug

Hans Gugelot

Hanno Kersting

Georg Leowald

Tomás Maldonado

Fritz Pfeil

Christian Staub

Friedrich Vordemberge-Gildewart

Walter Zeischegg

\section{Maestros de taller 1957-1958}

Paul Hindinger - Madera

Otto Schild - Yeso

Josef Schlecker - Metal

Wolfgang Siol - Fotografía

Cornelius Uittenhout - Plástico

\section{Docentes invitados 1957-1958}

Herman Von Baravalle - Visuelle Einführung

Max Bense - Información. Integración cultural

Peter Dressen

Gerhard Eichhorn - Mecánica general

Erich Franzen, K.I

Etienne Grandjean - Fisiología

Hermann Haan - Departamento de Construcción

Siegfried Haenle - Diseño técnico

Kate Hamburger - Dep. de Información

Bruno Hrosberger - Fisiología

Gert Kalow - Departamento de Información

Bruce Martin - Departamento de Construcción

Helenne Nonné-Schmidt - Curso Básico

Herbert Ohl - Departamento de Construcción

Dieter Oestreich -Departamento de Producto

Giulio Pizzeti - Estructuras

Fritz Querengässer - Comunicación Visual

Thomas Rago - Fotografía

Horst Rittel - Análisis de operaciones

Bernhard Rübenach - Radiodifusión

Jospeh Rykwert -Departamento de Construcción

Herbert Schober - Fisiología

Johannes Schön - Construcción técnica

Christian Norberg - Schulz - Dep. de Construcción

Hans-Günter Sperlich - Historia cultural

Matthew Wallis - Metodología de construcción

Martin Walser. Elisabeth Walter. - Información, integración cultural
130 Arquitetura y urbanismo (Architektur und stadtbau) cambia a "construción" Bauen con la salida de Max Bill. A comienzo del curso 1957 1958 no tiene asignado responsable.

131 Es significativa la carencia de material tanto en las donaciones como en los archivos en depósito acerca de los ejercicios de Grundlehre de este curso concreto. La revisión bibliográfica confirma este vacío que las fuentes consultadas -personal de archivo e investigadores- han sabido justificar.

\section{Materias 1957-1958 ${ }^{131}$}

En el panel dedicado al Grundlehre en la exposición de 1958 aparece un gráfico que sintetiza el reparto del tiempo entre las distintas disciplinas. Se refiere a un total de 1120 horas de las que aproximadamente, el $70 \%$ de dedica a la ejercitación práctica y el $30 \%$ a la teoría.

\section{Visuelle Methodik}

Nonné-Schmidt y Maldonado comparten el primer cuatrimestre de docencia y Maldonado continua solo en el segundo. Es el último año de docencia de la profesora bauhäusler. E breve repertorio que representa este año contiene algunas tareas que ya se habían comenzado a realizar en el 1955-1956, como son los Raster, Imprecisión y trabajos de Simetría. Anthony Froshaug, que había llegado invitado por Maldonado en el año 1957, comienza a trabajar con grafos. El grupo de materias relativas al color y la forma cambian en este periodo su denominación de Visuelle Einführung a Visuelle Methodik. Es probable que el cambio de denominación se deba a la incorporación de Anthony Froshaug , no obstante es el primer profesor que incorpora herramientas metodológicas al trabajo. En el panel dedicado a Grundlehre de la exposición de 1958 ya aparece este cambio de denominación.

Ohl y Baravalle reducen visiblemente su participación a un solo trimestre en las materias del Konstruktive Darstellung Methoden. El repertorio de Baravalle continúa desarrollando los 
temas de la geometría dinámica y Ohl también realiza ejercicios análogos al curso anterior en el estudio de detalles constructivos en distintos sistemas de representación.

Se dedica un total de 420 horas a Visuelle Methodik.

\section{Naturwissenschaften Fächer}

En este curso se amplía la presencia de las ciencias exactas a física y química, además de las matemáticas que se imparten a demanda, según las necesidades de los planteamientos de la escuela y ritmos de trabajo.

\section{Darstellungstechniken}

Aicher comienza el curso con sus seminarios de dibujo libre y escritura. Kalow enseña lengua tras la marcha de Gomringer, Querengasser tipografía. Los talleres de madera y metal se realizan en el primer trimestre, y el segundo el yeso. El taller de fotografía está presente en todo el curso dirigido por Siol y Rago. Es muy superior el tiempo dedicado al taller (280 horas) y que a las disciplinas que se utilizan como medios de representación (70).

\section{Allgemeinbildende und Theoretische Fächer -}

Max Bense, Elisabeth Walther y Horst Rittel se alternan en metodología. Kulturgeschichte - Historia cultural - ha variado poco en contenidos desde el comienzo y continúa con la historia de la arquitectura, pintura, escultura y literatura del siglo XX. Los profesores este curso son J. Rykwert y G. Sperlich. La materia sociología corre a cargo de Kesting y Franzen. Se dedica un total de 350 horas a las asignaturas teóricas que se suceden en bloques de 70 horas menos Kulturgeschichte que tiene el doble.

\section{Alumnos 1957-1958}

Wolfgang-D. Adler *construcción

Joahannes Beiter

Beric Brezinger

Werner Bührer * producto

A. Casillas de Alba

Karl-Achim Czemper *producto

Horacio Denot

Manfred Eisenbeis

Maria fraxedas

Max Gerzberg

Knut Jakob

Alexander Janowski

Gunnar Johnsson *producto

Lot Kalbermatter

Douglas Kelley

Gunthar Lamche

Hans Lieb

Roland Lindner *construcción

Norbert Linke *producto

Werner Lorenz

Kurt Mayer

Gian-Luca Meda

Siegfried Morgenegg

Uta Müller

Tsugio Nachi *producto
Christa Pohlsschröder

Joachim Rogmann

Jan Schleifer *producto

Günther Schmitz *construcción

Siegfruied Springer

Adolf Stibbe

Hans Ruedi Tobler

Rupert Urban *construcción

Ariberte Vahlenbreder *producto

E. Vargas-Stroller

Ann Walby

Madeline Wickham

Hans Eckhart Zahn 


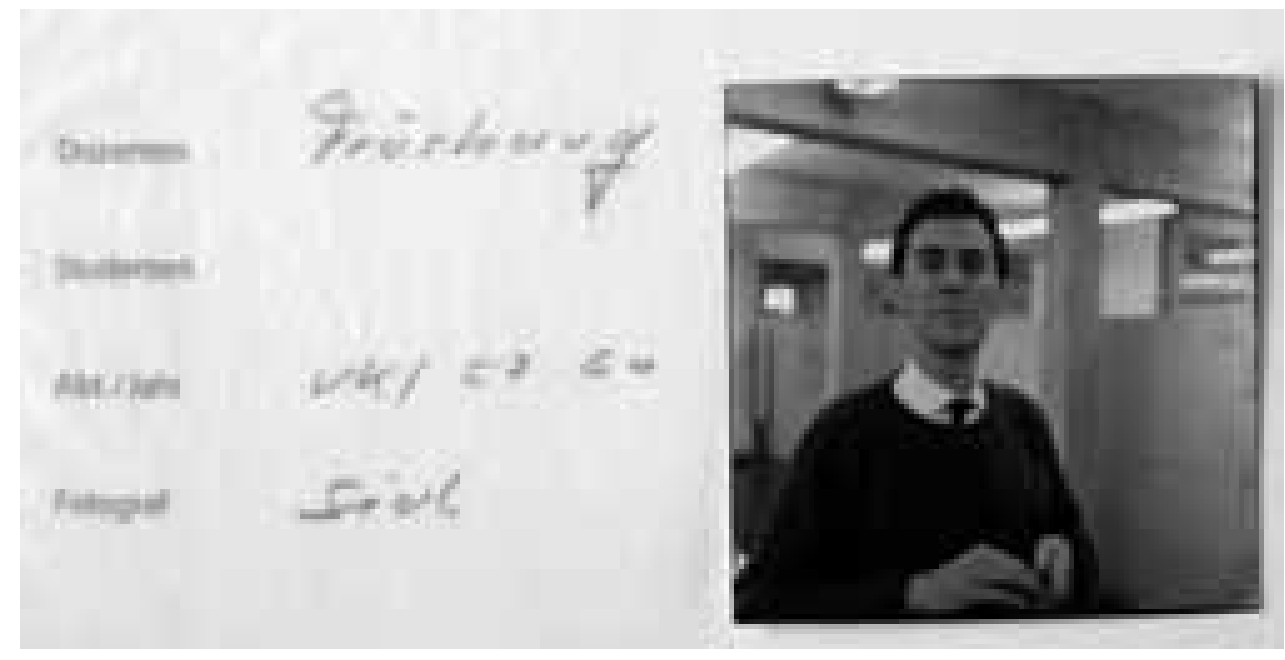

\subsubsection{Incorporación de Anthony Froshaug y el trabajo con grafos en Visuelle Methodik}

Anthony Froshaug proviene de Londres. Es tipógrafo y diseña exposiciones. Se había formado en la Central School of Arts and Crafts de Londres donde se encarga del departamento de tipografía en 1952-1953. Llega hasta Max Bill con una propuesta de publicación que no encuentra buena respuesta. ${ }^{132}$ Años más tarde, el 3 de agosto del año 1957 Maldonado viaja a Londres para entrevistarse con él y le propone un periodo de prueba de tres meses en el departamento de Comunicación Visual, de octubre a diciembre de 1957. Llega a Ulm el 1 de septiembre y se queda hasta 1960. En este periodo es el responsable del diseño de los números del 1 al 5 de la revista Ulm. De vuelta a Inglaterra, estudia arquitectura en la Architectural Association.

A su llegada a Ulm, Froshaug participa en el Curso Básico. Se refiere al bajo nivel de trabajo metodológico que encuentra en los alumnos del primer curso. Por este motivo, considera relevante la introducción de problemas de metodología visual que aporten una base mínima para el análisis del problemas con herramientas sistemáticas.

En la perspectiva general del Curso Básico, Anthony Froshaug tiene una importancia determinante, muy significativa y pocas veces reseñada en las publicaciones anteriores. Se puede afirmar que su llegada a la escuela representa un antes y un después en el Curso Básico y establece uno de los hitos de la didáctica del Grundlehre. Maldonado había incorporado la metodología como un aditamento de la práctica, una formación complementaria dentro de sus seminarios teóricos. Froshaug lleva la metodología a la práctica real y cotidiana en sus ejercicios de Visuelle Metodik. La inclusión de los grafos como tarea práctica anticipa las materias del Grafische Darstellungen y Methodische Übungen, en la que las herramientas analíticas forman parte indisoluble del proceso de resolución del ejercicio. i1/140 Anthony Froshaug en la HfG Fotógrafo: Siol F 58/0297
132 Froshaug había establecido contactos previos con Max Bill al igual que con Jan Tschichold, para publicar un libro sobre su obra En la monografía The Achitect's yearbook $n^{\circ} 5$, de 1953, Froshaug incluye el artículo "The Bauhaus idea. From Weimar to Ulm". A Max Bill le decepcionan algunos aspectos del diseño gráfico, como la sobrecubierta de la monografía, y rehusa a contar con él para la escuela en aquel primer momento.

En: Kinross, op. cit. pag 150 


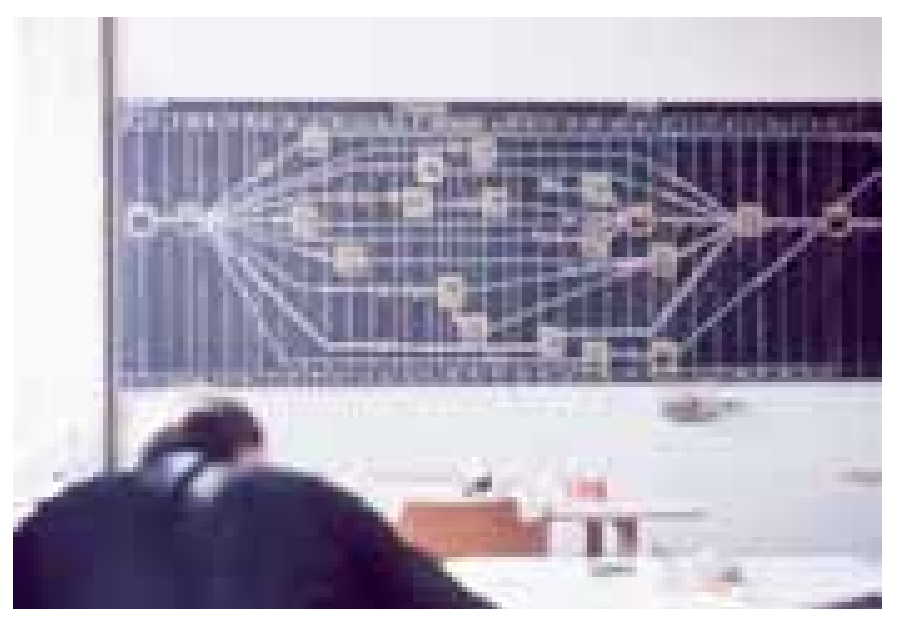

¡1/141 Metodología en la HfG

Fuente:

página de Facebook del HfG Archiv

i1/142, 143 Apuntes de metolología de las clases de Horst Rittel Alumno: Walter Eichenberger Dp 136.001

Fotografía: Sio

F 58.0310 F.B.S
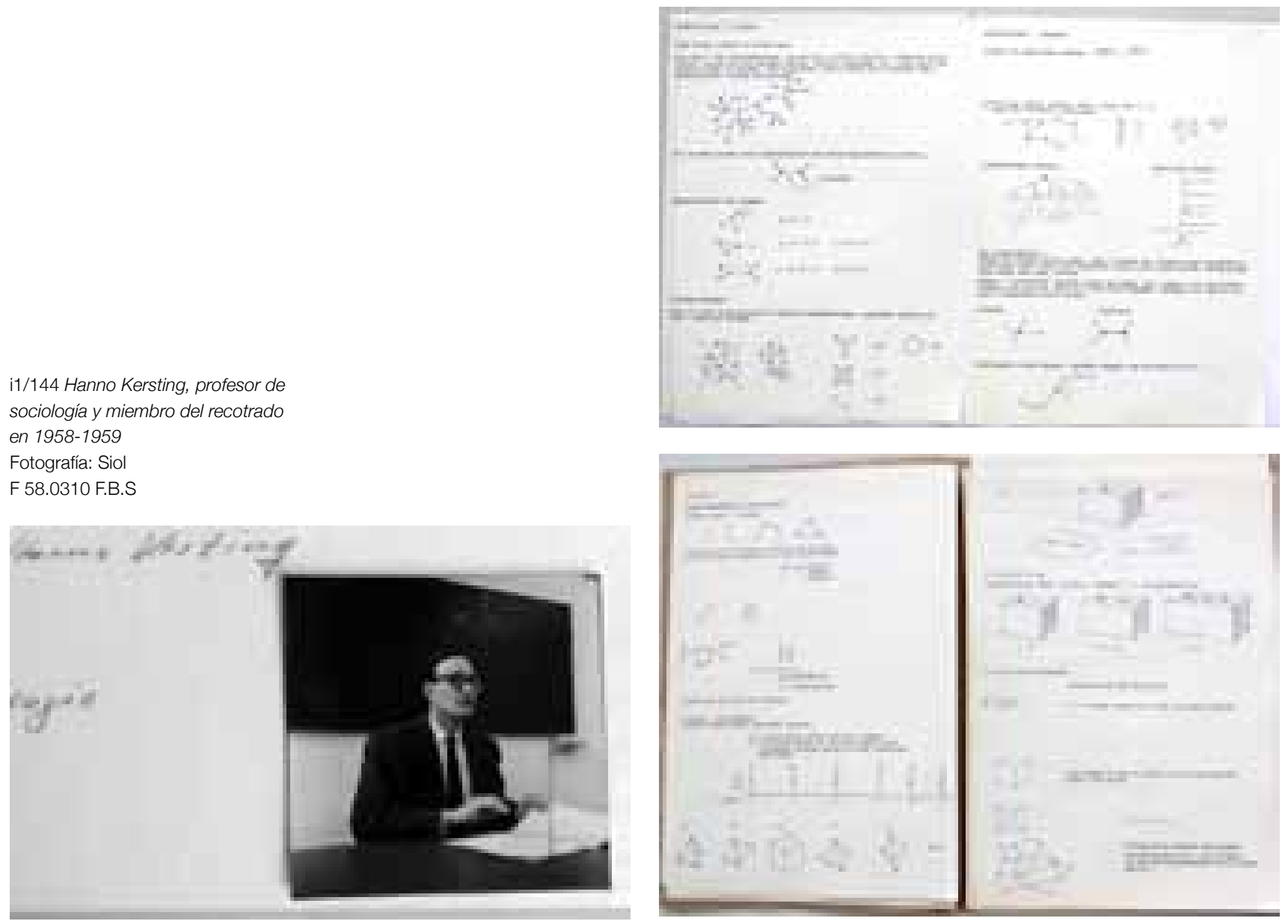

i1/145 Rittel, Kalow y Vordemberge Fuente: Spitz, René, op. cit pag 183

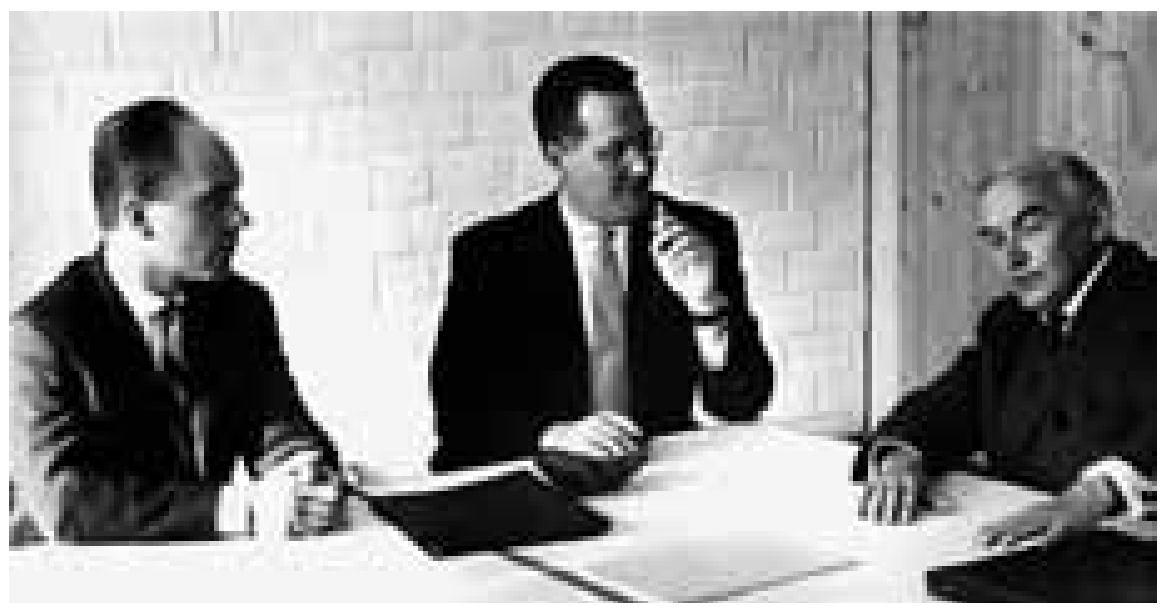




\subsection{Metodología en el Curso Básico}

\subsubsection{Contexto espacio-temporal de la etapa de la metodología}

Al finalizar el curso 1957-1958, Maldonado sigue impulsando su nueva formulación de la escuela a partir de los cambios científicos y tecnológicos de posguerra surgidos durante el conflicto con Max Bill y su marcha definitiva de la HfG. ${ }^{133}$ El 18 de septiembre de 1958, Maldonado ofrece una conferencia en el pabellón alemán de la Exposición Universal de Bruselas en la que sitúa al diseñador en un papel protagonista en la nueva "civilización industrial". ${ }^{134}$ Además de la capacidad inventiva, el diseñador necesitará unos métodos de pensamiento y de trabajo, conocimientos científicos y técnicos y una visión sociológica y cultural de su labor que le permita ser protagonista de esta nueva civilización.

En su informe al consejo ${ }^{135}$, Maldonado expone su creencia en un nuevo tipo de Formgeber que necesitará el planteamiento de los problemas y su análisis científico con herramientas metodológicas. Una de las herramientas que presenta Maldonado en este informe es el Análisis de Operaciones Matemáticas -MOA-, una disciplina desarrollada en EEUU que se sirve del método matemático para interpretar y analizar operaciones de cualquier tipo. La segunda asignatura sería teoría de la ciencia, que pretende sustituir la visión idealista del mundo por una de orientación científica. En estas nuevas materias se encuentra larvada una transformación del Curso Básico que toma en este periodo un rumbo nunca previsto por Maldonado.

El giro hacia la metodología iniciado en los seminarios de Maldonado y materializado por Froshaug, se consolida a lo largo de los cursos 1958-1959 y 1959-1960. En el primer curso de esta etapa, el Visuelle Methodik repite su planteamiento de análisis a partir de grafos e incorpora otras herramientas como los diagramas. Horst Rittel, que había llegado a la escuela en el año 1958, aumenta significativamente su presencia en el Curso Básico durante estos dos años y en ellos, despliega toda la enseñanza teórica de carácter científico y metodológico que alcanza sus cotas más elevadas hasta el momento en el Curso Básico. El Grundlehre conquista en estos dos cursos el polo opuesto en los planteamientos didácticos del inicio. Ha culminado su tránsito desde la intuición a la metodología.

Este periodo se inicia bajo un rectorado compartido por Aicher, Gugelot y Maldonado y finaliza con otro rectorado en el que ya no están Aicher y Maldonado, las figuras de peso en el grupo de los diseñadores de la escuela. El rectorado de 1960 está dirigido por Kalow, Rittel y Vordemberge-Gildewart. Gert Kalow es escritor y periodista, Rittel es físico teórico y matemático y Vordemberge-Gildewart es el único artista del grupo que además, no tiene participación como diseñador en ninguno de los grupos de desarrollo de la escuela. El cambio en la dirección de la escuela es sintomático de la nueva orientación científica de las enseñanzas y en particular, del Curso Básico. Horst Rittel dirige el departamento Grundlehre en el curso 1959-1960.

La revista Ulm nace en este periodo, los primeros dos números se editan en octubre de 1958 y su objetivo es la difusión de la actividad de la escuela. El diseño es de Anthony Froshaug y el editor es Hanno Kesting, un sociólogo que llega a la HfG en 1958. En los años siguientes, la revista se publica con más asiduidad. Se manifiesta una mayor voluntad de internacionalización con los viajes de Maldonado y Aicher a Brasilia y Rio de Janeiro en 1960.
133 Maldonado, Tomás. "Grundlehre" en: Petruschat, op. cit. pag 25

134 Maldonado, Tomás. "Los nuevos desarrollos en la industria y la formación del diseñador" en: Ulm 2, op. cit. p. 40.

135 Maldonado, Tomás. Bericht von Hern Tomás Maldonado in der Verwaltungsratsssitzung am 21 April 1958. (Informe de D. Tomás Maldonado en la reunión del consejo del 21 de abril de 1958). Discurso inédito. HfG Ulm, 1958 p. 4 
11/146 Propuesta de materias para

Grundlehre en el curso 1959/1960

Alumno: Walter Eichenberger Kal 9.03 F.B.S

$i 1 / 147,148,149$

Apuntes de clases teóricas: historia

cultural del siglo XX, fisiología, teoría

del color

Alumno: Walter Eichenberger

Dp 136.036 F.B.S
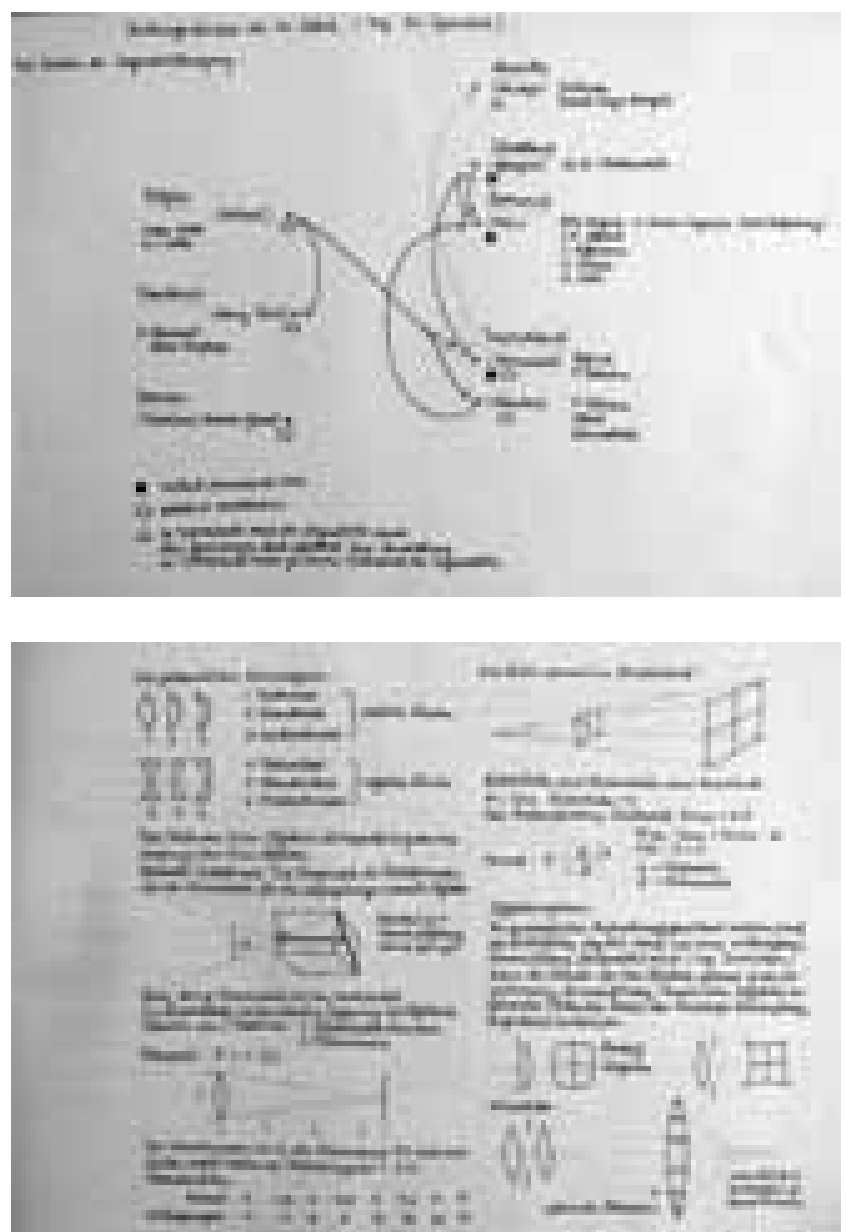
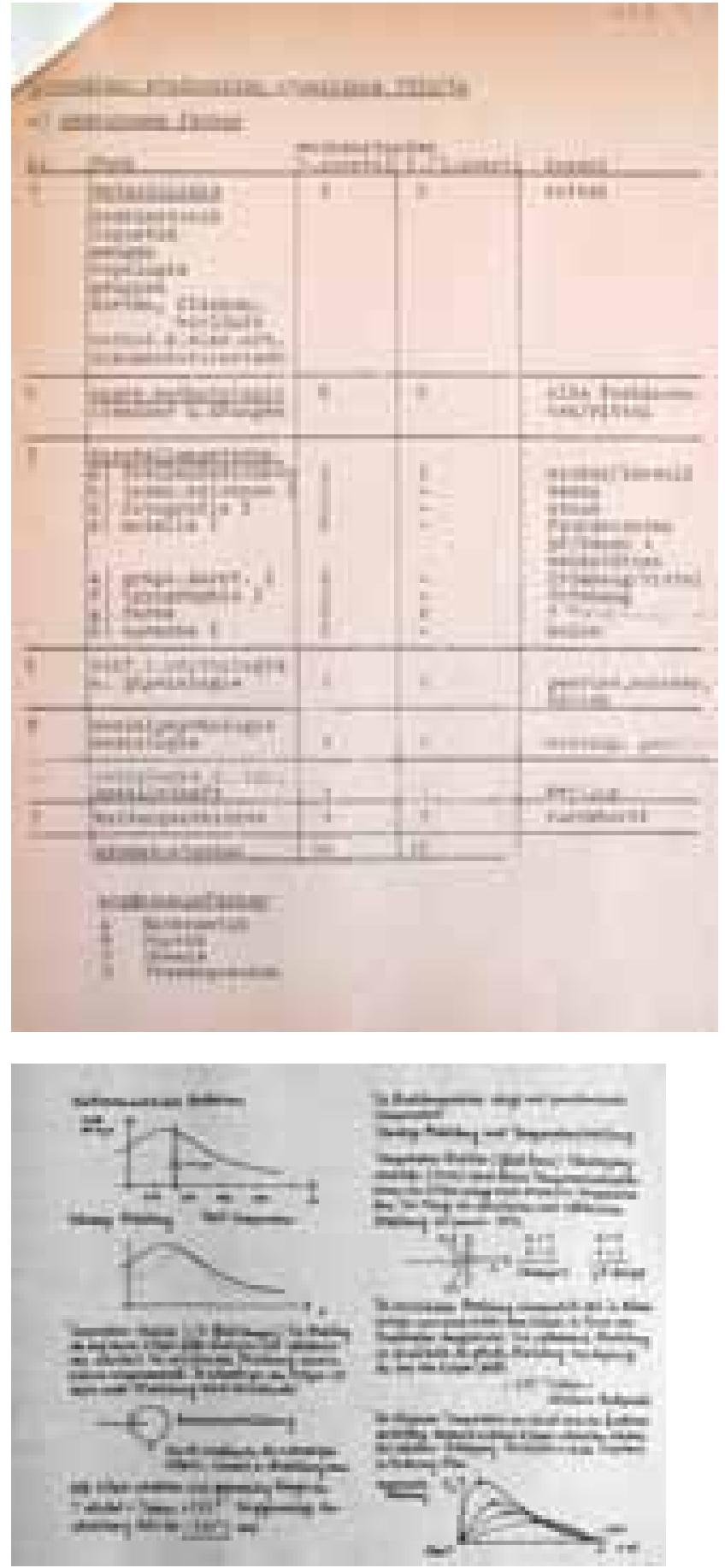


\subsubsection{Docentes, materias y alumnos del periodo}

\author{
Rectorado 1958-1959 \\ Otl Aicher \\ Hanno Kersting \\ Tomás Maldonado
}

\section{Departamentos 1958-1959}

Curso Básico - Tomás Maldonado

Producto - Georg Leowald, Walter Zeischegg, Hans Gugelot

Construcción- Herbert Ohl

Comunicación visual - Anthony Froshaug,

Christian Staub, Friedrich Vordemberge-

Gildewart

Información - Hanno Kersting

Asignaturas teóricas - Horst Rittel, Hans

Gugelot

Talleres - Otl Aicher, Paul Hindinger

\section{Docentes 1958-1959}

Otl Aicher

Anthony Froshaug

Hans Gugelot

Hanno Kersting

Georg Leowald

Tomás Maldonado

Herbert Ohl

Horst Rittel ${ }^{136}$

Christian Staub

Friedrich Vordemberge-Gildewart

Walter Zeischegg

\section{Maestros de taller 1958-1959}

Paul Hindinger - Madera

Otto Schild - Yeso

Josef Schlecker - Metal

Wolfgang Siol - Fotografía

Cornelius Uittenhout - Plástico
Docentes invitados 1958-1959

Josef Albers - seminario de color del C. Básico

Herman Von Baravalle - Visuelle Einführung

Max Bense - Dep. Información

Gerhard Eichhorn

Erich Franzen - Integración Cultural

Etienne Grandjean - Fisiología

Hermann Haan - Dep. de Construcción Ind.

Siegfried Haenle - Dibujo técnico

Kate Hamburger - Departamento de Información

Bruno Hrosberger - Fisiología

Gert Kalow - Departamento de Información

Bruce Martin - Departamento de Construcción Ind. Helenne Nonné-Schmidt - Curso Básico

Herbert Ohl - Departamento de Construcción Ind.

Dieter Oestreich - Departamento de Producto

Giulio Pizzeti - Estructuras.

Fritz Querengässer - Dep. Comunicación Visual

Thomas Rago - Fotografía

Horst Rittel - Análisis de operaciones

Bernhard Rübenach - Radiodifusión

Jospeh Rykwert - Dep. de Construcción Ind.

Herbert Schober - Fisiología

Johannes Schön - Construcción técnica

Christian Norberg - Schulz

Hans-Günter Sperlich - Historia Cultural

Matthew Wallis - Sistemas constructivos

Martin Walser. Elisabeth Walter. -

Dep. de Información.
136 Rittel se incorpora al cuerpo fijo de docentes de la escuela en el curso 1959-1960.

\section{Materias 1958-1959}

\section{Visuelle Methodik}

Tomás Maldonado se hace cargo de media parte de curso en la que continúa desarrollando su repertorio con una mayor depuración en sus resultados. Los trabajos de este curso son: proximidad-ambigüedad-semejanza, fondo-plano medio-primer plano, negro como color, simetría, precisión e imprecisión, tareas de raster, estructuras y campos de color en equilibrio. Se simplifica la ejecución de algunos ejercicios, como el caso del negro como color, que no aparece explícitamente vinculado a la curva de Peano.

La segunda parte está a cargo de Anthony Froshaug. También continúa el esquema llevado a cabo en el curso anterior, en el que se ejercita el análisis visual metodológico de redes viarias, redes de transportes públicos y las conexiones entre los cantones suizos. Se parte de una matriz, se halla el número de coincidencias entre los nodos y se define una retícula geométrica de base para el grafo que se ajuste al número máximo de coincidencias. 
Aparecen tareas en los que se emplean los diagramas que anticipan las Grafische Darstellung del curso posterior.

\section{Darstellungstechniken}

Gert Kalow imparte lengua, el dibujo técnico corre a cargo de Bauer y Aicher imparte una asignatura de dibujo libre y escritura. Froshaug tiene una asignatura de tipografía en este bloque. Los talleres de metal, madera y yeso se concentran en el primer trimestre; el taller de fotografía se realiza a lo largo de todo el curso.

\section{Naturwissenschaften Fächer}

Eichhorn es el profesor que imparte matemáticas durante los tres trimestres.

\section{Allgemeinbildende und Theoretische Fächer -}

Kulturgeschichte corre a cargo de G. Sperlich y Vordemberge. Kersting continúa en la materia sociología. Las dos novedades de este bloque de asignaturas está en la aparición de Rittel como profesor de MOA y metodología. Se estudia lógica matemática, combinatoria, permutaciones y topología. El plan de estudios de este año incluye una asignatura de fisiología y ergonomía a cargo de Schober. En psicología se enseña la teoría de la percepción.

\section{Alumnos 1958-1959}

Dagmar Arnold Otto Baumier

Gerhard Beigel *producto Karl Berthold *arquitectura Margarete Bienert

Reinhard Buter *producto

Susanne Eppinger *producto

Jüksel Eronat

Jürgen Freuer

Leonh. Fünfschilling *arquitectura

Wiktor Guirard *comunicación visual

Peter Heck

Marcel Herbert *arquitectura

Shinje Iwasaki

Moshe Kohnen *producto

Eberhard Köster

Gerda Krauspe *producto

Herbert Lee

Peter Lindinger

John-William Lottes

Hubert Matecki

\author{
Gian Luca Meda \\ Walter Müller *comunicación visual \\ Hans J. Nussbickel \\ Gudrun Otto *producto \\ Klaus Pfromm *arquitectura \\ Willi Ramstein *arquitectura \\ Bill Reavis \\ Gotho Rehfus \\ Rudolf Saas \\ René Sauter \\ Anneliese Schaefer \\ Kurt Schilicht \\ Leonhard Schmude \\ Hans-Jacob Schneider \\ Peter Schoeller *producto \\ Goran Wärff \\ Gernot Walsdorff \\ Alfred Weidmann \\ Oskar Wehling \\ Winfried Wurm *arquitectura
}


Rectorado 1959-1960

Tomás Maldonado

Herbert Ohl

Horst Rittel

\section{Departamentos 1959-1960}

Grundlehre - Horst Rittel

Produktform - Hans Gugelot, Walter Zeischegg

Bauen- Herbert Ohl

Comunicación visual - Friedrich Vordemberge-

Gildewart, Otl Aicher

Información - Hanno Kersting, Gert Kalow

Teoretische Fächer - Horst Rittel

\section{Docentes 1959-1960}

Otl Aicher

Anthony Froshaug

Hans Gugelot

Gert Kalow

Hanno Kersting

Georg Leowald

Tomás Maldonado

Herbert Ohl

Horst Rittel

Christian Staub

Friedrich Vordemberge-Gildewart

Walter Zeischegg

\section{Maestros de taller 1959-1960}

Paul Hindinger - Madera

Otto Schild - Yeso

Josef Schlecker - Metal

Wolfgang Siol - Fotografía

Cornelius Uittenhout - Plástico

\author{
desde abril 1960 \\ Gert Kalow \\ Horst Rittel \\ Friedrich Vordemberge-Gildewart
}

\section{Docentes invitados 1959-1960}

W. De Acosta - Construcción e instalaciones Harry P. Bahrick - Ingeniería humanística Herman Von Baravalle - Visuelle Einführung

Giuseppe Ciribini - Dep. Construcción Ind.

Gerhard Dressel - Análisis y forma de la obra

Siegfried Haenle - Ciencia de Materiales

Ewald Kapal - Fisiología Aplicada

Joachim Kaiser - Historia de la Literatura Moderna

Rudolf Knoll - Fabricación

Michael Leonard - Dep. Construcción Industrializada

Frei Otto - Dep. Construcción Industrializada

Eberhard Lusser - Estructuras

Mervyn W. Perrine - Teoría de la percepción

Giulio Pizzeti - Estructura

Hermann Reiher - Física Tec. para la construcción

Bernhard Rübenach - Radiodifusión

Claude Schnaidt - Historia y crítica de la Arq.

Herbert Schober - Fisiología

Johannes Schön - Construcción técnica

Christian Schutze - Comunicación de empresa

Hans Gunther Sperlich - Historia cultural

Fiedrich Tonne - Física Tec. para la construcción

Herbert v. Voss. Patentrecht - Construcción

Matthew Wallis - Metodología para la construcción

Z. Wasowsky - Técnica de cerramientos

Konrad Weller techn - Sistemas constructivos
137 Acta de la reunión pedagógica del 10 de noviembre de 1959.

II. Hochschule für Gestaltung

4 Scheilleitung

4.7 Pädagogische Konferenzen carpeta 150

Bauhaus Archiv.

\section{Materias 1959-1960}

El curso 1959-1960 experimenta un cambio significativo comparable al llevado a cabo por Maldonado en el año 1955-1956. Se puede afirmar que este curso alberga la segunda gran reforma del Curso Básico. Si Maldonado incorporó la ciencia, Horst Rittel introduce la metodología y el proyecto al Grundlehre.

Horst Rittel es el responsable del departamento Grundlehre en este periodo. Diseña un curriculum con orientación científica y una nueva organización de los profesores. En el Curso Básico se lleva a cabo una coordinación real de las materias teóricas y las prácticas con reuniones pedagógicas en las que se ponían en común los temas de proyecto y el contenido de las asignaturas teóricas. El horario se establece de acuerdo a estas reuniones. ${ }^{137}$

Los profesores de los departamentos de especialización se incorporan al primer curso de estudios para proponer ejercicios vinculados directamente a las tareas de sus departa- 
mentos: diseño industrial, comunicación visual, y construcción industrializada, tanto en las Methodische Übungen como en las Grafische Darstellung.

Desaparece el bloque de Visuelle Methodik como conjunto de disciplinas visuales. La función de la enseñanza y las materias se organizan en cuatro conjuntos de disciplinas: las Methodische Übungen o proyectos metodológicos, las Grafische Darstellungen, o representaciones gráficas, el Farbenlehre y las asignaturas teóricas.

La enseñanza del color es llevada a cabo por Rittel en coordinación con Vordemberge-Gildewart y Froshaug. El artista y el tipógrafo dirigen trabajos prácticos con el soporte teórico de Rittel, que explica la física, química, psicología y fisiología del color. Fomenta el empleo de herramientas matemáticas en el uso del color como la combinatoria, la probabilidad y las progresiones aritméticas en las escalas.

En las materias teóricas, se puede distinguir entre los contenidos apoyan los aspectos prácticos y las materias que tienen entidad en sí mismas y que forman la base general de los alumnos. En el primer grupo se encuentran las materias de Física Técnica, FisiologíaErgonomía, Psicología y Metodología. En el segundo, se sigue enseñando Kulturgeschichte que mantiene sus contenidos del principio, Sociología y Política, que aparece por primera vez en el curriculum.

El objetivo y contenido de las Methodische Übungen y las Grafische Darstellungen se explican en epígrafes a continuación.

\section{Alumnos 1959-1960}

Karlheinz Allgayer *arquitectura

Richard Bairseow

Peter Beck *producto

Jan Wilke Beenker

Gerhard Curdes *arquitectura

Rudi Dahlmann *arquitectura

Erich Dardel

Reinhold Deckelmann

Walter Eichenberger

Peter Paul Emmer *producto

Annemarie Fahr

Herbert Falk

Erika Fortner

Bernd-Gunter Franck *comunicación visual

Ursula Göritz

Robert Graeff *producto

Renate Grünwald *arquitectura

Dieter Hagermann

Ernst Haim

Robert Hohnstock

Alfred Jungreithmayr *comunicación visual

Walter Kielnecker *producto

Hans T. Kurtenbach

Hans Leinenbach *arquitectura

Gerhard Mayer

Larry Monk

\author{
Ernest Muchenberger *arquitectura \\ Herbert Oehm \\ Allison Peters \\ Alfons Poss \\ Cornelius Reeves *arquitectura \\ Dieter Reich *producto \\ Ulrich Rothfuss *producto \\ Erich Rufer \\ Hubert Ruiters *comunicación visual \\ Hermann Schäfer \\ Hannelore Schneidre \\ Ute von Seydlitz \\ Susanna Stich \\ Egon Tempel *arquitectura \\ Carlo Testa *arquitectura \\ Hajo Tochtermann *producto \\ Heinz Wäger *producto \\ Erdmann Wingert
}




\subsubsection{La llegada de Rittel y la introducción de la metodología ${ }^{138}$}

Horst Rittel ${ }^{139}$ llega como profesor invitado a la escuela con veintiocho años, recién licenciado en la universidad de Göttingen en Matemáticas puras y Física teórica. Del cuerpo docente de la HfG, Rittel es el único verdadero hombre de ciencia -tras la marcha de Max Bense-, y por eso se le confían todas aquellas asignaturas, tanto en el Grundkurs como en los departamentos, que tuvieran que ver con metodología, matemáticas o física. El objetivo de estas disciplinas era el de proporcionar un control del proceso de proyecto, una actividad que Rittel definió como acción planificadora.

Un recién llegado Rittel adquiere una insólita influencia que le lleva a integrar una nueva junta de gobierno junto a Tomás Maldonado y Herbert Ohl a final del curso 1958-1959. En la conferencia pedagógica final de este mismo curso, Rittel impone, apoyado por la mayoría del claustro, una nueva configuración del Curso Básico en la que la metodología adquiere un peso definitivo. Maldonado vio frustrada su voluntad de continuar con su configuración ya establecida. Las lecciones de Rittel habían sido un mero apoyo teórico a su curso de gramática visual, y a partir de ahora se erigían como la subestructura que daría soporte a la propedéutica del proyecto. Como consecuencia, Maldonado se refugia en el Departamento de Comunicación Visual y no vuelve a intervenir en el Curso Básico.

El Curso Básico de 1959-1960 está dominado por una omnipresencia sorprendente de Rittel en muchas de las materian que se imparten, bien en solitario o bien en colaboración con otros docentes.

Las asignaturas que imparte como profesor responsable de materia en el curso 1959-1960 son metodología -que incluye topología general, superficies topológicas, teoría de conjuntos, cálculo, lógica, teoría de grafos, matrices, simetría, retículas raster, superficies curvas, análisis operativo matemático, probabilidad y combinatoria-, MOA, teoría de la ciencia, cibernética y teoría de la información. El acrónimo MOA, como se ha mencionado, denomina una disciplina importada de Estados Unidos centrada en el análisis operativo matemático. En ella se enseña teoría de trabajo, procesos de comunicación, estudio de viabilidad, teoría de la ciencia, cibernética y teoría de la información. En la materia "teoría de la ciencia", explica las fases del proceso de trabajo aplicado a la disciplina de diseño.

También se hace cargo de una Methodische Übungen -máquina de votación, siendo el único docente no vinculado a ninguna rama del diseño que dirige un proyecto.

En colaboración con otros profesores, Rittel participa en la enseñanza del color, en la mitad de las Grafische Darstellung junto a Froshaug y en la mayoría de las Methodische Übungen que dirige también Froshaug.

El paso del joven físico por la HfG es breve. Abandona la escuela en 1963 y continúa su labor docente en el College of Enviromental Design de Berkeley. Junto con Christopher Alexander, Bruce Archer y John Christopher Jones contribuye a la fundación del movimiento "Design Methods Movement" ${ }^{140}$, que significa un cambio de paradigma en el proyecto y en la enseñanza del diseño, con un alta repercusión mundial. El Grundlehre de la HfG en sus últimos años fue un antecedente del surgimiento de la metodología en el diseño que estaba por llegar.

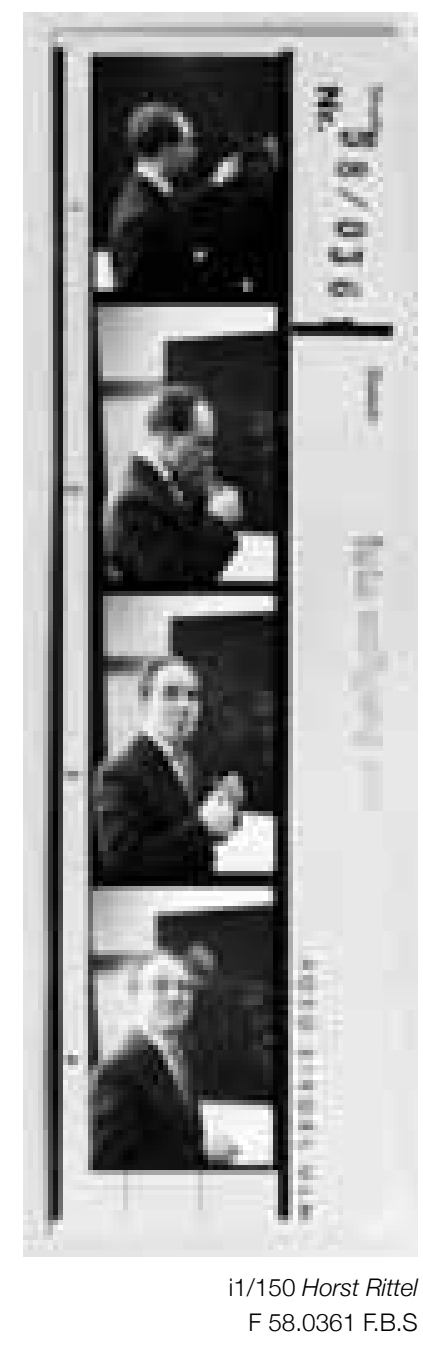

138 Parte del epígrafe es un extracto publicado anteriormente en: Fernández-Campos, Angel Luis; Sánchez Moya, María Dolores op. cit, 392-411.

139 "Horst Rittel enseñó un amplio rango de materias, incluyendo clases sobre metodología en todos los departamentos, MOA y en 1960-1961 matemáticas, física y física técnica. En el departamento de diseño industrial enseñó mecánica general, en el departamento de comunicación visual, teoría de la información, en el departamento de Arquitectura, metodología, teoría de estructuras, introducción a la matemática lógica, permutaciones y combinaciones, topología, teoría de la ciencia, y cibernética". REUTER, Wolf. ".. den Dualismus zwischen ratonaler und intuitiver Tätigkeit auflösen" Horst Rittel an der HfG UIm (“...disolviendo el dualismo entre la actividad intuitiva y racional". Horst Rittel en la HfG UIm. En Mantele, Martin. "Magos de la teoría" en: W.AA. Modelos de Ulm, op. cit. p. 94

140 Rith, Chanpory; Dubberly, Hugh. "Why Horst W.J. Rittel Matters". En: Design Issues, volumen 22, $\mathrm{n}^{\circ} 4$, otoño 2006. pp. 1-3 


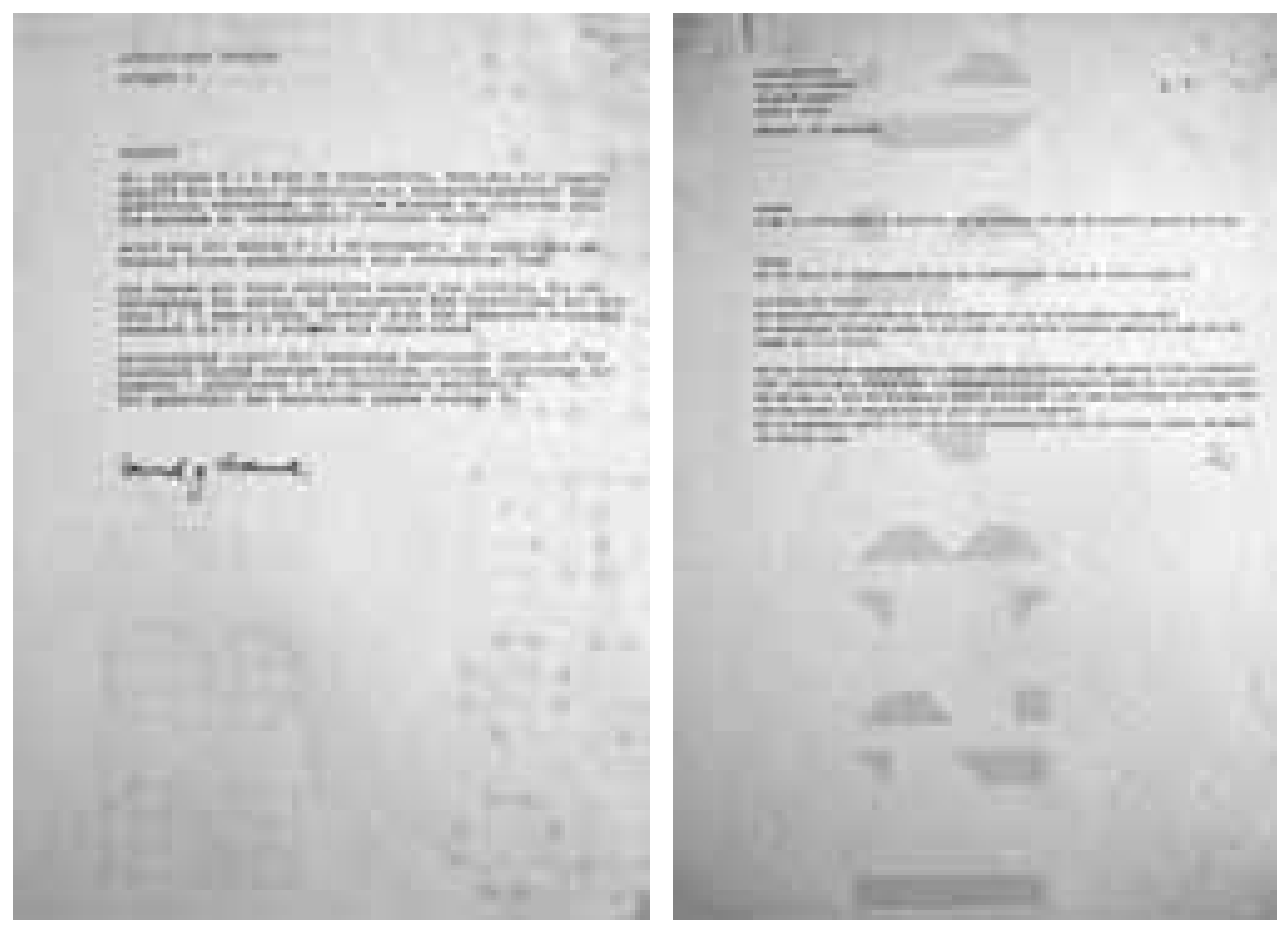

i1/151, 152, 153, 154, 155, 156

Enunciados y memorias de las

Methodische Übungen 1, 2, 3 y 4

Varios alumnos

Dp 136.036 F.B.S
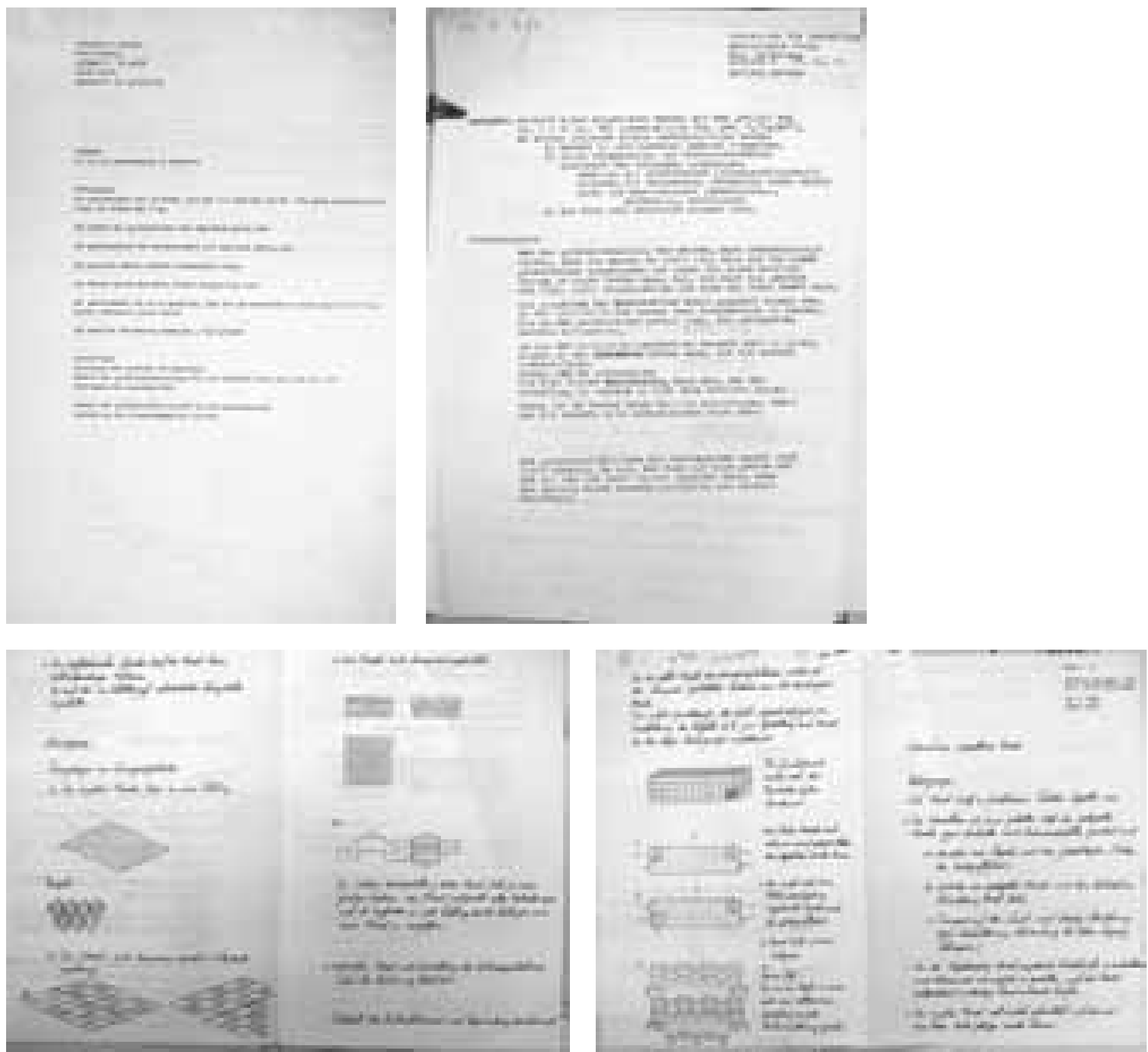


\subsubsection{Proyectos concretos: el abandono de la abstracción en las Methodische Übungen}

Las Methodische Übungen encarnan mejor que ningún otro ejercicio, la acción planificadora que enunciaba Rittel. A lo largo del 1959-1960, los alumnos del Grundkurs desarrollan dieciséis pequeños proyectos dirigidos por seis profesores procedentes de los departamentos: comunicación visual -Fröshaug-, diseño industrial -Gugelot y Zeischegg-y construcción -Herbert Ohl-. Como se ha mencionado, los profesores se alternaban en las enseñanzas y para planificar y coordinar esta secuenciación, celebraban reuniones pedagógicas.

Todos los ejercicios, de corta duración, comenzaban con un enunciado propuesto por el profesor. Dependiendo del docente, las instrucciones son más o menos concretas y acotan de forma diferente el campo de trabajo. De forma general, los alumnos debían registrar las fases en el desarrollo de las soluciones en un informe final sobre todo el trabajo, que debían entregar junto con la propuesta gráfica y las maquetas.

La exactitud en la ejecución gráfica queda en un segundo plano frente a la capacidad para ofrecer una respuesta que responda a múltiples variables. El virtuosismo en la ejecución de los dibujos que tanto había caracterizado el trabajo en el Visuelle Einführung queda desplazado.

Cada una de las dieciséis Methodische Übungen entraña una singularidad específica del área proyectual al que pertenece. Las que dirige Zeischegg se formulan desde el ámbito del diseño industrial. Fröshaug propone proyectos desde el campo de la comunicación visual, la mayoría en coordinación con Rittel. Herbert Ohl, del departamento de arquitectura, dirige un ejercicio denominado "estimación del espacio determinado mediante la organización de unidades de equipamiento". El departamento de información no proponía el desarrollo de ningún ejercicio.

Los proyectos que se desarrollaban en las Methodische Übungen partían de unas condiciones rigurosas con un margen de trabajo delimitado. Este es el caso del ejercicio núm.12 de Hans Gugelot. Los alumnos debían afrontar un problema constructivo muy acotado: diseñar el bastidor de soporte para una esfera de $10 \mathrm{~cm}$. de diámetro, manipulando una lámina cuadrada de hojalata de $20 \mathrm{~cm}$. de lado y $3 \mathrm{~mm}$. de espesor. A pesar de lo específico del enunciado, estudiantes desarrollan propuestas muy diferentes entre sí.

\section{Listado de las Methodische Übungen}

1 FROSHAUG: Zahlen - Leuchtschrift - Números de neón

2 FROSHAUG: Konstruktion von Ziffern. Konstruktion von mensch und maschinenlesbaren Ziffern - Construcción de dígitos. Construcción de dígitos legibles por humanos y máquinas.

3 FROSHAUG: Entwurf fur einen Gewichtstaz - Diseño para un juego de pesas
141 Froshaug abraza con entusiasmo esta nueva formulación del Curso Básico, en el que, al fin, las enseñanzas generan una urdimbre de conocimientos al servicio del proyecto: "El cambio fue saludable: por primera vez, las diferentes tareas estaban coordinadas entre sí y con el trabajo en los seminarios. Para aquellos problemas como el diseño del indicador de llegadas de una estación de tren con dígitos legibles por humanos y máquinas, tuve la posibilidad de referirme a las lecciones de Perrine en percepción, a las lecciones de Rittel de combinatoria, a varias consideraciones de requisitos económicos basadas en las conferencias de sociología de Kesting, y también fue útil saber qué tarea sucedería a la mía".

Kinross, Robin, op. cit, p. 144 
4 ZEISCHEGG: Aluminium Massel - Lingotes de aluminio

5 LEOWALD: Versandkartons - embalaje para envíos de productos farmacéuticos

6 GUGELOT: Verpackung für Diarahmen - Embalaje para diapositivas

7 ZEISCHEGG: Stapelbarer Würfel - Cubos apilables

$8 \mathrm{OHL}:$ Raumbestimmung durch Organisation der funktionellen Einrichtungseinheiten Espacio determinado por la organización de las unidades de dispositivos funcionales.

9 RITTEL: Entwurf einer Abstimmungsmaschine - Diseño de máquina de votación

10 LEOWALD: Polyeder. Tetraeder, Oktaeder, Dopppelsechseckpyramide - Poliedro. Tetraedro, octaedro, Pirámide hexagonal doble. Diseño de estructuras reticulares

11 ZEISCHEGG: Rotationskörper - Durchdringungskörper - Cuerpo de rotación - Cuerpo de penetración. Cuerpo generado por la intersección de varios cuerpos de rotación (cilindros)

12 GUGELOT: Tragegestell für eine Kugel - Patas para una esfera

13 FROSHAUG: Satzspiegel - Formato de composición para revista

14 FROSHAUG: Darstellung der kommunikations-verhältnisse innerhalb der hfg - ilustración de las relaciones de comunicación dentro de la HfG

15 ZEISCHEGG: Sperr-Rad - Rueda de trinquete

16 GUGELOT: Rachenlehre - Calibre de herradura o de mordazas 


\subsubsection{Pensamiento metodológico en las Grafische Darstellung}

Las Grafische Darstellungen -representaciones gráficas- se imparten como un método analítico y de conceptualización visual, mediante el desarrollo de diagramas. Los alumnos adquieren la capacidad analizar y sintetizar la información para la representación gráfica de la información asociada a un problema. La mayoría de los enunciados son propuestos por el tándem Froshaug-Rittel, salvo un ejercicio desarrollado por Aicher y otros dos por Schnaidt.

La representación de la realidad a través de pictogramas estaba experimentando un notable desarrollo en aquellos años. Otto Neurath es el precursor de la creación de un lenguaje, el Isotype -International System of Typographic Picture Education- que tuvo una gran difusión gracias a sus libros y su muestra en el Museo Social de Viena durante los años 20 y $30 .{ }^{142}$ En muchas otras partes del mundo se trató de seguir su ejemplo poniendo en marcha un lenguaje universal de códigos para traducir hechos de la realidad compleja en diagramas comprensibles por todo el mundo. El mundo de la publicidad y la televisión, en plena expansión en los años 50 y 60, fue otro de los impulsores de un lenguaje visual para la transmisión directa y clara de ideas.

El trabajo con diagramas en la HfG responde a ese interés normativo de búsqueda de un lenguaje común y objetivo. Entre los contenidos de la materia MOA se dedica un tema a la teoría del trabajo. En el análisis de los procesos de trabajo se estudian las herramientas de representación de los flujos de operaciones que constituyen una referencia para las Graphische Darstellung. En las tareas propuestas en la HfG se advierte, por encima de todas las cualidades de los pictogramas y diagramas, su utilización para el análisis de datos y para la representación de procesos. Un ejemplo de ello es el séptimo ejercicio, propuesto por Claude Schnaidt, que se centra en la representación de las secuencias de movimiento de la rutina diaria de un ama de casa en el interior de su vivienda. Los diagramas que elaboran los estudiantes tienen que contener los cambios de habitación, su frecuencia y la longitud de los caminos recorridos entre las diferentes estancias. Su potencial como herramienta de proyecto se intuye en el enunciado propuesto por Aicher, la representación gráfica no solo identifica los puntos de conflicto a resolver, sino también expone las posibles soluciones al problema planteado.

\section{Listado de las Grafische Darstellungen}

1. FROSHAUG \& RITTEL: Darstellung des Galton-Bretts bis 12-Reihe - Representación de un Tablero de Galton de 12 filas.

2. FROSHAUG \& RITTEL: Auswertung und Darstellung einer Messreihe - Análisis y visualización de una serie de medidas.

3. FROSHAUG \& RITTEL: Tick-Tack-Toe-Spiel - Grafische Darstellung aller möglichen Partien, Spielregeln, Untersuchung der Gewinnchancen - Juego de las tres en raya - Representación gráfica de todos los juegos posibles, reglas de juego, investigación de las probabilidades.
142 Ver Modley, Rudolf; Lowenstein, Dyno. Pictographs and graphs. How to make and use them. New York: Harper \& Brothers publishers, 1952, p. 6. 
143 En los trabajos recogidos en el HfG archiv no se ha localizado ninguna tarea con el número 6.

i1/157, 158

Graphische Darstellung 5 Alumna: Erika Fortner

Curso: Graphische Darstellungen

Aicher 1959-1960

Gs 061.007 F.B.S
4. FROSHAUG \& RITTEL: Klassifikation der Verkehrsmittel - Clasificación de los medios de Transporte.

5. AICHER: Untersuchung von Straßenkreuzung - Investigación de los cruces de carreteras.

7. SCHNAIDT:143 Darstellung von Bewegungsabläufen - Tageslauf einer hausfrau in ihrer Wohnung - Representación de los movimientos - rutina diaria de un ama de casa en su vivienda.

8. SCHNAIDT: Verteilung der erwerbstätigen Bevölkerung - Distribución de la población económicamente activa.
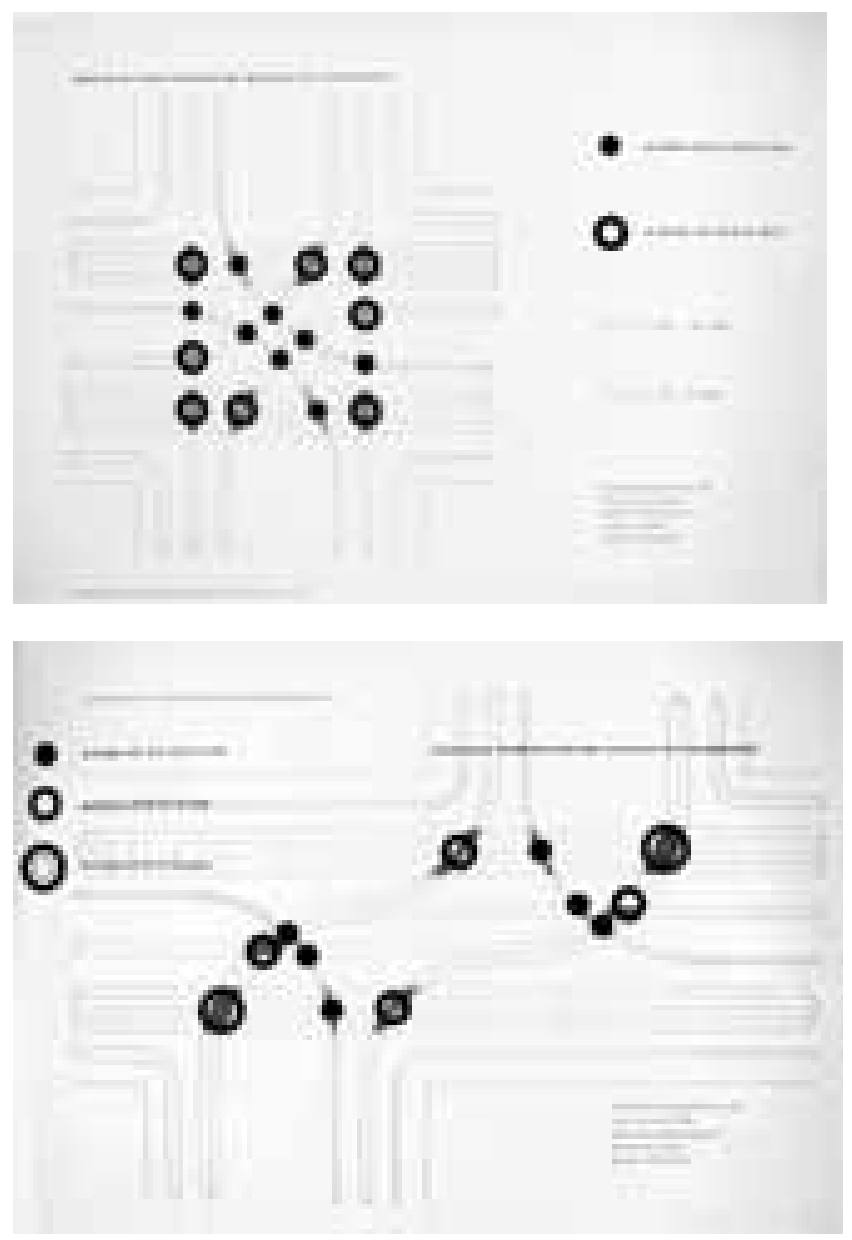


\subsection{La disolución del Curso Básico en el curriculum de los departamentos}

\subsubsection{Contexto espacio-temporal del fin del Grundlehre}

El desmembramiento del Grundlehre se inicia en el curso 1959-1960, comenzando por la transformación de su estructura. Una vez desvanecida su identidad, en el curso siguiente, pierde el nombre, pasándose a llamar 1.Studienjahre - primer año de estudios -. El curso 1960-1961 ve pasar a los últimos estudiantes que comparten un primer año conjunto. El siguiente, 1961-1962, por primera vez, los alumnos de primer curso ingresan en un departamento especializado. Desaparece el Grundlehre como departamento y como idea.

En septiembre de 1959, todos los estudiantes cursan las mismas asignaturas, como era habitual. Durante el primer cuatrimestre tuvieron lugar numerosas reuniones de coordinación donde se debatía la organización y mayor especialización del plan de estudios.

El cambio en cuestión tiene una larga prehistoria. No se pueden dar fechas exactas. Hasta donde puedo recordar, fue en 1956 o 1957 que se alzó la voz para incrementar una instrucción especializada en el seno de los departamentos. Fue esta activación de la formación especializada el tema principal en el debate sobre el Curso Básico. ${ }^{144}$

A partir de enero de 1960 los estudiantes se dividen en dos sectores: el sector "P" orientado a los departamentos de Diseño Industrial y Construcción y el sector "K" dirigido a la Comunicación Visual y la Información.

El plan de estudios mantiene un núcleo principal compuesto por las Methodische Übungen, las Graphische Darstellungen, la metodología y la Historia cultural del siglo XX. Al margen de estas materias comunes, la docencia se especializa por sectores y sirve específicamente a los intereses de cada grupo. Mientras que los alumnos del sector "P" reciben clases de dibujo técnico y física técnica, los del sector "K" aprenden fotografía y tipografía -ver horario en la página anterior-. ${ }^{145}$

Al finalizar el curso 1959-1960 en la conferencia pedagógica del 5 de Julio, Rittel informa de la evolución experimentada a lo largo de ese año que servirá como base para el debate y la organización del curso siguiente.

En este periodo, los profesores consideraban que la enseñanza en los departamentos era demasiado compleja y específica como para poder ser asimilada durante el segundo y tercer curso, antes de la ejecución del Diploma. Defendieron la necesidad de anticipar la preparación técnica, especialmente en las disciplinas que lo requerían. También argumentaron la imposibilidad de compatibilizar la enseñanza en el Grundlehere, que era un curso muy concurrido, con la enseñanza en el propio departamento y la dedicación a los grupos de desarrollo.

El curso 1960-1961 repite la organización ensayada en el año anterior: primer cuatrimestre conjunto; segundo con distinción de asignaturas entre los sectores "P" y "K" y en el tercer cuatrimestre aparece de nuevo la denominación de los departamentos a los que están orientados los sectores.
Entrevista a Hans Gugelot en la revista Output 6/7, 1961, pag. 18

144 Los representantes de los estudiantes que participan en la Conferencia Pedagógica manifiestan su satisfacción con algunos de los cambios experimentados aquel curso, en concreto, con el hecho de trabajar con proyectos pautados y la elaboración de memorias que justifiquen las tomas de decisiones. Sin embargo, acusan una falta de coordinación, de orientación en algunas tareas y la carencia de objetivos pedagógicos de algunos enunciados. Los estudiantes se pronuncian en contra de la separación entre sectores, por considerar que los estudiantes dirigidos a Diseño de Producto y Construcción industrializada reciben un mayor número de enunciados orientados a sus intereses.

145 Quijano, Marcela; Rinker, Dagmar HfG Ulm. Hochschule für Gestaltung Ulm 1953-1968 - hfg ulm. Ulm School of Design 1953-1968. Catálogo de la exposición permanente. Ulm: Ulmer Museum/HfG Archiv, 2007.p. 44 


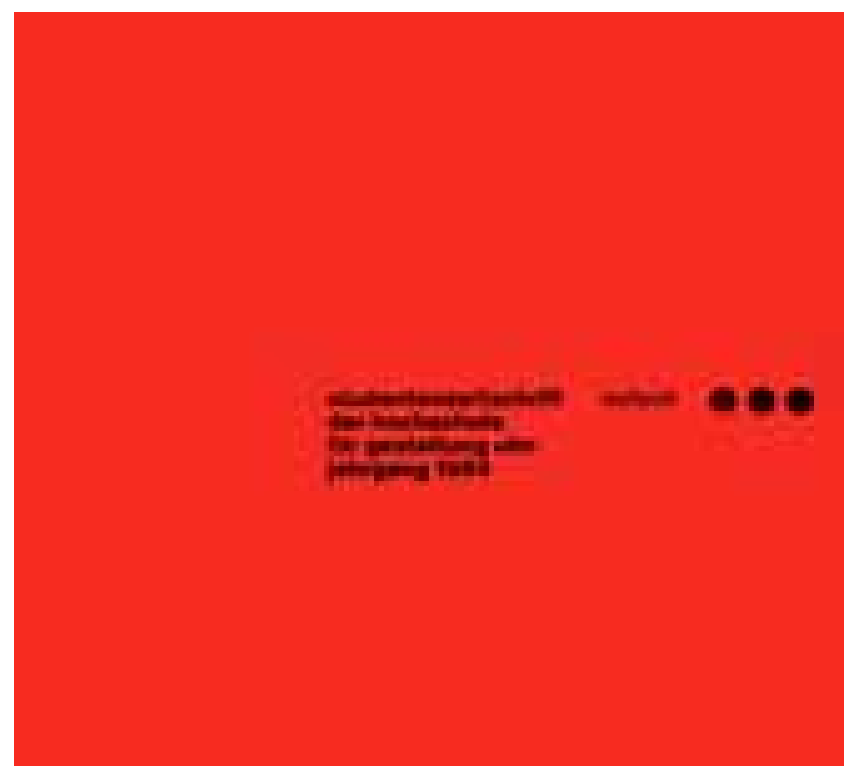

i1/159, 160, 161

Interior y exterior de la revista outup,

editada por los alumnos de la HfG a

partir de 1961

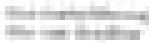

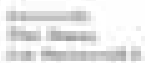
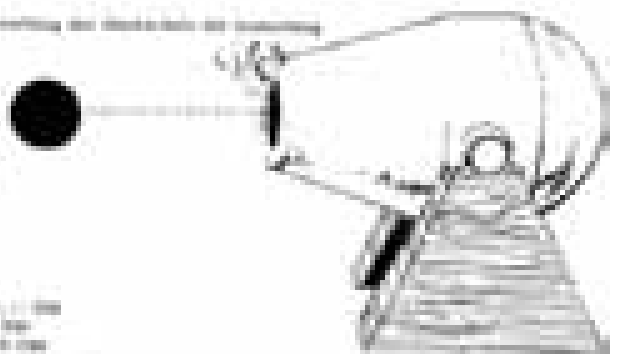

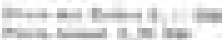

$4+2$

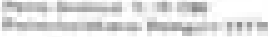

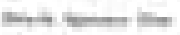

tiat

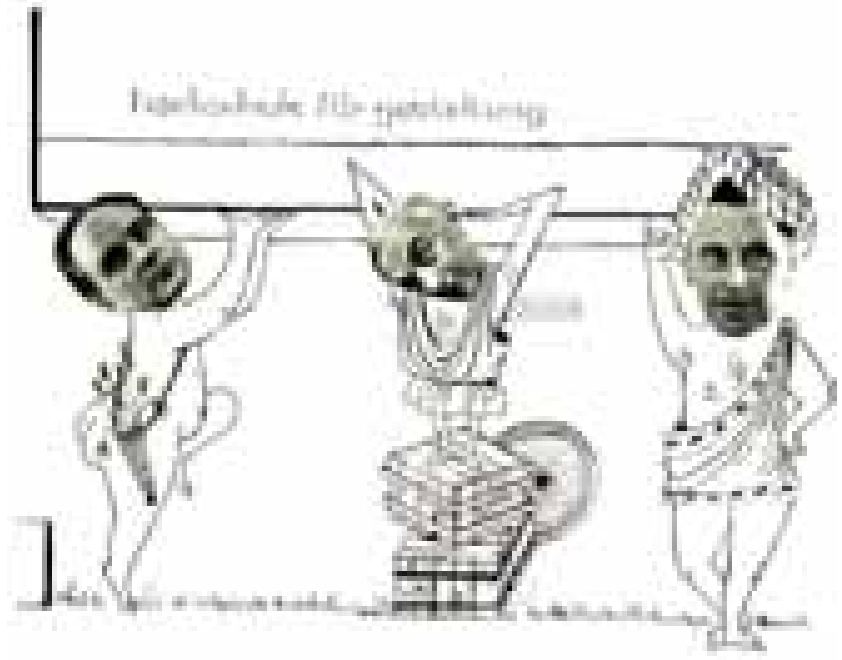

Fint dost 
En abril de 1960, Aicher, Gugelot y Maldonado participan en el congreso mundial de diseño de Tokyo. En julio de ese mismo, la escuela obtiene la medalla de plata de la Trienal de Milán.

El primer número de Output, la revista editada por los estudiantes, se publica en marzo de 1961. El número doble $6+7$, de noviembre de 1961, se dedica a una monografía sobre la desaparición del Grundlehre. La revista tiene un tono general que no apoya el fin del Curso Básico y ni la disolución en los departamentos. Czemper, delegado de los estudiantes, recoge la opinión de los alumnos en un artículo. Éstos no creen conveniente la separación en sectores de alumnos el primer curso porque establece diferencias entre ellos. También se publica el artículo de Gugelot que expone los problemas que presentaba el Grundlehre en el conjunto de las titulaciones. La revista aporta unas tablas comparativas que desgranan el número de horas dedicadas a las materias en el último curso de Grundlehre 19601961 y primer curso en los departamentos 1961-1962. Estas tablas ponen de manifiesto la disminución del número de horas dedicado a teoría que revierte en un incremento de trabajo en proyectos específicos de los departamentos.

\subsubsection{Docentes, materias y alumnos del periodo}

\section{Rectorado 1960-1961}

Gert Kalow

Horst Rittel

Friedrich Vordemberge-Gildewart

\section{Departamentos 1960-1961}

Curso Básico - Horst Rittel

Diseño de Producto - Hans Gugelot

Construcción Industrializada- Herbert Ohl

Comunicación visual - Anthony Froshaug

Información - Gert Kalow

Cine

\section{Docentes 1960-1961}

Otl Aicher

Anthony Froshaug

Hans Gugelot

Gert Kalow

Tomás Maldonado

Herbert Ohl

Horst Rittel

Christian Staub

Friedrich Vordemberge-Gildewart

Walter Zeischegg

\section{Maestros del taller 1960-1961}

Paul Hindinger - Madera

Otto Schild - Yeso

Josef Schlecker - Metal

Wolfgang Siol - Fotografía

Cornelius Uittenhout - Plástico

\section{Docentes invitados 1960-1961}

Bruce Archer - Diseño de producto Grundlehre

Hans Werner Arndt

Wilfried Berghan - Cine

Harald Berns - Diseño de producto Grundlehre

Rudolf Doernach - Construcción Industrializada

Gerhard Dressel - Análisis y forma de los edificios

Iring Fetscher - Sociología

Kurt-Joachim Foische - Cine

Siegfried Haenle - Dibujo Técnico

Herman Henn - Estructuras

Harn Hennecke - Historia de la literatura moderna

Joachim Kaiser - Historia de la literatura moderna

Ewald Kapal - Fisiología aplicada

Rainer Mackensen - Sociología

Mervyn W. Perrine - Teoría de la percepción

Hermann Reiher - Física Técnica para la construcción

Bernhard Rübenach - Radiodifusión

Arnold Schonfeld - Ingeniería de Embalajes

Hans Sukopp - Tecnología del cine

Firedrich Tonne - Física Técnica para la construcción

D. G Thornley - Arquitectura y proyecto cívico

Herert v. Voss. - Patentes en construcción

Klaus Wegener - Psicología

Z. Wasowski. - Instalaciones de la vivienda

Konrad Weller. - Construcción Industrializada 


\section{Materias 1960-1961}

La organización de las enseñanzas durante el curso 1960-1061 se amolda a la sectorización del Grundlehre iniciada en el curso anterior. Las Methodische Übungen desaparecen como tareas de proyecto omnipresentes a lo largo de todo el curso. Lo mismo sucede con las Grafische Darstellungen como ejercicios para el análisis visual. En el plan de estudios para el Grundlehre de este curso se introduce la materia Grundlehrearbeit -tareas de formación básica- durante los dos primeros cuatrimestres.

El proyecto se instaura definitivamente como disciplina en el tercer cuatrimestre. El debate generado en la Conferencia Pedagógica de julio de 1960 pone de relieve el enfrentamiento entre Rittel y los profesores de los departamentos. Se habla sobre la carga de las asignaturas teóricas y el protagonismo de las Methodische Übungen. El debate conduce a la aparición de los Abteilungsarbeiten -tareas de departamento- como materia de proyectos de tercer cuatrimestre. Las tareas que se suceden este año se organizan a partir de reuniones de coordinación a las que asisten profesores de todos los departamentos para la secuenciación de los ejercicios. La especialización también continúa con las materias teóricas.

\section{Alumnos 1960-1961}

Ann Bayard

Sigrid Bender

Herwig Birg

Irene Bornhauser *producto

Karl-Dieter Bodack

Marie-Luise Budde

Michael Conrad *producto

Hans Dahlström

Terence Dalley

Per Arne Darnell

Sven Diehl

Horst Diener *producto

Konrad Diepolder

Heinz Dobrinski *arquitectura

Elmar Fetscher

Heinz Grueber

Gudrun Haegele

Jürgen Hauffe

Winfried Jokisch

Harry Kaas

Lieselotte Kappier

Egbert Koch

Gerd Krammling

Horst Kretschmar *producto

Jan McLaren

Lars Lidman
William Linder

Pio Manzoni *producto

Peter Matouch *producto

Horst Müller *producto

Rolf Müller

Katlen Paterna

Tonci Pelikan

Eric Rossicci

Ernst Rottefusser

Jan Sargeant

Konrad Schäfer

Kurth Scheidegger

Gerhard Schlüter

Horst-Josef Schu *arquitectura

Harmurt Seeger

Jörg Stumpf *producto

Karin Teufel

J. Tochterman

Hans Werner *producto

Alfons Willeke

Günter Wotzka 


\subsubsection{Los departamentos en el Curso Básico y el papel de Bruce Archer}

Los Abteilung Arbeiten aparecen en el curso 1960-1961 como una transformación de las Methodische Übungen. Comparten con estas últimas el carácter y el planteamiento de la tarea: los profesores distribuyen un enunciado orientado a un proyecto con una serie de condicionantes que los alumnos tienen que resolver. En una memoria, deben argumentar la toma de decisiones que les lleva a concretar su propuesta. Hasta tal punto comparte los fines de las Methodische Übungen, que algunos enunciados se repiten, como el lingote de alumnio y el sistema de lectura hombre-máquina.

Sin embargo, algunas tareas comienzan a profundizar en la especialización de los alumnos. Por ejemplo, el proyecto para el sistema de juguete constructivo, establece en su enunciado condiciones específicas para los estudiantes del departamento de Diseño Industrial, de Construcción Industrializada y de Comunicación Visual.

En el segundo cuatrimestre se llevan a cabo seminarios específicos por sectores para la profundización en conocimientos tecnológicos de los que carecían los estudiantes. Esto permite asumir proyectos como el lingote de aluminio o el sistema de juguete constructivo con el bagaje suficiente, y los alumnos valoran de forma positiva esta coordinación estrecha entre teoría y práctica. ${ }^{146}$

El profesor Bruce Archer es un docente fundamental en los seminarios. Llegado este mismo curso desde Londres, ejercía la docencia de Diseño industrial en la Central School of Arts \& Crafts y publicaba periódicamente artículos en la revista Design. Antes de llegar a UIm, Archer difundía estudios sistemáticos sobre objetos fabricados por la industria que destacaban por su actitud crítica y por presentar un enfoque del diseño como una disciplina con sistemas de investigación propios. Este factor es precisamente lo que aporta a la escuela, la incorporación de los métodos de investigación en diseño, justo en el momento en el que este debate se está incorporando al curso básico. Junto con Rittel y Froshaug, idea enunciados en los que el objetivo principal del proyecto no es un objeto final sino un proceso -identificación de una muestra radiactiva, ordenación de una serie de objetos-. Los estudiantes valoran este giro positivamente porque significa la aportación de una nueva forma de pensar. ${ }^{147}$

\section{Listado de los Abteilungsarbeiten: ${ }^{148}$}

1 FROSHAUG, ARCHER, RITTEL: Clasificación de una colección de 6 objetos

2 FROSHAUG : Rótulo para una estación

4 ARCHER: Código de conducta

5 BERNS: Investigación de los límites de una imagen realista en axonometría general, el ejemplo de un cubo

6 FROSHAUG: Alfabeto legible por hombres y máquinas

8 ARCHER: Identificación de error en 12 muestras radioactivas

\section{ZEISCHEGG: Lingote de aluminio}

10 FROSHAUG: Sistema de juego constructivo

11 MALDONADO: Similitud de los campos por brillo y / o valor de color

12 ARCHER: Diseño de insignias para congreso de diseño
146 Czemper, K. A. "Die pädagogischen Ziele der Grundlehre", output. 6+7, 1961. Hg. v. Studentenselbstverwaltung der Hochschule für Gestaltung UIm. (revista editada por los estudiantes de la HfG) p. 6.

147 íbidem

148 En el HfG Archiv no se ha podido localizar la tarea número 3 y la número 7 por no quedar constancia de enunciados ni trabajos de alumnos. 

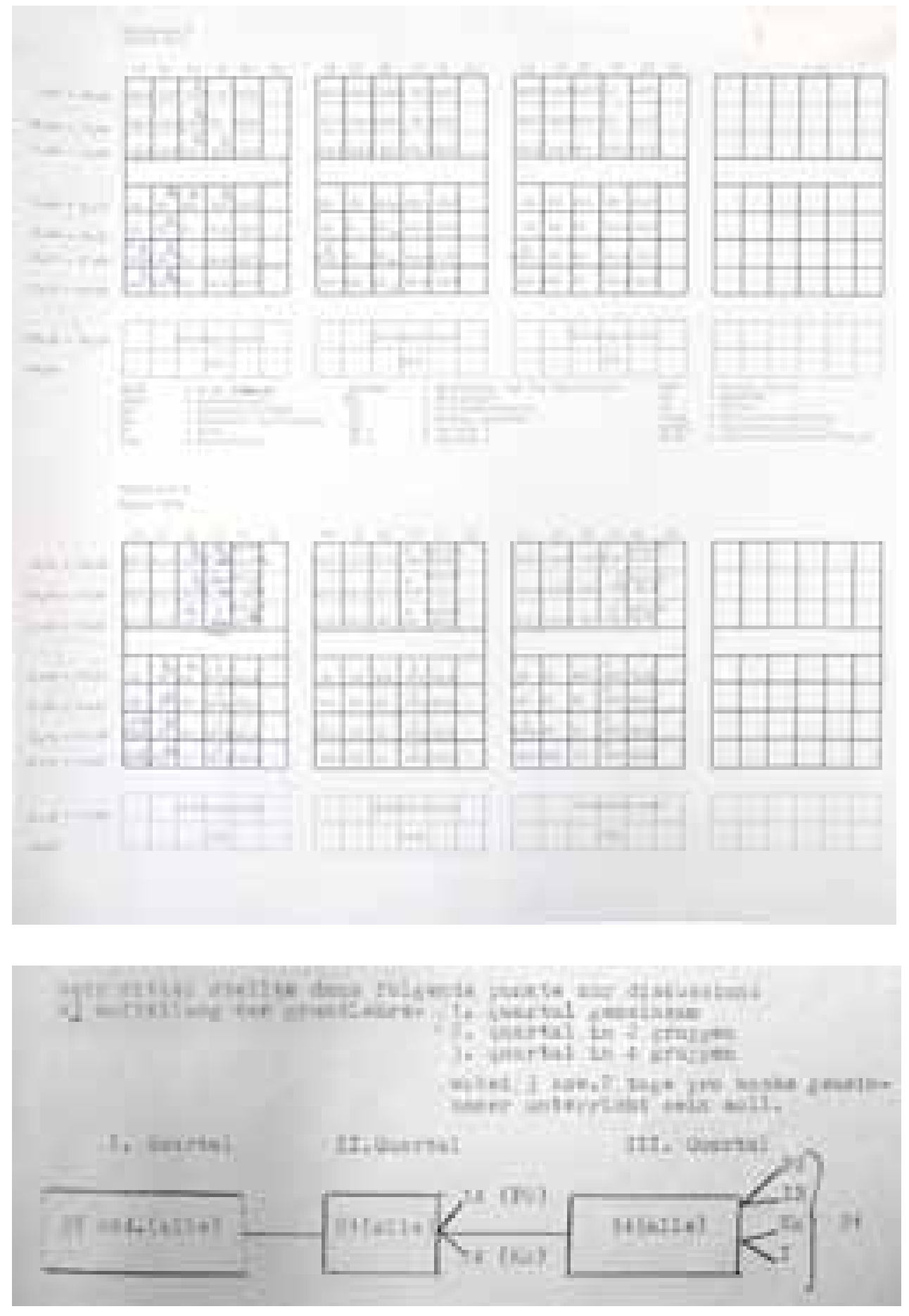

i1/162, 163

Propuesta del plan de estudios para

el departemento de Construcción

Industrializada, Diseño Industrial,

Comunicación visual e Información.

Se 191.57 F.B.S 


\subsubsection{La madurez del Curso Básico en los departamentos}

El año 1961 acaba con un enfrentamiento declarado entre los profesores que apostaban por repetir la misma secuencia del Grundlehre de curso anterior y los responsables de los departamentos, que reclamaban el comienzo de los Abteilungsarbeiten desde la primera semana. Los enunciados estarían adaptados a los problemas de diseño de cada departamento y los estudiantes realizarían la inmersión en su disciplina desde el primer día. Se plantean alternativas que preserven el espíritu colectivo que caracterizaba el Curso Básico y que los propios alumnos habían defendido como una de las riquezas del primer año de estudios. Sin embargo, el acta recoge la decisión final que opta por un nuevo comienzo del Curso Básico en los departamentos. ${ }^{149}$

Abteilungsarbeiten es la denominación de los ejercicios en los departamentos que podían tener cualquier cometido, desde el desarrollo de cuestiones de color, creación de forma en dos y tres dimensiones, y proyectos específicos de introducción a la disciplina, con aplicaciones concretas.

Una secuencia de 12 Abteilungsarbeiten configura el primer curso de departamento de Construcción Industrializada. Esta secuencia se sigue repitiendo hasta el cierre de la escuela y consiste en un repertorio evolutivo, en el que aparecen temas que buscan familiarizar al alumno con la sistematización de la construcción prefabricada. La presencia de la retícula como sistema de ordenación en dos y tres dimensiones, caracteriza los cursos siguientes. ${ }^{150}$

El departamento de Comunicación Visual reserva el proyecto al último cuatrimestre. Los dos anteriores se dedican al análisis de los signos y su entorno y las relaciones entre elementos. La secuencia de ejercicios del departamento son los que guardan mayor relación con el Visuelle Einführung propuesto por Maldonado. Los proyectos del tercer cuatrimestre se dedican a la tipografía, la fotografía y la maquetación editorial. ${ }^{151}$

Lo abstracto, sin la intervención de la función ni el uso, es la directriz que se sigue el primer año en el departamento de Diseño Industrial. Los ejercicios están más cerca del análisis y de la generación formal, sin otros condicionantes, que de la aplicación directa. Se trabajaba en la generación de redes y sus ensamblajes; se analizan utensilios sencillos. Es la disciplina en la que la morfología permanece con mayor predominancia. ${ }^{152}$

Las Abteilungsarbeiten del Departamento de Información guardan relación con las de Comunicación Visual. La semiótica está muy presente en la ejercitación de los estudiantes.

La propuesta con la que se inicia esta misma etapa refleja una proporción entre el número de horas dedicada a los proyectos y al bloque teórico igual para todos los departamentos. Mientras que Comunicación Visual, Información y Diseño Industrial mantienen las mismas asignaturas teóricas -con diferente carga horaria-, el departamento de Construcción Industrializada desplaza asignaturas históricas como Lenguaje, Tipografía y Semiótica y las sustituye por materias específicas como Física Técnica o Resistencia de Materiales.

Se supera el idealismo del Curso Básico Universal y al mismo tiempo se pierde un espacio común de aprendizaje, en el que predomina una visión holística de la formación de los estudiantes.
149 Acta de la Conferencia Pedagógica del 3 de julio de 1961. Se 191.57.

150 Schmitz, Gunter "Ergebnisse aus dem Unterricht. Grundlagen der Gestaltung für Architekten, en: Revista Ulm 19/20, op. cit. p. 41

151 Lindinger, Herbert, "Ergebnisse aus dem Unterricht. Abteilung Visuelle Kommunikation 1. Studienjahr. en: Revista UIm, 17/18, op. cit p. 40

152 Bonsiepe, Gui, "Ergebnisse aus dem Unterricht. Dreidimensionale formale Übungen”, en: UIm, 17/18, op. cit, pp. 21, 22. 


\section{2 atlas color}

\subsection{Color óptico}

2.1.1 Orden

Luz, oscuridad. Color. Círculo cromático

Colores primarios, secundarios, terciarios, cuaternarios

Sólido de color de Ostwald

\subsubsection{Escalas}

Escalas acromáticas y monocromáticas

Escalas polícromas

Escalas de colores con blanco y negro

\subsubsection{Teoría de contrastes}

\subsection{Color visual}

2.2.1 Color visual: enfoque experimental

Un color parece dos

Dos colores parecen uno

Mezcla ilusoria y transparencia

Colores progenitores

Intersección de colores

Transformaciones

Cantidad de color

Borde vibrante

Intensidad luminosa

Estudios libres

2.2.2 Color visual: enfoque sistemático

Proximidad-ambigüedad-semejanza

Fondo-ambigüedad-primer plano

Estructuras y superficies de color en equilibrio visual

Cantidad de color

\subsection{Color háptico}

\subsection{Color sintáctico}

2.4.1 Topología y color

Triángulo de Sierpinski

La Curva de Peano como activadora del color

Curva de Peano: sistema de brillo de color equivalente, sistema

de figura-fondo positivo-negativo

Escala de color lineal sobre la Curva de Peano

Relatividad del color en la Supercie de Peano

Superficie de Peano: negro como color

2.4.2 Distribución sistemática o aleatoria

Distribución de color mediante un patrón

Comparativa entre campos coloreados al azar

Similitud de los campos por brillo y/o color

Escala de grises aleatoria / sistemática y con dado al azar

2.5 Contribución a la didáctica del color conclusiones parciales atlas color 

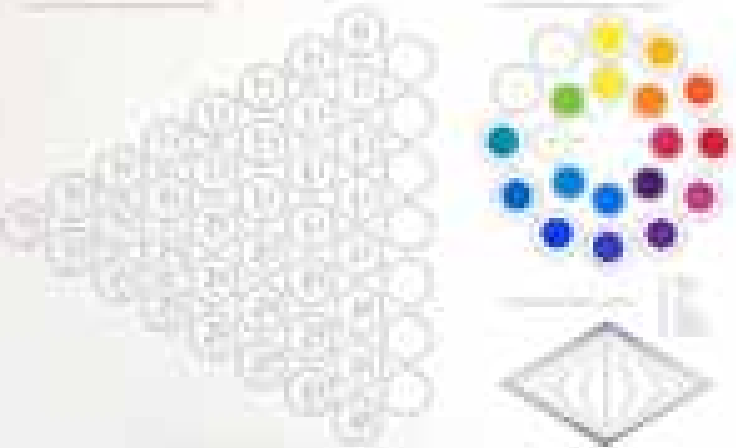

i2/1. Color como variable o fenómeno óptico

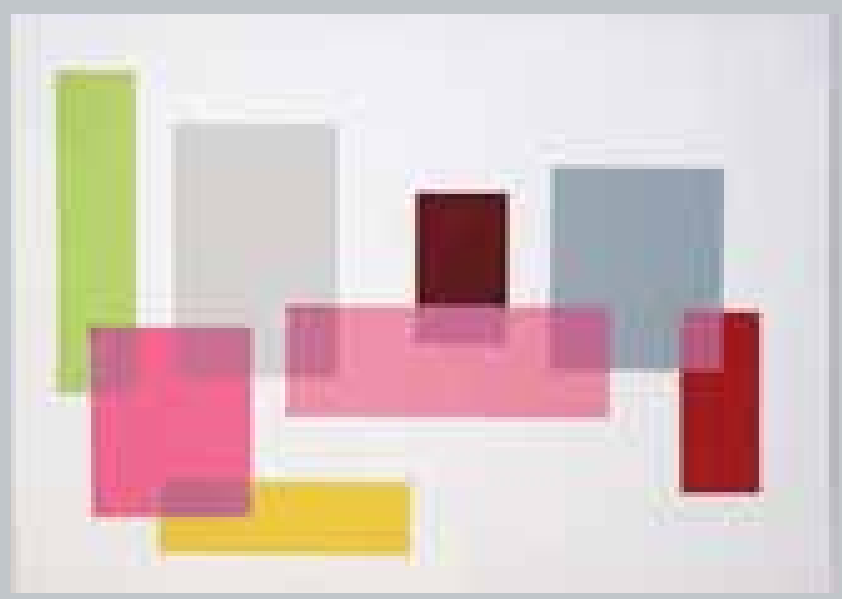

i2/2 Color como variable visual

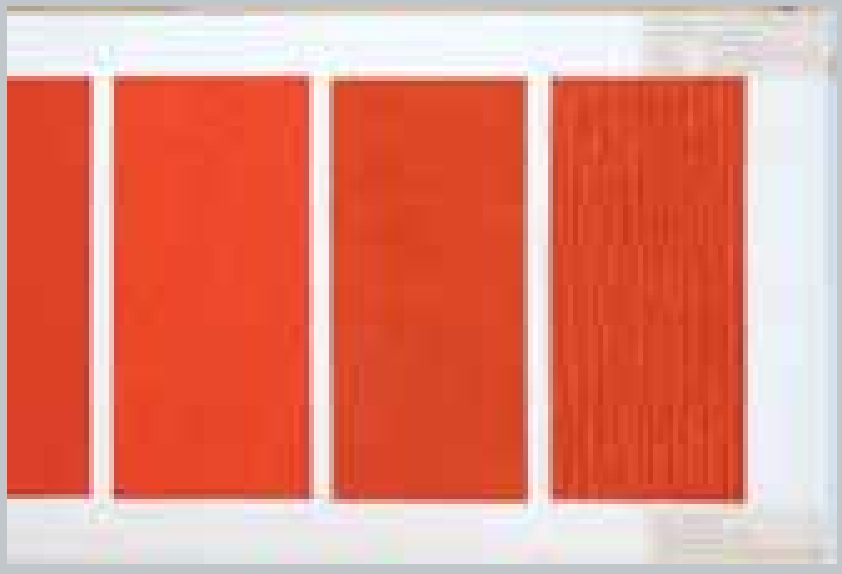

i2/3 Color como variable háptica

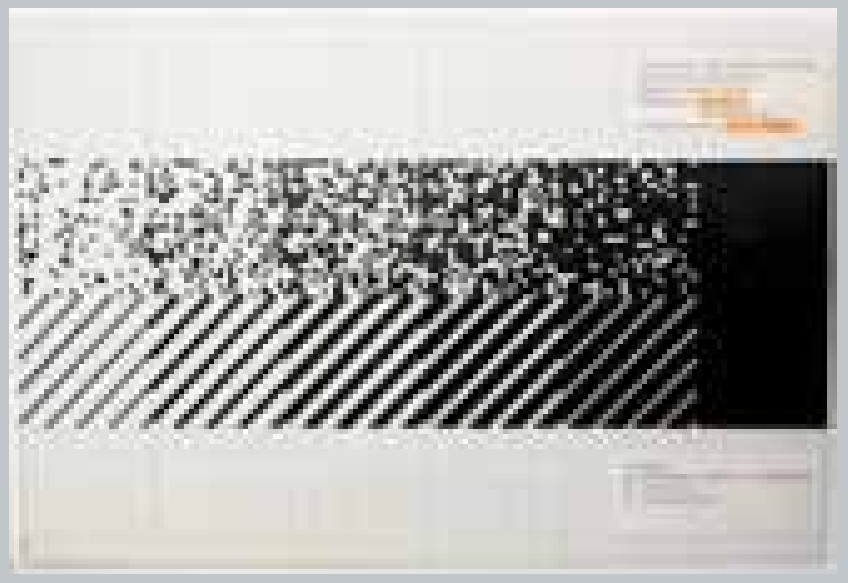


En los ejercicios recogidos en este atlas, el color es un elemento del lenguaje visual independiente de otros elementos formales. Define composiciones visuales por sí mismo y puede alcanzar una autonomía suficiente para ejercitar su práctica y su estudio. La naturaleza física y el tratamiento plástico del color tiene un interés pedagógico intrínseco. Así se reconoce en las didácticas propias de los docentes que intervienen en curso básico de la $\mathrm{HfG}$ y en sus precedentes.

La reconstrucción de una didáctica del color parte de la selección de aquellos ejercicios en los que éste constituye la variable dominante y fundamental de trabajo. El resultado de los enunciados, y la solución que se ofrece a los problemas que se plantean en ellos, están basados en el color como elemento plástico que orquesta los procesos de intervención y da forma al resultado de manera determinante, como elemento primigenio, completo y expresivo a partir del cual se construyen las soluciones.

Con el fin de sintetizar una didáctica del color a partir del análisis de los diferentes enunciados, se establecen cuatro variables que atienden a aspectos concretos del color como elemento autónomo: el color como variable o fenómeno óptico, el color como variable perceptual, el color como variable háptica y el color como variable sintáctica.

En el color como variable o fenómeno óptico se organizan los ejercicios relativos a su organización en el círculo cromático y sus propiedades tono, luminosidad y saturación. La labor de Helenne Nonné-Schmidt en la enseñanza del color como variable óptica fue comparativamente la más amplia en relación a la de los profesores Vordemberge-Gildewart y Albers. Los referentes que desarrollan los ejercicios propuestos por Nonné-Schmidt son las teorías planteadas por Paul Klee y posteriormente el sistema de Ostwald y el sistema de Munsell. A partir de estos referentes se analizan la generación del color, sus propiedades y cualidades, y su interrelación con otros colores mediante escalas y gradientes. Los ejercicios propuestos pertenecen a Helene Nonné-Schmidt y Friedrich Vordemberge-Gildewart.

El color como variable visual define el ámbito de los ejercicios en los que la variable dominante es el color frente a otros elementos formales. Las soluciones a los enunciados se abordan desde la consideración del color y su interacción como fenómeno gestáltico y como condicionante para la ejecución de los ejercicios. Los casos que se exponen son ejercicios del curso de Color Básico de Josef Albers, algunas de las tareas propuestas por Tomás Maldonado en el Visuelle Einführung y una serie de ejercicios del Farbenlehre de Vordemberge-Gildewart. Se distinguen dos enfoques, uno experimental llevado a cabo por Josef Albers y otro planificado desarrollado en los cursos de Maldonado y VordembergeGildewart.

El color como variable háptica estudia una serie de ejercicios en los que el color es un elemento formal que activa otras variables sensoriales relacionadas con la percepción táctil, interactuando éstas de forma conjunta en la solución al ejercicio propuesto, en este caso por Vordemberge-Gildewart.

Finalmente, se exponen una serie de ejercicios en los que el color tiene consideración de variable sintáctica. Esto significa que el color aparece como una variable subordinada dentro del conjunto de mecanismos, estrategias y decisiones del proceso de realización del ejercicio, pero inherente a la solución adoptada. 


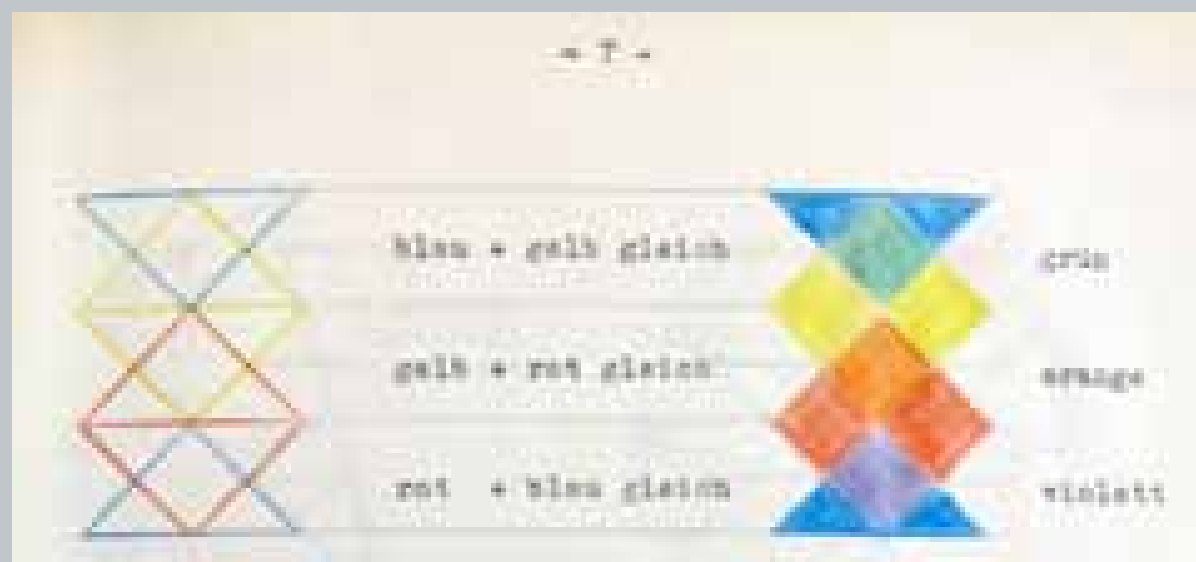

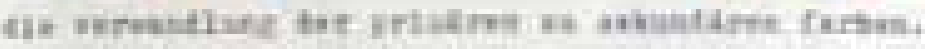
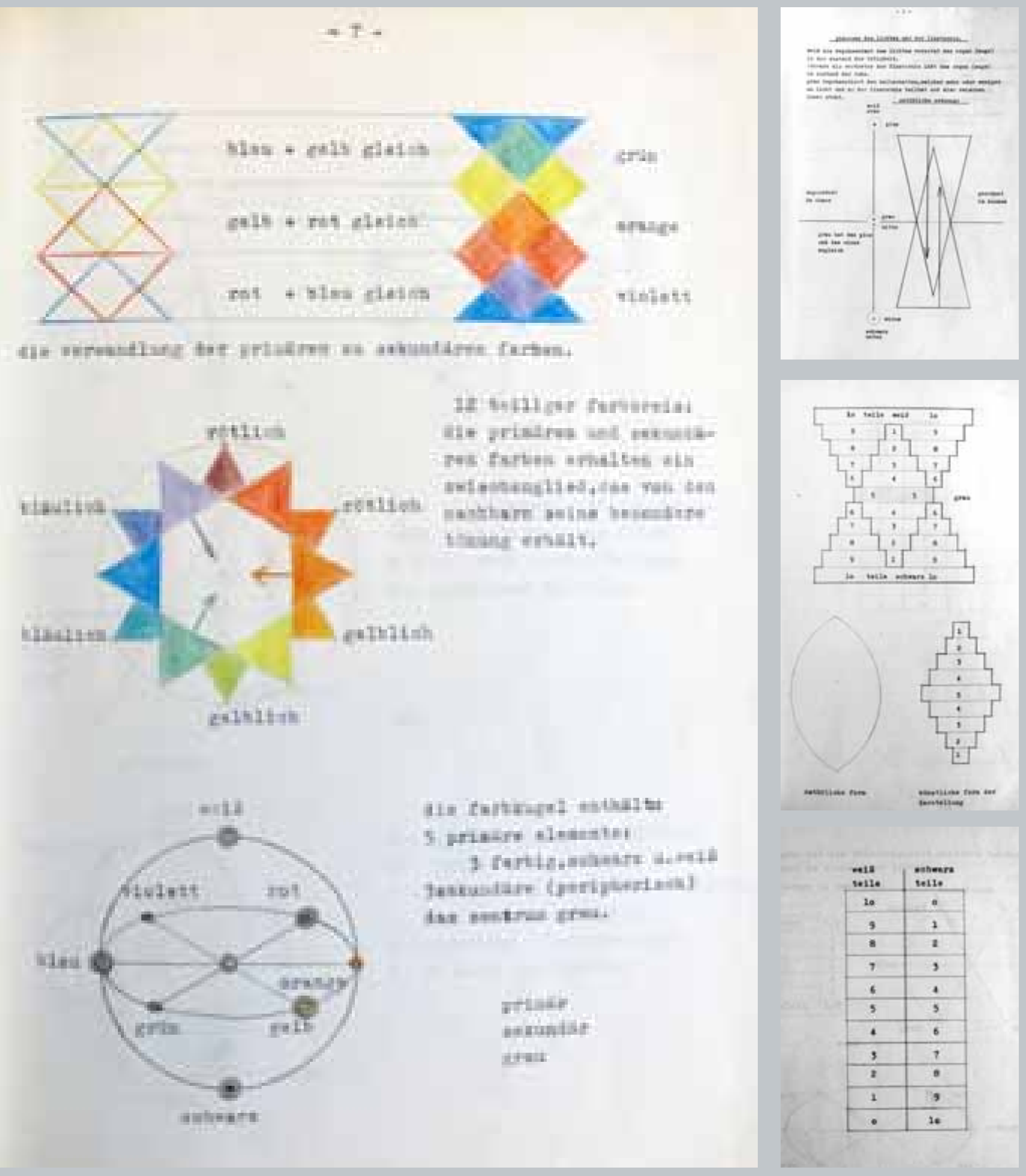

i2/5 Apuntes de la enseñanza del color de Paul Klee.

i2/6, 7, 8 Apuntes de la enseñanza del color de Paul Klee.

Curso: Nonné-Schmidt (1955-1956)

Curso: Nonné-Schmidt (1955-1956)

Alumno: Christopher Naske

Dp 024.01. FBS

Dp 024.01. FBS 


\subsection{Color óptico}
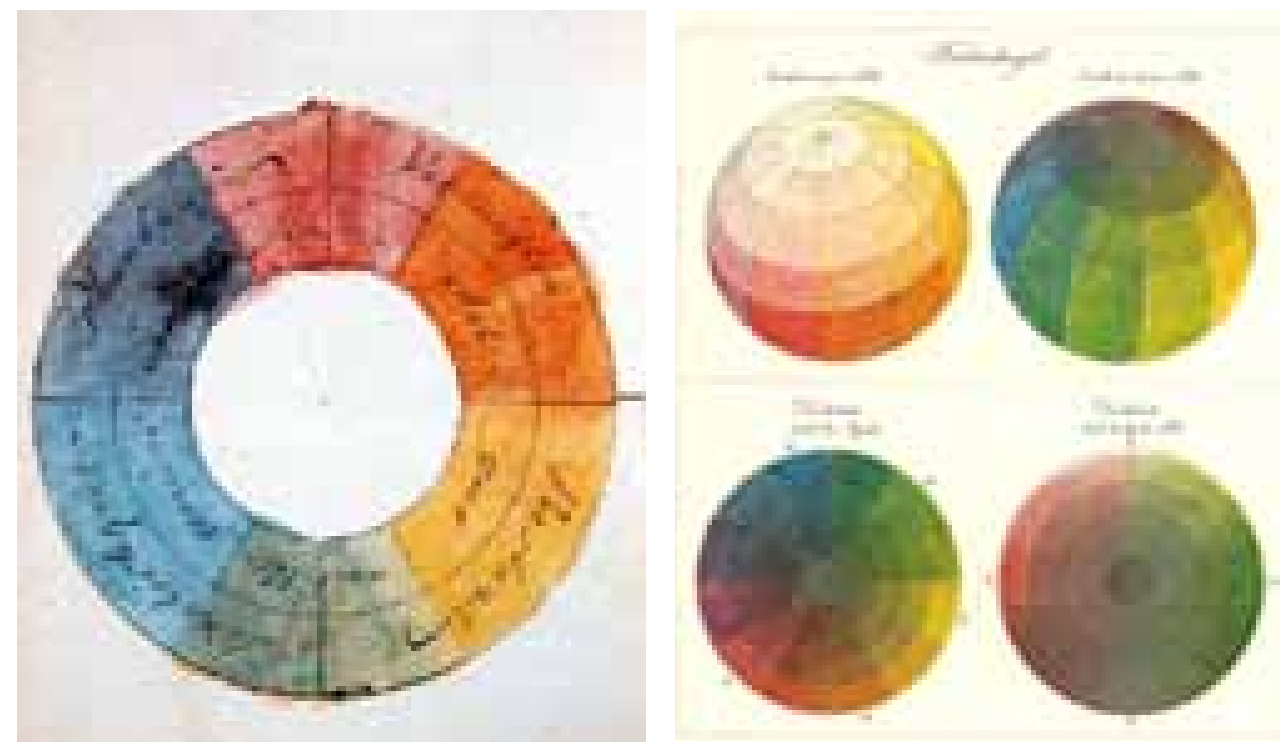

i2/9 Círculo cromático de Goethe, 1809. Distribución de los espectros de color en el círculo.

i2/10 Esfera de color de Runge, 1810. Distribución en una esfera del esquema de color polarizado entre luz y oscuridad.

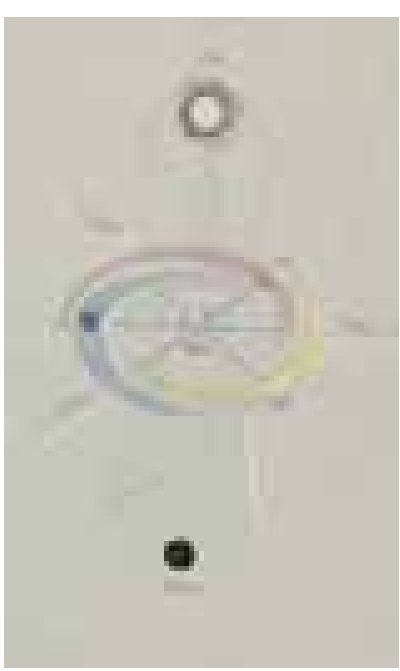

La didáctica del color como variable óptica se organiza en dos grandes bloques temáticos de ejercicios basados en sus cualidades como fenómeno óptico.

En el primer bloque, denominado orden, se representa la distribución sistematizada de los colores según un espectro cromático en un sistema en el que éstos se ordenan según sus dimensiones o atributos de tono, luminosidad y saturación. En este caso, la tarea es abordada por Nonné-Schmidt, y los referentes utilizados serán las teorías propuestas por Paul Klee ${ }^{1}$ en la Bauhaus y el sólido de Ostwald ${ }^{2}$, con algunas referencias al sólido de Munsell. $^{3}$

A partir de esa ordenación sistemática se analiza en el segundo bloque de ejercicios las relaciones que se establecen entre los colores en función de sus atributos o sus combinaciones. Estas relaciones se dibujan mediante escalas en las que se representan intervalos obtenidos por las mezclas de uno o varios colores entre sí o con el blanco y el negro. Dentro de este grupo de ejercicios se recopilan varios tipos en función del aspecto estudiado que determina el número de colores que se emplean:

- Escalas del blanco al negro, como representación del gradiente de luminosidad. - Escalas de un color mezclado con blanco y negro, para representar los valores de luminosidad del color.

- Escalas del espectro completo con blanco y negro, para obtener los valores de luminosidad del espectro.

- Escalas de dos colores, en este caso colores complementarios.

- Escalas de tres colores: de tres colores cualesquiera o bien de un color y su complementario y los adyacentes a éste.

i2/11 El color en el orden primoridal. Paul Klee. http://www. kleegestaltungslehre.zpk.org/ee/ZPK BG/2012/01/02/156/.

Este esquema sintetiza el dinamismo del color de Goethe y la polaridad del luz y oscuridad de la esfera de Runge.

1 "La base del trabajo teórico de Itten, Klee y Kandisnky sobre el color estaba formada principalmente por las teorías del color de Goethe, Runge y Hölzel. En los talleres de pintura mural y carteles, por el contrario, Hinnerk Scheper y Joost Schmidt, se apoyaban en gran parte en la estricta ordenación esquemática del color del premio Nobel de Química Wilhelm Ostwald."

Schuster, Britta. "La enseñanza de color en la Bauhaus". en: Fiedler, Jeaninne (ed.) Bauhaus. Köln: Könemann, 2000, pp. 392- 399.

2 El sólido de Ostwald consiste en la definición de una gama de tonos equiseparados artiméticamente. Ostwald, Wilhelm. Die Farbenfibel. Leipzig: Verlag Unesma, 1917.

3 El sólido de Munsell consiste en una gamas de tonos equiseparados perceptualmente. Munsell, A. H. Atlas of the Munsell color system. Massachusetts: Malden, 2015. 


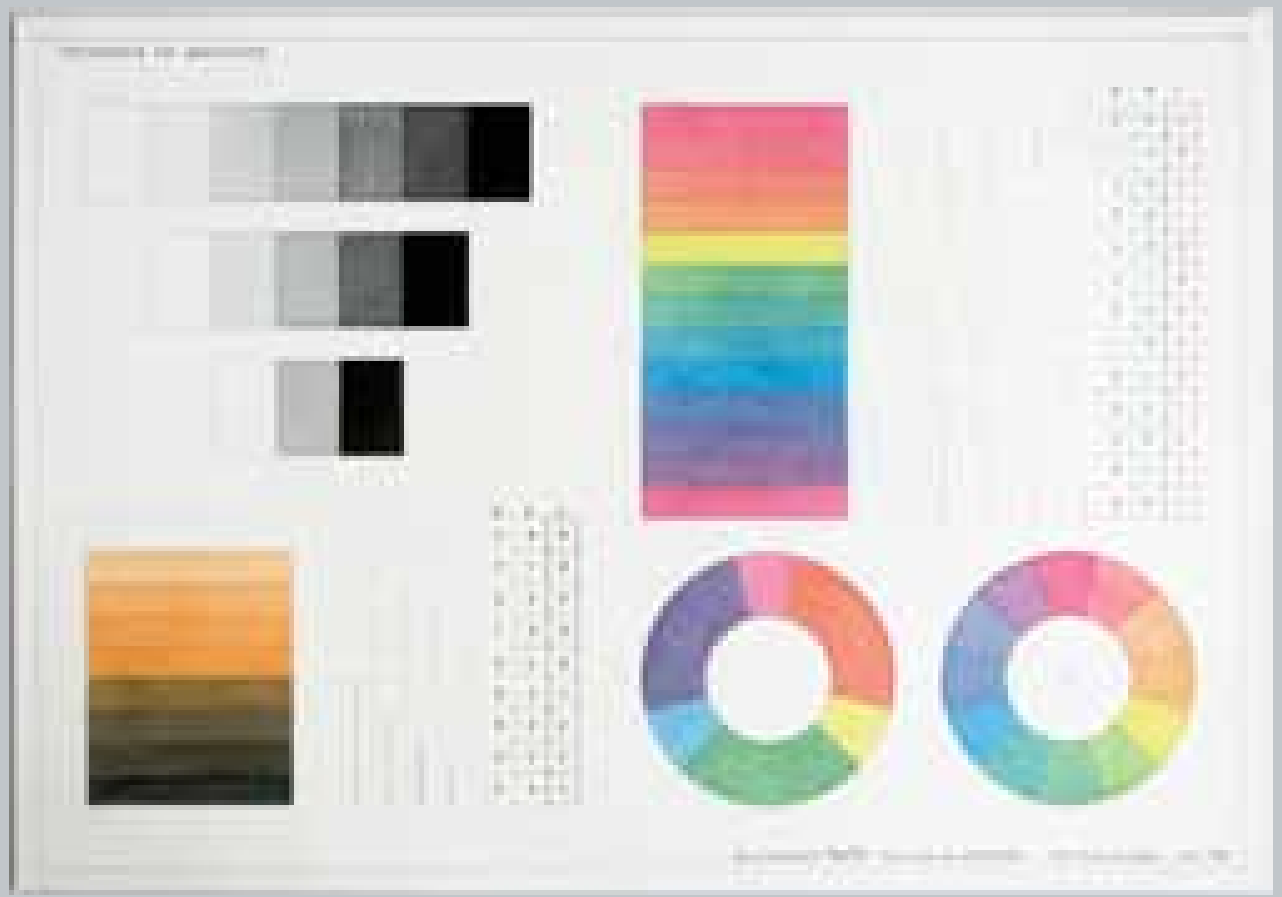

i2/12 Luz oscuridad. Color puro: espectro y círculo cromático

Alumno: Bethus Mulder.

Curso: Visuelle Einführung

Enseñanza del color de NonnéSchmidt. (1956-1957)

Gs_043.001. F.B.S
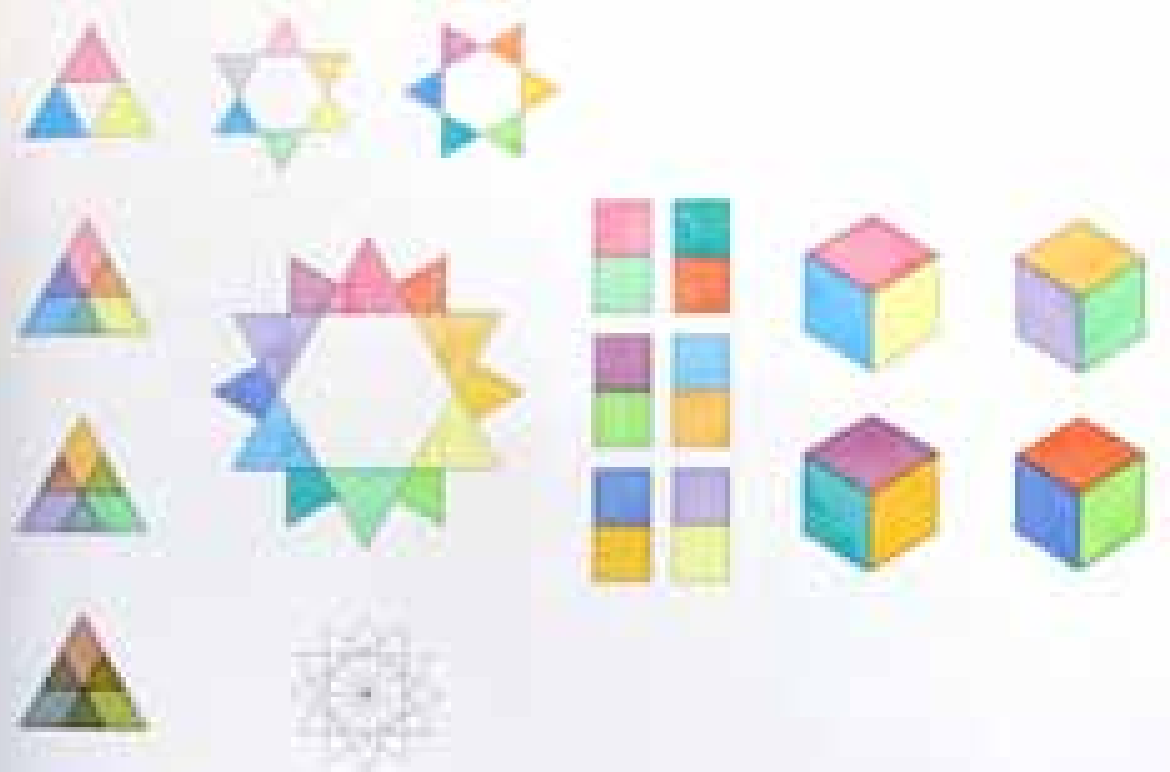

i2/13 Círculo cromático. Colores primarios, secundarios, terciarios y cuaternarios. Pares de complementarios. Triadas

\section{Alumno: Hans von Klier.}

Curso: Visuelle Einführung Enseñanza del color de Nonné-Schmidt. (19551956).

Dp_017.001. F.B.S 

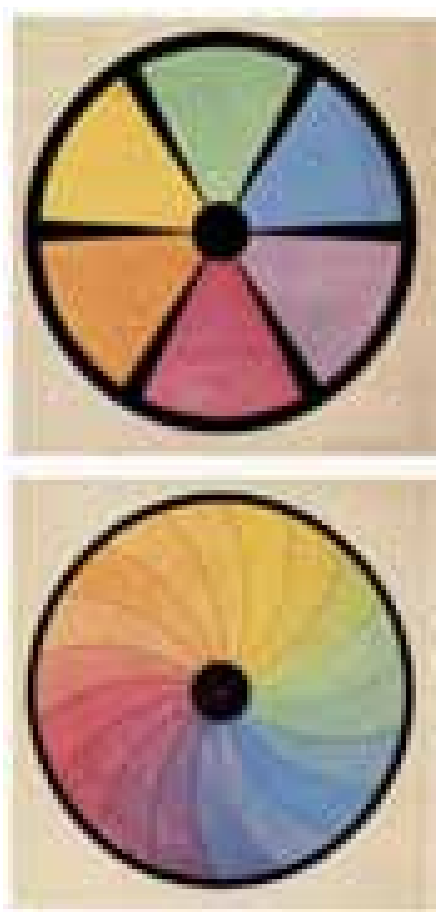

\subsubsection{Orden}

La labor fundamental en este tema se desarrolló en los ejercicios planteados por Helene Nonné-Schmidt en su enseñanza del color dentro del Visuelle Einführung entre 1953 y 1957. A través de su vínculo con Paul Klee y Joost Schmidt desarrolla la enseñanza del color teniendo a ambos profesores como referentes en este tema.

En sus enseñanzas, Nonné-Schmidt incluye referencias a los sistemas principales sobre el orden del color. Así, cita a Newton, Chevreul, Schopenhauer y finalmente se refiere al sólido de Ostwald. ${ }^{4}$ Se refiere a las tres cualidades del color tono, luminosidad y saturación y cómo éstas se organizan en láminas en las distintas secciones del sólido. En este sentido la enseñanza de Nonné se encuentra más próxima a la de Joost Schmidt.

En estos ejercicios los alumnos estudian el fenómeno de la luz y la oscuridad, el color y su ordenación en sistemas. La representación de estos fenómenos les permite conocer la organización del color a partir de los colores primarios, y la generación y distribución de todo el espectro cromático. En los ejercicios sobre el color de los alumnos de NonnéSchmidt entre 1953 y 1957 se puede apreciar una unidad en los temas con variantes en la ejecución técnica de los mismos.

Nonné-Schmidt, partiendo de sus apuntes de las clases de Paul Klee, comienza por explicar el fenómeno de la luz y la oscuridad ${ }^{5}$ estableciendo la contraposición entre blanco y negro ubicándolos en los extremos de un eje vertical y el gris en el centro (i2/5). Para explicar el fenómeno del color se basa asimismo en la concepción de Klee del círculo cromático configurado por pares de colores opuestos por un eje y del movimiento oscilante de los colores para la generación del espectro. Klee, a su vez, se basaba en la esfera de Runge y en el movimiento de los colores en los meridianos y el ecuador: luminosidad arriba-abajo; temperatura: izquierda-derecha (i2/9-i2/11). ${ }^{6}$ i2/14 K. Afanaśyev. Círculos espectrales en el Taller de color de Klutsis. 1er año. 1926/1927.

Procedencia del libro: Form Vkhutemas to Markhi 1920-1936. Architectural projects form the Collection of the MARKhl Museum. Moscú: A-Fond Publishers 2005. Pág. 106.

Publicado en: Britta Kaiser Schuster. La enseñanza del color en la Bauhaus. En: Jeanine Fiedler y Peter Feierabdend eds. Bauhaus. Colonia: Konemann, 1999.pág 395

i2/15 Johannes Itten. Esfera de color en 7 niveles de luminosidad y 12 tonos. 1921. Publicada en : Adler, Bruno. Utopia Dokumente der Wirklichkeit.Weimar: Utopia, 1921.

4 Apuntes de clase de Klaus Krippendorff, curso 1956-57, Visuelle Einführung VE-1, Kurs NonnéSchmidt, Dp/18.

5 Eggelhöfer, F., \& Keller-Tschirren, M. Paul Klee. Maestro de la Bauhaus. [cat. expo., Fundación Juan March, Madrid]. Madrid: Fundación Juan March, 2003, p.56.

6 Ibidem. 


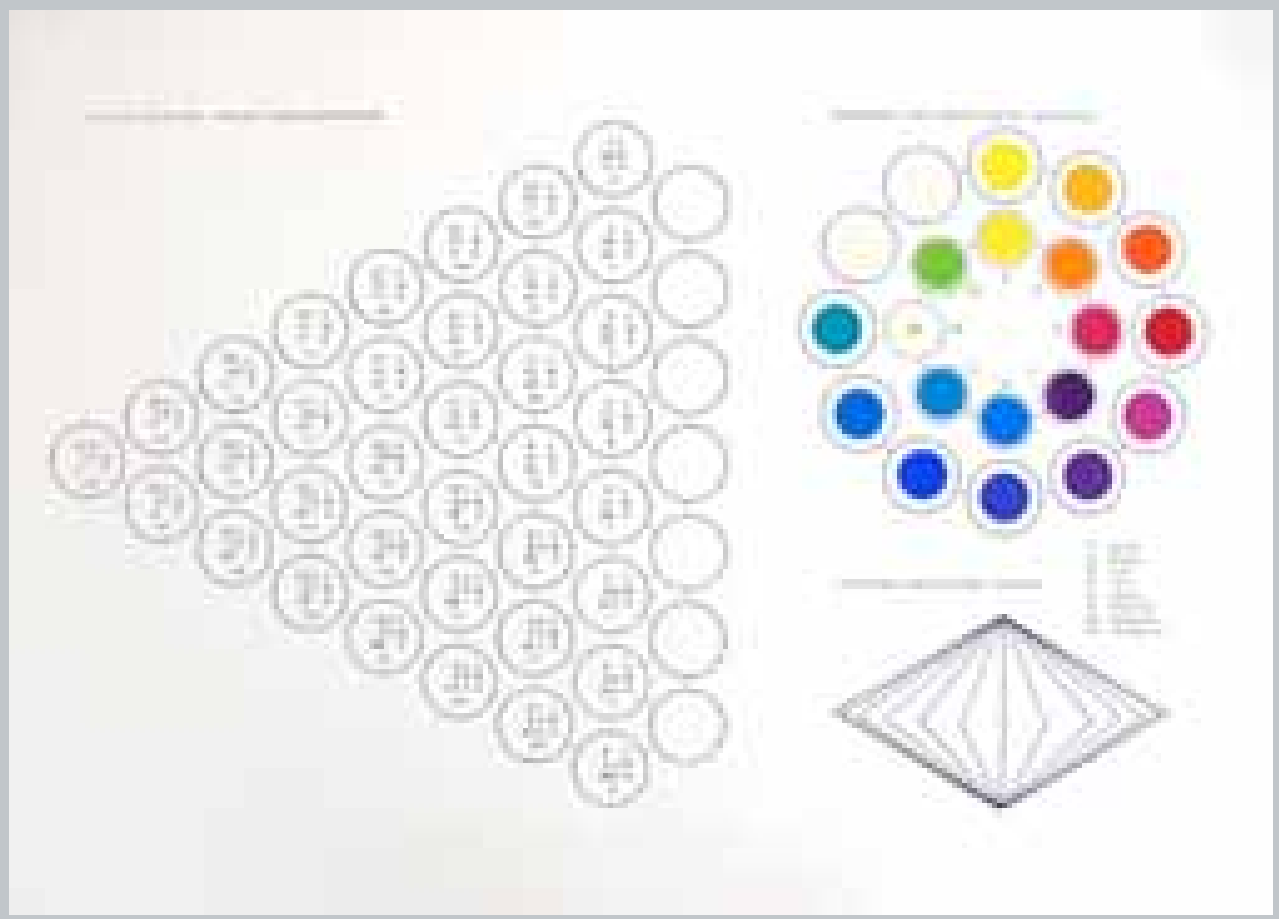

i2/16 Sólido de Ostwald.

Alumno: Ulrich Burandt.

Curso: Visuelle Einführung.

Enseñanza del color de Nonné-

Schmidt. (1956-1957)

Gs_033.038. F.B.S

Representación de la sección del doble cono de Ostwald. Comparativa en la distribución de colores primarios

y secundarios en el ecuador entre Ostwald y Munsell
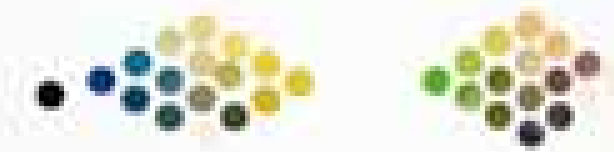

Enseñanza del color de NonnéSchmidt. (1955-1956).

Gs_038.004. F.B.S
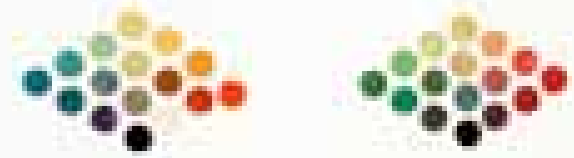

i2/17 Teoría del Color de Ostwald. Sección por el sólido de color.

Alumno: Peter Disch. 

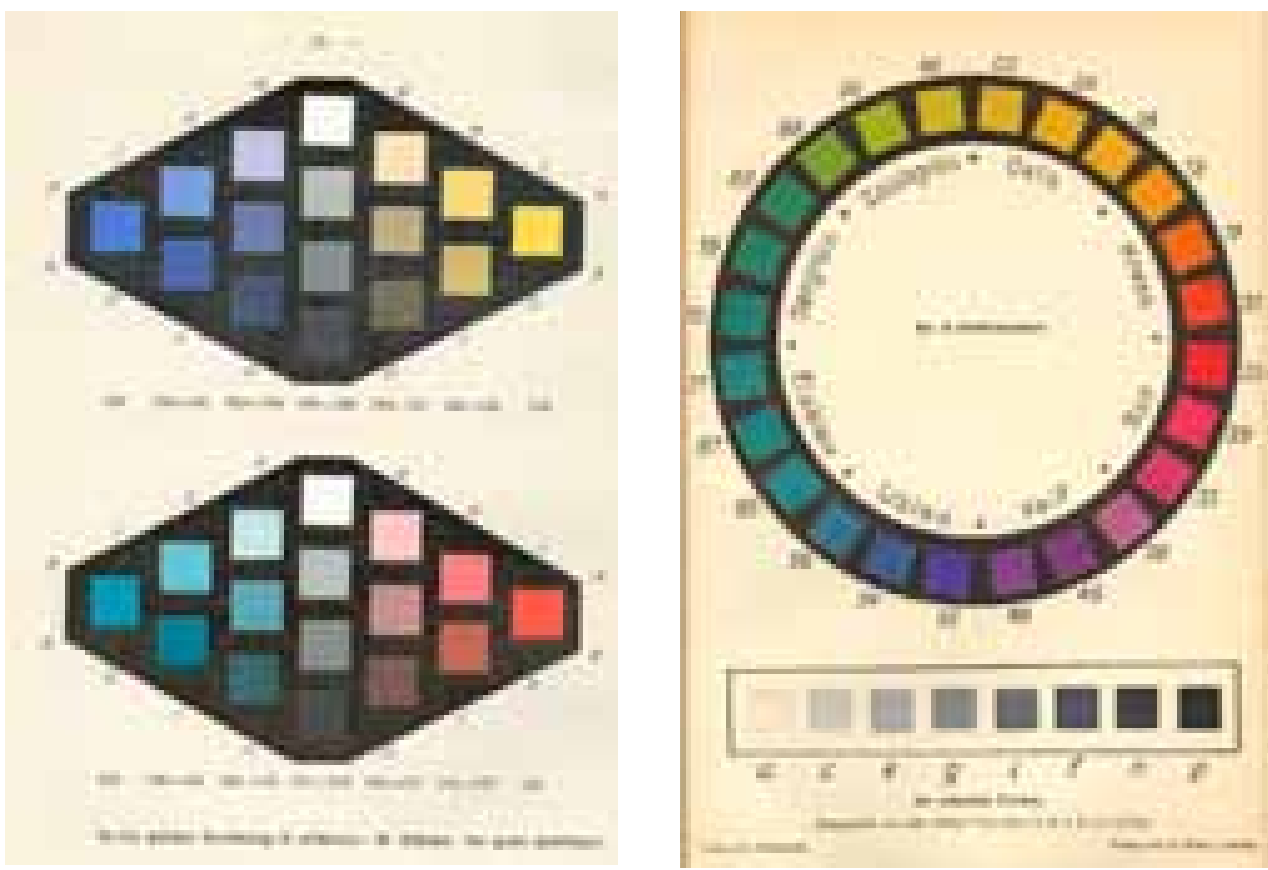

i2/18, 19 Marco teórico: Sólido de Ostwald. 24 tonos en el ecuador del sólido y eje acromático de 8 valores con su nomenclatura.

\section{Luz, oscuridad. Color. Círculo cromático}

Los esquemas del círculo elaborados por Nonné-Schimidt se centran en la organización de los colores a partir de los primarios y secundarios, frente al caso de Itten en la Bauhaus en el que se estudia la intensidad del color al incorporar el blanco y el negro (i2/5). La primera lámina recoge varios esquemas entre los que se encuentra el círculo cromático de doce tonos a partir de Goethe (i2/12). Junto a éste se representa en una franja rectangular una escala con los doce valores y un esquema gráfico y numérico con la proporción graduada de 0 a 4 de cada uno de los valores de los colores primarios que intervienen para obtener respectivamente las mezclas de los colores secundarios y terciarios. Para ello representan esquemas tanto del círculo cromático como escalas de valor del blanco al negro. ${ }^{7}$

\section{Colores primarios, secundarios, terciarios, cuaternarios}

En la siguiente lámina se realizan esquemas del círculo cromático de doce valores y se construyen triángulos en los que mediante las mezclas por veladuras de acuarelas de los colores primarios, se obtienen sucesivamente los secundarios, terciarios y cuaternarios (i2/13). Junto a éstos se representan los pares de colores complementarios y las tríadas de colores equidistantes en el círculo cromático.

\section{Sólido de color de Ostwald}

El interés por el uso del sólido de Ostwald como sistema de referencia en el que el color se ordena científicamente para su estudio se remonta a la enseñanza de Joost Schmidt en el periodo de la Bauhaus de Dessau y al "afán de normativización de la época de Dessau". ${ }^{8}$ Este ejercicio se realiza en los cursos 1955-56 y 1956-57. En las láminas se representa un dibujo esquemático del doble cono del sólido de Ostwald, junto con la distribución de colores en el ecuador del sólido y una sección del sólido limitada por el eje de acromático conocida que representa la mitad del plano de valores en forma de triángulo (i2/16, i2/17). En dicho esquema se describe la notación de cada color en función de las coordenadas. En el curso 1954-1955 se desarrollan parcialmente varios planos del sólido de Ostwald. Este ejercicio es singular en el conjunto de las disciplinas visuales en el curso básico ya que no se volverá a repetir una vez que Nonné-Schmidt abandone la HfG.
7 Durante el curso 1954-1955. Albers se refirió al circulo cromático eventualmente para tratar el problema de la "temperatura" del color. Este ejercicio se incluye en el siguiente epígrafe "teorías".

8 Britta Kaiser Schuster, op. cit. p.39. 
i2/20 Escala de luminosidad del rojo.

$$
\text { (detalle) }
$$

Alumno: Hans von Klier.

Curso: enseñanza del color de

Nonné-Schmidt. (1955-1956).

Dp_017.001. F.B.S

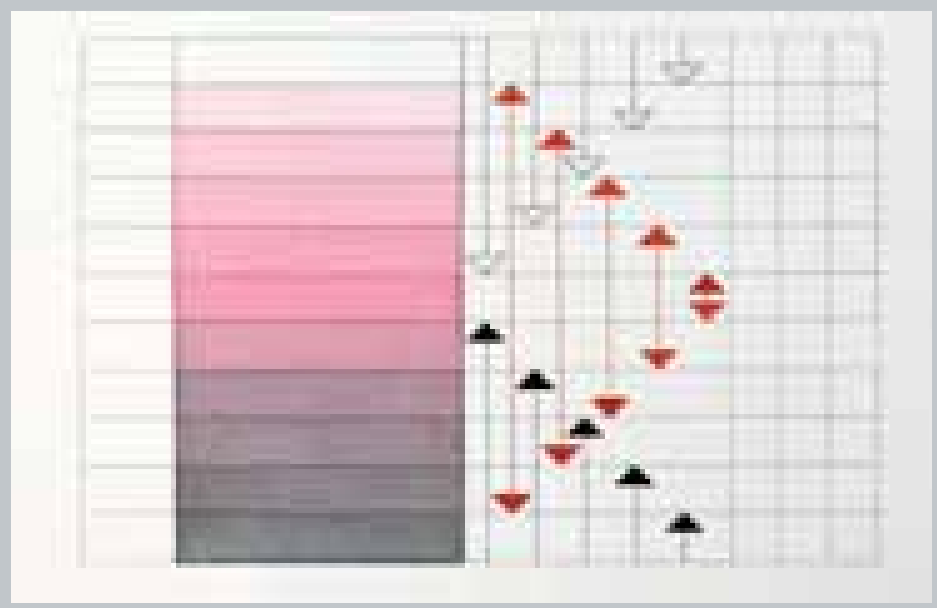

i2/21 Escalas monocromáticas

Alumno: Christoph Naske.

Curso: Enseñanza del color de Nonné-Schmidt. (1953-1954)

Dp_024.001. F.B.S

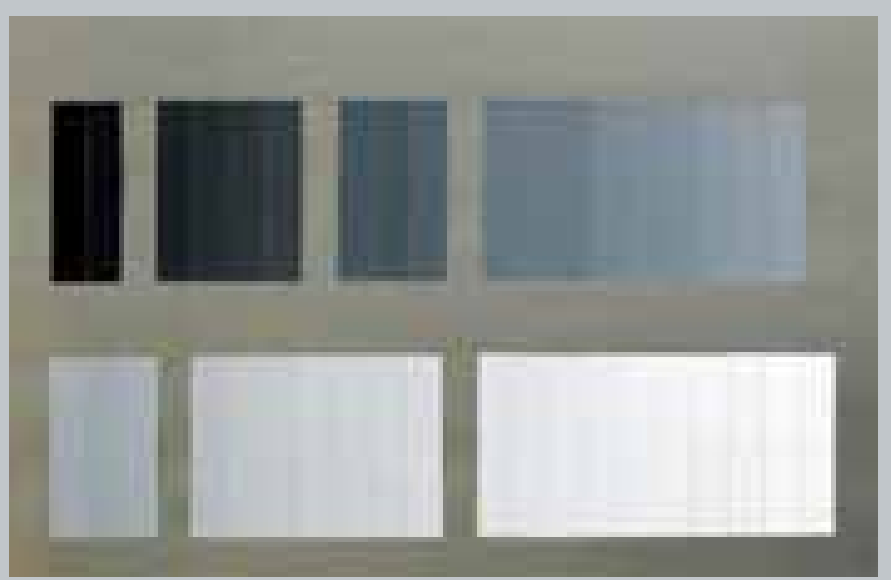

\section{i2/22 Escala de grises}

Alumno: Christoph Naske.

Curso: Enseñanza del color de Nonné-Schmidt.(1953-1954).

Dp_024.001. F.B.S

i2/23 Escala de grises

Alumno: Eric Rossicci

Curso: Enseñanza del color de Vordemberge-Gildewart (19601961) G 59. F.B.S
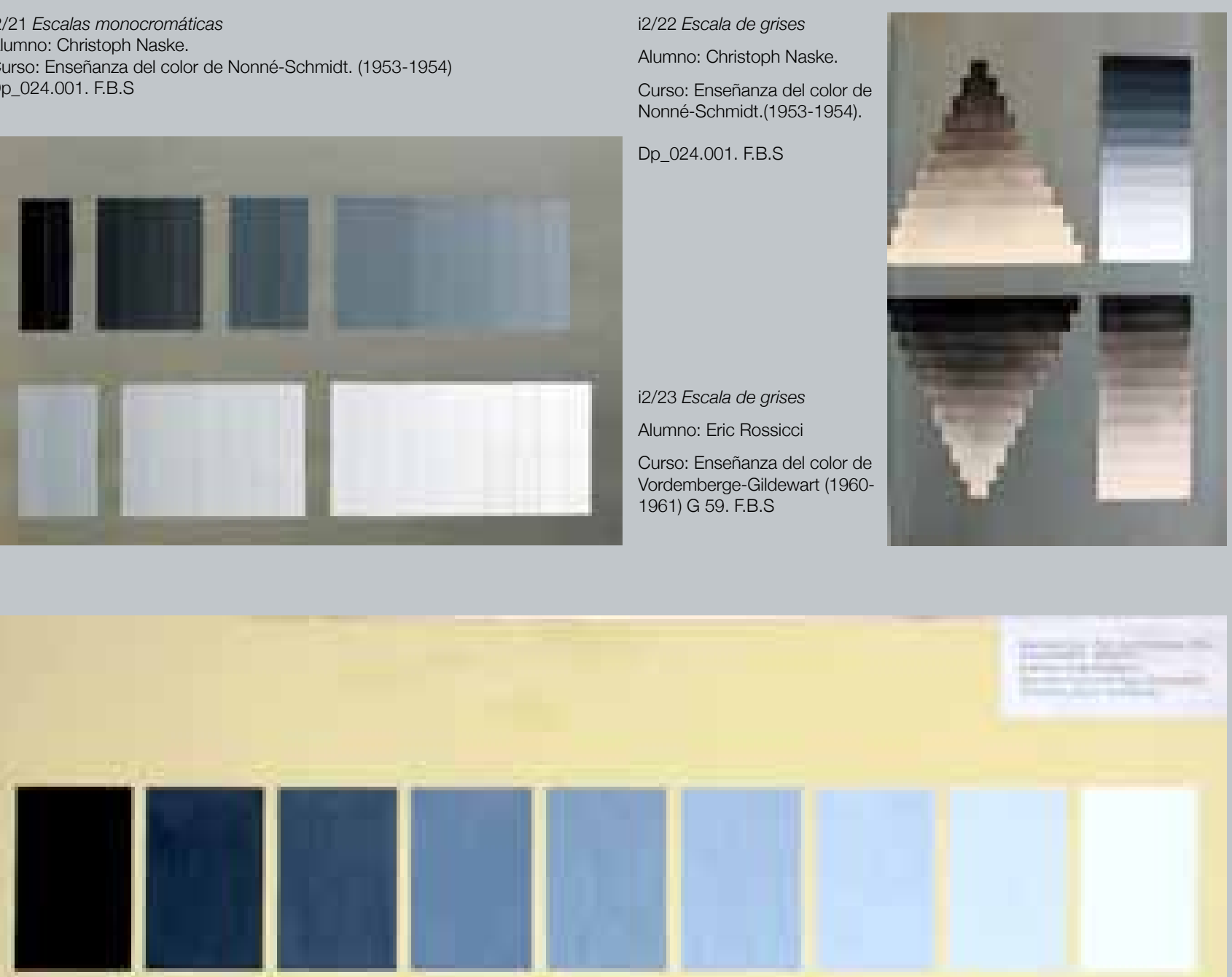

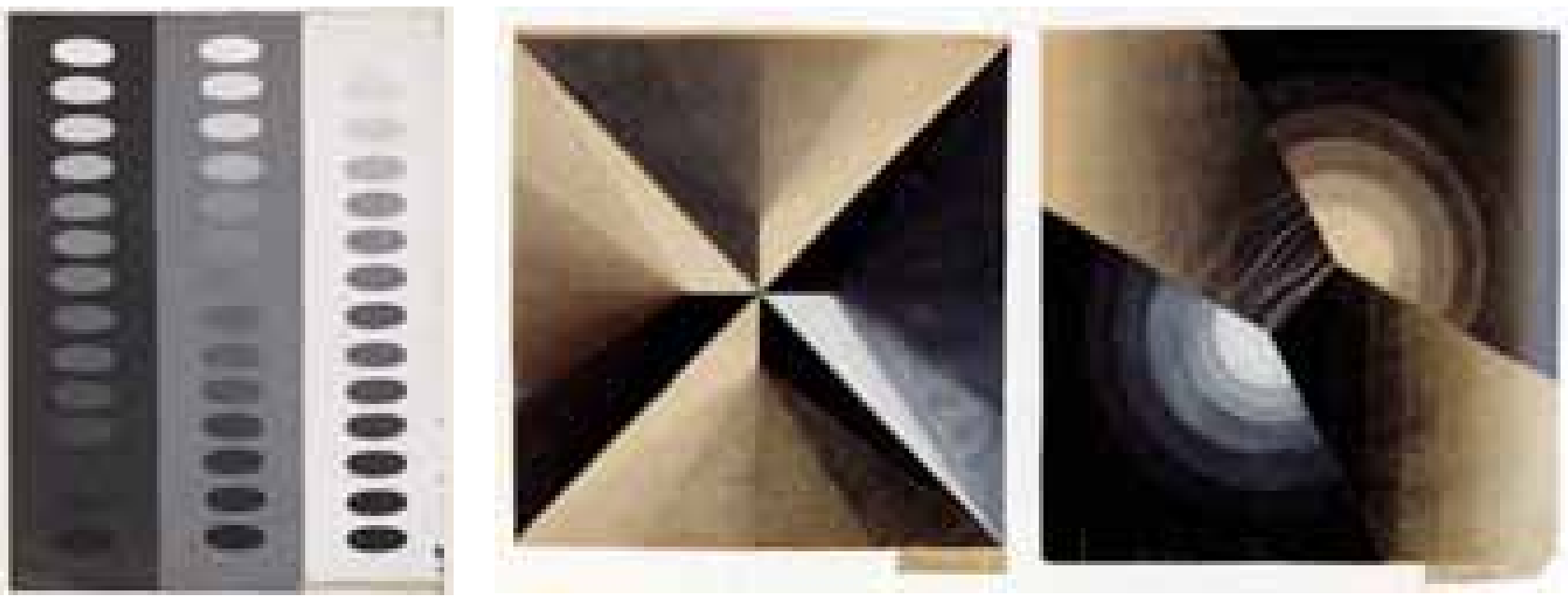

\subsubsection{Escalas}

Se propone como ejercicio la realización de escalas de intervalos con el objetivo de estudiar la relación de los colores entre sí y con el blanco y el negro. Es el segundo aspecto a tratar una vez que se conoce cómo se organiza el espectro cromático. Esta tarea en principio, no tiene tanto un enfoque pictórico como metodológico. Se trata de la utilización de unas variables partiendo de dos -blanco y negro- y su manipulación mediante distintos mecanismos como la interpolación de valores de dos colores. ${ }^{9}$ Los alumnos elaboran escalas de intervalos de colores mediante su mezcla para experimentar con sus atributos: tono, luminosidad, saturación. Se realizan escalas acromáticas del blanco al negro o monocromáticas variando la luminosidad de un color. Por otro lado las escalas policromáticas permiten experimentar la creación de colores secundarios, terciarios, cuaternarios y las mezclas de éstos entre sí. Los valores pictóricos como la transparencia, las veladuras y la saturación, se consideran a posteriori como consecuencia de la interpolación entre las variables cromáticas.

La metodología viene respaldada por el dominio de la técnica gráfica y cuenta con el soporte del estudio y referencias al modelo cromático visto con anterioridad. Muchos de los ejercicios se pueden asimilar a ejercitaciones a partir de secciones del modelo de Oswald.

\section{Escalas acromáticas y monocromáticas}

\section{Escalas acromáticas}

Las escalas acromáticas son escalas de grises que van del blanco al negro, modulando una serie de valores cuyo número varía. Las plantea Nonné-Schmidt entre 1953 y 1957 con distintas técnicas y formatos. Por su parte Vordemberge-Gildewart incluye la escala de grises de nueve intervalos como tarea inicial en su curso de color en los cursos 195960 y 1960-61. Los ejercicios elaborados por Nonné-Schmidt y Vordemberge-Gildewart se centran exclusivamente en la ejecución de la transición de valores entre el blanco y el negro. Son ejercicios cuyo objetivo es la mera representación de esta escala. No plantean otras cuestiones, desde enfoque experimentales o analíticos, relativas relación entre las distintas etapas de la escala con el comportamiento visual de los colores -escala de Hirschfeld Mack (i2/24)- o con la influencia de la técnica gráfica aplicada -escalas de alumno de Klutsis (i2/25). i2/24 Experimentos para el avanceretroceso según la variación del tono de color. Las formas mismas son idénticas en tamaño y color. 19221923. Ludwig Hirschfeld-Mak

https://www.harvardartmuseums.org/ art/225130

i2/25 V. Kolpakova y N. Kolpakova Taller de color de Gustav Klutsis. Análisis de niveles acromáticos de cuatro materiales: tinta china, gouache, tiza negra y grafito. Primer año, 1927/1928.

Procedencia del libro: IvanovaVeen L.I; Ovsiannikova E. B. From Vkhutemas to Markhi 1920-1936. Architectural projects form the Collection of the MARKhl Museum. Moscú: A-Fond Publishers 2005. Pág. 106.

9 En la teoría de Klee la escala del blanco al negro representa el movimiento arriba-abajo en el eje de la esfera de Runge. En el caso de Ostwald se trata del eje del sólido desde el valor superior blanco al inferior negro. 
i2/26 Tarea 2: escala horizontal y diagonal a partir de tres colores dados: blanco, negro y rojo.

Alumno: Eric Rosicci.

Curso: Enseñanza del color de Vordenberge-Gildewart.(1960-1961) G56. FBS.

||||||||||||||||||||||||||||

\section{||||||||||||||||||||||||||||}

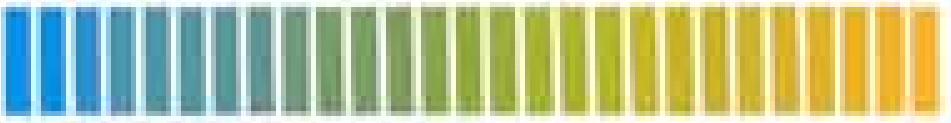

i2/27 Superposición de colores primarios, secundarios y terciarios,

Escala de 2 colores con témpera

Alumno: Urs Beutler.

Curso: Enseñanza del color de Nonné-Schmidt (1956-1957).

Dp_032.006.

FBS.

i2/28. Escalas acromáticas con acuarela.

Alumno: Klier

Curso: Enseñanza del color de Nonné-Schmidt.1(956-1957).

Dp_032.006. FBS. 

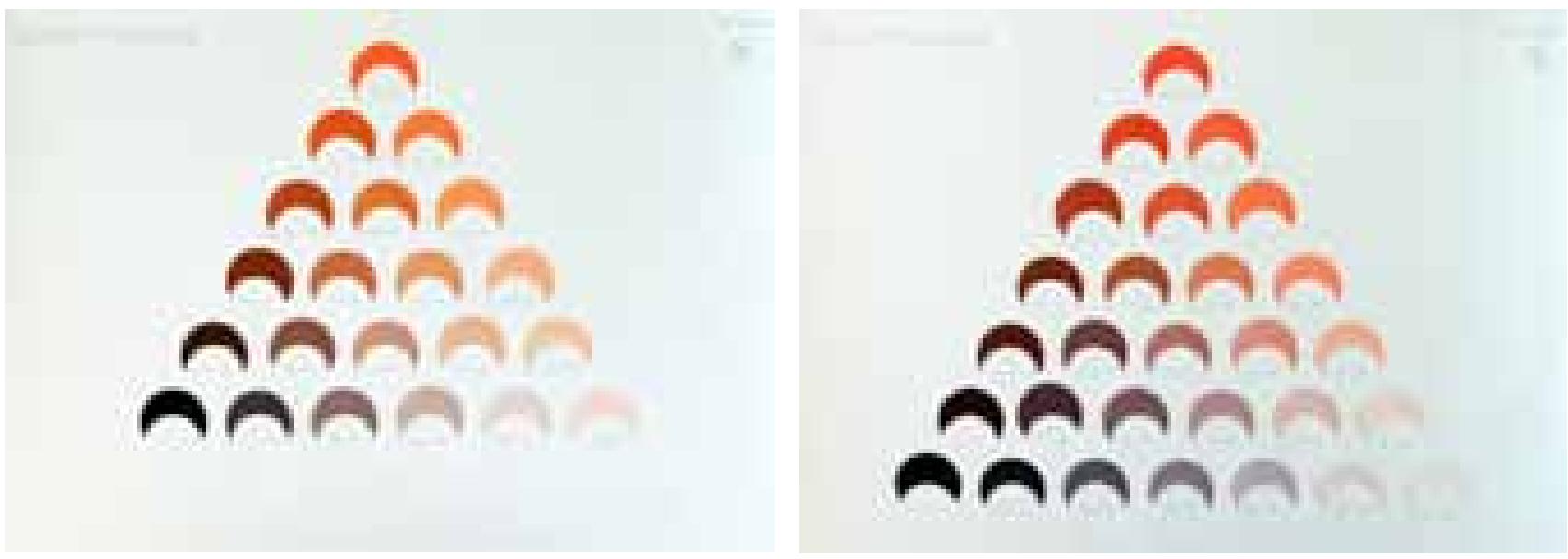

\section{Escalas monocromáticas o de luminosidad}

Las escalas de luminosidad consisten en la mezcla de un color con blanco y negro para modular su claridad u oscuridad. Nonné-Schmidt lo aborda junto a la escala cromática. En los primeros años del Grundlehre los alumnos lo realizan en láminas independientes, mientras que a partir del curso 1955-1956 estas escalas aparecen integradas junto a otros gráficos en la misma lámina.

Vordemberge-Gildewart propone en los cursos 1959-1960 y 1960-1961 el ejercicio "Escala horizontal y diagonal a partir de tres colores dados: blanco, rojo, negro". (i2/26) Para ello dispone una serie 15 de círculos de color colocados tangentes entre sí en forma triángulo equilátero. En los vértices se colorean los círculos con blanco negro y rojo. El ejercicio consiste en completar el resto de círculos interpolando los valores de los colores entre sí en sentido diagonal y en horizontal respectivamente. Si bien este ejercicio no trata explícitamente sobre la teoría del color de Ostwald su procedimiento de ejecución es bastante similar a la ordenación del triángulo del mismo tono en el sólido si los comparamos entre sí. El referente más directo a este ejercicio es Der mobile Farbkörper (i2/29, i2/30).

\section{Escalas polícromas}

Los alumnos realizan escalas utilizando varios colores de acuerdo al objetivo a desarrollar. De esta forma se proponen ejercicios para la ejecución de escalas de dos, tres y cuatro colores

\section{Escalas de dos colores}

En el curso de Nonné-Schmidt se desarrollan este tipo de escalas de dos colores en los casos de mezclas de colores primarios entre sí para obtener mezclas progresivas de los secundarios, y en las escalas entre colores complementarios para obtener los intervalos de saturación entre estos colores. (i2/27, 28)

\section{Escalas de tres colores}

La directriz para las mezclas son el círculo cromático y las mezclas sucesivas a partir del triángulo de los primarios.

En primer lugar, se realizan cuatro superposiciones de colores equidistantes entre sí según las triadas de primarios, secundarios y terciarios del círculo cromático. A continuación, en otra lámina, se realiza otra serie de seis oscurecimientos progresivos basados en seis ternas de colores. Los tres primeros oscurecimientos están formados por cada primario y sus secundarios adyacentes. Los otros tres por un secundario y los terciarios adyacentes a éste, según el esquema de oscurecimientos a partir de los colores primarios. (i2/ 30, 31)

i2/29, 30 Láminas del Album de Aemilius Mueller, Der mobile Farbkörper.

Fuente: Mueller, Aemilius. Der mobile Farbkörper. 743. Zur vermittlung allgem. Winterthur, Chromos Verlag, 1953.

Aemilius Muller había asisitido como profesor del Curso básico del 195455 después de la publicación de su teoría, en un seminario de breve duración, al igual que J. Itten.

El vértice está coronado por un color que aparece consignado con un número en la parte derecha de la página. Aparecen después los gradientes hacia el negro y el blanco, en los vértices opuestos a través de 10 o 12 pasos. Bajo los cartones, que pueden moverse, aparece la proporción de mezcla de blanco o negro sobre el color, siendo 0-0 el color, 12-0 (10-0) el más oscuro y 0-12 (0-10) el más claro. Esta progresión en el color está relacionada con el sólido de Ostwald. 


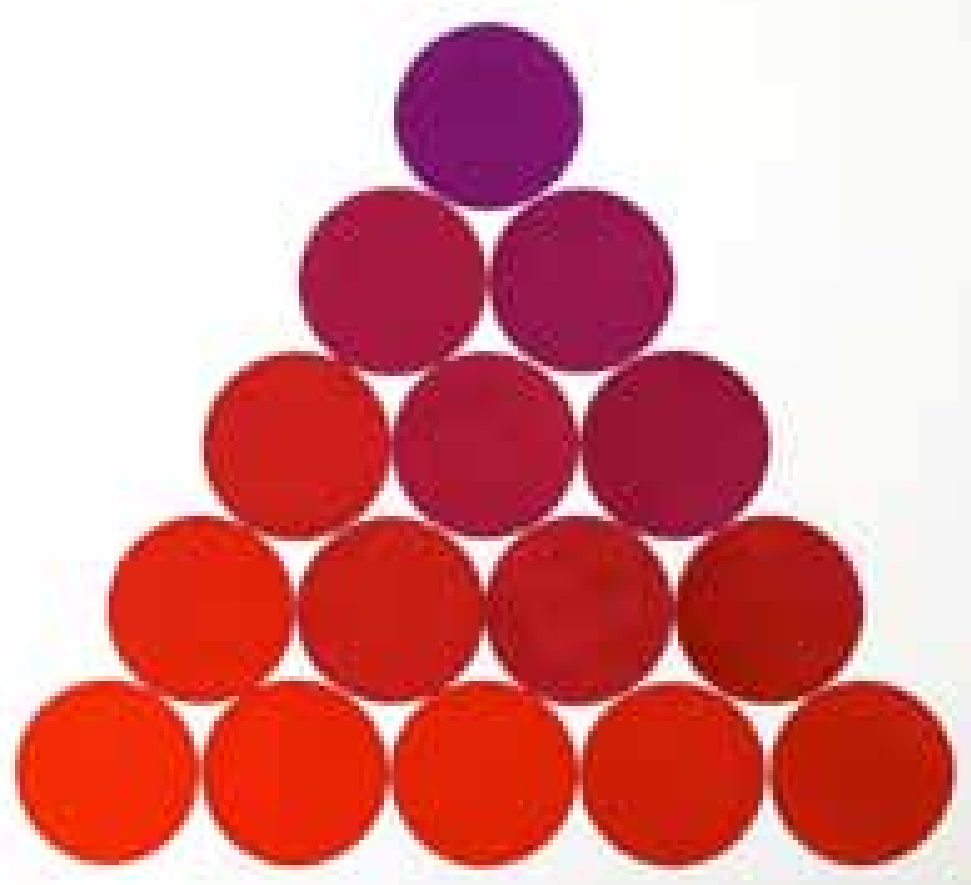

i2/31 Tarea 4: escala horizontal y

diagonal a partir de tres colores

dados: bermellón, carmín, púrpura.

Alumna: Erika Fortner.

Curso: Enseñanza del color de

Vordenberge-Gildewart (1959-1960)

Gs_061.023. FBS.

i2/32 Tarea 4: escala horizontal y diagonal a partir de tres colores dados: rojo, amarillo, azul.

Alumno: Hans T. Kurtenbach.

Curso: Enseñanza del color de Vordenberge-Gildewart (1959-60).

Gs_061.023. FBS. 


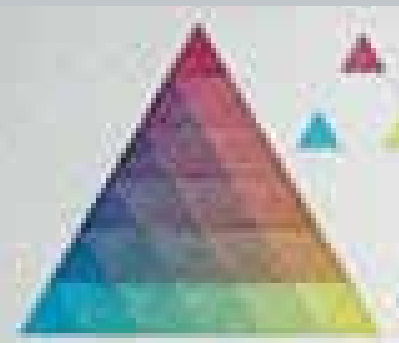

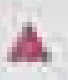

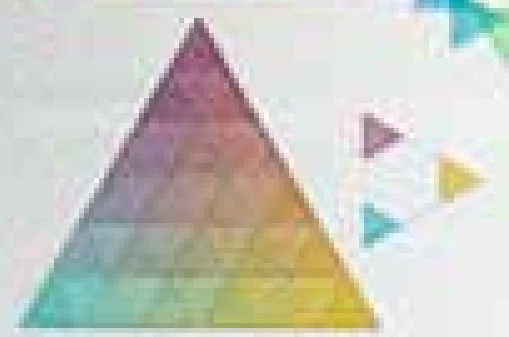

A.

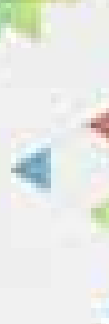

i2/33 Escalas de tres colores: color rojo, anaranjado y amarillo con el azul

Alumno: Klaus Krippendorff Curso: Enseñanza del color de Nonné-Schmidt (1956-1957).

Dp_018.023

i2/34 Superposición de triadas.

Alumno: Ulrich Burandt.

Curso: Enseñanza del color de Nonné-Schmidt. (1956-1957).

Gs_033.038.FSB 

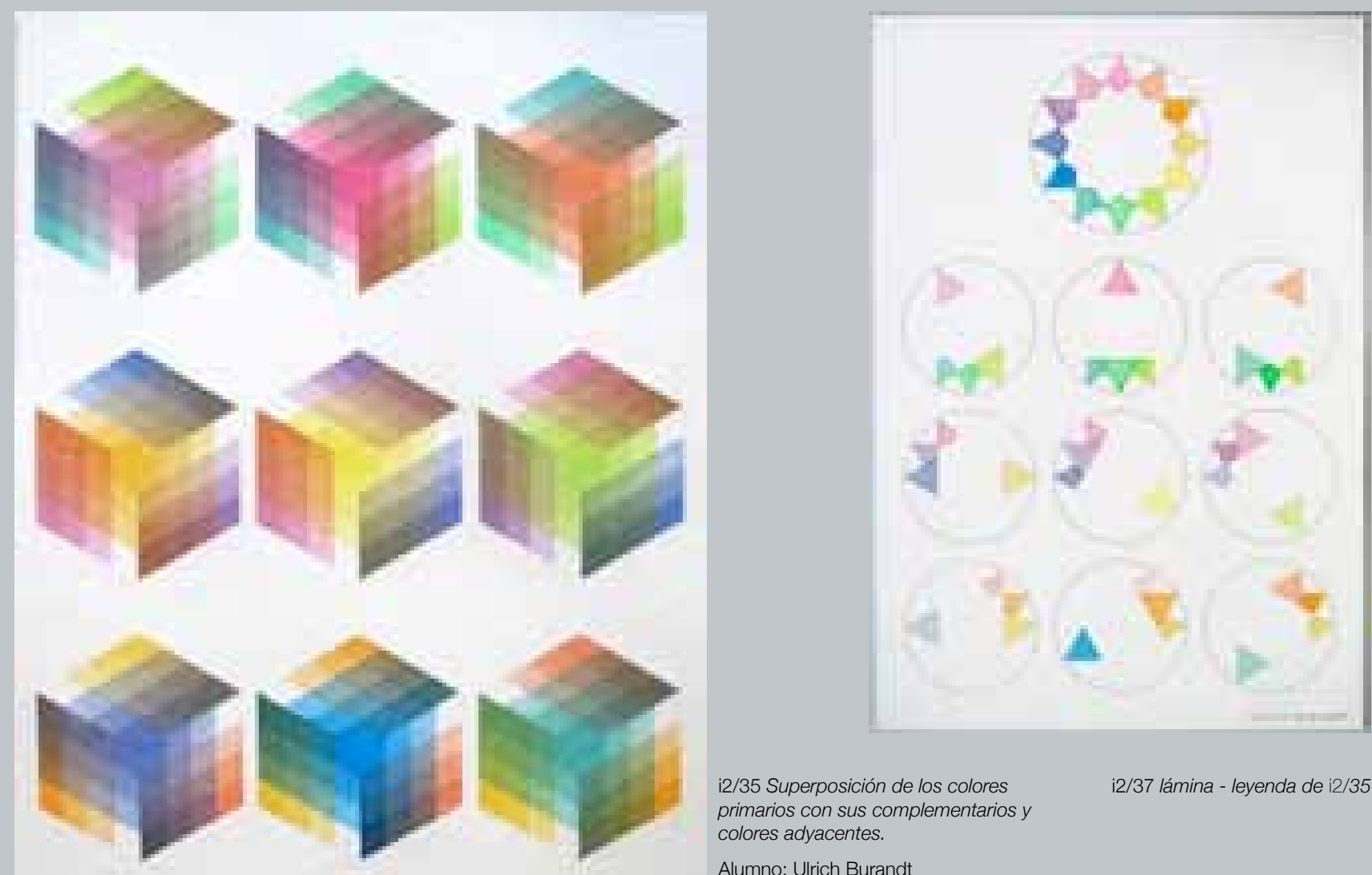

i2/35 Superposición de los colores primarios con sus complementarios y colores adyacentes.

Alumno: Ulrich Burandt

Curso: Nonné-Schmidt (1956-57).

Gs_033.038

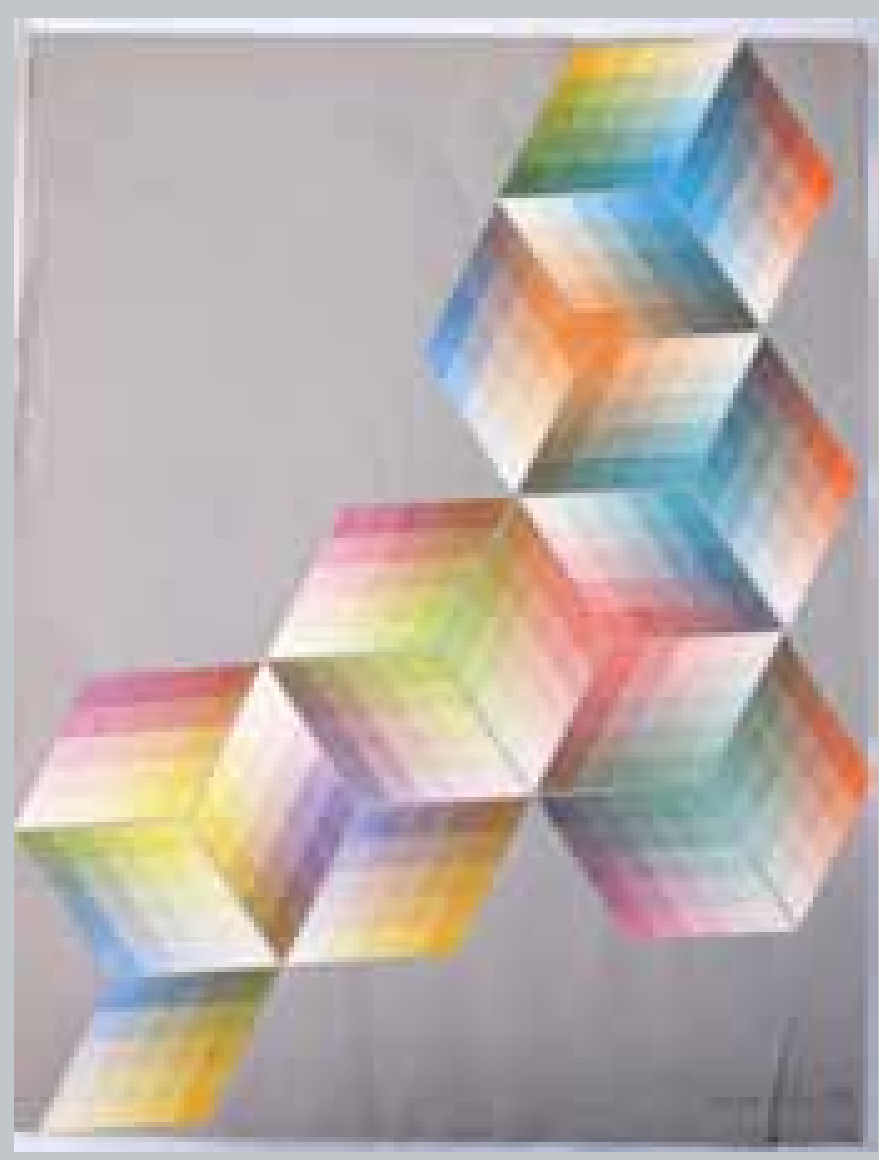

\section{i2/36 Superposición de los colores}

primarios con sus complementarios y

colores adyacentes.

Alumno: Müller Kuhn

Curso: Nonné-Schmidt (1956-57).

Dp 066.004 
Vordemberge-Gildewart aborda esta variante en los cursos 1959-60 y 1960-61 en las tareas 4 y 3 , numeradas así según cada curso, "Escala horizontal y diagonal a partir de tres colores dados". (121/31, 32).

Como en el caso análogo anterior, se trata de obtener intervalos interpolando en sentido horizontal y diagonal. Los resultados de estos ejercicios permiten apreciar la distribución de intervalos con colores opacos - frente a los ejercicios de Nonné-Schmidt - a partir de la triada de primarios o de una terna de colores afines.

\section{Escalas de cuatro colores}

Este tipo de escalas son tratadas únicamente en el curso de Nonné-Schmidt, basados en ejercicios de su marido. En este caso, los estudiantes se sirven de una superficie hexagonal compuesta por tres rombos, en el centro de la cual se ubica el color. Los colores complementarios correspondientes se disponen respectivamente encima del color central mediante sucesivas veladuras. En cada caso el rombo superior muestra una escala de grises en dirección horizontal, con esta condición el conjunto de rombos adopta la apariencia de un cubo cuyas caras contienen los degradados de color (i2/35, i2/36)

El ejercicio principal se acompaña de una segunda lámina en la que se representa los correspondientes esquemas de combinación entre colores complementarios y adyacentes. Este ejercicio puede considerarse de naturaleza pictórica en el sentido del estudio de las armonías entre colores (i2/37). 


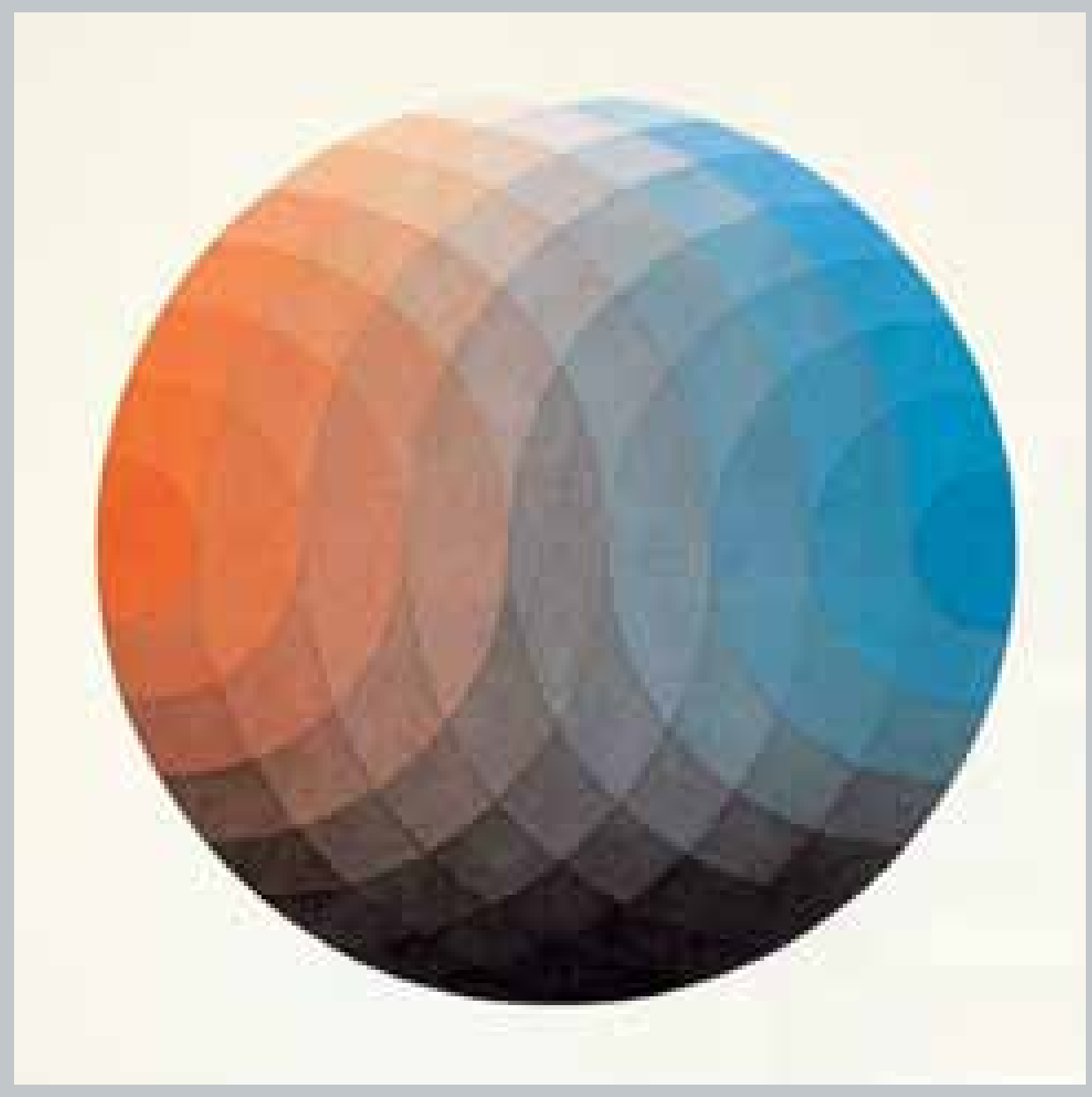

i2/38 Sección de una esfera de color. (Ejercicio realizado a partir del sólido de Ostwald).

Alumno: Hellmut Müller-Kuhn.

Enseñanza del color de NonnéSchmidt. (1953-54)

Deposita 066 F.B.S

i2/39 Oscurecimientos de los colores con blanco y negro. (secciones del sólido de Ostwald)

\section{Alumno: Hans von Klier.}

Curso: Enseñanza del color de Nonné-Schmidt. (1955/1956)

Dp_017.001. FBS i2/40 Oscurecimientos de los colores con blanco y negro. (secciones del sólido de Ostwald) -variante en la disposición respecto de $\mathrm{i} 2 / 39$ -

\section{Alumno: Beutler}

Curso: Enseñanza del color de Nonné-Schmidt. (1956/1957)

Dp_032.006 F.B.S

\section{9.}

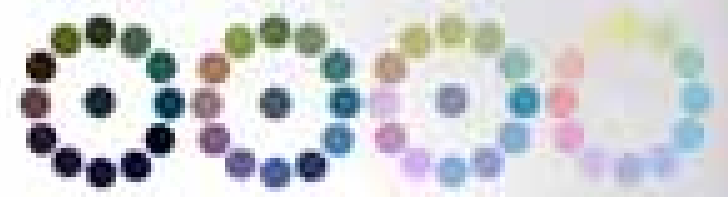

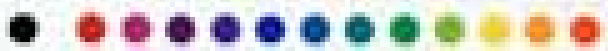

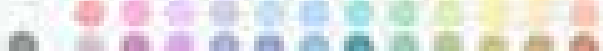

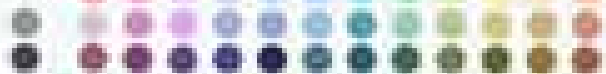
8.898986898 

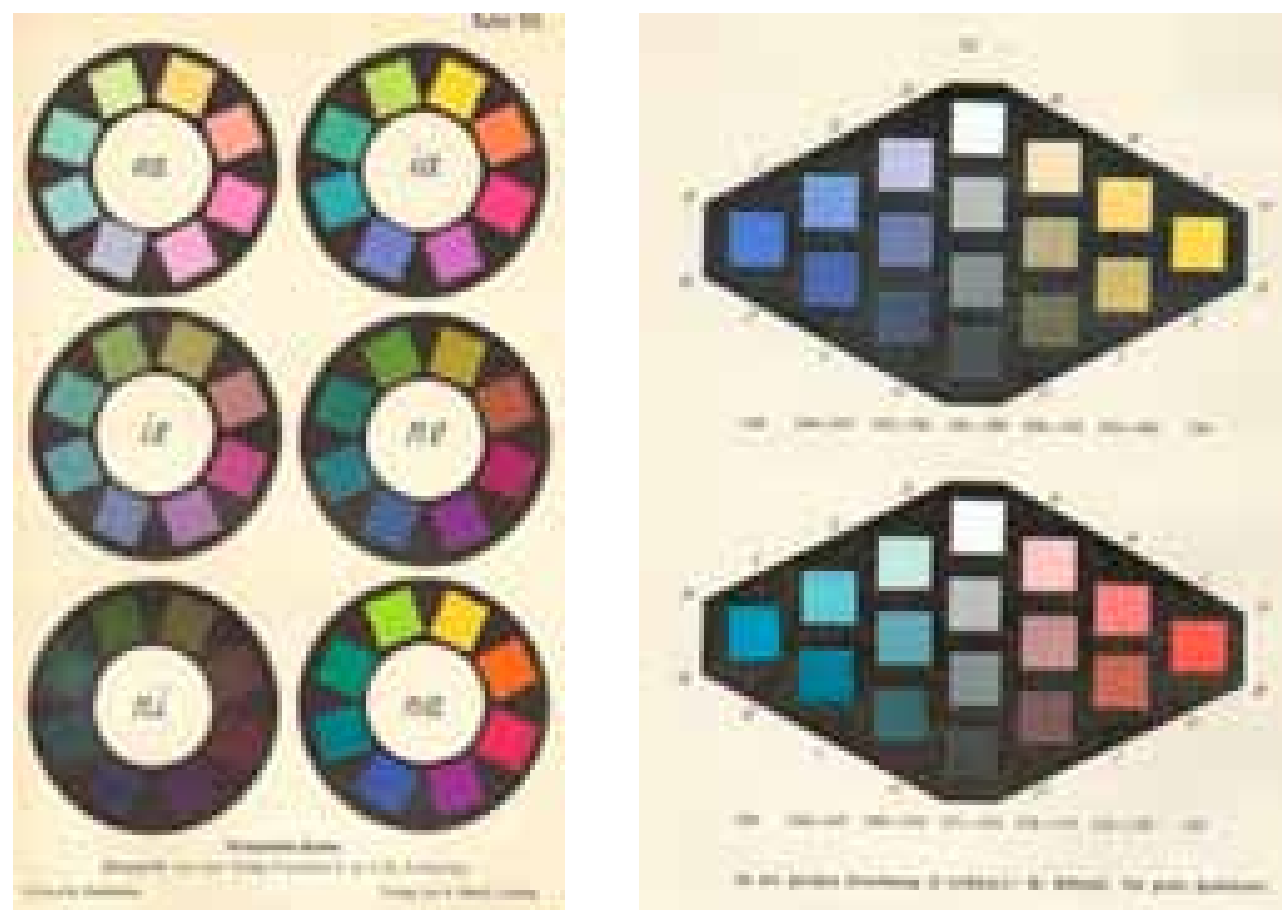

i2/41, 42 Sólido de Ostwald.

Círculos del mismo valor

\section{Escalas de colores con blanco y negro}

Este tipo de ejercicios propone la organización de escalas de color en el que intervienen el blanco y el negro. En el curso de Nonné-Schmidt encontramos tres casos. En el primero de ellos se realiza el oscurecimiento progresivo del espectro cromático aplicando una escala de grises (i2/39, i2/40). Esto puede considerarse como una aplicación de la teoría de color de Ostwald que se correspondería con la representación simplificada de los círculos del mismo valor del sólido.

En el segundo caso se puede interpretar de nuevo como una aplicación de la teoría de Ostwald, como representación del perímetro envolvente de una sección del sólido. La disposición de los intervalos de color se realizan formando circunferencias o bandas en los que realizan la transición que describe el borde de la sección del sólido: del blanco al color puro, de éste al negro; del negro al complementario del anterior y, finalmente de nuevo hacia el blanco (i2/43, i2/45). Un ejercicio análogo es la sección del sólido de Ostwald con la forma de una esfera mediante la superposición de veladuras de acuarela (i2/37).

En el tercer caso encontramos dos ejemplos de oscurecimiento con blanco y negro que podemos considerar como experimentos a partir de la teoría de Ostwald, más que aplicaciones en sí. Hans von Klier en el curso 1955-56 realiza un "Experimento para determinar el efecto de diferentes valores de brillo en los pares de colores complementarios" (i2/44).

Finalmente, en la enseñanza del color de los cursos finales del Grundlehre, Vordemberge propone en su curso la mezcla en diagonal de un color con blanco y negro análoga a la anterior. 

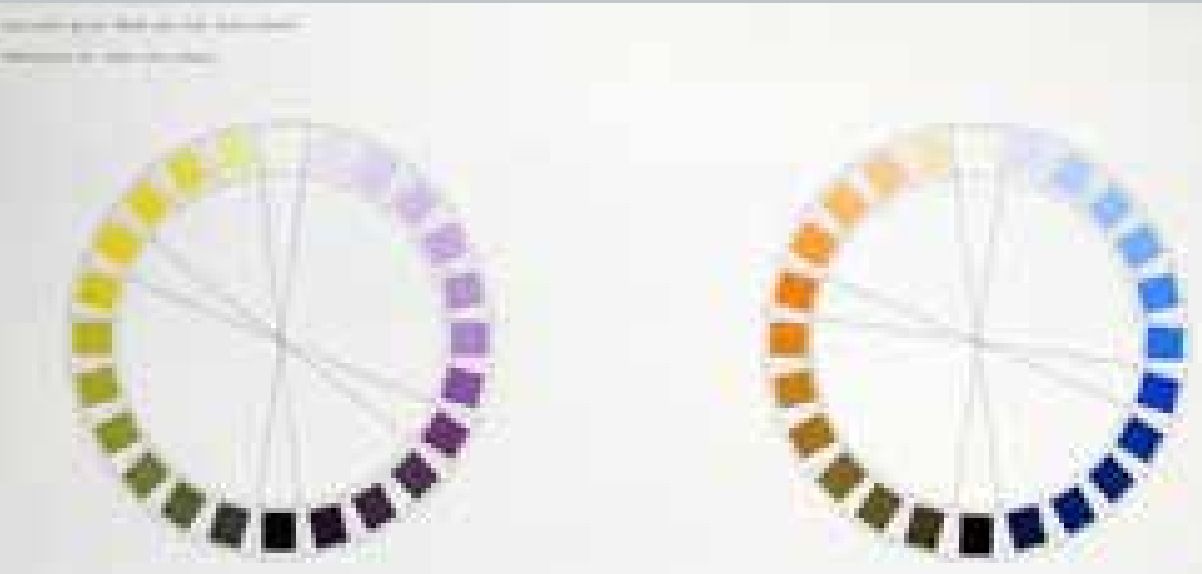

i2/43 Escalas de color con blanco y negro

Alumno: Beutler

Curso: Visuelle Einführung.

Enseñanza del color de Nonné-

Schmidt. (1956-1957).

Dp 032.006 F.B.S

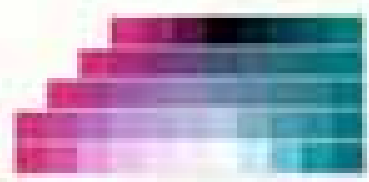

complementarios.

\section{Alumno: Hans von Klier.}

Curso: Enseñanza del color de

Nonné-Schmidt. (1955-1956)

Dp_017.001. FBS
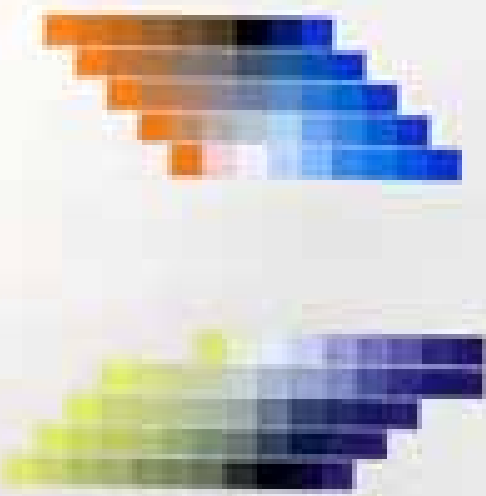


$$
\text { ח }
$$




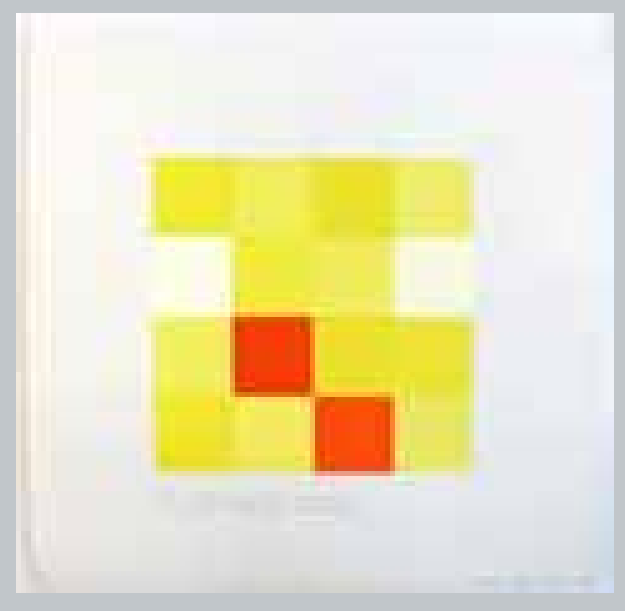

i2/46 Contraste claro oscuro amarillo - oscuro amarillo -

rojo expansión en vertical.

Alumno: Max Graf

Curso: Itten (1954-1955)

Dp 012.003 F.B.S

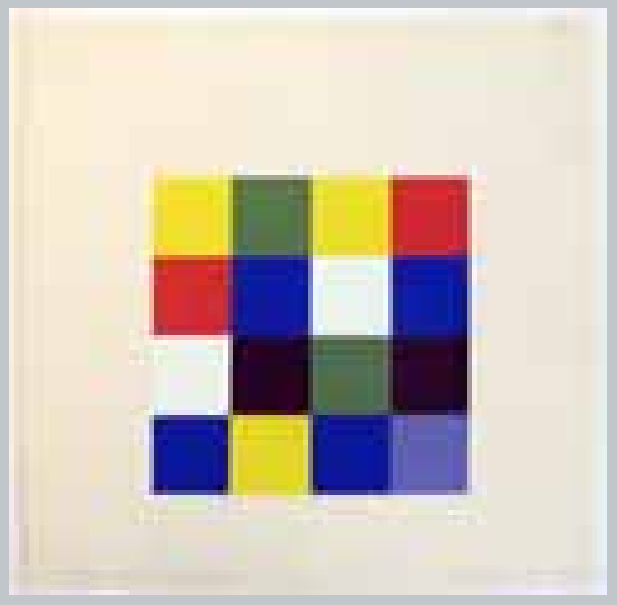

i2/47 Contraste color en símismo

Alumno: Hans von Klier.

Curso: Enseñanza del color de Nonné-Schmidt (1955-1956) Dp 012.003 F.B.S

i2/48 Contraste frio-cálido claro y oscuro, azul-rojo

Alumno: Max Graf

Curso: Itten (1954-1955)

Dp 012.003 F.B.S

i2/49 Contraste frío-cálido con colores fríos

Alumno: Hans von Klier

Curso: Enseñanza del color de Nonné-Schmidt. (1955-1956) Dp 012.003 F.B.S 


\subsubsection{Teoría de contrastes}

\author{
El problema estético de los colores se puede contemplar desde un triple punto de \\ vista: \\ sensible y óptico (impresión del color), psíquico (expresión del color), intelectual y \\ simbólico (construcción del color) ${ }^{10}$
}

Si atendemos a la brevedad temporal del paso de Itten por la HfG, su participación puede considerarse como una anécdota en la historia de la escuela, sin embargo, su contribución a la enseñanza del color, fue significativa en lo que concierne a la enseñanza de la oposición entre colores basados en su teoría general de contrastes. Itten define el contraste "cuando se puede constatar entre dos efectos de colores que se comparan unas diferencias o unos intervalos sensibles"11. Para Itten, la ejercitación en el color para llegar a alcanzar su dominio, pasa por la experimentación de las infinitas combinaciones y divergencias que existen entre todos los colores. La singularidad de la enseñanza de Itten es relevante en la HfG porque la noción de contraste no se aborda en los cursos de Nonné-Schmidt ni de Albers.

Durante el seminario intensivo que imparte en la $\mathrm{HfG}$ los alumnos trabajan tres de los siete tipos de contrastes que recoge en su libro Kunst der Farbe. En su manual, Itten define siete tipos de contrastes en función del parámetro a considerar: 1. contraste del color en sí mismo, 2. contraste de claro-oscuro, 3. contraste frío-cálido, 4. contraste entre complementarios, 5. contraste simultáneo, 6. contraste cualitativo y 7 . contraste cuantitativo. En la HfG se realizan ejercicios sobre los tres primeros tipos.

Itten define el contraste del color en si mismo como un recurso habitual en la pintura en todas sus épocas, presente en el arte popular -trajes, cerámicas, bordados -. Atribuye a este tipo de contraste la capacidad de representación de un amplio espectro de emociones vitales, desde la la vida bulliciosa y el brote de una fuerza luminosa hasta la tristeza más profunda. $^{12}$

El contraste claro-oscuro estudia la gradación entre los dos extremos más poderosos de la expresión pictórica: el blanco y el negro, entre el negro más negro -terciopelo puro- y el blanco más blanco -sulfato de barita-. El escalonamiento depende de la agudeza visual del que lo ejecuta, por lo que su ejercitación está orientada al fomento de la sensibilidad de los alumnos. Las escalas de contraste entre el claro y el oscuro también se aplican a las escalas de color. En la HfG Itten trabaja el contraste de claro a oscuro con escalas de color mientras que en el Vorkurs Bauhaus, ${ }^{13}$ este trabajo se lleva a cabo con escalas de tono entre el gris más claro y el negro más profundo (i2/50).

Los ejercicios de contraste frío-cálido ${ }^{14}$ trabajan gradaciones de color de un mismo tono -por ejemplo, rojo-anaranjado- o azul-verde que tienen aplicación en la representación de la perspectiva, la lejanía o proximidad y la textura.

Itten establece varios métodos para trabajar con el contraste; el contraste de color en las categorías que se han mencionado y, en un segundo término, introduciendo la variable de la proporción que añade la complejidad de la cantidad de color. De esta forma, encontraremos dameros de superficies cuadradas de contrastes y tiras con rectángulos de diferentes proporciones.

El Itten que llega a la $\mathrm{HfG}$ es un artista y docente maduro que ha llegado a formular su propia teoría sobre el color, treinta años después de su docencia en la Bauhaus. Por tanto, la docencia en la HfG es en mayor medida el resultado de la decantación de unos saberes por el paso del tiempo y su experiencia docente más que la importación de un curso de la Bauhaus.

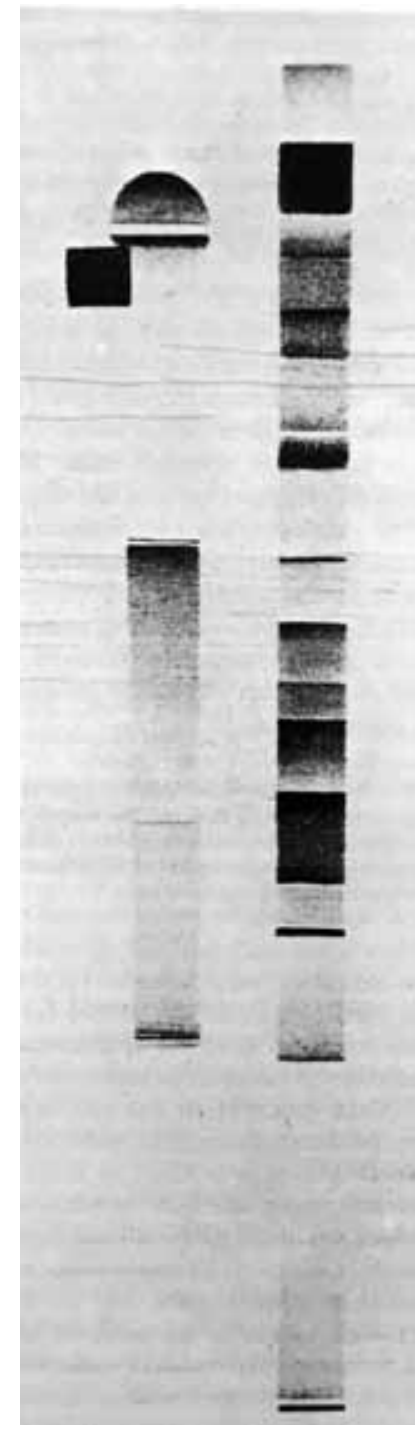

i2/50 Contraste claro-oscuro. Ejercicio del curso preliminar de Itten en la Bauhaus de Weimar, 1927.

10 Itten, Johannes. Kunst der Farbe, Ravensburg: Otto Maier, 1962.

11 ĺbid, p.33.

12 Íbid, p.36

13 Itten imparte docencia en la Bauhaus de Weimar hasta 1923. Wick, Reiner. Op. cit. p. 91.

14 Por su parte, Albers propone un ejercicio en el que estudia la idea de temperatura de color desde la confrontación de pares de colores entre si (i2/55). 
Curso: Itten (1954-1955)

Dp 012.003 F.B.S
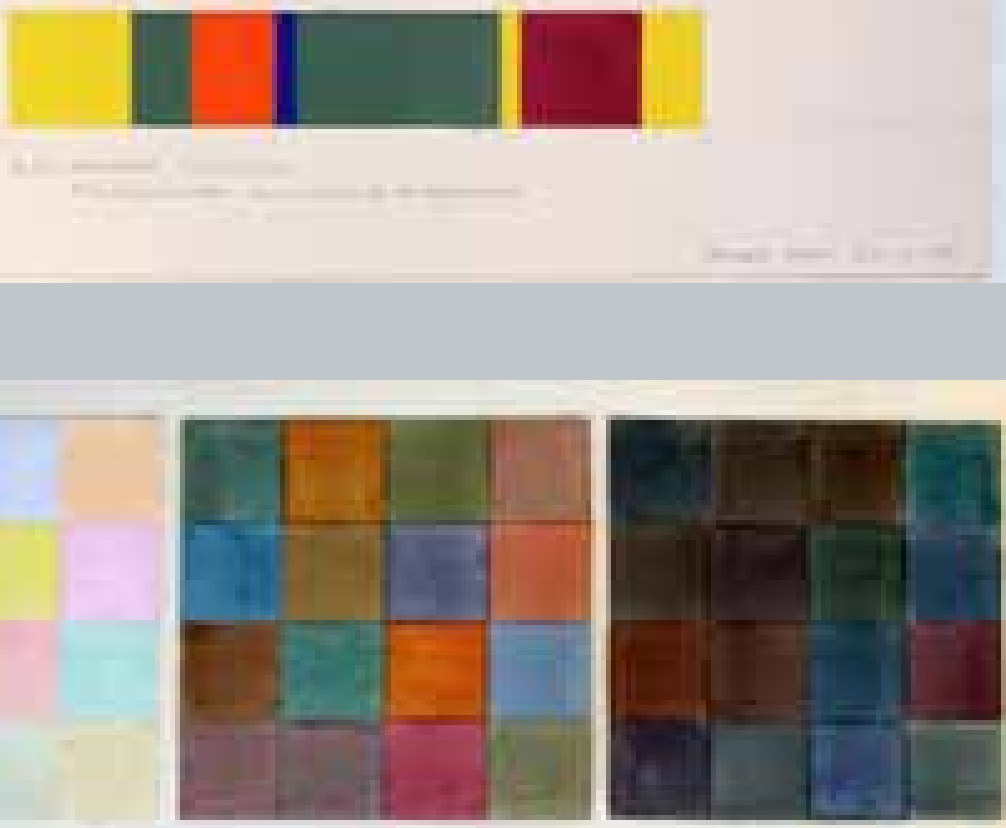

luminosidad

Alumno: Max Graf

Curso: Itten (1954-1955)

Dp 012.003 F.B.S

i2/53 Claro-oscuro amarillo-azul en expansión horizontal y vertical Alumno: Max Graf

Curso: Itten (1954-1955)

Dp 012.003 F.B.S

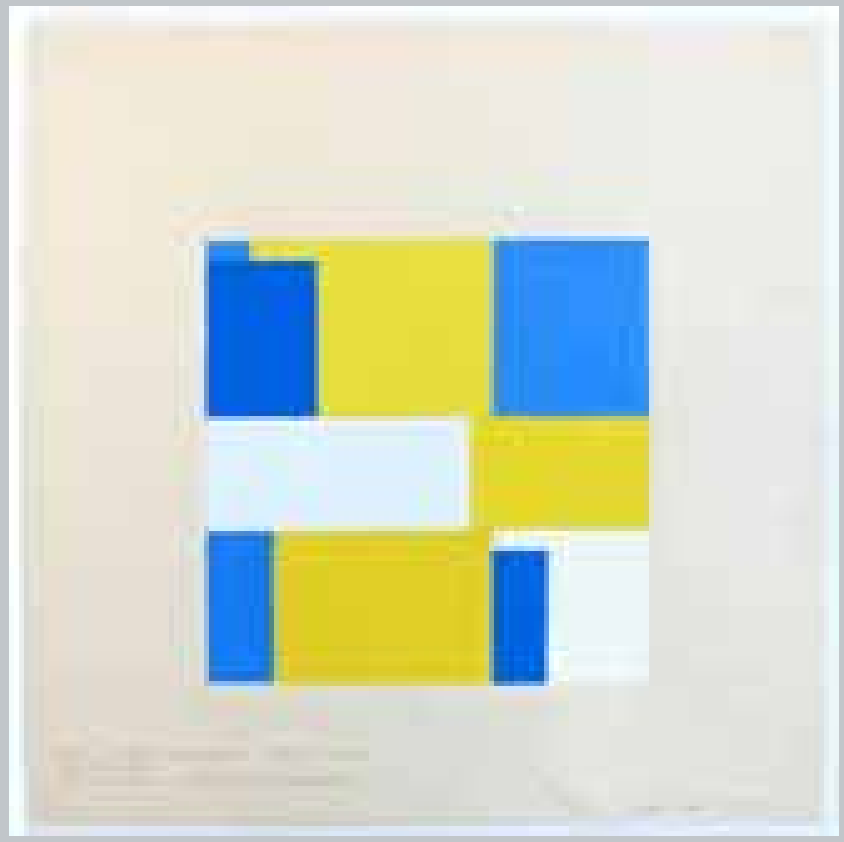

i2/54 Claro-oscuro con color y líneas negras

Alumno: Max Graf

Curso: Itten (1954-1955)

Dp 012.003 F.B.S

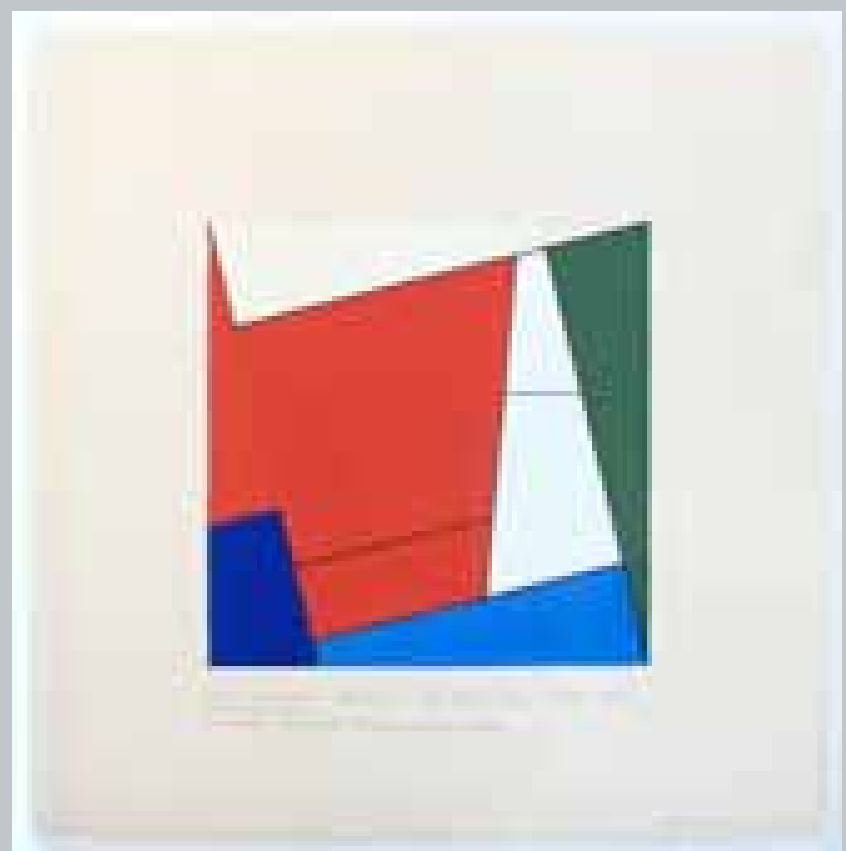




\section{$\square$}
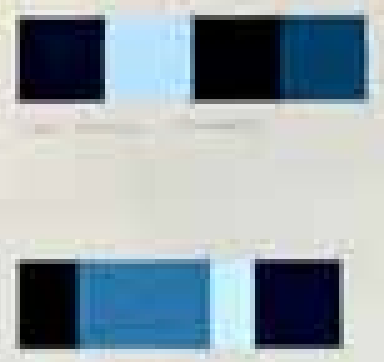

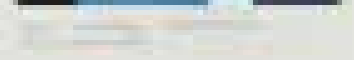
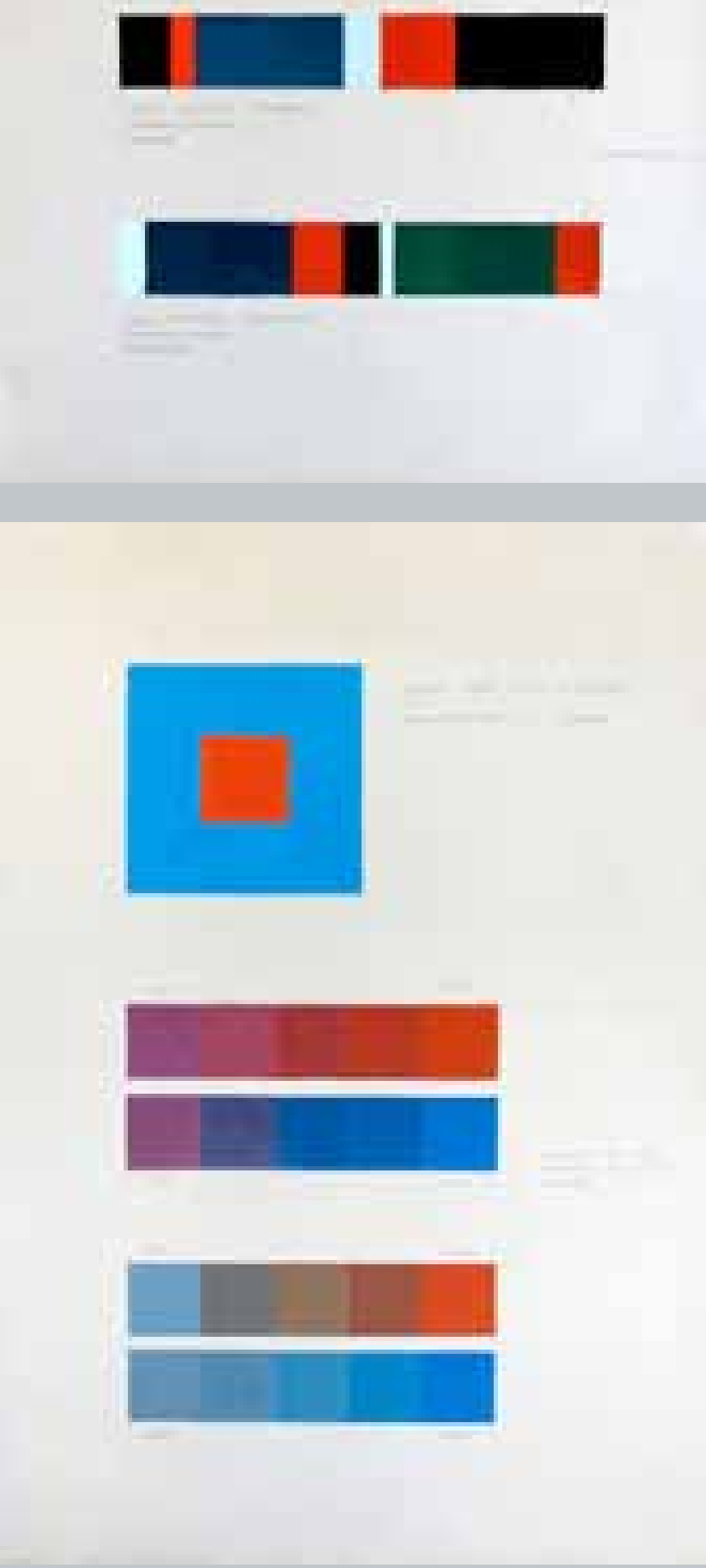

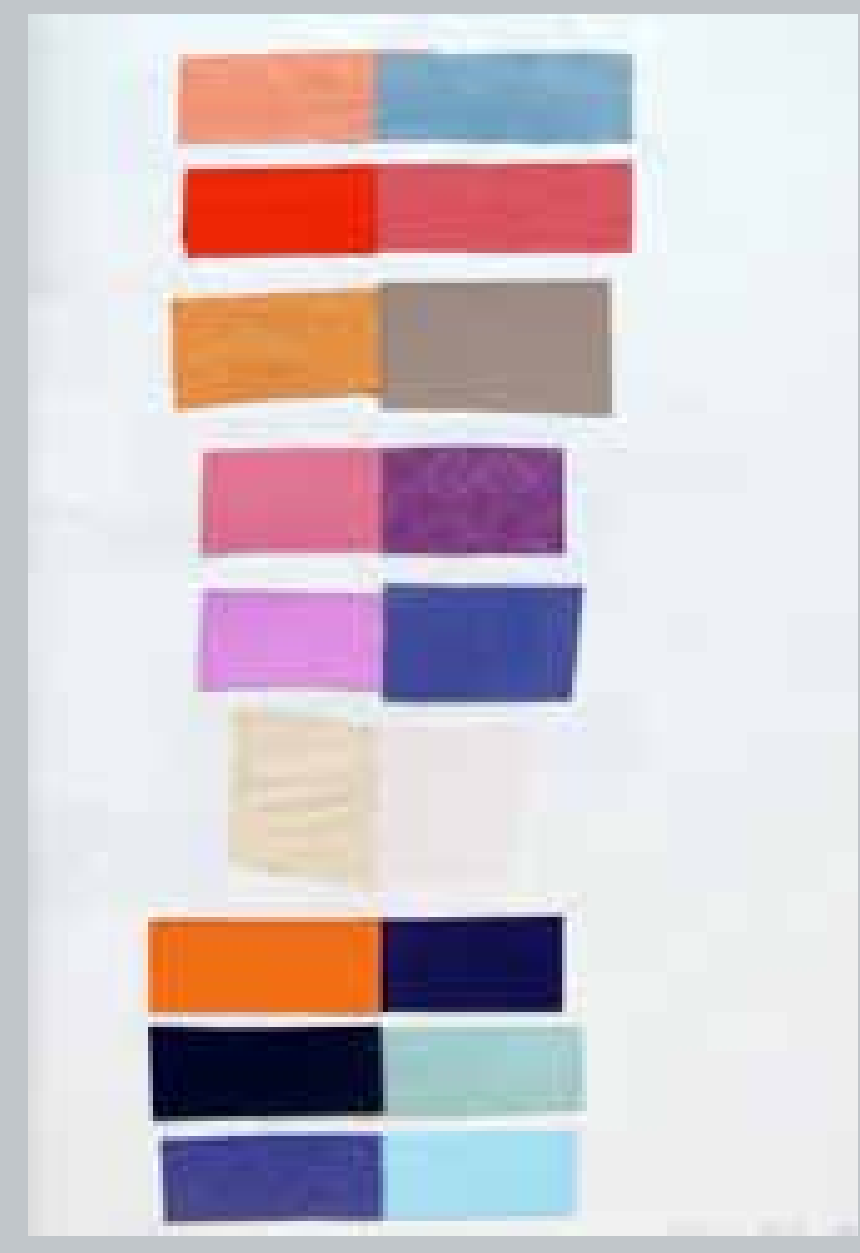

i2/55 Claro-oscuro con uno y dos colores.

Alumno: Max Graf

Curso: Itten (1954-1955)

Dp 012.003 F.B.S

Temperatura con color Alumno: Max Graf

Curso: Albers (1954-1955) Dp 012.003 F.B.S i2/57 Contraste frío-cálido

Alumno: Max Graf

Curso: Itten (1954-1955)

Dp 012.003 F.B.S 


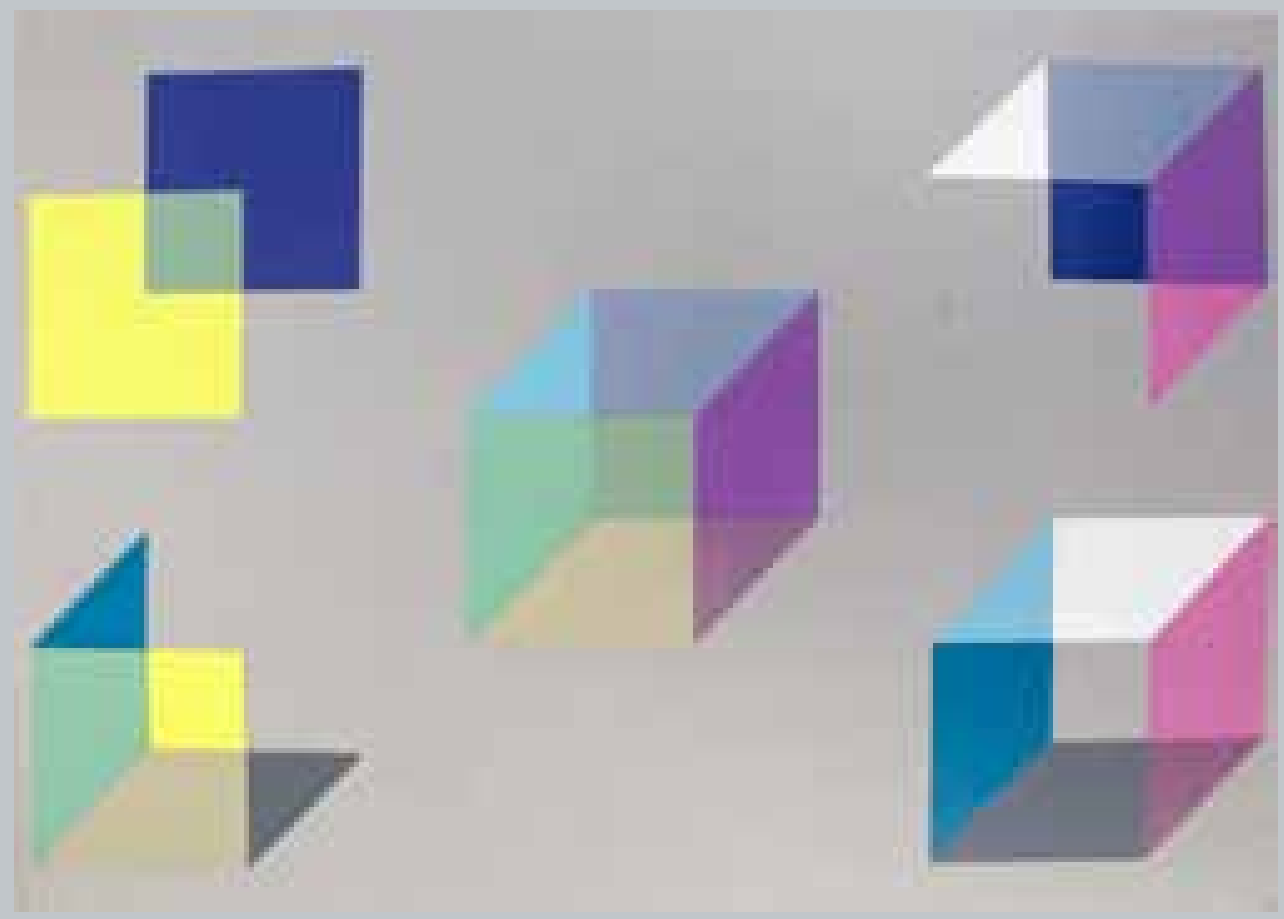

i2/58 Color visual: enfoque

experimental
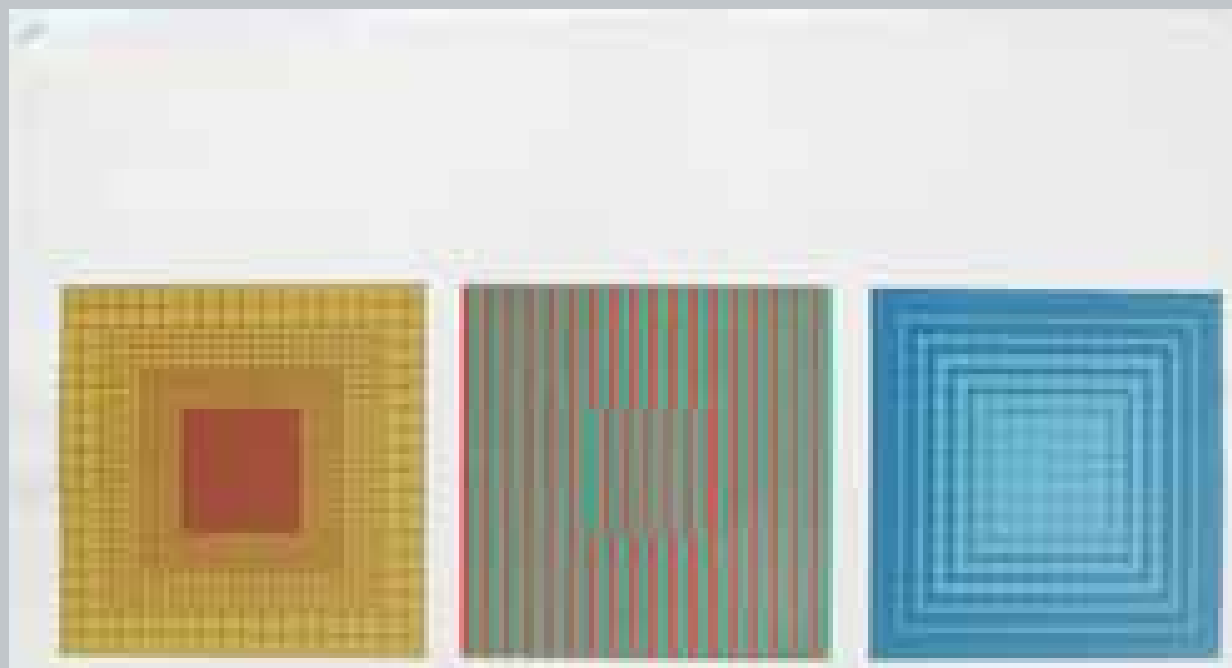

i2/59 Color visual: enfoque 


\subsection{Color visual}

La consideración del color como variable visual engloba un conjunto de ejercicios en los que el uso del mismo viene dado por la voluntad de desencadenar su potencial perceptivo para la configuración de las tareas. Se puede apreciar que una de las corrientes de la enseñanza del color en la HfG parte de la consideración del color como la variable para la articulación en los ejercicios propuestos desde el punto de vista perceptivo, en relación a aspectos relacionados con las leyes de la Gestalt frente a condiciones de otra naturaleza.

El uso del color sobre la superficie del plano desencadena la irritación visual. En este caso las variables las constituyen la superficie del color en sí misma y la cantidad de color en relación a la confrontación intencional con otros colores entre sí o con otros patrones visuales.

El curso del color básico que desarrolla Albers, dos ejercicios de Vordemberge-Gildewart y dos ejercicios del curso Visuelle Einführung de Maldonado, son los cursos en los que mejor se aprecia el desarrollo de esta cualidad.

Las ejercitaciones que realizan los alumnos se basan en las relaciones entre masas de colores y en cómo condicionar la percepción del conjunto, manipulando como variables operativas su relación de cantidad, posición, proximidad o vecindad. En este sentido, la articulación del color se puede llevar a cabo de dos maneras: la exploratoria, mediante tanteos visuales, o bien orientada por unas premisas de partida.

Los trabajos se desarrollan mediante dos tipos de técnicas gráficas. Los ejercicios de color de Albers se construyen mediante el recorte y fijación de papel de color. Para Albers, este medio permite mantener la homogeneidad de la superficie. Las piezas de color se yuxtaponen en el plano dando lugar a unidades visuales a partir de directrices gestálticas. Los ejercicios de Maldonado y Vondemberge-Gildewart se realizan con gouache.

\subsubsection{Color visual: enfoque experimental}

Albers describía su curso de color como un laboratorio. Frente al la postura de NonnéSchmidt, continuadora de la aplicación de las teorías de Oswald, Albers propone un acercamiento empírico al color, un "aprendizaje a través de la experiencia, en lugar de un aprendizaje a través de la aplicación de la teoría y las reglas". ${ }^{15}$

En su informe sobre los primeros cursos en la $\mathrm{HfG}$ describe los aspectos que trabaja en los experimentos visuales con el color y revela las variables para ejecutarlos:

Casi nunca vemos en nuestras mentes qué es físicamente el color, porque el color es el medio artístico más relativo. Es el resultado tanto de la interacción como de la interdependencia entre color y color, entre color y forma, entre color y cantidad. ${ }^{16}$

Este enfoque es primordial para su didáctica. Albers considera que la capacidad de ver el color, saber ver el color, es un fin necesario por delante de saber sobre el color. ${ }^{17}$ Este orden de importancia le distancia de la didáctica de Nonné-Schmidt quien parte de los sistemas de color, de la teoría científica y los manuales.
15 Albers, Josef. "Mis cursos en la Hochschule für Gestaltung de Ulm" 1954 En: Josef Albers Medios mínimos, efecto máximo. (Exposición celebrada en Madrid, Fundación Juan March 28-III-2014 al 6-VIII-2014) Madrid: Fundación Juan March, 2014, p. 276.

El color fue uno de los temas que dio lugar a controversias entre Albers y los otros docentes que desarrollaron esta labor junto a él en la HfG como Nonné-Schmidt y Johannes Itenn.

16 Ibidem.

17 Ibidem. 


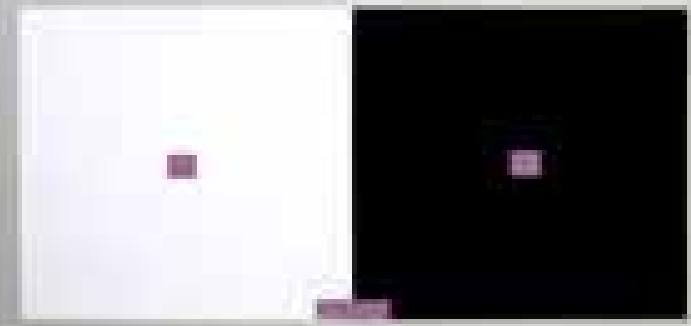

i2/60 Un color parece dos / Alumno: Imno Krumrey

/Curso: Albers (1953-1954) Dp 019.006 F.B.S

i2/62 Mezcla ilusoria y transparencia / Alumna: Ingela Albers

Curso: Albers (1953-1954) / Albers, Ingela; Quinano, Marcela; \& Wachsmann, Christianne. Baushaüsler in Ulm. Die Grundlehre an der Ulmer HfG zwischen 1953 und 1955. Ulm: HfG-Archiv Ulm, 1993.

i2/63 Mezcla ilusoria y transparencia / Alumno: Moeckl /Curso: Albers (19541955) / G 10 F.B.S
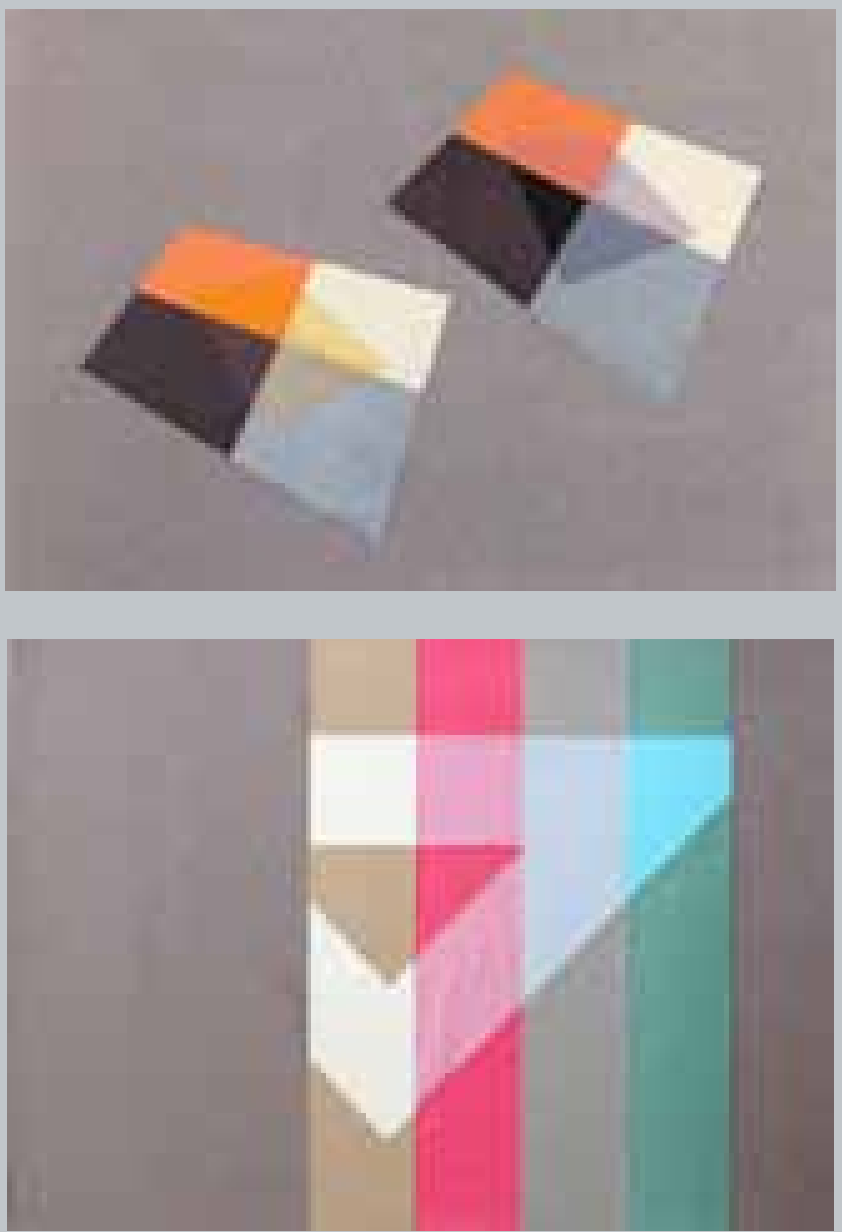
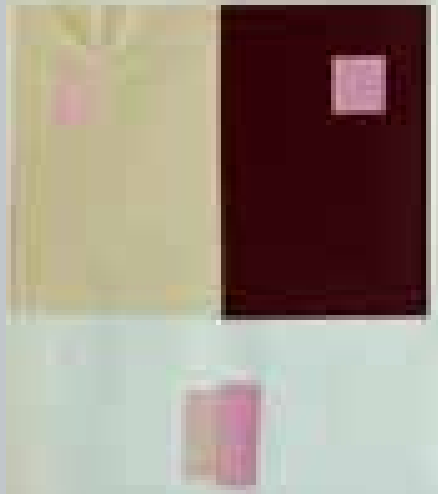

i2/61 Dos colores parecen uno.

Alumno: Max Graf /Curso:

Albers (1954-1955) Dp 012.003 F.B.S

i2/64 Mezcla ilusoria y transparencia / Alumno: Christoph Naske

Curso: Albers (1954-1955) / Dp 012.003 F.B.S

i2/65 Mezcla ilusoria y transparencia. / Alumno: Moeckl

Curso: Albers (1954-1955) / G 10 F.B.S
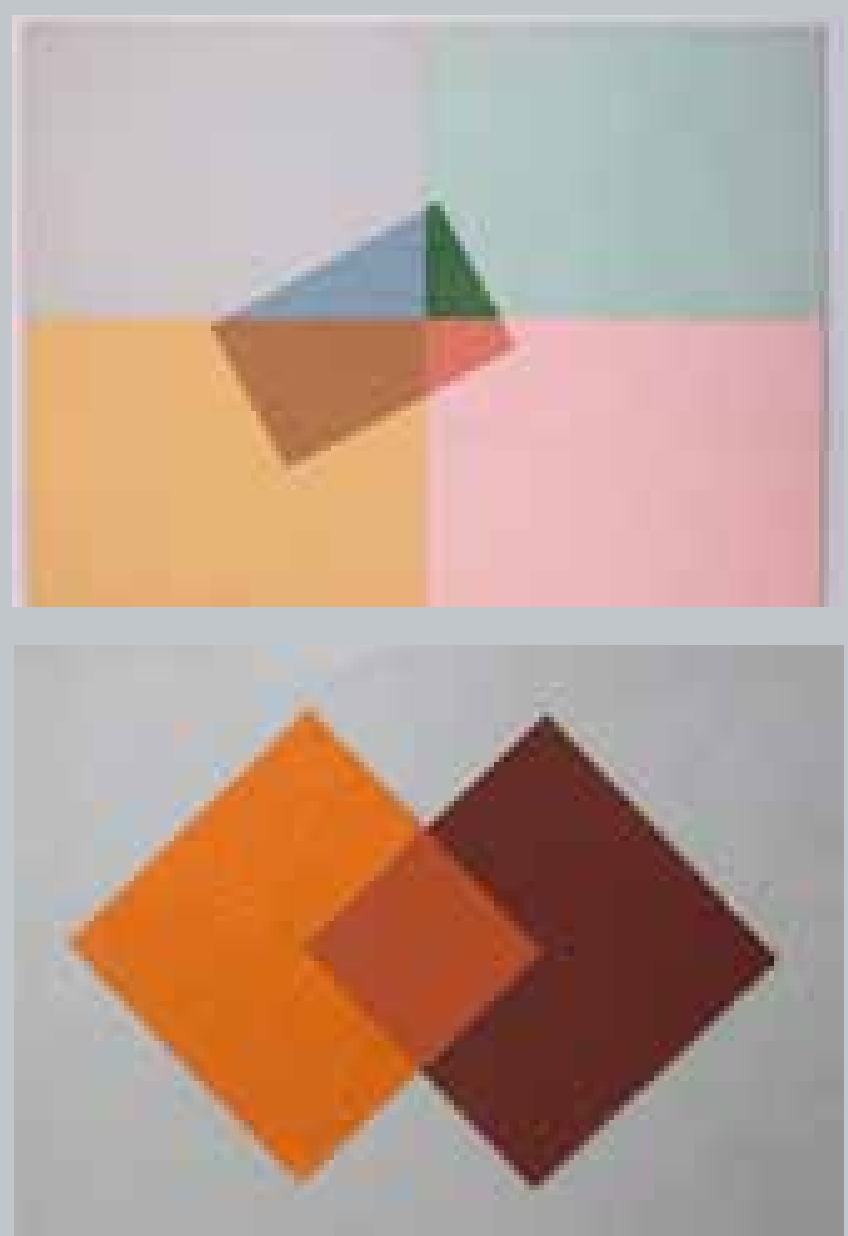

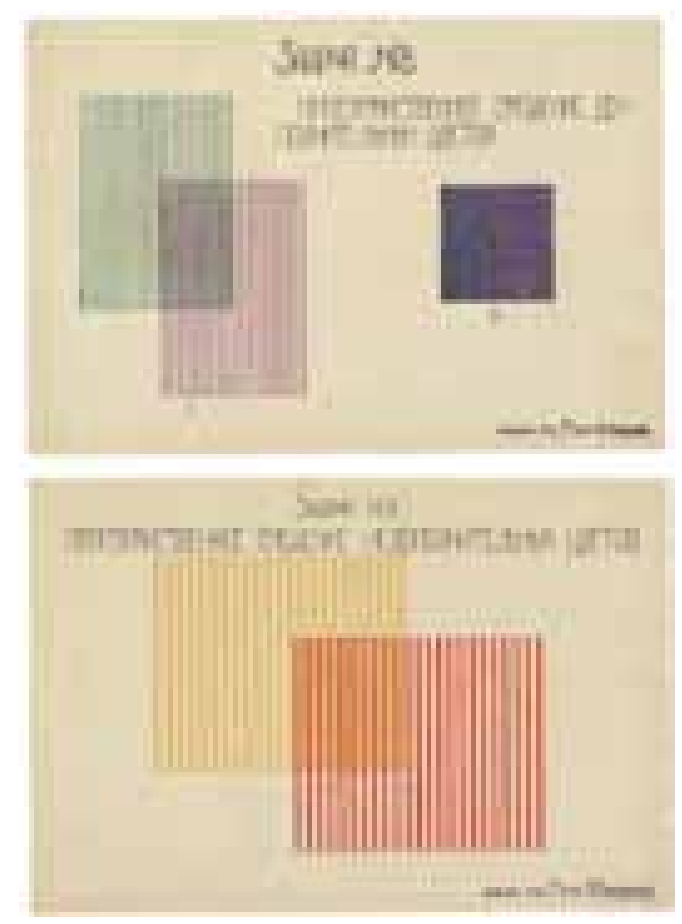

En los ejercicios de color de Albers en la HfG encontramos los temas fundamentales que más adelante serán el núcleo futuro de su obra publicada en Yale "interacción del color". ${ }^{18}$ Tanto Itten como Albers desarrollan su docencia en la HfG en un momento de madurez previo a la escritura de los manuales representativos de su legado didáctico.

Albers desarrolla en Ulm el repertorio de ejercicios que ha comenzado en el Black Mountain College y consolida en Yale. El curso de color en Yale consta de cinco partes: ejercicios preliminares, ejercicios de cambio de color, ejercicios de mezclas, ejercicios varios y estudios libres. Esta estructura sigue un itinerario aproximativo hacia la relatividad del color en el que, a través de exploraciones entre colores, se comprueba su interacción. ${ }^{19}$

Los recursos y herramientas que Albers despliega para activar la apreciación del color pertenecen al mundo de las ilusiones perceptuales, es decir, a la ilusión y seducción del ojo para dar lugar situaciones intencionadas.

El repertorio de ejercicios que Albers desarrolla en la HfG en sus estancias del 1953-1954 y 1954-1955 consiste en una secuencia progresiva en la que las relaciones entre colores adquiere una mayor complejidad en función del número de variables que intervienen en la unidad visual.

La secuencia de ejercicios es la siguiente: un color parece dos, dos colores parecen uno, mezclas ilusorias y transparencia, colores progenitores, intersección de colores, mezclas, transformaciones, cantidad de color, borde vibrante, intensidad luminosa y estudios libres.

\section{Un color parece dos}

Dos piezas del mismo tamaño y color situadas sobre fondos de color distinto hacen que el mismo color parezca dos colores diferentes (i2/60). Esta ilusión se origina por el contraste que se produce entre la relación recorte y fondo mediante la manipulación de la luminosidad y el tono del fondo: si los fondos son opuestos, mayor será la diferencia, si uno de los tonos es complementario del recorte, enfatizará el tono del mismo. Por ejemplo, un recorte naranja sobre fondo azul complementario hará que percibamos un naranja más intenso y vibrante que el recorte naranja que se encuentra al lado y sobre otro fondo. i2/66 Kolpakova, V. Taller de Klutsis, primer año, curso 1926/27. Mezcla escapial de colores no complementarios.

Ivanova-Veen L.I; Ovsiannikova E. B. op. cit. p. 103.

18 Albers, Josef. Interaction of Color. New Haven: Yale University Press, 1963.

19 Krampen, Martin. Beobachten und formulieren. Grundkurs mit Übungen, nach einem Filmskript von Josef Albers. Zentrum für Kunst und Medientechnologie Karlsruhe ZKM, 2009.

El dvd que edita Martin Krampen es un documento de reconstrucción del curso básico de Albers en la HfG realizado por el antiguo alumno de la promoción 1953-1954 Martin Krampen, basado en un guión de Josef Albers. El dvd explica el repertorio que Albers desarrolló mediante la recreación de los casos y la ilustración de éstos con ejemplos elaborados por los alumnos. Este apartado en la tesis sigue el mismo esquema del dvd y aporta algún ejemplo inédito. 

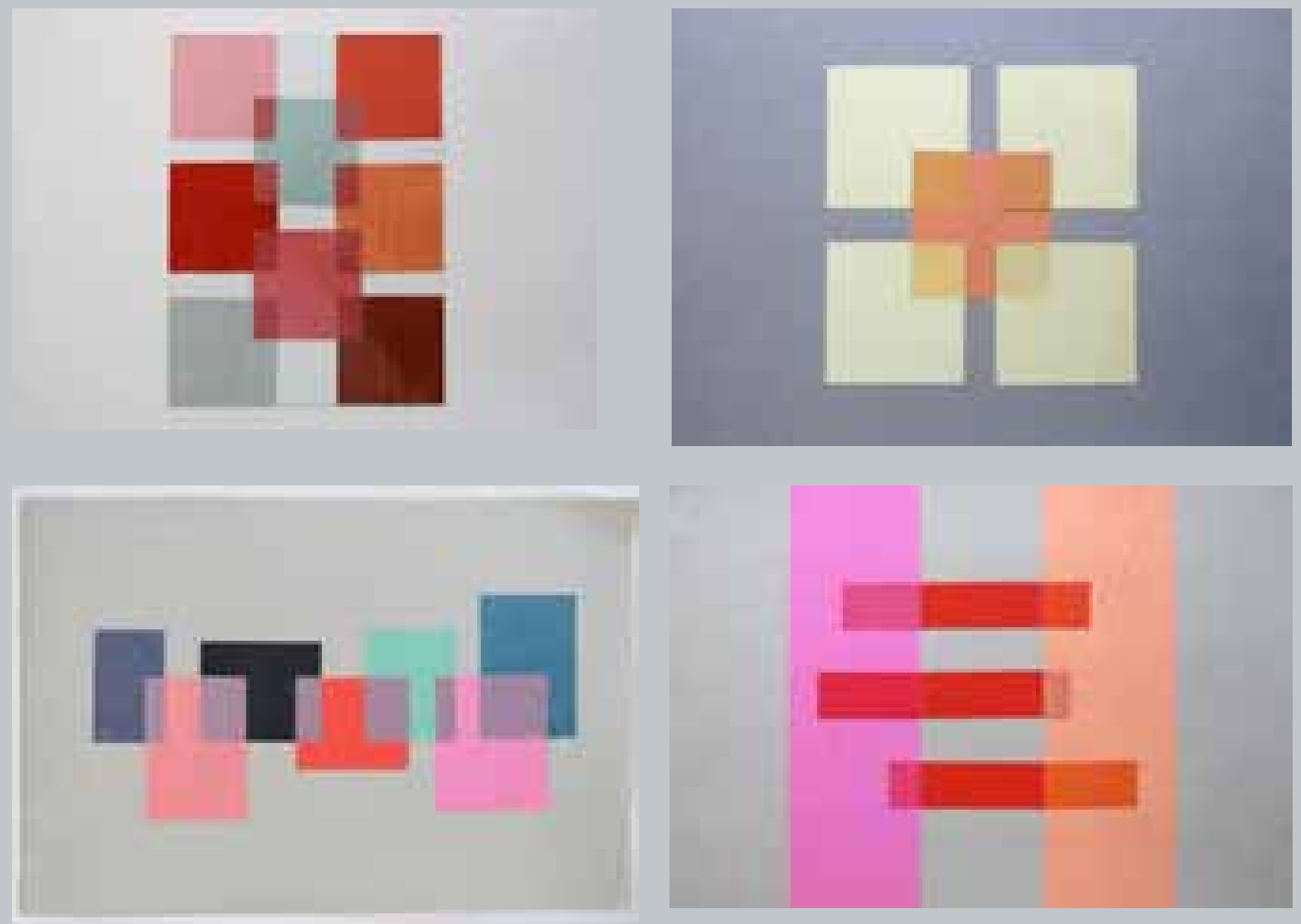

i2/67 Colores progenitores / Alumno: Imno Krumrey

Curso: Albers (1954-1955) / Dp 019.006 F.B.S

i2/68 Colores progenitores / Alumna: Christel Stankovits

Curso: Albers (1953-1954) / G6 F.B.S i2/69 Colores progenitores / Alumno: Imno Krumrey

Curso: Albers (1954-1955) / Dp 019.008 F.B.S

i2/70 Colores progenitores / Alumno: Imno Krumrey

Curso: Albers (1954-1955) / Dp 019.008 F.B.S

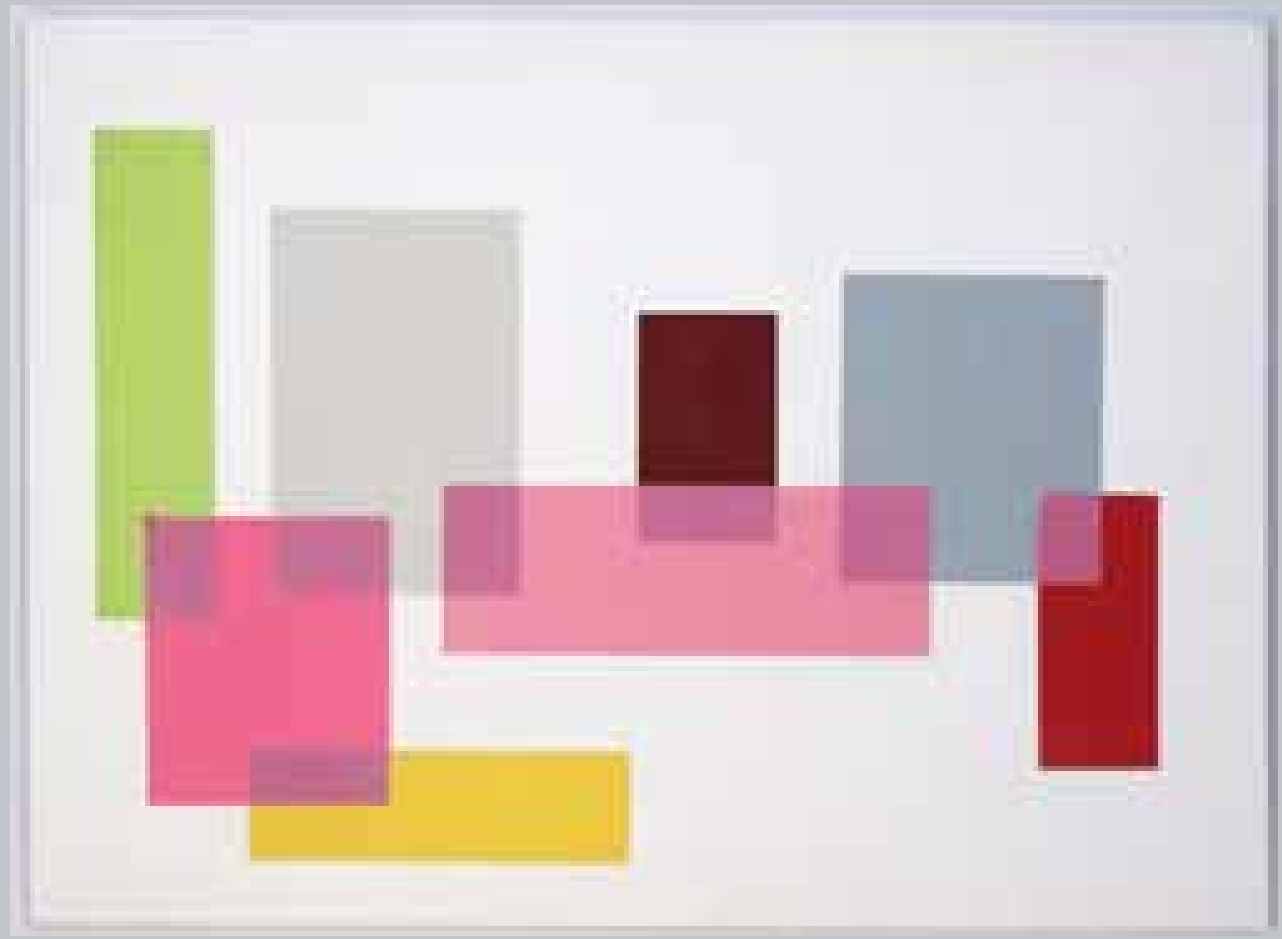

i2/71 Colores progenitores Alumno: Christoph Naske Curso: Albers (1953-1954) Dp 024.002 F.B.S 


\section{Dos colores parecen uno}

Dos piezas del mismo tamaño y colores sutilmente diferentes son colocadas sobre fondos de colores diferentes a su vez a los anteriores (i2/61). Este fenómeno, análogo al anterior, busca la semejanza entre colores que equilibran cromáticamente la percepción. El efecto es posible porque los recortes son del mismo tono

\section{Mezcla ilusoria y trasparencia}

Albers juega con la afinidad y la luminosidad entre colores para crear la ilusión simultánea de mezcla y superposición entre superficies. Los papeles coloreados son opacos y no permiten la transparencia como tal, por lo que se crea es una ilusión que se lleva a cabo buscando un tono afín y de transición entre las superficies de color que se mezclan virtualemente. (i2/62-i2/65).

\section{Colores progenitores}

Se parte de dos colores llamados progenitores, que se mezclan mediante un tercer componente. Este tercer componente hará que se perciban los progenitores situados uno sobre el otro, o bien en una mitad óptica. La intención es la de alternar el efecto de superposición entre las dos superficies progenitoras en función del color seleccionado y crear, además una situación de ambigüedad.

El efecto extremo de esta ambigüedad se consigue superponiendo de forma concatenada varias superficies de colores muy distintos de entre sí, de manera que la mezcla virtual sea siempre la misma (i2/68-71).

El ejercicio tiene variantes. La más frecuente consiste en distribuir una serie de rectángulos de colores muy distintos entre sí -progenitores- a los que se superponen otros rectángulos que dan como resultado la misma mezcla a pesar de la disparidad entre los tonos progenitores. Otra variante, menos frecuente, está formada por dos colores progenitores a los que se superpone el mismo color y da lugar a una mezcla diferente y en escala cromática. (i2/69, i2/70)

\section{Intersección de colores}

Este tipo de ejercicio se denomina "colores intersecantes" en el libro la interacción del color. ${ }^{20}$ Se puede considerar que este ejercicio es un ejemplo singular de mezcla. Dados dos colores similares el objetivo consiste en buscar un tercero que sea la mezcla intermedia. El recorte intermedio considerado mezcla genera una ilusión conectora o ligante de los dos tonos en los extremos (i2/72, i2/73).

\section{Transformaciones}

Las transformaciones son estudios en los que se exploran las posibilidades de combinación a partir de una familia de colores tomada como base de referencia. Sobre esta familia se superponen otra serie de colores afines entre sí y uno a uno a los anteriores. El resultado crea ilusiones de transparencia directa o de mezcla y transparencia de forma simultánea. El patrón de cuatro colores afines o dos parejas de colores se colocan como base formando un cuadrado. En el centro de la composición y sobre cada uno de estos se coloca otra pieza sustitutoria de un color afín a aquel sobre el que se superpone virtualmente. Los efectos logrados pueden ser de transparencia y bien un aclarado de los colores o bien un oscurecimiento de los mismos (i2/74-i2/76). 


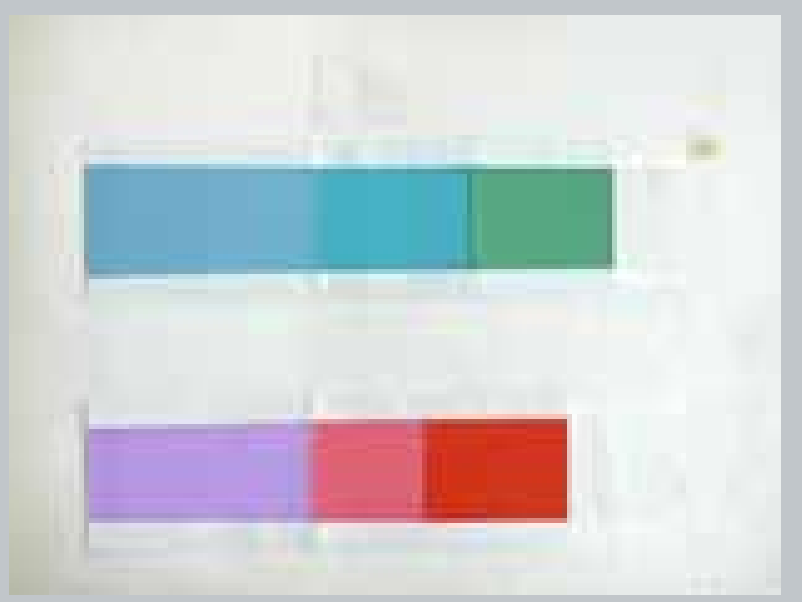

12/72 Intersecciones / Alumno: Max Graf

Curso: Albers (1954-1955) / Dp 019.008 F.B.S

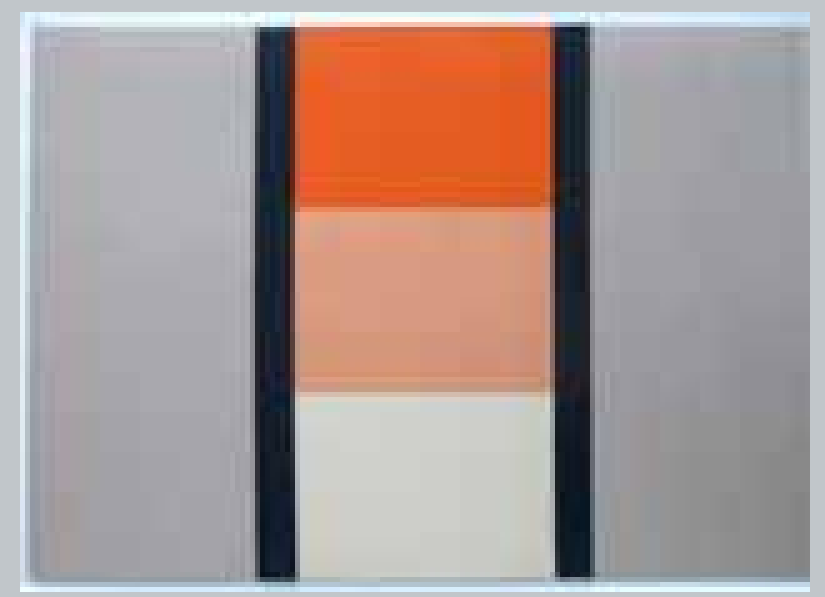

i2/73 Intersecciones / Alumno: Imno Krumrey Curso: Albers (1954-1955) / Dp 019.008 F.B.S
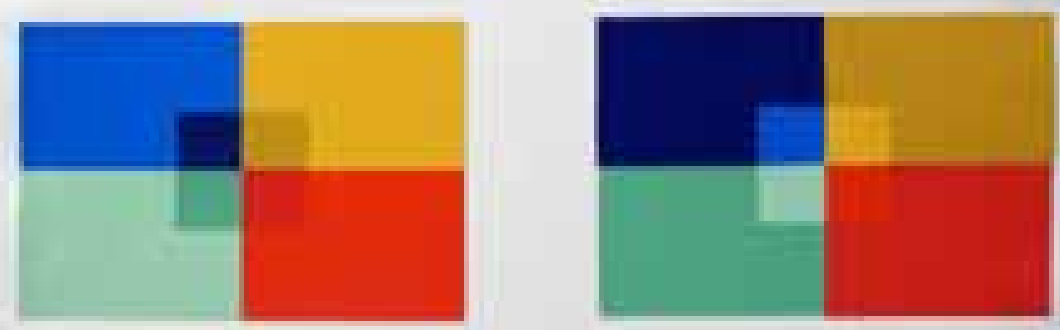

i2/74 Transformaciones

Alumno: Ernst Möckl

Curso: Albers (1954-1955)

G10 F.B.S

i2/75 Transformaciones

Alumno: Frauke Koch-Weser

Curso: Albers (1954-1955)

Dp_042.023G F.B.S

i2/76 Transformaciones

Alumno: Imno Krumrey

Curso: Albers (1954-1955)

Dp_019.008 F.B.S
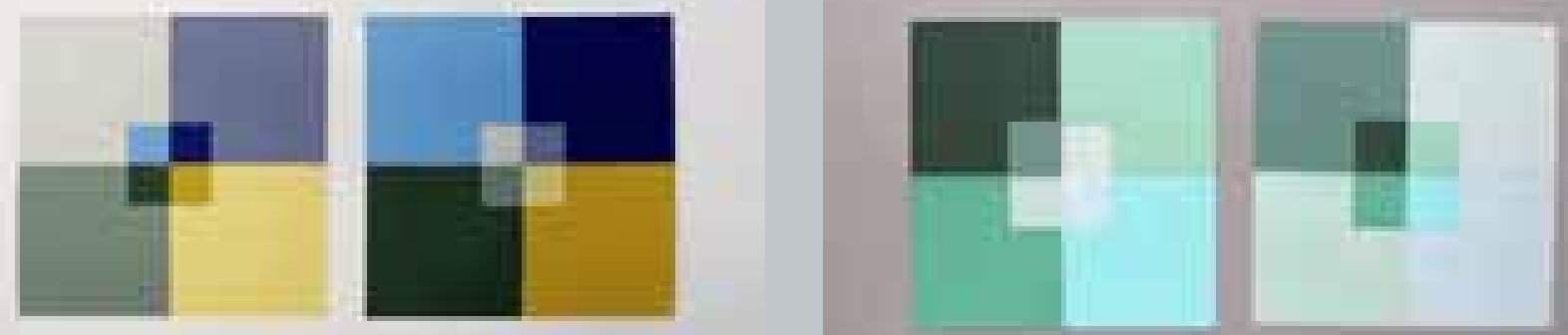


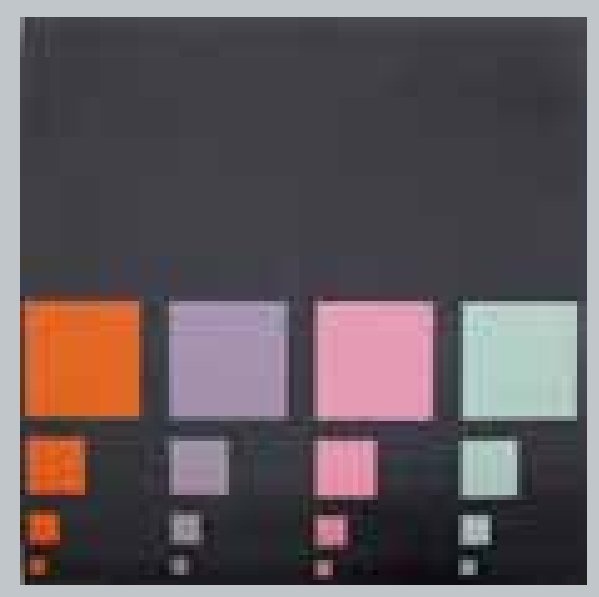

i2/77 (serie de 4) cantidad de color

Alumno: Maurice Goldring

Curso: Albers (1953-1954)

G7 F.B.S
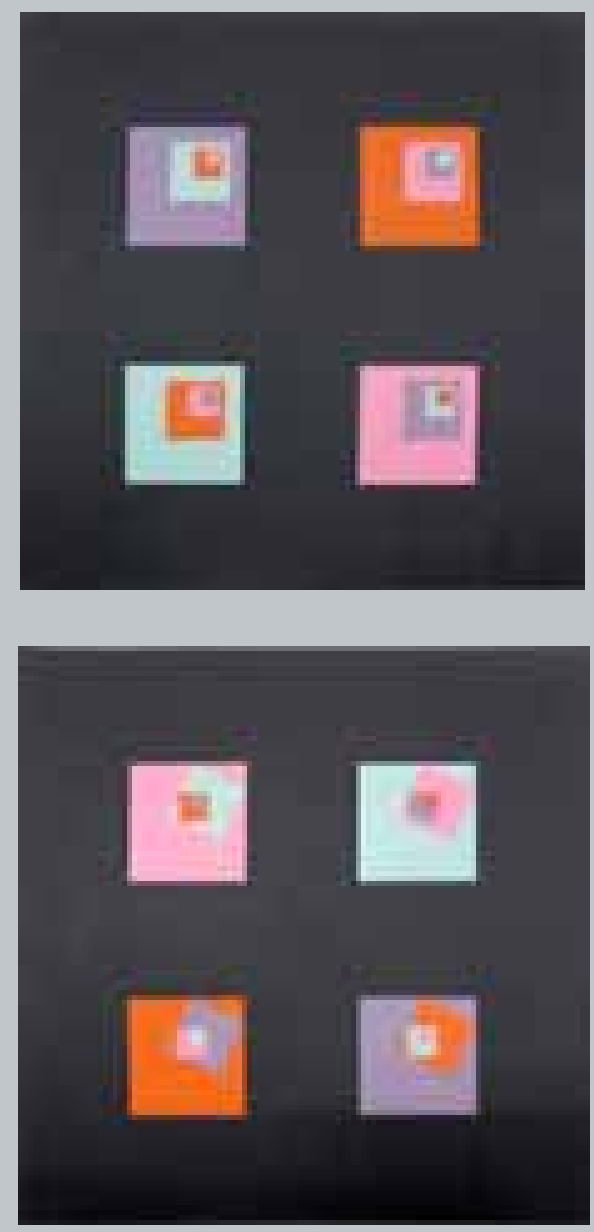

i2/78 Cantidad de color

Alumno: Christoph Naske

Curso: Albers (1953-1954)

G6 F.B.S
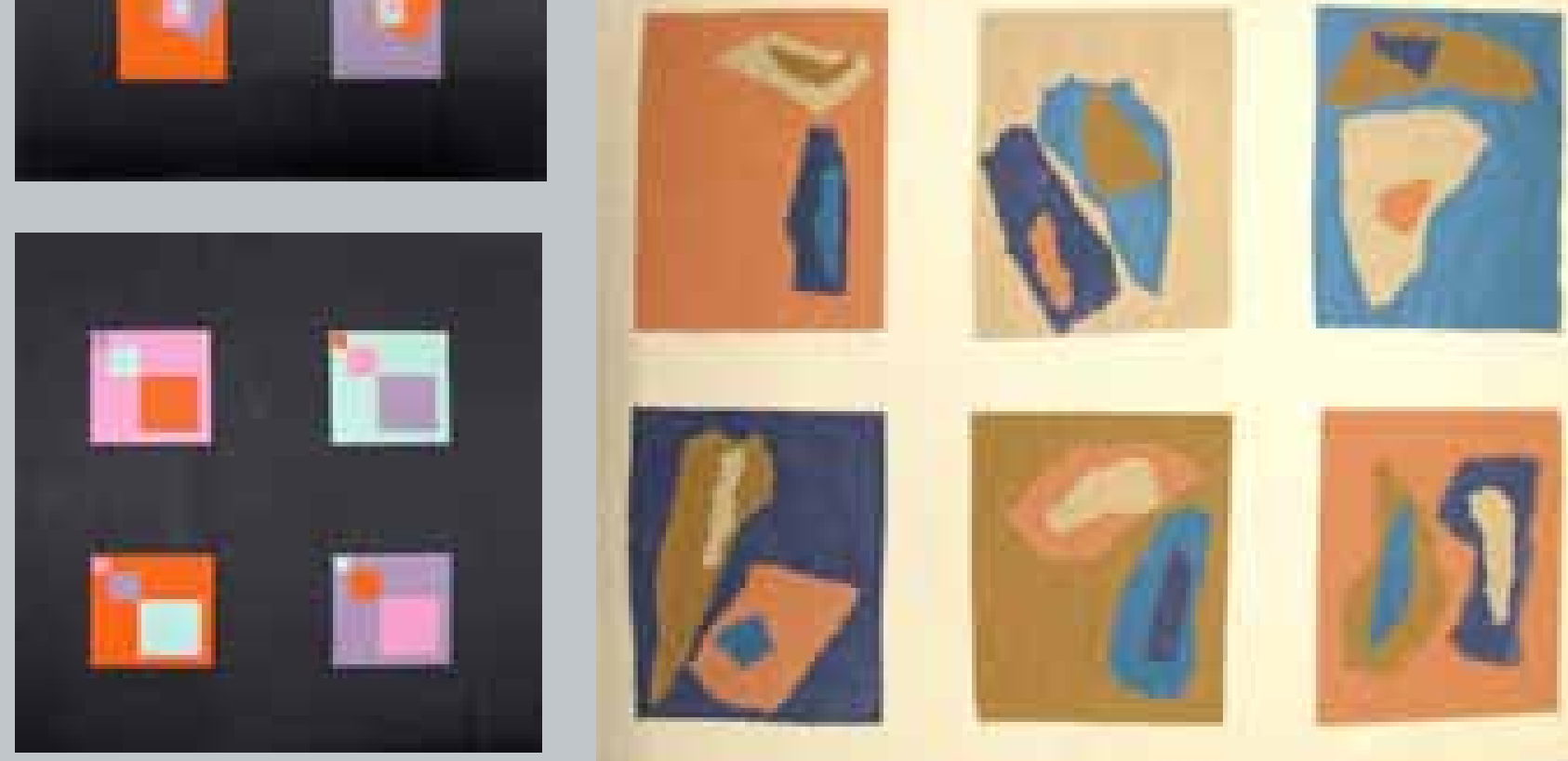


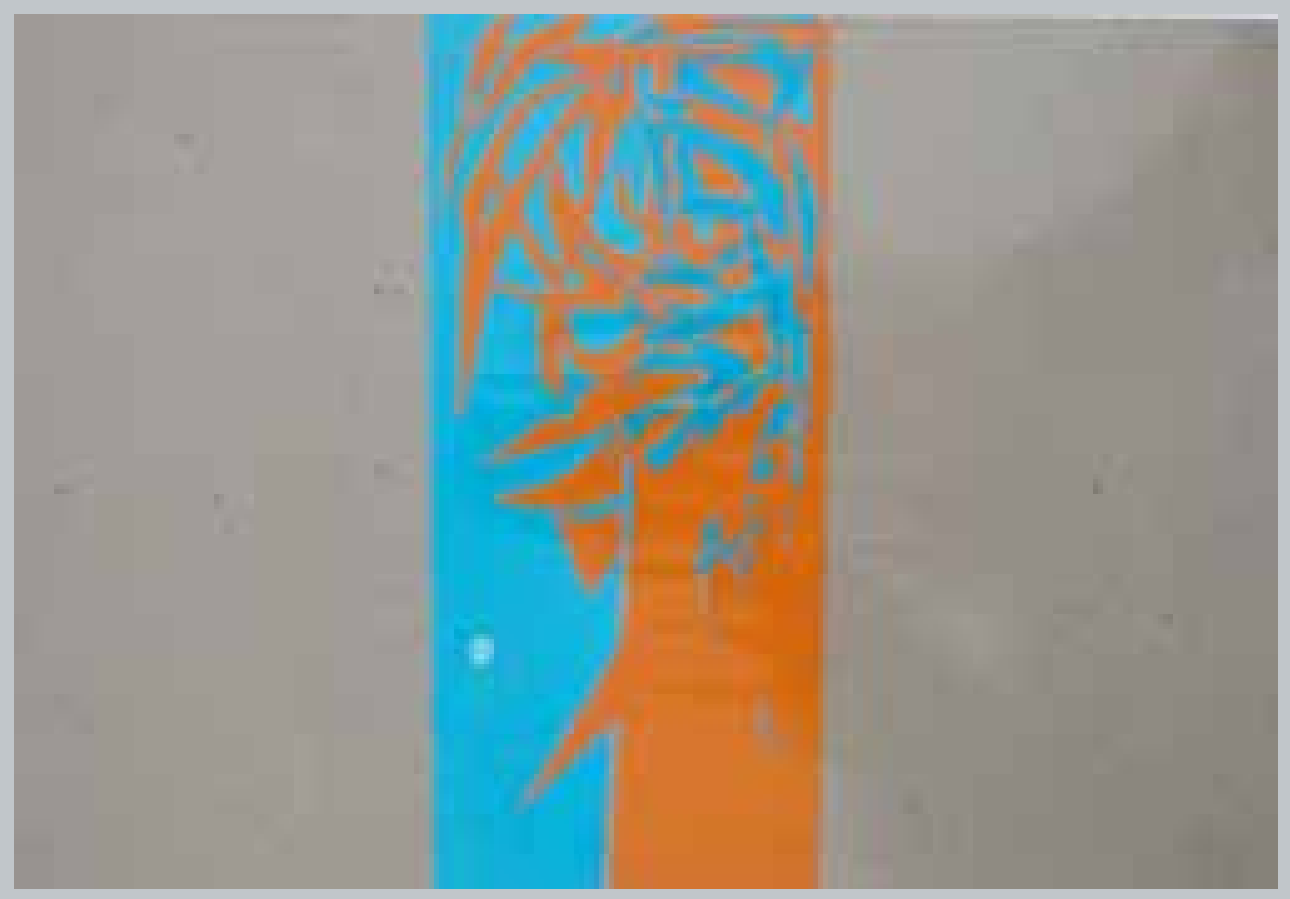

Curso: Albers (1953-1954)

Dp.019.008 F.B.S

i2/80 Borde vibrante

Alumno: Imno Krumrey

Curso: Albers (1953-1954)

Dp 019.006 F.B.S

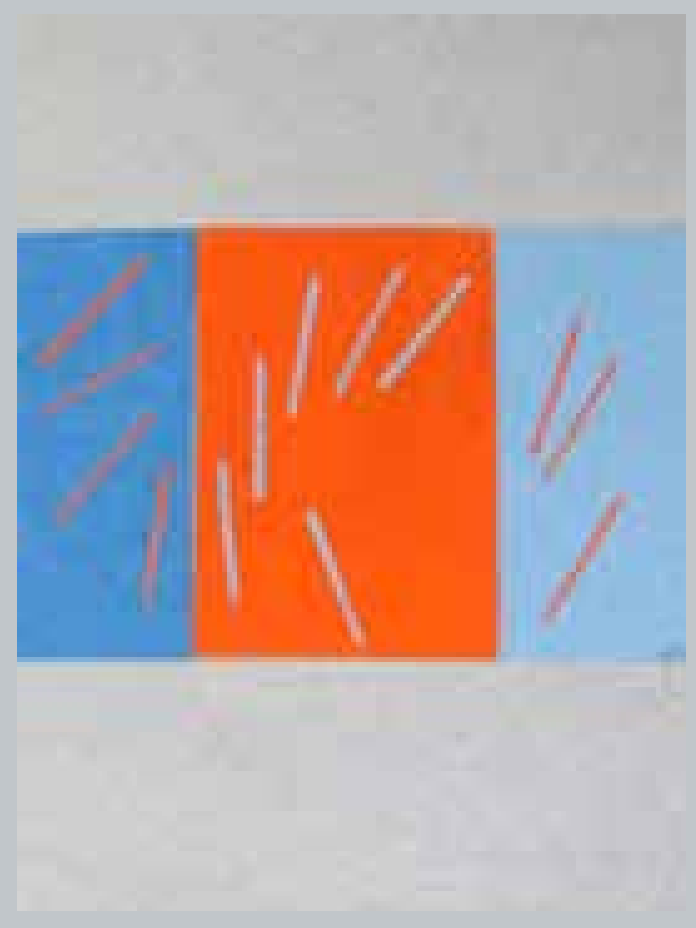

i2/81 Borde vibrante

Alumno: Imno Krumrey

Curso: Albers (1953-1954)

Dp 019.006 F.B.S

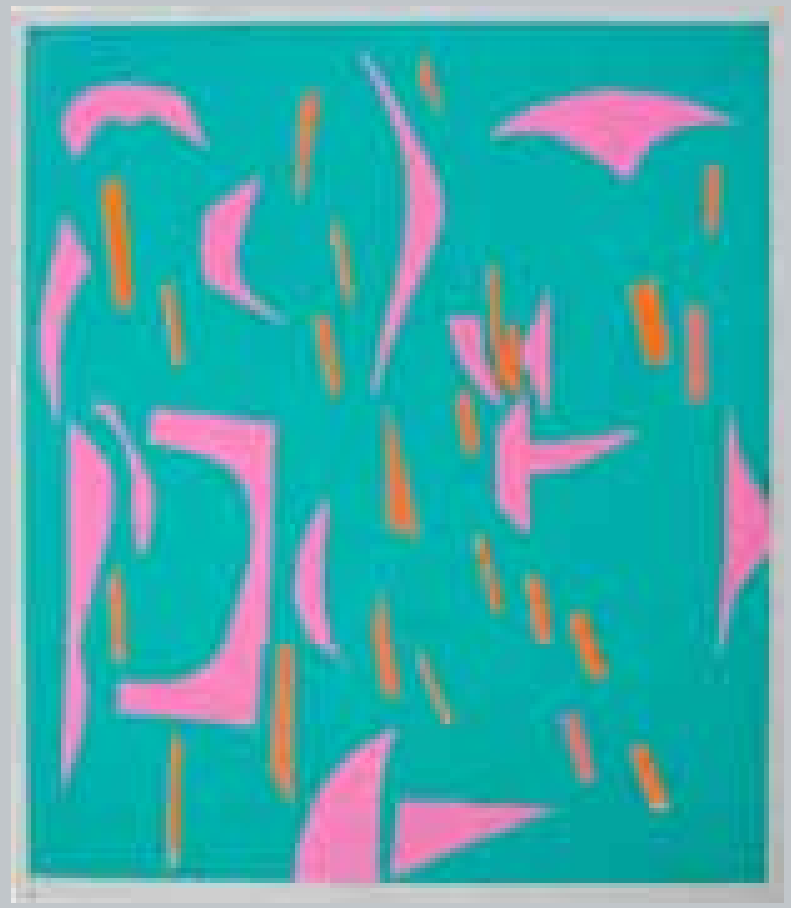




\section{Cantidad de color}

Este ejercicio consiste en una serie de superposiciones que forman una secuencia. En función de la orquestación de los grupos de colores se producen diferentes climas de color. Se parte del mismo patrón formal de figuras concéntricas, regulares o irregulares. Se repite el patrón inicial alternando sucesivamente los colores para así tener una serie de respuestas visuales análogas estructuralmente pero diferentes por la cantidad de color en cada caso (i2/77, i2/78).

\section{Borde vibrante}

En estos ejercicios se utilizan pares de colores complementarios, uno como fondo y el otro distribuido de forma fragmentaria sobre el anterior, de modo que esta configuración añadida al efecto de contraste simultáneo conduce a la situación vibrátil. La disposición de los recortes en el fondo sigue una configuración dinámica. La ilusión de movimiento de la unidad visual está acentuada por la vibración entre los colores que se produce en los límites de las figuras. Por tanto, resulta clave en la ejecución la adecuada proximidad entre recortes que de lugar a este efecto óptico (i2/79-i2/81).

\section{Intensidad luminosa}

Se parte de la definición de una forma pregnante angulosa con dos recortes de colores con la misma intensidad luminosa. La coexistencia en yuxtaposición de estas superficies tiene la capacidad de destruir la forma por la imposibilidad del ojo de dibujar un límite entre ambas, así como se anula la capacidad de percepción de su propio color. Este caso vendría a ser la anulación del principio fondo-figura mediante el uso del color (i2/83).

\section{Estudios libres}

En los estudios libres los alumnos ponen en práctica situaciones que tienen como referencia las experimentaciones anteriores. En Ulm se emplean en mayor medida la transparencia virtual junto a la cantidad de color y los colores progenitores. Encontramos un caso singular en el que se estudia la prevalencia de un color en un conjunto en función de la proporción de la superficie de ese color respecto del resto de colores del conjunto (i2/82, i2/84, i2/85-i2/88). 

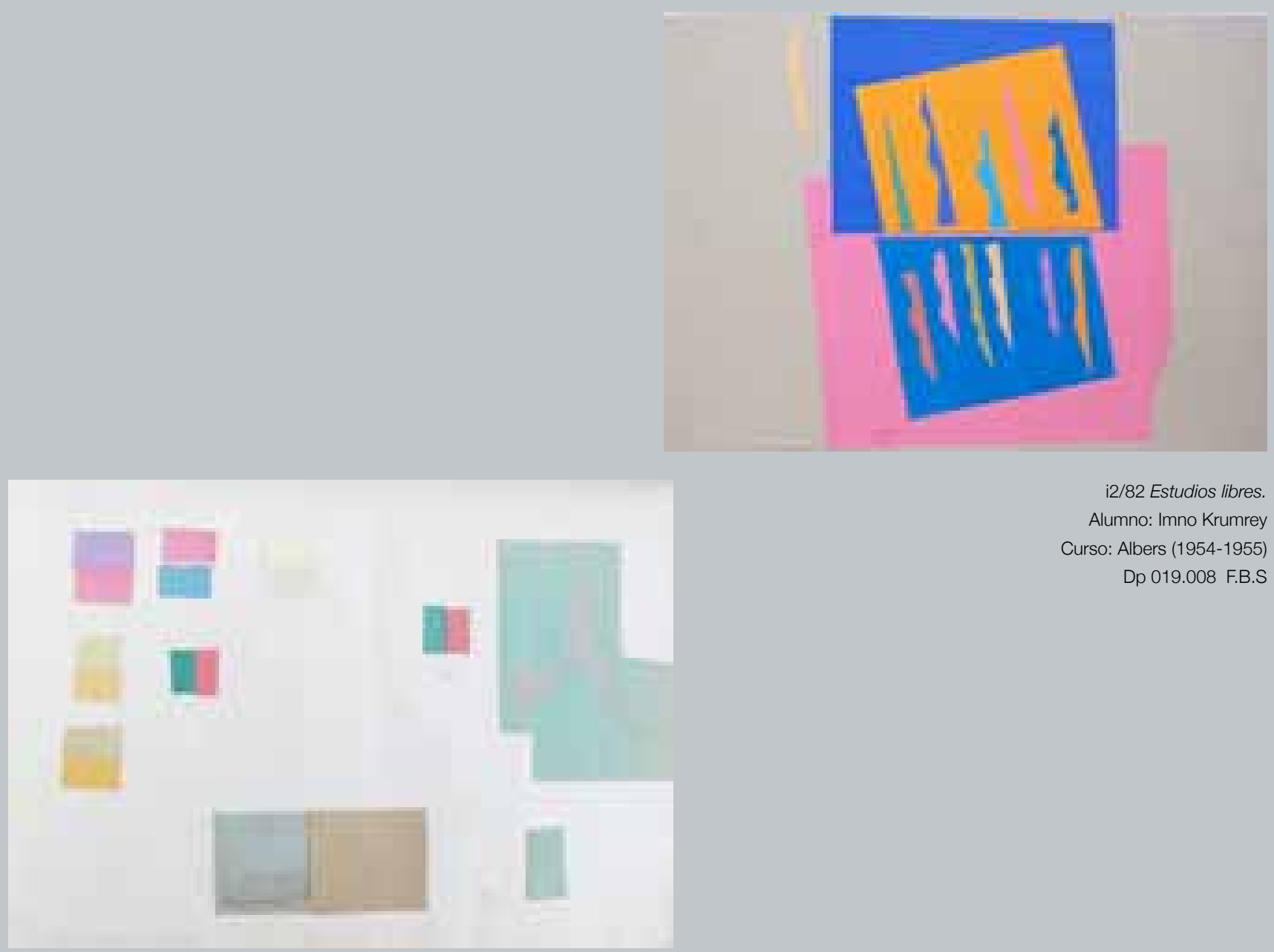

i2/82 Estudios libres.

Alumno: Imno Krumrey

Curso: Albers (1954-1955)

Dp 019.008 F.B.S

i2/83 Intensidad luminosa

Alumno: Max Graf

Curso: Albers (1954-1955)

Dp 012.003 F.B.S
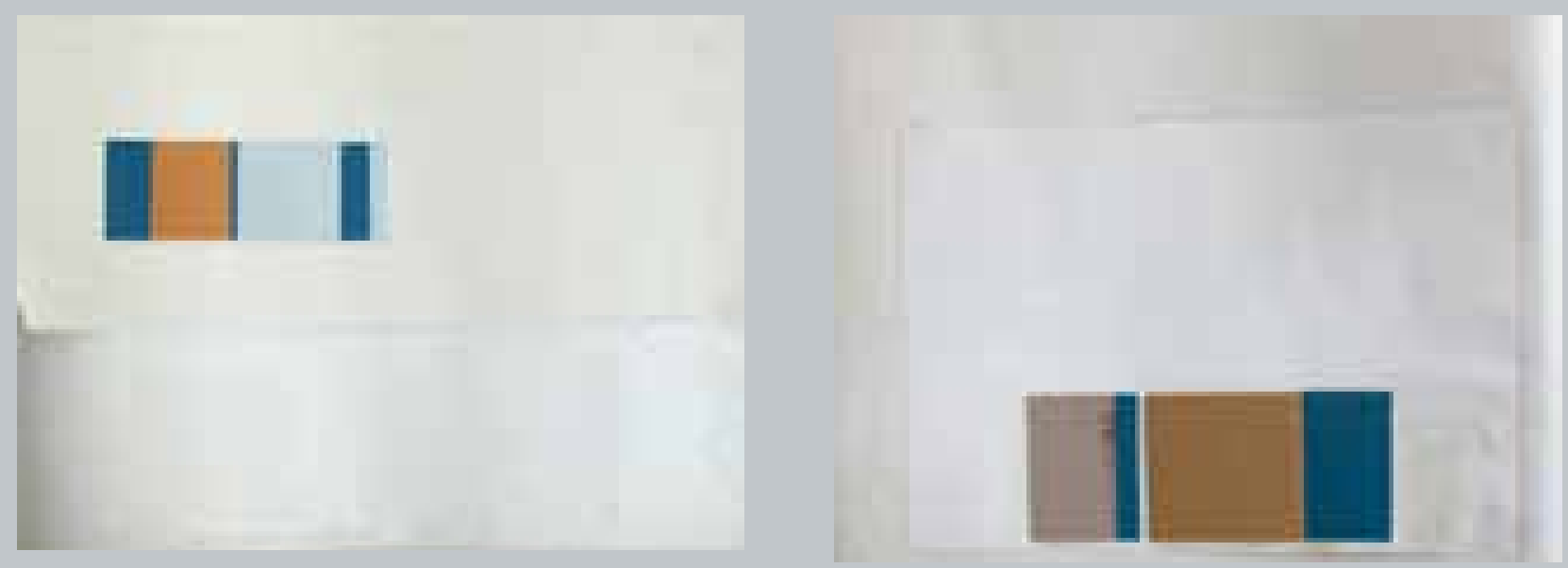

i2/84 Estudios libres, prevalencia,

ninguno prevalece

i2/85 Estudios libres, prevalencia,

Alumno: Max Graf

ninguno prevalece

Curso: Albers (1954-1955)

Dp 012.003 F.B.S

Alumno: Max Graf

Curso: Albers (1954-1955)

Dp 012.003 F.B.S 


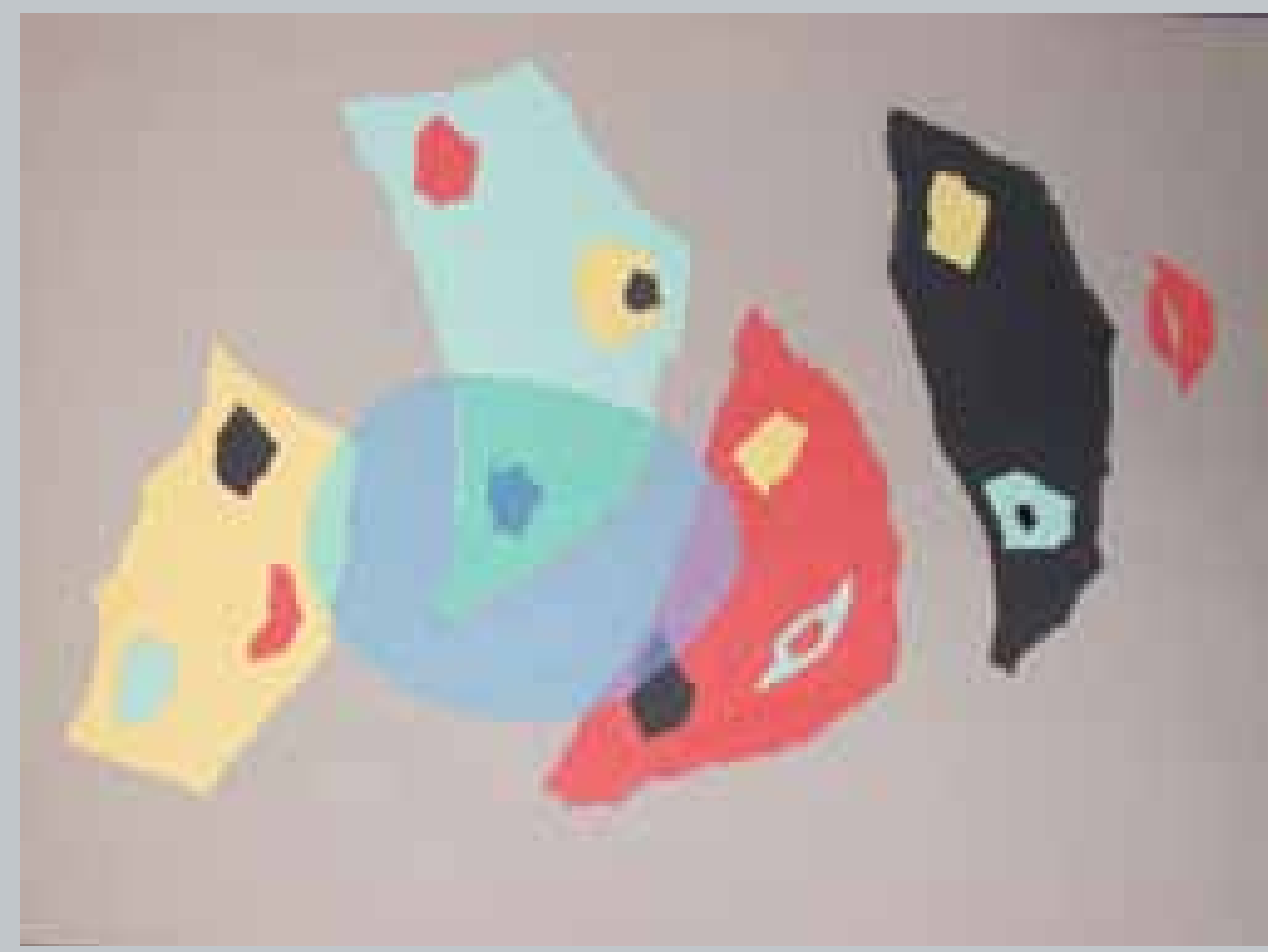

2/86 Estudio libre.

Dp 042.023G F.B.S

i2/88 Estudio libre

Alumno: Frauke Koch Weser

Curso: Albers (1954-1955)

Dp 042.023G F.B.S
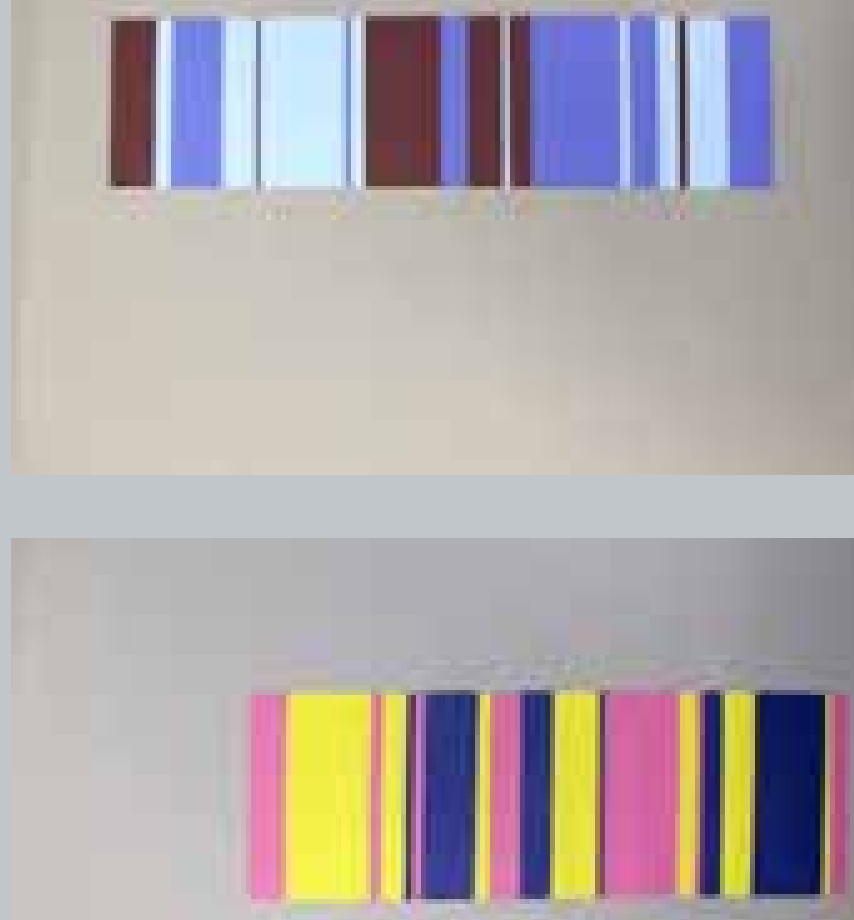


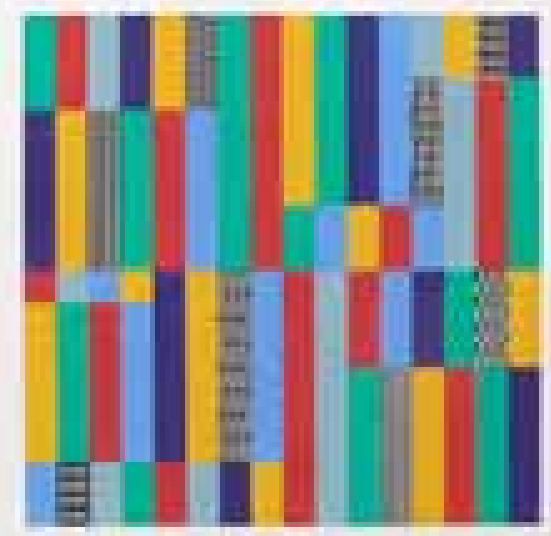

i2/89 Estructuras en superficie de color

Alumno: Klaus Kripendorff

Curso: Maldonado (1956-1957)

Dp 018.026 F.B.S
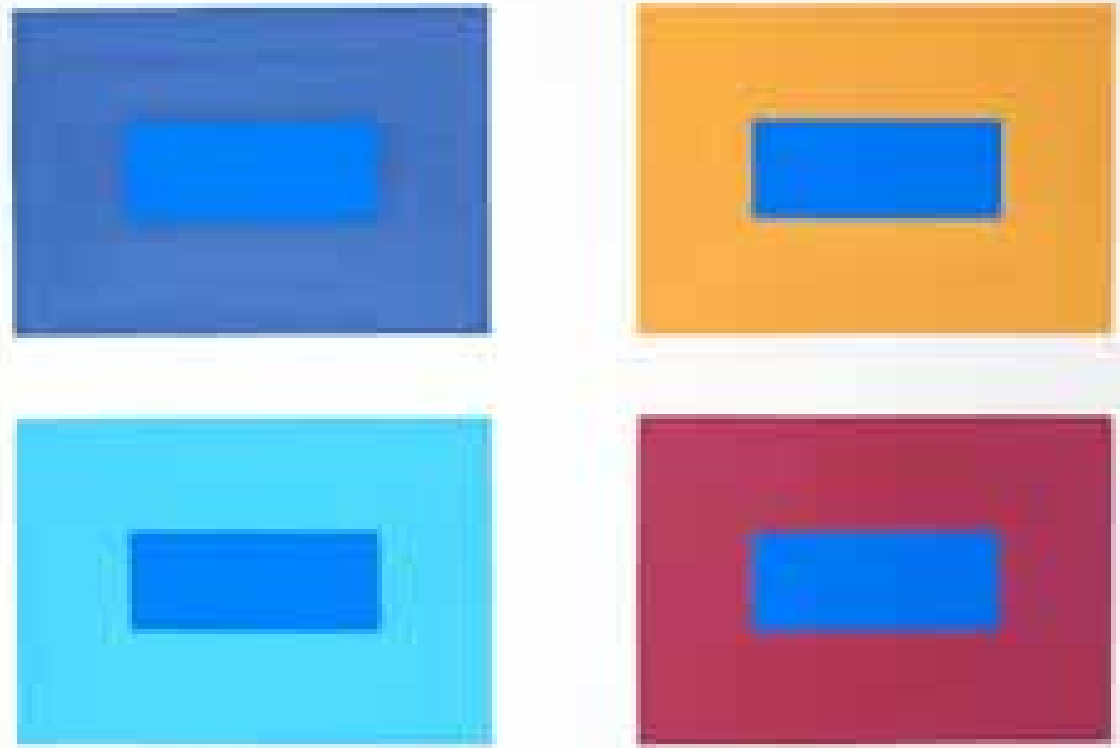

i2/90 Tarea 5

Alumno: Hans T. Kurtenbach

Curso: Vordemberge (1959-1960)

Gs 061.023 F.B.S 

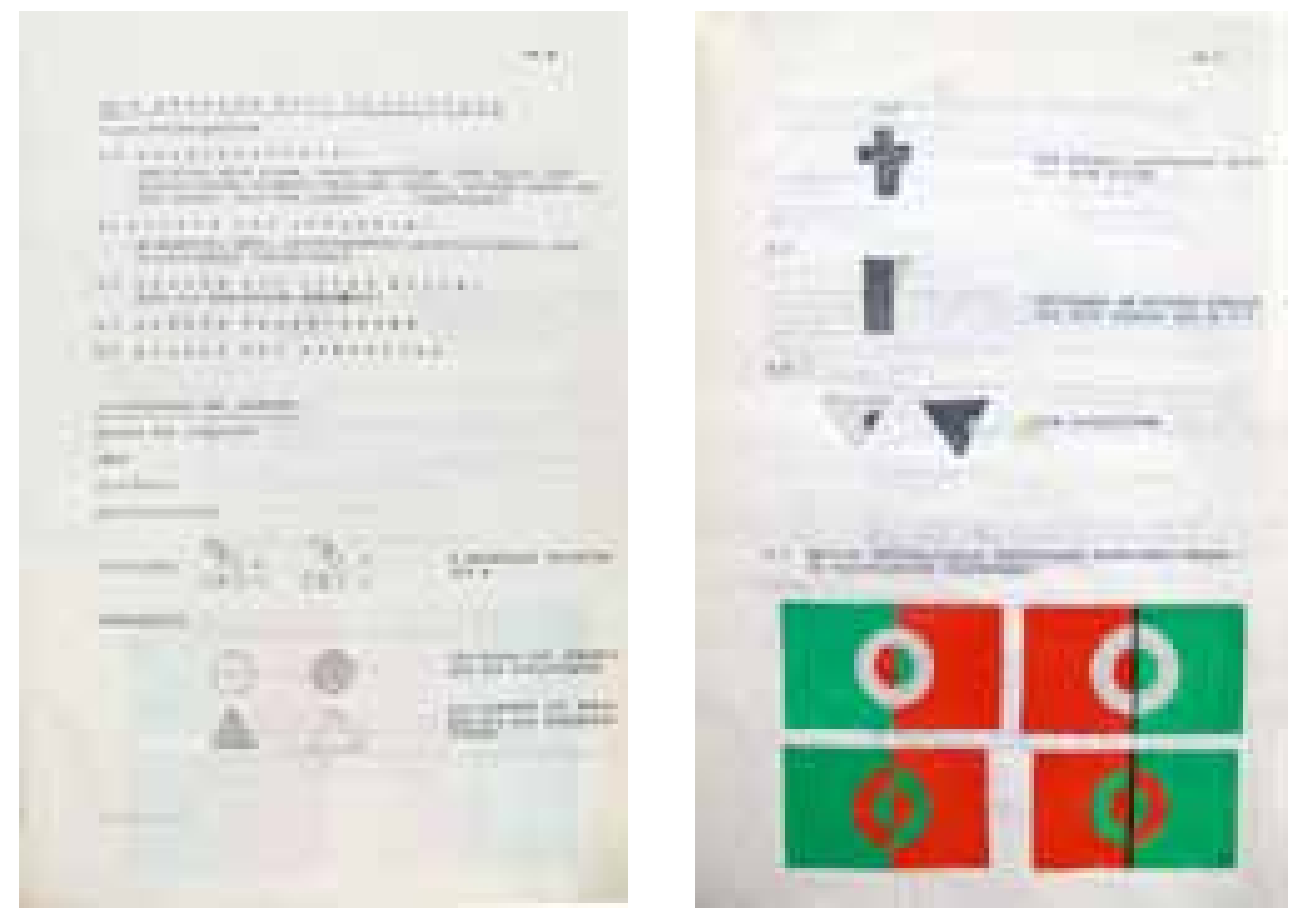

\subsubsection{Color visual: enfoque sistemático}

La enseñanza del color desarrollada por Tomás Maldonado y Friedrich VordembergeGildewart en el curso básico se plantea como una investigación. Los ejercicios persiguen responder a retos visuales propuestos desde preceptos gestálticos. Si bien Albers ejemplifica algunas leyes de la Gestalt con los ejercicios que propone, es Maldonado quien lo fundamenta de una manera científica en su curso (i2/91, i2/92). Maldonado proviene de un grupo de vanguardia argentino vinculado al arte concreto. Ha madurado una posición por su praxis artística en la que la psicología de la Gestalt constituye una certeza en el problema práctico de la percepción del espacio y del tiempo en la pintura. Enuncia que el problema fundamental del arte concreto es el tema de la figura versus fondo y el espacio ilusorio que surge de esta relación. ${ }^{21}$ La psicología de la Gestalt por tanto, se considera como un referente para el estudio y la base para la experimentación y la invención. "Ni buscar ni encontrar: inventar". ${ }^{22}$

Frente a los desarrollos de Albers, los ejercicios de Maldonado planteados en su curso dentro del Visuelle Einführung, constituyen una investigación para dar respuesta a unos preceptos de partida de tipo artístico así como la constatación de preceptos científicos, como son las cinco leyes de la Gestalt que desarrolla en su "introducción elemental a la enseñanza de la percepción." ${ }^{23}$ La didáctica del color de Albers ostenta un carácter experimental y exploratorio. Maldonado, sin embargo, propone de forma explícita la teoría y las reglas para, a través de ellas, constatar de manera planificada los principios de la Gestalt. La metodología llevada a cabo por Maldonado parte de una clase magistral impartida por la mañana sobre cuestiones de la percepción y en paralelo, propone trabajos prácticos en los que los alumnos investigan a partir de los preceptos teóricos enunciados previamente. Más allá de representar los principios de la Gestalt a modo de las ilustraciones científicas, los ejercicios son investigaciones al proponer de forma simultánea factores perceptivos y pictóricos para configurar unidades visuales en sí mismas.

Los trabajos que propone Maldonado son unidades visuales, es decir, conjuntos unitarios de composición plástica. La configuración del todo visual se produce gracias al fenómeno de irritación perceptiva, es decir, la interdependencia y la lectura que genera la confrontación de determinados colores. Por tanto, el color es el elemento visual principal y la variable de configuración esencial de esta serie de ejercicios. Los otros elementos formales de

2/ 91, 92 Apuntes Kurs Maldondado

Alumno: Ulrich Burandt

Curso: Maldonado (1956-1957)

Dp 033.012 F.B.S

21 Maldonado, Tomás. "El arte concreto y el problema de lo ilimitado. Notas para un estudio teórico". Zürich 1948 en : Gradowczyk, Mario y S. Huff, William (ed) . El arte concreto y el problema de lo ilimitado (edición facsímil), Buenos Aires: Ramona, 2003.

"Figura versus fondo el problema fundamental del arte concreto. Toda figura sobre un fondo determina un espacio. Si esto sucede dentro de un plano en su superficie el espacio es ilusorio. La pregunta concretista qué hacer con este espacio, cómo destruirlo. El arte concreto es un esfuerzo permantente por destruir este espacio ilusorio.

22 Maldonado, Tomás. "Manifiesto invencionista". publicado originalmente en: Revista Arte Concreto-Invención, Buenos Aires, 1946. Recogido en: Maldonado, Tomás, Escritos Preulmianos. Buenos Aires: Infinito, 1997, pp. 39-40.

23 Cuaderno de apuntes de Ulrich Burandt, alumno del curso básico 1956-57 


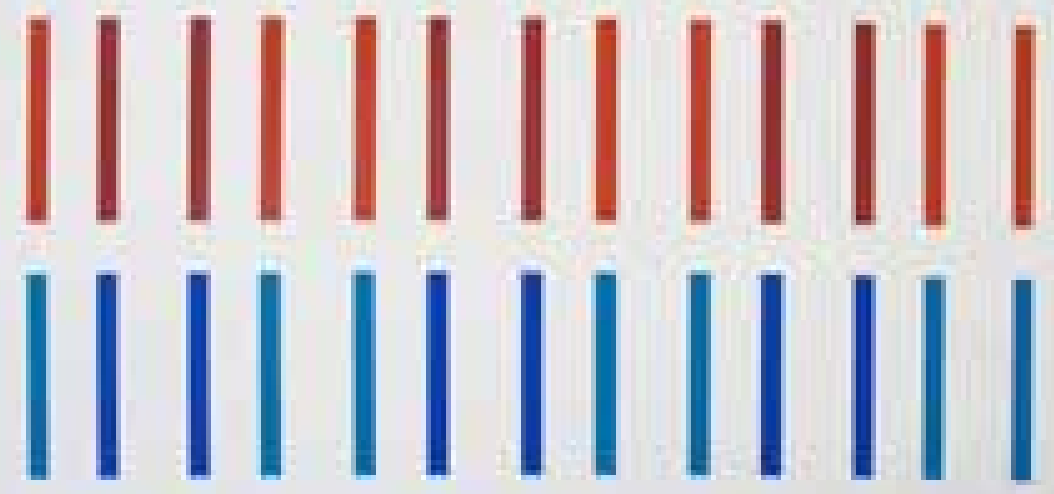

Alumno: Walter Müller.

Curso: Visuelle Einführung. Kurs

Maldonado (1958-1959).

Dp_022.002. FBS

i2/94 Proximidad-ambiguedad-semejanza.

Alumno: Walter Müller / Curso: Visuelle Einführung. Kurs Maldonado (1958-1959) Dp_022.002. FBS

i2/95 Proximidad-ambiguedad-semejanza.

Alumno: Klaus Krippendorf. Visuelle Einführung. Kurs Maldonado. (1956-1957). Dp_018.006. FBS

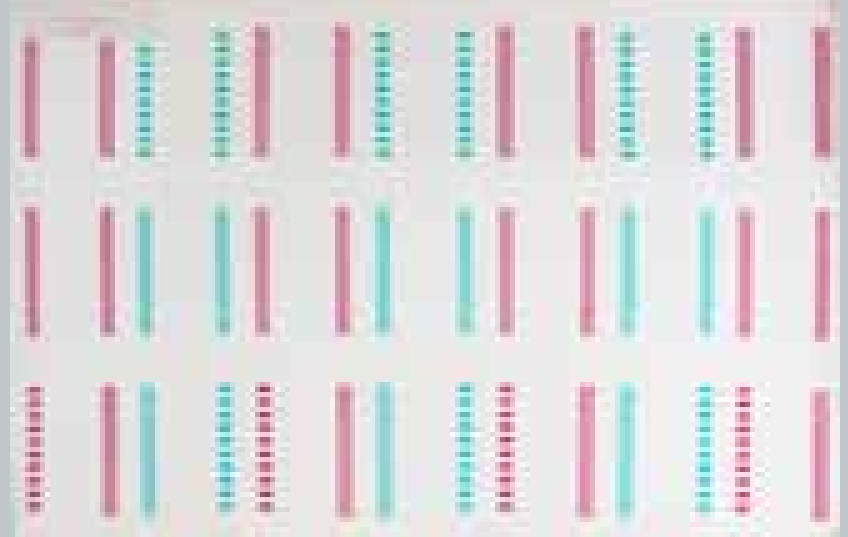

i2/96 Proximidad-ambiguedad-semejanza.

Alumno: Edgar Decurtins / Curso: Visuelle Einführung. Kurs Maldonado (1956-1957). Dp_042.035 FBS

i2/97 Proximidad-ambigüedad-semejanza.

Alumna: Mónica Buch / Curso: Visuelle Einführung Kurs Maldonado (1956-1957). Dp_045.005 FBS
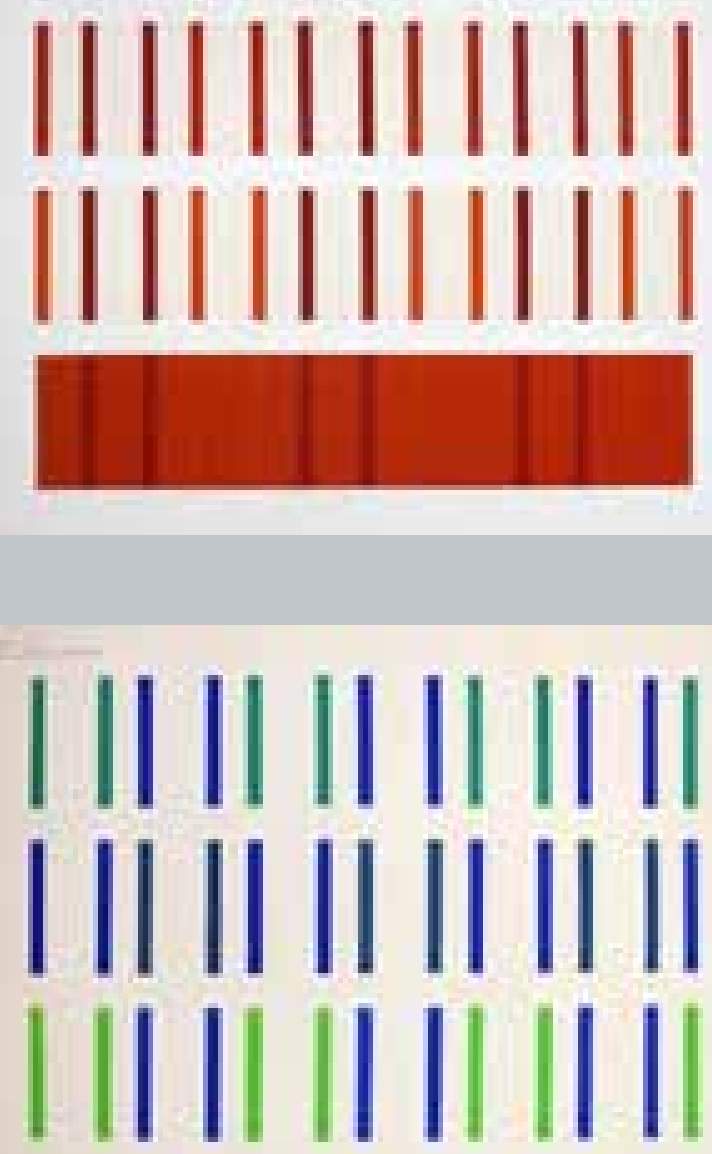


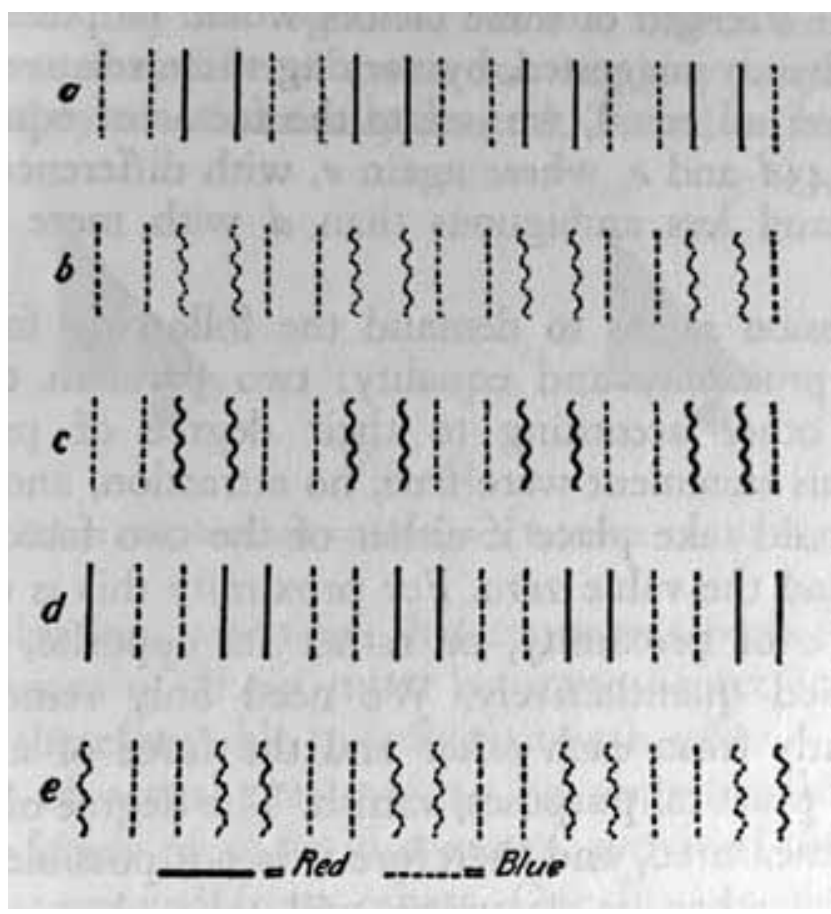

estos ejercicios serán la forma o configuración estructural de los propios elementos y su ubicación.

Las tareas se proponen como investigaciones en torno a las cinco leyes de la Gestalt. Este capítulo se centra solo en aquellas en las que el color es la variable principal. Los principios gestálticos que subyacen en los ejercicios están relacionados fundamentalmente con la percepción de la figura y el fondo, la pregnancia y la percepción de la proximidad y continuidad entre figuras.

Vordemberge-Gildewart también proviene del ámbito del arte concreto. No trabaja de forma explícita con las leyes de Gestalt, pero sus ejercicios muestran analogías en la manera de operar con las superficies de color modificando la cantidad y calidad de éste para lograr efectos visuales.

Bajo esta consideración del color como un elemento para el trabajo sistemático con el color según las leyes de la Gestalt, se analizan tres ejercicios del curso de Maldonado -proximidad-ambigüedad-semejanza, fondo-ambigüedad-primer plano y estructuras y superficies de color en equilibrio visual- y tres ejercicios del curso de VordembergeGildewart -efecto tridimensional intencionado, cambios cualitativos en la superficie de color, y cambios en un mismo color-.

\section{Proximidad-ambigüedad-semejanza}

Este ejercicio se realiza en los cursos 1955-1956 y 1956-1957 y 1958-1959. Por su semejanza con ilustraciones técnicas, puede considerarse como la representación de un ensayo clásico gestáltico frente a los siguientes ejercicios cuya apariencia es más figurativa. Maldonado propone la utilización de las leyes gestálticas de proximidad y ambigüedad en un duelo experimental, una en contra de la otra. Para ello el estudiante utilizará como variables una serie de franjas verticales y alargadas que se corresponden con la representación clásica de estas leyes (i2/99). Las franjas se distribuyen en dos rangos de distancias, como variable de la proximidad y el color como la variable que condicionará la semejanza: la confrontación de ambas desencadenará el juego visual.

El ejercicio consiste en la creación de tres bandas horizontales o campos visuales en los que se distribuirán las tiras de colores (i2/92). Cada una de estas franjas tiene que cumplir

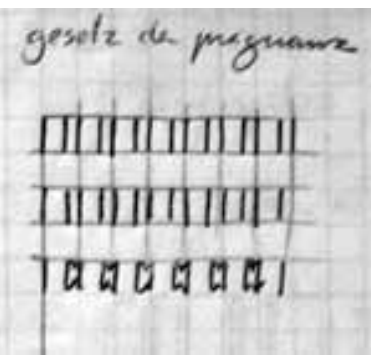

i2/98 Proximidad-ambigüedadsemejanza. Apuntes del alumno Klaus Krippendorff.

i2/99 Proximidad e igualdad. llustración del manual: Koffka, Kurt "Principles of Gestalt Psychology". New York: Harcourt, Brace \& World, Inc, 1935. 

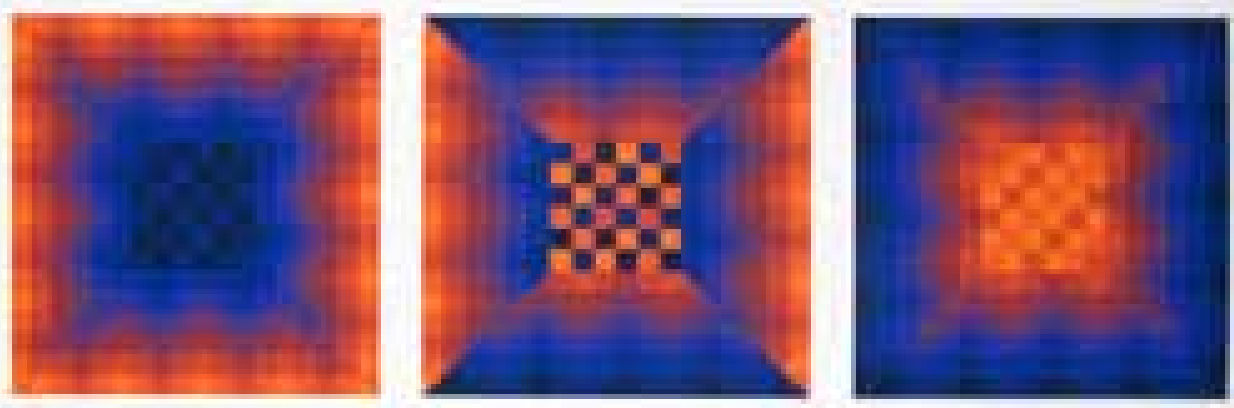

i2/100 Fondo-ambiguedad-primer plano.

Alumno: Edgar Decurtins

Curso: Visuelle Einführung.

Kurs Maldonado. (1956-1957)

Dp_042.035 F.B.S

i2/101 Fondo-ambiguedad-primer

plano.

Alumno: Berthus Mulder.

Curso: Visuelle Einführung. Kurs

Maldonado (1956-1957)

Dp_044.001 F.B.S
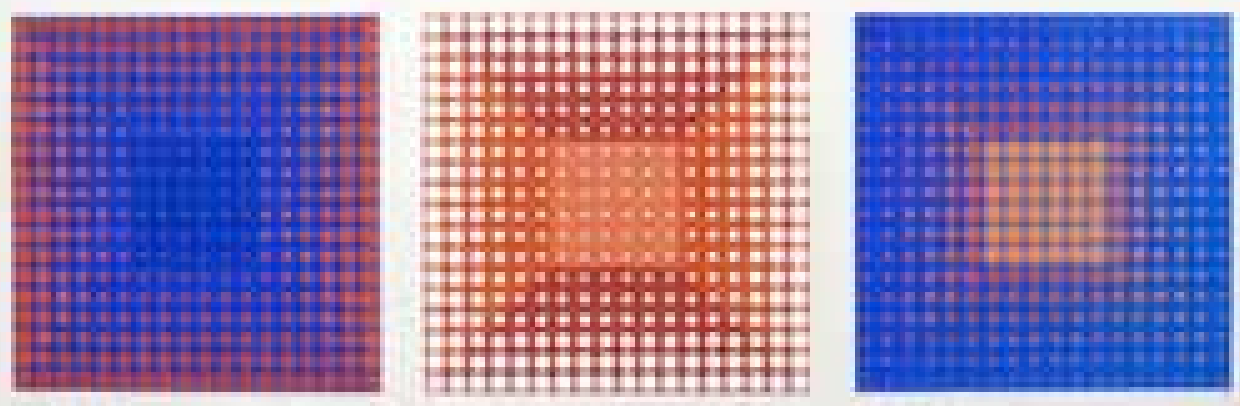
uno de los requisitos que Maldonado propone en el título del ejercicio, de tal manera que la fila superior tiene que ofrecer la percepción de proximidad, la central de ambigüedad y la última, de semejanza. El uso del color distribuido en las tiras verticales es el que facilita el cumplimiento del requisito.

En la banda superior, la proximidad prevalece a la semejanza. Es decir, se perciben en primer lugar las lineas que están más próximas como una unidad visual a pesar de que estas líneas tienen colores diferentes. En la banda inferior, el efecto es el opuesto, la semejanza prevalece sobre la proximidad, es decir, se perciben como unidad visual las bandas del mismo color con mayor intensidad que las que se sitúan a menor distancia. En la banda central se produce una situación de ambigüedad, es decir, pueden percibirse como unidad visual tanto las tiras del mismo color como las que están más próximas. En algunos casos los alumnos utilizaron patrones visuales reticulares o a modo de tramas de líneas como una variable más para condicionar los resultados -figuras i2/91 y i2/92-. En la banda superior el patrón juega en contra de la proximidad. En la banda inferior, juega en contra de la semejanza. En la intermedia contribuye a la ambigüedad.

\section{Fondo-ambigüedad-primer plano}

Este ejercicio se realiza en los cursos 1956-57 y 1958-59. Se puede considerar como un conjunto de experimentaciones visuales con referencias claras a los homenajes al cuadrado obra de Josef Albers pero con un enfoque distinto, en el que Maldonado investiga sobre la variabilidad en la percepción de los planos de profundidad que, según Huff, alcanzó una solución gracias a la estructura de retícula.

El objetivo del ejercicio consiste en lograr que la condición de primer plano y de fondo sean intercambiables en la misma unidad visual, es decir, que el fondo se perciba como figura y la figura como fondo en virtud de la creación de un estado de ambigüedad. En este caso, las variables de ejecución son el color, la ubicación, la configuración del conjunto mediante la estructura de retícula ${ }^{24}$ como mecanismo configurador y los códigos visuales de profundidad para alcanzar el resultado propuesto en cada caso. A su vez, la retícula se materializa en forma de cuadrados o de círculos (i2/100, i2/101). De este modo, en el primer campo visual en el que destaca el fondo de la figura, la distribución de colores es tal que se percibe como más iluminada, mientras que en el perímetro se distribuyen los colores más apagados. En los resultados de estos ejercicios se manifiesta uno de los intereses pictóricos tempranos de Maldonado a los que se refiere Huff como parte de la problemática artística de Maldonado: la disolución entre fondo y la figura. ${ }^{25}$

Se realizan tres estudios en la misma lámina, con el mismo formato empleando un patrón de colores. Como en el ejercicio anterior, el alumno tiene que conseguir que en cada uno de los estudios predomine la percepción de uno de los componentes visuales: el fondo, el plano intermedio o el primer plano.

\section{Estructuras y superficies de color en equilibrio visual}

Bajo este título se agrupan una serie de ejercicios realizados en los curso 1955-1956, 19561957 y 1958-1959 que abordan el tema del equilibrio visual como respuesta al concepto de pregnancia. El objetivo es mantener el equilibrio de la composición como unidad visual procurando que ninguno de los elementos del conjunto destaque de manera imprevista
24 La retícula como mecanismo configurador se analiza en el capítulo "Atlas forma". En estos ejercicios es el soporte estructural el que de forma sinérgica con el color configuran la solución.

25 Este aspecto pictórico se estudia en el capítulo "Atlas forma" como tema subyacente en los ejercicios "exactoinexacto" e "inexacto-exacto". 


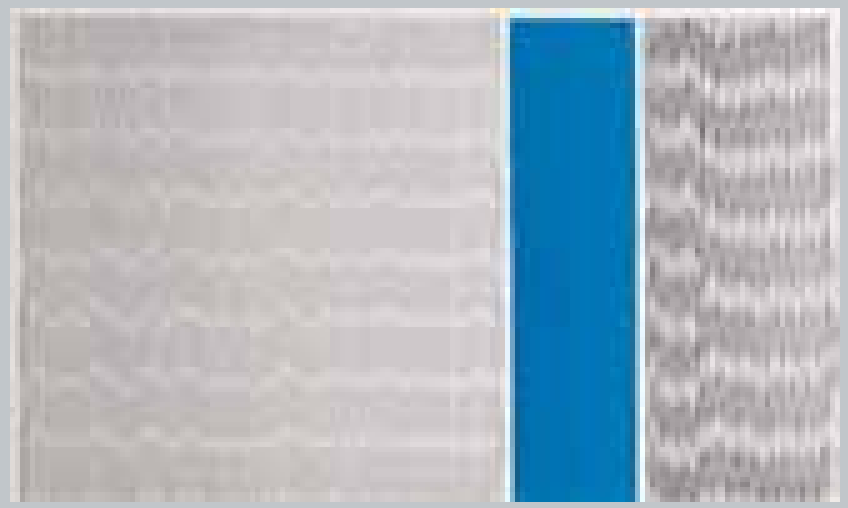

i2/102 Tres planos en equilibrio Alumno: Peter Disch

Curso: Visuelle Einführung. Kurs Maldonado (1955-1956).

Gs 038.003 F.B.S

i2/103 Tres planos en equilibrio Alumno: Peter Disch Curso: Visuelle Einführung. Kurs Maldonado (1955-1956) Gs 038.003 F.B.S

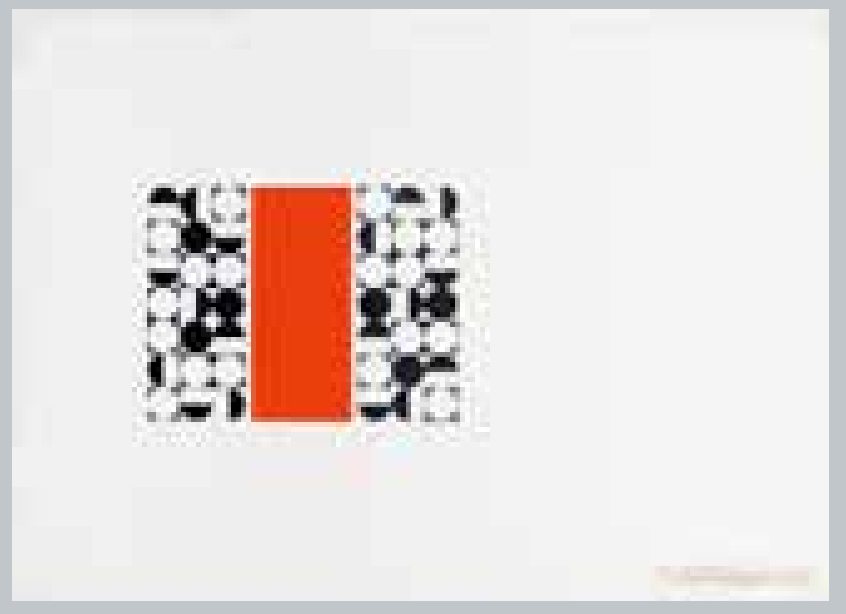

i2/104 Non prima donna

Alumno: Gerhard Curdes

Curso: Visuelle Einführung. Kurs Maldonado (1959-1960) Dp 039.019 F.B.S

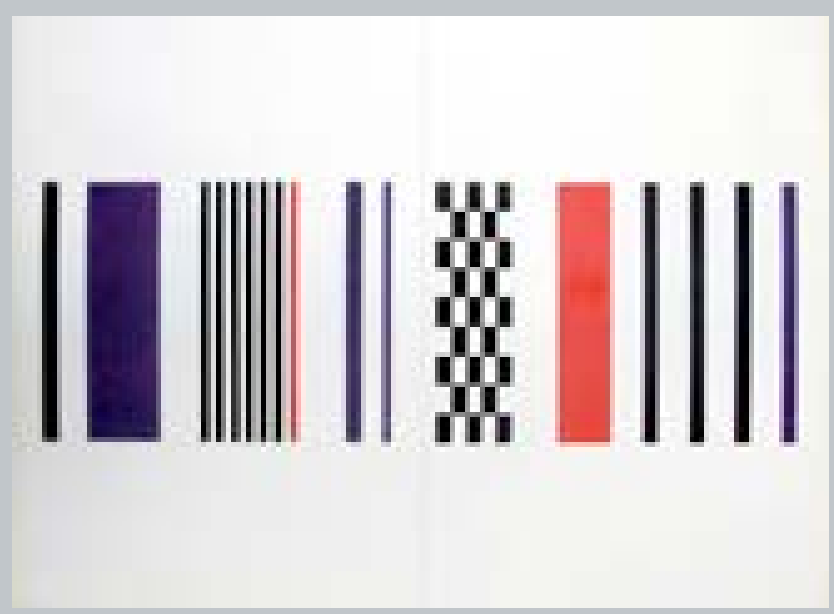

i2/105 Superficie sin dominante Alumno: Edgar Decurtins

Curso: Visuelle Einführung. Kurs Maldonado (1956-1957) Dp 042.035 F.B.S i2/106 Superficie sin dominante Alumna: Monika Buch Curso: Visuelle Einführung. Kurs Maldonado (1956-1957) Dp 045.005 F.B.S 
por su preeminencia sobre los demás. Esta premisa conlleva una estudio sistemático de la ubicación de los elementos y su coloración.

En los ejercicios recogidos en este periodo se pueden apreciar distintas variables formales y una progresión y concreción de éstas. Los primeros ejercicios se plantean sobre una banda de proporción horizontal (i2/102 y i2/103). El color se sitúa en una posición intermedia en la que actúa como elemento articulador de las "estructuras" visuales de rectángulos blancos y negros o los patrones de líneas y puntos. En el curso 1956-1957 la banda se sustituye por un lienzo cuadrado como campo visual que se fragmenta para albergar los elementos constitutivos de la unidad visual, franjas coloreadas o estructuradas (i2/105 y i2/106). Finalmente, en el curso 1958-1959 se retoma la banda horizontal, pero con un énfasis en el papel de las estructuras de modo que se configura una unidad compactada (i2/104). Este formato será el que adopten versiones posteriores practicadas por Maldonado bajo el título de "Non primma donna". El alumno tiene que lograr un equilibrio visual de nuevo utilizando el color como variable. En este caso se incorporan la forma y los patrones visuales de líneas y puntos.

\section{Cantidad de color}

En su enseñanza del color de los cursos 1959-1960 y 1960-1961 Vordemberge-Gildewart propone dos series de ejercicios en los que mejor se sintetiza su propuesta didáctica sobre el color: el conflicto entre calidad y cantidad. El enfoque de los ejercicios tiene un matiz implícitamente gestáltico.

En el primero de esta tipología se denomina "efecto tridimensional intencionado (primer plano, centro, fondo), mediante seis colores seleccionados y aplicados en igual cantidad" (i2/107, i2/109 y i2/111). Las variables de trabajo son los rectángulos con los colores selecionados, la separación entre éstos y la determinante será la ubicación de cada color en el conjunto para conseguir el efecto de ilusión de profundidad.

El título completo de este ejercicio es "cambio cuantitativo de las superficies de color a favor de un efecto bidimensional". (i2/108, i2/110 y i2/112) Este ejercicio se puede entender como un segundo paso del primero. Se mantienen los colores del ejercicio anterior y el orden en el que están dispuestos. El objetivo es modificar el ancho de los rectángulos y su separación para neutralizar el efecto tridimensional ofrecido por el contraste entre las bandas de color.

En el curso 1960-1961 Vordemberge-Gildewart propone el ejercicio "Cambios en un mismo color para la misma cantidad por la variación del color de su entorno" en un planteamiento análogo a los primeros experimentos de Albers sobre la modificación de la apariencia del color (i2/113 y i2/114). El objetivo del ejercicio es variar la percepción del rectángulo interior de color en sus cualidad de tono. A diferencia de los ejercicios del curso de Albers, en este ejercicio se utiliza gouache en lugar de papel de color. Además, se realizan en una serie de cuatro combinaciones en la misma lámina, a modo de los esquemas del contraste simultáneo de Chevréul. ${ }^{26}$

En el curso básico se desarrollaron una serie de ejercicios que trabajaban la relación entre el color y la superficie de la materia, un vínculo a la que pertenece a lo háptico y sus cualidades, reales o sugeridas, perceptibles por el tacto.
26 Michel Ėugene Chevréul fue un químico del siglo XIX que desarrolla una teoría sobre el color y que publica con el título: De la loi du contraste simultané des couleurs et de l'assortiment des objets colorés considéré d'après cette loi dans ses rapports avec la peinture, les tapisseries des gobelins, les tapisseries de Beauvais pour meubles, les tapis, la mosaique, les vitraux colorés, l'impression des étoffes, l'imprimerie, l'enluminure, la decoration des édifices, l'habillement et l'horticulture. Paris : Imprimerie nationale : Librairie Gauthier-Villars et fils, quai des Grands-Augustins, 55, 1889.

Este libro expone el concepto de contraste simultáneo, basado en la idea de que dos colores adyacentes se percibirán tan distintos como pueda el ojo. A partir de este concepto, se estudian las variables de contraste entre colores que influyen en la diferente percepción de colores adyacentes. 


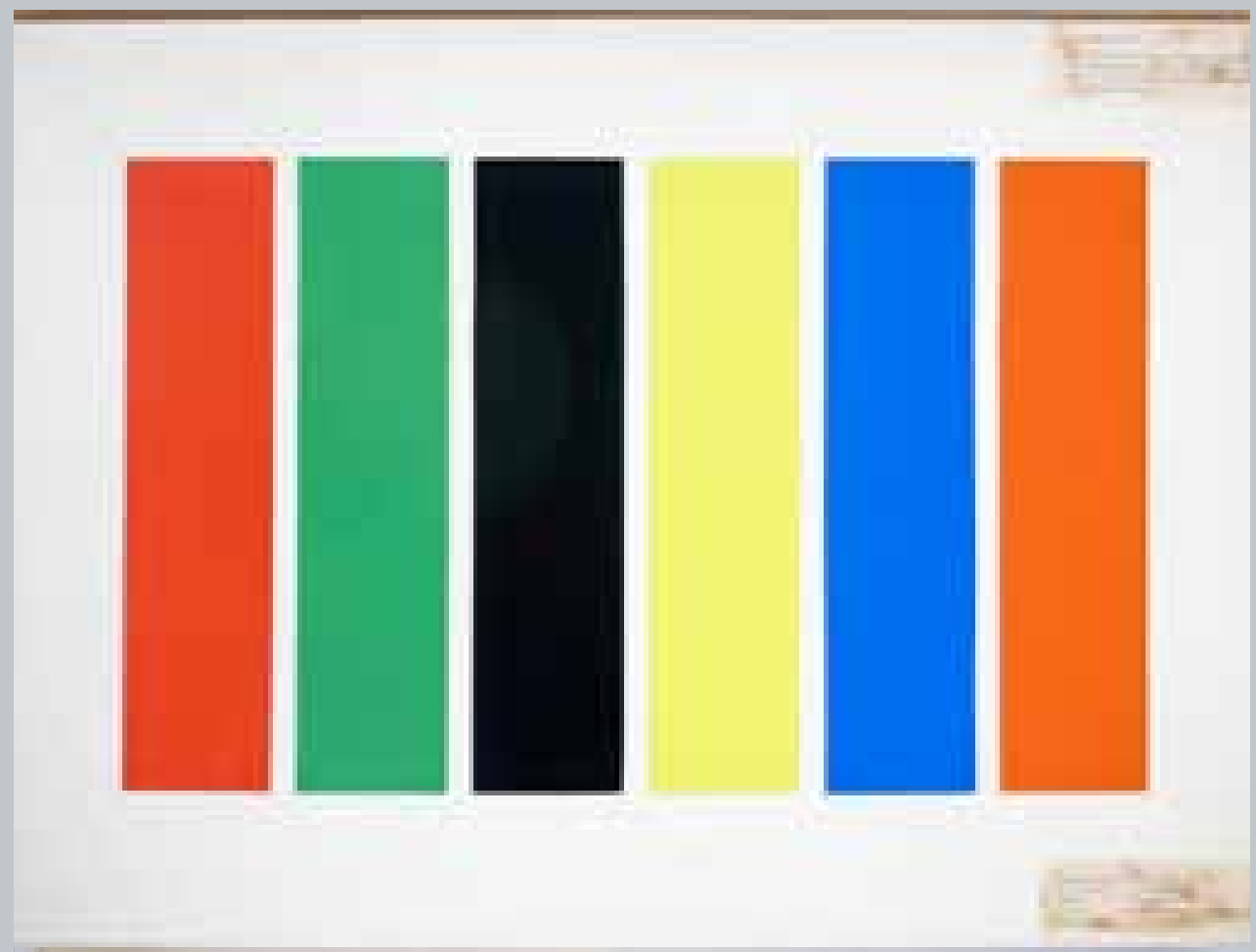

i2/117 Efecto deliberadamente tridimensional debido a seis colores

cuantitativamente idénticos

Alumna: Erika Fortner.

Curso: Rittel Vordemberge

Farbenlehre (1959-1960)

Gs 061.021 F.B.S
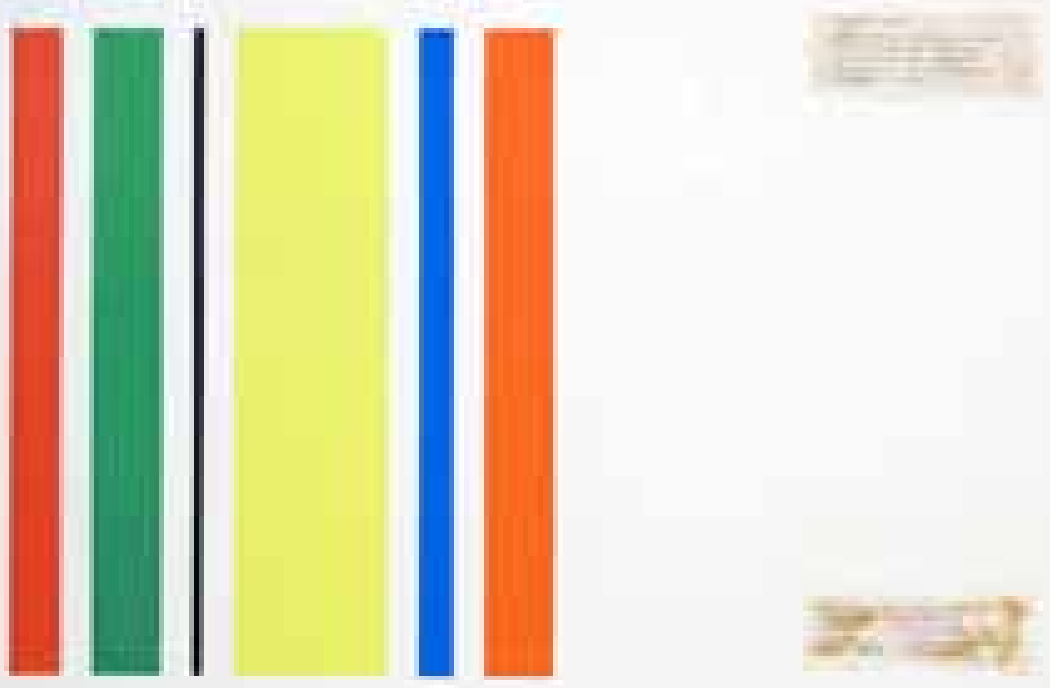

i2/108 Cambio cuantitativo de las superficies de color a favor de un efecto bidimensional

Alumna: Erika Fortner.

Curso: Rittel Vordemberge

Farbenlehre (1959-1960)

Gs 061.021 F.B.S 

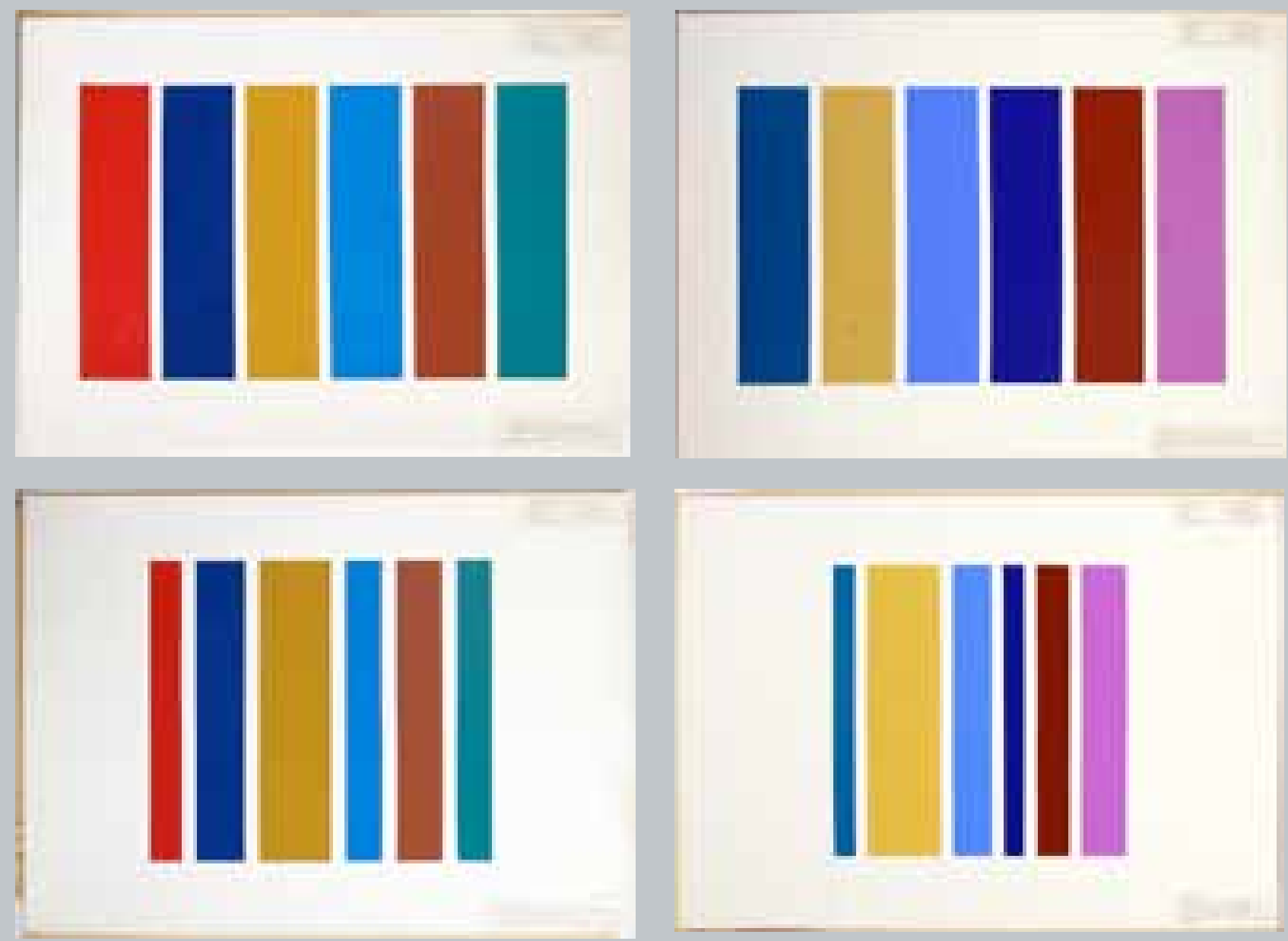

i2/109 Efecto deliberadamente tridimensional debido a seis colores cuantitativamente idénticos / Alumno: Reinhold Deckelmann Curso: Rittel Vordemberge Farbenlehre (1959-1960) / Dp 047.005 F.B.S

i2/110 Cambio cuantitativo de las superficies de color a favor de un efecto bidimensional / Alumno: Reinhold Deckelmann

Curso: Rittel Vordemberge Farbenlehre (1959-1960) / Dp 047.005 F.B.S

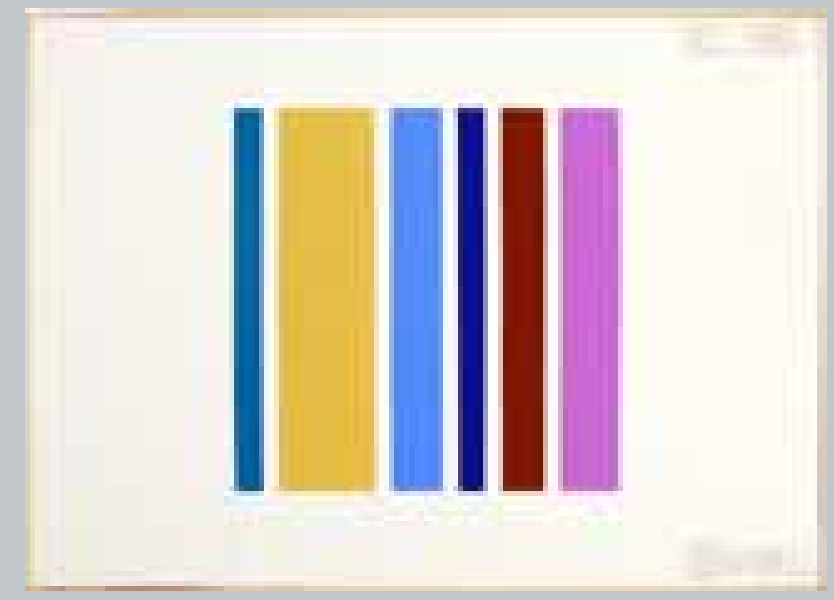

i2/111 Efecto deliberadamente tridimensional debido a seis colores cuantitativamente idénticos / Alumno: Heinz Wager.

Curso: Rittel Vordemberge Farbenlehre (1959-1960) / Gs 048.014 F.B.S

i2/112 Cambio cuantitativo de las superficies de color a favor de un efecto bidimensional / Alumno: Heinz Wager.

Curso: Rittel Vordemberge Farbenlehre (1959-1960) / Gs 048.014 F.B.S
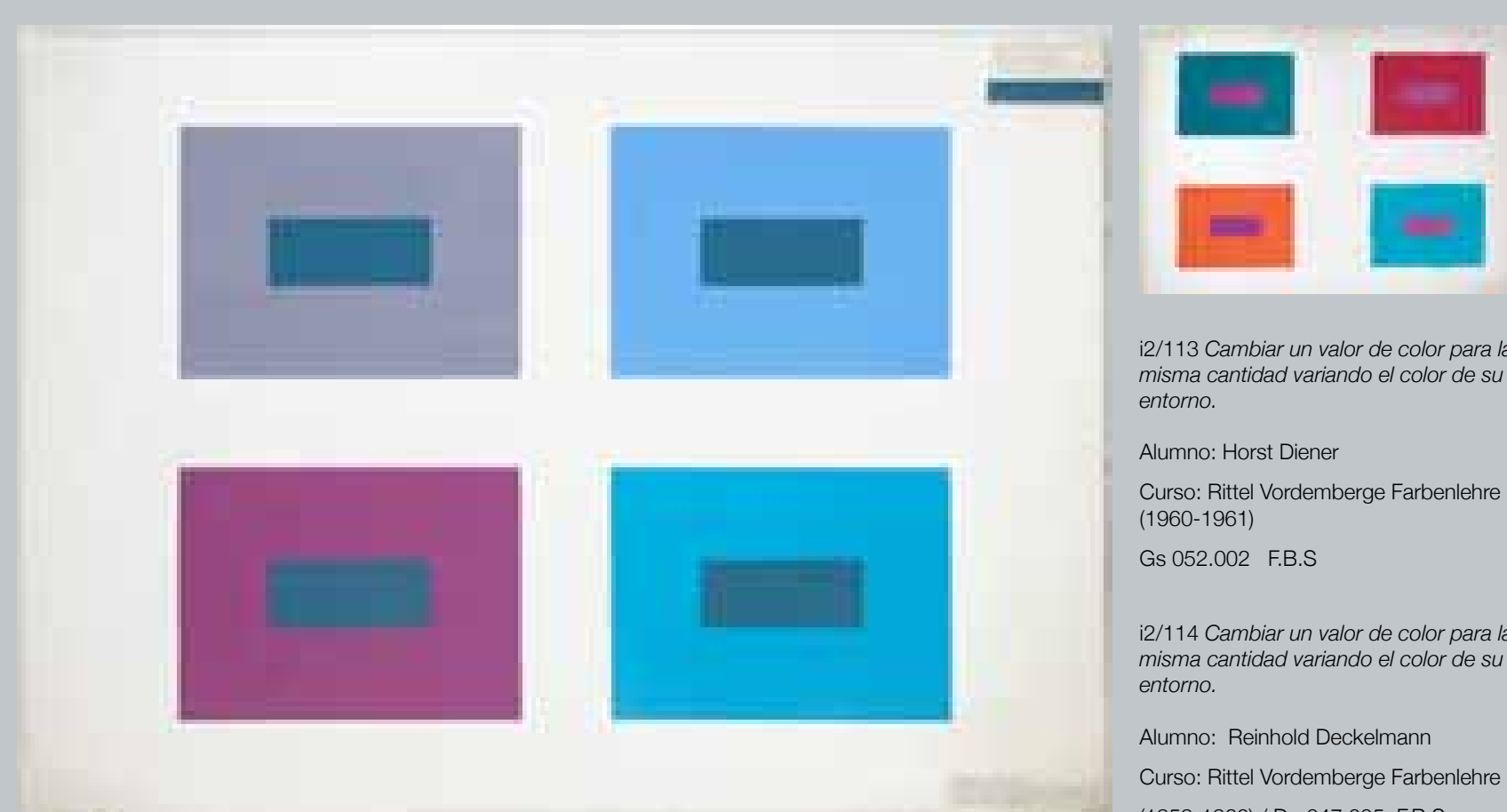

i2/113 Cambiar un valor de color para la misma cantidad variando el color de su entorno.

Alumno: Horst Diener

Curso: Rittel Vordemberge Farbenlehre (1960-1961)

Gs 052.002 F.B.S

i2/114 Cambiar un valor de color para la misma cantidad variando el color de su entorno.

Alumno: Reinhold Deckelmann

Curso: Rittel Vordemberge Farbenlehre (1959-1960) / Dp 047.005 F.B.S 


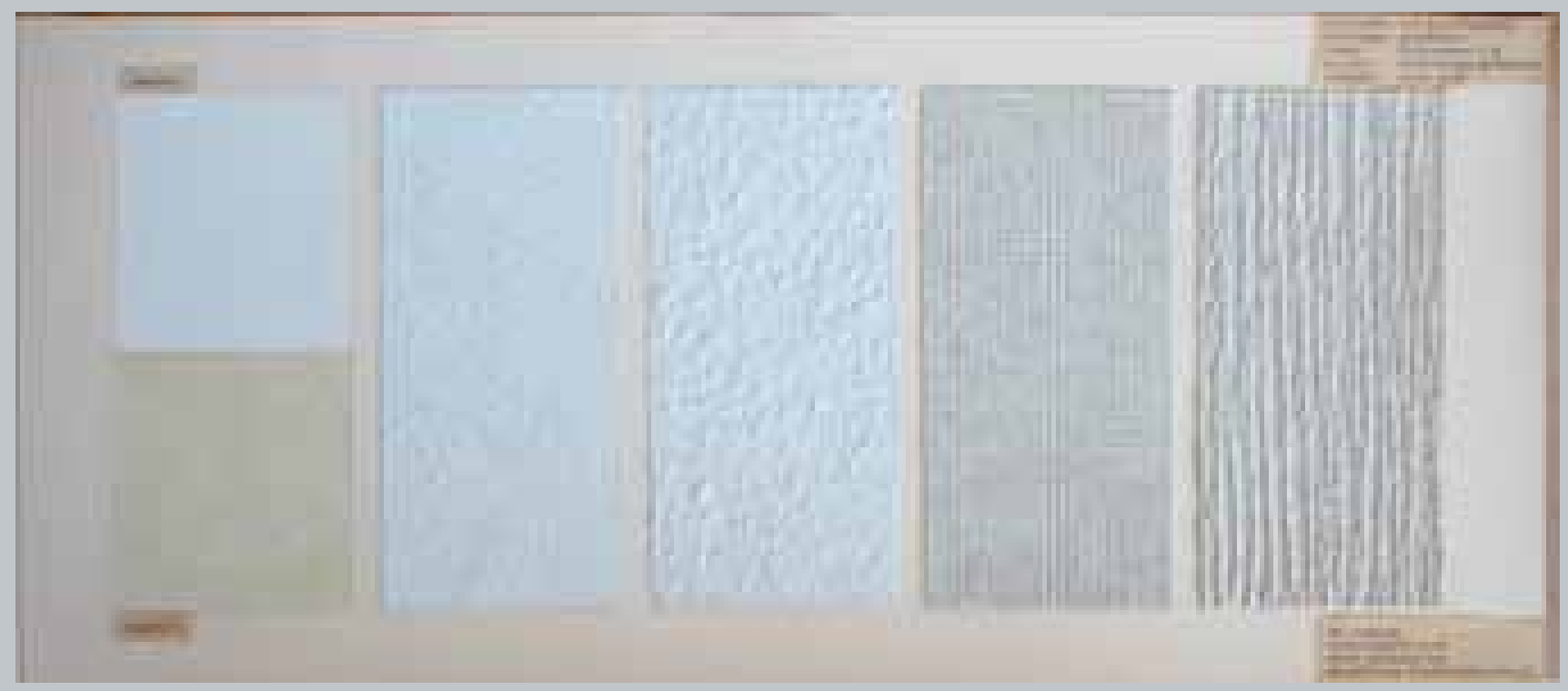

i2/115 Escala de valores de color blanco mediante la faktura de la superficie / Alumno: Peter Beck / Curso: Rittel Vordemberge Farbenlehre (1959-1960) / Dp 003.033

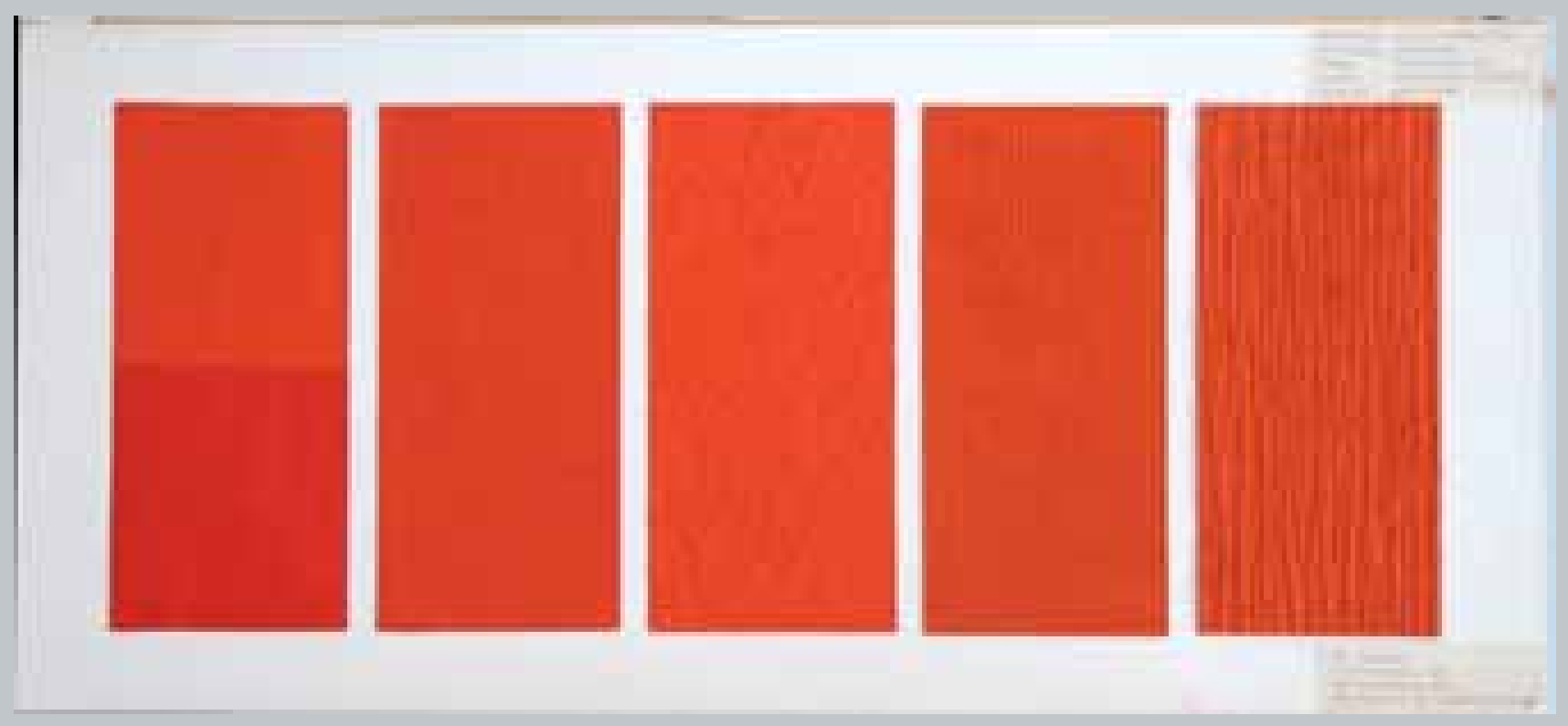

i2/116 Escala de valores de color rojo mediante la faktura de la superficie / Alumno: Peter Beck / Curso: Rittel Vordemberge Farbenlehre (1959-1960) / Dp 003.033
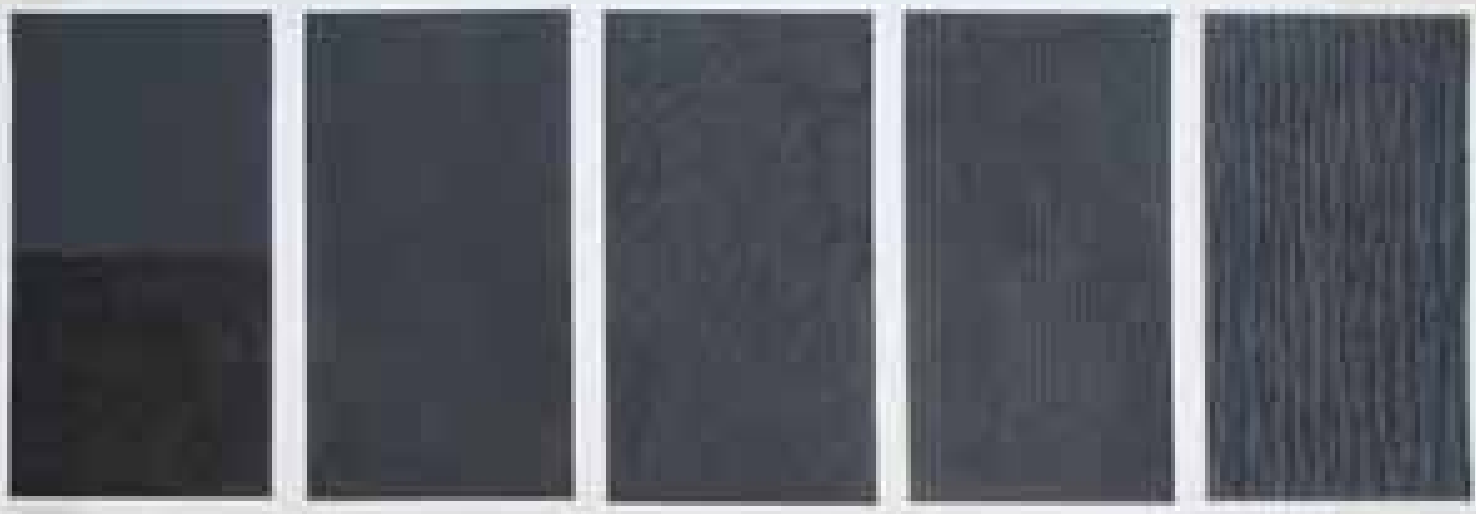

i2/117 Escala de valores de color negro mediante la faktura de la superficie / Alumno: Peter Beck / Curso: Rittel Vordemberge Farbenlehre (1959-1960) / Dp 003.033 


\subsection{Color háptico}

La textura y el color se explora de forma simultánea en los cursos 1959-1960 y 1960-1961 en el curso de Vordemberge-Gildewart. Se ejecutan tres variaciones sobre el mismo tema bajo el título "escala de valores de blanco - rojo y negro, respectivamente- mediante la faktura $^{27}$ de la superficie -refracción del color-" (i2/115, i2/116 y i2/117).

En cada una de las tres láminas se disponen cinco rectángulos de distintos materiales que se colorean con el mismo tono: blanco, rojo y negro respectivamente. En la primera pieza cuya superficie es lisa, la mitad inferior se barniza. La segunda y tercera presentan una textura granulada. La cuarta presenta una textura textil y la quinta, una superficie estriada. El ejercicio se plantea como una escala de color, es decir, los rectángulos están ordenados en virtud del oscurecimiento del tono, de forma que la superficie lisa es la más clara y la más rugosa la más oscura.

El recurso pictórico-plástico de la "faktura" se remonta al constructivismo. Las escalas táctiles han sido empleadas por las vanguardias como método de experimentación con la materia. Como dispositivo didáctico, este ejercicio nos remite directamente a la tradición de la Bauhaus por el carácter exploratorio de los materiales - la didáctica de Itten - y a los estudios sensoriales de Moholy Nagy en la Bauhaus y la New Bauhaus de Chicago. Frente al enfoque de objeto artístico que le otorgaban los futuristas -el tactilismo de Marinetti-, Moholy centró el objetivo de estos ejercicios en la activación de los aspectos sensoriales, como una introducción a la educación de la percepción óptica. ${ }^{28}$ Había una intención de sistematización en los ejercicios de Moholy, que a veces se acompañaban de gráficos que pretendían representar los niveles de intensidad vinculados de las sensaciones provocadas al tacto.

El ejercicio de Vordemberge en la HfG se distancia de los experimentos materiales de Itten y se acerca a los planteamientos de Moholy por la sistematización del proceso: frente a la intervención deliberada de materiales diversos, se realiza una operación sistemática. Sin embargo, mientras que para Moholy el parámetro principal del ejercicio es la textura, para Vordemberge la textura solo es un medio para alcanzar otro objetivo: la variación del tono en el color. Aquí reside el interés del ejercicio de la HfG como evolución de los ejercicios con textura: la simultaneidad de la percepción visual y táctil.

Si bien este ejercicio de Vordemberge redunda en un planteamiento pictórico visual, podemos ampliar la dimensión didáctica del mismo hacia aspectos de la componente pragmática de la semiótica. Desde este punto de vista, el ejercicio puede considerarse una carta de colores, un estudio de la interacción entre color y superficie táctil con una transferencia más allá de lo visual, con una componente háptica de los objetos en la que la componente material es la que determina el valor del conjunto.

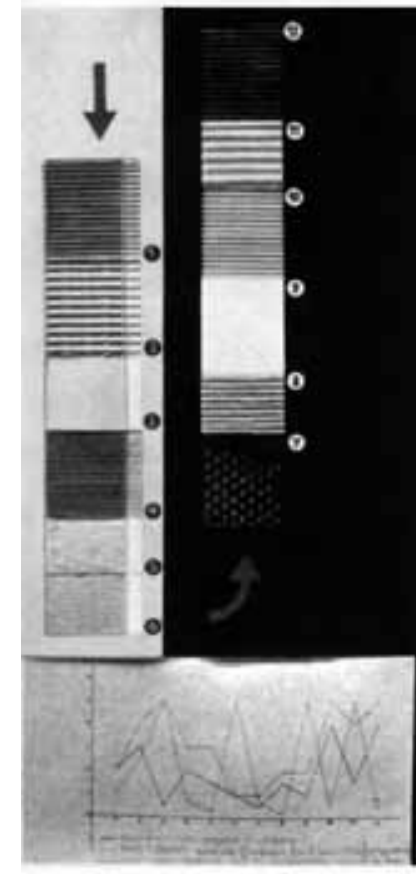

i2/118 Panel táctil de papel con valores estructurados en sensaciones de presión y vibración.

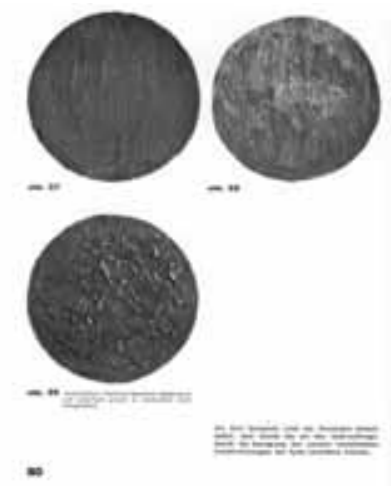

i2/119 Varias facturas del mismo óleo sobre el mismo lienzo fotografiadas bajo la misma luz. ${ }^{30}$ Ejemplos de facturas pictóricas: el color de la pintura influido por el movimiento del pincel. Un mal necesario si uno quiere conjurar a la luz. ${ }^{29}$

27 "Faktura" es un término usado por Moholy-Nagy que desarrolla una terminología propia con el objetivo de precisar y definir el modo de presentación de los materiales. Factura, que tiene su raíz en el lacín "facere: hacer", es según Moholy la epidermis, la forma y apariencia de la superficie del material transformado. Wick, Rainer. Pedagogía de la Bauhaus. Madrid: Alianza Editorial, 1982, p. 135.

28 Íbid, p. 133.

29 Moholy-Nagy, Lázsló. Von Material zu Architektur. München: Langen, 1929, pp. 80-81.

30 lbidem. 


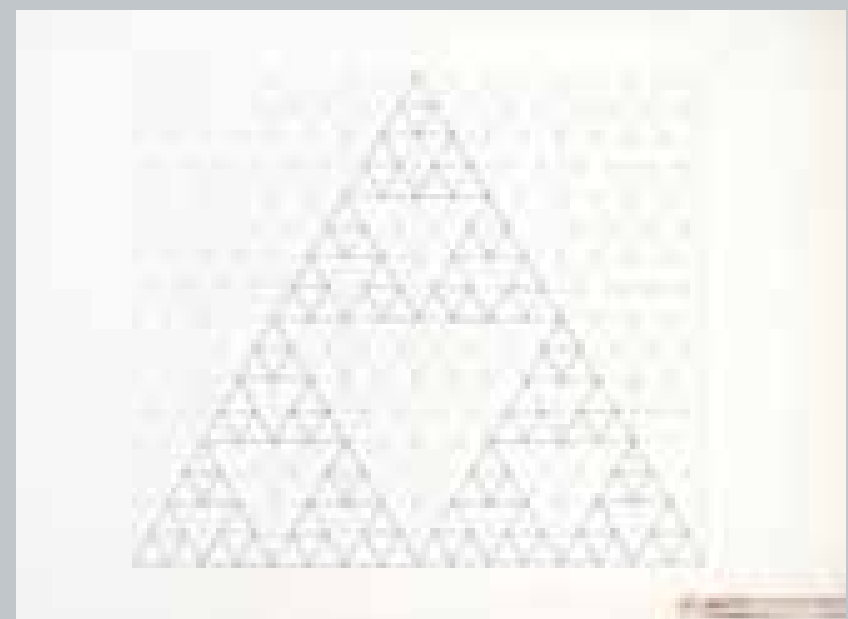

i2/120 Triángulo de Sierpinski - sistema de ordenación

Alumno: Peter Disch.

Curso: Visuelle Einführung. Kurs Maldonado (1955-1956).

Gs_038.003 F.B.S

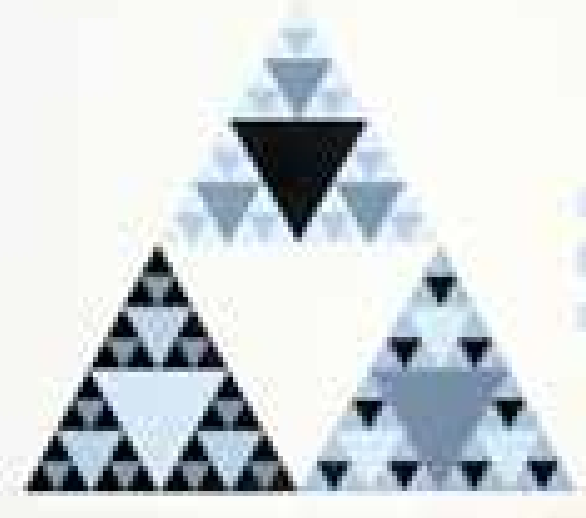

i2/122 Triángulo de Sierpinski blanco/negro corrección Alumno: Peter Disch.

Curso: Visuelle Einführung. Kurs Maldonado (1955-1956).

Gs_038.003 F.B.S

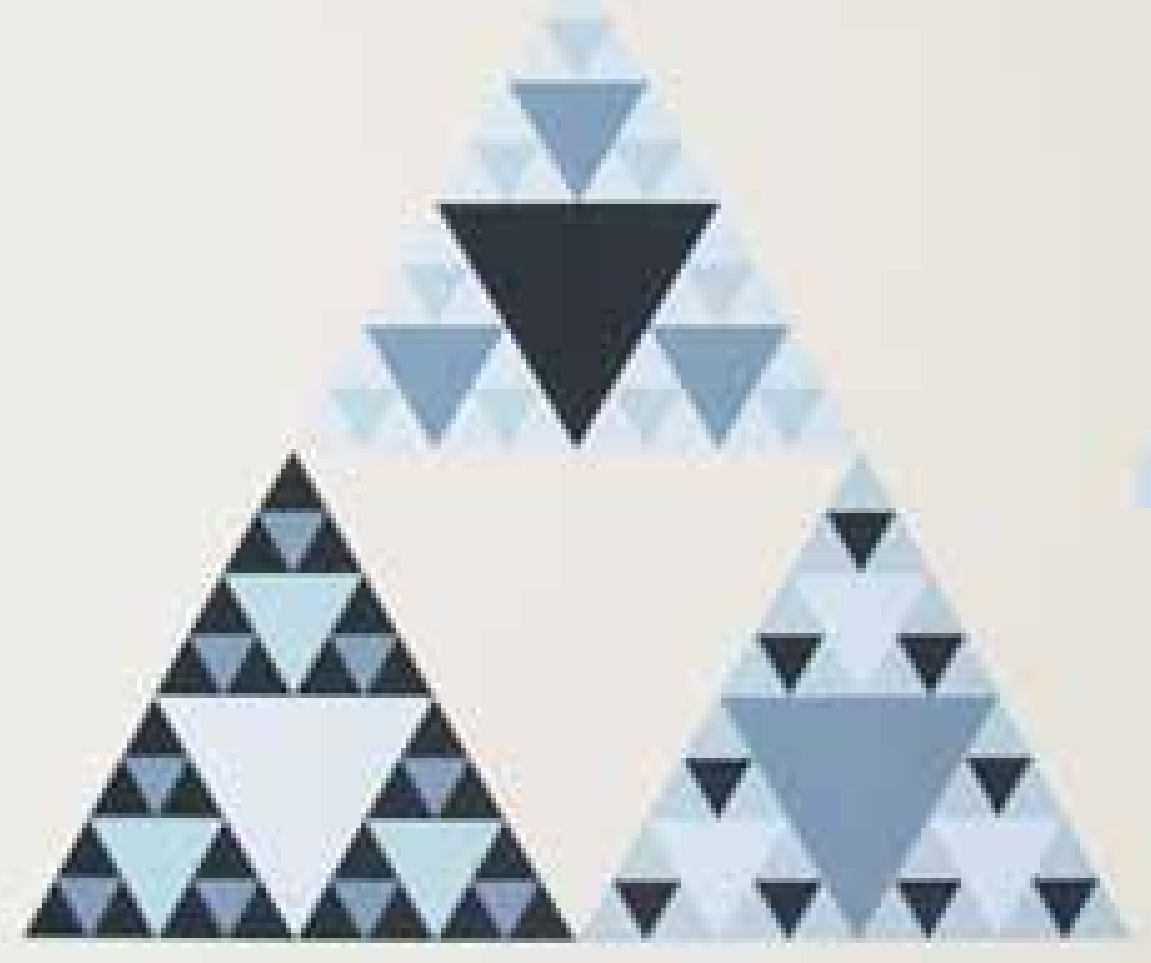




\subsection{Color sintáctico}

En los ejercicios de este epígrafe, el color es una variable integrada en el conjunto de procesos de configuración de la tarea mediante la articulación coordinada de todas las variables como si se tratara de la construcción de una sintaxis. En estos ejercicios, el color pasa a convertirse en un elemento de ejercitación visual subordinada a otras de orden superior que pueden ser una norma, condición o patrón que determina la propia ejercitación y configura la solución del ejercicio. En este sentido se estudian una serie de ejercicios cuyas variables principales o condicionantes son de tres naturalezas: geométricas, combinatorias y proyectuales.

\subsubsection{Topología y color}

Maldonado utiliza la topología como uno de los elementos de la sintaxis compositivo-visual en una serie de ejercicios en su curso dentro del Visuelle Einführung, en paralelo a su enseñanza sobre la percepción. Huff considera que la labor de Maldonado al incorporar la simetría, la topología y la teoría de la percepción en sus ejercicios de Diseño Básico, ya imbuidos del Arte Concreto, le permite "someter el artefacto geométrico a la experiencia perceptual". ${ }^{31}$

Sin embargo, una revisión panorámica de los ejercicios del Visuelle Einführung permite apreciar un proceso en el que la topología se incorpora a la solución del problema del fondo y figura. Las herramientas de la topología - curva de Peano, triángulo de Sierpinskise convierten en el medios para evidenciar la relación fondo-figura al margen de las representaciones habituales empleadas por la psicología de la Gestalt. Esta valoración prioritaria de la topología frente al resultado perceptual da lugar a esta serie de ejercicios en los que el color se subordina a las directrices de la topología.

Maldonado incorpora sus clases magistrales sobre topología tras las de percepción y las de simetría, sin embargo introduce el triángulo de Sierpinski y la curva de Peano como mecanismos de articulación de las configuraciones gestálticas, como patrones o supraestructuras organizadora de la solución visual a los ejercicios. De hecho la curva de Peano le permite experimentar en varios ejercicios las cuestiones relativas a la percepción de la figura y el fondo.

\section{Triángulo de Sierpinski}

Este ejercicio se realizó como experiencia única en el curso 1955-1956 y no se vuelve a repertir en cursos posteriores. Maldonado utiliza la triángulo de Sierpinski como mecanismo gráfico para integrar el color de manera que se diluya su percepción del conjunto (i2/120-i2/122).

Mediante la distribución del color en tres zonas diferenciadas del triángulo de Sierpinski²2 y la variación en la distribución en cada una de las tres áreas se altera la percepción del conjunto teniendo en cuenta que parte de la misma escala de colores:

"Resulta que el efecto óptico de los grados de color en cada uno de los 3 triángulos es diferente y no es consistente con la escala" (i2/125-i2/127).
31 Huff, William S. "Grundlehre at the $\mathrm{HfG}$ - with a focus on the "Visuelle Grammatik" publicado en : W.AA. Ulmer modelle - Modelle nach ulm, hochschule für gestaltung ulm 1953-1968. Ostfildern-Ruit: Hatje Cantz Verlag, 2003. (Catálogo de exposición)., p. 182.

32 Waclaw Sierpinski es un matemático polaco que describe el triángulo que lleva su mismo nombre como una superficie fractal en 1915. El triángulo se construye por iteración, es decir, en sucesivos repartos del área en triángulos equiláteros invertidos. El proceso necesitaría infinitas iteraciones para construirse.

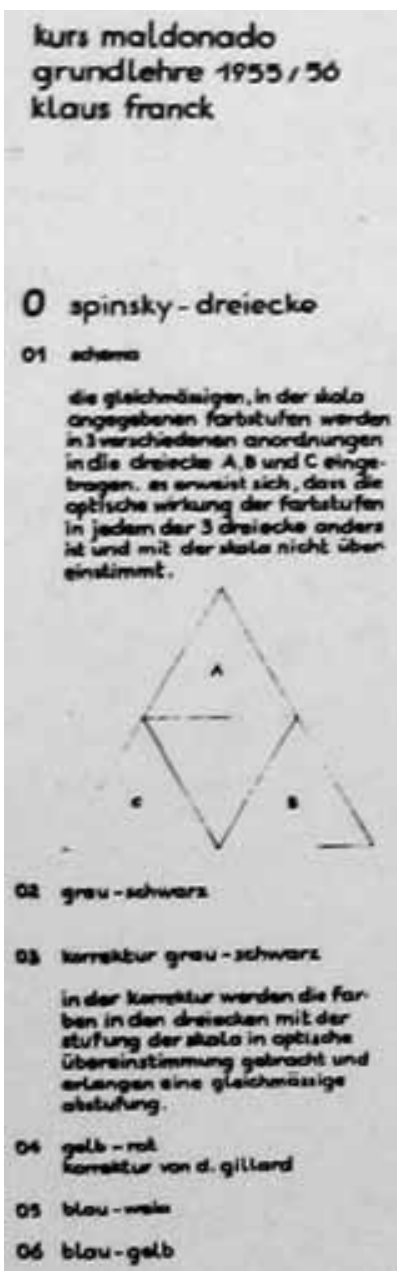

i2/123 Lámina resumen del Kurs Maldonado realizada por el alumno Claus Franck (1955-56) 


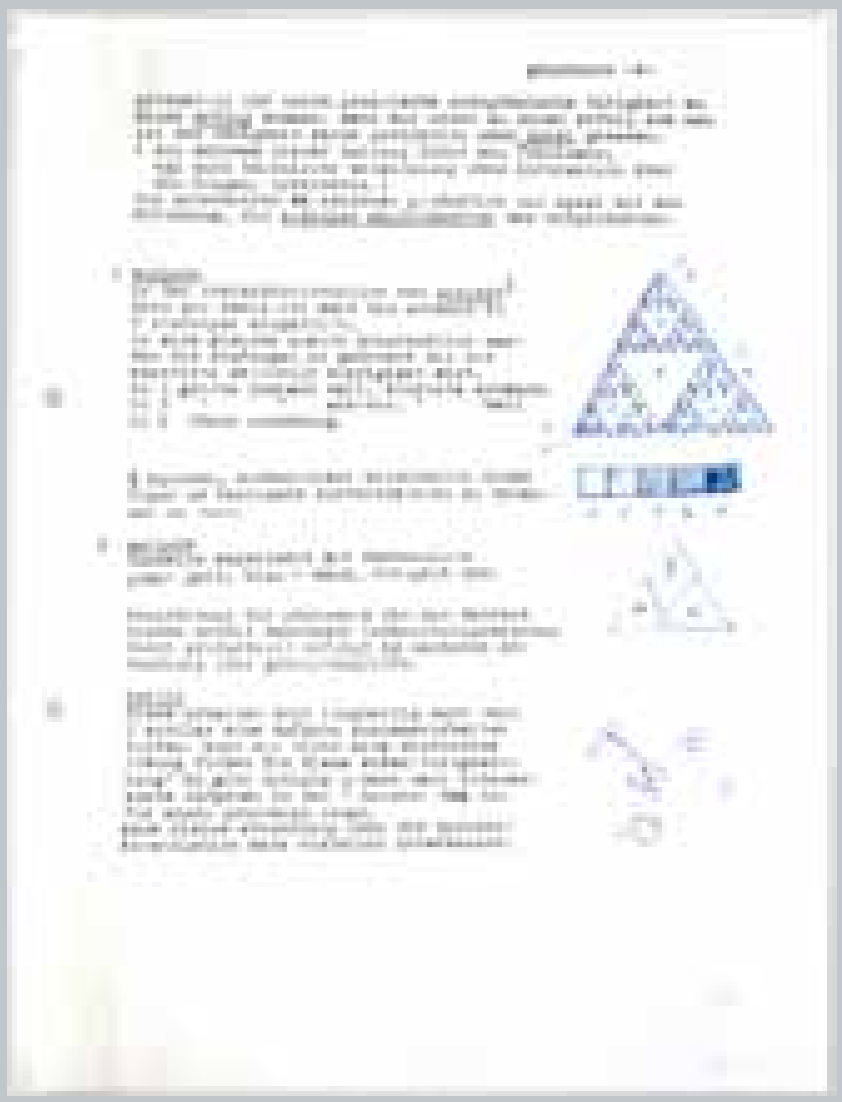

i2/124 Triángulo de Sierpinski apuntes de clase.

Alumno: Andries Van Onck

Curso: Visuelle Einführung. Kurs Maldonado (1955-1956).

Azs 193 F.B.S

i2/125 Triángulo de Sierpinski - del blanco al azul, triple escala de color. Alumno: Peter Disch.

Curso: Visuelle Einführung. Kurs Maldonado (1955-1956).

Gs_038.003 F.B.S

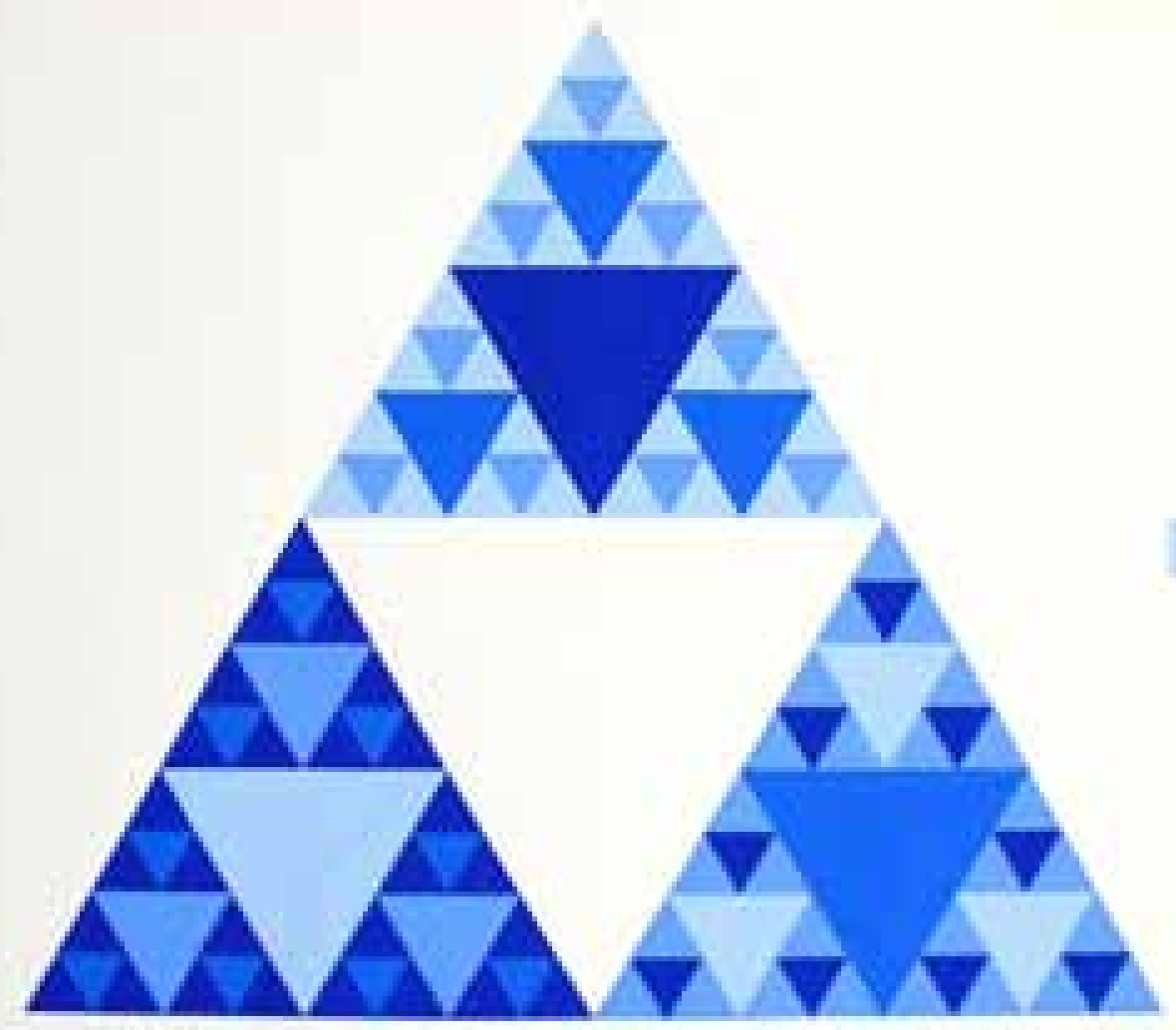




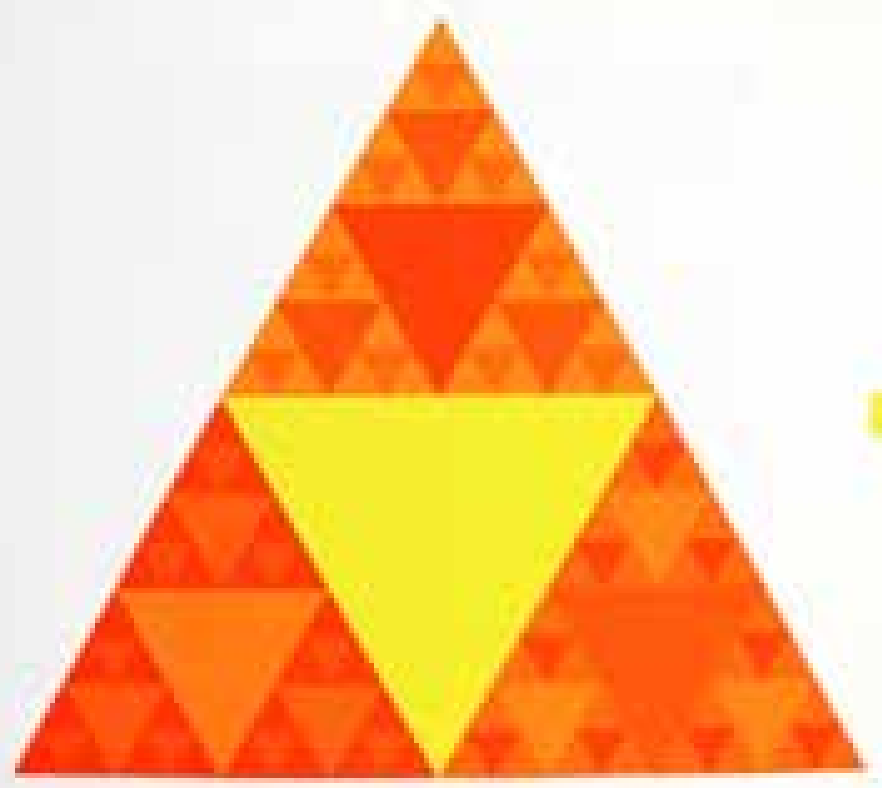

i2/126 Triángulo de Sierpinski - del amarillo al naranja

Alumno: Peter Disch.

Curso: Visuelle Einführung. Kurs

Maldonado (1955-1956).

Gs_038.003 F.B.S

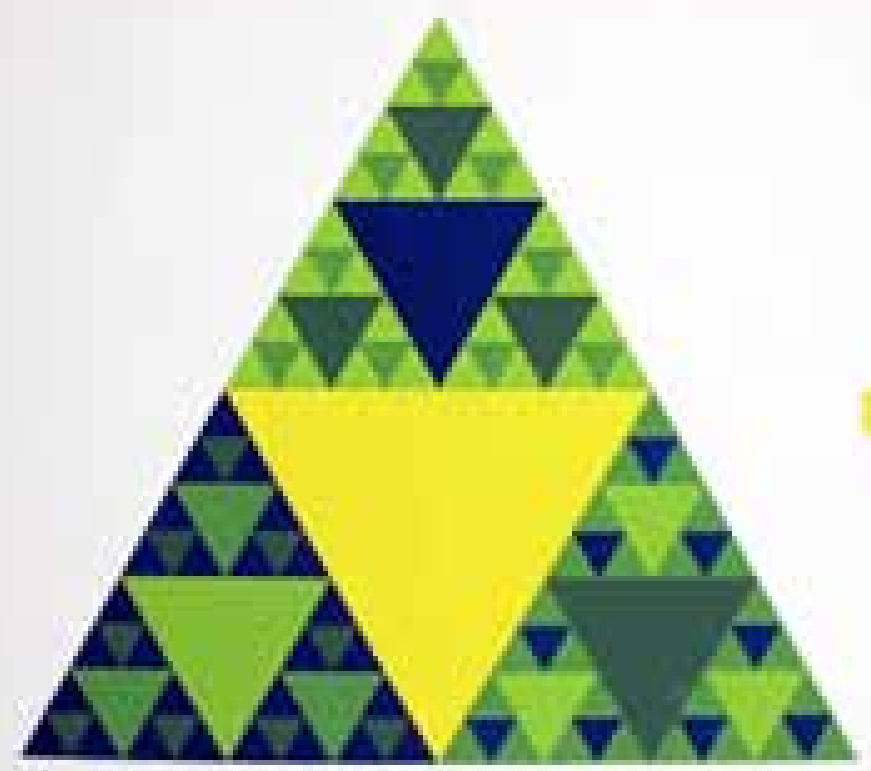

i2/127 Triángulo de Sierpinski - del amarillo al azul.

Alumno: Peter Disch.

Curso: Visuelle Einführung. Kurs Maldonado (1955-1956).

Gs_038.003 F.B.S 
i2/128 Fondo-figura positivo-

negativo / Alumno: Peter

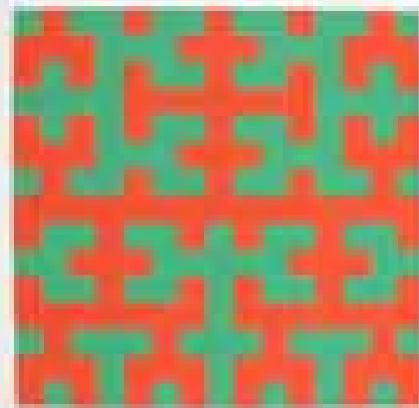

Disch.

Curso: Visuelle Einführung. Kurs Maldonado (1955-1956) / Gs_038.003

F.B.S
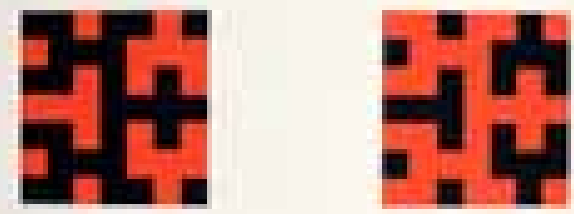

i2/130 Fondo-figura positivo-

negativo / Alumno: Peter Disch

Curso: Visuelle Einführung.

Kurs Maldonado (1955-1956). I

Gs_038.003

F.B.S

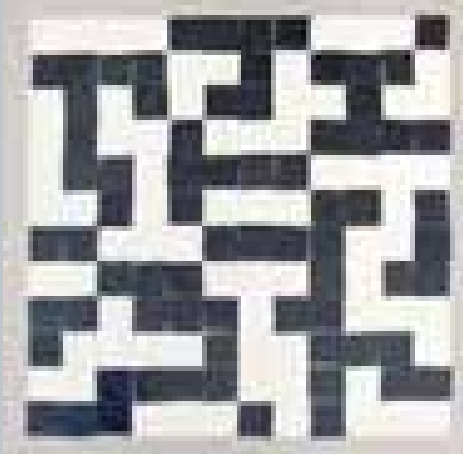

i2/129 Tarea $n^{\circ} 1$. Fondo y figura/ Alumno: Christopher

Naske

Curso: Diseño básico Albers (1953-1954). / Dp_024.003

F.B.S

i2/131 Escala de color lineal sobre curva de peano / Alumno: Peter Disch. Curso: Visuelle Einführung. Kurs Maldonado (1955-1956). / Gs_038.003 F.B.S
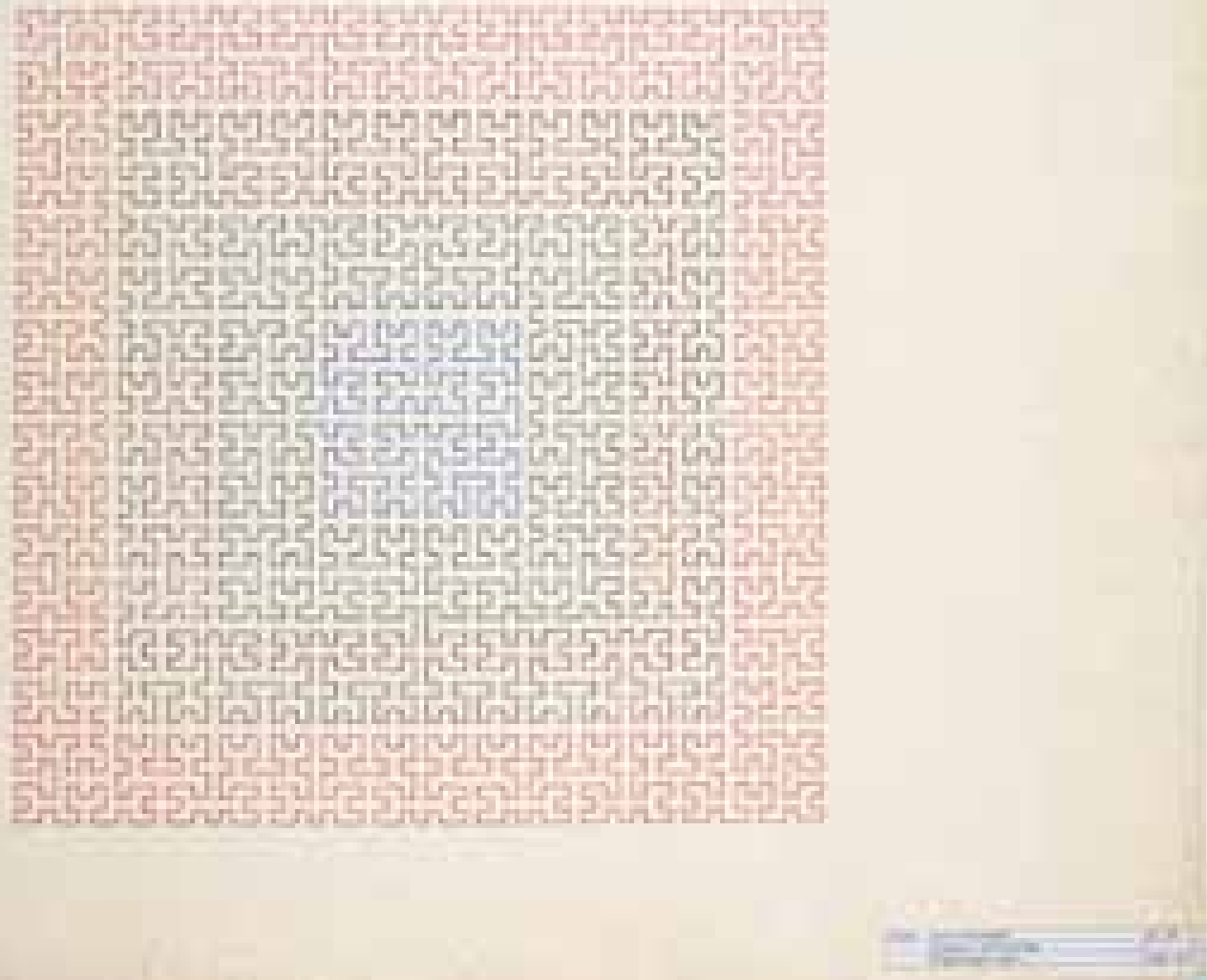
El ejercicio comprende hasta 5 casos: dos ejercitaciones con escala de grises - una de ella con corrección óptica- y otras tres en diferentes escalas de color: amarillo a rojo, amarillo a verde y blanco a azul. Se estudia la configuración de los conjuntos parciales a partir de la distribución de colores que conlleva la percepción de los triángulos en distintos niveles de profundidad aparente. En este sentido son una anticipación del ejercicio primer planomedio-fondo. Las correcciones ópticas propuestas y las ejercitaciones con diferentes tonos, pueden contemplarse en el contexto de la búsqueda personal de Maldonado sobre la disolución del fondo-figura.

En la corrección los colores de los triángulos se distribuyen con la escala de grises corregida para una buena consistencia óptica y se alcanza una gradación uniforme. En este caso el triángulo de Sierpinski se ha empleado para la detección del fenómeno perceptivo y su posterior mejora.

\section{La Curva de Peano como activadora del color}

Durante los cursos 1955-1956 y 1956-1957 Maldonado incorpora la curva de Peano como mecanismo gráfico en sus ejercicios con la misma finalidad del anterior: la percepción unitaria del plano visual. Al igual que la triángulo de Sierpinski, la curva de Peano tiene una naturaleza fractal y está recogida en los manuales de topología por ser la primera curva continua que puede rellenar todo el plano. Esta curva se emplea como subestructura en varias modalidades de ejercicios en los que estudia la relación fondo figura, la relatividad del color y el uso del negro como color procurando que no se perciba como un orificio. En este último caso encontramos dos variantes como veremos a continuación.

\section{Curva de Peano: sistema de brillo de color equivalente, sistema de figura- fondo positivo-negativo}

Como precedente, se puede mencionar el estudio fondo figura de Albers (i2/129).

Estos ejercicios son ensayos sobre la aplicación de los principios gestálticos fondofigura y colores complementarios. En el primero, la curva de Peano se muestra como un mecanismo para diluir el constraste entre el rojo y el verde permitiendo una percepción uniforme (i2/128). En el segundo, se aprecia como la geometría fractal de la curva de Peano aún en secciones parciales permite una percepción dual y ambigua del principio figura-fondo (i2/130).

\section{Escala de color lineal sobre la Curva de Peano}

Se utiliza la geometría fractal de la Curva de Peano para subordinar una escala de color y lograr un efecto de dilución homogénea de la figura. De esta forma, no resalta ninguna parte de la curva en concreto y el aspecto global resulta homogéneo. (i2/131).

33 Parchomenko, A. S. Was ist eine Kurve? Berlin: Deutscher Verlag der WissenschafteN, 1957, pp. 14, 15, 16. 

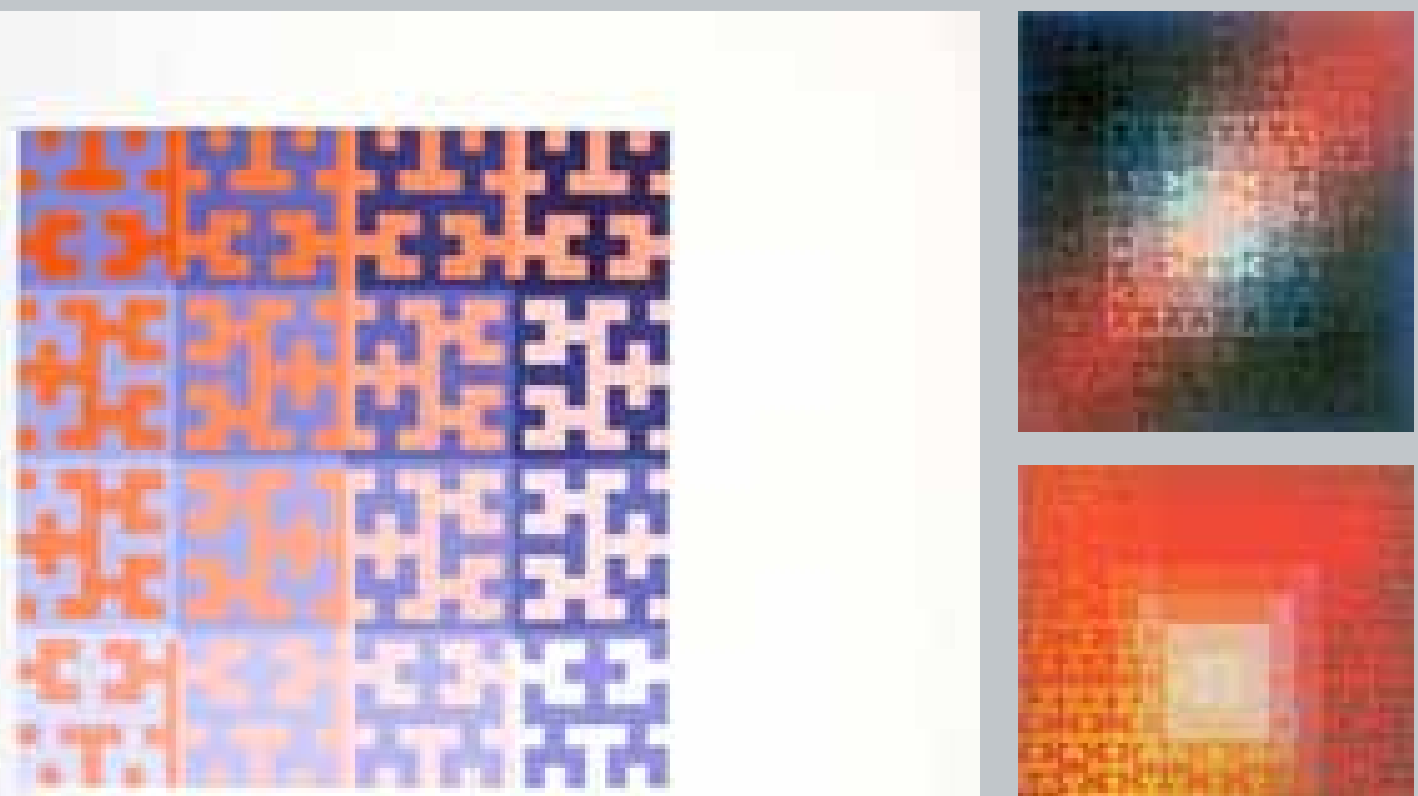

i2/133 Un shock como curva de Peano / Alumno: Hans von Klier / Curso: Visuelle Einführung.

Kurs Maldonado (1955-1956). / Dp 017.002 F.B.S

i2/134,135 Relatividad del color en una superficie de Peano.

Alumno: Andries van Onck

Curso: Visuelle Einführung. Kurs

Maldonado (1955-1956).

i2/137 Negro como color. Alumno: Von Klier. / Curso: Visuelle Einführung. Kurs Maldonado (1956-1957). Dp 017.002. F.B.S

i2/138 Negro como color. Alumna: Meret Lindinger / Curso: Visuelle Einführung. Kurs Maldonado (19561957). Dp 119.001. F.B.S

Gs 038.003 F.B.S
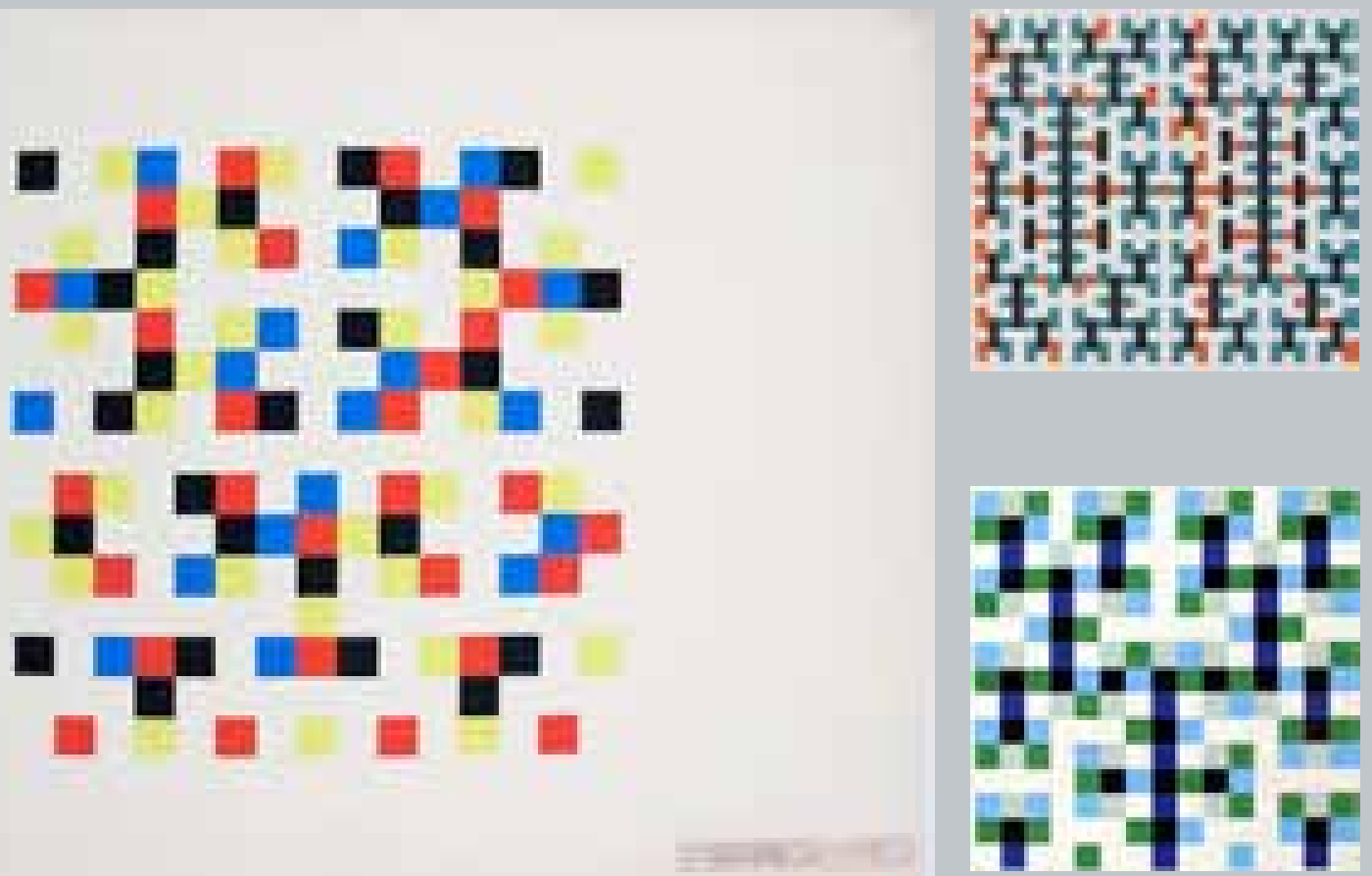


\section{Relatividad del color en la Supercie de Peano}

Bajo esta denominación general se engloban una de las aplicaciones del color sobre la superficie de Peano como mecanismo de activación de la relatividad del color. En los ejemplos mostrados (i2/133) realizados por Hans Von Klier y de Adries Van Onck (i2/134, i2/135), ambos del curso 1955-1956, se trabajan los efectos de profundidad y de fondofigura.

En el ejercicio de Von Klier representa un efecto visual de profundidad configurado mediante el color. En la búsqueda de la disolución de la figura y el fondo los colores se aplican de modo que se alcanza una situación de ambigüedad visual mediante el patrón de la curva de Peano y la coloración de las superficies delimitadas por ésta.

En el caso de los ejercicios de Van Onck se incorpora una variable más a la ambigüedad perceptiva que ya se trabajó en el primer plano-ambigüedad-fondo. Van Onck focaliza la composición en el centro geométrico del cuadrado mediante los gradientes de luminosidad en las distintas regiones que delimita la curva de Peano.

\section{Superficie de Peano: negro como color}

Este ejercicio se realiza en los curso 1955-1956 y 1956-1957 con la curva de Peano como herramienta. El objetivo consiste en encontrar la solución óptima para que el negro se perciba en una imagen como un color más y no como un agujero, es decir, restar al negro su cualidad de profundidad. Para ello se ponen en práctica dos enfoques distintos.

En el curso 1955-1956 el problema se resuelve aplicando el color sobre la figura ramificada que describe la curva de Peano. La curva se utiliza como guía de unas celdillas cuadradas. El color se distribuye mediante un patrón que evita que las superficies coloreadas de negro se perciban como orificios.

El alumno Klaus Franck divide el cuadrado de partida en una retícula de 32 x 32 celdillas y lo organiza en cuatro bandas verticales, de modo que "la segunda representa la inversión del color de la primera. En la tercera hay un cambio de colores y en la cuarta una inversión de la tercera." ${ }^{34}$

El ejercicio propone la organización de la composición de manera que la distribución del color contribuya a que no se produzcan esos agujeros a los que se refiere el título. Es un ejercicio de naturaleza gestáltica opuesto a "Fondo-medio-primer plano", en tanto que rompe el esquema perceptivo del conjunto mediante la disolución de los indicadores de profundidad. Esta disolución se alcanza mediante una distribución del color a partir de la superficie de Peano que permite la iteración del color en cada celdilla de la retícula de manera que el color negro no se focaliza perceptivamente.

La segunda solución se desarrolla en el curso 1956-1957. El ejercicio se aborda de una manera más compleja en la distribución del color, como se aprecia ya en el título "Negro como color no como agujero sobre una superficie de Peano modificada" (i2/139, i2/140).
34 Recogido textualmente en la lámina resumen del Kurs Maldonado dibujado por el alumno del curso 1955-56 Klaus Frank. 
i2/139 Negro como color no como agujero sobre una superfiice de

Peano modificada

Alumno: Ulrich Burandt

Curso: Visuelle Einführung. Kurs

Maldonado (1956-1957)

Gs_033.035 F.B.S

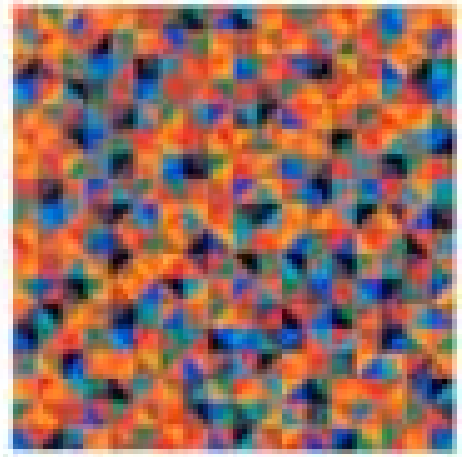

i2/140 Negro como color no como agujero sobre una superfiice de Peano modificada

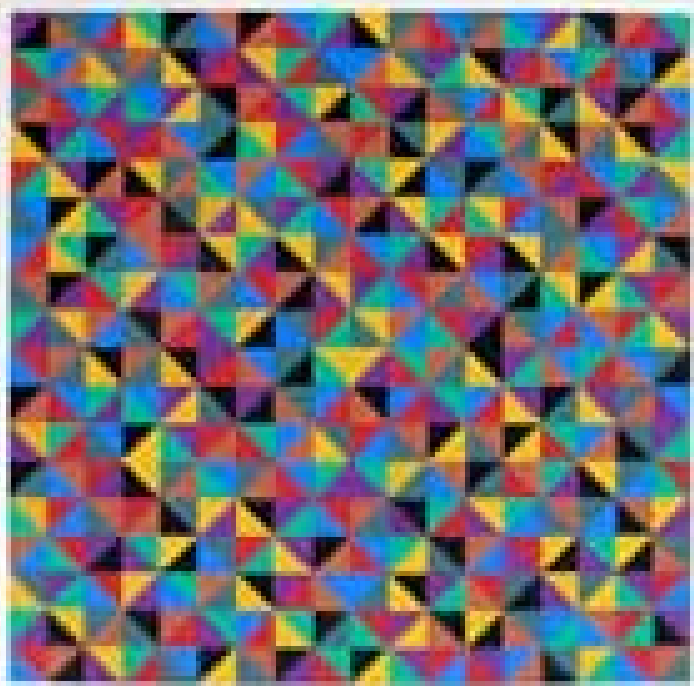

Alumno: Klaus Krippendorff Curso: Visuelle Einführung. Kurs Maldonado (1956-1957) 

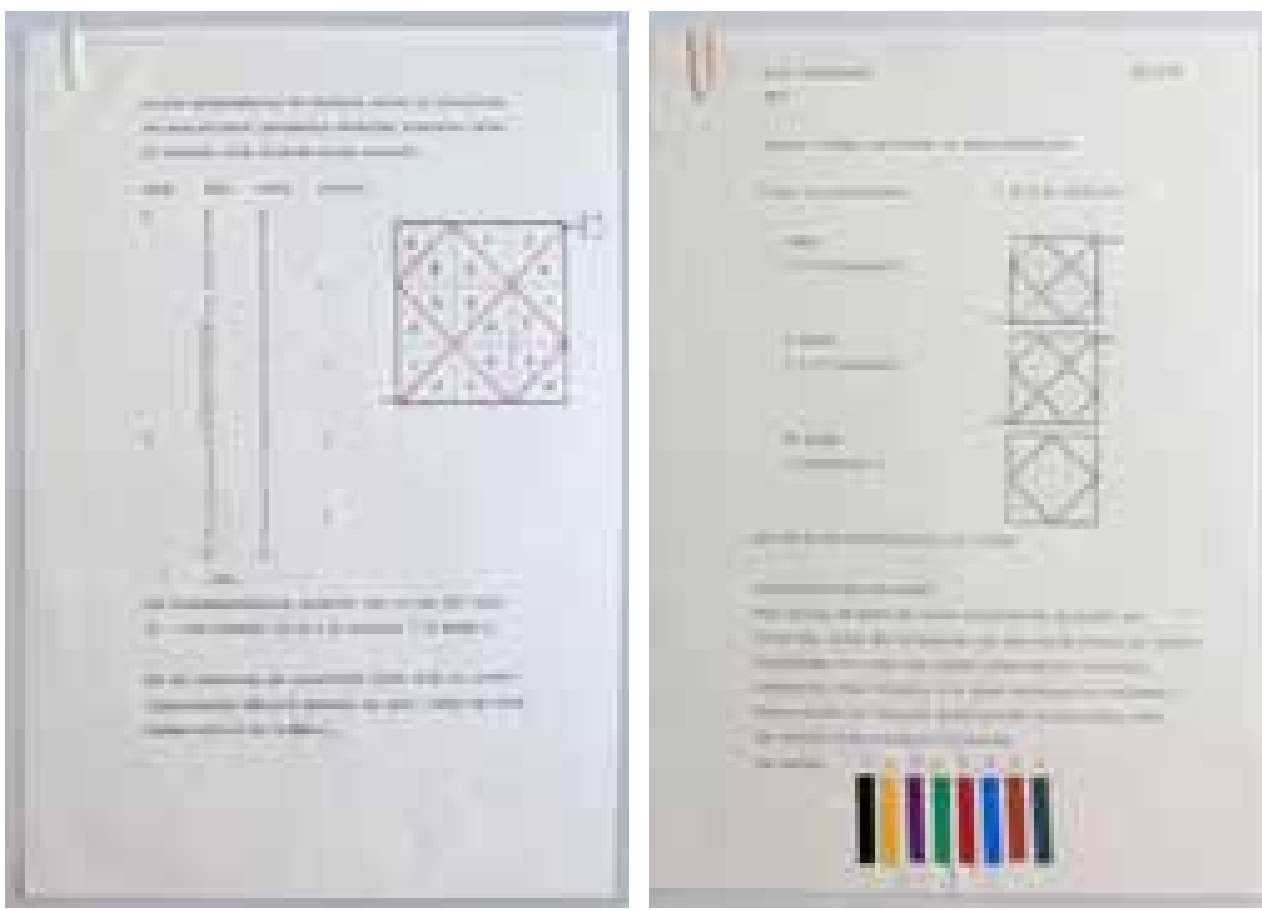

i2/141, 142 Apuntes del ejercicio "negro como color"

Alumno: Klaus Krippendorf

Curso: Visuelle Einführung. Kurs Maldonado (1955-1956).

El cuadrado se divide en una retícula de $18 \times 18$ unidades. Las unidades se agrupan en células de $3 \times 3$ unidades que será la referencia para la distribución del color. Sobre esta retícula principal se dibuja una curva de Peano modificada que llena todo el espacio del cuadrado de modo que al recorrer las células de 3x3 unidades, cada una de estas unidades se subdivide en dos campos. A cada campo se le aplica un color siguiendo un patrón de asignación de pares: a cada campo le corresponde uno de los 8 colores. De cada uno de estos colores se irá eliminando progresivamente uno de ellos para dar lugar a la iteración en el cubrimiento de la superficie y la percepción difusa de los colores y con ello la del negro como uno más y no como un vacío. (Ver apuntes en las imágenes i2/141, i2/142). 

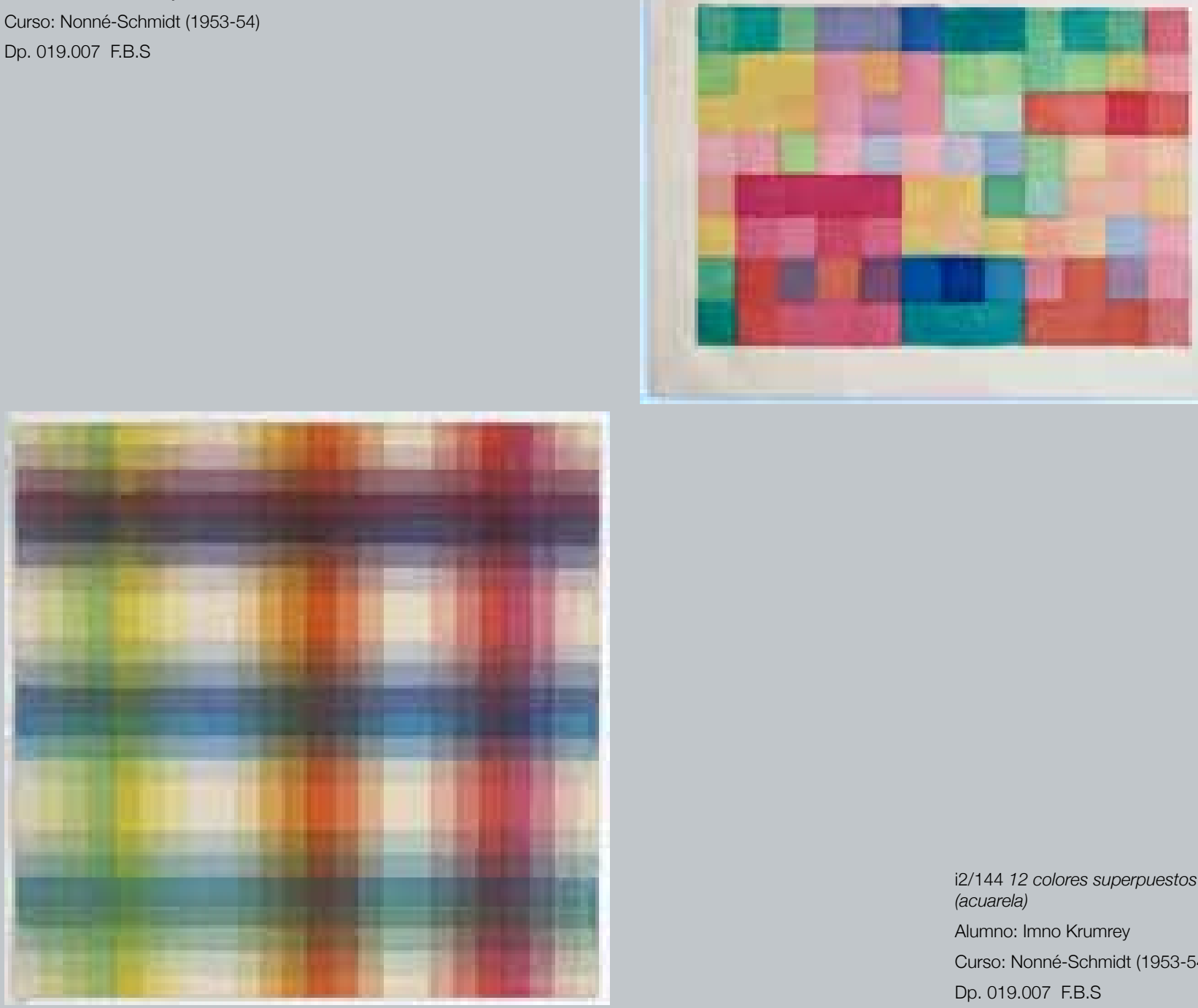

i2/144 12 colores superpuestos

(acuarela)

Alumno: Imno Krumrey

Curso: Nonné-Schmidt (1953-54)

Dp. 019.007 F.B.S

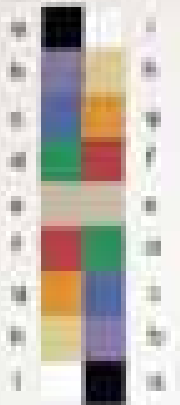

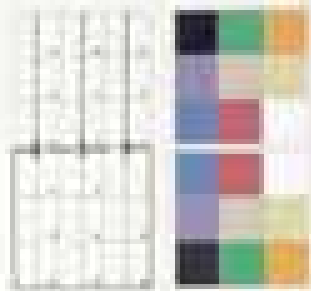
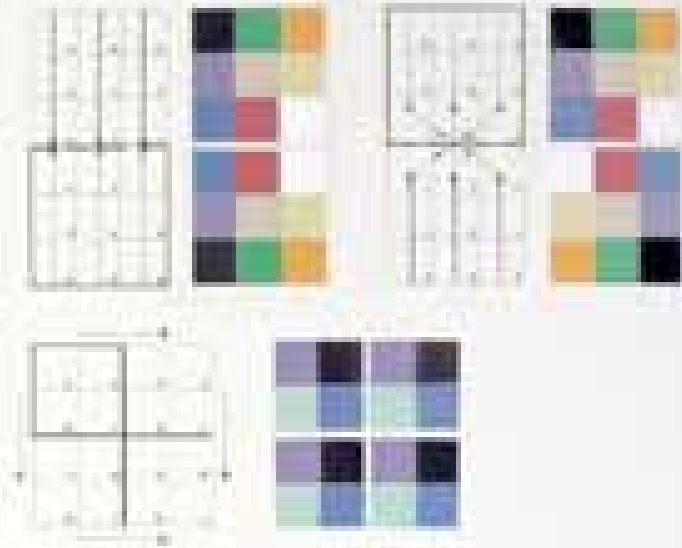

i2/145 Desplazamientos de color sistemáticos (papel de color)

Alumno: Imno Krumrey

Curso: Nonné-Schmidt (1953-54) Dp. 019.007 F.B.S 


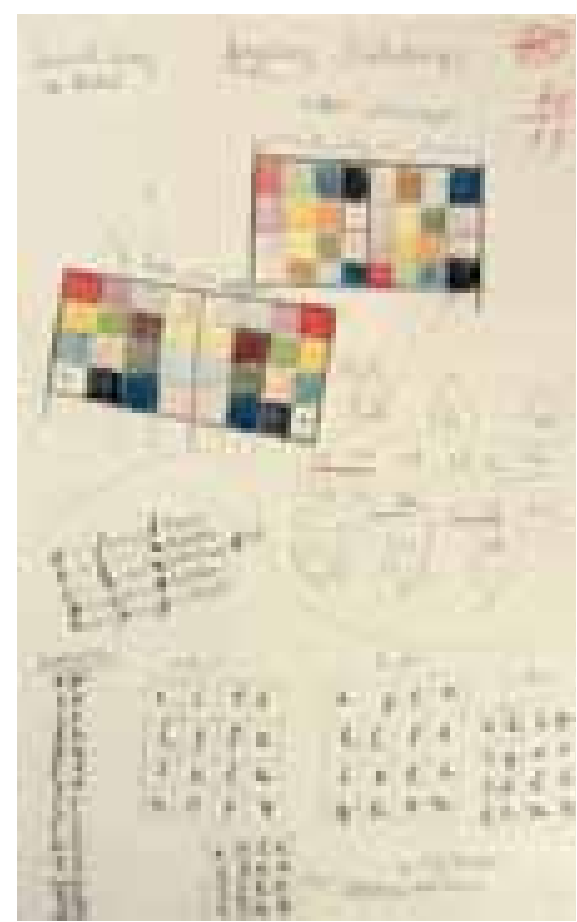

\subsubsection{Distribución sistemática o aleatoria}

Los planteamientos de la enseñanza del color descritos hasta este punto se basan en la distribución del color a partir de sus condicionantes perceptivos, mediante mecanismos ilusorios o de la mano de la topología. Junto a estos planteamientos, se emplean otros mecanismos que proporcionan una distribución visual uniforme del color a partir de parámetros numéricos, con una sistemática concreta o aleatoria.

Los enunciados relativos a este último planteamiento metodológico, salvo el desarrollado por Nonné-Schmidt, pertenecen a los últimos años de existencia del curso básico como departamento independiente. Este periodo, como ya se ha mencionado, estuvo caracterizado por una confianza en la metodología científica apoyada en gran parte en la figura de Horst Rittel. Recordemos que Rittel fue contratado en el curso 1957-1958 y que provenía de una licenciatura en matemática pura y física técnica en la Universidad de Göttingen. Se involucra en la docencia de varias materias entre ellas la teoría del color. Pero lo que es más importante, a Rittel se le atribuye ${ }^{35}$ la introducción de métodos para manejar los datos de un problema y llegar a la solución con técnicas relativas a la recopilación de datos, análisis, síntesis y optimización. La enseñanza del color en los últimos años del Grundlehre no es ajena a esta confianza en las herramientas para manejar los datos de partida y surgen ejercicios afines a esta didáctica.

\section{Distribución de color mediante un patrón}

En el primer curso del Grundlehre, Nonné-Schmidt realiza el ejercicio de distribución del color en una retícula que planteaba Klee en la Bauhaus. Klee realizaba el ejercicio "orden especial" 36 en el que se analizaba la cuestión del equilibrio de los colores en una configuración. El ejercicio abordaba las modificaciones de densidad de las partes que consigue una expansión o estrechamiento del plano por efecto del claroscuro. La modificación en la densidad se conseguía mediante progresiones numéricas cardinales (1, 2, 4, 8...) o series de números enteros (1, 2, 3, 4, 5...). (i2/146) 
Alumno: Terence Daley

Curso: Froshaug farbenlehre (1960-61)

G 62 F.B.S

i2/148 Comparativa entre campos

coloreados al azar.

Alumno: Hans T. Kurtenbach

Curso: Rittel Froshaug farbenlehre

(1959-60)

Gs 061.024 F.B.S
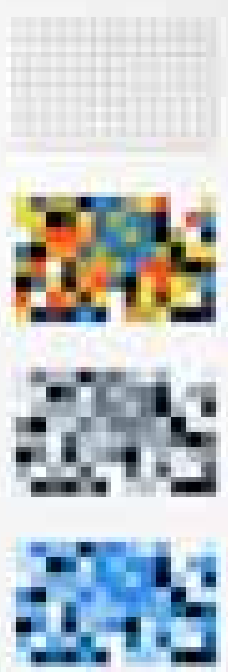
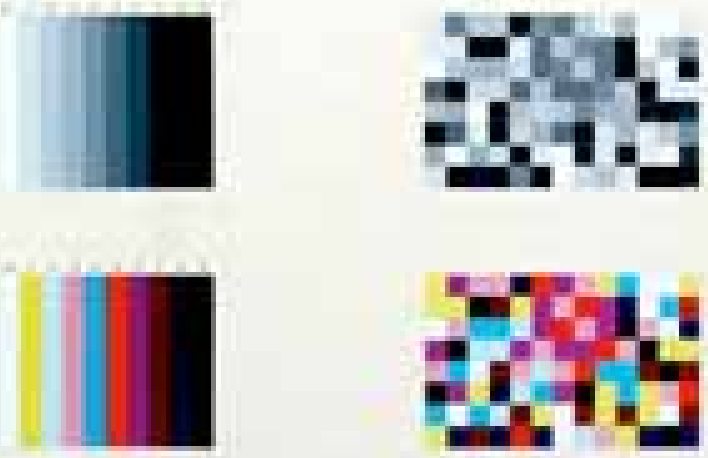

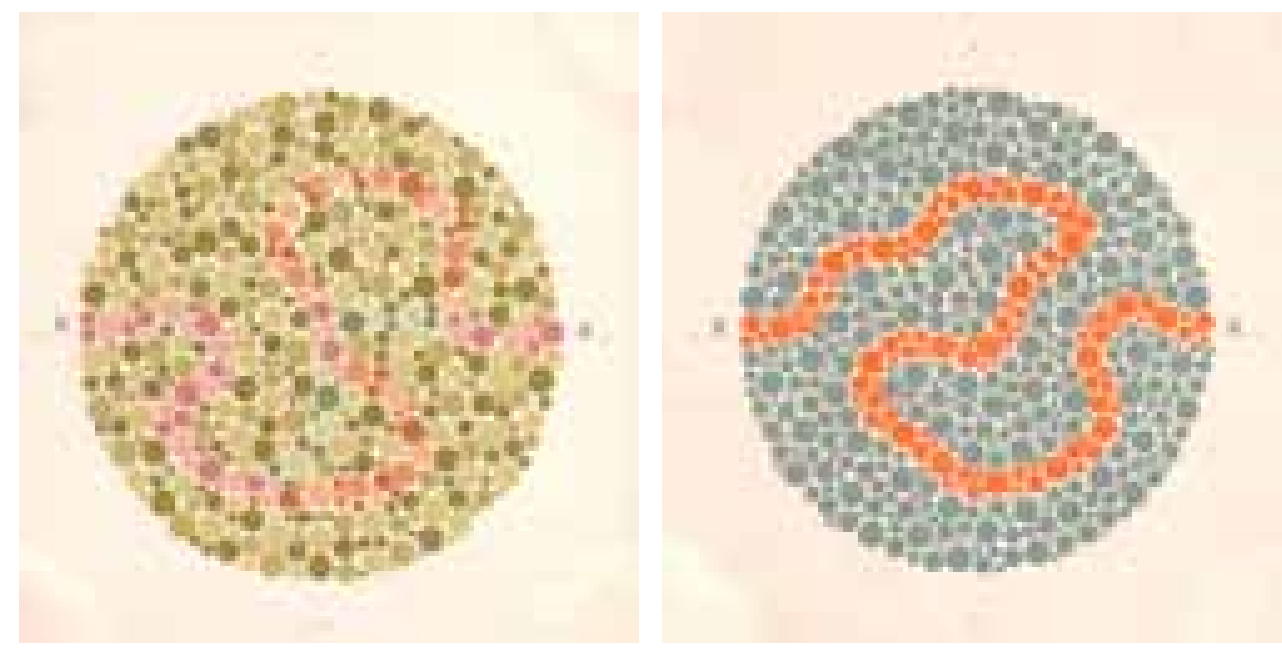

\section{i2/149, i2/150}

Láminas del test del doctor Shimobu Ishihara para el diagnóstico de las discromatopsias.

Nonné-Schmidt titula el ejercicio "Desplazamientos de color sistemáticos" que este caso, están elaborados con papeles de colores (i2/145). Las variables de trabajo son la escala de colores, su codificación alfabética y los patrones de distribución sobre una retícula cuadrada en las que los cuadrados de papel se ubican en cada campo según cada patrón, a partir de su simetría y desplazamiento.

Al mismo tiempo y, una vez que se han estudiado el orden y las escalas de color, Nonné propone la distribución sistemática de los 12 colores del circulo cromático en capas superpuestas de tres intensidades distintas. Esta distribución permite generar una matriz ordenada de valores tonales que son el punto de partida de distribuciones aleatorias en los ejercicios de acuarela (i2/143, i2/144).

\section{Comparativa entre campos coloreados al azar}

Froshaug propuso este ejercicio en los cursos 1959-1960 y 1960-1961. Se trata de una comparativa entre dos o tres campos que están coloreados con escalas de valores tonales similares mediante un patrón aleatorio. Froshaug se basó en el Test de Daltonismo Isihara, en el que se "camuflan" una serie de puntos de un color sobre un conjunto de otro color dominante. ${ }^{37}$

El punto de partida consiste en la definición de una escala de grises de nueve tonos numerados del 0 al 9 . A esta escala se le hace corresponder una escala de color del blanco al negro en la que los ocho campos intermedios deben rellenarse con colores brillantes diferentes, de modo que los colores en su gradación correspondan a las gradaciones grisáceas. Los campos están definidos por un rectángulo integrado por una retícula de $8 \times$ 13 celdas. Las celdas están numeradas al azar con cifras del 0 al 9 . En el curso 1960-1961 se incluye una escala tonal de un color como tercera variable, como el azul que puede verse en la imagen (i2/147, i2/148).

La tarea consiste en colorear cada celda de cada rectángulo con el color que le corresponda de cada escala tonal. El conjunto se organiza de manera que se pueda comparar con facilidad. 

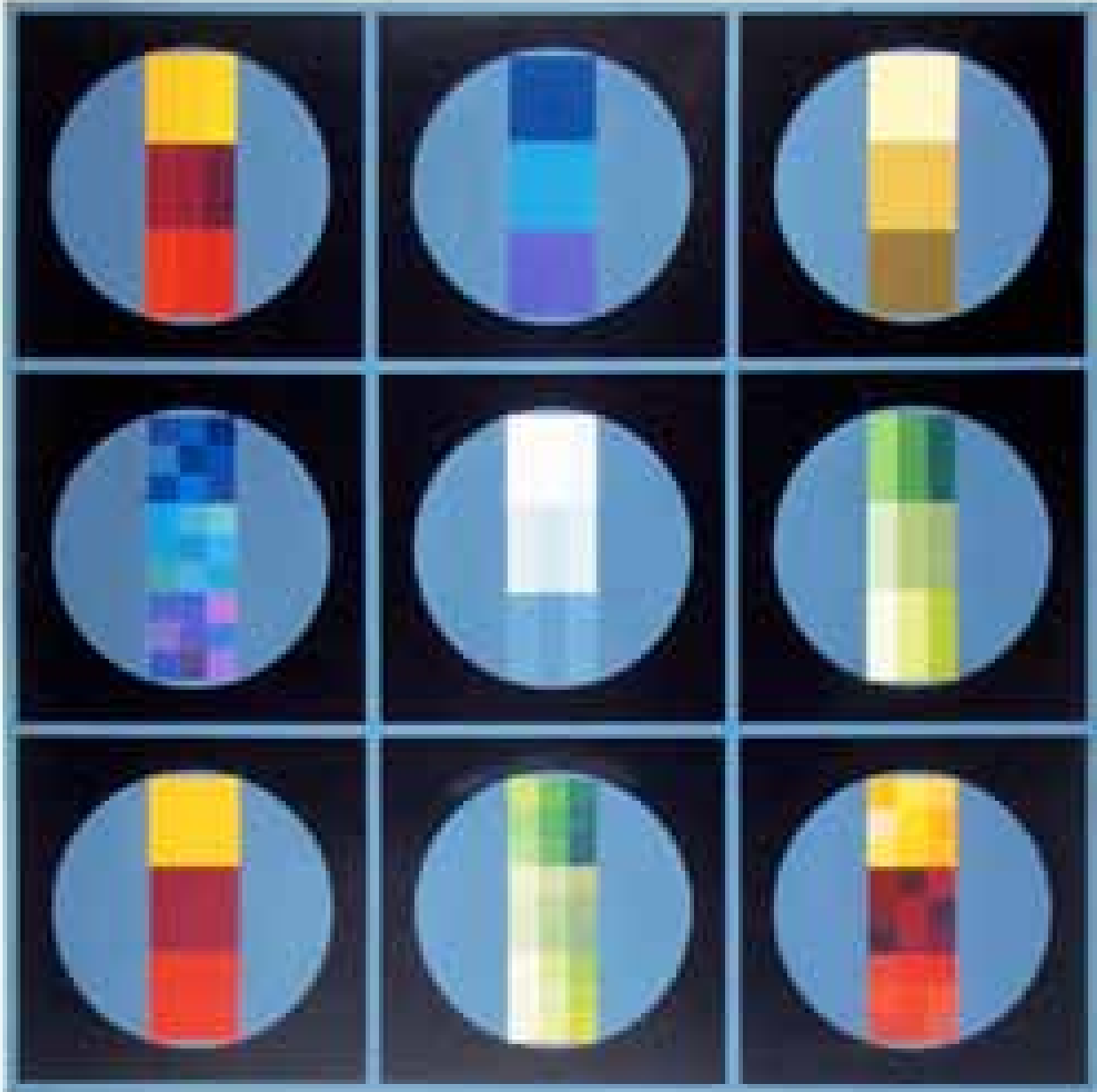

i2/151 Similitud de los campos de brillo y color

Alumno: Rolf Müller

Curso: Maldonado (1960-61)

Abteilungsarbeit-11 F.B.S 


\section{Similitud de los campos por brillo y/o color}

Maldonado a finales del curso 1960-1961 propone la Abteilungsarbeit $\mathrm{n}^{\circ} 11$ que busca la similitud de los campos tratados con diferentes variables. (i2/151) Sobre tres cuadrados situados en una fila, se establecen tres posibles variables de distribución de color: (a) el cuadrado completo; (b) el cuadrado dividido en tres franjas verticales y (c) el cuadrado dividido en nueve cuadrados. A estos cuadrados se le superponen tres colores de la misma familia que se irán descomponiendo a medida que la variable de composición del cuadrado se subdivida, teniendo que quedar el aspecto final (c) similar al de partida (a).

\section{Escala aritmética de grises aleatoria, sistemática y con dado al azar}

La generación de escalas de grises mediante técnicas sistemáticas y aleatorias tienen lugar en dos cursos sucesivos, el 1959-1960 y 1960-1961. La primera de ellas surge como una propuesta de ejercicio conjunta de Horst Rittel, que imparte metodología y VordembergeGildewart, encargado del curso de color junto a Froshaug en la que se comparan dos escalas de grises generadas de forma sistemática y aleatoria. La propuesta del curso siguiente, 1960/1961, se realiza en el curso de color de Froshaug.

La escala de Rittel y Vordemberge-Gildewart propone una comparativa entre dos escalas aritméticas de grises cuya intensidad varía progresivamente en cinco etapas. Las escalas de grises se organizan sobre sendas franjas divididas en seis cuadrados iguales. Cada uno de ellos corresponde a una etapa de la escala de grises. El último está totalmente coloreado de negro, mientras que los cinco restantes se completan ocupando con el color negro la superficie de manera proporcional. La primera escala, en la franja superior, se completa de manera aleatoria. La segunda se completa siguiendo un patrón formal, a partir de franjas de sombreado. La premisa fundamental del ejercicio es que ambas etapas -aleatoria y sistemática- deben tener la misma proporción de trama sombreada. El trabajo se realiza con papel milimetrado y rotulador calibrado. (i2/152)

En el curso siguiente, Froshaug desarrolla una escala de grises determinada por el azar. La escala tiene seis etapas, siendo la primera el blanco y la última el negro. Las cinco etapas intermedias de la escala son el resultado de una progresión aritmética de los resultados proporcionados por la tirada de un dado. Cada etapa está representada por un cuadrado dibujado sobre papel milimetrado que contiene en su superficie 900 celdillas de $1 \times 1 \mathrm{~mm}$.

El ejercicio comienza con 900 tiradas de dado y el registro del resultado -del 1 al 6- en un estadillo (i2/153). El cuadrado de la primera etapa representa el gris más claro de la escala. En él, se trasladan los 900 resultados del estadillo a cada una de las celdillas, se rellenan solo aquellas en los que haya salido el 6 . En la segunda etapa de la escala, se rellenan aquellas celdillas en las que haya salido el 5 o 6 . En la tercera, las celdillas en las que haya salido el 4 o un número mayor que 4 , y así sucesivamente. Al aumentar el rango de números aumenta la probabilidad y por tanto, la superficie de la escala se va oscureciendo sucesivamente en virtud de una progresión aritmética y del azar (i2/154). 


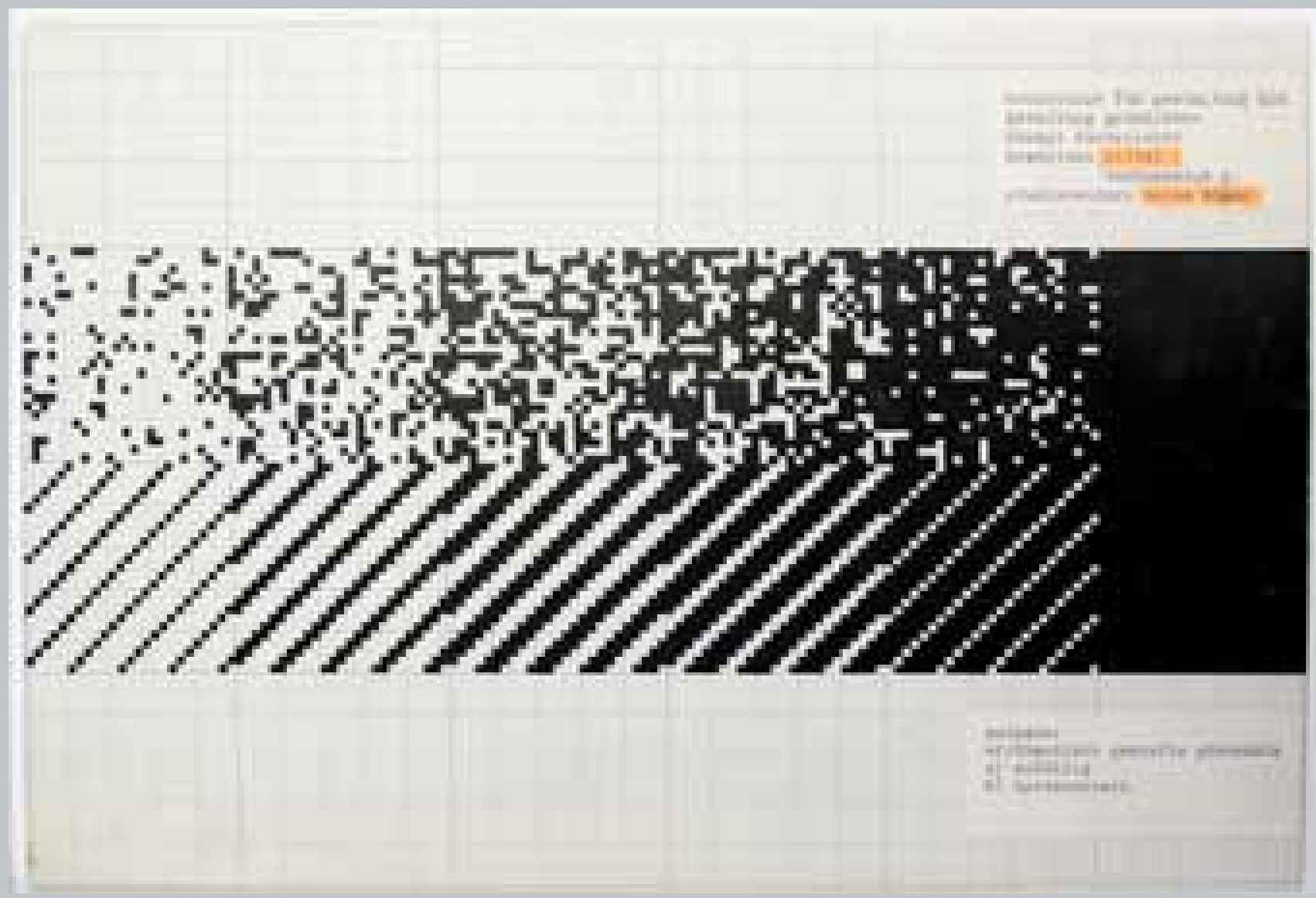




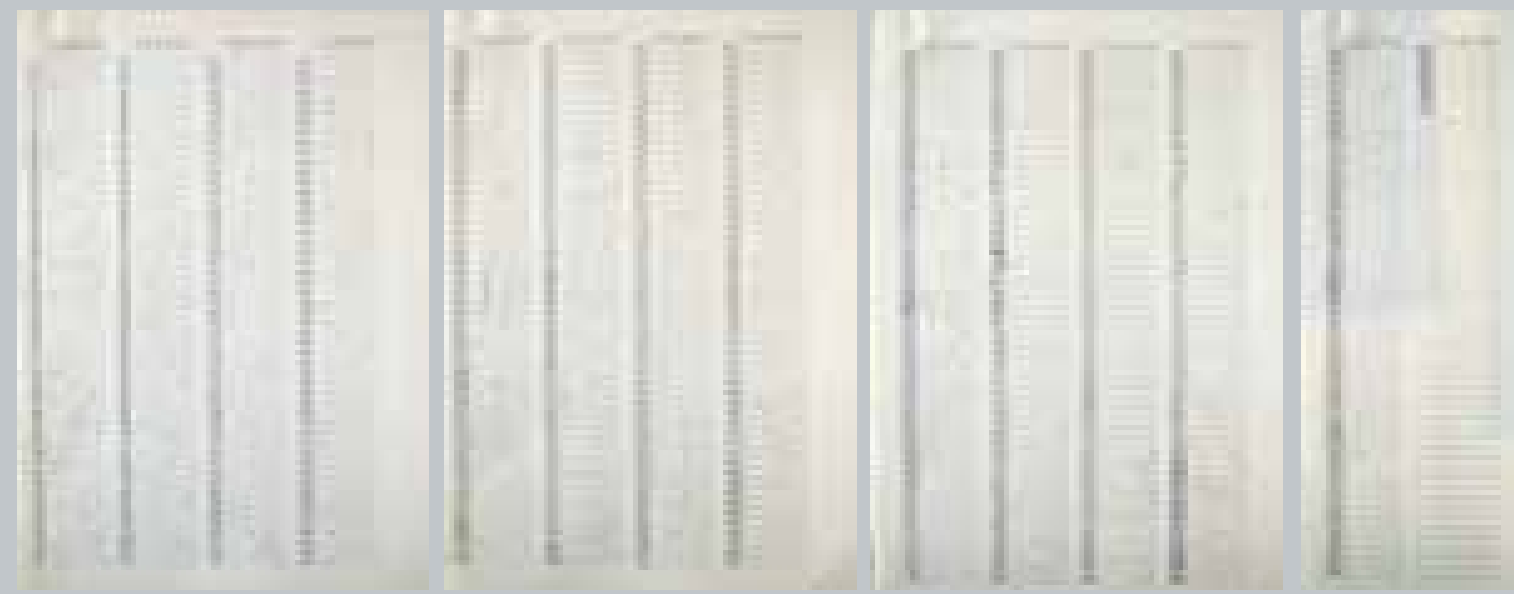

i2/153 Estadillos que recogen los resultados de la tirada del dado para la elaboración de la escla de grises. Alumno: Eric Rossicci

Curso: Froshaug (1960-61) / G63

i2/154 Escala de grises generada por la tirada de un dado

Alumno: Eric Rossicci

Curso: Froshaug (1960-61) / G63
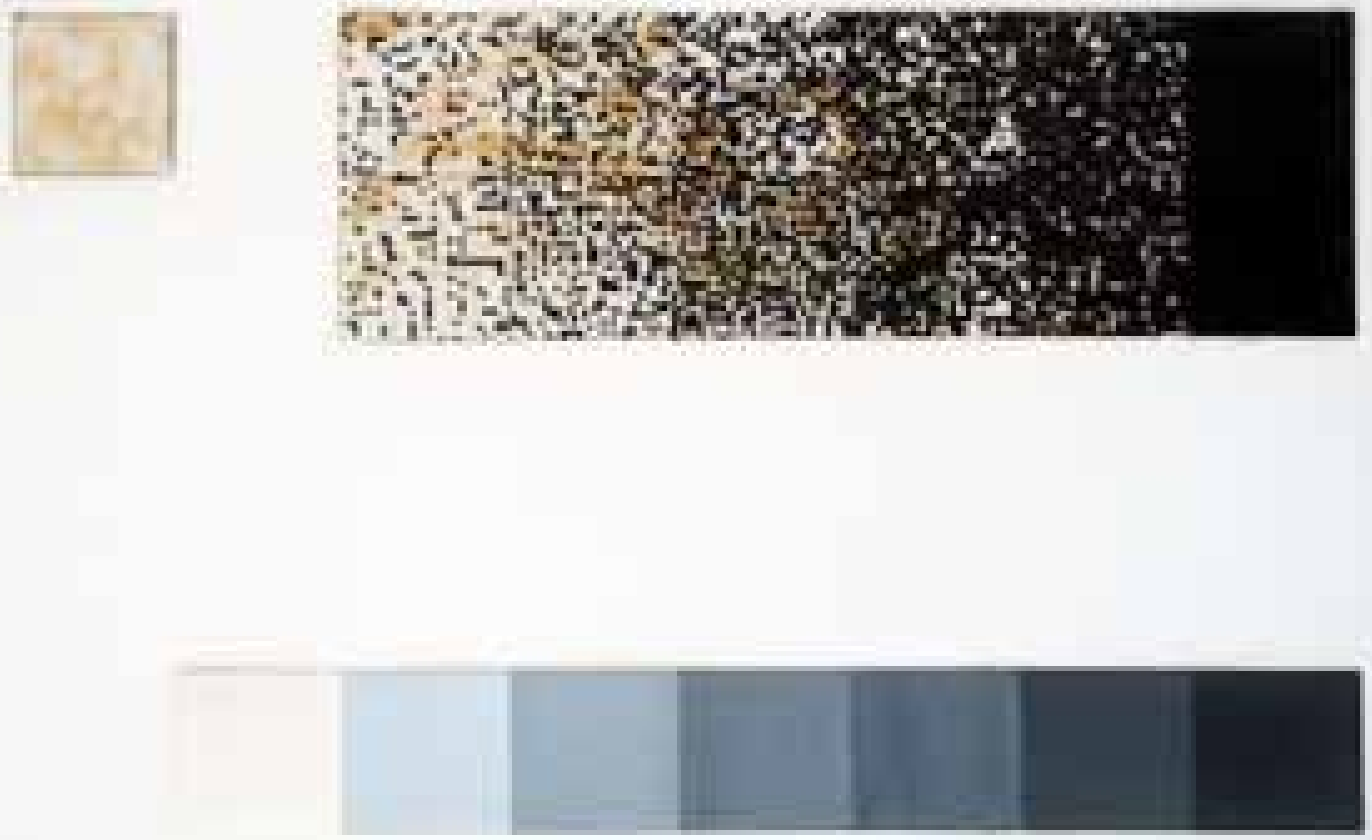


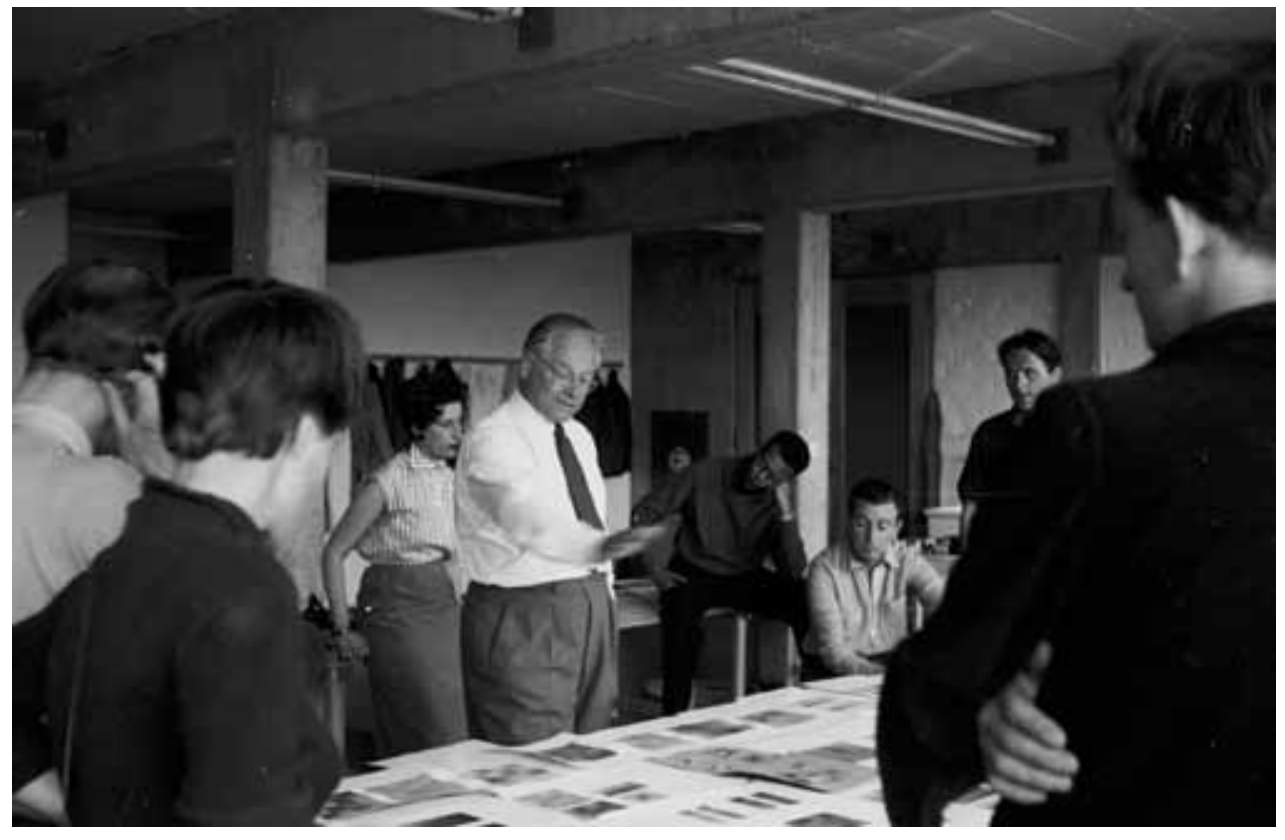

i2/155 Grundlehre 1954-1955. Seminario de Color de Johannes Itten.

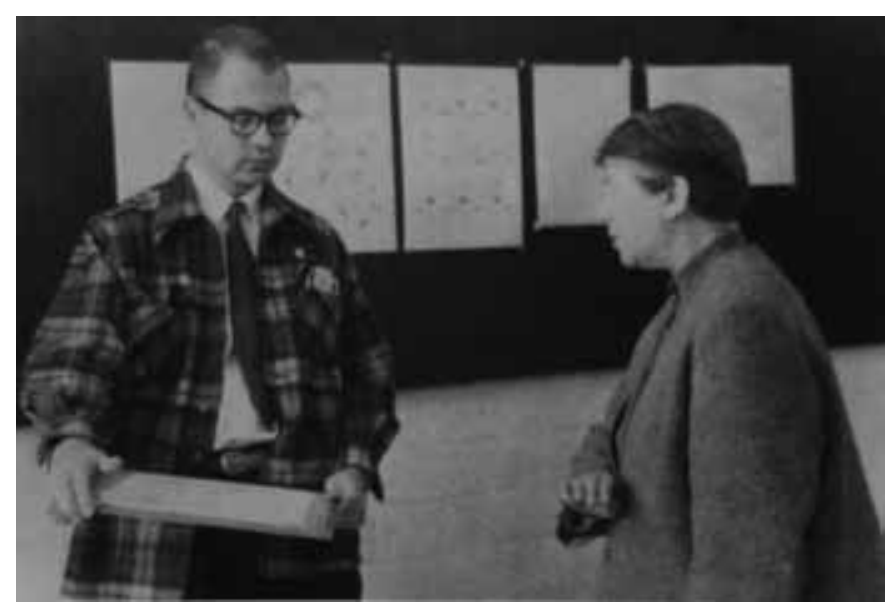

i2/156 Grundlehre 1956-1957. Helene Nonné-Schmidt con William S. Huff.

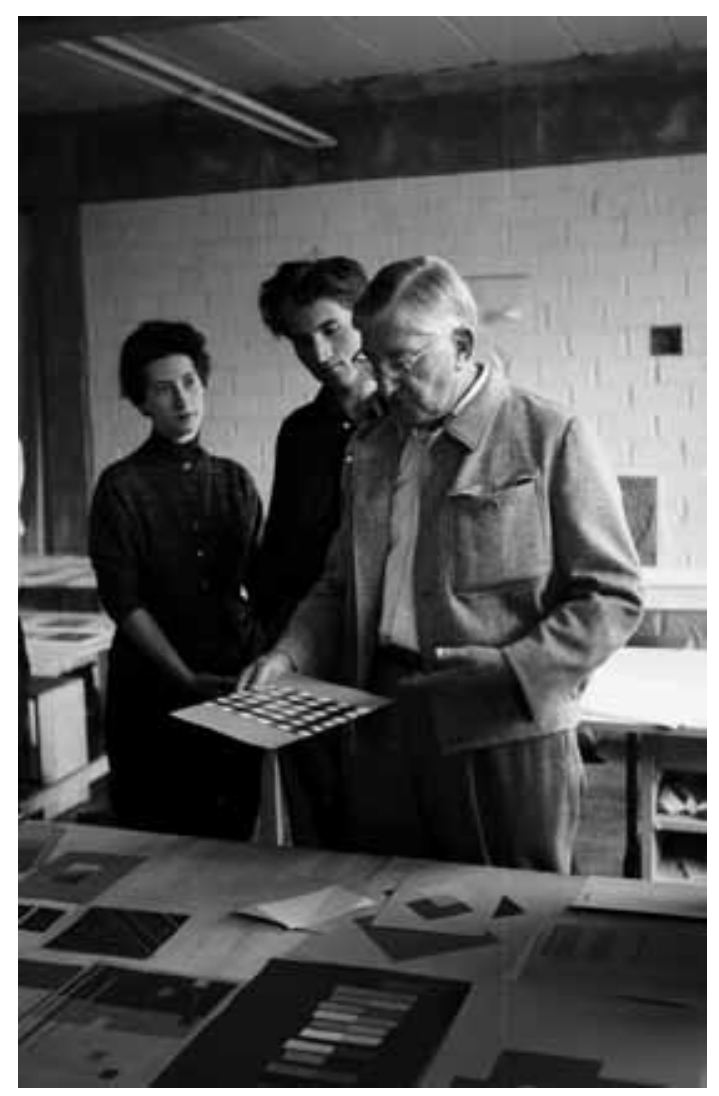

i2/157 Grundlehre 1954-1955. Josef Albers en la presentación de resultados de Color Básico. 


\subsection{Contribución a la didáctica del color conclusiones parciales atlas color}

\section{El color pragmático}

El factor más significativo que aporta la HfG a la didáctica del color en el contexto de los cursos básicos experimentados hasta aquel momento se basa en que la dimensión visual y pictórica de éste progresa hacia una visión práctica en los proyectos.

En la Bauhaus, la enseñanza del color estudia los sistemas de organización en varios modelos y su articulación en el círculo cromático. Como formación preparatoria se circunscribe fundamentalmente a los cursos pintura de Paul Klee y Kandisnky. Sus enseñanzas están basadas en una didáctica de elaboración propia orientada hacia la práctica del color como medio artístico de carácter simbólico y retórico.

Mención aparte merecen las experiencias del Taller de Pintura de Ludwig Hirschfeld-Mack que anticipan posibilidades del color como medio visual. Los materiales documentados del Institute of Design se encuentran próximos a esta enseñanza del color, en la que se parte del estudio del círculo y las escalas cromáticas.

La enseñanza del Color en el Curso Básico de los Vhkutemas parte de su organización sistemática. Las propuestas más relevantes, a cargo de Gustav Klutsis se aproximan al estudio del color como variable visual que se desarrolla en el Grundlehre. A partir de esta base, en el segundo curso se llevan a cabo experiencias prácticas de aplicación de color sobre volúmenes arquitectónicos. Los Vhkutemas por tanto, es la escuela que más se acerca a la HfG en cuanto al alcance de la didáctica del color y su aplicación a los proyectos.

\section{El color $\mathbf{y}$ otras variables}

La estrategia dominante en el Grundlehre es aquella en la que el color participa de forma conjunta con otras variables. El estudio de los sistemas de color y su organización se localizan en los cuatro primeros cursos, coincidiendo con la docencia de Nonné-Schmidt. En el resto de enfoques que sucedieron con posterioridad, el color actúa como variable junto a otras y prevalece en la configuración de las soluciones.

El Color no tuvo una asignatura propia en el Grundlehre de forma constante. Su didáctica se incluye como parte del Visuelle Einführung y solo en el tramo final se distingue una dedicación específica a estas enseñanzas denominada Farbenlehre, en los ejercicios desarrollados por Vordemberge-Gildewart y Froshaug en coordinación con las enseñanzas teóricas de Rittel.

A pesar de ello, su presencia fue constante a lo largo de este periodo. La profusión de los ejercicios centrados en el color pone de manifiesto su valor como medio para el aprendizaje. Para todos los docentes representa una herramienta a la que recurren en mayor o menor medida. 
i2/158 Curso Básico de Josef Albers. 1955. Ejercicios de Color Básico.

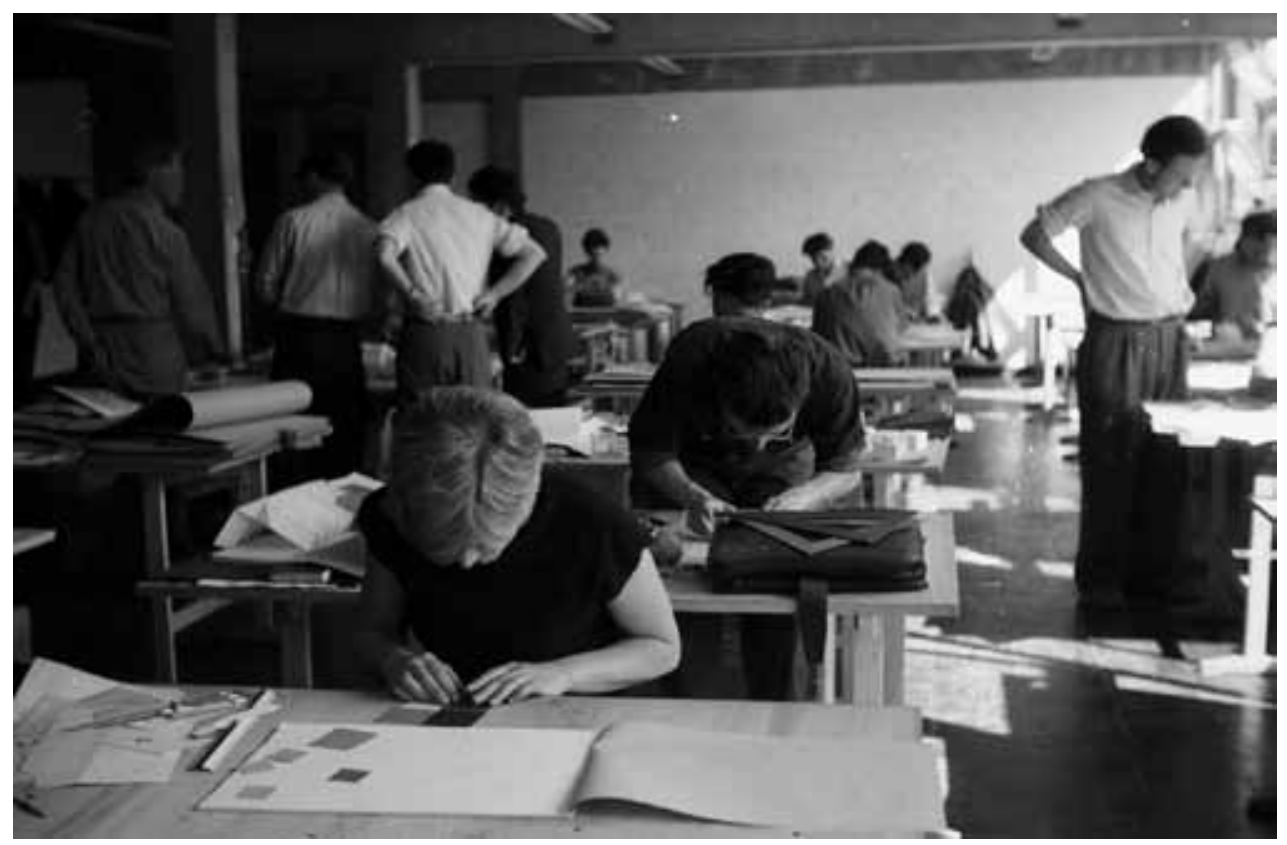

\section{Entre procesos exploratorios y sistemáticos}

38 "La magia del color" es la denominación del seminario de Josef Albers que aparece en los documentos de la escuela relativos al curso 1953-1954. Az31.1 FBS
En el Grundlehre los colores se mezclan de forma intuitiva o se ordenan en escalas partir de un patrón programado. La enseñanza oscila entre estos dos polos: intuición y metodología. Albers practica de forma consciente con el color de forma lúdica. Nonné-Schmidt es la primera que introduce el estudio sistemático a partir de modelos científicos. Mientras que Itten y Bill recurren a su valor convencional como elemento plástico.

Maldonado es la figura en la que confluyen ambos procesos, exploratorio y sistemático. Maldonado se apropia de la "magia del color"38 de Albers y la codifica para transformar los experimentos del bauhäusler en artefactos visuales programados. Para ello recurre a la topología, la simetría y las leyes de la percepción. La experiencia directa con el maestro de la Bauhaus le permite identificar una mecánica de trabajo para desarrollar el potencial visual del color y la relatividad de su percepción. Maldonado encuentra en las tareas de Albers la fórmula para la codificación del color: la creación ilusoria y ambigua de efectos visuales -profundidad, transparencia, solapamiento, continuidad, espacio y umbrales de percepción.

En algunos ejercicios sobre color de la fase final del Grundlehre se hacen evidentes las estrategias propias de trabajo vinculadas a la Metodología como materia teórica que caracteriza esta etapa. En las escalas de grises, los mecanismos para alcanzar progresividad entre cada etapa de la escala se basan en el azar o en la sistematización de un patrón.

Los ejercicios que plantea Froshaug en los últimos años que combinan progresión aritmética y la combinatoria parten de un método muy acotado y concreto en el que tiene cabida la variable del azar. Estos ejercicios transcurren entre el proceso sistemático y el exploratorio. Se parte de un método definido y universal para todos los alumnos, en el que no tiene cabida la acción personal, en el que el resultado no se vislumbra como algo preestablecido. De esta manera se investiga el grado de similitud que tiene la generación de escalas y patrones por medio del azar frente a las escalas de grises tradicionales de color sólido. Si bien la panorámica cronológica del curso de color parece trazar un camino desde la exploración hacia la sistematización, se puede comprobar que en los últimos años este mismo camino se recorre de manera inversa. 

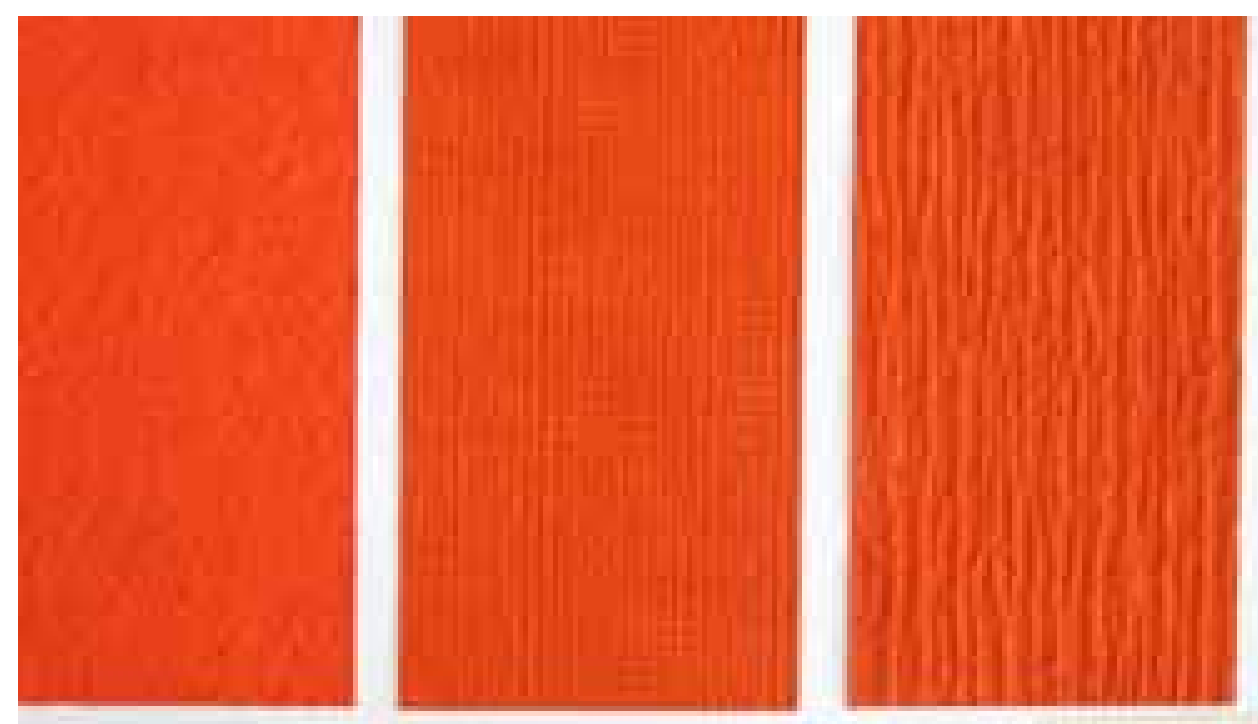

\section{Color, función y significado}

El color en el Grundlehre es un elemento plástico del lenguaje visual. En el Curso Básico apenas hay casos en los que los profesores se adentren en su capacidad para transmitir funciones o significados.

En este sentido, el caso más singular lo representan las escalas de color y textura de Vordemberge-Gildewart. Las faktura de color son un episodio inconexo y sin mayor continuidad, pero constituyen un modelo didáctico con un gran potencial por su planteamiento transversal: combinar el color con la textura en una serie de ejercitaciones sistemáticas, unificar la percepción visual con la percepción háptica. Como la mayoría de ejercicios, es una tarea abstracta y sin una aplicación práctica. Sin embargo, se puede relacionar con el estudio de las cualidades de los materiales en el Diseño de Productos. Las posibilidades didácticas de este ejercicio y esta relación no se desarrollan con todo su potencial. No se profundiza en la relación simbólica entre el color y la textura de una superficie o en la relación entre el significado del color de un objeto - calor, peligro- y las cualidades táctiles del material del que está hecho - rugosidad, lisura, suavidad, aspereza.

Pero este ejercicio no es el único caso de ejercicios que plantean la posibilidad de incorporar otras variables de tipo conceptual. Itten y Albers plantean la relación entre color y temperatura en los ejercicios desarrollados en Ulm. Ambos incluyen el binomio frío-cálido dentro de sus repertorios didácticos. Pero tampoco profundizan en las connotaciones de estos conceptos o en el simbolismo de esta dicotomía.

En relación a la semiótica, el tratamiento del color de estos ejercicios se puede asimilar con aspectos semánticos, el significado del color asociado a la temperatura, o aspectos pragmáticos, la asociación de un color a un material o un uso. Estos aspectos tampoco fueron considerados por Maldonado en su planteamiento del Grundlehre ya que los consideraba propios de cursos posteriores.

La llegada del proyecto en la fase final del Grundlehre acaba con esta carencia en el tratamiento del color y lo incorpora a las herramientas de proyecto. Froshaug lo introduce como una de los elementos que los estudiantes del sector de comunicación Visual tienen que considerar específicamente en el diseño del juguete constructivo. El color forma parte de las reglas del juego y es un material más para la construcción del juguete. i2/159 Escala de valores de color rojo mediante la faktura de la superficie Alumno: Peter Beck

Curso:Rittel/Vordemberge-Gildewart Farbenlehre (1959-1960)

Dp 003.033 

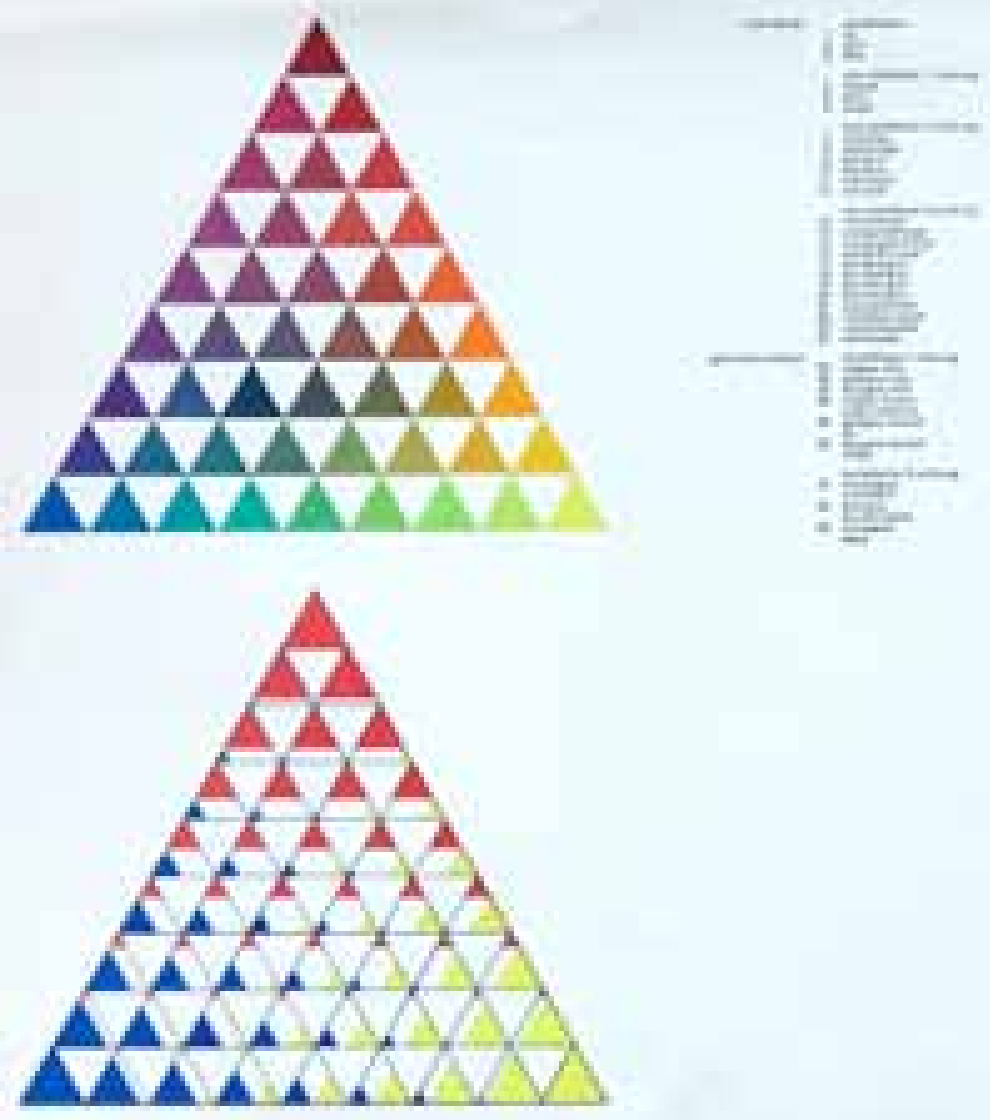

$i 2 / 160$

Ejercicio del primer año de estudios en el del Departamento de Comunicación Visual, curso 1965-1966.

Materia: Abteilungsarbeit

Triángulo cromático

Profesor: William S. Huff

Alumno: Ursula Wenzel

GS_030.003 FBS

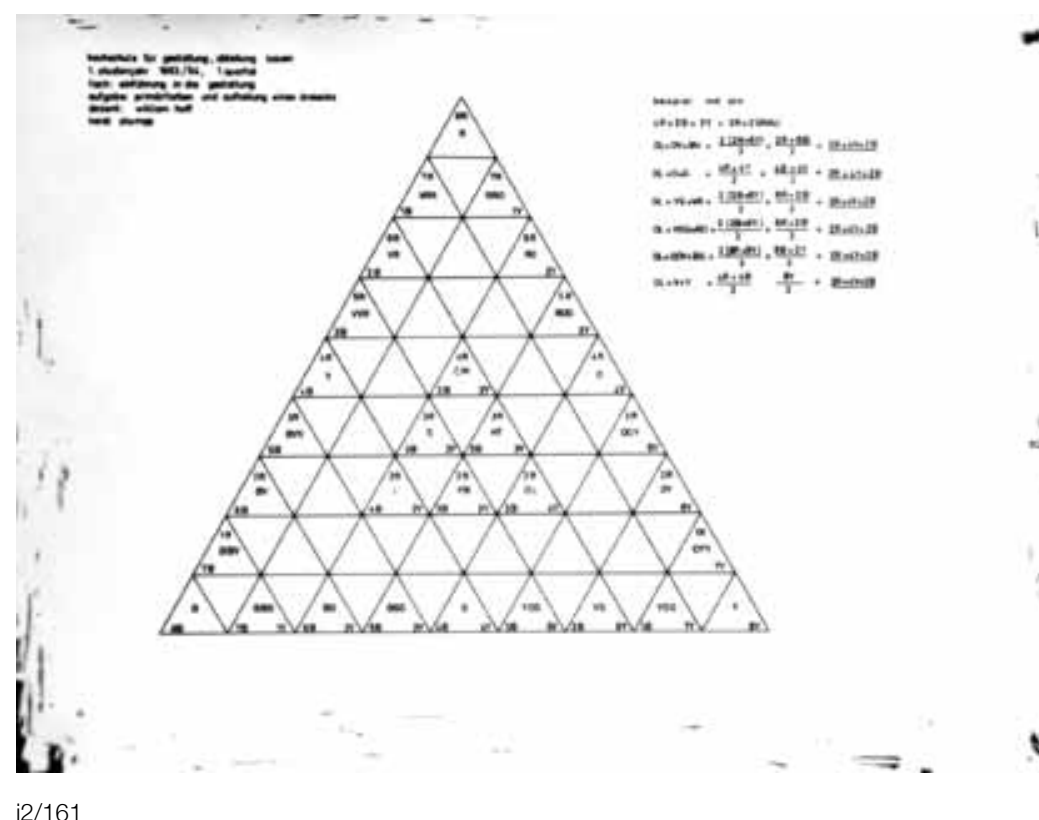

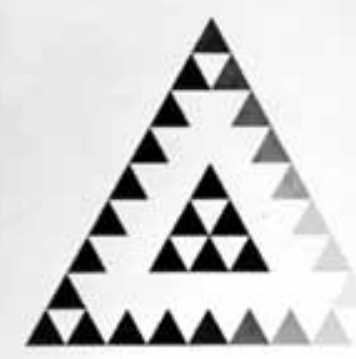

i2/162

Ejercicio del primer año de estudios en el del Departamento de Construcción, curso 1963-1964.

Materia: Einführung in die Gestaltung

Colores primarios y división de un triángulo.

Profesor: William S. Huff

Alumno: Horst Stumpp

F_64-0724 FBS 

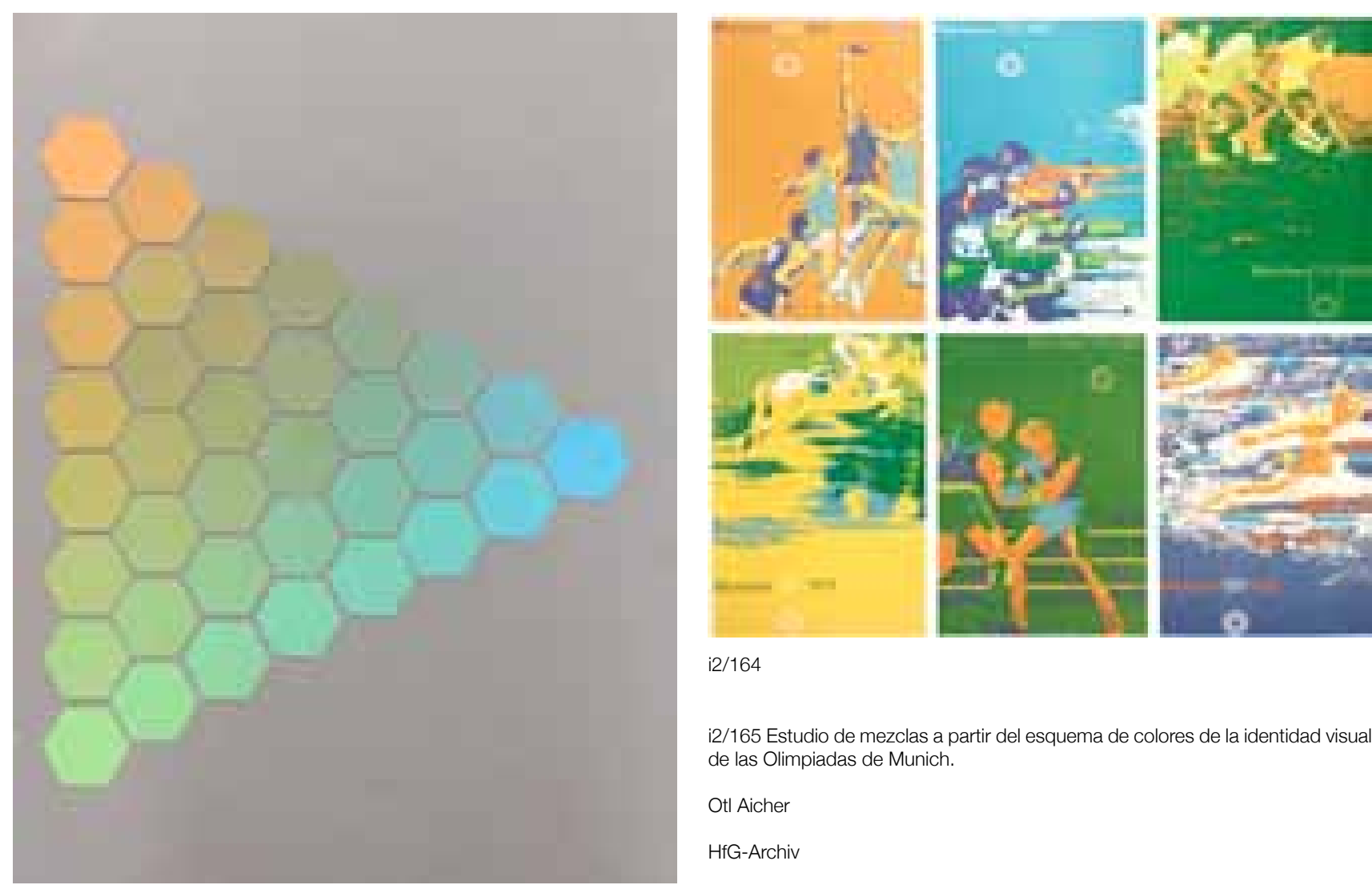

i2/164

i2/165 Estudio de mezclas a partir del esquema de colores de la identidad visual de las Olimpiadas de Munich.

Otl Aicher

HfG-Archiv

\section{La disección del color}

La labor de Nonné-Schmidt en el Grundlehre destaca como la única iniciativa para su estudio científico y sistemático del color a partir de sus cualidades. Su curso disecciona los sistemas de color; sus estudiantes desgajan los sólidos de color de Ostwald y Munsell para estudiar su naturaleza y comportamiento. Los ejercicios les permiten la aprehensión de los mecanismos de interpolación y relación entre los colores como variables mediante la manipulación gráfica de las mismas. El color se analiza para construir mapeados de relaciones, que se exploran a partir de la programación de las mezclas. El proceso está sistematizado, porque se establecen las etapas y los vértices de partida. La faceta experimental reside en que los resultados no se conocen de antemano. Estos ejercicios no tuvieron un relevo directo por parte de otros docentes.

La disección del color reaparece con fuerza en la etapa final del Grundlehere y en los primeros años tras la desaparición de éste en los ejercicios de Vordemberge-Gildewart con una analogía directa al Mobile Körper de Aemilius Müller.

La labor de Nonné-Schmidt y de Vordemberge-Gildewart se quedan en un plano teórico, sin aplicación. Sin embargo, esta forma de abordar el color se revela como una herramienta fundamental de proyecto. El estudio de los sistemas de color y los mecanismos de interpolación permanecen como ejercicios en el primer curso de los departamentos y se utiliza para la creación de cartas de color en proyectos de identidad visual como en el de las Olimpiadas de Munich. (i2/163-i2/165). 


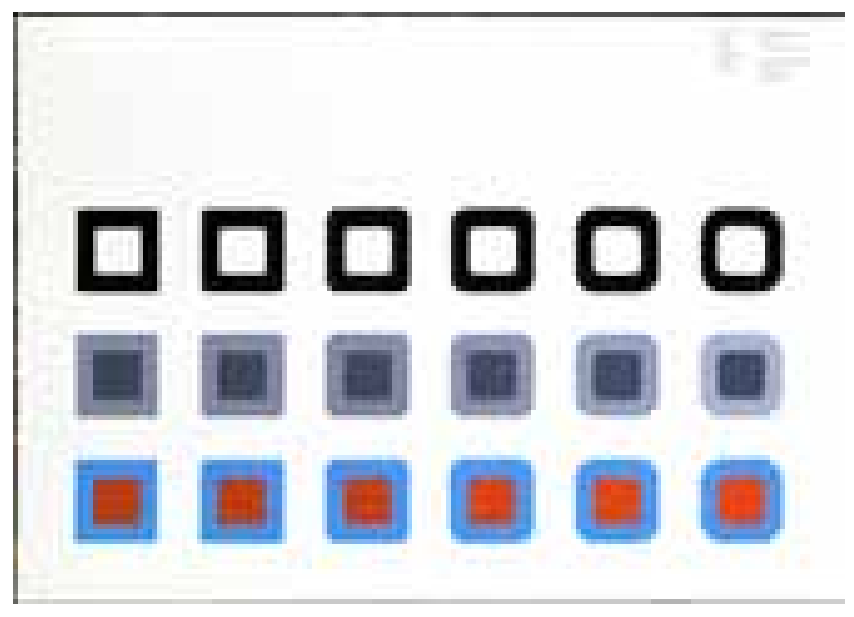

i2/166

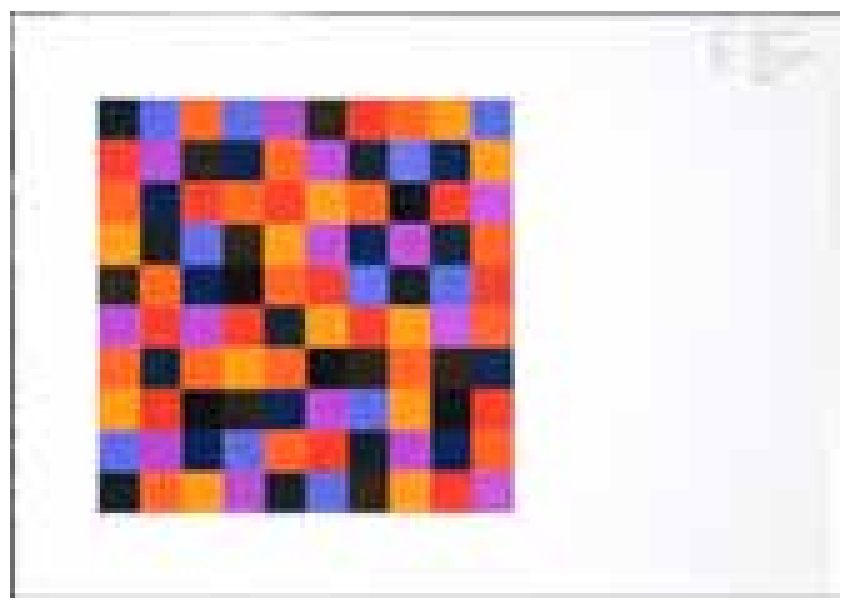

i2/168

i2/166 Ejercicio del primer año de estudios en el del Departamento de Diseño Industrial, curso 1962-1963.

Materia: Design

Tratamiento del radio

Profesor: Tomás Maldonado

Alumno: Heinrich Roth

Dp_054.037 FBS

i2/168 Ejercicio del primer año de estudios en el del Departamento de Diseño Industrial, curso 1962-1963.

Materia: Design

Ordenación. Distribución y equilibrio

Profesor: Tomás Maldonado

Alumno: Heinrich Roth

Dp_054.037 FBS

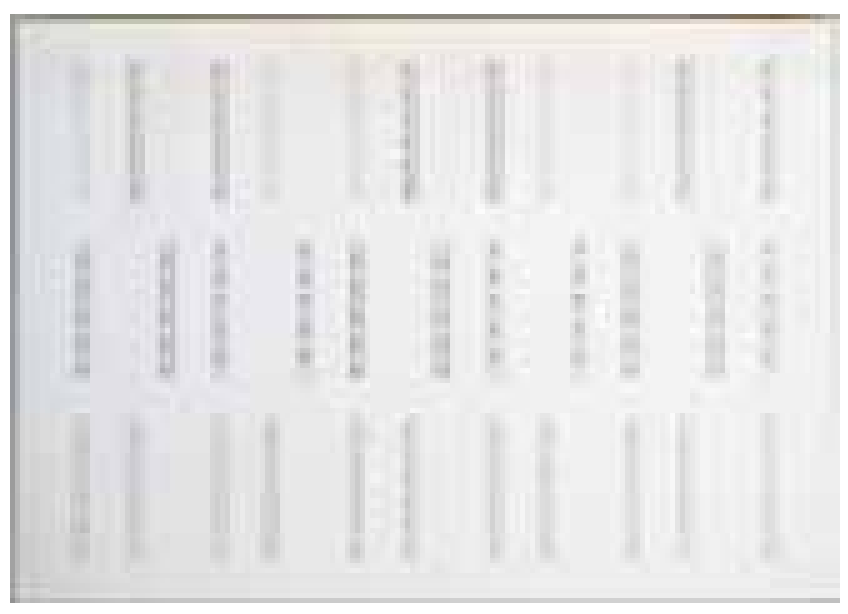

i2/167

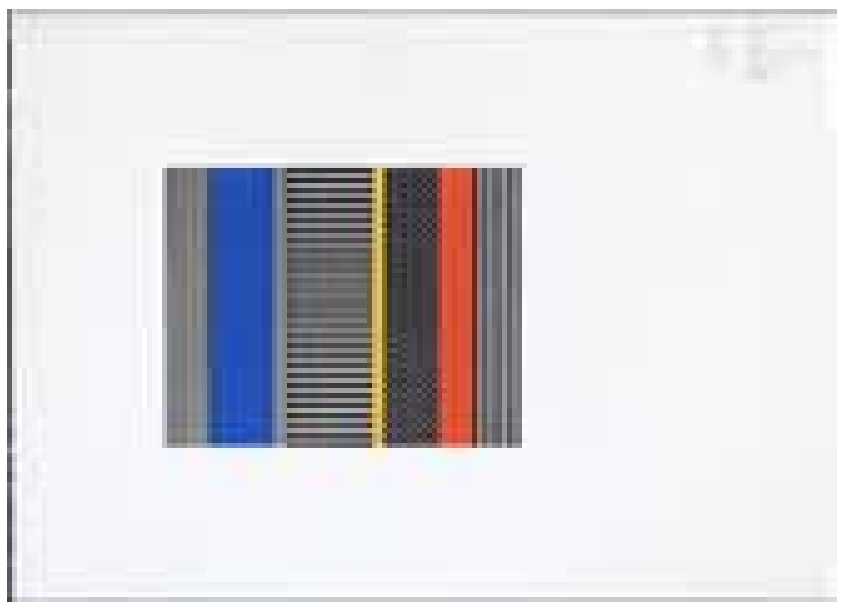

i2/169

i2/167 Ejercicio del primer año de estudios en el del Departamento de Comunicación Visual, curso 1964-1965.

Materia: Visuelle Methodik

Proximidad-ambigüedad-semejanza

Profesor: William S. Huff

Alumno: Gunther Elstner

Dp_088.019 FBS

i2/169 Ejercicio del primer año de estudios en el del Departamento de Diseño Industrial, curso 1962-1963.

Materia: Design

Distribución

Profesor: Tomás Maldonado

Alumno: Heinrich Roth

Dp_054.037 FBS 


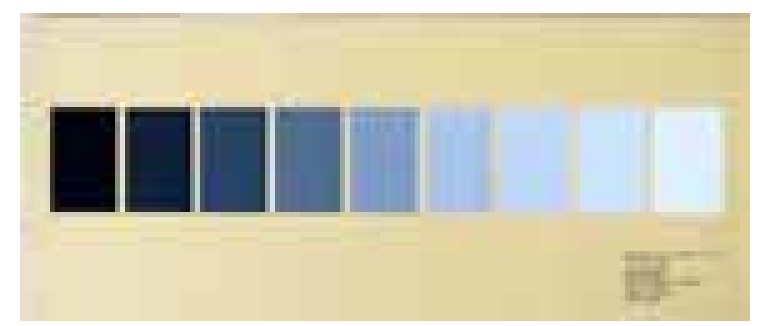

i2/170

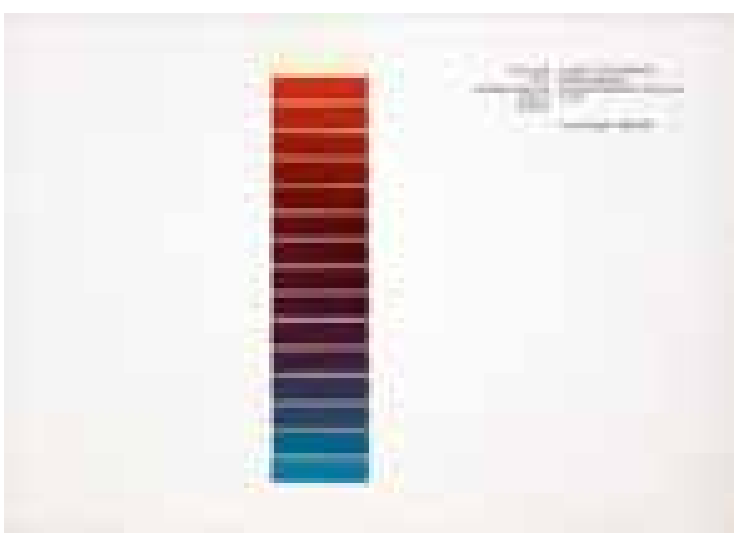

i2/171

i2/170 Ejercicio del primer año de estudios en el del Departamento de Construcción, curso 1961-1962.

Materia: Farbübungen

Escala de grises

Profesor: Friedrich Vondemberge-Gildewart

Alumno: Rolf Berner

Dp_005.042 FBS

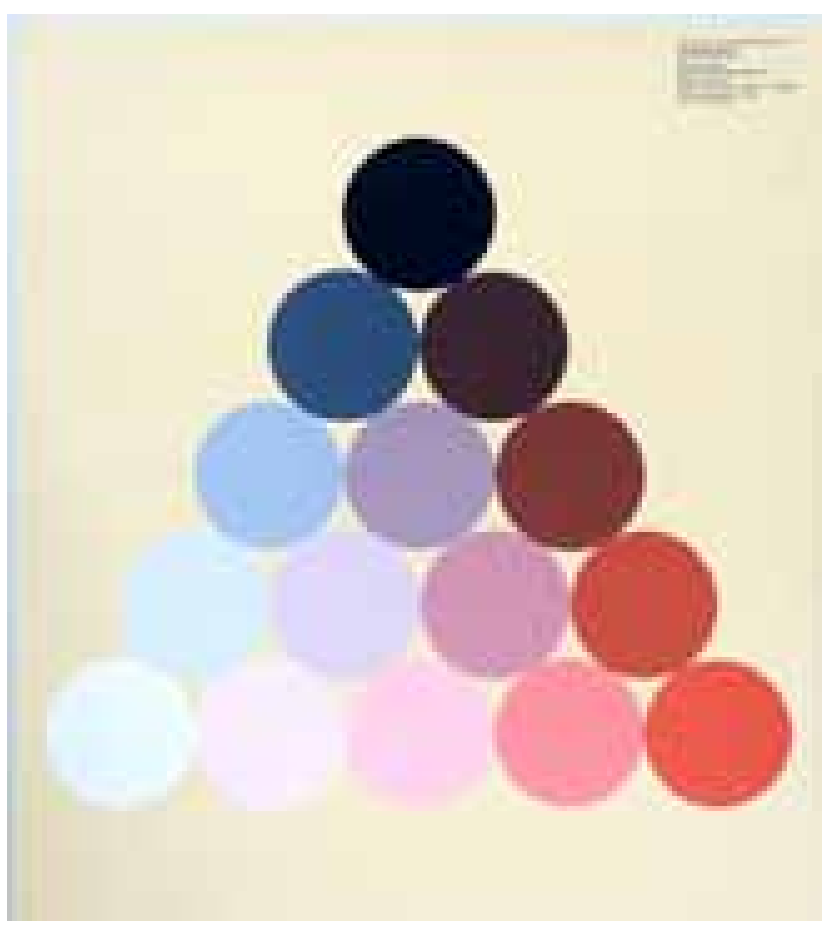

i2/172

i2/172 Ejercicio del primer año de estudios en el del Departamento de Construcción, curso 1961-1962.

Materia: Farbübungen

Escala horizontal-diagonal de tres colores dados: blanco, rojo, negro. Profesor: Friedrich Vondemberge-Gildewart

Alumno: Rolf Berner

Dp_005.042 FBS

i2/171 Ejercicio del primer año de estudios en el del Departamento de Comunicación Visual, curso 1965-1966.

Materia: Abteilungsarbeit

Mezcla de complementarios

Profesor: William S. Huff

Alumno: Ursula Wenzel

GS_030.002 FBS

\section{Arquetipos didácticos en la enseñanza del color}

La didáctica del color del Grundlehre pervive tras su desaparición. En los departamentos, sus valores se transmiten de dos modos: como temas de investigación o como modelos de ejercicios. Entre los temas de investigación, se profundiza en los aspectos visuales que se exploran mediante el color: la relación fondo figura, las leyes de la Gestalt, la ambigüedad en la percepción o la relatividad del uso del color.

Entre los modelos de ejercicios, las escalas de color continúan como herramienta para adiestrar la capacidad técnica en la interpolación de colores. (i2/710-i2/172). Por otro lado los "artefactos" de Maldonado se han instituido en modelos de referencia en los Departamentos. (i2/166-i2/169) Los ejercicios de parquetería se repiten en Comunicación Visual y Diseño Industrial y se transforman en ejercicios de aplicación del color en el departamento de Construcción. Significativamente, el legado bauhäusler regresa una década después a la enseñanza preparatoria del color. La "magia del color" de Albers reaparece en la HfG de la mano de William S. Huff tras la publicación de "Interacción del Color" en 1963. (i2/177-i2/180). 


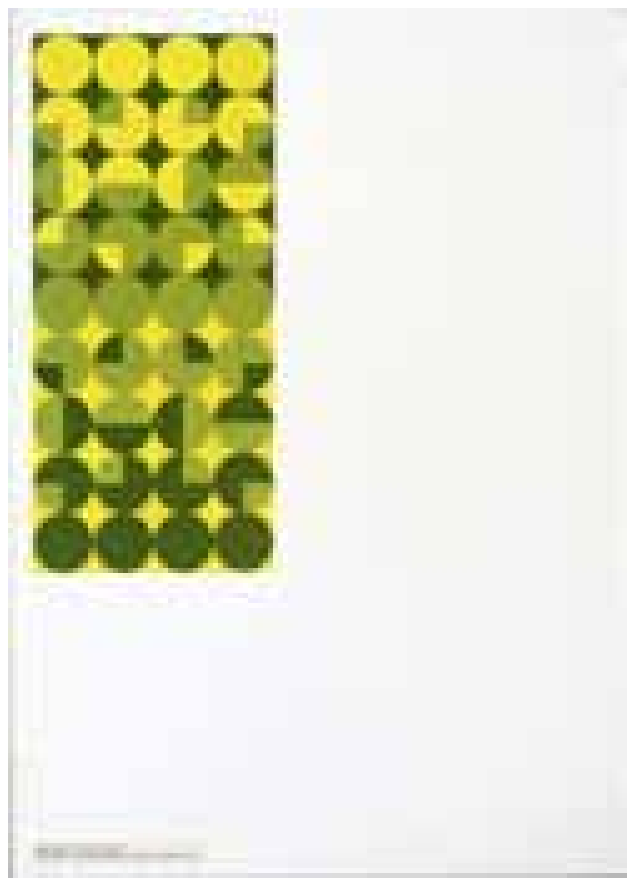

i2/173
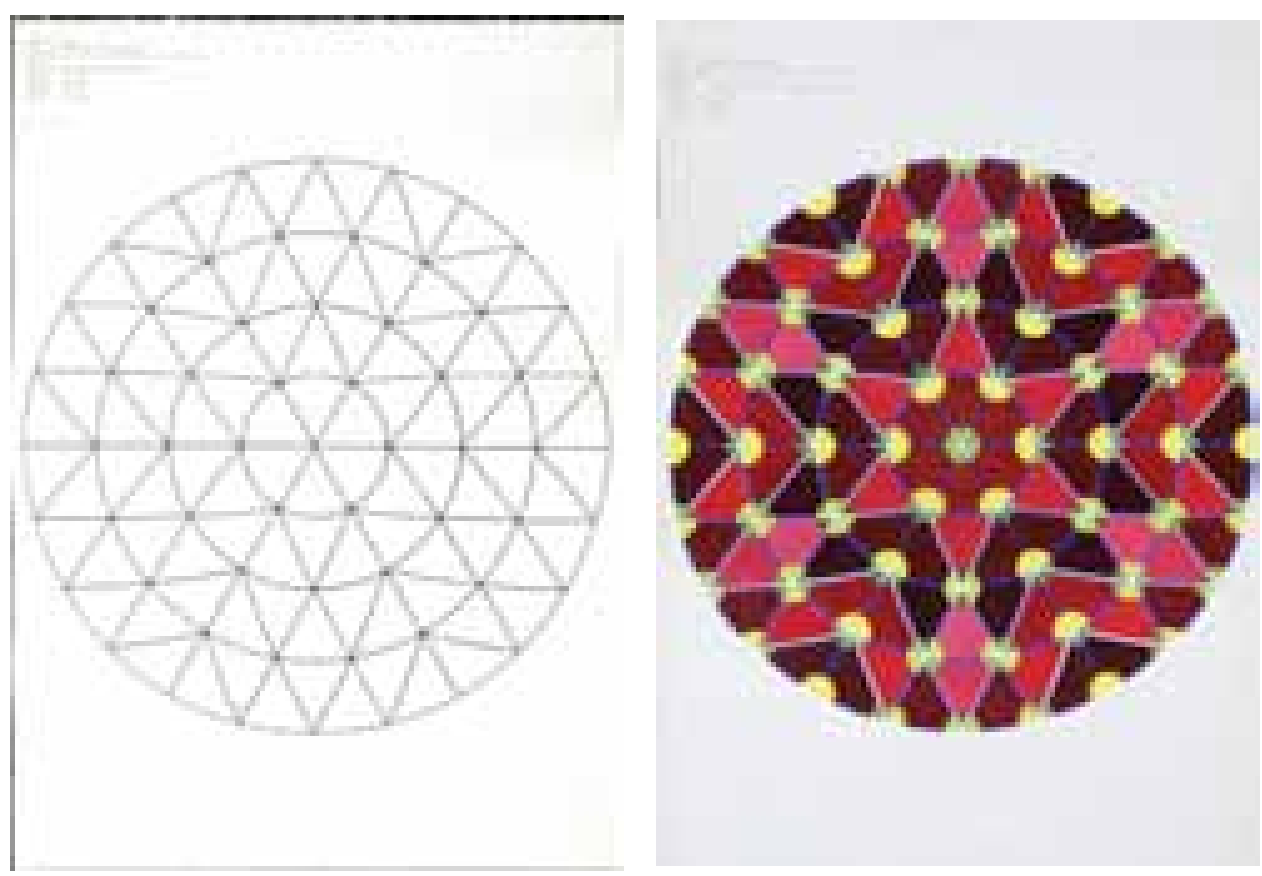

La relación fondo figura es el ejercicio que inicia el estudio del color como elemento visual. Se incluye en los leporellos o carpetas de tareas preparatorias en las que se elaboran series de ejercicios que exploran con el color, la forma, la textura y el encuadre en la imagen fotográfica.

i2/173, 174 Ejercicio del primer año de estudios en el del Departamento de Comunicación Visual, curso 19651966.

Relación fondo-figura. (Pasividad actividad del fondo y la figura)

Profesor: Herbert Lindinger Alumno: Urs Fanger

Dp_082.018 FBS

Ejercicio del primer año de estudios en el del Departamento de Construcción, curso 1962-1963. Estudios de aplicación como la relación entre forma y color en elementos con una ubicación análoga.

Materia: introducción al diseño

Color como interpretación de la división de un círculo.

i2/175 Lámina 1 División del círculo. i2/176 Lámina 2 interpretación a color de los segmentos, ángulos y superficies.

Profesor: Herbert Oh

Alumno: Isao Fukuwatari

Dp_120.003 FBS 

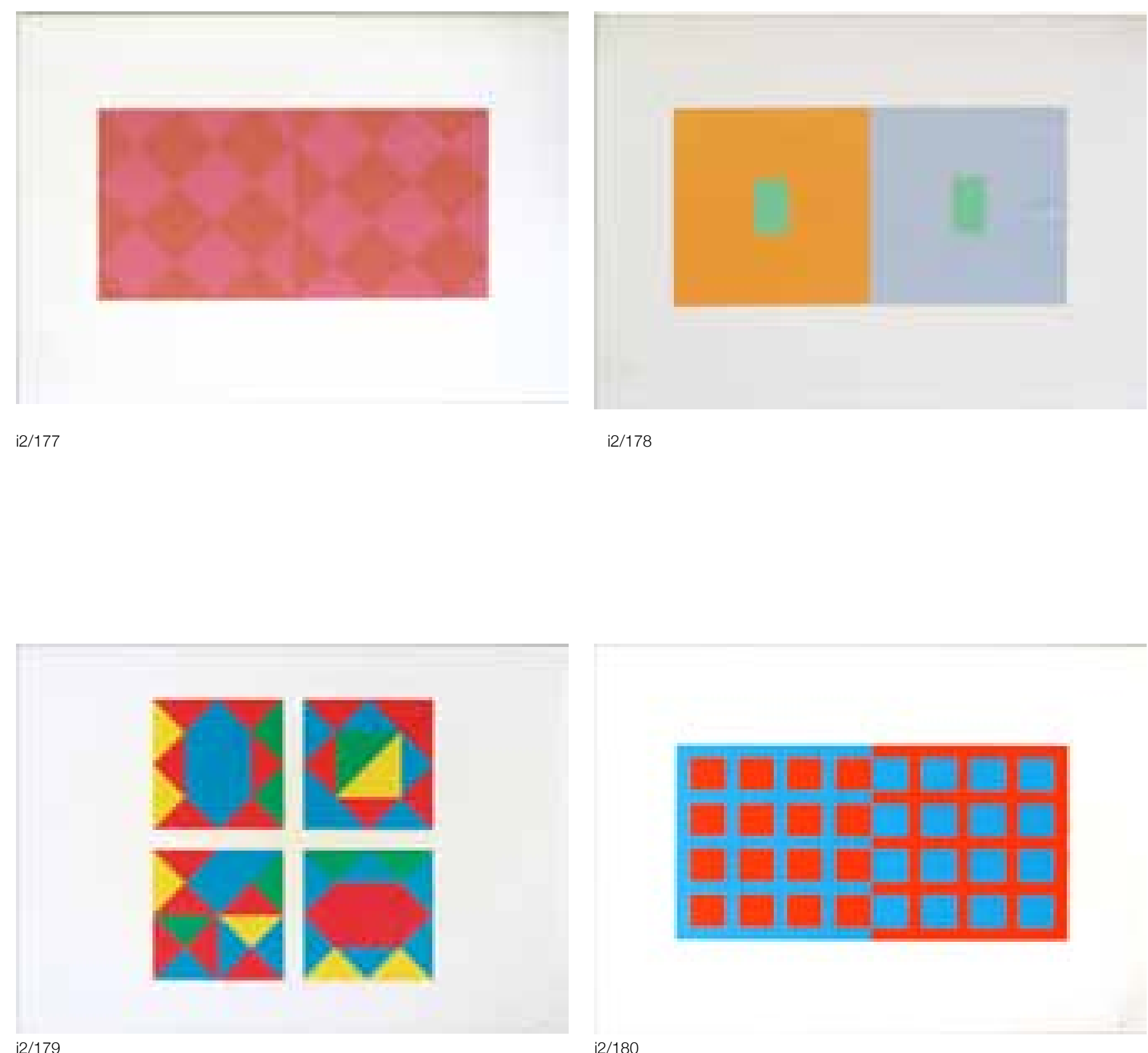

Ejercicio del primer año de estudios en el del Departamento de Comunicación Visual, curso 1964-1965. Estudios de color a partir de las láminas de "Interacción del color".

\section{i2/177 Dos colores, misma luminosidad}

i2/178 Tres colores parecen cuatro

2/179 Cuatro ambientes

i2/180 Vibración

\section{Materia: Visuelle Methodik}

Trabajos de color a partir de Albers

Profesor: William S. Huff

Alumno: Gunther Elstner

Dp_088.009 FBS 